$\operatorname{Argonne} \underset{\bigotimes}{\bigotimes}$

\title{
Annual Report of Monitoring at Morrill, Kansas, in 2010
}

\section{Environmental Science Division}


About Argonne National Laboratory

Argonne is a U.S. Department of Energy laboratory managed by UChicago Argonne, LLC under contract DE-AC02-06CH11357. The Laboratory's main facility is outside Chicago, at 9700 South Cass Avenue, Argonne, Illinois 60439. For information about Argonne and its pioneering science and technology programs, see www.anl.gov.

\section{Availability of This Report}

This report is available, at no cost, at http://www.osti.gov/bridge. It is also available on paper to the U.S. Department of Energy and its contractors, for a processing fee, from:

U.S. Department of Energy

Office of Scientific and Technical Information

P.O. Box 62

Oak Ridge, TN 37831-0062

phone (865) 576-8401

fax (865) 576-5728

reports@adonis.osti.gov

\section{Disclaimer}

This report was prepared as an account of work sponsored by an agency of the United States Government. Reference herein to any specific commercial product, process, or service by trade name, trademark, manufacturer, or otherwise, does not necessarily constitute or imply its endorsement, recommendation, or favoring by the United States Government or any agency thereof. The views and opinions of document authors expressed herein do not necessarily state or reflect those of the United States Government or any agency thereof, Argonne National Laboratory, or UChicago Argonne, LLC. 


\section{Annual Report of Monitoring at Morrill, Kansas, in 2010}

by

Applied Geosciences and Environmental Management Section

Environmental Science Division, Argonne National Laboratory

June 2011

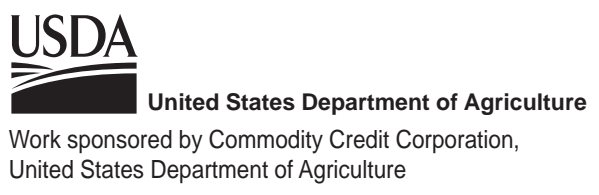




\section{Contents}

Notation.

1 Introduction and Background .............................................................................. 1-1

2 Sample Collection and Analysis Activities.......................................................................... 2- 2-1

2.1 Measurement of Groundwater Levels.............................................................. 2-1

2.2 Monitoring Well and Private Well Sampling and Analyses .................................. 2-1

2.3 Surface Water and Sediment Sampling and Analyses ........................................... 2-2

2.4 Vegetation Sampling and Analyses .................................................................... 2-3

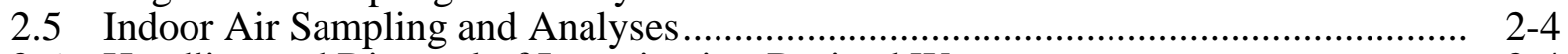

2.6 Handling and Disposal of Investigation-Derived Waste ...................................... 2-4

2.7 Quality Control for Sample Collection, Handling, and Analysis ........................... 2-5

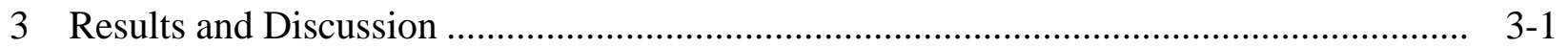

3.1 Groundwater Level Data......................................................................... $3-1$

3.2 Groundwater Analysis Results............................................................................ 3-2

3.3 Surface Water and Sediment Analysis Results ................................................... 3-3

3.4 Vegetation Analysis Results and Observations .................................................. $3-3$

3.5 Indoor Air Analysis Results........................................................................... 3-4

3.6 Comparison of Analytical Results for Samples Collected after Low-Flow Purging and after Purging of Three Well Volumes ............................................. $3-4$

4 Conclusions and Recommendations ................................................................ $4-1$

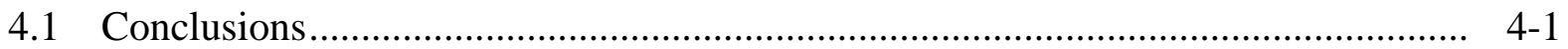

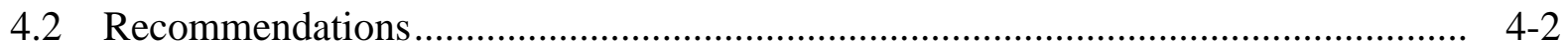

5 References .............................................................................................. $5-1$

Appendix A: Sampling Activities at Morrill in 2010 ……….............................................. A-1

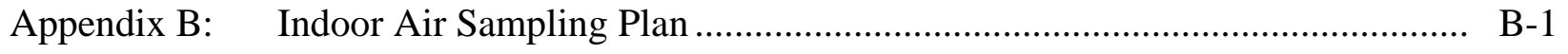

Appendix C: $\quad$ Results from AGEM Laboratory for Dual Analyses of Samples Collected at Morrill in 2010 and for Quality Control Samples ................... C-1

Supplement 1: Waste Characterization Data.................................................................. on CD

Supplement 2: Automatically Recorded Water Level Data..............................................on CD

Supplement 3: Sample Documentation from TestAmerica Laboratories, Inc., for Groundwater Verification Samples on CD

Supplement 4: Sample Documentation from TestAmerica Laboratories, Inc., for Indoor Air Samples Samples 


\section{Figures}

$1.1 \quad$ Monitoring network at Morrill, as of September 2010 ......................................... 1-4

2.1 Locations of surface water and creek bed sediment sampling along

Terrapin Creek at Morrill in September 2010.

2.2 Locations of native vegetation sampling downgradient of the former CCC/USDA facility and along Terrapin Creek at Morrill in July 2010

2.3 Locations of indoor air sampling downgradient of the former CCC/USDA facility in August 2010.

3.1a Potentiometric surface at Morrill, based on water levels measured manually on April 28, 2010

3.1b Potentiometric surface at Morrill, based on water levels measured manually on October 1, 2010.

3.2a Hydrographs summarizing results of long-term water level monitoring from January 1, 2009, to December 31, 2009.

3.2b Hydrographs summarizing results of long-term water level monitoring from January 1, 2010, to October 1, 2010

3.3a Carbon tetrachloride concentrations in groundwater at Morrill, April 2010

3.3b Carbon tetrachloride concentrations in groundwater at Morrill, September 2010 ....... 3-31

3.4a Carbon tetrachloride concentrations in groundwater at Morrill, April 2009

3.4b Carbon tetrachloride concentrations in groundwater at Morrill, September 2009 .....

3.5 Carbon tetrachloride concentrations in vegetation at Morrill, July 2010 3-34

3.6 Carbon tetrachloride concentrations in indoor air at Morrill, August 2010 3-35 


\section{Tables}

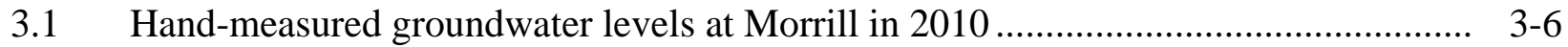

3.2 Results of analyses at the AGEM Laboratory for volatile organic compounds in groundwater samples collected at Morrill, October 2003 to September 2010

3.3 Field measurements for groundwater samples collected at Morrill, October 2003 to September 2010

3.4 Results of analyses at the AGEM Laboratory for volatile organic compounds in surface water and sediment samples collected at Morrill, March 2007 to September 2010

3.5 Results of analyses at the AGEM Laboratory for carbon tetrachloride and chloroform in vegetation samples collected at Morrill, October 2006 to July 2010

3.6 Analytical results for in indoor air samples collected at Morrill, August 2010............ 3-25

A.1 Sequence of sampling activities in April-September 2010 at Morrill, Kansas

C.1 Analytical results from the AGEM Laboratory for quality control samples collected to monitor sample collection and handling activities at Morrill in 2010

C.2 Analytical results from the AGEM Laboratory for dual analyses of samples collected at Morrill in 2010.

C.3 Analytical results from TestAmerica and the AGEM Laboratory for groundwater samples collected at Morrill in 2010 and submitted for verification analysis . 


\section{Notation}

AGEM Applied Geosciences and Environmental Management

AMSL above mean sea level

BGL below ground level

${ }^{\circ} \mathrm{C} \quad$ degree(s) Celsius

CCC Commodity Credit Corporation

CD compact disc

DOE U.S. Department of Energy

DO dissolved oxygen

EPA U.S. Environmental Protection Agency

$\mathrm{ft} \quad$ foot (feet)

gal gallon(s)

hr hour(s)

in. inch(es)

KDHE Kansas Department of Health and Environment

L liter(s)

$\mu \mathrm{g} / \mathrm{kg} \quad$ microgram(s) per kilogram

$\mu \mathrm{g} / \mathrm{L} \quad$ microgram(s) per liter

$\mu \mathrm{S} / \mathrm{cm} \quad$ microsiemen(s) per centimeter

$\mathrm{mg} / \mathrm{L} \quad$ milligram(s) per liter

mi mile(s)

min minute(s)

$\mathrm{mV} \quad$ millivolt(s)

ORP oxidation-reduction potential

RPD relative percent difference

SOP standard operating procedure

TOC top of casing

USDA U.S. Department of Agriculture

VOC volatile organic compound 


\section{Annual Report of Monitoring at Morrill, Kansas, in 2010}

\section{Introduction and Background}

Carbon tetrachloride contamination in groundwater at Morrill, Kansas, was initially identified in 1985 during statewide testing of public water supply wells for volatile organic compounds (VOCs). High levels of nitrate were also present in the wells. The city of Morrill is located in Brown County in the northeastern corner of the state, about $7 \mathrm{mi}$ east of Sabetha. The population of Morrill as of the 2000 census was approximately 277. All residents of Morrill now obtain their drinking water from the Sabetha municipal water system via a pipeline constructed in 1991. Starting in 1922, eight different public wells formerly served the Morrill municipal system at some time. Because of poor water quality, including high nitrate levels attributed to numerous animal feeding operations in the vicinity and application of fertilizer on agricultural lands, use of the local groundwater from any public well for municipal supply purposes was terminated in 1991 in favor of obtaining water from the Sabetha municipal water system.

Investigations of the carbon tetrachloride and nitrate contamination by the Kansas Department of Health and Environment (KDHE) in 1989, 1994, and 1996 (KDHE 1989; GeoCore 1994a-e, 1996) identified a localized plume of carbon tetrachloride in groundwater extending downgradient from a grain storage facility located in the northwestern section of Morrill. The facility was formerly operated by the Commodity Credit Corporation (CCC), an agency of the U.S. Department of Agriculture (USDA), from 1950 to 1971. Since termination of the CCC/USDA grain storage operations in 1971, the property and existing grain bins have been used for private grain storage up to the present time. Prior to 1986, commercial grain fumigants were commonly used by the CCC/USDA, as well as private and commercial grain storage operations, to preserve grain.

Because the identified carbon tetrachloride contamination could in part be linked to historical use of carbon tetrachloride-based fumigants at its former facility, in 2003 the CCC/USDA assumed responsibility for the site investigation of the carbon tetrachloride contamination. The CCC/USDA involvement began with development and implementation of a work plan for a Phase I expedited site characterization (Argonne 2003). That investigation and subsequent investigations (Argonne 2004, 2005a) were performed by the Environmental Science Division of Argonne National Laboratory. Argonne is a nonprofit, multidisciplinary research center operated by UChicago Argonne, LLC, for the U.S. Department of Energy (DOE). The 
CCC/USDA has entered into an interagency agreement with DOE, under which Argonne continues to provide technical assistance to the CCC/USDA with environmental site characterization and remediation at its former grain storage facilities.

The initial investigation by the CCC/USDA in 2003 determined that soils at the former facility have not been impacted by grain fumigation activities. Neither carbon tetrachloride nor chloroform was detected in near-surface soils or in subsurface soils collected to bedrock or to a depth of $15 \mathrm{ft}$ below ground level (BGL). Therefore, no identifiable human health risk is associated with either carbon tetrachloride or chloroform in shallow soils, which additionally pose no further threat of contamination to groundwater. High carbon tetrachloride concentrations in groundwater (maximum $390 \mu \mathrm{g} / \mathrm{L}$ in a sample collected from monitoring well MW3S located on the former CCC/USDA property — in 1995) have declined significantly during longterm monitoring by the KDHE and currently by the CCC/USDA. Maximum levels within the plume of $<50 \mu \mathrm{g} / \mathrm{L}$ at present confirm that no continuing soil source remains at the former CCC/USDA facility. Nevertheless, carbon tetrachloride concentrations exceeding the KDHE Tier 2 risk-based screening level of $5.0 \mu \mathrm{g} / \mathrm{L}$ remain.

In September 2005, the CCC/USDA initiated periodic sampling of groundwater at Morrill, in accord with a monitoring program approved by the state (KDHE 2005), to monitor carbon tetrachloride concentrations in the groundwater.

Under the KDHE-approved monitoring plan (Argonne 2005b), groundwater was initially sampled twice yearly for a period of two years. The samples were analyzed for VOCs, as well as for selected geochemical parameters to aid in the evaluation of possible natural contaminant degradation (reductive dechlorination) processes in the subsurface environment. The data have been inconclusive overall, possibly because of the relatively low contaminant concentrations in the plume. Nevertheless, consistently low levels of dissolved oxygen (DO) and oxidationreduction potential (ORP) at monitoring well MW1D (in the deepest portion of the contaminated aquifer) and the presence of chloroform (the primary degradation product of carbon tetrachloride) suggest that some degree of reductive dechlorination is occurring.

The analytical results for groundwater sampling events at Morrill from September 2005 to September 2009 were documented previously (Argonne 2006, 2007a,b, 2008a,b, 2009, 2010). Those results consistently demonstrated the presence of carbon tetrachloride contamination, at concentrations exceeding the KDHE Tier 2 risk-based screening level of $5.0 \mu \mathrm{g} / \mathrm{L}$ for this 
compound, in a groundwater plume extending generally southward from the former CCC/USDA facility, toward Terrapin Creek at the south edge of the town. Low concentrations $(\leq 2 \mu \mathrm{g} / \mathrm{L})$ of carbon tetrachloride have been detected persistently at monitoring well MW8S, on the bank of an intermittent tributary to Terrapin Creek. This observation suggests a possible risk of contamination of the surface waters of the creek. That concern is the regulatory driver for ongoing monitoring.

In light of the early findings, in 2006 the CCC/USDA recommended expansion of the approved monitoring program to include the collection and analysis of surface water samples along Terrapin Creek (Argonne 2007a). At the request of the KDHE (2007a), locations for both surface water and shallow sediment sampling were discussed with the KDHE in January 2007. An addendum to the existing monitoring plan (Appendix A in the report of 2009 monitoring [Argonne 2010]) and a standard operating procedure (SOP AGEM-15; Appendix B in Argonne 2010) for sediment sampling were submitted to the KDHE on the basis of these discussions and were subsequently approved (KDHE 2008b). To supplement the original scope of the monitoring, Argonne also samples natural vegetation at locations within the contaminant plume and along Terrapin Creek for analyses for VOCs.

In August 2010, indoor air sampling was conducted at seven residences, one church, and one business located within the contaminant plume to evaluate the potential for vapor intrusion. Carbon tetrachloride contamination was not detected.

The April and September 2010 groundwater sampling events reported here represent a continuation of the approved monitoring program, as requested by the KDHE (2007b). The groundwater sampling is presently conducted, in accord with the monitoring plan (Argonne 2005b) and the addendum to that plan (Appendix A in Argonne 2010), in a network of 12 monitoring wells and 3 private wells (Figure 1.1), at locations approved by the KDHE (2008b). In addition, since 2008, overflow from the Grimm irrigation well (installed in 2008 just south of Terrapin Creek) has also been sampled. 


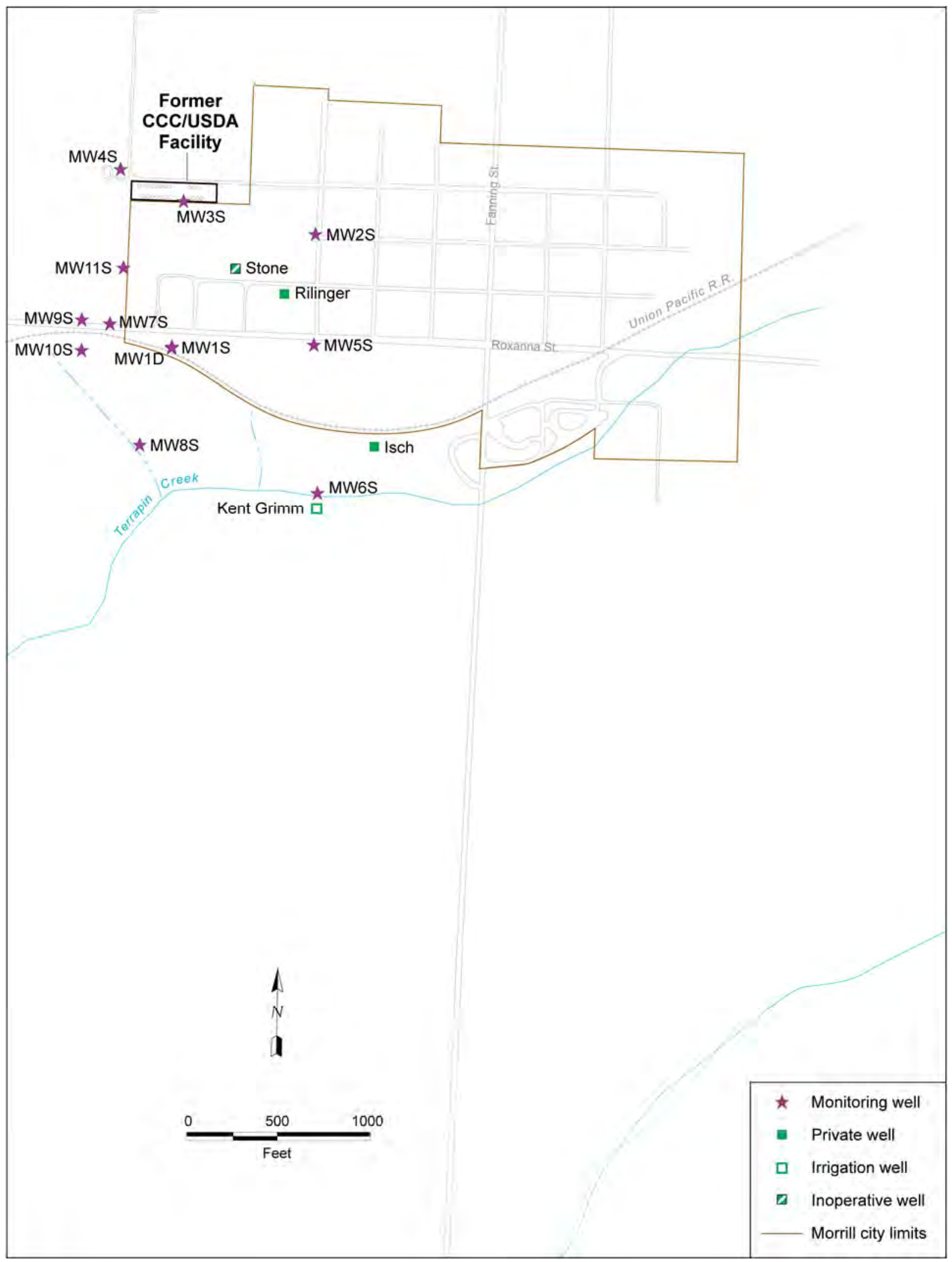

FIGURE 1.1 Monitoring network at Morrill, as of September 2010. 


\section{Sample Collection and Analysis Activities}

\subsection{Measurement of Groundwater Levels}

Data recorders currently installed in wells MW1S-MW4S and MW6S-MW8S are gathering long-term data on the groundwater elevation and gradient at Morrill in order to evaluate daily-to-seasonal variation. In addition, to calibrate the long-term data and to define the potentiometric surface, depths to groundwater and total well depths from the tops of the well casings are measured periodically (in conjunction with the data recorder downloads) and also during each groundwater sampling event, with an accuracy of $\pm 0.01 \mathrm{ft}$.

During the current reporting period, the data recorders were downloaded on April 28, 2010, and October 1, 2010. Water levels were measured manually in all monitoring wells on these dates, as well as during the sampling events on April 6-7, 2010, and September 22-23, 2010.

The groundwater level data are discussed in Section 3.1.

\subsection{Monitoring Well and Private Well Sampling and Analyses}

Monitoring wells MW1D and MW1S-MW11S and the Stone, Isch, and Rilinger private wells (Figure 1.1) were sampled on April 6-7, 2010, and September 22-23, 2010.

Except as noted otherwise, samples were collected from monitoring wells by using a lowflow bladder pump. After measurement of water levels, each monitoring point was purged of a small volume, in accord with U.S. Environmental Protection Agency (EPA) procedure EPA/540/S-95/504 (Puls and Barcelona 1996; Yeskis and Zavala 2002) and the equipment manufacturers' instructions. Field measurements of temperature, $\mathrm{pH}$, conductivity, DO, and ORP were taken during purging until the measurements stabilized. Field measurements of iron(II) were made as outlined in the monitoring plan (Argonne 2005b), in accord with procedures in the Master Work Plan (Argonne 2002). Samples from the Isch and Rilinger private wells were collected after a 5-min purge with the dedicated pump. The sample from the Stone private well was collected after purging of the well by bailing. 
Prior sampling at MW1S, which is located near the center of the contaminant plume and is screened over a 40-ft interval, has indicated that a representative sample is not collected by the low-flow sampling procedure. During additional sampling in April 2010, low-flow samples were collected at three depths within the screen (at positions near the top, middle, and bottom of the screen). A sample was also collected after purging of three well volumes. This comparison sampling is discussed further in Section 3.5.

The sequence of activities during the 2010 sampling events is summarized in Appendix A, Table A.1.

Groundwater samples for VOCs analyses were collected in appropriate laboratory containers, labeled, packaged, and chilled to $4^{\circ} \mathrm{C}$ by placement in ice-filled coolers. The samples were shipped by an overnight delivery service to the Applied Geosciences and Environmental Management (AGEM) Laboratory at Argonne for VOCs analyses with EPA Method 524.2 (EPA 1995). Separate aliquots of selected samples (chosen in the field) were shipped to TestAmerica Laboratories, Inc., South Burlington, Vermont, for verification VOCs analysis.

The groundwater analysis results are presented and discussed in Section 3.2.

\subsection{Surface Water and Sediment Sampling and Analyses}

At the request of the KDHE (2007a), surface water samples and corresponding samples of the underlying shallow sediments in the creek bed are routinely collected for VOCs analyses at five locations along Terrapin Creek (Figure 2.1), as outlined in the monitoring plan addendum (Appendix A in Argonne 2010). The sampling was conducted in accord with procedures in the Master Work Plan (Argonne 2002) and SOP AGEM-15 (Appendix B in Argonne 2010). Surface water flow in Terrapin Creek south of Morrill originates at the outfall from an earthen dam and retention pond approximately $1,900 \mathrm{ft}$ southwest of the former CCC/USDA facility (Figure 2.1). Surface water and sediment sampling location SMB, which is directly downstream from this outfall, is believed to lie upgradient, or cross-gradient, to groundwater flow (and hence possible contaminant migration) from the vicinity of the former CCC/USDA facility. (See Section 3.1.) Sampling locations SM1-SM4 were selected to lie downgradient and downstream from the carbon tetrachloride detections previously identified at MW8S and elsewhere in the monitoring well network. 
Samples of surface water were collected in appropriate containers, labeled, preserved at $4^{\circ} \mathrm{C}$, and shipped by an overnight delivery service to the AGEM Laboratory for VOCs analyses with EPA Method 524.2 (EPA 1995). Samples of the shallow creek bed sediments were collected by scooping the materials directly into appropriate laboratory containers. The samples were labeled, preserved on dry ice, and shipped to the AGEM Laboratory for sample preparation and VOCs analyses with modified EPA Methods 5030B and 8260B.

The surface water and sediment analysis results are presented and discussed in Section 3.3.

\subsection{Vegetation Sampling and Analyses}

Vegetation sampling has been proven to provide an accurate indication of contaminants in near-surface groundwater; it is used at Morrill and at other CCC/USDA sites for tracking the movement of carbon tetrachloride plumes through the uptake of the contaminant into tree and vegetation tissue. The sampling at Morrill serves as an early warning for potential movement of carbon tetrachloride into Terrapin Creek, downgradient from the former CCC/USDA facility.

Vegetation samples are collected at locations within the contaminant plume and along Terrapin Creek and its tributaries south and southwest of the former CCC/USDA facility. Vegetation sampling locations, which have expanded over time, have been selected along the apparent direction of groundwater flow from the former facility. Vegetation samples were collected at 18 locations in July 2007 (Argonne 2008a), at 25 locations in July 2008 (Argonne 2009), and at 22 locations in August 2009 (Argonne 2010). In July 2010, during the current reporting period, branch tissue samples were collected at 42 locations from mature ash, cottonwood, elm, hackberry, juniper, maple, mulberry, oak, Osage orange, pear, pine, walnut, and willow trees.

Figure 2.2 illustrates the locations of the vegetation sampling conducted on July 27-28, 2010. The sequence of sampling activities, including descriptions of sample locations and identifications of the trees sampled, is summarized in Table A.1, Appendix A. Analytical results are presented and discussed in Section 3.4. 
The tree tissue samples were collected in appropriate laboratory containers, labeled, preserved on dry ice, and shipped to the AGEM Laboratory for carbon tetrachloride and chloroform analyses by a headspace technique based on a modification of EPA Method 5021 (http://www.epa.gov/epahome/index/; Alvarado and Rose 2004).

\subsection{Indoor Air Sampling and Analyses}

At the request of the KDHE (2009a,b), a work plan for indoor air sampling was submitted and approved (KDHE 2010b) in spring 2010. The scope of the plan involved the collection of indoor air samples in structures directly overlying or within $100 \mathrm{ft}$ laterally of the identified carbon tetrachloride plume emanating from the former CCC/USDA facility at Morrill. The depths to groundwater in this area are generally $40 \mathrm{ft}$ BGL or less. An ambient air sample was also collected.

The purpose of the indoor air sampling was to assess the risk to human health due to potential upward migration of vapors of carbon tetrachloride and its degradation products into potentially affected homes. The investigation of vapor intrusion was not a defined objective at the time of Argonne's previous investigations, which predated issuance of the KDHE's vapor intrusion guidance (KDHE 2007c).

The KDHE-approved work plan for the indoor air sampling is in Appendix B. The KDHE (2010a) allowed the use of its internal procedure BER-33 (KDHE 2000) for this sampling event and another at Everest, Kansas. Samples were collected in 24-hr canisters on August 11-12, 2010, and were analyzed for carbon tetrachloride and chloroform by EPA Method TO-15 at TestAmerica Laboratories, Inc., South Burlington, Vermont. Sampling for radon was also conducted by using sampling kits and analysis provided through the National Radon Program Services at Kansas State University. The locations of the seven homes, one church, and one business sampled are shown in Figure 2.3. The results are discussed in Section 3.5.

\subsection{Handling and Disposal of Investigation-Derived Waste}

The small volume of purge water generated as potentially contaminated investigationderived waste was containerized on-site. The accumulated purge water was sampled and analyzed for VOCs (including ethylene dibromide) and nitrates by a KDHE-certified laboratory 
(Pace Analytical Services, Lenexa, Kansas). The analytical results (Supplement 1, on the compact disc [CD] inside the back cover of this report) indicated no detectable concentrations of carbon tetrachloride, chloroform, ethylene dibromide, or nitrate. On December 17, 2010, the wastewater was received at the Sabetha municipal water treatment facility for disposal.

\subsection{Quality Control for Sample Collection, Handling, and Analysis}

The quality control/quality assurance procedures followed during the 2010 monitoring events are described in detail in the Master Work Plan (Argonne 2002) and SOP AGEM-15 (Appendix B in Argonne 2010). These procedures are summarized as follows:

- Sample collection and handling activities were monitored by the documentation of samples as they were collected and the use of chain-ofcustody forms and custody seals to ensure sample integrity during handling and shipment.

- Samples designated for VOCs analyses were received with custody seals intact and at the appropriate preservation temperature. All samples were analyzed within the required holding times.

- Quality control samples (a field blank, equipment rinsates, and trip blanks) collected to monitor sample collection and handling activities were free of carbon tetrachloride contamination. Method blanks used to monitor analytical methodologies were free of carbon tetrachloride and chloroform contamination. Analytical results for quality control samples collected to monitor sample-handling activities are in Appendix C, Table C.1.

- Groundwater, surface water, and sediment samples were analyzed for VOCs at the AGEM Laboratory with the purge-and-trap method on a gas chromatograph-mass spectrometer system. Calibration checks with each sample delivery group were within the required range of $\pm 20 \%$ of the standard. Surrogate standard determinations performed on samples and blanks were within the specified range of $80-120 \%$ for all samples, in either the initial analysis or a successful reanalysis. The low relative percent difference 
(RPD) values for duplicate analyses of selected samples (approximately 3\%) demonstrated consistency in the analytical methodology. Results for dual analyses at the AGEM Laboratory are in Appendix C, Table C.2.

- In accordance with the procedures defined in the Master Work Plan (Argonne 2002), the analyses of water samples at the AGEM Laboratory were verified by a second laboratory. Groundwater samples collected during each of the 2010 monitoring events were also submitted to TestAmerica for analysis according to the EPA's Contract Laboratory Program methodology. Complete analytical results for seven groundwater samples and a trip blank collected in April 2010 are in sample delivery group 136730 in Supplement 2 (on CD). Complete analytical results for five groundwater samples and one trip blank collected in September 2010 are in sample delivery group 200-1702 in Supplement 2 (on CD). The results are summarized in Appendix C, Table C.3. Good agreement is evident over the range of contaminant concentrations detected, with average RPD values of $13 \%$ for carbon tetrachloride and $8 \%$ for chloroform.

- Vegetation samples were analyzed for carbon tetrachloride and chloroform at the AGEM Laboratory by using a gas chromatograph with electron capture detection to achieve the low detection limits required. An 11-point calibration of the gas chromatograph system was established on the basis of the mass of known quantities of carbon tetrachloride and chloroform.

- Indoor air samples were analyzed for carbon tetrachloride and chloroform at TestAmerica with EPA Method TO-15. Initial and continuing calibration standards met the $30 \%$ difference criterion, and each of the analyses exhibited acceptable internal standard performance. Acceptable recovery of target analytes was achieved in a laboratory control sample. The method blank associated with the sample delivery group was free of contamination by the analytes of interest. 


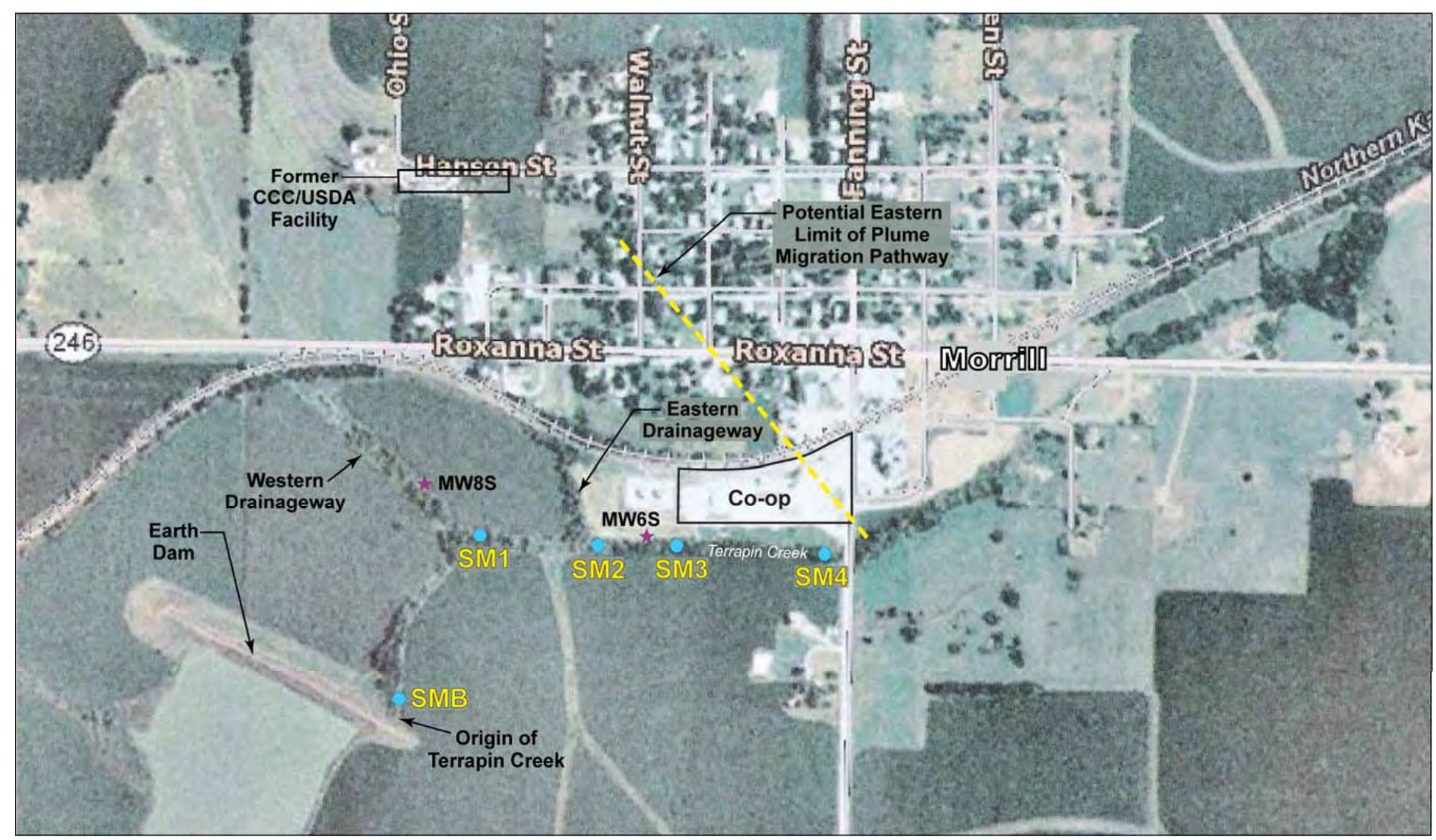

FIGURE 2.1 Locations of surface water and creek bed sediment sampling along Terrapin Creek at Morrill in September 2010. 


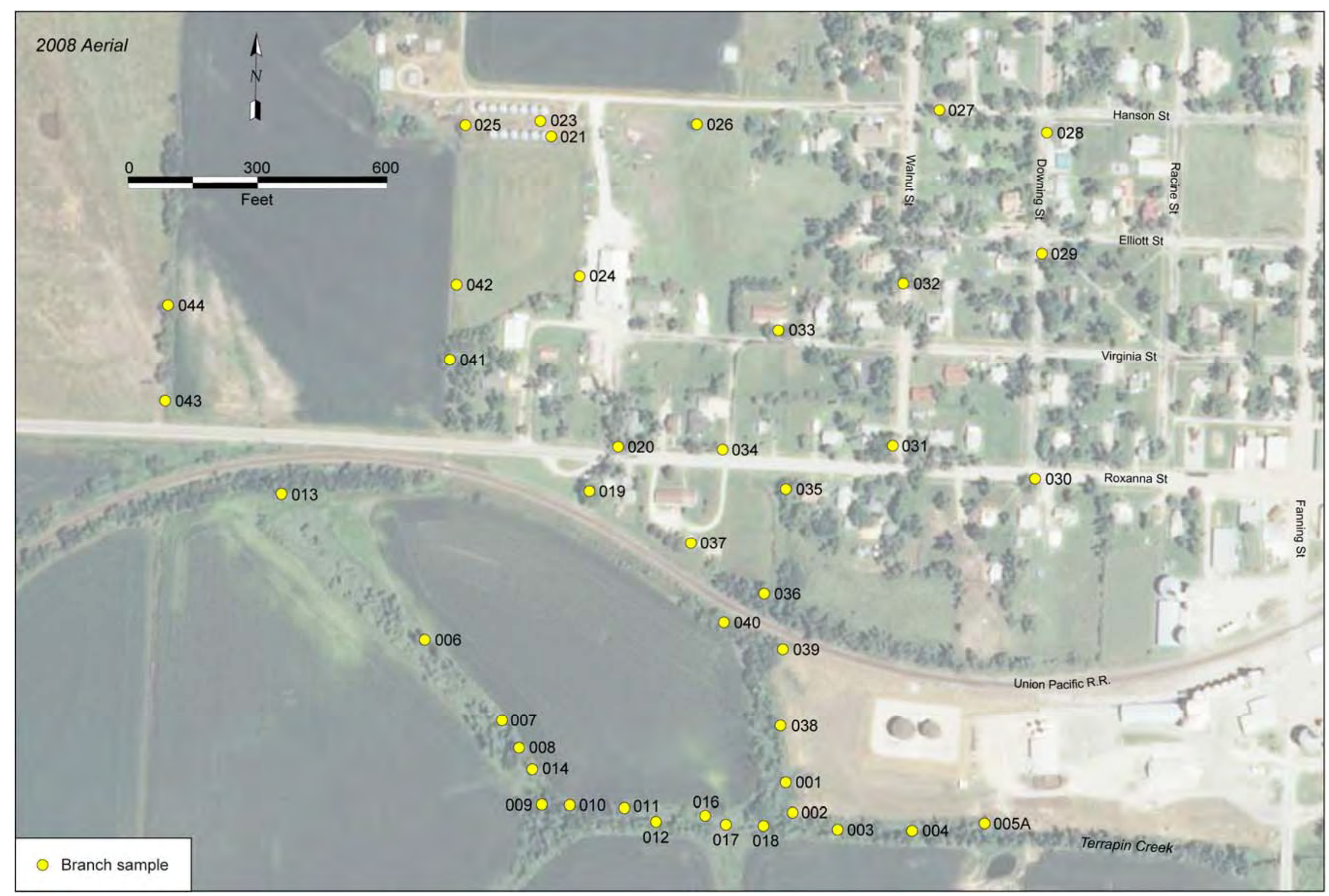

FIGURE 2.2 Locations of native vegetation sampling downgradient of the former CCC/USDA facility and along Terrapin Creek at Morrill in July 2010. 


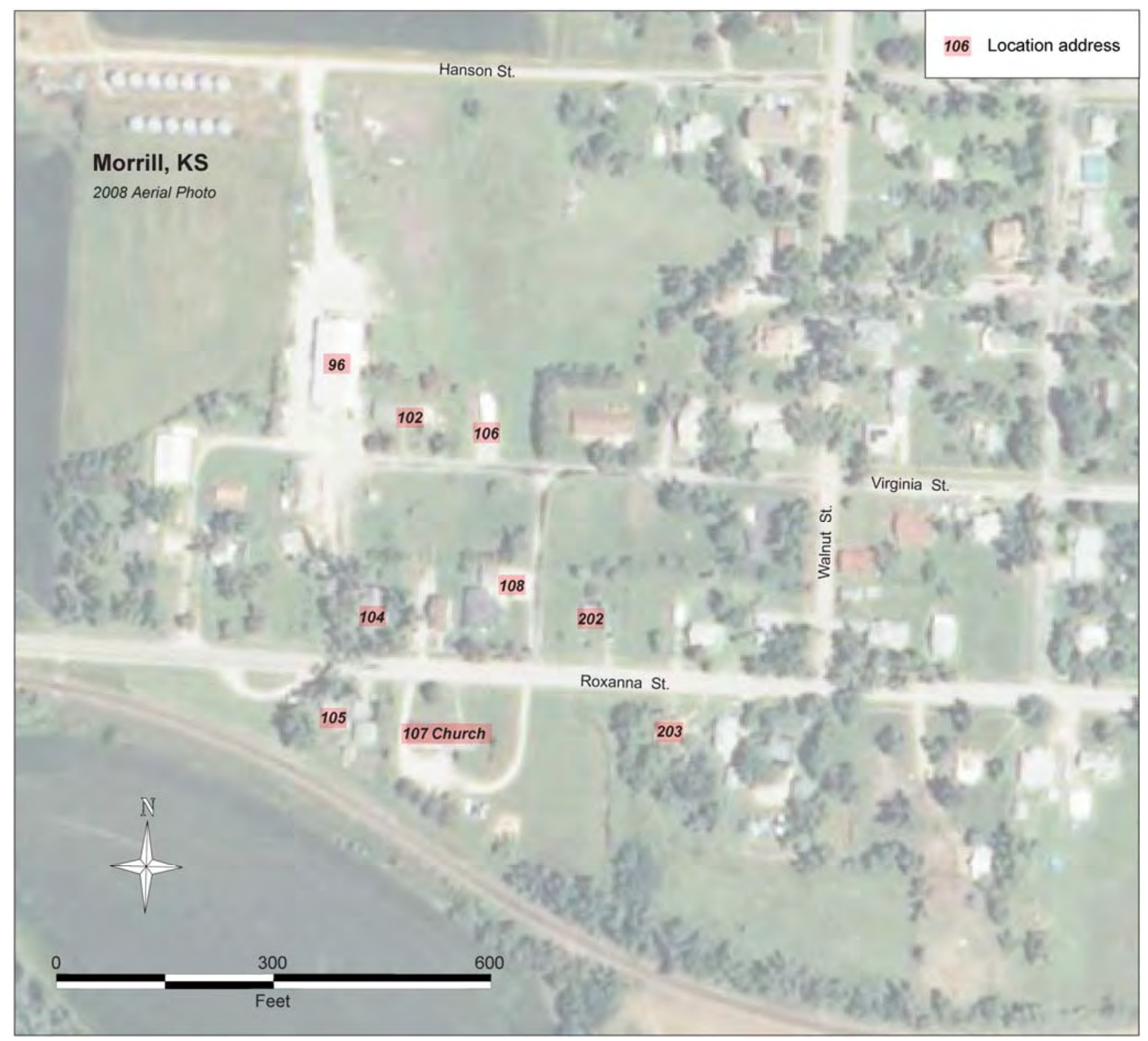

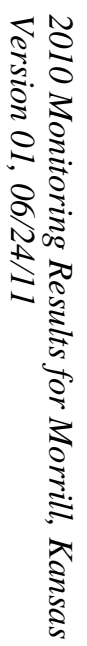

FIGURE 2.3 Locations of indoor air sampling downgradient of the former CCC/USDA facility in August 2010. 


\section{Results and Discussion}

\subsection{Groundwater Level Data}

Depths to groundwater were measured manually in all available monitoring wells on April 28, 2010, and October 1, 2010. These hand-measured water level data, along with handmeasured levels from the April 6-7, 2010, and September 22-23, 2010, sampling events, are in Table 3.1.

Estimates of the potentiometric surface at Morrill in previous monitoring reports have persistently shown low water levels at location MW11S, empirically suggesting the apparent presence of a groundwater "sink" southwest of the former facility and in the vicinity of this monitoring well. This observation is likely an artifact associated with the greater depth of completion of well MW11S in the aquifer unit and the local groundwater flow system (both horizontal and vertical) at this location, relative to nearby wells MW3S, MW4S, and MW7S. To eliminate this influence on the interpreted groundwater flow directions, the estimated potentiometric surfaces presented in Figures 3.1a-b for April 28, 2010, and October 1, 2010, respectively, were generated by excluding the anomalous water level measurements from well MW11S.

Groundwater flow during the early spring (April 28; Figure 3.1a) and the fall (October 1; Figure 3.1b) was predominantly to the south, from the vicinity of the former CCC/USDA facility toward Terrapin Creek. A slight shift toward the southwest in the immediate vicinity of the intermittent tributary that flows into Terrapin Creek (directly south of the former CCC/USDA facility) is apparent in conjunction with significantly higher groundwater levels in the potentiometric surface for April 28 (Figure 3.1a).

Hydrographs recorded in 2009-2010 for the Morrill monitoring wells (Figures 3.2a and 3.2b) illustrate that the observed May 2009 and March 2010 rises in water levels reflect seasonal responses to spring precipitation and recharge, followed by generally declining groundwater levels during the remainder of each year. Similar seasonal responses have been observed annually (to varying extents) throughout Argonne’s investigations at the Morrill site.

The hydrographs in Figures 3.2a and 3.2b for monitoring well MW6S are marked by a series of sharp downward "spikes" in the water level at this location during July and August of 
both 2009 and 2010. The observed events are believed to reflect transient drawdown in response to pumping of the Grimm irrigation well (location TD12), which was installed just south of the MW6S location in March 2008 (Argonne 2008b). Little or no distinct response to the pumping of this well is apparent at the other monitoring well locations; however, the operation of the Grimm well empirically coincides with the decline in water levels observed at all locations in the summer and fall of 2009 and 2010. A similar seasonal decline in water levels was also observed at Morrill in summer and fall 2007, in the absence of the Grimm well pumping, suggesting that spring precipitation and recharge represent the predominant factors affecting the local groundwater level patterns at Morrill.

\subsection{Groundwater Analysis Results}

The analytical data for VOCs in the groundwater samples collected in April and September 2010 are in Table 3.2, together with data for the previous sampling events conducted under the KDHE-approved monitoring plan (Argonne 2005b). The results of field measurements on the groundwater samples are in Table 3.3. The April and September 2010 data for carbon tetrachloride in groundwater are illustrated in Figures 3.3a and 3.3b, respectively. For comparison, the results of the groundwater sampling performed in April and September 2009 are in Figures 3.4a and 3.4b, respectively.

In April 2010 (Figure 3.3a), carbon tetrachloride was detected at 8 of the 15 monitoring locations, at concentrations ranging from $<1 \mu \mathrm{g} / \mathrm{L}$ at the Stone and Rilinger private wells to a maximum of $38 \mu \mathrm{g} / \mathrm{L}$ at well MW11S. Low levels of chloroform $(<1-1.2 \mu \mathrm{g} / \mathrm{L})$ were detected in four wells (Table 3.2).

In September 2010 (Figure 3.3b), carbon tetrachloride was again detected at 8 of the 15 monitoring locations, at concentrations ranging from $<1 \mu \mathrm{g} / \mathrm{L}$ (in the Stone private well) to a maximum of $47 \mu \mathrm{g} / \mathrm{L}$ at well MW3S. Low levels of chloroform $(<1 \mu \mathrm{g} / \mathrm{L}$ to a maximum of $9.4 \mu \mathrm{g} / \mathrm{L}$ at MW5S) were detected in six wells (Table 3.2).

The results in Table 3.2, Figures 3.3a,b, and Figures 3.4a,b indicate that no significant changes were observed in the concentrations or distribution of carbon tetrachloride in the groundwater at Morrill during the 2010 review period, or in comparison to the results of the spring and fall 2009 monitoring events. 


\subsection{Surface Water and Sediment Analysis Results}

Table 3.4 presents the results of VOCs analyses of the surface water and shallow sediment samples collected (at the request of the KDHE [2007a]) along Terrapin Creek. No carbon tetrachloride was detected in the surface water samples (locations shown in Figure 2.1) at an analytical method detection limit of $0.1 \mu \mathrm{g} / \mathrm{L}$. Similarly, no carbon tetrachloride was identified in the associated sediment samples at an analytical method detection limit of $1.0 \mu \mathrm{g} / \mathrm{kg}$. The 2010 results therefore indicate that the surface waters and underlying sediments of Terrapin Creek remain uncontaminated by carbon tetrachloride.

\subsection{Vegetation Analysis Results and Observations}

The July 2008 vegetation sampling event involved an expansion of the database to include locations at and directly downgradient from the former CCC/USDA facility (locations MR019 to MR024; Figure 2.2). Essentially the same locations were sampled in August 2009. The sampling area was expanded further in July 2010 to verify the assumed potential for identification of the plume extending from the former facility and toward Terrapin Creek, on the basis of evidence of contaminant uptake in vegetation. This proven methodology has been successful at other CCC/USDA sites.

Analytical data for carbon tetrachloride and chloroform in tree branch samples collected in July 2010 (and in previous years) are shown in Table 3.5. In the July 2010 sampling, trace concentrations of carbon tetrachloride were identified at 3 of 42 locations sampled (MR031, MR041, and MR044; Figure 3.5). The analytical method detection limit was $0.1 \mu \mathrm{g} / \mathrm{kg}$.

Sampling at Morrill and elsewhere has demonstrated that carbon tetrachloride can be taken up seasonally — at detectable levels - in tissues of trees whose roots intercept low concentrations of carbon tetrachloride in groundwater. We hypothesize that the low concentrations in groundwater along the contaminant plume extending toward Terrapin Creek are sufficient for uptake by the trees. The low-level detections in vegetation are consistent with the observation, at other sites, that vegetation sampling is a valuable early indicator of plume expansion, and they validate the assumption made in areal expansion of the vegetation sampling effort at Morrill. 


\subsection{Indoor Air Analysis Results}

Table 3.6 summarizes the analytical results for the indoor air samples collected at selected residences and buildings located downgradient of the former CCC/USDA grain storage facility. Carbon tetrachloride was not detected in any of the samples collected. Chloroform was detected at levels consistent with indoor air sources such as use of chlorinated water and household cleaning products. No evidence of upward migration of vapors from the low-level contaminant groundwater plume to indoor air is therefore indicated. The results for carbon tetrachloride are illustrated in Figure 3.6. Analytical results from TestAmerica for carbon tetrachloride and chloroform in indoor air samples are in Supplement 4 (on CD).

Indoor air samples were also analyzed for radon by using sampling kits and analysis provided through the National Radon Program Services at Kansas State University. Analytical results from the Kansas State University program are also in Supplement 4.

\subsection{Comparison of Analytical Results for Samples Collected after Low-Flow Purging and after Purging of Three Well Volumes}

At the request of the KDHE (2008a), selected wells were sampled in October 2008 by using both the low-flow purging technique and the three-well-volume purging technique to confirm the suitability of the low-flow method for groundwater sampling at Morrill. Of particular concern was the applicability of the low-flow method for the wells installed by the KDHE in 1995, with screen intervals of 30-40 ft. As reported previously (Argonne 2009), in October 2008 samples were collected by both methods from wells MW1S, MW2S, and MW3S (installed by the KDHE in 1995 with 30- to 40-ft screens), as well as from well MW11S (installed by Argonne in 2004 with a 15-ft screen). Result for all pairs except the MW1S samples compared favorably; the low-flow results for well MW1S were dramatically lower than three-volumepurge results. Low-flow results for MW1S in October 2008 were therefore considered nonrepresentative, and the value for the sample obtained after purging of three well volumes was honored instead (Argonne 2009). Sampling conducted in later years has continued this evaluation of the suitability of the low-flow sampling method.

In April 2010, monitoring well MW1S was sampled three times by using the low-flow method, with the intake pump positioned at three distinct depths - near the bottom, middle, and top of the screened interval. Carbon tetrachloride was not detected in any of the samples. For 
comparison, a sample was subsequently collected after purging of three well volumes. In that sample, carbon tetrachloride was detected at $21 \mu \mathrm{g} / \mathrm{L}$. The observed concentration differences between methods reflect hydraulic and/or contaminant flow properties that result in higher concentrations in three-well-volume samples than in low-flow samples from any of the depths sampled.

In September 2010, when water levels were significantly reduced across the site, sampling of well MW1S with the low-flow procedure (with the pump positioned near the middle of the screen) resulted in a carbon tetrachloride detection at $1.6 \mu \mathrm{g} / \mathrm{L}$. Further multi-depth lowflow or no-purge sampling could improve understanding of the contaminant and groundwater flow conditions and might suggest a more suitable low-flow sampling depth that would provide representative analytical results. 
TABLE 3.1 Hand-measured groundwater levels at Morrill in 2010.

\begin{tabular}{|c|c|c|c|c|c|c|c|c|c|}
\hline \multirow[b]{2}{*}{ Well } & \multirow[b]{2}{*}{$\begin{array}{l}\text { Top of Casing } \\
\text { Elevation } \\
\text { (ft AMSL) }\end{array}$} & \multicolumn{2}{|c|}{ April 6-7, 2010} & \multicolumn{2}{|c|}{ April 28, 2010} & \multicolumn{2}{|c|}{ September 22-23, 2010} & \multicolumn{2}{|c|}{ October 1, 2010} \\
\hline & & $\begin{array}{l}\text { Depth to } \\
\text { Water } \\
\text { (ft BGL) }\end{array}$ & $\begin{array}{c}\text { Groundwater } \\
\text { Elevation } \\
\text { (ft AMSL) }\end{array}$ & $\begin{array}{l}\text { Depth to } \\
\text { Water } \\
\text { (ft BGL) }\end{array}$ & $\begin{array}{c}\text { Groundwater } \\
\text { Elevation } \\
\text { (ft AMSL) }\end{array}$ & $\begin{array}{l}\text { Depth to } \\
\text { Water } \\
\text { (ft BGL) }\end{array}$ & $\begin{array}{l}\text { Groundwater } \\
\text { Elevation } \\
\text { (ft AMSL) }\end{array}$ & $\begin{array}{l}\text { Depth to } \\
\text { Water } \\
\text { (ft BGL) }\end{array}$ & $\begin{array}{c}\text { Groundwater } \\
\text { Elevation } \\
\text { (ft AMSL) }\end{array}$ \\
\hline MW1S & 1124.68 & 11.65 & 1113.03 & 16.09 & 1108.59 & 19.89 & 1104.79 & 23.05 & 1101.63 \\
\hline MW1D & 1124.63 & 24.70 & 1099.93 & 28.10 & 1096.53 & 27.92 & 1096.71 & 30.90 & 1093.73 \\
\hline MW3S & 1135.76 & 12.14 & 1123.62 & 16.36 & 1119.40 & 23.73 & 1112.03 & 24.61 & 1111.15 \\
\hline MW4S & 1143.61 & 21.80 & 1121.81 & 25.37 & 1118.24 & 33.15 & 1110.46 & 33.75 & 1109.86 \\
\hline MW5S & 1122.21 & 8.32 & 1113.89 & 10.39 & 1111.82 & 19.25 & 1102.96 & 20.16 & 1102.05 \\
\hline MW6S & 1090.97 & 6.15 & 1084.82 & 5.19 & 1085.78 & 5.53 & 1085.44 & 5.38 & 1085.59 \\
\hline MW7S & 1119.86 & 6.38 & 1113.48 & 8.30 & 1111.56 & 14.15 & 1105.71 & 14.63 & 1105.23 \\
\hline MW8S & 1098.53 & 1.10 & 1097.43 & 0.60 & 1097.93 & 2.53 & 1096.00 & 2.85 & 1095.68 \\
\hline MW9S & 1118.31 & 16.50 & 1101.81 & 16.96 & 1101.35 & 21.15 & 1097.16 & 21.23 & 1097.08 \\
\hline MW10S & 1110.78 & 7.50 & 1103.28 & 8.85 & 1101.93 & 11.69 & 1099.09 & 12.43 & 1098.35 \\
\hline MW11S & 1133.08 & 29.45 & 1103.63 & 30.51 & 1102.57 & 34.75 & 1098.33 & 25.63 & 1107.45 \\
\hline
\end{tabular}


TABLE 3.2 Results of analyses at the AGEM Laboratory for volatile organic compounds in groundwater samples collected at Morrill, October 2003 to September 2010. Shading indicates sample collection with the low-flow procedure.

\begin{tabular}{|c|c|c|c|c|c|c|c|c|c|c|c|c|}
\hline \multirow[b]{2}{*}{ Location } & \multirow{2}{*}{$\begin{array}{l}\text { Screen } \\
\text { Interval } \\
\text { (ft BGL) }\end{array}$} & \multirow[b]{2}{*}{$\begin{array}{l}\text { Sample } \\
\text { Date }\end{array}$} & \multirow{2}{*}{$\begin{array}{l}\text { Depth to } \\
\text { Water } \\
\text { (ft TOC) }^{\mathrm{a}}\end{array}$} & \multirow{2}{*}{$\begin{array}{c}\text { Depth of } \\
\text { well } \\
\text { (ft TOC) })^{a}\end{array}$} & \multirow[b]{2}{*}{$\begin{array}{l}\text { Volume } \\
\text { Purged }\end{array}$} & \multirow[b]{2}{*}{$\begin{array}{l}\text { Purge } \\
\text { Units }\end{array}$} & \multirow{2}{*}{$\begin{array}{c}\text { Pump } \\
\text { Intake } \\
\text { Position } \\
\text { (ft BGL) }\end{array}$} & \multicolumn{3}{|c|}{ Concentration $(\mu \mathrm{g} / \mathrm{L})$} & \multirow[b]{2}{*}{ Comment } & \multirow[b]{2}{*}{ Sample } \\
\hline & & & & & & & & $\begin{array}{c}\text { Carbon } \\
\text { Tetrachloride }\end{array}$ & Chloroform & $\begin{array}{c}\text { Methylene } \\
\text { Chloride }\end{array}$ & & \\
\hline MW1S & $11-51$ & $10 / 23 / 03$ & 30.36 & 54.04 & 70 & gal & - & 33 & 1.6 & $N D^{b}$ & & MRMW1S-W-16422 \\
\hline MW1S & $11-51$ & $6 / 2 / 04$ & 26.97 & 53.94 & 53 & gal & - & 19 & 0.9 & ND & & MRMW1S-W-16461 \\
\hline MW1S & $11-51$ & $9 / 13 / 05$ & 24.16 & 53.9 & 57 & gal & - & 35 & 1.7 & ND & & MRMW1S-W-19259 \\
\hline MW1S & $11-51$ & $3 / 22 / 06$ & 29 & 53.95 & 48 & gal & - & 40 & 1.8 & ND & & MRMW1S-W-20008 \\
\hline MW1S & $11-51$ & 9/20/06 & 26.82 & 53.97 & 55 & gal & - & 23 & $0.9 \mathrm{~J}^{\mathrm{c}}$ & ND & & MRMW1S-W-22495 \\
\hline MW1S & $11-51$ & $3 / 21 / 07$ & 25.8 & 53.98 & 55 & gal & - & 23 & 1.1 & ND & & MRMW1S-W-16488 \\
\hline MW1S & $11-51$ & $10 / 1 / 07$ & 21.65 & 53.95 & 63 & gal & - & 56 & 2.7 & ND & & MRMW1S-W-16595 \\
\hline MW1S & $11-51$ & $4 / 14 / 08$ & 16.2 & 54 & 5.5 & $\mathrm{~L}$ & - & $0.3 \mathrm{~J}$ & ND & ND & April monitoring. & MRMW1S-W-23230 \\
\hline MW1S & $11-51$ & $4 / 22 / 08$ & 16 & 54 & 6.25 & L & - & $0.2 \mathrm{~J}$ & ND & ND & $\begin{array}{l}\text { Confirm low carbon } \\
\text { tetrachloride. }\end{array}$ & MRMW1S-W-23259 \\
\hline MW1S & $11-51$ & $5 / 1 / 08$ & - & - & 3.24 & $\mathrm{~L}$ & 22 & ND & ND & ND & Top of screen. & MRMW1S-22-W-23275 \\
\hline MW1S & $11-51$ & $5 / 1 / 08$ & - & - & 3.24 & L & 27 & ND & ND & ND & Middle of screen. & MRMW1S-27-W-23276 \\
\hline MW1S & $11-51$ & $5 / 1 / 08$ & - & - & 4.25 & L & 48 & $0.3 \mathrm{~J}$ & ND & ND & Bottom of screen. & MRMW1S-48-W-23277 \\
\hline MW1S & $11-51$ & $10 / 20 / 08$ & 25.8 & 54 & 6 & L & 31 & $0.7 \mathrm{~J}$ & ND & ND & Low flow. & MRMW1S-W-27620 \\
\hline MW1S & $11-51$ & $10 / 21 / 08$ & - & - & - & - & - & 35 & 1.8 & ND & Full purge. & MRMW1S-W-27649 \\
\hline MW1S & $11-51$ & $4 / 24 / 09$ & 24.4 & 54 & 5 & $\mathrm{~L}$ & 39.2 & ND & ND & ND & & MRMW1S-W-27652 \\
\hline MW1S & $11-51$ & $9 / 3 / 09$ & 19 & 54 & 8 & L & 35 & ND & ND & ND & Low flow. & MRMW1S-W-29942 \\
\hline MW1S & $11-51$ & $9 / 4 / 09$ & 19.3 & 51.2 & 244 & L & 50 & 34 & 1.7 & ND & Three well volumes. & MRMW1S-W-29971 \\
\hline MW1S & $11-51$ & $4 / 7 / 10$ & 11.65 & 51.3 & 7 & L & 16.6 & ND & ND & ND & Top of screen. & MRMW1ST-W-29981 \\
\hline MW1S & $11-51$ & $4 / 7 / 10$ & 11.57 & 51.31 & 6 & L & 31.5 & ND & ND & ND & Middle of screen. & MRMW1SM-W-29980 \\
\hline MW1S & $11-51$ & $4 / 7 / 10$ & 11.65 & 51.31 & 6 & $\mathrm{~L}$ & 46.3 & ND & ND & ND & Bottom of screen. & MRMW1SB-W-29979 \\
\hline MW1S & $11-51$ & $4 / 7 / 10$ & 11.48 & 51.3 & 80 & gal & 49 & 21 & 1.2 & ND & Three well volumes. & MRMW1S3X-W-29982 \\
\hline MW1S & $11-51$ & $9 / 22 / 10$ & 19.89 & 54 & 10 & $\mathrm{~L}$ & 31 & 1.6 & ND & ND & Low flow. & MRMW1S-W-30010 \\
\hline MW1D & $63-88$ & $10 / 22 / 03$ & 28.39 & 88.5 & 92 & gal & - & ND & ND & ND & & MRMW1D-W-16421 \\
\hline MW1D & $63-88$ & $6 / 2 / 04$ & 26.82 & 88.6 & 140 & gal & - & ND & ND & ND & & MRMW1D-W-16458 \\
\hline MW1D & $63-88$ & $9 / 13 / 05$ & 23.72 & 88.6 & 200 & gal & - & ND & ND & ND & & MRMW1D-W-16518 \\
\hline MW1D & $63-88$ & $3 / 19 / 06$ & 26.85 & 88.6 & 112 & gal & _- & ND & ND & $0.4 \mathrm{~J} \mathrm{~B}^{\mathrm{d}}$ & & MRMW1D-W-19986 \\
\hline MW1D & $63-88$ & $9 / 20 / 06$ & 25.52 & 88.8 & 125 & gal & _- & ND & ND & ND & & MRMW1D-W-16532 \\
\hline MW1D & $63-88$ & $3 / 21 / 07$ & 25.79 & 88.8 & 125 & gal & _- & ND & ND & ND & & MRMW1D-W-16487 \\
\hline MW1D & $63-88$ & $10 / 1 / 07$ & 22.75 & 89.38 & 130 & gal & _- & ND & ND & ND & & MRMW1D-W-16596 \\
\hline MW1D & $63-88$ & $4 / 14 / 08$ & 29.51 & 89 & 6 & $\mathrm{~L}$ & - & ND & ND & ND & & MRMW1D-W-23231 \\
\hline MW1D & $63-88$ & $10 / 20 / 08$ & 30.4 & 89 & 7 & L & 75.5 & ND & ND & ND & & MRMW1D-W-27621 \\
\hline MW1D & $63-88$ & $4 / 24 / 09$ & 31 & 89 & 7 & L & 75.5 & ND & ND & ND & & MRMW1D-W-27653 \\
\hline MW1D & $63-88$ & $9 / 3 / 09$ & 27.05 & 89 & 6.5 & L & 75.5 & ND & ND & ND & & MRMW1D-W-29943 \\
\hline MW1D & $63-88$ & $4 / 6 / 10$ & 24.7 & 89 & 8.5 & L & 75.5 & ND & ND & ND & & MRMW1D-W-29983 \\
\hline MW1D & $63-88$ & $9 / 22 / 10$ & 27.92 & 89 & 9 & L & 75.5 & ND & ND & ND & & MRMW1D-W-30011 \\
\hline MW2S & $13-53$ & $10 / 22 / 03$ & 42.21 & 53.35 & Purc & $d$ dry, se & pled. & ND & ND & ND & & MRMW02-W-16419 \\
\hline MW2S & $13-53$ & $6 / 2 / 04$ & 37.44 & 53.3 & 31 & gal & - & ND & ND & ND & & MRMW2S-W-16459 \\
\hline MW2S & $13-53$ & $9 / 14 / 05$ & 33.68 & 53.33 & 38 & gal & - & ND & ND & ND & & MRMW2S-W-19264 \\
\hline MW2S & $13-53$ & $3 / 21 / 06$ & 40.87 & 53.32 & 27 & gal & - & ND & ND & ND & & MRMW2S-W-19992 \\
\hline MW2S & $13-53$ & $9 / 18 / 06$ & 36.53 & 53.3 & 28 & gal & - & ND & ND & ND & & MRMW2S-W-22488 \\
\hline MW2S & $13-53$ & $3 / 22 / 07$ & 35.77 & 53.3 & 35 & gal & - & ND & ND & ND & & MRMW2S-W-16559 \\
\hline
\end{tabular}


TABLE 3.2 (Cont.)

\begin{tabular}{|c|c|c|c|c|c|c|c|c|c|c|c|c|}
\hline \multirow[b]{2}{*}{ Location } & \multirow{2}{*}{$\begin{array}{l}\text { Screen } \\
\text { Interval } \\
\text { (ft BGL) }\end{array}$} & \multirow[b]{2}{*}{$\begin{array}{l}\text { Sample } \\
\text { Date }\end{array}$} & \multirow{2}{*}{$\begin{array}{l}\text { Depth to } \\
\text { Water } \\
\text { (ft TOC) })^{a}\end{array}$} & \multirow{2}{*}{$\begin{array}{l}\text { Depth of } \\
\text { well } \\
(\mathrm{ft} T O C)^{\mathrm{a}}\end{array}$} & \multirow[b]{2}{*}{$\begin{array}{l}\text { Volume } \\
\text { Purged }\end{array}$} & \multirow[b]{2}{*}{$\begin{array}{l}\text { Purge } \\
\text { Units }\end{array}$} & \multirow{2}{*}{$\begin{array}{l}\text { Pump } \\
\text { Intake } \\
\text { Position } \\
\text { (ft BGL) }\end{array}$} & \multicolumn{3}{|c|}{ Concentration $(\mu \mathrm{g} / \mathrm{L})$} & \multirow[b]{2}{*}{ Comment } & \multirow[b]{2}{*}{ Sample } \\
\hline & & & & & & & & $\begin{array}{c}\text { Carbon } \\
\text { Tetrachloride }\end{array}$ & Chloroform & $\begin{array}{l}\text { Methylene } \\
\text { Chloride }\end{array}$ & & \\
\hline MW5S & $15-55$ & 9/13/05 & 22.66 & 54.2 & 75 & gal & - & 6.3 & $0.2 \mathrm{~J}$ & ND & & MRMW5S-W-19260 \\
\hline MW5S & $15-55$ & $3 / 22 / 06$ & 28.64 & 54.51 & 50 & gal & - & 7.3 & $0.2 \mathrm{~J}$ & ND & & MRMW5S-W-19996 \\
\hline MW5S & $15-55$ & 9/20/06 & 25.43 & 54.63 & 52 & gal & - & 6.4 & $0.3 \mathrm{~J}$ & ND & & MRMW5S-W-22493 \\
\hline MW5S & $15-55$ & $3 / 22 / 07$ & 25.14 & 54.56 & 58 & gal & - & 6.5 & $0.4 \mathrm{~J}$ & ND & & MRMW5S-W-16569 \\
\hline MW5S & $15-55$ & $10 / 3 / 07$ & 19.55 & 54.65 & 68 & gal & - & 4.0 & $0.3 \mathrm{~J}$ & ND & & MRMW5S-W-16588 \\
\hline MW5S & $15-55$ & $4 / 14 / 08$ & 11.2 & 54.6 & 6 & $\mathrm{~L}$ & - & ND & ND & ND & April monitoring. & MRMW5S-W-23235 \\
\hline MW5S & $15-55$ & $4 / 23 / 08$ & 11.3 & 54.6 & 6.5 & L & - & ND & ND & ND & $\begin{array}{l}\text { Confirm low carbon } \\
\text { tetrachloride. }\end{array}$ & MRMW5S-W-23266 \\
\hline MW5S & $15-55$ & $5 / 1 / 08$ & - & - & 3.7 & L & 20 & ND & ND & ND & Vary pump intake. & MRMW5S-20-W-23272 \\
\hline MW5S & $15-55$ & $5 / 1 / 08$ & - & - & 3.4 & L & 28 & ND & ND & ND & Vary pump intake. & MRMW5S-28-W-23273 \\
\hline MW5S & $15-55$ & $5 / 1 / 08$ & - & - & 4 & L & 52 & ND & ND & ND & Vary pump intake. & MRMW5S-52-W-23274 \\
\hline MW5S & $15-55$ & $10 / 21 / 08$ & 22.5 & 54.6 & 7 & L & 35 & 1.7 & ND & ND & & MRMW5S-W-27625 \\
\hline MW5S & $15-55$ & $4 / 24 / 09$ & 22.1 & 54.6 & 5.5 & L & 38.35 & ND & ND & ND & & MRMW5S-W-27657 \\
\hline MW5S & $15-55$ & $9 / 3 / 09$ & 17.6 & 54.6 & 5.5 & L & 36.3 & ND & ND & ND & & MRMW5S-W-29947 \\
\hline MW5S & $15-55$ & $4 / 7 / 10$ & 8.32 & 54.5 & 5.5 & L & 35 & ND & ND & ND & & MRMW5S-W-29987 \\
\hline MW5S & $15-55$ & 9/22/10 & 19.25 & 55 & 6.5 & L & 35 & ND & 9.4 & ND & & MRMW5S-W-30015 \\
\hline MW6S & $10-25$ & $6 / 3 / 04$ & 3.34 & 26.9 & 45 & gal & - & ND & ND & ND & & MRMW6S-W-16465 \\
\hline MW6S & $10-25$ & 9/14/05 & 4.7 & 26.9 & 43 & gal & - & ND & ND & ND & & MRMW6S-W-19263 \\
\hline MW6S & $10-25$ & $3 / 20 / 06$ & 5.35 & 26.91 & 43 & gal & - & ND & ND & ND & & MRMW6S-W-19990 \\
\hline MW6S & $10-25$ & $9 / 18 / 06$ & 5.48 & 26.92 & 27 & gal & - & ND & ND & ND & & MRMW6S-W-22486 \\
\hline MW6S & $10-25$ & $3 / 21 / 07$ & 5.42 & 26.92 & 30 & gal & - & ND & ND & ND & & MRMW6S-W-16486 \\
\hline MW6S & $10-25$ & $10 / 2 / 07$ & 5 & 26.9 & 31 & gal & - & ND & ND & ND & & MRMW6S-W-16583 \\
\hline MW6S & $10-25$ & $4 / 15 / 08$ & 5.15 & 26.9 & 2.5 & L & - & ND & ND & ND & & MRMW6S-W-23236 \\
\hline MW6S & $10-25$ & $10 / 20 / 08$ & 5.7 & 26.9 & 5 & L & 17.5 & ND & ND & ND & & MRMW6S-W-27626 \\
\hline MW6S & $10-25$ & $4 / 24 / 09$ & 6.2 & 26.9 & 12 & L & 17.5 & ND & ND & ND & & MRMW6S-W-27658 \\
\hline MW6S & $10-25$ & 9/4/09 & 5.85 & 26.9 & 5.4 & $\mathrm{~L}$ & 17.5 & ND & ND & ND & & MRMW6S-W-29948 \\
\hline MW6S & $10-25$ & $4 / 6 / 10$ & 6.15 & 26.9 & 8 & L & 17.5 & ND & ND & ND & & MRMW6S-W-29988 \\
\hline MW6S & $10-25$ & $9 / 22 / 10$ & 5.53 & 26.9 & 8.75 & L & 17.5 & ND & ND & ND & & MRMW6S-W-30016 \\
\hline MW7S & $20-45$ & $6 / 3 / 04$ & 26.68 & 46.98 & 40 & gal & - & 18 & ND & ND & & MRMW7S-W-16466 \\
\hline MW7S & $20-45$ & 9/12/05 & 17.57 & 46.94 & 55 & gal & - & 43 & 1.1 & ND & & MRMW7S-W-19258 \\
\hline MW7S & $20-45$ & $3 / 22 / 06$ & 22.45 & 46.98 & 48 & gal & - & 21 & $0.4 \mathrm{~J}$ & ND & & MRMW7S-W-20000 \\
\hline MW7S & $20-45$ & $9 / 19 / 06$ & 20.94 & 47.02 & 56 & gal & - & 38 & $0.7 \mathrm{~J}$ & ND & & MRMW7S-W-22490 \\
\hline MW7S & $20-45$ & 3/20/07 & 18.01 & 47.02 & 50 & gal & - & 16 & $0.4 \mathrm{~J}$ & ND & & MRMW7S-W-16481 \\
\hline MW7S & $20-45$ & $10 / 1 / 07$ & 12.4 & 47 & 70 & gal & - & 8.1 & $0.2 \mathrm{~J}$ & ND & & MRMW7S-W-16581 \\
\hline MW7S & $20-45$ & $4 / 14 / 08$ & 7.72 & 47 & 1.82 & $\mathrm{~L}$ & - & 10 & $0.3 \mathrm{~J}$ & ND & & MRMW7S-W-23237 \\
\hline MW7S & $20-45$ & $4 / 23 / 08$ & 7.8 & 47 & 11 & L & - & 8.3 & $0.2 \mathrm{~J}$ & ND & & MRMW7S-W-23265 \\
\hline MW7S & $20-45$ & $10 / 20 / 08$ & 17.2 & 47 & 6.3 & L & 32.5 & 7.9 & ND & ND & & MRMW7S-W-27627 \\
\hline MW7S & $20-45$ & $4 / 23 / 09$ & 16.7 & 47 & 7 & L & 32.5 & 9.5 & ND & ND & & MRMW7S-W-27659 \\
\hline MW7S & $20-45$ & $9 / 3 / 09$ & 13.8 & 47 & 9 & L & 32.5 & 8.0 & ND & ND & & MRMW7S-W-29949 \\
\hline MW7S & $20-45$ & $4 / 6 / 10$ & 6.38 & 47 & 5.4 & L & 32.5 & 15 & $0.4 \mathrm{~J}$ & ND & & MRMW7S-W-29989 \\
\hline MW7S & $20-45$ & $9 / 23 / 10$ & 14.15 & 45 & 6 & L & 32.5 & 6.6 & ND & ND & & MRMW7S-W-30017 \\
\hline MW8S & $10-25$ & $6 / 3 / 04$ & 3.7 & 26.75 & 45 & gal & - & ND & ND & ND & & MRMW8S-W-16464 \\
\hline MW8S & $10-25$ & 9/14/05 & 4.02 & 26.82 & 57 & gal & - & $0.9 \mathrm{~J}$ & ND & ND & & MRMW8S-W-19265 \\
\hline MW8S & $10-25$ & 3/20/06 & 4.57 & 26.41 & 43 & gal & - & $0.6 \mathrm{~J}$ & ND & $0.4 \mathrm{~J} \mathrm{~B}$ & & MRMW8S-W-19991 \\
\hline
\end{tabular}


TABLE 3.2 (Cont.)

\begin{tabular}{|c|c|c|c|c|c|c|c|c|c|c|c|c|}
\hline \multirow[b]{2}{*}{ Location } & \multirow{2}{*}{$\begin{array}{l}\text { Screen } \\
\text { Interval } \\
\text { (ft BGL) }\end{array}$} & \multirow{2}{*}{$\begin{array}{l}\text { Sample } \\
\text { Date }\end{array}$} & \multirow{2}{*}{$\begin{array}{l}\text { Depth to } \\
\text { Water } \\
\text { (ft TOC) }^{\mathrm{a}}\end{array}$} & \multirow{2}{*}{$\begin{array}{l}\text { Depth of } \\
\text { well } \\
\text { (ft TOC) }^{\mathrm{a}}\end{array}$} & \multirow{2}{*}{$\begin{array}{l}\text { Volume } \\
\text { Purged }\end{array}$} & \multirow{2}{*}{$\begin{array}{l}\text { Purge } \\
\text { Units }\end{array}$} & \multirow{2}{*}{$\begin{array}{l}\text { Pump } \\
\text { Intake } \\
\text { Position } \\
\text { (ft BGL) }\end{array}$} & \multicolumn{3}{|c|}{ Concentration $(\mu \mathrm{g} / \mathrm{L})$} & \multirow[b]{2}{*}{ Comment } & \multirow[b]{2}{*}{ Sample } \\
\hline & & & & & & & & Tetrachloride & Chloroform & Chloride & & \\
\hline MW8S & $10-25$ & 9/19/06 & 4.76 & 26.83 & 45 & gal & - & 1.3 & ND & ND & & MRMW8S-W-22492 \\
\hline MW8S & $10-25$ & 3/20/07 & 2.63 & 26.82 & 49 & gal & - & $0.6 \mathrm{~J}$ & ND & ND & & MRMW8S-W-16483 \\
\hline MW8S & $10-25$ & $10 / 2 / 07$ & 2.2 & 26.8 & 48 & gal & - & $0.8 \mathrm{~J}$ & ND & ND & & MRMW8S-W-16584 \\
\hline MW8S & $10-25$ & $4 / 15 / 08$ & 0.7 & 26.8 & 5.5 & L & - & 1.1 & ND & ND & & MRMW8S-W-23238 \\
\hline MW8S & $10-25$ & $10 / 20 / 08$ & 3.6 & 26.8 & 8 & $\bar{L}$ & 17.5 & 1.3 & ND & ND & & MRMW8S-W-27628 \\
\hline MW8S & $10-25$ & $4 / 23 / 09$ & 2.3 & 26.8 & 6 & L & 17.5 & ND & ND & ND & & MRMW8S-W-27660 \\
\hline MW8S & $10-25$ & $9 / 3 / 09$ & 2.9 & 26.8 & 8.5 & L & 17.5 & 1.9 & ND & ND & & MRMW8S-W-29950 \\
\hline MW8S & $10-25$ & $4 / 6 / 10$ & 1.1 & 26.8 & 8 & L & 17.5 & 1.7 & ND & ND & & MRMW8S-W-29990 \\
\hline MW8S & $10-25$ & $9 / 22 / 10$ & 2.53 & 26.8 & 9 & L & 17.5 & 1.6 & ND & ND & & MRMW8S-W-30018 \\
\hline MW9S & $38.83-53.83$ & $3 / 22 / 06$ & 20.2 & 58.62 & 20 & gal & - & ND & ND & ND & & MRMW9S-W-20004 \\
\hline MW9S & $38.83-53.83$ & $9 / 19 / 06$ & 18.87 & 59 & 22 & gal & - & ND & ND & ND & & MRMW9S-W-22494 \\
\hline MW9S & $38.83-53.83$ & $3 / 20 / 07$ & 16.69 & 59 & 22 & gal & - & ND & ND & ND & & MRMW9S-W-16480 \\
\hline MW9S & $38.83-53.83$ & $10 / 1 / 07$ & 14 & 58.6 & 23 & gal & - & ND & ND & ND & & MRMW9S-W-16582 \\
\hline MW9S & $38.83-53.83$ & $4 / 14 / 08$ & 16.58 & 58.63 & 2.29 & L & - & $0.8 \mathrm{~J}$ & ND & ND & & MRMW9S-W-23239 \\
\hline MW9S & $38.83-53.83$ & $10 / 20 / 08$ & 21.5 & 58.5 & 11 & L & 46.33 & 1.1 & ND & ND & & MRMW9S-W-27629 \\
\hline MW9S & $38.83-53.83$ & $4 / 23 / 09$ & 21.9 & 58.5 & 5.5 & L & 46.33 & 1.0 & ND & ND & & MRMW9S-W-27661 \\
\hline MW9S & $38.83-53.83$ & $9 / 4 / 09$ & 19.95 & 58.5 & 5 & L & 46.33 & 1.4 & ND & ND & & MRMW9S-W-29951 \\
\hline MW9S & $38.83-53.83$ & $4 / 6 / 10$ & 16.5 & 58.5 & 6 & L & 46.33 & 1.9 & ND & ND & & MRMW9S-W-29991 \\
\hline MW9S & $38.83-53.83$ & $9 / 22 / 10$ & 21.15 & 53.83 & 7 & L & 46.33 & 1.9 & ND & ND & & MRMW9S-W-30019 \\
\hline MW10S & $30-45$ & $3 / 21 / 06$ & 12.3 & 49.6 & 19 & gal & - & ND & ND & ND & & MRMW10S-W-19999 \\
\hline MW10S & $30-45$ & 9/18/06 & 11.08 & 49.61 & 20 & gal & - & ND & ND & ND & & MRMW10S-W-22489 \\
\hline MW10S & $30-45$ & $3 / 21 / 07$ & 10.77 & 49.61 & 20 & gal & - & ND & ND & ND & & MRMW10S-W-16485 \\
\hline MW10S & $30-45$ & $10 / 1 / 07$ & 6.95 & 49.65 & 20 & gal & - & ND & ND & ND & & MRMW10S-W-16593 \\
\hline MW10S & $30-45$ & $4 / 14 / 08$ & 9.82 & 49.7 & 1.9 & L & - & ND & ND & ND & & MRMW10S-W-23240 \\
\hline MW10S & $30-45$ & $10 / 20 / 08$ & 13.7 & 49.7 & 5.4 & L & 37.5 & ND & ND & ND & & MRMW10S-W-27630 \\
\hline MW10S & $30-45$ & $4 / 23 / 09$ & 13.6 & 45 & 7.5 & L & 37.5 & ND & ND & ND & & MRMW10S-W-27662 \\
\hline MW10S & $30-45$ & $9 / 3 / 09$ & 12 & 49.7 & 7.5 & L & 37.5 & ND & ND & ND & & MRMW10S-W-29952 \\
\hline MW10S & $30-45$ & $4 / 6 / 10$ & 7.5 & 49.7 & 9 & L & 37.5 & ND & ND & ND & & MRMW10S-W-29992 \\
\hline MW10S & $30-45$ & $9 / 22 / 10$ & 11.69 & 49.7 & 11 & L & 37.5 & ND & $0.3 \mathrm{~J}$ & ND & & MRMW10S-W-30020 \\
\hline MW11S & $53-68$ & 3/22/06 & 35.2 & 72.5 & 20 & gal & - & 39 & $0.9 \mathrm{~J}$ & ND & & MRMW11S-W-20001 \\
\hline MW11S & $53-68$ & 9/19/06 & 36 & 73.14 & 20 & gal & - & 53 & 1.0 & ND & & MRMW11S-W-22491 \\
\hline MW11S & $53-68$ & $3 / 20 / 07$ & 34.65 & 73.14 & 20 & gal & - & 37 & $0.8 \mathrm{~J}$ & ND & & MRMW11S-W-16479 \\
\hline MW11S & $53-68$ & $10 / 1 / 07$ & 31.55 & 73 & 20 & gal & - & 54 & 1.2 & ND & & MRMW11S-W-16594 \\
\hline MW11S & $53-68$ & $4 / 15 / 08$ & 29.9 & 72.7 & 5.5 & L & - & 35 & $0.8 \mathrm{~J}$ & ND & April monitoring. & MRMW11S-W-23241 \\
\hline MW11S & $53-68$ & $4 / 22 / 08$ & 30.2 & 72.7 & 7.2 & $\bar{L}$ & - & 42 & $0.9 \mathrm{~J}$ & ND & $\begin{array}{l}\text { Confirm low carbon } \\
\text { tetrachloride. }\end{array}$ & MRMW11S-W-23261 \\
\hline MW11S & $53-68$ & $10 / 20 / 08$ & 37.1 & 72.7 & 9 & L & 60.5 & 42 & $0.9 \mathrm{~J}$ & ND & Low flow. & MRMW11S-W-27631 \\
\hline MW11S & $53-68$ & $10 / 21 / 08$ & - & - & - & - & - & 45 & $0.9 \mathrm{~J}$ & ND & Full purge. & MRMW11S-W-27651 \\
\hline MW11S & $53-68$ & $4 / 23 / 09$ & 38.1 & 72.7 & 5 & L & 60.5 & 46 & 1.0 & ND & & MRMW11S-W-27663 \\
\hline MW11S & $53-68$ & $9 / 3 / 09$ & 34.7 & 72.7 & 7.5 & L & 60.5 & 39 & $0.9 \mathrm{~J}$ & ND & Low flow. & MRMW11S-W-29953 \\
\hline MW11S & $53-68$ & $9 / 4 / 09$ & 34.95 & 72.7 & 72 & L & 67 & 41 & $0.9 \mathrm{~J}$ & ND & Three well volumes. & MRMW11S-W-29973 \\
\hline MW11S & $53-68$ & $4 / 6 / 10$ & 29.45 & 72.7 & 6.5 & L & 60.5 & 38 & 1.0 & ND & & MRMW11S-W-29993 \\
\hline MW11S & $53-68$ & $9 / 23 / 10$ & 34.75 & 72.7 & 7 & L & 60.5 & 28 & 1.0 & ND & & MRMW11S-W-30021 \\
\hline
\end{tabular}


TABLE 3.2 (Cont.)

\begin{tabular}{|c|c|c|c|c|c|c|c|c|c|c|c|c|}
\hline \multirow[b]{2}{*}{ Location } & \multirow{2}{*}{$\begin{array}{l}\text { Screen } \\
\text { Interval } \\
\text { (ft BGL) }\end{array}$} & \multirow{2}{*}{$\begin{array}{l}\text { Sample } \\
\text { Date }\end{array}$} & \multirow{2}{*}{$\begin{array}{l}\text { Depth to } \\
\text { Water } \\
\text { (ft TOC) })^{\mathrm{a}}\end{array}$} & \multirow{2}{*}{$\begin{array}{l}\text { Depth of } \\
\text { well } \\
\text { (ft TOC) })^{a}\end{array}$} & \multirow{2}{*}{$\begin{array}{l}\text { Volume } \\
\text { Purged }\end{array}$} & \multirow{2}{*}{$\begin{array}{l}\text { Purge } \\
\text { Units }\end{array}$} & \multirow{2}{*}{$\begin{array}{l}\text { Pump } \\
\text { Intake } \\
\text { Position } \\
\text { (ft BGL) }\end{array}$} & \multicolumn{3}{|c|}{ Concentration $(\mu \mathrm{g} / \mathrm{L})$} & \multirow[b]{2}{*}{ Comment } & \multirow[b]{2}{*}{ Sample } \\
\hline & & & & & & & & Tetrachloride & Chloroform & Chloride & & \\
\hline Isch & - & 2/19/04 & - & - & Pump ${ }^{f}$ & - & - & ND & ND & ND & & MRJR-W-16502 \\
\hline Isch & - & $9 / 14 / 05$ & - & - & Pump & - & - & ND & ND & ND & & MRPRISCH-W-16513 \\
\hline Isch & - & $3 / 23 / 06$ & - & - & 20 & gal & - & ND & ND & ND & & MRISCH-W-19989 \\
\hline Isch & - & $9 / 19 / 06$ & - & - & Pump & - & - & ND & ND & ND & & MRISCH-W-16531 \\
\hline Isch & - & $3 / 22 / 07$ & - & - & Pump & - & - & ND & ND & ND & & MRISCH-W-16564 \\
\hline Isch & - & $10 / 3 / 07$ & - & - & Pump & - & - & ND & ND & ND & & MRISCH-W-16590 \\
\hline Isch & - & $4 / 15 / 08$ & - & - & Pump & - & - & $0.4 \mathrm{~J}$ & ND & ND & & MRISCH-W-23242 \\
\hline Isch & - & $4 / 22 / 08$ & - & - & Pump & - & - & ND & ND & ND & & MRISCH-W-23262 \\
\hline Isch & - & $10 / 21 / 08$ & - & - & Pump & - & - & ND & ND & ND & & MRISCH-W-27632 \\
\hline Isch & - & $4 / 22 / 09$ & - & - & Pump & - & - & ND & ND & ND & & MRISCH-W-27664 \\
\hline Isch & - & $9 / 2 / 09$ & - & - & Pump & - & - & ND & ND & ND & & MRISCH-W-29954 \\
\hline Isch & - & $4 / 7 / 10$ & - & - & Pump & - & - & ND & ND & ND & & MRISCH-W-29994 \\
\hline Isch & - & $9 / 22 / 10$ & - & - & Pump & - & - & ND & ND & ND & & MRISCH-W-30022 \\
\hline Rillinger & - & $6 / 4 / 04$ & - & - & Pump & - & - & ND & ND & ND & & MRPRIVRIL-W-16471 \\
\hline $\begin{array}{l}\text { Rillinger } \\
\text { Rer }\end{array}$ & - & $9 / 14 / 05$ & _- & - & Pump & _- & _- & 2.6 & $0.1 \mathrm{~J}$ & ND & & MRPRILL-W-16512 \\
\hline Rillinger & - & 3/19/06 & - & - & Pump & - & - & ND & ND & $0.4 \mathrm{~J} \mathrm{~B}$ & & MRRILINGER-W-19988 \\
\hline Rillinger & - & 9/19/06 & - & - & Pump & - & - & ND & ND & ND & & MRRILI-W-16530 \\
\hline Rillinger & - & $3 / 29 / 07$ & - & - & Pump & - & - & 1.3 & 1.1 & ND & & MRRILINGER-W-16561 \\
\hline Rillinger & _- & $10 / 3 / 07$ & _- & _- & Pump & _- & _- & $13^{\mathrm{g}}$ & $0.4 \mathrm{~J}$ & ND & & MRRILINGER-W-16591 \\
\hline Rillinger & - & $10 / 8 / 07$ & - & - & Pump & - & - & $0.4 \mathrm{~J}$ & ND & ND & & MRRILINGER-W-16592 \\
\hline Rillinger & - & $1 / 11 / 08$ & - & - & Pump & - & - & 6.2 & $0.5 \mathrm{~J}$ & ND & & MORIL-W-11108 \\
\hline Rillinger & - & $4 / 15 / 08$ & - & - & Pump & - & - & 9.9 & $0.4 \mathrm{~J}$ & ND & & MRRILINGER-W-23243 \\
\hline Rillinger & - & $10 / 21 / 08$ & - & - & Pump & - & - & $0.9 \mathrm{~J}$ & ND & ND & & MRRILLINGER-W-27633 \\
\hline Rillinger & - & $4 / 22 / 09$ & - & - & Pump & - & - & 1.2 & ND & ND & & MRRILLINGER-W-27665 \\
\hline Rillinger & - & 9/2/09 & - & - & Pump & - & - & 1.0 & ND & ND & & MRRILLINGER-W-29955 \\
\hline Rillinger & - & $4 / 7 / 10$ & - & - & Pump & - & - & $0.8 \mathrm{~J}$ & ND & ND & & MRRILLINGER-W-29995 \\
\hline Rillinger & - & $9 / 22 / 10$ & - & - & Pump & - & - & 1.3 & ND & ND & & MRRILLINGER-W-30023 \\
\hline Stone & $43^{h}$ & $6 / 4 / 04$ & 23.35 & - & \multicolumn{2}{|c|}{ Purged dry. } & - & 10 & ND & ND & & MRPRIVSTON-W-16475 \\
\hline Stone & 43 & $9 / 14 / 05$ & 17.18 & 40 & - & - & - & 2.6 & $0.3 \mathrm{~J}$ & ND & & MRPRSTON-W-16511 \\
\hline Stone & 43 & $3 / 19 / 06$ & 17.42 & 40 & 100 & gal & - & 14 & $0.8 \mathrm{~J}$ & $0.4 \mathrm{~J} \mathrm{~B}$ & & MRSTONE-W-19987 \\
\hline Stone & 43 & 9/19/06 & 18.55 & 38.8 & 41 & gal & - & 2.1 & ND & ND & & MRSTONE-W-16529 \\
\hline Stone & 43 & $3 / 22 / 07$ & 20.62 & 38.8 & 56 & gal & - & 5.4 & $0.3 \mathrm{~J}$ & ND & & MRSTONE-W-16560 \\
\hline Stone & 43 & $10 / 3 / 07$ & 14.6 & 38.6 & 72 & gal & - & 2.8 & ND & ND & & MRSTONE-W-16589 \\
\hline Stone & 43 & $4 / 15 / 08$ & - & 38.86 & - & - & - & $0.9 \mathrm{~J}$ & ND & ND & & MRSTONE-W-23244 \\
\hline Stone & 43 & $10 / 21 / 08$ & - & - & 5 & gal & - & 3.0 & ND & ND & & MRSTONE-W-27634 \\
\hline Stone & 43 & $4 / 23 / 09$ & - & - & 5 & gal & - & 1.1 & ND & ND & & MRSTONE-W-27666 \\
\hline Stone & 43 & $9 / 2 / 09$ & - & - & 5 & gal & - & $0.9 \mathrm{~J}$ & ND & ND & & MRSTONE-W-29956 \\
\hline Stone & 43 & $4 / 7 / 10$ & - & - & 5 & gal & - & $0.5 \mathrm{~J}$ & ND & ND & & MRSTONE-W-29996 \\
\hline Stone & 43 & $9 / 22 / 10$ & - & - & 5 & gal & - & $0.6 \mathrm{~J}$ & 1.8 & ND & & MRSTONE-W-30024 \\
\hline
\end{tabular}




\begin{tabular}{|c|c|c|c|c|c|c|c|c|c|c|c|c|}
\hline \multirow[b]{2}{*}{ Location } & \multirow{2}{*}{$\begin{array}{l}\text { Screen } \\
\text { Interval } \\
\text { (ft BGL) }\end{array}$} & \multirow[b]{2}{*}{$\begin{array}{l}\text { Sample } \\
\text { Date }\end{array}$} & \multirow{2}{*}{$\begin{array}{l}\text { Depth to } \\
\text { Water } \\
\text { (ft TOC) })^{a}\end{array}$} & \multirow{2}{*}{$\begin{array}{l}\text { Depth of } \\
\text { well } \\
\text { (ft TOC) })^{a}\end{array}$} & \multirow[b]{2}{*}{$\begin{array}{l}\text { Volume } \\
\text { Purged }\end{array}$} & \multirow[b]{2}{*}{$\begin{array}{l}\text { Purge } \\
\text { Units }\end{array}$} & \multirow{2}{*}{$\begin{array}{c}\text { Pump } \\
\text { Intake } \\
\text { Position } \\
\text { (ft BGL) }\end{array}$} & \multicolumn{3}{|c|}{ Concentration $(\mu \mathrm{g} / \mathrm{L})$} & \multirow[b]{2}{*}{ Comment } & \multirow[b]{2}{*}{ Sample } \\
\hline & & & & & & & & $\begin{array}{c}\text { Carbon } \\
\text { Tetrachloride }\end{array}$ & Chloroform & $\begin{array}{l}\text { Methylene } \\
\text { Chloride }\end{array}$ & & \\
\hline TD12 & $27-67$ & $4 / 22 / 08$ & - & - & - & - & - & ND & ND & ND & $\begin{array}{l}\text { Grimm irrigation } \\
\text { well. }\end{array}$ & MRTD12-W-23264 \\
\hline TD12 & $27-67$ & $10 / 20 / 08$ & - & - & - & - & - & ND & ND & ND & $\begin{array}{l}\text { Grimm irrigation } \\
\text { well. }\end{array}$ & MRTD12-W-27635 \\
\hline TD12 & $27-67$ & $4 / 23 / 09$ & - & - & - & - & - & ND & ND & ND & $\begin{array}{l}\text { Tile drain into creek. } \\
\text { Overflow before }\end{array}$ & MRTD12-W-27667 \\
\hline TD12 & $27-67$ & 9/3/09 & - & - & - & - & - & ND & ND & ND & catchment. & MRTD12-W-29957 \\
\hline TD12 & $27-67$ & $4 / 6 / 10$ & - & - & - & - & - & ND & ND & ND & & MRTD12-W-29997 \\
\hline TD12 & $27-67$ & $9 / 22 / 10$ & - & - & - & - & - & ND & ND & ND & & MRTD12-W-30025 \\
\hline
\end{tabular}

a Depths were measured from the top of the casing (TOC).

b ND, not detected at an instrument detection limit of $0.1 \mu \mathrm{g} / \mathrm{L}$.

c Qualifier J indicates an estimated concentration below the method quantitation limit of $1.0 \mu \mathrm{g} / \mathrm{L}$.

d Qualifier B indicates that the compound was present in the associated method blank.

e Qualifier R indicates that the contaminant was present in the associated equipment rinsate. Resampling confirmed that the well was free of contamination.

f The well's dedicated pump was used for sampling. The pump was allowed to run before the sample was collected.

g Sample collected after recent reactivation of well. Well resampled on 1/8/07 and 1/11/08.

h Total depth. 
TABLE 3.3 Field measurements for groundwater samples collected at Morrill, October 2003 to September 2010. Shading indicates sample collection with the low-flow procedure.

\begin{tabular}{|c|c|c|c|c|c|c|c|c|c|c|c|c|c|c|c|}
\hline Location & $\begin{array}{l}\text { Screen } \\
\text { Interval } \\
\text { (ft BGL) }\end{array}$ & $\begin{array}{l}\text { Sample } \\
\text { Date }\end{array}$ & $\begin{array}{l}\text { Depth to } \\
\text { Water } \\
\text { (ft TOC) }\end{array}$ & $\begin{array}{l}\text { Depth of } \\
\text { well } \\
\text { (ft TOC) }\end{array}$ & $\begin{array}{l}\text { Volume } \\
\text { Purged }\end{array}$ & $\begin{array}{l}\text { Purge } \\
\text { Units }\end{array}$ & $\begin{array}{l}\text { Pump } \\
\text { Intake } \\
\text { Position } \\
\text { (ft BGL) }\end{array}$ & $\begin{array}{c}\text { Temperature } \\
\left({ }^{\circ} \mathrm{C}\right)\end{array}$ & $\mathrm{pH}$ & $\begin{array}{l}\text { Conductivity } \\
(\mu \mathrm{S} / \mathrm{cm})\end{array}$ & $\begin{array}{c}\text { Dissolved } \\
\text { Oxygen } \\
\text { (mg/L) }\end{array}$ & $\begin{array}{l}\text { ORP } \\
(\mathrm{mV})\end{array}$ & $\begin{array}{l}\text { Iron II } \\
\text { (mg/L) }\end{array}$ & $\begin{array}{l}\text { Carbon } \\
\text { Dioxide } \\
\text { (mg/L) }\end{array}$ & Sample \\
\hline MW1S & $11-51$ & $10 / 23 / 03$ & 30.36 & 54.04 & 70 & gal & - & 14.6 & 7.14 & 933 & $-^{a}$ & 13 & - & - & MRMW1S-W-16422 \\
\hline MW1S & $11-51$ & $6 / 2 / 04$ & 26.97 & 53.94 & 53 & gal & - & 14.4 & 7.16 & 970 & - & - & - & - & MRMW1S-W-16461 \\
\hline MW1S & $11-51$ & $9 / 13 / 05$ & 24.16 & 53.9 & 57 & gal & - & 15.3 & 6.95 & 1174 & 7.17 & 200 & 0 & 55 & MRMW1S-W-19259 \\
\hline MW1S & $11-51$ & $3 / 22 / 06$ & 29 & 53.95 & 48 & gal & - & 15.5 & 7.23 & 927 & 9.94 & 220 & 0.01 & 40 & MRMW1S-W-20008 \\
\hline MW1S & $11-51$ & $9 / 20 / 06$ & 26.82 & 53.97 & 55 & gal & - & 15.7 & 7.12 & 973 & 7.52 & - & 0.03 & 40 & MRMW1S-W-22495 \\
\hline MW1S & $11-51$ & $3 / 21 / 07$ & 25.8 & 53.98 & 55 & gal & - & 16.6 & 6.48 & 960 & 5.45 & 88 & 0 & 40 & MRMW1S-W-16488 \\
\hline MW1S & $11-51$ & $10 / 1 / 07$ & 21.65 & 53.95 & 63 & gal & - & 16.0 & 6.80 & 886 & 6.79 & 128 & 0 & 30 & MRMW1S-W-16595 \\
\hline MW1S & $11-51$ & $4 / 14 / 08$ & 16.2 & 54 & 5.5 & $\mathrm{~L}$ & - & 13.9 & 7.09 & 1237 & 6.38 & 118 & 0.02 & - & MRMW1S-W-23230 \\
\hline MW1S & $11-51$ & $4 / 22 / 08$ & 16 & 54 & 6.25 & $\mathrm{~L}$ & - & 15.6 & 6.96 & 1230 & 6.10 & 133 & - & - & MRMW1S-W-23259 \\
\hline MW1S & $11-51$ & $5 / 1 / 08$ & - & - & 3.24 & L & 22 & 17.1 & 7.11 & 801 & 3.34 & 104 & - & - & MRMW1S-22-W-23275 \\
\hline MW1S & $11-51$ & $5 / 1 / 08$ & - & - & 3.24 & L & 27 & 17.0 & 7.10 & 820 & 3.40 & 102 & - & - & MRMW1S-27-W-23276 \\
\hline MW1S & $11-51$ & $5 / 1 / 08$ & - & - & 4.25 & L & 48 & 16.3 & 7.00 & 1301 & 3.03 & 118 & - & - & MRMW1S-48-W-23277 \\
\hline MW1S & $11-51$ & $10 / 20 / 08$ & 25.8 & 54 & 6 & $\bar{L}$ & 31 & 14.0 & 6.84 & 1265 & 5.40 & 103 & 0 & - & MRMW1S-W-27620 \\
\hline MW1S & $11-51$ & $10 / 21 / 08$ & - & - & $T W V^{b}$ & - & - & 14.8 & 7.02 & 978 & - & - & 0 & - & MRMW1S-W-27649 \\
\hline MW1S & $11-51$ & $4 / 24 / 09$ & 24.4 & 54 & 5 & L & 39.2 & 16.2 & 7.00 & 986 & 8.93 & 75 & 0.04 & - & MRMW1S-W-27652 \\
\hline MW1S & $11-51$ & $9 / 3 / 09$ & 19 & 54 & 8 & $\bar{L}$ & 35 & 16.0 & 6.94 & 1334 & 7.08 & 28 & 0.04 & - & MRMW1S-W-29942 \\
\hline MW1S & $11-51$ & $9 / 4 / 09$ & 19.3 & 51.2 & 244 & L & 50 & 15.0 & 6.92 & 950 & 7.73 & 61 & - & - & MRMW1S-W-29971 \\
\hline MW1S & $11-51$ & $4 / 7 / 10$ & 11.65 & 51.3 & 7 & L & 16.6 & 9.6 & 7.01 & 695 & 8.66 & - & 233 & 0 & MRMW1ST-W-29981 \\
\hline MW1S & $11-51$ & $4 / 7 / 10$ & 11.57 & 51.31 & 6 & L & 31.5 & 11.9 & 6.96 & 918 & 8.42 & - & 246 & 0 & MRMW1SM-W-29980 \\
\hline MW1S & $11-51$ & $4 / 7 / 10$ & 11.65 & 51.31 & 6 & $\mathrm{~L}$ & 46.3 & 11.7 & 6.95 & 965 & 8.29 & - & 251 & 0.01 & MRMW1SB-W-29979 \\
\hline MW1S & $11-51$ & $4 / 7 / 10$ & 11.48 & 51.3 & 80 & gal & 49 & 14.0 & 7.18 & 687 & 8.86 & - & 211 & 0.03 & MRMW1S3X-W-29982 \\
\hline MW1S & $11-51$ & $9 / 22 / 10$ & 19.89 & 54 & 10 & $\mathrm{~L}$ & 31 & 17.9 & 7.01 & 1337 & 6.17 & - & 134 & 0.07 & MRMW1S-W-30010 \\
\hline MW1D & $63-88$ & $10 / 22 / 03$ & 28.39 & 88.5 & 92 & gal & - & 14.9 & 6.87 & 2620 & - & 25 & - & - & MRMW1D-W-16421 \\
\hline MW1D & $63-88$ & $6 / 2 / 04$ & 26.82 & 88.6 & 140 & gal & - & 13.9 & 6.87 & 2460 & - & - & - & - & MRMW1D-W-16458 \\
\hline MW1D & $63-88$ & 9/13/05 & 23.72 & 88.6 & 200 & gal & - & 15.5 & 6.56 & 2470 & - & - & - & - & MRMW1D-W-16518 \\
\hline MW1D & $63-88$ & $3 / 19 / 06$ & 26.85 & 88.6 & 112 & gal & - & 12.9 & 6.95 & 2460 & 5.11 & 230 & 0 & - & MRMW1D-W-19986 \\
\hline MW1D & $63-88$ & 9/20/06 & 25.52 & 88.8 & 125 & gal & - & 12.5 & 6.93 & 2690 & - & - & - & - & MRMW1D-W-16532 \\
\hline MW1D & $63-88$ & $3 / 21 / 07$ & 25.79 & 88.8 & 125 & gal & - & 15.3 & 6.39 & 2540 & 0.08 & 12 & 0.39 & 40 & MRMW1D-W-16487 \\
\hline MW1D & $63-88$ & $10 / 1 / 07$ & 22.75 & 89.38 & 130 & gal & - & 16.3 & 6.60 & 2230 & 6.79 & 5 & 0.44 & 45 & MRMW1D-W-16596 \\
\hline MW1D & $63-88$ & $4 / 14 / 08$ & 29.51 & 89 & 6 & $\mathrm{~L}$ & - & 14.6 & 6.99 & 2637 & 0.50 & 32 & 0.73 & - & MRMW1D-W-23231 \\
\hline MW1D & $63-88$ & $10 / 20 / 08$ & 30.4 & 89 & 7 & L & 75.5 & 13.4 & 6.83 & 2556 & 0.24 & 21 & 0.37 & - & MRMW1D-W-27621 \\
\hline MW1D & $63-88$ & $4 / 24 / 09$ & 31 & 89 & 7 & L & 75.5 & 16.7 & 6.92 & 2419 & 0.37 & 22 & 0.28 & - & MRMW1D-W-27653 \\
\hline MW1D & $63-88$ & $9 / 3 / 09$ & 27.05 & 89 & 6.5 & L & 75.5 & 16.9 & 7.00 & 2200 & 0.59 & 16 & 0.27 & - & MRMW1D-W-29943 \\
\hline MW1D & $63-88$ & $4 / 6 / 10$ & 24.7 & 89 & 8.5 & L & 75.5 & 17.8 & 6.39 & 2274 & 0.15 & - & 32.8 & 0.01 & MRMW1D-W-29983 \\
\hline MW1D & $63-88$ & $9 / 22 / 10$ & 27.92 & 89 & 9 & $\mathrm{~L}$ & 75.5 & 17.6 & 7.01 & 2492 & 0.31 & - & 61.1 & 0.04 & MRMW1D-W-30011 \\
\hline MW2S & $13-53$ & $10 / 22 / 03$ & 42.21 & 53.35 & $\mathrm{PDS}^{\mathrm{c}}$ & - & & 16.2 & 6.86 & 875 & - & 20 & - & - & MRMW02-W-16419 \\
\hline MW2S & $13-53$ & $6 / 2 / 04$ & 37.44 & 53.3 & 31 & gal & - & 16.9 & 7.07 & 861 & - & - & - & - & MRMW2S-W-16459 \\
\hline MW2S & $13-53$ & $9 / 14 / 05$ & 33.68 & 53.33 & 38 & gal & - & 15.2 & 6.94 & 801 & 7.85 & 142 & - & 65 & MRMW2S-W-19264 \\
\hline MW2S & $13-53$ & $3 / 21 / 06$ & 40.87 & 53.32 & 27 & gal & - & 13.0 & 7.07 & 863 & 9.40 & 262 & 0.14 & 25 & MRMW2S-W-19992 \\
\hline MW2S & $13-53$ & 9/18/06 & 36.53 & 53.3 & 28 & gal & - & 13.6 & 6.99 & 844 & 6.81 & 69 & 0 & 80 & MRMW2S-W-22488 \\
\hline MW2S & $13-53$ & $3 / 22 / 07$ & 35.77 & 53.3 & 35 & gal & _- & 15.2 & 6.40 & 790 & 5.82 & 69 & 0 & 30 & MRMW2S-W-16559 \\
\hline MW2S & $13-53$ & $10 / 3 / 07$ & 31.17 & 53.4 & 44 & gal & - & 16.8 & 6.97 & 703 & 6.70 & 269 & 0.01 & 30 & MRMW2S-W-16587 \\
\hline MW2S & $13-53$ & $4 / 15 / 08$ & 23.55 & 53.4 & 2.22 & $\mathrm{~L}$ & - & 11.7 & 7.23 & 742 & 3.22 & 75 & 0 & - & MRMW2S-W-23232 \\
\hline MW2S & $13-53$ & $10 / 21 / 08$ & 33.7 & 53.5 & 5 & L & 33 & 13.4 & 7.08 & 745 & 8.55 & 104 & 0 & - & MRMW2S-W-27622 \\
\hline MW2S & $13-53$ & $10 / 21 / 08$ & - & - & TWV & - & - & - & - & - & - & - & - & - & MRMW2S-W-27652 \\
\hline
\end{tabular}


TABLE 3.3 (Cont.)

\begin{tabular}{|c|c|c|c|c|c|c|c|c|c|c|c|c|c|c|c|}
\hline Location & $\begin{array}{l}\text { Screen } \\
\text { Interval } \\
\text { (ft BGL) }\end{array}$ & $\begin{array}{l}\text { Sample } \\
\text { Date }\end{array}$ & $\begin{array}{l}\text { Depth to } \\
\text { Water } \\
\text { (ft TOC) }\end{array}$ & $\begin{array}{l}\text { Depth of } \\
\text { well } \\
\text { (ft TOC) }\end{array}$ & $\begin{array}{l}\text { Volume } \\
\text { Purged }\end{array}$ & $\begin{array}{l}\text { Purge } \\
\text { Units }\end{array}$ & $\begin{array}{l}\text { Pump } \\
\text { Intake } \\
\text { Position } \\
\text { (ft BGL) }\end{array}$ & $\begin{array}{c}\text { Temperature } \\
\left({ }^{\circ} \mathrm{C}\right)\end{array}$ & $\mathrm{pH}$ & $\begin{array}{l}\text { Conductivity } \\
(\mu \mathrm{S} / \mathrm{cm})\end{array}$ & $\begin{array}{c}\text { Dissolved } \\
\text { Oxygen } \\
\text { (mg/L) }\end{array}$ & $\begin{array}{l}\text { ORP } \\
(\mathrm{mV})\end{array}$ & $\begin{array}{l}\text { Iron II } \\
(\mathrm{mg} / \mathrm{L})\end{array}$ & $\begin{array}{l}\text { Carbon } \\
\text { Dioxide } \\
\text { (mg/L) }\end{array}$ & Sample \\
\hline MW2S & $13-53$ & $4 / 23 / 09$ & 33.2 & 53.5 & 6.5 & $\mathrm{~L}$ & 43.35 & 15.2 & 6.97 & 755 & 9.19 & 26 & 0 & - & MRMW2S-W-27654 \\
\hline MW2S & $13-53$ & 9/3/09 & 29.4 & 53.5 & 5.4 & L & 41.2 & 15.1 & 7.15 & 690 & 8.80 & 32 & 0.01 & - & MRMW2S-W-29944 \\
\hline MW2S & $13-53$ & $4 / 6 / 10$ & 18.57 & 52.42 & 6.5 & $\mathrm{~L}$ & 36 & 17.1 & 6.95 & 681 & 6.85 & - & 37.3 & 0.12 & MRMW2S-W-29984 \\
\hline MW2S & $13-53$ & $9 / 22 / 10$ & 31.25 & 53 & 7 & $\mathrm{~L}$ & 33 & 16.1 & 7.03 & 723 & 7.16 & - & 142 & 0 & MRMW2S-W-30012 \\
\hline MW3S & $18-48$ & $10 / 23 / 03$ & 36.47 & 47.79 & 73 & gal & - & 16.8 & 7.23 & 655 & - & 6 & - & - & MRMW03-W-16423 \\
\hline MW3S & $18-48$ & $6 / 2 / 04$ & 30.67 & 47.5 & 34 & gal & - & 14.2 & 7.23 & 664 & - & - & - & - & MRMW3S-W-16462 \\
\hline MW3S & $18-48$ & $9 / 13 / 05$ & 25.6 & 47.6 & 50 & gal & - & 14.6 & 7.13 & 663 & 8.82 & 223 & 0 & 100 & MRMW3S-W-19261 \\
\hline MW3S & $18-48$ & $3 / 23 / 06$ & 35.62 & 47.74 & 28 & gal & - & 8.9 & 7.16 & 662 & 6.74 & 269 & 0.08 & 25 & MRMW3S-W-19994 \\
\hline MW3S & $18-48$ & 9/20/06 & 29.44 & 47.75 & 22 & gal & - & 12.9 & 7.15 & 669 & 7.64 & 105 & 0 & - & MRMW3S-W-22496 \\
\hline MW3S & $18-48$ & $3 / 22 / 07$ & 26.19 & 47.75 & 45 & gal & - & 15.0 & 6.44 & 578 & 5.90 & 261 & 0.17 & 30 & MRMW3S-W-16563 \\
\hline MW3S & $18-48$ & $10 / 3 / 07$ & 22.7 & 47.9 & 50 & gal & - & 15.3 & 6.97 & 594 & 0.38 & 282 & 0 & 20 & MRMW3S-W-16585 \\
\hline MW3S & $18-48$ & $4 / 14 / 08$ & 16.95 & 47.8 & 3.31 & L & - & 13.7 & 7.17 & 693 & 3.52 & 165 & 0 & - & MRMW3S-W-23233 \\
\hline MW3S & $18-48$ & $4 / 22 / 08$ & 15.8 & 47.8 & 6.5 & L & - & 16.0 & 6.99 & 685 & 6.71 & 155 & - & - & MRMW3S-W-23260 \\
\hline MW3S & $18-48$ & $5 / 1 / 08$ & - & - & 2.62 & $\bar{L}$ & 26 & 13.2 & 7.17 & 675 & 3.83 & 161 & - & - & MRMW3S-26-W-23269 \\
\hline MW3S & $18-48$ & $5 / 1 / 08$ & - & - & 2.75 & L & 38 & 12.7 & 7.12 & 671 & 4.21 & 193 & - & - & MRMW3S-38-W-23270 \\
\hline MW3S & $18-48$ & $5 / 1 / 08$ & - & - & 3.17 & $\bar{L}$ & 45 & 12.6 & 7.03 & 675 & 4.57 & 205 & - & - & MRMW3S-45-W-23271 \\
\hline MW3S & $18-48$ & $10 / 21 / 08$ & 27 & 47.8 & 4.2 & L & 33 & 12.6 & 7.17 & 673 & 6.42 & 115 & 0 & - & MRMW3S-W-27623 \\
\hline MW3S & $18-48$ & $10 / 21 / 08$ & - & - & TWV & - & - & 14.3 & 7.11 & 522 & - & - & 0 & - & MRMW3S-W-27650 \\
\hline MW3S & $18-48$ & $4 / 23 / 09$ & 26.7 & 47.8 & 5 & L & 37.3 & 17.0 & 7.06 & 662 & 9.40 & -63 & 0.01 & - & MRMW3S-W-27655 \\
\hline MW3S & $18-48$ & $9 / 3 / 09$ & 22.4 & 47.8 & 5.5 & L & 35.2 & 16.3 & 7.28 & 640 & 7.69 & 12 & 0.01 & - & MRMW3S-W-29945 \\
\hline MW3S & $18-48$ & $9 / 4 / 09$ & 22.55 & 47.8 & 190 & L & 46 & 14.2 & 6.57 & 659 & 9.09 & 95 & - & - & MRMW3S-W-29972 \\
\hline MW3S & $18-48$ & $4 / 6 / 10$ & 12.14 & 47.8 & 6 & L & 33 & 16.0 & 6.99 & 611 & 8.20 & 8 & 0 & - & MRMW3S-W-29985 \\
\hline MW3S & $18-48$ & $9 / 23 / 10$ & 23.73 & 47.8 & 7 & L & 33 & 15.8 & 7.22 & 674 & 11.00 & 150 & 0.05 & - & MRMW3S-W-30013 \\
\hline MW4S & $17-47$ & $10 / 21 / 03$ & 46.4 & 47.8 & PDS & - & & - & 7.17 & 758 & - & - & - & - & MRMW04-W-16418 \\
\hline MW4S & $17-47$ & $6 / 4 / 04$ & 43.21 & 47.8 & 10 & gal & - & 15.4 & 6.93 & 769 & - & - & - & - & MRMW4S-W-16470 \\
\hline MW4S & $17-47$ & $9 / 14 / 05$ & 36.21 & 47.81 & 8 & gal & - & 15.4 & 7.30 & 751 & 8.00 & 174 & 0 & 50 & MRMW4S-W-19262 \\
\hline MW4S & $17-47$ & $3 / 21 / 06$ & 44.55 & 47.72 & 6 & gal & - & 6.7 & 7.25 & 729 & 10.90 & 154 & 0 & 25 & MRMW4S-W-19993 \\
\hline MW4S & $17-47$ & $9 / 18 / 06$ & 41.56 & 47.75 & 5.25 & gal & - & 13.1 & 7.25 & 728 & 8.05 & 41 & 0 & 50 & MRMW4S-W-22487 \\
\hline MW4S & $17-47$ & $3 / 22 / 07$ & 38.74 & 47.75 & 6 & gal & - & 14.2 & 6.53 & 765 & 5.91 & 78 & 0.1 & 25 & MRMW4S-W-16562 \\
\hline MW4S & $17-47$ & $10 / 3 / 07$ & 31.11 & 47.73 & 30 & gal & - & 16.4 & 6.95 & 715 & 7.40 & 281 & 0.1 & 30 & MRMW4S-W-16586 \\
\hline MW4S & $17-47$ & $1 / 11 / 08$ & - & - & - & - & - & 11.3 & 7.56 & 757 & - & - & - & - & MOMW4S-W-011108 \\
\hline MW4S & $17-47$ & $4 / 14 / 08$ & 26.32 & 47.85 & 2.48 & $\mathrm{~L}$ & - & 13.1 & 7.28 & 783 & 3.80 & 213 & 0 & - & MRMW4S-W-23234 \\
\hline MW4S & $17-47$ & $10 / 20 / 08$ & 36.7 & 47.8 & 8 & gal & - & 14.5 & 7.16 & 756 & 8.71 & 104 & 0 & - & MRMW4S-W-27624 \\
\hline MW4S & $17-47$ & $4 / 23 / 09$ & 41.5 & 47.8 & 5 & L & 44.65 & 15.8 & 7.16 & 717 & 9.45 & 22 & 0.05 & - & MRMW4S-W-27656 \\
\hline MW4S & $17-47$ & $9 / 4 / 09$ & 31.6 & 47.8 & 6 & L & 39.3 & 15.0 & 7.13 & 731 & 8.23 & 80 & 0.04 & - & MRMW4S-W-29946 \\
\hline MW4S & $17-47$ & $4 / 6 / 10$ & 21.8 & 47.85 & 5.5 & L & 34.83 & 16.5 & 7.07 & 629 & 8.07 & - & -1.2 & 0 & MRMW4S-W-29986 \\
\hline MW4S & $17-47$ & $9 / 22 / 10$ & 33.15 & 47.8 & 6 & L & 32 & 16.3 & 7.07 & 732 & 7.28 & - & 90.4 & 0 & MRMW4S-W-30014 \\
\hline MW5S & $15-55$ & $10 / 22 / 03$ & 31.4 & 55.72 & 48 & gal & - & 15.3 & 7.10 & 816 & - & 6 & - & - & MRMW05-W-16420 \\
\hline MW5S & $15-55$ & $6 / 2 / 04$ & 26.33 & 55.65 & $>57$ & gal & - & 14.3 & 7.21 & 817 & - & - & - & - & MRMW5S-W-16460 \\
\hline MW5S & $15-55$ & 9/13/05 & 22.66 & 54.2 & 75 & gal & - & 16.0 & 7.04 & 763 & 13.90 & 228 & 0 & 60 & MRMW5S-W-19260 \\
\hline MW5S & $15-55$ & $3 / 22 / 06$ & 28.64 & 54.51 & 50 & gal & - & 13.9 & 7.25 & 781 & 4.52 & 234 & 0.06 & 35 & MRMW5S-W-19996 \\
\hline MW5S & $15-55$ & 9/20/06 & 25.43 & 54.63 & 52 & gal & - & 13.9 & 7.19 & 787 & 5.82 & 73 & 0 & 35 & MRMW5S-W-22493 \\
\hline MW5S & $15-55$ & $3 / 22 / 07$ & 25.14 & 54.56 & 58 & gal & - & 15.5 & 6.50 & 436 & 3.98 & 159 & 0.08 & 30 & MRMW5S-W-16569 \\
\hline MW5S & $15-55$ & $10 / 3 / 07$ & 19.55 & 54.65 & 68 & gal & - & 16.5 & 7.18 & 850 & 1.87 & 268 & 0.04 & 25 & MRMW5S-W-16588 \\
\hline MW5S & $15-55$ & $4 / 14 / 08$ & 11.2 & 54.6 & 6 & $\mathrm{~L}$ & - & 14.1 & 6.90 & 1008 & 3.73 & 143 & 0.02 & - & MRMW5S-W-23235 \\
\hline
\end{tabular}


TABLE 3.3 (Cont.)

\begin{tabular}{|c|c|c|c|c|c|c|c|c|c|c|c|c|c|c|c|}
\hline Location & $\begin{array}{l}\text { Screen } \\
\text { Interval } \\
\text { (ft BGL) }\end{array}$ & $\begin{array}{l}\text { Sample } \\
\text { Date }\end{array}$ & $\begin{array}{l}\text { Depth to } \\
\text { Water } \\
\text { (ft TOC) }\end{array}$ & $\begin{array}{l}\text { Depth of } \\
\text { well } \\
\text { (ft TOC) }\end{array}$ & $\begin{array}{l}\text { Volume } \\
\text { Purged }\end{array}$ & $\begin{array}{l}\text { Purge } \\
\text { Units }\end{array}$ & $\begin{array}{l}\text { Pump } \\
\text { Intake } \\
\text { Position } \\
\text { (ft BGL) }\end{array}$ & $\begin{array}{c}\text { Temperature } \\
\left({ }^{\circ} \mathrm{C}\right)\end{array}$ & $\mathrm{pH}$ & $\begin{array}{l}\text { Conductivity } \\
(\mu \mathrm{S} / \mathrm{cm})\end{array}$ & $\begin{array}{l}\text { Dissolved } \\
\text { Oxygen } \\
\text { (mg/L) }\end{array}$ & $\begin{array}{l}\text { ORP } \\
(\mathrm{mV})\end{array}$ & $\begin{array}{l}\text { Iron II } \\
\text { (mg/L) }\end{array}$ & $\begin{array}{c}\text { Carbon } \\
\text { Dioxide } \\
\text { (mg/L) }\end{array}$ & Sample \\
\hline MW5S & $15-55$ & $4 / 23 / 08$ & 11.3 & 54.6 & 6.5 & $\mathrm{~L}$ & - & 14.9 & 6.88 & 1009 & 3.27 & 184 & - & - & MRMW5S-W-23266 \\
\hline MW5S & $15-55$ & $5 / 1 / 08$ & - & - & 3.7 & $\mathrm{~L}$ & 20 & 15.2 & 6.92 & 1014 & 2.99 & 126 & - & - & MRMW5S-20-W-23272 \\
\hline MW5S & $15-55$ & $5 / 1 / 08$ & - & - & 3.4 & $\mathrm{~L}$ & 28 & 15.1 & 6.90 & 997 & 2.38 & 124 & - & - & MRMW5S-28-W-23273 \\
\hline MW5S & $15-55$ & $5 / 1 / 08$ & - & - & 4 & L & 52 & 15.1 & 6.89 & 989 & 2.06 & 128 & - & - & MRMW5S-52-W-23274 \\
\hline MW5S & $15-55$ & $10 / 21 / 08$ & 22.5 & 54.6 & 7 & L & 35 & 13.2 & 7.04 & 818 & 5.54 & 180 & 0 & - & MRMW5S-W-27625 \\
\hline MW5S & $15-55$ & $4 / 24 / 09$ & 22.1 & 54.6 & 5.5 & L & 38.35 & 15.2 & 6.98 & 817 & 6.68 & 77 & 0 & - & MRMW5S-W-27657 \\
\hline MW5S & $15-55$ & $9 / 3 / 09$ & 17.6 & 54.6 & 5.5 & L & 36.3 & 16.5 & 7.10 & 873 & 3.07 & 23 & 0.02 & - & MRMW5S-W-29947 \\
\hline MW5S & $15-55$ & $4 / 7 / 10$ & 8.32 & 54.5 & 5.5 & L & 35 & 12.6 & 6.76 & 844 & 4.46 & - & 149 & 0 & MRMW5S-W-29987 \\
\hline MW5S & $15-55$ & $9 / 22 / 10$ & 19.25 & 55 & 6.5 & L & 35 & 16.4 & 6.81 & 891 & 1.69 & - & 112 & 0 & MRMW5S-W-30015 \\
\hline MW6S & $10-25$ & $6 / 3 / 04$ & 3.34 & 26.9 & 45 & gal & - & 15.1 & 6.89 & 2410 & - & - & - & - & MRMW6S-W-16465 \\
\hline MW6S & $10-25$ & $9 / 14 / 05$ & 4.7 & 26.9 & 43 & gal & - & 14.1 & 7.06 & 2350 & 0.01 & 54 & 0 & 60 & MRMW6S-W-19263 \\
\hline MW6S & $10-25$ & $3 / 20 / 06$ & 5.35 & 26.91 & 43 & gal & - & 9.8 & 6.91 & 2360 & 1.37 & 89 & 0.38 & 60 & MRMW6S-W-19990 \\
\hline MW6S & $10-25$ & $9 / 18 / 06$ & 5.48 & 26.92 & 27 & gal & - & 12.5 & 6.96 & 2410 & 0.08 & -29 & 0.35 & 85 & MRMW6S-W-22486 \\
\hline MW6S & $10-25$ & $3 / 21 / 07$ & 5.42 & 26.92 & 30 & gal & - & 18.0 & 6.34 & 2450 & 0.12 & 75 & 0.78 & 40 & MRMW6S-W-16486 \\
\hline MW6S & $10-25$ & $10 / 2 / 07$ & 5 & 26.9 & 31 & gal & - & 17.1 & 7.33 & 2280 & 0.20 & 61 & 0.19 & 35 & MRMW6S-W-16583 \\
\hline MW6S & $10-25$ & $4 / 15 / 08$ & 5.15 & 26.9 & 2.5 & $\mathrm{~L}$ & - & 8.7 & 6.99 & 2485 & 0.31 & -76 & 0.41 & - & MRMW6S-W-23236 \\
\hline MW6S & $10-25$ & $10 / 20 / 08$ & 5.7 & 26.9 & 5 & L & 17.5 & 14.3 & 6.84 & 2380 & 0.36 & 18 & 0.28 & - & MRMW6S-W-27626 \\
\hline MW6S & $10-25$ & $4 / 24 / 09$ & 6.2 & 26.9 & 12 & L & 17.5 & 15.2 & 6.93 & 2270 & 0.19 & -39 & 0.63 & - & MRMW6S-W-27658 \\
\hline MW6S & $10-25$ & $9 / 4 / 09$ & 5.85 & 26.9 & 5.4 & L & 17.5 & 13.8 & 6.88 & 2302 & 0.64 & 79 & 0.32 & - & MRMW6S-W-29948 \\
\hline MW6S & $10-25$ & $4 / 6 / 10$ & 6.15 & 26.9 & 8 & $\mathrm{~L}$ & 17.5 & 15.2 & 6.87 & 2141 & 0.05 & - & -19.0 & 0.21 & MRMW6S-W-29988 \\
\hline MW6S & $10-25$ & $9 / 22 / 10$ & 5.53 & 26.9 & 8.75 & $\mathrm{~L}$ & 17.5 & 15.6 & 6.92 & 2354 & 0.42 & - & 46.9 & 0.24 & MRMW6S-W-30016 \\
\hline MW7S & $20-45$ & $6 / 3 / 04$ & 26.68 & 46.98 & 40 & gal & - & 13.8 & 7.19 & 763 & - & - & - & - & MRMW7S-W-16466 \\
\hline MW7S & $20-45$ & $9 / 12 / 05$ & 17.57 & 46.94 & 55 & gal & - & 15.0 & 7.26 & 760 & 8.35 & 240 & 0 & 50 & MRMW7S-W-19258 \\
\hline MW7S & $20-45$ & $3 / 22 / 06$ & 22.45 & 46.98 & 48 & gal & - & 15.2 & 7.32 & 740 & 5.52 & 268 & 0.03 & 25 & MRMW7S-W-20000 \\
\hline MW7S & $20-45$ & 9/19/06 & 20.94 & 47.02 & 56 & gal & - & 13.2 & 7.15 & 764 & 7.37 & 114 & 0 & 25 & MRMW7S-W-22490 \\
\hline MW7S & $20-45$ & $3 / 20 / 07$ & 18.01 & 47.02 & 50 & gal & - & 14.6 & 6.43 & 750 & 5.31 & 95 & 0 & 30 & MRMW7S-W-16481 \\
\hline MW7S & $20-45$ & $10 / 1 / 07$ & 12.4 & 47 & 70 & gal & - & 15.6 & 6.99 & 725 & 7.76 & 269 & 0.01 & 35 & MRMW7S-W-16581 \\
\hline MW7S & $20-45$ & $4 / 14 / 08$ & 7.72 & 47 & 1.82 & $\mathrm{~L}$ & - & 13.4 & 7.21 & 811 & 2.50 & 276 & 0 & - & MRMW7S-W-23237 \\
\hline MW7S & $20-45$ & $4 / 23 / 08$ & 7.8 & 47 & 11 & L & - & 14.0 & 7.00 & 822 & 7.41 & 191 & - & - & MRMW7S-W-23265 \\
\hline MW7S & $20-45$ & $10 / 20 / 08$ & 17.2 & 47 & 6.3 & L & 32.5 & 14.9 & 7.02 & 802 & 6.38 & 87 & 0 & - & MRMW7S-W-27627 \\
\hline MW7S & $20-45$ & $4 / 23 / 09$ & 16.7 & 47 & 7 & L & 32.5 & 16.1 & 7.01 & 727 & 9.48 & -53 & 0.01 & - & MRMW7S-W-27659 \\
\hline MW7S & $20-45$ & $9 / 3 / 09$ & 13.8 & 47 & 9 & $\mathrm{~L}$ & 32.5 & 17.4 & 7.58 & 814 & 9.86 & 102 & 0.02 & - & MRMW7S-W-29949 \\
\hline MW7S & $20-45$ & $4 / 6 / 10$ & 6.38 & 47 & 5.4 & L & 32.5 & 14.7 & 7.04 & 718 & 7.92 & - & 22.8 & 0 & MRMW7S-W-29989 \\
\hline MW7S & $20-45$ & $9 / 23 / 10$ & 14.15 & 45 & 6 & L & 32.5 & 16.0 & 6.91 & 772 & 8.39 & - & 201 & 0 & MRMW7S-W-30017 \\
\hline MW8S & $10-25$ & $6 / 3 / 04$ & 3.7 & 26.75 & 45 & gal & - & 12.8 & 7.12 & 941 & - & _- & - & - & MRMW8S-W-16464 \\
\hline MW8S & $10-25$ & $9 / 14 / 05$ & 4.02 & 26.82 & 57 & gal & - & 14.1 & 7.30 & 853 & 0.02 & 65 & 0 & 40 & MRMW8S-W-19265 \\
\hline MW8S & $10-25$ & $3 / 20 / 06$ & 4.57 & 26.41 & 43 & gal & - & 12.5 & 7.04 & 954 & 0.90 & 153 & 0.05 & 30 & MRMW8S-W-19991 \\
\hline MW8S & $10-25$ & 9/19/06 & 4.76 & 26.83 & 45 & gal & - & 11.8 & 7.09 & 903 & 0.58 & 284 & 0.13 & 50 & MRMW8S-W-22492 \\
\hline MW8S & $10-25$ & $3 / 20 / 07$ & 2.63 & 26.82 & 49 & gal & - & 11.0 & 6.52 & 1026 & 0.77 & 76 & 0 & 30 & MRMW8S-W-16483 \\
\hline MW8S & $10-25$ & $10 / 2 / 07$ & 2.2 & 26.8 & 48 & gal & - & 15.2 & 6.76 & 607 & 2.66 & 209 & 0.02 & 25 & MRMW8S-W-16584 \\
\hline MW8S & $10-25$ & $4 / 15 / 08$ & 0.7 & 26.8 & 5.5 & $\mathrm{~L}$ & - & 10.2 & 7.27 & 1067 & 1.58 & 170 & 0 & - & MRMW8S-W-23238 \\
\hline MW8S & $10-25$ & $10 / 20 / 08$ & 3.6 & 26.8 & 8 & L & 17.5 & 14.0 & 6.91 & 1002 & 0.93 & 69 & 0 & - & MRMW8S-W-27628 \\
\hline MW8S & $10-25$ & $4 / 23 / 09$ & 2.3 & 26.8 & 6 & L & 17.5 & 11.4 & 6.88 & 825 & 1.76 & -35 & 0.02 & - & MRMW8S-W-27660 \\
\hline MW8S & $10-25$ & 9/3/09 & 2.9 & 26.8 & 8.5 & $\mathrm{~L}$ & 17.5 & 14.1 & 7.52 & 890 & 2.09 & 115 & 0 & - & MRMW8S-W-29950 \\
\hline MW8S & $10-25$ & $4 / 6 / 10$ & 1.1 & 26.8 & 8 & L & 17.5 & 13.7 & 7.00 & 843 & 2.60 & - & 212 & 0 & MRMW8S-W-29990 \\
\hline
\end{tabular}


TABLE 3.3 (Cont.)

\begin{tabular}{|c|c|c|c|c|c|c|c|c|c|c|c|c|c|c|c|}
\hline Location & $\begin{array}{l}\text { Screen } \\
\text { Interval } \\
\text { (ft BGL) }\end{array}$ & $\begin{array}{l}\text { Sample } \\
\text { Date }\end{array}$ & $\begin{array}{l}\text { Depth to } \\
\text { Water } \\
\text { (ft TOC) }\end{array}$ & $\begin{array}{c}\text { Depth of } \\
\text { well } \\
\text { (ft TOC) }\end{array}$ & $\begin{array}{l}\text { Volume } \\
\text { Purged }\end{array}$ & $\begin{array}{l}\text { Purge } \\
\text { Units }\end{array}$ & $\begin{array}{l}\text { Pump } \\
\text { Intake } \\
\text { Position } \\
\text { (ft BGL) }\end{array}$ & $\begin{array}{c}\text { Temperature } \\
\left({ }^{\circ} \mathrm{C}\right)\end{array}$ & $\mathrm{pH}$ & $\begin{array}{c}\text { Conductivity } \\
(\mu \mathrm{S} / \mathrm{cm})\end{array}$ & $\begin{array}{l}\text { Dissolved } \\
\text { Oxygen } \\
\text { (mg/L) }\end{array}$ & $\begin{array}{l}\text { ORP } \\
(\mathrm{mV})\end{array}$ & $\begin{array}{l}\text { Iron II } \\
(\mathrm{mg} / \mathrm{L})\end{array}$ & $\begin{array}{c}\text { Carbon } \\
\text { Dioxide } \\
\text { (mg/L) }\end{array}$ & Sample \\
\hline MW8S & $10-25$ & $9 / 22 / 10$ & 2.53 & 26.8 & 9 & $\mathrm{~L}$ & 17.5 & 16.2 & 7.12 & 865 & 1.51 & - & 100 & 0.04 & MRMW8S-W-30018 \\
\hline MW9S & $38.83-53.83$ & $3 / 22 / 06$ & 20.2 & 58.62 & 20 & gal & - & 14.6 & 7.17 & 715 & 0.41 & 25 & 0 & 35 & MRMW9S-W-20004 \\
\hline MW9S & $38.83-53.83$ & 9/19/06 & 18.87 & 59 & 22 & gal & - & 13.0 & 7.08 & 707 & 0.10 & 113 & 0 & 55 & MRMW9S-W-22494 \\
\hline MW9S & $38.83-53.83$ & $3 / 20 / 07$ & 16.69 & 59 & 22 & gal & - & 14.2 & 6.39 & 714 & 0.21 & 40 & 0 & 20 & MRMW9S-W-16480 \\
\hline MW9S & $38.83-53.83$ & $10 / 1 / 07$ & 14 & 58.6 & 23 & gal & - & 15.5 & 7.05 & 664 & 5.50 & 191 & 0 & 30 & MRMW9S-W-16582 \\
\hline MW9S & $38.83-53.83$ & $4 / 14 / 08$ & 16.58 & 58.63 & 2.29 & $\mathrm{~L}$ & - & 12.6 & 7.33 & 709 & 1.93 & 266 & 0.07 & - & MRMW9S-W-23239 \\
\hline MW9S & $38.83-53.83$ & $10 / 20 / 08$ & 21.5 & 58.5 & 11 & L & 46.33 & 15.1 & 7.15 & 690 & 6.18 & 106 & 0 & - & MRMW9S-W-27629 \\
\hline MW9S & $38.83-53.83$ & $4 / 23 / 09$ & 21.9 & 58.5 & 5.5 & L & 46.33 & 16.0 & 7.17 & 669 & 5.99 & -65 & 0.07 & - & MRMW9S-W-27661 \\
\hline MW9S & $38.83-53.83$ & $9 / 4 / 09$ & 19.95 & 58.5 & 5 & L & 46.33 & 17.3 & 7.18 & 684 & 5.33 & 52 & 0.03 & - & MRMW9S-W-29951 \\
\hline MW9S & $38.83-53.83$ & $4 / 6 / 10$ & 16.5 & 58.5 & 6 & $\mathrm{~L}$ & 46.33 & 15.3 & 7.19 & 650 & 5.50 & - & -9.7 & 0.02 & MRMW9S-W-29991 \\
\hline MW9S & $38.83-53.83$ & $9 / 22 / 10$ & 21.15 & 53.83 & 7 & L & 46.33 & 17.1 & 7.12 & 704 & 6.07 & - & 151 & 0 & MRMW9S-W-30019 \\
\hline MW10S & $30-45$ & $3 / 21 / 06$ & 12.3 & 49.6 & 19 & gal & - & 6.3 & 7.11 & 701 & 2.10 & 88 & 0.01 & 40 & MRMW10S-W-19999 \\
\hline MW10S & $30-45$ & 9/18/06 & 11.08 & 49.61 & 20 & gal & - & 14.3 & 7.17 & 701 & 0.04 & 24 & 0.08 & 60 & MRMW10S-W-22489 \\
\hline MW10S & $30-45$ & $3 / 21 / 07$ & 10.77 & 49.61 & 20 & gal & - & 14.5 & 6.51 & 720 & 0.88 & 11 & 0 & 30 & MRMW10S-W-16485 \\
\hline MW10S & $30-45$ & $10 / 1 / 07$ & 6.95 & 49.65 & 20 & gal & - & 16.3 & 6.97 & 664 & 0.35 & 248 & 0.04 & 35 & MRMW10S-W-16593 \\
\hline MW10S & $30-45$ & $4 / 14 / 08$ & 9.82 & 49.7 & 1.9 & $\mathrm{~L}$ & - & 16.0 & 7.25 & 723 & 1.25 & 181 & 0 & - & MRMW10S-W-23240 \\
\hline MW10S & $30-45$ & $10 / 20 / 08$ & 13.7 & 49.7 & 5.4 & L & 37.5 & 14.3 & 7.03 & 710 & 1.02 & 56 & 0 & - & MRMW10S-W-27630 \\
\hline MW10S & $30-45$ & $4 / 23 / 09$ & 13.6 & 45 & 7.5 & L & 37.5 & 15.1 & 7.05 & 668 & 1.78 & -57 & 0.07 & - & MRMW10S-W-27662 \\
\hline MW10S & $30-45$ & 9/3/09 & 12 & 49.7 & 7.5 & $\mathrm{~L}$ & 37.5 & 14.3 & 7.59 & 731 & 2.03 & 86 & 0.07 & - & MRMW10S-W-29952 \\
\hline MW10S & $30-45$ & $4 / 6 / 10$ & 7.5 & 49.7 & 9 & L & 37.5 & 16.1 & 7.06 & 649 & 2.91 & - & 221 & 0 & MRMW10S-W-29992 \\
\hline MW10S & $30-45$ & $9 / 22 / 10$ & 11.69 & 49.7 & 11 & L & 37.5 & 16.7 & 7.29 & 723 & 5.05 & - & 131 & 0 & MRMW10S-W-30020 \\
\hline MW11S & $53-68$ & $3 / 22 / 06$ & 35.2 & 72.5 & 20 & gal & - & 14.8 & 7.33 & 762 & 9.40 & 237 & 0.06 & 30 & MRMW11S-W-20001 \\
\hline MW11S & $53-68$ & 9/19/06 & 36 & 73.14 & 20 & gal & - & 13.0 & 7.24 & 764 & 1.42 & 158 & 0.02 & 30 & MRMW11S-W-22491 \\
\hline MW11S & $53-68$ & $3 / 20 / 07$ & 34.65 & 73.14 & 20 & gal & - & 14.6 & 6.33 & 782 & 3.90 & 76 & 0 & 30 & MRMW11S-W-16479 \\
\hline MW11S & $53-68$ & $10 / 1 / 07$ & 31.55 & 73 & 20 & gal & - & 16.4 & 6.49 & 624 & 6.57 & 241 & 0.04 & 35 & MRMW11S-W-16594 \\
\hline MW11S & $53-68$ & $4 / 15 / 08$ & 29.9 & 72.7 & 5.5 & $\mathrm{~L}$ & - & 13.9 & 7.30 & 785 & 6.14 & 152 & 0 & - & MRMW11S-W-23241 \\
\hline MW11S & $53-68$ & $4 / 22 / 08$ & 30.2 & 72.7 & 7.2 & L & - & 15.1 & 7.25 & 790 & 6.22 & 163 & - & - & MRMW11S-W-23261 \\
\hline MW11S & $53-68$ & $10 / 20 / 08$ & 37.1 & 72.7 & 9 & L & 60.5 & 14.3 & 7.16 & 756 & 8.95 & 104 & 0 & - & MRMW11S-W-27631 \\
\hline MW11S & $53-68$ & $10 / 21 / 08$ & - & - & TWV & - & - & 14.8 & 7.19 & 766 & - & - & 0 & - & MRMW11S-W-27651 \\
\hline MW11S & $53-68$ & $4 / 23 / 09$ & 38.1 & 72.7 & 5 & $\mathrm{~L}$ & 60.5 & 16.5 & 7.19 & 722 & 9.03 & -62 & 0.09 & - & MRMW11S-W-27663 \\
\hline MW11S & $53-68$ & $9 / 3 / 09$ & 34.7 & 72.7 & 7.5 & $\mathrm{~L}$ & 60.5 & 13.9 & 7.63 & 777 & 9.35 & 102 & 0.05 & - & MRMW11S-W-29953 \\
\hline MW11S & $53-68$ & $9 / 4 / 09$ & 34.95 & 72.7 & 72 & L & 67 & 16.2 & 6.69 & 721 & 8.78 & 100 & - & - & MRMW11S-W-29973 \\
\hline MW11S & $53-68$ & $4 / 6 / 10$ & 29.45 & 72.7 & 6.5 & L & 60.5 & 15.0 & 7.14 & 700 & 7.20 & - & -20.1 & 0 & MRMW11S-W-29993 \\
\hline MW11S & $53-68$ & $9 / 23 / 10$ & 34.75 & 72.7 & 7 & L & 60.5 & 15.7 & 7.40 & 756 & 12.62 & - & 179 & 0.33 & MRMW11S-W-30021 \\
\hline Isch & - & $2 / 19 / 04$ & - & - & Pump $^{d}$ & - & - & - & - & - & - & - & - & - & MRJR-W-16502 \\
\hline Isch & - & 9/14/05 & - & - & Pump & - & - & 20.4 & 6.73 & 2300 & - & - & - & - & MRPRISCH-W-16513 \\
\hline Isch & - & $3 / 23 / 06$ & - & - & 20 & gal & - & 13.0 & 7.23 & 9400 & - & - & - & - & MRISCH-W-19989 \\
\hline Isch & - & $9 / 19 / 06$ & - & - & Pump & - & - & - & - & - & - & - & - & - & MRISCH-W-16531 \\
\hline Isch & - & $3 / 22 / 07$ & - & - & Pump & - & - & - & - & - & - & - & - & - & MRISCH-W-16564 \\
\hline Isch & - & $10 / 3 / 07$ & - & - & Pump & - & - & - & - & - & - & - & - & - & MRISCH-W-16590 \\
\hline Isch & - & $4 / 15 / 08$ & - & - & Pump & - & - & 12.6 & 7.33 & 3160 & - & - & 0.28 & - & MRISCH-W-23242 \\
\hline Isch & - & $10 / 21 / 08$ & - & - & Pump & - & - & - & - & - & - & - & - & - & MRISCH-W-27632 \\
\hline Isch & - & $4 / 22 / 09$ & - & - & Pump & - & - & 15.3 & 6.70 & 2389 & - & - & 0.04 & - & MRISCH-W-27664 \\
\hline Isch & - & $9 / 2 / 09$ & - & - & Pump & - & - & 13.9 & 7.18 & 2600 & - & - & - & - & MRISCH-W-29954 \\
\hline
\end{tabular}


TABLE 3.3 (Cont.)

\begin{tabular}{|c|c|c|c|c|c|c|c|c|c|c|c|c|c|c|c|}
\hline Location & $\begin{array}{l}\text { Screen } \\
\text { Interval } \\
\text { (ft BGL) }\end{array}$ & $\begin{array}{l}\text { Sample } \\
\text { Date }\end{array}$ & $\begin{array}{l}\text { Depth to } \\
\text { Water } \\
\text { (ft TOC) }\end{array}$ & $\begin{array}{l}\text { Depth of } \\
\text { well } \\
\text { (ft TOC) }\end{array}$ & $\begin{array}{l}\text { Volume } \\
\text { Purged }\end{array}$ & $\begin{array}{l}\text { Purge } \\
\text { Units }\end{array}$ & $\begin{array}{l}\text { Pump } \\
\text { Intake } \\
\text { Position } \\
\text { (ft BGL) }\end{array}$ & $\begin{array}{c}\text { Temperature } \\
\left({ }^{\circ} \mathrm{C}\right)\end{array}$ & $\mathrm{pH}$ & $\begin{array}{c}\text { Conductivity } \\
(\mu \mathrm{S} / \mathrm{cm})\end{array}$ & $\begin{array}{c}\text { Dissolved } \\
\text { Oxygen } \\
\text { (mg/L) }\end{array}$ & $\begin{array}{l}\text { ORP } \\
(\mathrm{mV})\end{array}$ & $\begin{array}{l}\text { Iron II } \\
(\mathrm{mg} / \mathrm{L})\end{array}$ & $\begin{array}{c}\text { Carbon } \\
\text { Dioxide } \\
\text { (mg/L) }\end{array}$ & Sample \\
\hline Isch & - & $4 / 7 / 10$ & - & - & Pump & - & - & 11.8 & 6.86 & 2326 & - & - & - & 0.05 & MRISCH-W-29994 \\
\hline Rillinger & - & $6 / 4 / 04$ & - & - & Pump & - & - & 15.9 & 6.99 & 2450 & - & - & - & - & MRPRIVRIL-W-16471 \\
\hline Rillinger & - & 9/14/05 & - & - & Pump & - & - & - & - & - & - & - & - & - & MRPRILL-W-16512 \\
\hline Rillinger & - & $3 / 19 / 06$ & - & - & Pump & - & - & 11.9 & 7.05 & 2550 & - & - & - & - & MRRILINGER-W-19988 \\
\hline Rillinger & - & 9/19/06 & - & - & Pump & - & - & - & - & - & - & - & - & - & MRRILI-W-16530 \\
\hline Rillinger & - & $3 / 29 / 07$ & - & - & Pump & - & - & - & - & - & - & - & - & - & MRRILINGER-W-16561 \\
\hline Rillinger & - & $10 / 3 / 07$ & - & - & Pump & - & - & - & - & - & - & - & - & - & MRRILINGER-W-16591 \\
\hline Rillinger & - & $1 / 11 / 08$ & - & - & Pump & - & - & 12.2 & 7.46 & 884 & - & - & - & - & MORIL-W-11108 \\
\hline Rillinger & - & $4 / 15 / 08$ & - & - & Pump & - & - & 12.0 & 7.56 & 868 & - & - & 0 & - & MRRILINGER-W-23243 \\
\hline Rillinger & - & $10 / 21 / 08$ & - & - & Pump & - & - & - & - & - & - & - & - & - & MRRILLINGER-W-27633 \\
\hline Rillinger & - & $4 / 22 / 09$ & - & - & Pump & - & - & 14.5 & 7.14 & 2279 & - & - & 0.01 & - & MRRILLINGER-W-27665 \\
\hline Rillinger & - & $9 / 2 / 09$ & - & - & Pump & - & - & 14.8 & 7.60 & 809 & - & - & - & - & MRRILLINGER-W-29955 \\
\hline Rillinger & - & $4 / 7 / 10$ & - & - & Pump & - & - & 13.1 & 7.08 & 2287 & - & - & - & - & MRRILLINGER-W-29995 \\
\hline Rillinger & - & 9/22/10 & - & - & Pump & - & - & 14.9 & 7.04 & 2337 & - & - & - & 0.07 & MRRILLINGER-W-30023 \\
\hline Stone & $43^{\mathrm{e}}$ & $6 / 4 / 04$ & 23.35 & - & PDS & - & - & 17.1 & 7.35 & 682 & - & - & - & - & MRPRIVSTON-W-16475 \\
\hline Stone & 43 & $9 / 14 / 05$ & 17.18 & 40 & - & _- & - & 17.3 & 6.81 & 638 & - & - & - & - & MRPRSTON-W-16511 \\
\hline Stone & 43 & $3 / 19 / 06$ & 17.42 & 40 & 100 & gal & - & 12.9 & 6.42 & 650 & - & 213 & 0 & - & MRSTONE-W-19987 \\
\hline Stone & 43 & 9/19/06 & 18.55 & 38.8 & 41 & gal & - & 16.7 & 7.12 & 639 & - & - & - & - & MRSTONE-W-16529 \\
\hline Stone & 43 & $3 / 22 / 07$ & 20.62 & 38.8 & 56 & gal & - & 16.7 & 6.58 & 679 & 4.71 & 19 & 0.28 & 35 & MRSTONE-W-16560 \\
\hline Stone & 43 & $10 / 3 / 07$ & $\begin{array}{l}14.6 \\
14.6\end{array}$ & 38.6 & 72 & gal & - & 16.1 & 6.97 & 564 & 7.07 & 225 & 0.07 & 25 & MRSTONE-W-16589 \\
\hline Stone & 43 & $4 / 15 / 08$ & - & 38.86 & - & - & - & 11.3 & 7.45 & 557 & - & - & 0 & - & MRSTONE-W-23244 \\
\hline Stone & 43 & $10 / 21 / 08$ & - & & 5 & gal & - & - & - & - & - & - & - & - & MRSTONE-W-27634 \\
\hline Stone & 43 & $4 / 23 / 09$ & - & - & 5 & gal & - & 13.9 & 7.12 & 588 & - & - & - & - & MRSTONE-W-27666 \\
\hline Stone & 43 & 9/2/09 & - & - & 5 & gal & - & 13.8 & 7.40 & 623 & - & - & - & - & MRSTONE-W-29956 \\
\hline Stone & 43 & $4 / 7 / 10$ & - & - & 5 & gal & - & 10.9 & 6.83 & 468 & - & - & - & - & MRSTONE-W-29996 \\
\hline Stone & 43 & 9/22/10 & - & - & 5 & gal & - & 15.3 & 7.21 & 552 & - & - & - & - & MRSTONE-W-30024 \\
\hline
\end{tabular}

Not measured.

b TWV, three well volumes.

c PDS, purged dry and then sampled.

d The well's dedicated pump was used for sampling. The pump was allowed to run before the sample was collected.

e Total depth. 
TABLE 3.4 Results of analyses at the AGEM Laboratory for volatile organic compounds in surface water and sediment samples collected at Morrill, March 2007 to September 2010. ${ }^{a}$

\begin{tabular}{|c|c|c|c|c|c|c|c|}
\hline \multirow[b]{2}{*}{ Location } & \multirow[b]{2}{*}{ Sample } & \multirow[b]{2}{*}{$\begin{array}{l}\text { Sample } \\
\text { Date }\end{array}$} & \multirow[b]{2}{*}{ Medium } & \multicolumn{4}{|c|}{ Concentration ( $\mu \mathrm{g} / \mathrm{L}$ in water; $\mu \mathrm{g} / \mathrm{kg}$ in sediment) } \\
\hline & & & & $\begin{array}{c}\text { Carbon } \\
\text { Tetrachloride }\end{array}$ & Chloroform & $\begin{array}{l}\text { Methylene } \\
\text { Chloride }\end{array}$ & $\begin{array}{l}\text { Quantitation } \\
\text { Limit }\end{array}$ \\
\hline SM1 & MRSM1-W-16572 & $3 / 22 / 07$ & Water & $N D^{b}$ & ND & ND & 1 \\
\hline SM1 & MRSM1-S-16573 & $3 / 22 / 07$ & Sediment & ND & ND & ND & 10 \\
\hline SM1 & MRSM1-W-16583 & $10 / 8 / 07$ & Water & ND & ND & ND & 1 \\
\hline SM1 & MRSM1-S-16584 & $10 / 8 / 07$ & Sediment & ND & ND & ND & 10 \\
\hline SM1 & MRSM1-W-23254 & $4 / 14 / 08$ & Water & ND & ND & ND & 1 \\
\hline SM1 & MRSM1-S-23254 & $4 / 14 / 08$ & Sediment & ND & ND & ND & 10 \\
\hline SM1 & MRSM1-W-27644 & $10 / 20 / 08$ & Water & ND & ND & ND & 1 \\
\hline SM1 & MRSM1-S-27644 & $10 / 20 / 08$ & Sediment & ND & ND & ND & 10 \\
\hline SM1 & MRSM1-W-27676 & $4 / 22 / 09$ & Water & ND & ND & ND & 1 \\
\hline SM1 & MRSM1-S-27676 & $4 / 22 / 09$ & Sediment & ND & ND & ND & 10 \\
\hline SM1 & MRSM1-W-29966 & $9 / 2 / 09$ & Water & ND & ND & ND & 1 \\
\hline SM1 & MRSM1-S-29966 & $9 / 2 / 09$ & Sediment & ND & ND & ND & 10 \\
\hline SM1 & MRSM1-W-29974 & $4 / 6 / 10$ & Water & ND & ND & ND & 1 \\
\hline SM1 & MRSM1-S-29974 & $4 / 6 / 10$ & Sediment & ND & ND & ND & 10 \\
\hline SM1 & MRSM1-W-30005 & $9 / 22 / 10$ & Water & ND & ND & ND & 1 \\
\hline SM1 & MRSM1-S-30005 & $9 / 22 / 10$ & Sediment & ND & ND & ND & 10 \\
\hline SM2 & MRSM2-W-16574 & $3 / 22 / 07$ & Water & ND & ND & ND & 1 \\
\hline SM2 & MRSM2-S-16575 & $3 / 22 / 07$ & Sediment & ND & ND & ND & 10 \\
\hline SM2 & MRSM2-W-16585 & $10 / 8 / 07$ & Water & ND & ND & ND & 1 \\
\hline SM2 & MRSM2-S-16586 & $10 / 8 / 07$ & Sediment & ND & ND & ND & 10 \\
\hline SM2 & MRSM2-W-23255 & $4 / 14 / 08$ & Water & ND & ND & ND & 1 \\
\hline SM2 & MRSM2-S-23255 & $4 / 14 / 08$ & Sediment & ND & ND & ND & 10 \\
\hline SM2 & MRSM2-W-27645 & $10 / 20 / 08$ & Water & ND & ND & ND & 1 \\
\hline SM2 & MRSM2-S-27645 & $10 / 20 / 08$ & Sediment & ND & ND & ND & 10 \\
\hline SM2 & MRSM2-W-27677 & $4 / 22 / 09$ & Water & ND & ND & ND & 1 \\
\hline SM2 & MRSM2-S-27677 & $4 / 22 / 09$ & Sediment & ND & ND & ND & 10 \\
\hline SM2 & MRSM2-W-29967 & $9 / 2 / 09$ & Water & ND & ND & ND & 1 \\
\hline SM2 & MRSM2-S-29967 & $9 / 2 / 09$ & Sediment & ND & ND & ND & 10 \\
\hline SM2 & MRSM2-W-29975 & $4 / 6 / 10$ & Water & ND & ND & ND & 1 \\
\hline SM2 & MRSM2-S-29975 & $4 / 6 / 10$ & Sediment & ND & ND & ND & 10 \\
\hline SM2 & MRSM2-W-30006 & $9 / 22 / 10$ & Water & ND & ND & ND & 1 \\
\hline SM2 & MRSM2-S-30006 & $9 / 22 / 10$ & Sediment & ND & ND & ND & 10 \\
\hline SM3 & MRSM3-W-16576 & $3 / 22 / 07$ & Water & ND & ND & ND & 1 \\
\hline SM3 & MRSM3-S-16577 & $3 / 22 / 07$ & Sediment & ND & ND & ND & 10 \\
\hline SM3 & MRSM3-W-16587 & $10 / 8 / 07$ & Water & ND & ND & ND & 1 \\
\hline SM3 & MRSM3-S-16588 & $10 / 8 / 07$ & Sediment & ND & ND & ND & 10 \\
\hline SM3 & MRSM3-W-23256 & $4 / 14 / 08$ & Water & ND & ND & ND & 1 \\
\hline SM3 & MRSM3-S-23256 & $4 / 14 / 08$ & Sediment & ND & ND & ND & 10 \\
\hline SM3 & MRSM3-W-27646 & $10 / 20 / 08$ & Water & ND & ND & ND & 1 \\
\hline SM3 & MRSM3-S-27646 & $10 / 20 / 08$ & Sediment & ND & ND & ND & 10 \\
\hline SM3 & MRSM3-W-27678 & $4 / 22 / 09$ & Water & ND & ND & ND & 1 \\
\hline SM3 & MRSM3-S-27678 & $4 / 22 / 09$ & Sediment & ND & ND & ND & 10 \\
\hline SM3 & MRSM3-W-29968 & $9 / 2 / 09$ & Water & ND & ND & ND & 1 \\
\hline SM3 & MRSM3-S-29968 & $9 / 2 / 09$ & Sediment & ND & ND & ND & 10 \\
\hline SM3 & MRSM3-W-29976 & $4 / 6 / 10$ & Water & ND & ND & ND & 1 \\
\hline SM3 & MRSM3-S-29976 & $4 / 6 / 10$ & Sediment & ND & ND & ND & 10 \\
\hline SM3 & MRSM3-W-30007 & $9 / 22 / 10$ & Water & ND & ND & ND & 1 \\
\hline SM3 & MRSM3-S-30007 & $9 / 22 / 10$ & Sediment & ND & ND & ND & 10 \\
\hline
\end{tabular}


TABLE 3.4 (Cont.)

\begin{tabular}{|c|c|c|c|c|c|c|c|}
\hline \multirow[b]{2}{*}{ Location } & \multirow[b]{2}{*}{ Sample } & \multirow[b]{2}{*}{$\begin{array}{l}\text { Sample } \\
\text { Date }\end{array}$} & \multirow[b]{2}{*}{ Medium } & \multicolumn{4}{|c|}{ Concentration ( $\mu \mathrm{g} / \mathrm{L}$ in water; $\mu \mathrm{g} / \mathrm{kg}$ in sediment) } \\
\hline & & & & $\begin{array}{c}\text { Carbon } \\
\text { Tetrachloride }\end{array}$ & Chloroform & $\begin{array}{l}\text { Methylene } \\
\text { Chloride }\end{array}$ & $\begin{array}{l}\text { Quantitation } \\
\text { Limit }\end{array}$ \\
\hline SM4 & MRSM4-W-16578 & $3 / 22 / 07$ & Water & ND & ND & ND & 1 \\
\hline SM4 & MRSM4-S-16579 & $3 / 22 / 07$ & Sediment & ND & ND & ND & 10 \\
\hline SM4 & MRSM4-W-16589 & $10 / 8 / 07$ & Water & ND & ND & ND & 1 \\
\hline SM4 & MRSM4-S-16590 & $10 / 8 / 07$ & Sediment & ND & ND & ND & 10 \\
\hline SM4 & MRSM4-W-23257 & $4 / 14 / 08$ & Water & ND & ND & ND & 1 \\
\hline SM4 & MRSM4-S-23257 & 4/14/08 & Sediment & ND & ND & ND & 10 \\
\hline SM4 & MRSM4-W-27647 & 10/20/08 & Water & ND & ND & ND & 1 \\
\hline SM4 & MRSM4-S-27647 & 10/20/08 & Sediment & ND & ND & ND & 10 \\
\hline SM4 & MRSM4-W-27679 & $4 / 22 / 09$ & Water & ND & ND & ND & 1 \\
\hline SM4 & MRSM4-S-27679 & $4 / 22 / 09$ & Sediment & ND & ND & ND & 10 \\
\hline SM4 & MRSM4-W-29969 & 9/2/09 & Water & ND & ND & ND & 1 \\
\hline SM4 & MRSM4-S-29969 & $9 / 2 / 09$ & Sediment & ND & ND & ND & 10 \\
\hline SM4 & MRSM4-W-29977 & $4 / 6 / 10$ & Water & ND & ND & ND & 1 \\
\hline SM4 & MRSM4-S-29977 & $4 / 6 / 10$ & Sediment & ND & ND & ND & 10 \\
\hline SM4 & MRSM4-W-30008 & 9/22/10 & Water & ND & ND & ND & 1 \\
\hline SM4 & MRSM4-S-30008 & $9 / 22 / 10$ & Sediment & ND & ND & ND & 10 \\
\hline SMB & MRSMB-W-16570 & $3 / 22 / 07$ & Water & ND & ND & ND & 1 \\
\hline SMB & MRSMB-S-16571 & $3 / 22 / 07$ & Sediment & ND & ND & ND & 10 \\
\hline SMB & MRSMB-W-16581 & $10 / 8 / 07$ & Water & ND & ND & ND & 1 \\
\hline SMB & MRSMB-S-16582 & $10 / 8 / 07$ & Sediment & ND & ND & ND & 10 \\
\hline SMB & MRSMB-W-23258 & $4 / 14 / 08$ & Water & ND & ND & ND & 1 \\
\hline SMB & MRSMB-S-23258 & $4 / 14 / 08$ & Sediment & ND & ND & ND & 10 \\
\hline SMB & MRSMB-W-27648 & $10 / 20 / 08$ & Water & ND & ND & ND & 1 \\
\hline SMB & MRSMB-S-27648 & $10 / 20 / 08$ & Sediment & ND & ND & ND & 10 \\
\hline SMB & MRSMB-W-27680 & $4 / 22 / 09$ & Water & ND & ND & ND & 1 \\
\hline SMB & MRSMB-S-27680 & $4 / 22 / 09$ & Sediment & ND & ND & ND & 10 \\
\hline SMB & MRSMB-W-29970 & $9 / 2 / 09$ & Water & ND & ND & ND & 1 \\
\hline SMB & MRSMB-S-29970 & $9 / 2 / 09$ & Sediment & ND & ND & ND & 10 \\
\hline SMB & MRSMB-W-29978 & $4 / 6 / 10$ & Water & ND & ND & ND & 1 \\
\hline SMB & MRSMB-S-29978 & $4 / 6 / 10$ & Sediment & ND & ND & ND & 10 \\
\hline SMB & MRSMB-W-30009 & $9 / 22 / 10$ & Water & ND & ND & ND & 1 \\
\hline SMB & MRSMB-S-30009 & 9/22/10 & Sediment & ND & ND & ND & 10 \\
\hline
\end{tabular}

a Analyses conducted at the AGEM Laboratory by EPA Method 524.2 for surface water samples or by modified EPA Method 5030B/8260B for sediment samples.

b ND, not detected at the instrument detection limit of $0.1 \mu \mathrm{g} / \mathrm{L}$ for surface water samples or $1.0 \mu \mathrm{g} / \mathrm{kg}$ for sediment samples. 
TABLE 3.5 Results of analyses at the AGEM Laboratory for carbon tetrachloride and chloroform in vegetation samples collected at Morrill, October 2006 to July $2010 .^{\text {a }}$

\begin{tabular}{|c|c|c|c|c|c|}
\hline \multirow[b]{2}{*}{ Location } & \multirow[b]{2}{*}{ Sample } & \multirow[b]{2}{*}{$\begin{array}{l}\text { Sample } \\
\text { Date }\end{array}$} & \multirow[b]{2}{*}{ Type } & \multicolumn{2}{|c|}{ Concentration $(\mu \mathrm{g} / \mathrm{kg})$} \\
\hline & & & & $\begin{array}{c}\text { Carbon } \\
\text { Tetrachloride }\end{array}$ & Chloroform \\
\hline MR001 & MR001-B-18959 & 10/14/06 & Branch & $N D^{b}$ & 1.6 \\
\hline MR001 & MR001-L-18958 & 10/14/06 & Leaf & ND & 3.3 \\
\hline MR001 & MR001-B-23173 & $4 / 2 / 07$ & Branch & ND & ND \\
\hline MR001 & MR001-B-23213 & $7 / 26 / 07$ & Branch & ND & ND \\
\hline MR001 & MR001-L-23212 & $7 / 26 / 07$ & Leaf & ND & ND \\
\hline MR001 & MR001-B-16623 & $7 / 24 / 08$ & Branch & ND & ND \\
\hline MR001 & MR001-B-29924 & $8 / 27 / 09$ & Branch & ND & ND \\
\hline MR001 & MR001-B-31929 & $7 / 27 / 10$ & Branch & ND & ND \\
\hline MR001A & MR001A-B-16622 & $7 / 24 / 08$ & Branch & ND & ND \\
\hline MR002 & MR002-B-18961 & $10 / 14 / 06$ & Branch & ND & ND \\
\hline MR002 & MR002-L-18960 & $10 / 14 / 06$ & Leaf & ND & ND \\
\hline MR002 & MR002-B-23174 & $4 / 2 / 07$ & Branch & ND & 1.5 \\
\hline MR002 & MR002-B-23211 & $7 / 26 / 07$ & Branch & ND & ND \\
\hline MR002 & MR002-L-23210 & $7 / 26 / 07$ & Leaf & ND & 1.3 \\
\hline MR002 & MR002-B-16621 & $7 / 24 / 08$ & Branch & ND & ND \\
\hline MR002 & MR002-B-29923 & $8 / 27 / 09$ & Branch & ND & ND \\
\hline MR002 & MR002-B-31928 & $7 / 27 / 10$ & Branch & ND & ND \\
\hline MR003 & MR003-B-18963 & $10 / 14 / 06$ & Branch & ND & 1.5 \\
\hline MR003 & MR003-L-18962 & $10 / 14 / 06$ & Leaf & ND & 1.8 \\
\hline MR003 & MR003-B-23175 & $4 / 2 / 07$ & Branch & ND & ND \\
\hline MR003 & MR003-B-23209 & $7 / 26 / 07$ & Branch & ND & ND \\
\hline MR003 & MR003-L-23208 & $7 / 26 / 07$ & Leaf & ND & 0.8 \\
\hline MR003 & MR003-B-16620 & $7 / 24 / 08$ & Branch & ND & ND \\
\hline MR003 & MR003-B-29922 & $8 / 27 / 09$ & Branch & ND & ND \\
\hline MR003 & MR003-B-31927 & $7 / 27 / 10$ & Branch & ND & ND \\
\hline MR004 & MR004-B-18965 & $10 / 14 / 06$ & Branch & ND & 2.1 \\
\hline MR004 & MR004-L-18964 & $10 / 14 / 06$ & Leaf & ND & ND \\
\hline MR004 & MR004-B-23176 & $4 / 2 / 07$ & Branch & ND & ND \\
\hline MR004 & MR004-B-23205 & $7 / 26 / 07$ & Branch & ND & ND \\
\hline MR004 & MR004-L-23204 & $7 / 26 / 07$ & Leaf & ND & ND \\
\hline MR004 & MR004-B-16619 & $7 / 24 / 08$ & Branch & ND & ND \\
\hline MR004 & MR004-B-29921 & $8 / 27 / 09$ & Branch & ND & ND \\
\hline MR004 & MR004-B-31926 & $7 / 27 / 10$ & Branch & ND & ND \\
\hline MR005 & MR005-B-18967 & $10 / 14 / 06$ & Branch & ND & ND \\
\hline MR005 & MR005-L-18966 & $10 / 14 / 06$ & Leaf & ND & 1.2 \\
\hline MR005 & MR005-B-23177 & $4 / 2 / 07$ & Branch & ND & ND \\
\hline MR005 & MR005-B-23207 & $7 / 26 / 07$ & Branch & ND & ND \\
\hline MR005 & MR005-L-23206 & $7 / 26 / 07$ & Leaf & ND & 1.4 \\
\hline MR005A & MR005-B-16618 & $7 / 24 / 08$ & Branch & ND & ND \\
\hline MR005A & MR005A-B-29920 & 8/27/09 & Branch & ND & ND \\
\hline MR005A & MR005A-B-31925 & $7 / 27 / 10$ & Branch & ND & ND \\
\hline MR006 & MR006-B-18969 & $10 / 14 / 06$ & Branch & ND & 3.8 \\
\hline MR006 & MR006-L-18968 & $10 / 14 / 06$ & Leaf & ND & ND \\
\hline MR006 & MR006-B-23161 & $4 / 2 / 07$ & Branch & ND & ND \\
\hline
\end{tabular}


TABLE 3.5 (Cont.)

\begin{tabular}{|c|c|c|c|c|c|}
\hline \multirow[b]{2}{*}{ Location } & \multirow[b]{2}{*}{ Sample } & \multirow[b]{2}{*}{$\begin{array}{l}\text { Sample } \\
\text { Date }\end{array}$} & \multirow[b]{2}{*}{ Type } & \multicolumn{2}{|c|}{ Concentration $(\mu \mathrm{g} / \mathrm{kg})$} \\
\hline & & & & $\begin{array}{c}\text { Carbon } \\
\text { Tetrachloride }\end{array}$ & Chloroform \\
\hline MR006 & MR006-B-23181 & $7 / 26 / 07$ & Branch & ND & ND \\
\hline MR006 & MR006-L-23180 & $7 / 26 / 07$ & Leaf & ND & 1.3 \\
\hline MR006 & MR006-B-16625 & $7 / 24 / 08$ & Branch & ND & ND \\
\hline MR006 & MR006-B-29930 & $8 / 27 / 09$ & Branch & ND & ND \\
\hline MR006 & MR006-B-31931 & $7 / 27 / 10$ & Branch & ND & ND \\
\hline MR007 & MR007-B-18971 & $10 / 14 / 06$ & Branch & ND & 1.6 \\
\hline MR007 & MR007-L-18970 & 10/14/06 & Leaf & ND & 2.0 \\
\hline MR007 & MR007-B-23162 & $4 / 2 / 07$ & Branch & ND & ND \\
\hline MR007 & MR007-B-23183 & $7 / 26 / 07$ & Branch & 0.1 & ND \\
\hline MR007 & MR007-L-23182 & $7 / 26 / 07$ & Leaf & ND & 1.3 \\
\hline MR007 & MR007-B-16626 & $7 / 24 / 08$ & Branch & ND & ND \\
\hline MR007 & MR007-B-29932 & $8 / 27 / 09$ & Branch & 0.7 & 0.8 \\
\hline MR007 & MR007-B-31932 & $7 / 27 / 10$ & Branch & ND & ND \\
\hline MR008 & MR008-B-18973 & $10 / 14 / 06$ & Branch & ND & 1.3 \\
\hline MR008 & MR008-L-18972 & $10 / 14 / 06$ & Leaf & ND & 2.5 \\
\hline MR008 & MR008-B-23163 & $4 / 2 / 07$ & Branch & ND & 0.8 \\
\hline MR008 & MR008-B-23185 & $7 / 26 / 07$ & Branch & ND & ND \\
\hline MR008 & MR008-L-23184 & $7 / 26 / 07$ & Leaf & ND & ND \\
\hline MR008 & MR008-B-16627 & $7 / 24 / 08$ & Branch & ND & ND \\
\hline MR008 & MR008-B-29933 & 8/27/09 & Branch & ND & ND \\
\hline MR008 & MR008-B-31933 & $7 / 27 / 10$ & Branch & ND & ND \\
\hline MR009 & MR009-B-18975 & $10 / 14 / 06$ & Branch & ND & 0.9 \\
\hline MR009 & MR009-L-18974 & $10 / 14 / 06$ & Leaf & ND & 2.5 \\
\hline MR009 & MR009-B-23165 & $4 / 2 / 07$ & Branch & ND & ND \\
\hline MR009 & MR009-B-23189 & $7 / 26 / 07$ & Branch & ND & ND \\
\hline MR009 & MR009-L-23188 & $7 / 26 / 07$ & Leaf & ND & 1.5 \\
\hline MR009 & MR009-B-16629 & $7 / 24 / 08$ & Branch & ND & ND \\
\hline MR009 & MR009-B-29935 & $8 / 27 / 09$ & Branch & ND & ND \\
\hline MR009 & MR009-B-31935 & $7 / 27 / 10$ & Branch & ND & ND \\
\hline MR010 & MR010-B-18977 & $10 / 14 / 06$ & Branch & ND & 3.4 \\
\hline MR010 & MR010-L-18976 & $10 / 14 / 06$ & Leaf & ND & ND \\
\hline MR010 & MR010-B-23167 & $4 / 2 / 07$ & Branch & ND & ND \\
\hline MR010 & MR010-B-23193 & 7/26/07 & Branch & ND & 0.8 \\
\hline MR010 & MR010-L-23192 & $7 / 26 / 07$ & Leaf & ND & 1.4 \\
\hline MR010 & MR010-B-16631 & $7 / 24 / 08$ & Branch & 0.1 & 1.4 \\
\hline MR010 & MR010-B-29936 & $8 / 27 / 09$ & Branch & ND & ND \\
\hline MR010 & MR010-B-31936 & $7 / 27 / 10$ & Branch & ND & ND \\
\hline MR011 & MR011-B-18979 & 10/14/06 & Branch & ND & 2.2 \\
\hline MR011 & MR011-L-18978 & 10/14/06 & Leaf & ND & 2.6 \\
\hline MR011 & MR011-B-23168 & $4 / 2 / 07$ & Branch & ND & ND \\
\hline MR011 & MR011-B-23195 & $7 / 26 / 07$ & Branch & ND & ND \\
\hline MR011 & MR011-L-23194 & $7 / 26 / 07$ & Leaf & ND & 1.7 \\
\hline MR011 & MR011-B-16632 & $7 / 24 / 08$ & Branch & ND & ND \\
\hline MR011 & MR011-B-29937 & $8 / 27 / 09$ & Branch & ND & ND \\
\hline MR011 & MR011-B-31937 & $7 / 27 / 10$ & Branch & ND & ND \\
\hline MR012 & MR012-B-18981 & 10/14/06 & Branch & ND & 2.1 \\
\hline MR012 & MR012-L-18980 & $10 / 14 / 06$ & Leaf & ND & 2.9 \\
\hline
\end{tabular}


TABLE 3.5 (Cont.)

\begin{tabular}{|c|c|c|c|c|c|}
\hline \multirow[b]{2}{*}{ Location } & \multirow[b]{2}{*}{ Sample } & \multirow[b]{2}{*}{$\begin{array}{l}\text { Sample } \\
\text { Date }\end{array}$} & \multirow[b]{2}{*}{ Type } & \multicolumn{2}{|c|}{ Concentration $(\mu \mathrm{g} / \mathrm{kg})$} \\
\hline & & & & $\begin{array}{c}\text { Carbon } \\
\text { Tetrachloride }\end{array}$ & Chloroform \\
\hline MR012 & MR012-B-23169 & $4 / 2 / 07$ & Branch & ND & ND \\
\hline MR012 & MR012-B-23197 & $7 / 26 / 07$ & Branch & ND & ND \\
\hline MR012 & MR012-L-23196 & $7 / 26 / 07$ & Leaf & ND & 1.4 \\
\hline MR012 & MR012-B-16633 & $7 / 24 / 08$ & Branch & ND & ND \\
\hline MR012 & MR012-B-29938 & $8 / 27 / 09$ & Branch & ND & ND \\
\hline MR012 & MR012-B-31938 & $7 / 27 / 10$ & Branch & ND & ND \\
\hline MR013 & MR013-B-18983 & $10 / 14 / 06$ & Branch & ND & ND \\
\hline MR013 & MR013-L-18982 & $10 / 14 / 06$ & Leaf & ND & 2.2 \\
\hline MR013 & MR013-B-23160 & $4 / 2 / 07$ & Branch & ND & ND \\
\hline MR013 & MR013-B-23179 & $7 / 26 / 07$ & Branch & ND & ND \\
\hline MR013 & MR013-L-23178 & $7 / 26 / 07$ & Leaf & ND & ND \\
\hline MR013 & MR013-B-16624 & $7 / 24 / 08$ & Branch & ND & ND \\
\hline MR013 & MR013-B-29931 & $8 / 27 / 09$ & Branch & ND & ND \\
\hline MR013 & MR013-B-31930 & $7 / 27 / 10$ & Branch & ND & ND \\
\hline MR014 & MR014-B-23164 & $4 / 2 / 07$ & Branch & ND & ND \\
\hline MR014 & MR014-B-23187 & $7 / 26 / 07$ & Branch & 0.3 & ND \\
\hline MR014 & MR014-L-23186 & $7 / 26 / 07$ & Leaf & ND & ND \\
\hline MR014 & MR014-B-16628 & $7 / 24 / 08$ & Branch & ND & ND \\
\hline MR014 & MR014-B-29934 & $8 / 27 / 09$ & Branch & ND & ND \\
\hline MR014 & MR014-B-31934 & $7 / 27 / 10$ & Branch & ND & ND \\
\hline MR015 & MR015-B-23166 & $4 / 2 / 07$ & Branch & ND & 0.8 \\
\hline MR015 & MR015-B-23191 & $7 / 26 / 07$ & Branch & ND & ND \\
\hline MR015 & MR015-L-23190 & $7 / 26 / 07$ & Leaf & ND & ND \\
\hline MR015 & MR015-B-16630 & $7 / 24 / 08$ & Branch & ND & ND \\
\hline MR016 & MR016-B-23170 & $4 / 2 / 07$ & Branch & ND & 1.1 \\
\hline MR016 & MR016-B-23199 & $7 / 26 / 07$ & Branch & ND & ND \\
\hline MR016 & MR016-L-23198 & $7 / 26 / 07$ & Leaf & ND & ND \\
\hline MR016 & MR016-B-16634 & $7 / 24 / 08$ & Branch & ND & ND \\
\hline MR016 & MR016-B-29939 & $8 / 27 / 09$ & Branch & ND & ND \\
\hline MR016 & MR016-B-31939 & $7 / 27 / 10$ & Branch & ND & ND \\
\hline MR017 & MR017-B-23171 & 4/2/07 & Branch & ND & ND \\
\hline MR017 & MR017-B-23203 & $7 / 26 / 07$ & Branch & ND & ND \\
\hline MR017 & MR017-L-23202 & $7 / 26 / 07$ & Leaf & ND & ND \\
\hline MR017 & MR017-B-16635 & $7 / 24 / 08$ & Branch & ND & ND \\
\hline MR017 & MR017-B-29940 & $8 / 27 / 09$ & Branch & 0.1 & ND \\
\hline MR017 & MR017-B-31940 & $7 / 27 / 10$ & Branch & ND & ND \\
\hline MR018 & MR018-B-23172 & $4 / 2 / 07$ & Branch & ND & ND \\
\hline MR018 & MR018-B-23201 & $7 / 26 / 07$ & Branch & ND & ND \\
\hline MR018 & MR018-L-23200 & $7 / 26 / 07$ & Leaf & ND & 3.2 \\
\hline MR018 & MR018-B-16636 & $7 / 24 / 08$ & Branch & ND & ND \\
\hline MR018 & MR018-B-29941 & $8 / 27 / 09$ & Branch & 0.2 & ND \\
\hline MR018 & MR018-B-31941 & $7 / 27 / 10$ & Branch & ND & ND \\
\hline MR019 & MR019-B-16637 & $7 / 24 / 08$ & Branch & 0.1 & ND \\
\hline MR019 & MR019-B-29929 & $8 / 27 / 09$ & Branch & ND & ND \\
\hline MR019 & MR019-B-31924 & $7 / 27 / 10$ & Branch & ND & ND \\
\hline
\end{tabular}


TABLE 3.5 (Cont.)

\begin{tabular}{|c|c|c|c|c|c|}
\hline \multirow[b]{2}{*}{ Location } & \multirow[b]{2}{*}{ Sample } & \multirow[b]{2}{*}{$\begin{array}{l}\text { Sample } \\
\text { Date }\end{array}$} & \multirow[b]{2}{*}{ Type } & \multicolumn{2}{|c|}{ Concentration $(\mu \mathrm{g} / \mathrm{kg})$} \\
\hline & & & & $\begin{array}{c}\text { Carbon } \\
\text { Tetrachloride }\end{array}$ & Chloroform \\
\hline MR020 & MR020-B-20021 & $7 / 24 / 08$ & Branch & ND & ND \\
\hline MR020 & MR020-B-29928 & $8 / 27 / 09$ & Branch & ND & ND \\
\hline MR020 & MR020-B-31923 & $7 / 27 / 10$ & Branch & ND & ND \\
\hline MR021 & MR021-B-20022 & $7 / 24 / 08$ & Branch & ND & ND \\
\hline MR021 & MR021-B-29926 & $8 / 27 / 09$ & Branch & ND & ND \\
\hline MR021 & MR021-B-31920 & $7 / 27 / 10$ & Branch & ND & ND \\
\hline MR022 & MR022-B-20023 & $7 / 24 / 08$ & Branch & ND & ND \\
\hline MR023 & MR023-B-20024 & $7 / 24 / 08$ & Branch & ND & ND \\
\hline MR023 & MR023-B-29925 & $8 / 27 / 09$ & Branch & 0.2 & ND \\
\hline MR023 & MR023-B-31921 & $7 / 27 / 10$ & Branch & ND & ND \\
\hline MR024 & MR024-B-20025 & $7 / 24 / 08$ & Branch & ND & ND \\
\hline MR024 & MR024-B-29927 & $8 / 27 / 09$ & Branch & ND & ND \\
\hline MR024 & MR024-B-31922 & $7 / 27 / 10$ & Branch & ND & ND \\
\hline MR025 & MR025-B-31942 & $7 / 28 / 10$ & Branch & ND & ND \\
\hline MR026 & MR026-B-31943 & $7 / 28 / 10$ & Branch & ND & ND \\
\hline MR027 & MR027-B-31944 & $7 / 28 / 10$ & Branch & ND & ND \\
\hline MR028 & MR028-B-31945 & $7 / 28 / 10$ & Branch & ND & ND \\
\hline MR029 & MR029-B-31946 & $7 / 28 / 10$ & Branch & ND & ND \\
\hline MR030 & MR030-B-31947 & $7 / 28 / 10$ & Branch & ND & ND \\
\hline MR031 & MR031-B-31948 & $7 / 28 / 10$ & Branch & 0.7 & ND \\
\hline MR032 & MR032-B-31949 & $7 / 28 / 10$ & Branch & ND & ND \\
\hline MR033 & MR033-B-31950 & $7 / 28 / 10$ & Branch & ND & ND \\
\hline MR034 & MR034-B-31951 & $7 / 28 / 10$ & Branch & ND & ND \\
\hline MR035 & MR035-B-31952 & $7 / 28 / 10$ & Branch & ND & ND \\
\hline MR036 & MR036-B-31953 & $7 / 28 / 10$ & Branch & ND & ND \\
\hline MR037 & MR037-B-31954 & $7 / 28 / 10$ & Branch & ND & ND \\
\hline MR038 & MR038-B-31955 & $7 / 28 / 10$ & Branch & ND & ND \\
\hline MR039 & MR039-B-31956 & $7 / 28 / 10$ & Branch & ND & ND \\
\hline MR040 & MR040-B-31957 & $7 / 28 / 10$ & Branch & ND & ND \\
\hline MR041 & MR041-B-31958 & $7 / 28 / 10$ & Branch & 2.2 & ND \\
\hline MR042 & MR042-B-31959 & 7/28/10 & Branch & ND & ND \\
\hline
\end{tabular}


TABLE 3.5 (Cont.)

\begin{tabular}{cccccc}
\hline & & & & \multicolumn{2}{c}{ Concentration $(\mu \mathrm{g} / \mathrm{kg})$} \\
\cline { 5 - 6 } Location & Sample & $\begin{array}{c}\text { Sample } \\
\text { Date }\end{array}$ & Type & $\begin{array}{c}\text { Carbon } \\
\text { Tetrachloride }\end{array}$ & Chloroform \\
\hline MR043 & MR043-B-31960 & $7 / 28 / 10$ & Branch & ND & ND \\
MR044 & MR044-B-31961 & $7 / 28 / 10$ & Branch & 0.5 & ND \\
\hline
\end{tabular}

a Analyses conducted at the AGEM Laboratory by modified EPA Method 5021 (headspace analysis on a gas chromatograph with electron capture detection)

b ND, not detected at the method reporting limit of $0.1 \mu \mathrm{g} / \mathrm{kg}$ for carbon tetrachloride or $0.75 \mu \mathrm{g} / \mathrm{kg}$ for chloroform. 
TABLE 3.6 Analytical results for indoor air samples collected at Morrill, August 2010.

\begin{tabular}{|c|c|c|c|}
\hline \multirow[b]{2}{*}{ Street Address } & \multirow[b]{2}{*}{ Location } & \multicolumn{2}{|c|}{ Concentration $^{\mathrm{a}}\left(\mu \mathrm{g} / \mathrm{m}^{3}\right)$} \\
\hline & & $\begin{array}{l}\text { Carbon } \\
\text { Tetrachloride }\end{array}$ & Chloroform \\
\hline \multirow[t]{2}{*}{104 Roxanna Street } & First floor & $N D^{c}$ & 1.1 \\
\hline & Basement & ND & 1.3 \\
\hline 105 Roxanna Street & First floor & ND & 1.0 \\
\hline \multirow[t]{3}{*}{107 Roxanna Street } & Back hallway & ND & ND \\
\hline & Office & ND & ND \\
\hline & Main room & ND & ND \\
\hline \multirow[t]{2}{*}{108 Roxanna Street } & First floor & ND & 3.6 \\
\hline & Basement & ND & 4.6 \\
\hline \multirow[t]{2}{*}{202 Roxanna Street } & First floor & ND & 3.5 \\
\hline & Basement & ND & ND \\
\hline 203 Roxanna Street & First floor & ND & 8.7 \\
\hline 96 Virginia Street & Shop office & ND & 26 \\
\hline \multirow[t]{2}{*}{102 Virginia Street } & First floor & ND & 3.8 \\
\hline & Basement & ND & 4.1 \\
\hline 106 Virginia Street & First floor & ND & 2.2 \\
\hline Ambient, MW1 & & ND & ND \\
\hline
\end{tabular}

a Laboratory analysis by TestAmerica Laboratories, South Burlington, Vermont, by EPA Method TO-15.

b Analysis for radon at Air Chek, Inc., Naples, North Carolina.

c ND, not detected at a method reporting limit of $1.3 \mu \mathrm{g} / \mathrm{m}^{3}$ for carbon tetrachloride or $0.98 \mu \mathrm{g} / \mathrm{m}^{3}$ for chloroform. 


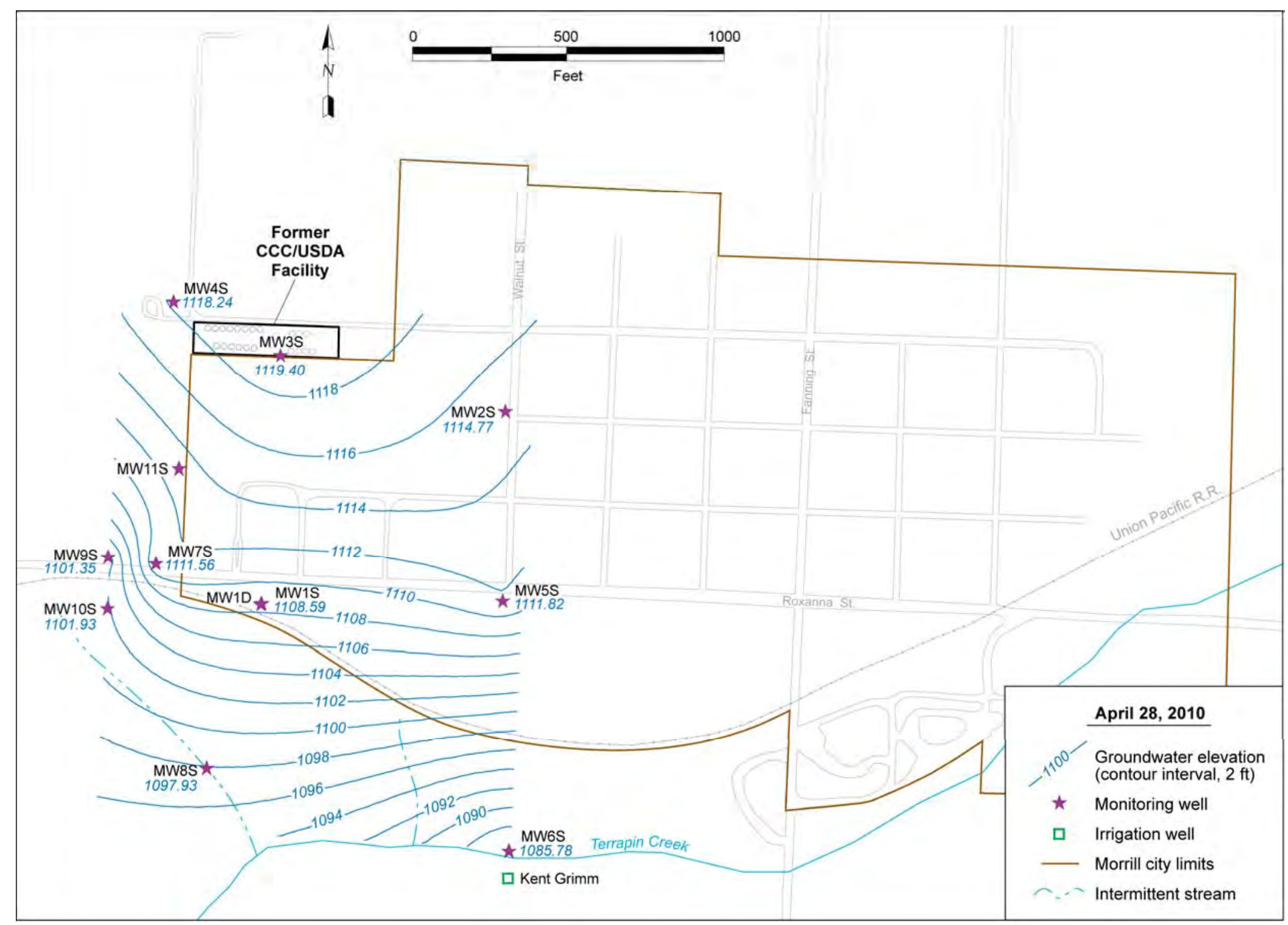

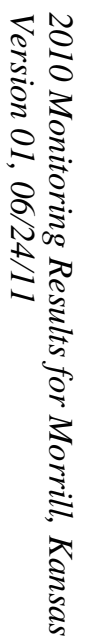

FIGURE 3.1a Potentiometric surface at Morrill, based on water levels measured manually on April 28, 2010. 


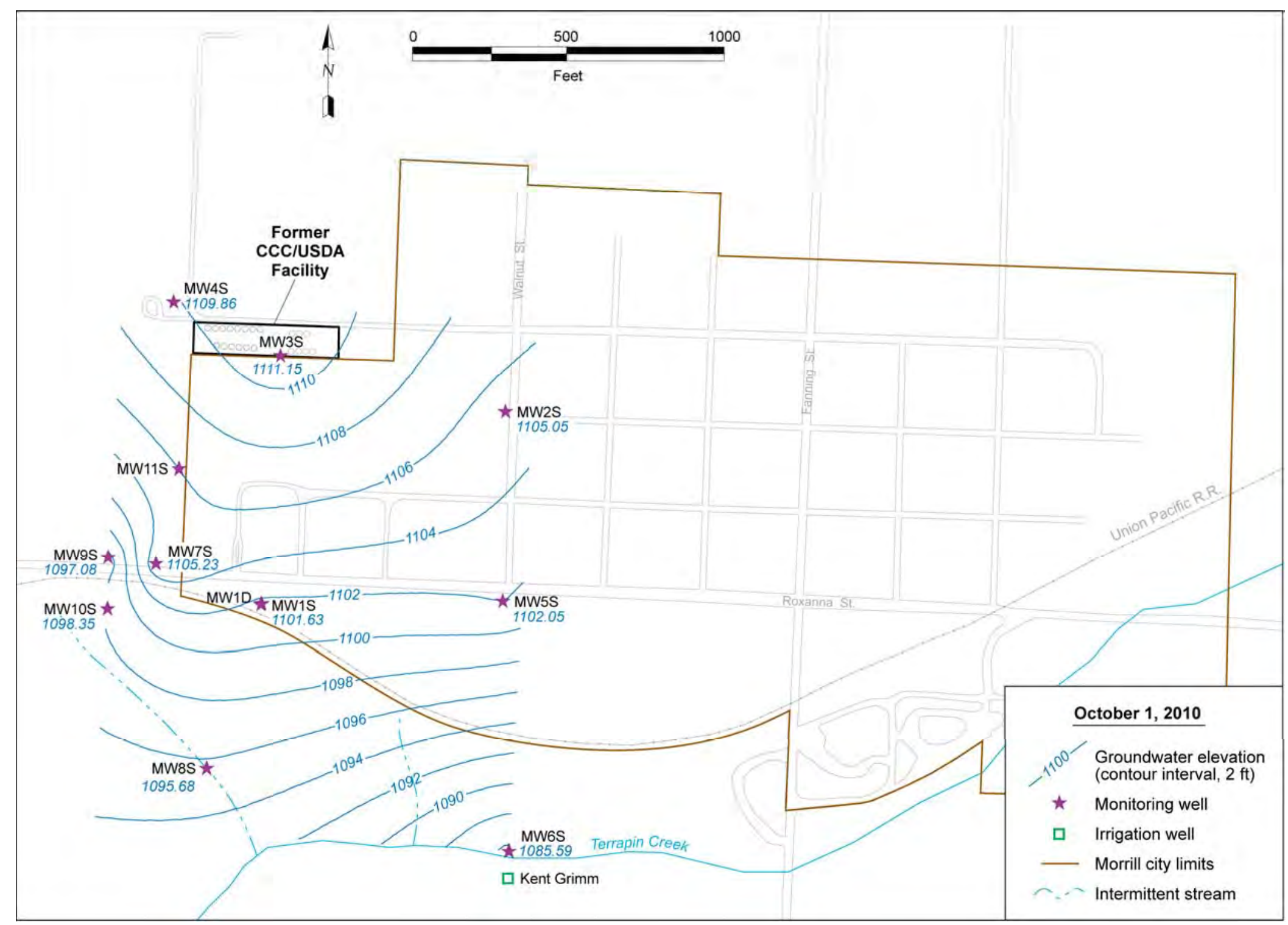

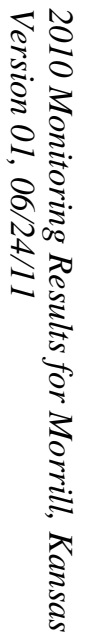

FIGURE 3.1b Potentiometric surface at Morrill, based on water levels measured manually on October 1, 2010. 


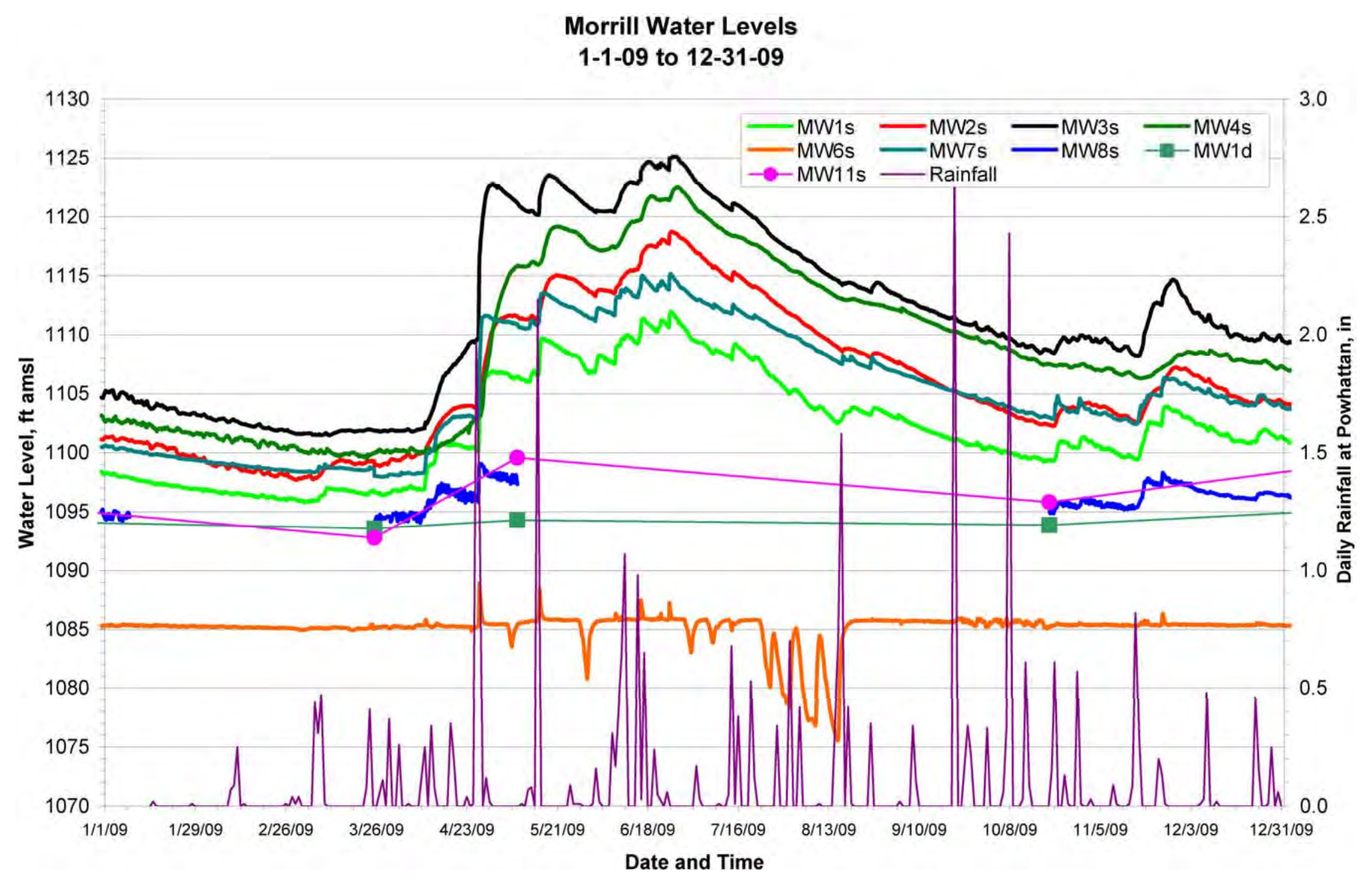

FIGURE 3.2a Hydrographs summarizing results of long-term water level monitoring from January 1, 2009, to December 31, 2009. 


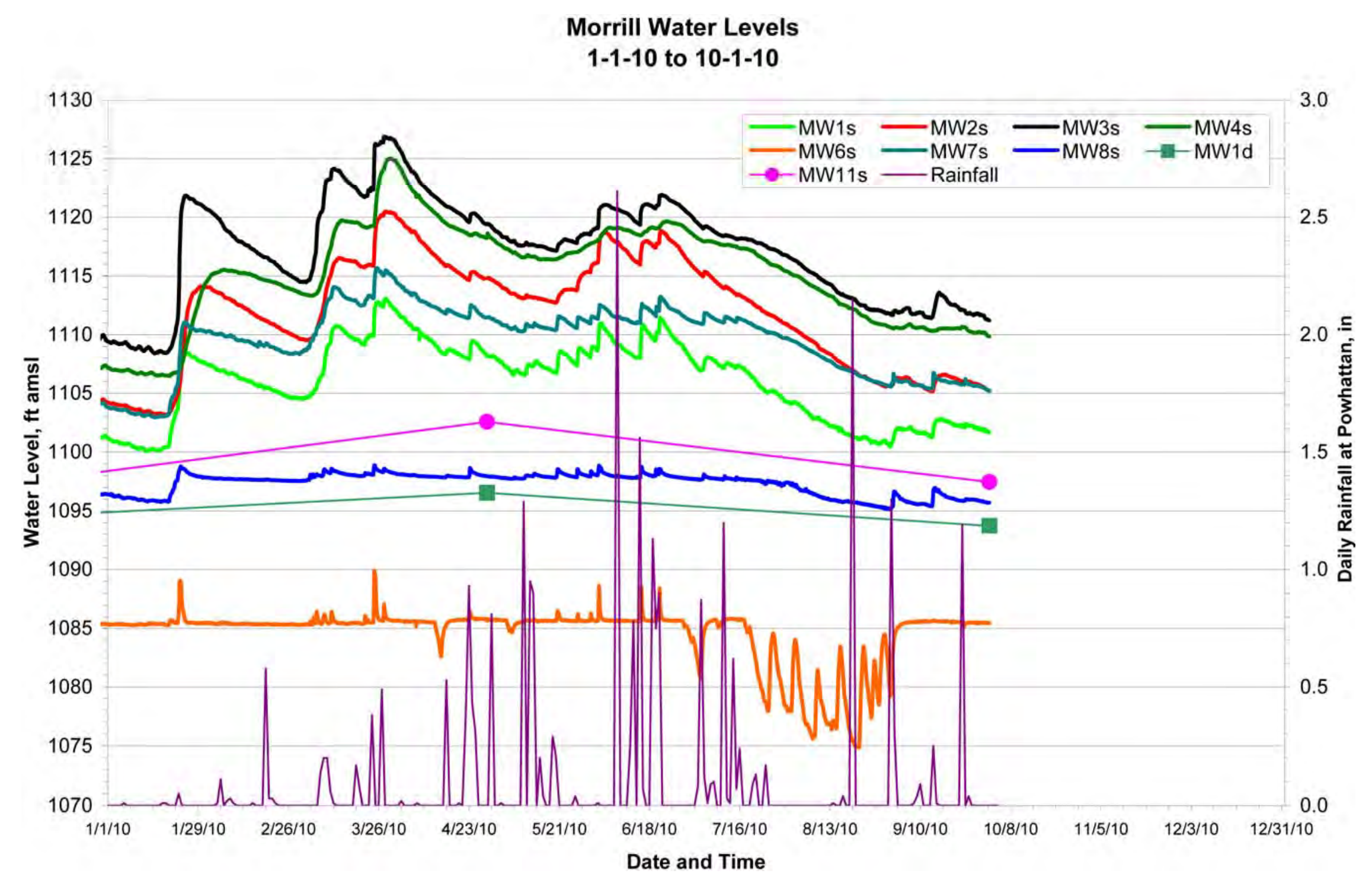

FIGURE 3.2b Hydrographs summarizing results of long-term water level monitoring from January 1, 2010, to October 1, 2010. 


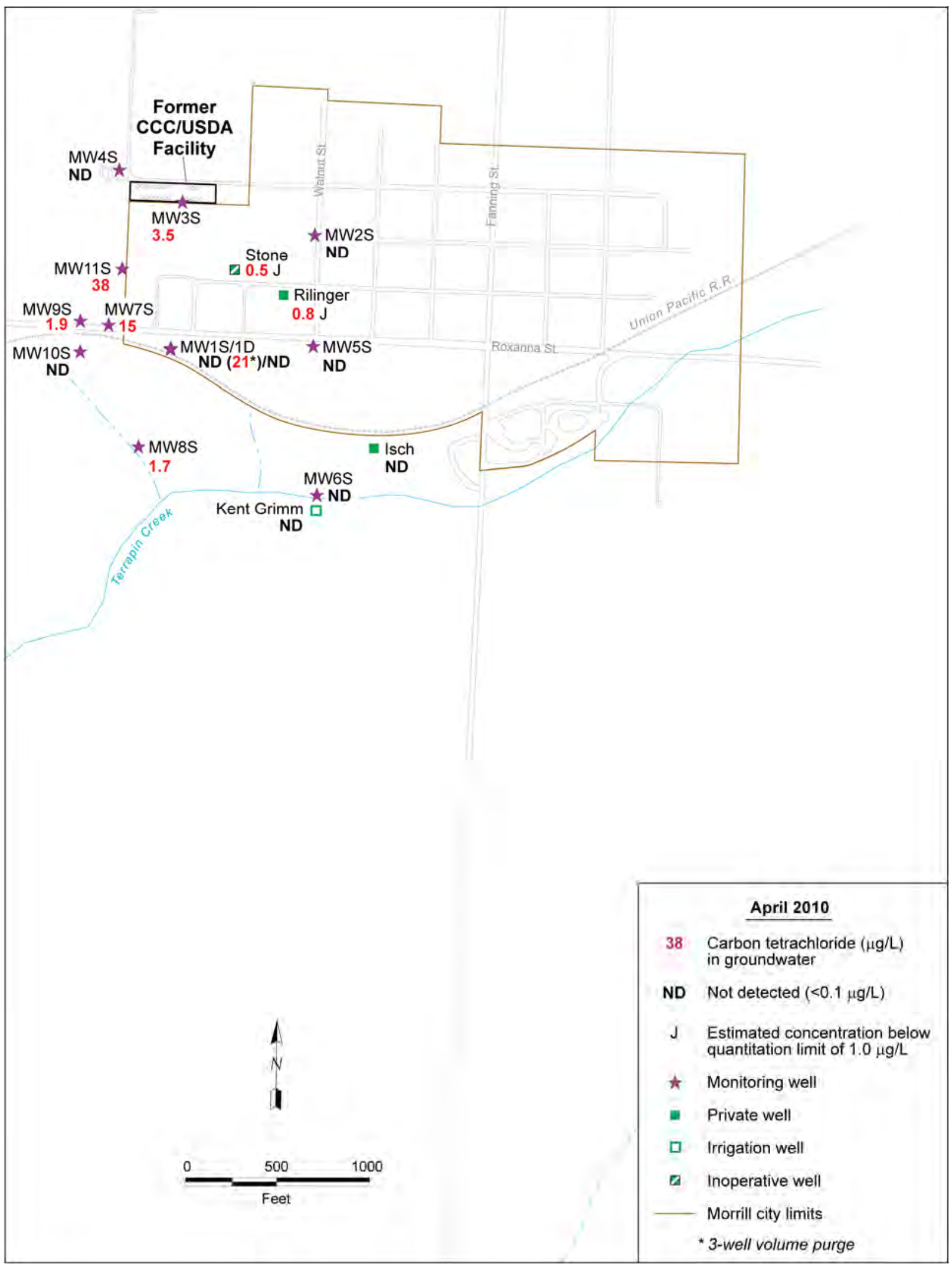

FIGURE 3.3a Carbon tetrachloride concentrations in groundwater at Morrill, April 2010. 


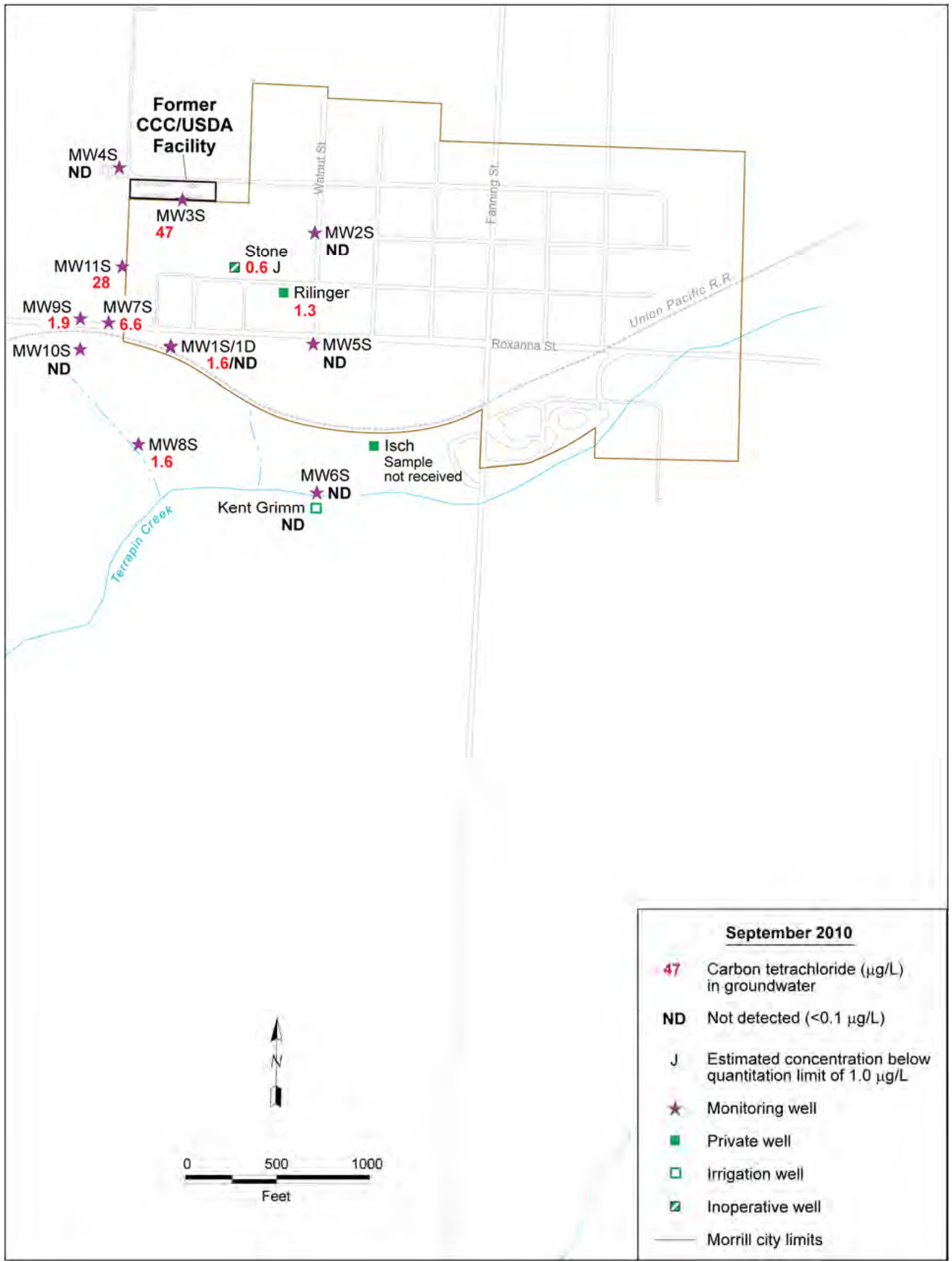

FIGURE 3.3b Carbon tetrachloride concentrations in groundwater at Morrill, September 2010. 


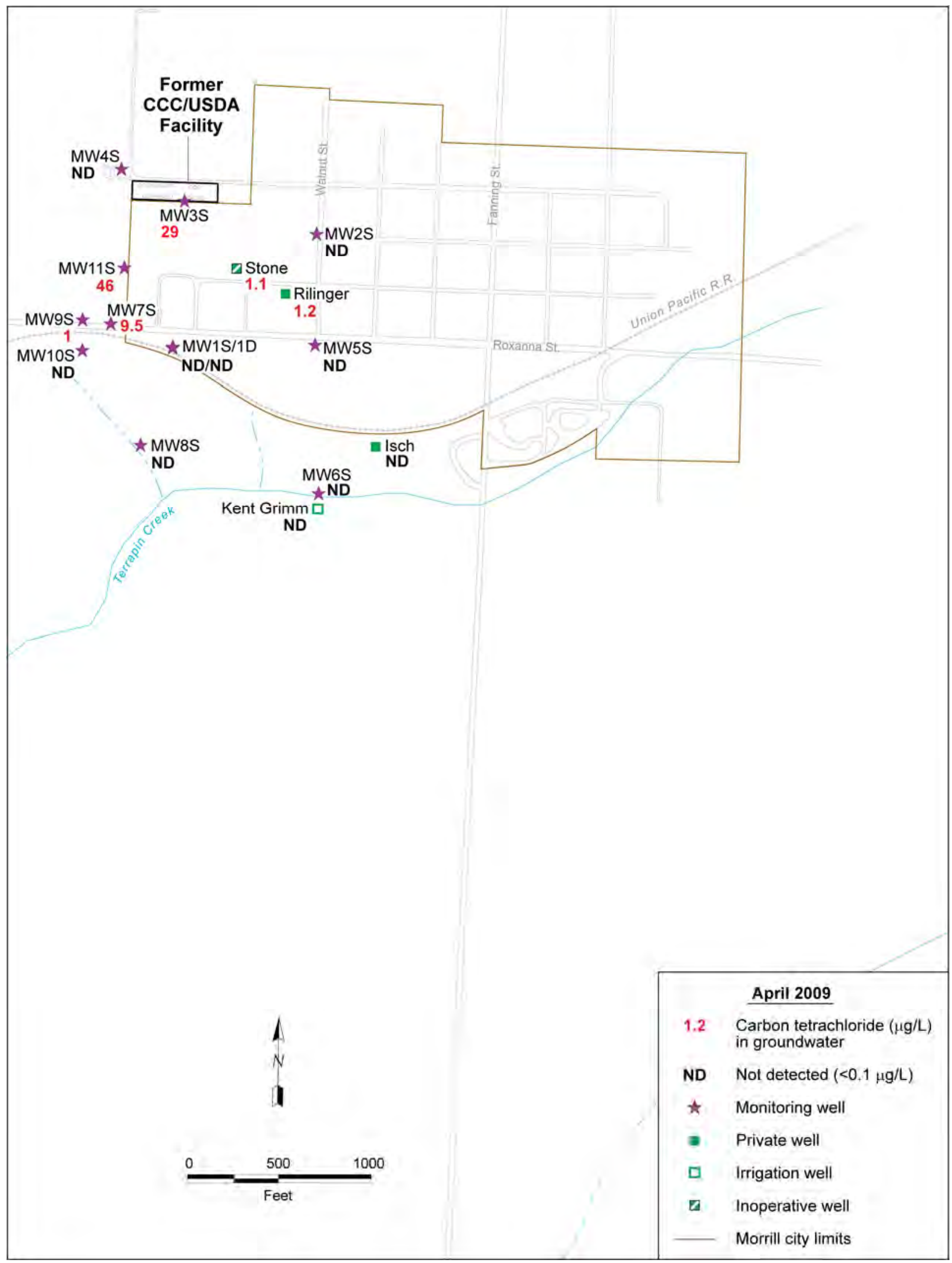

FIGURE 3.4a Carbon tetrachloride concentrations in groundwater at Morrill, April 2009. 


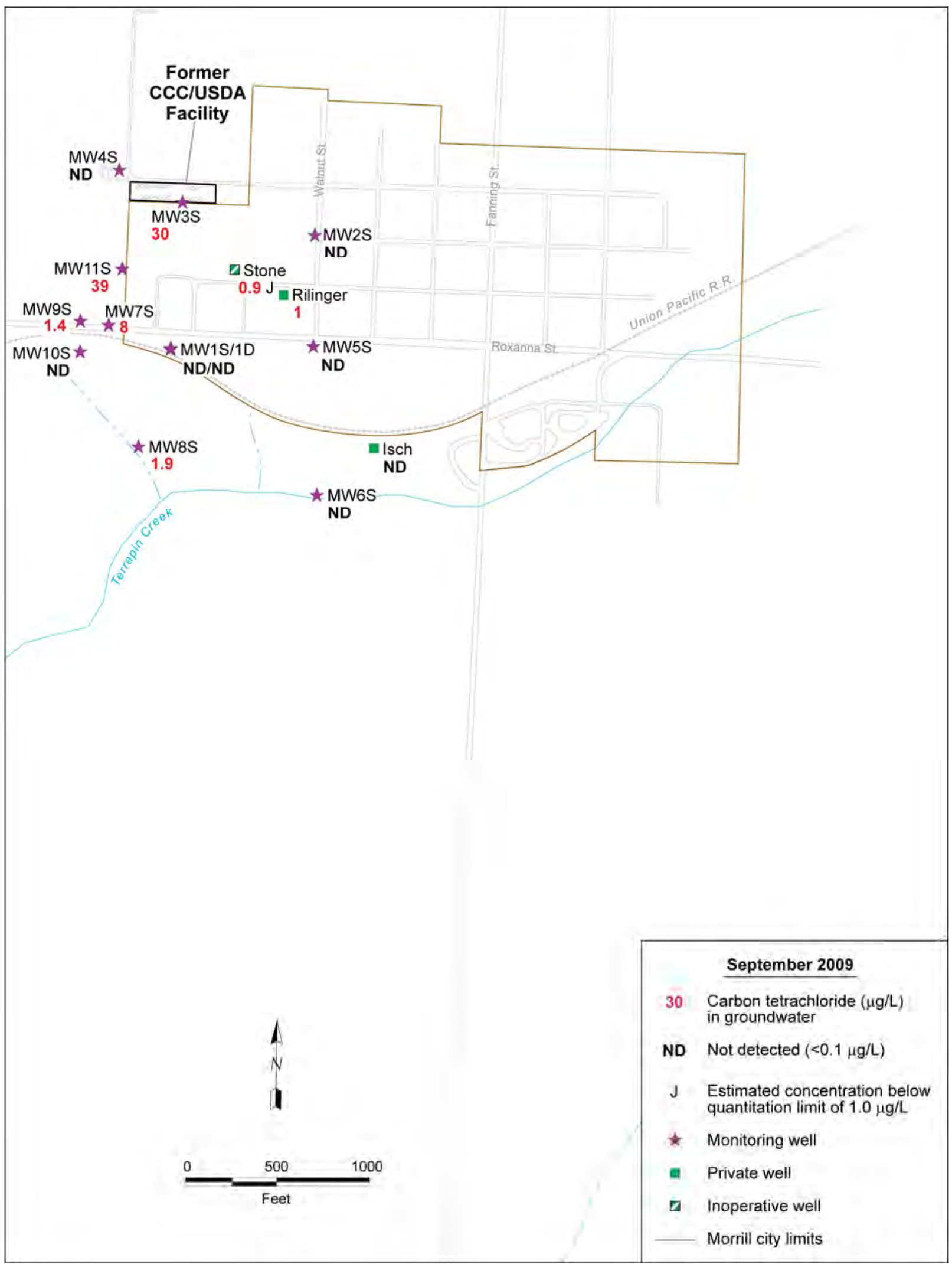

FIGURE 3.4b Carbon tetrachloride concentrations in groundwater at Morrill, September 2009. 


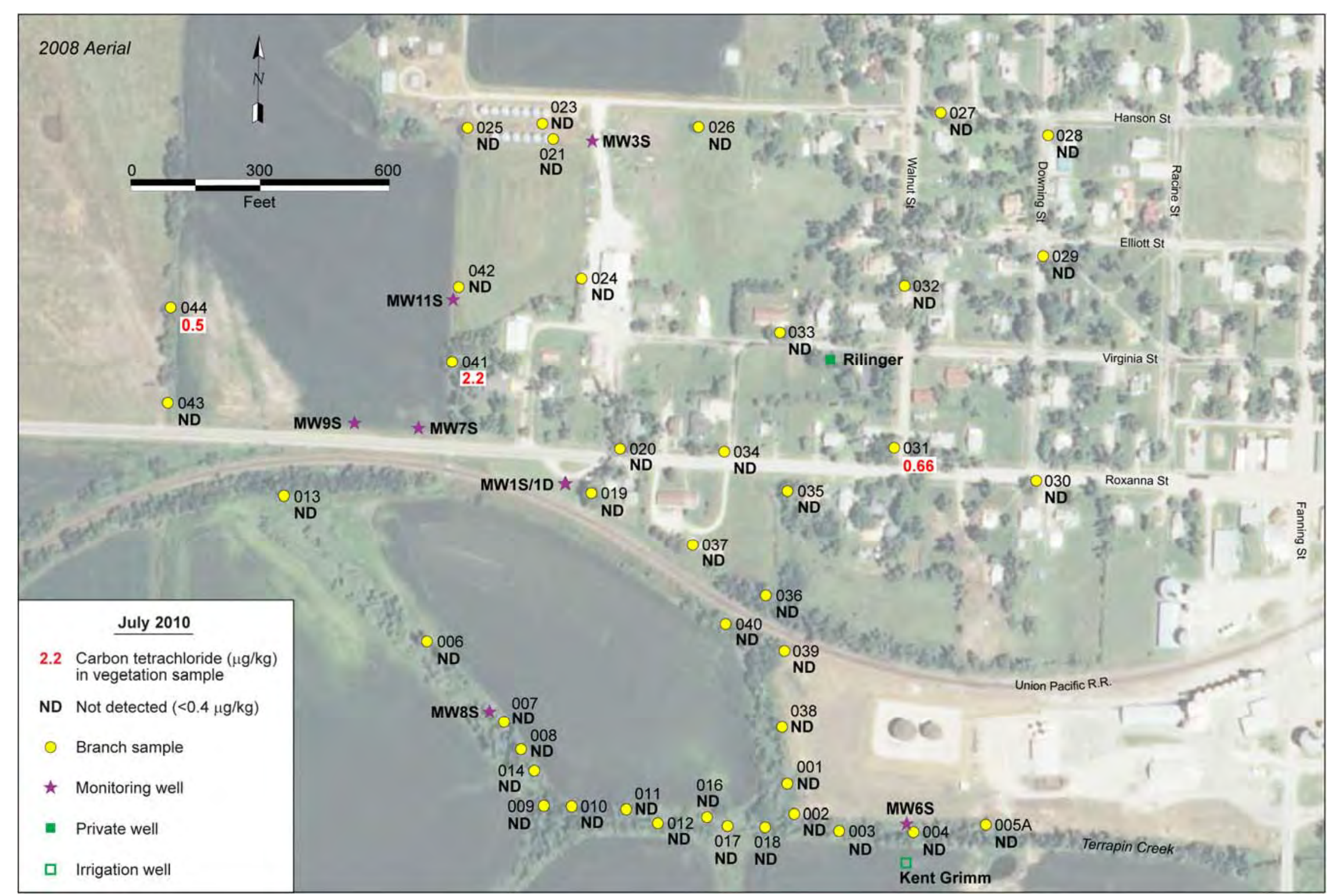

FIGURE 3.5 Carbon tetrachloride concentrations in vegetation at Morrill, July 2010. 


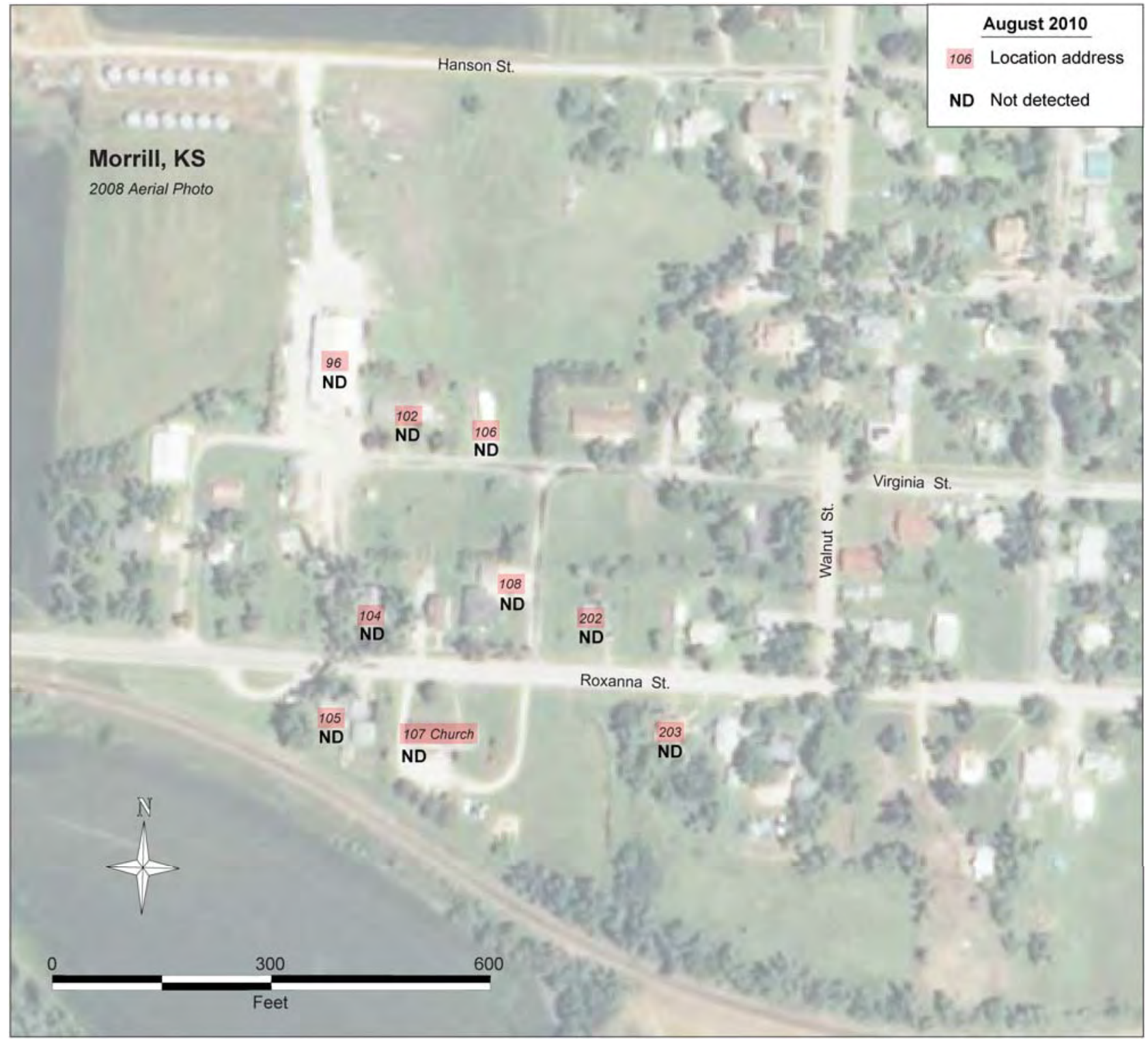

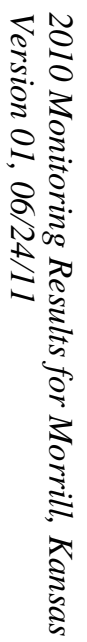

FIGURE 3.6 Carbon tetrachloride concentrations in indoor air at Morrill, August 2010. 


\section{Conclusions and Recommendations}

\subsection{Conclusions}

The findings of the April 2010 and September 2010 monitoring events at Morrill support the following conclusions:

- Groundwater flow during the early spring and the later part of this review period was predominantly to the south, from the vicinity of the former CCC/USDA facility toward Terrapin Creek. In late spring, a slight shift occurred toward the southwest in the immediate vicinity of the intermittent tributary that flows into Terrapin Creek. This shift in the late spring reflected transient seasonal precipitation and recharge that resulted in higher groundwater levels at this time.

- No significant changes were observed in the concentration or distribution of carbon tetrachloride in the groundwater at Morrill during 2010, or in comparison to the results of the spring and fall 2009 monitoring events. A maximum carbon tetrachloride concentration of $47 \mu \mathrm{g} / \mathrm{L}$ was identified in groundwater - at well MW3S — during the September 2010 sampling event.

- No carbon tetrachloride contamination was detected in surface waters or shallow streambed sediments sampled at five locations along Terrapin Creek, downgradient from the former CCC/USDA facility, indicating that Terrapin Creek remains unaffected by the contaminant plume.

- Sampling of tree branch tissues from existing trees for VOCs analyses can be an indicator of shallow subsurface groundwater contamination. Detections of carbon tetrachloride in vegetation at the Morrill site to date have been generally consistent with the documented location of the groundwater plume. In July 2010, trace concentrations of carbon tetrachloride were detected in vegetation samples collected from trees at 3 of the 42 sampled locations south (downgradient) of the former CCC/USDA facility. The low-level detections in vegetation in the absence of detectable contamination in surface water and 
sediment confirm the value of vegetation sampling as an early indicator of contaminant migration.

- A work plan for the investigation of the potential for vapor intrusion into homes along the identified extent of the carbon tetrachloride plume and extending $100 \mathrm{ft}$ laterally was submitted and approved by the KDHE (2010b). Sampling of indoor air was conducted in August 2010. Carbon tetrachloride was not detected in any of the indoor air samples. Low concentrations of chloroform, indicative of indoor air sources, were detected. Additionally, low radon levels were detected.

\subsection{Recommendations}

- Comparisons of the low-flow and three-well-volume purging methods indicate that low-flow sampling provides representative results at the majority of the Morrill monitoring wells, except for MW1S, which is located in the heart of the plume and is screened over a $40-\mathrm{ft}$ interval. Investigation of modified or alternative sampling methods will continue to ensure that representative samples for analysis can be reliably obtained from this well.

- A significant decline in the maximum detected concentration of carbon tetrachloride has been evident since the initial sampling in 1995, when the contaminant was detected in groundwater at the former CCC/USDA facility at $390 \mu \mathrm{g} / \mathrm{L}$. The current maximum levels are $<50 \mu \mathrm{g} / \mathrm{L}$. The accumulated results of 12 monitoring events since 2004 have demonstrated little change in the carbon tetrachloride contamination in terms of migration, concentration, or internal distribution in the affected area. At the request of KDHE (2010c), semi-annual groundwater sampling will continue.

- Because of the consistency of analytical results for vegetation samples collected since 2006 and the very low levels of carbon tetrachloride detected in only a few locations, the frequency of this sampling should be decreased to once every two years. Sampling at the height of the growing season is a sensitive indicator of contaminant migration and uptake by trees. Sampling 
every two years will be protective, in view of the slow contaminant migration rate at Morrill. 


\section{References}

Alvarado, J.S., and C. Rose, 2004, "Static Headspace Analysis of Volatile Organic Compounds in Soil and Vegetation Samples for Site Characterization,” Talanta 62:17-23.

Argonne, 2002, Final Master Work Plan: Environmental Investigations at Former CCC/USDA Facilities in Kansas, 2002 Revision, ANL/ER/TR-02/004, prepared for the Commodity Credit Corporation, U.S. Department of Agriculture, Washington, D.C., by Argonne National Laboratory, Argonne, Illinois, December.

Argonne, 2003, Final Work Plan: Phase I Expedited Site Characterization, Morrill, Kansas, ANL/ER/TR-03/001, prepared for the Commodity Credit Corporation, U.S. Department of Agriculture, Washington, D.C., by Argonne National Laboratory, Argonne, Illinois, September.

Argonne, 2004, Final Phase I-Phase II Interim Report: Expedited Site Characterization, Morrill, Kansas, ANL/ER/TR-04/001, prepared for the Commodity Credit Corporation, U.S. Department of Agriculture, Washington, D.C., by Argonne National Laboratory, Argonne, Illinois, December.

Argonne, 2005a, Final Report, Monitoring Well Installation and Sampling, 2004, at Morrill, Kansas, ANL/ER/TR-04/010, prepared for the Commodity Credit Corporation, U.S. Department of Agriculture, Washington, D.C., by Argonne National Laboratory, Argonne, Illinois, November.

Argonne, 2005b, Final Work Plan: Groundwater Monitoring at Morrill, Kansas, ANL/ER/TR05/003, prepared for the Commodity Credit Corporation, U.S. Department of Agriculture, Washington, D.C., by Argonne National Laboratory, Argonne, Illinois, August.

Argonne, 2006, September 2006 Monitoring Results for Morrill, Kansas, ANL/EVS/AGEM/CHRON-1016, prepared for the Commodity Credit Corporation, U.S. Department of Agriculture, Washington, D.C., by Argonne National Laboratory, Argonne, Illinois, December.

Argonne, 2007a, Final Report: Groundwater Monitoring at Morrill, Kansas, in September 2005 and March 2006, with Expansion of the Monitoring Network in January 2006, 
ANL/EVS/AGEM/TR-06-09, prepared for the Commodity Credit Corporation, U.S. Department of Agriculture, Washington, D.C., by Argonne National Laboratory, Argonne, Illinois, June.

Argonne, 2007b, March-April 2007 Monitoring Results for Morrill, Kansas, ANL/EVS/AGEM/TR-07-06, prepared for the Commodity Credit Corporation, U.S. Department of Agriculture, by Argonne National Laboratory, Argonne, Illinois, May.

Argonne, 2008a, October 2007 Monitoring Results for Morrill, Kansas, ANL/EVS/AGEM/TR08-02, prepared for the Commodity Credit Corporation, U.S. Department of Agriculture, Washington, D.C., by Argonne National Laboratory, Argonne, Illinois, March.

Argonne, 2008b, April 2008 Monitoring Results for Morrill, Kansas, ANL/EVS/AGEM/TR-0815, prepared for the Commodity Credit Corporation, U.S. Department of Agriculture, Washington, D.C., by Argonne National Laboratory, Argonne, Illinois, July.

Argonne, 2009, October 2008 Monitoring Results for Morrill, Kansas, ANL/EVS/AGEM/TR09-03, prepared for the Commodity Credit Corporation, U.S. Department of Agriculture, Washington, D.C., by Argonne National Laboratory, Argonne, Illinois, March.

Argonne, 2010, Annual Report of Monitoring at Morrill, Kansas, ANL/EVS/AGEM/TR-10-04, prepared for the Commodity Credit Corporation, U.S. Department of Agriculture, Washington, D.C., by Argonne National Laboratory, Argonne, Illinois, August.

EPA, 1995, Method 524.2: Measurement of Purgeable Organic Compounds in Water by Capillary Column Gas Chromatography/Mass Spectrometry, Revision 4.1, edited by J.W. Munch, National Exposure Research Laboratory, Office of Research and Development, U.S. Environmental Protection Agency, Cincinnati, Ohio.

GeoCore, 1994a, Work Plan: Phase I Sampling and Site Visit: Brown County Groundwater Investigation, prepared for the Kansas Department of Health and Environment, Bureau of Environmental Remediation, Topeka, Kansas, by GeoCore Services, Inc., Salina, Kansas, April 12. 
GeoCore, 1994b, Well Survey Material: Brown County Groundwater Investigation: Morrill PWS Well \#5 Site, field notes made by GeoCore Services, Inc., Salina, Kansas, during Phase I sampling of private wells, May 19.

GeoCore, 1994c, Work Plan: Phase II Drilling and Sampling: Brown County Groundwater Investigation, prepared for the Kansas Department of Health and Environment, Bureau of Environmental Remediation, Topeka, Kansas, by GeoCore Services, Inc., Salina, Kansas, July 14.

GeoCore, 1994d, Preliminary Report: Phase I Sampling: Brown County Groundwater Investigation, prepared for the Kansas Department of Health and Environment, Bureau of Environmental Remediation, Topeka, Kansas, by GeoCore Services, Inc., Salina, Kansas, July 19.

GeoCore, 1996, Environmental Site Investigation Report: Phase II Drilling and Sampling: Morrill Public Water Supply Well \#5, Morrill, Kansas, prepared for the Kansas Department of Health and Environment, Bureau of Environmental Remediation, Topeka, Kansas, by GeoCore Services, Inc., Salina, Kansas, February 29.

KDHE, 1989, Preliminary Assessment of the Morrill Public Water Supply Well \#5, Morrill, Kansas (Site Identification Number: KS D981710288), Kansas Department of Health and Environment, Bureau of Environmental Remediation, Topeka, Kansas, June 6.

KDHE, 2000, Procedures for Sampling and Analysis of Indoor Air Samples, Standard Operating Procedure BER-33, Bureau of Environmental Remediation, Kansas Department of Health and Environment, Topeka, Kansas, December 27.

KDHE, 2005, letter from C. Carey (Bureau of Environmental Remediation, Kansas Department of Health and Environment, Topeka, Kansas) to C. Roe (Commodity Credit Corporation, U.S. Department of Agriculture, Washington, D.C.) regarding Final Work Plan: Groundwater Monitoring at Morrill, Kansas, October 31.

KDHE, 2007a, letter from C. Carey (Bureau of Environmental Remediation, Kansas Department of Health and Environment, Topeka, Kansas) to C. Roe (Commodity Credit Corporation, 
U.S. Department of Agriculture, Washington, D.C.) regarding draft Morrill sampling report, January 5.

KDHE, 2007b, letter from C. Carey (Bureau of Environmental Remediation, Kansas Department of Health and Environment, Topeka, Kansas) to C. Roe (Commodity Credit Corporation, U.S. Department of Agriculture, Washington, D.C.) regarding March-April 2007 Monitoring Results for Morrill, Kansas, August 1.

KDHE, 2007c, Kansas Vapor Intrusion Guidance: Chemical Vapor Intrusion and Residential Indoor Air, Bureau of Environmental Remediation, Kansas Department of Health and Environment, Topeka, Kansas, June (http://www.kdheks.gov/ber/download/ Ks_VI_ Guidance.pdf).

KDHE, 2008a, letter from E. McWilliams (Bureau of Environmental Remediation, Kansas Department of Health and Environment, Topeka, Kansas) to C. Roe (Commodity Credit Corporation, U.S. Department of Agriculture, Washington, D.C.) regarding April 2008 Monitoring Report for Morrill, Kansas, August 6.

KDHE, 2008b, letter from C. Carey (Bureau of Environmental Remediation, Kansas Department of Health and Environment, Topeka, Kansas) to C. Roe (Commodity Credit Corporation, U.S. Department of Agriculture, Washington, D.C.) regarding revisions to the Morrill sampling report for September 2005 and March 2006, February 6.

KDHE, 2009a, electronic mail message from E. Finzer (Bureau of Environmental Remediation, Kansas Department of Health and Environment, Topeka, Kansas) to C. Roe (Commodity Credit Corporation, U.S. Department of Agriculture, Washington, D.C.), regarding a prioritization list for 2010-2011, October 14.

KDHE, 2009b, letter from E. Finzer (Bureau of Environmental Remediation, Kansas Department of Health and Environment, Topeka, Kansas) to C. Roe (Commodity Credit Corporation, U.S. Department of Agriculture, Washington, D.C.), regarding the CCC/USDA's vapor intrusion site prioritization summary, November 9.

KDHE, 2010a, letter from C. Carey (Bureau of Environmental Remediation, Kansas Department of Health and Environment, Topeka, Kansas) to C. Roe (Commodity Credit Corporation, 
U.S. Department of Agriculture, Washington, D.C.), regarding the plan for indoor air sampling at Everest, March 2.

KDHE, 2010b, letter from M. Townsend (Bureau of Environmental Remediation, Kansas Department of Health and Environment, Topeka, Kansas) to C. Roe (Commodity Credit Corporation, U.S. Department of Agriculture, Washington, D.C.) regarding work plan for indoor air sampling at Morrill, May 6.

KDHE, 2010c, letter from M. Townsend (Bureau of Environmental Remediation, Kansas Department of Health and Environment, Topeka, Kansas) to C. Roe (Commodity Credit Corporation, U.S. Department of Agriculture, Washington, D.C.) regarding Annual Report of Monitoring at Morrill, Kansas, in 2009, September 28.

Puls, R.W., and Barcelona, M.J., 1996, "Low-Flow (Minimal Drawdown) Ground-Water Sampling Procedures,” EPA/540/S-95/504, in Ground Water Issue, Superfund Technology Support Center for Ground Water, National Risk Management Research Laboratory, Ada, Oklahoma, April (www.epa.gov/tio/tsp/download/lwflw2a.pdf).

Yeskis, D., and B. Zavala, 2002, Ground-Water Sampling Guidelines for Superfund and RCRA Project Managers: Ground Water Forum Issue Paper, EPA 542-S-02-001, Technology Innovative Office, Office of Solid Waste and Emergency Response, U.S. Environmental Protection Agency, Washington, D.C., May (http://www.epa.gov/tio/tsp/download/ gw_sampling_guide.pdf). 
Appendix A:

Sampling Activities at Morrill in 2010 
TABLE A.1 Sequence of sampling activities in April-September 2010 at Morrill, Kansas.

\begin{tabular}{|c|c|c|c|c|c|c|c|c|c|}
\hline $\begin{array}{l}\text { Sample } \\
\text { Date }\end{array}$ & Time & Sample & Medium ${ }^{\mathrm{a}}$ & Type $^{a}$ & Location & $\begin{array}{l}\text { Depth } \\
\text { (ft BGL) }\end{array}$ & $\begin{array}{l}\text { Chain of } \\
\text { Custody }\end{array}$ & $\begin{array}{l}\text { Shipping } \\
\text { Date }\end{array}$ & Sample Description \\
\hline \multicolumn{10}{|c|}{ April 2010 groundwater, surface water, and sediment sampling } \\
\hline $4 / 6 / 10$ & $11: 24$ & MRMW7S-W-29989 & Water & MW & MW7S & $20-45$ & 2621 & $4 / 6 / 10$ & $\begin{array}{l}\text { Depth to water }=6.38 \mathrm{ft} \text {. Depth of } 4 \text {-in. } \\
\text { well }=47 \mathrm{ft} \text {. Sample collected by } \\
\text { using low-flow bladder pump after } \\
\text { purgiing of } 5.4 \mathrm{~L} \text {. Pump intake } \\
\text { positioned at } 32.5 \mathrm{ft} \text {. Aliquots } \\
\text { collected for verification analysis. }\end{array}$ \\
\hline $4 / 6 / 10$ & $12: 02$ & MRMW10S-W-29992 & Water & MW & MW10S & $30-45$ & 2621 & $4 / 6 / 10$ & $\begin{array}{l}\text { Depth to water }=7.5 \mathrm{ft} \text {. Depth of } 2 \text {-in. } \\
\text { well }=49.7 \mathrm{ft} . \text { Sample collected by } \\
\text { using low-flow bladder pump after } \\
\text { purgiing of } 9 \mathrm{~L} \text {. Pump intake } \\
\text { positioned at } 37.5 \mathrm{ft} \text {. Aliquots } \\
\text { collected for verification analysis. }\end{array}$ \\
\hline $4 / 6 / 10$ & $12: 08$ & MRMW9S-W-29991 & Water & MW & MW9S & $38.83-53.83$ & 2621 & $4 / 6 / 10$ & $\begin{array}{l}\text { Depth to water }=16.5 \mathrm{ft} \text {. Depth of } 2 \text {-in. } \\
\text { well }=58.5 \mathrm{ft} . \text { Sample collected by } \\
\text { using low-flow bladder pump after } \\
\text { purgiing of } 6 \mathrm{~L} \text {. Pump intake } \\
\text { positioned at } 46.33 \mathrm{ft} \text {. }\end{array}$ \\
\hline $4 / 6 / 10$ & $12: 09$ & MRMW9SDUP-W-29998 & Water & MW & Mw9S & $38.83-53.83$ & 2621 & $4 / 6 / 10$ & $\begin{array}{l}\text { Replicate of sample MRMW9S-W- } \\
29991 .\end{array}$ \\
\hline $4 / 6 / 10$ & $12: 50$ & MRMW11S-W-29993 & Water & MW & MW11S & $53-68$ & 2621 & $4 / 6 / 10$ & $\begin{array}{l}\text { Depth to water }=29.45 \mathrm{ft} \text {. Depth of } 2 \text {-in. } \\
\text { well }=72.7 \mathrm{ft} \text {. Sample collected by } \\
\text { using low-flow bladder pump after } \\
\text { purgiing of } 6.5 \mathrm{~L} \text {. Pump intake } \\
\text { positioned at } 60.5 \mathrm{ft} \text {. }\end{array}$ \\
\hline $4 / 6 / 10$ & $13: 00$ & MRQCIR-W-30000 & Water & RI & $\mathrm{QC}$ & - & 2620 & $4 / 6 / 10$ & $\begin{array}{l}\text { Rinsate of decontaminated sampling line } \\
\text { after collection of sample MRMW11S- } \\
\text { W-29993. }\end{array}$ \\
\hline $4 / 6 / 10$ & $13: 14$ & MRMW8S-W-29990 & Water & MW & MW8S & $10-25$ & 2621 & $4 / 6 / 10$ & $\begin{array}{l}\text { Depth to water }=1.1 \mathrm{ft} \text {. Depth of } 4 \text {-in. } \\
\text { well }=26.8 \mathrm{ft} \text {. Sample collected by } \\
\text { using low-flow bladder pump after } \\
\text { purgiing of } 8 \mathrm{~L} \text {. Pump intake } \\
\text { positioned at } 17.5 \mathrm{ft} \text {. }\end{array}$ \\
\hline $4 / 6 / 10$ & $14: 00$ & MRSM4-W-29977 & Water & SW & SM4 & - & 2620 & $4 / 6 / 10$ & Water sample from Terrapin Creek. \\
\hline $4 / 6 / 10$ & $14: 01$ & MRSM4-S-29977 & Soil & Sed & SM4 & - & 2625 & $4 / 6 / 10$ & Sediment sample from Terrapin Creek. \\
\hline $4 / 6 / 10$ & $14: 06$ & MRTD12-W-29997 & Water & DW & TD12 & $27-67$ & 2621 & $4 / 6 / 10$ & $\begin{array}{l}\text { Collected from Grimm well overflow } \\
\text { before catch pond. }\end{array}$ \\
\hline $4 / 6 / 10$ & $14: 14$ & MRSM2-W-29975 & Water & SW & SM2 & _- & 2620 & $4 / 6 / 10$ & Water sample from Terrapin Creek. \\
\hline $4 / 6 / 10$ & $14: 15$ & MRSM2-S-29975 & Soil & Sed & SM2 & - & 2625 & $4 / 6 / 10$ & Sediment sample from Terrapin Creek. \\
\hline $4 / 6 / 10$ & $14: 22$ & MRSM1-W-29974 & Water & SW & SM1 & - & 2620 & $4 / 6 / 10$ & Water sample from Terrapin Creek. \\
\hline $4 / 6 / 10$ & $14: 23$ & MRSM1-S-29974 & Soil & Sed & SM1 & - & 2625 & $4 / 6 / 10$ & Sediment sample from Terrapin Creek. \\
\hline $4 / 6 / 10$ & $14: 30$ & MRSMB-W-29978 & Water & SW & SMB & - & 2620 & $4 / 6 / 10$ & Water sample from Terrapin Creek. \\
\hline
\end{tabular}


TABLE A.1 (Cont.)

\begin{tabular}{|c|c|c|c|c|c|c|c|c|c|}
\hline $\begin{array}{l}\text { Sample } \\
\text { Date }\end{array}$ & Time & Sample & Medium $^{\mathrm{a}}$ & Type $^{\mathrm{a}}$ & Location & $\begin{array}{c}\text { Depth } \\
\text { (ft BGL) }\end{array}$ & $\begin{array}{l}\text { Chain of } \\
\text { Custody }\end{array}$ & $\begin{array}{l}\text { Shipping } \\
\text { Date }\end{array}$ & Sample Description \\
\hline \multicolumn{10}{|c|}{ April 2010 groundwater, surface water, and sediment sampling (cont.) } \\
\hline $4 / 6 / 10$ & $14: 31$ & MRSMB-S-29978 & Soil & Sed & SMB & - & 2625 & $4 / 6 / 10$ & Sediment sample from Terrapin Creek. \\
\hline $4 / 6 / 10$ & $14: 44$ & MRSM3-W-29976 & Water & SW & SM3 & - & 2620 & $4 / 6 / 10$ & Water sample from Terrapin Creek. \\
\hline $4 / 6 / 10$ & $14: 45$ & MRSM3-S-29976 & Soil & Sed & SM3 & - & 2625 & $4 / 6 / 10$ & Sediment sample from Terrapin Creek. \\
\hline $4 / 6 / 10$ & $14: 48$ & MRMW4S-W-29986 & Water & MW & MW4S & $17-47$ & 2621 & $4 / 6 / 10$ & $\begin{array}{l}\text { Depth to water }=21.8 \mathrm{ft} \text {. Depth of } 4 \text {-in. well } \\
=47.85 \mathrm{ft} \text {. Sample collected by using } \\
\text { bailer after purging of } 5.5 \mathrm{~L} \text {. Pump } \\
\text { intake positioned at } 34.83 \mathrm{ft} \text {. Aliquots } \\
\text { collected for verification analysis. }\end{array}$ \\
\hline $4 / 6 / 10$ & $15: 26$ & MRMW6S-W-29988 & Water & MW & MW6S & $10-25$ & 2621 & $4 / 6 / 10$ & $\begin{array}{l}\text { Depth to water }=6.15 \mathrm{ft} \text {. Depth of } 4 \text {-in. well } \\
=26.9 \mathrm{ft} \text {. Sample collected by using low- } \\
\text { flow bladder pump after purgiing of } 8 \mathrm{~L} \text {. } \\
\text { Pump intake positioned at } 17.5 \mathrm{ft} \text {. }\end{array}$ \\
\hline $4 / 6 / 10$ & $15: 45$ & MRMW3S-W-29985 & Water & MW & MW3S & $18-48$ & 2621 & $4 / 6 / 10$ & $\begin{array}{l}\text { Depth to water }=12.14 \mathrm{ft} \text {. Depth of } 4 \text {-in. } \\
\text { well }=47.80 \mathrm{ft} \text {. Sample collected by } \\
\text { using low-flow bladder pump after } \\
\text { purgiing of } 6 \mathrm{~L} \text {. Pump intake positioned } \\
\text { at } 33 \mathrm{ft} \text {. }\end{array}$ \\
\hline $4 / 6 / 10$ & $16: 45$ & MRMW2S-W-29984 & Water & MW & MW2S & $13-53$ & 2621 & $4 / 6 / 10$ & $\begin{array}{l}\text { Depth to water }=18.57 \mathrm{ft} \text {. Depth of } 4 \text {-in. } \\
\text { well }=53.42 \mathrm{ft} \text {. Sample collected by } \\
\text { using low-flow bladder pump after } \\
\text { purgiing of } 6.5 \mathrm{~L} \text {. Pump intake } \\
\text { positioned at } 36 \mathrm{ft} \text {. }\end{array}$ \\
\hline $4 / 6 / 10$ & $16: 50$ & MRQCTB-W-30003 & Water & TB & $\mathrm{QC}$ & - & 2620 & $4 / 6 / 10$ & $\begin{array}{l}\text { Trip blank sent to the AGEM Laboratory for } \\
\text { organic analysis with water samples } \\
\text { listed on chain-of-custody forms (COCs) } \\
2621 \text { and } 2620 \text {. }\end{array}$ \\
\hline $4 / 6 / 10$ & $17: 09$ & MRMW1D-W-29983 & Water & MW & MW1D & $63-88$ & 2621 & $4 / 6 / 10$ & $\begin{array}{l}\text { Depth to water }=24.7 \mathrm{ft} \text {. Depth of } 4 \text {-in. well } \\
=89 \mathrm{ft} . \text { Sample collected by using low- } \\
\text { flow bladder pump after purgiing of } \\
8.5 \mathrm{~L} \text {. Pump intake positioned at } 75.5 \mathrm{ft} \text {. }\end{array}$ \\
\hline $4 / 7 / 10$ & $11: 15$ & MRMW5S-W-29987 & Water & MW & MW5S & $15-55$ & 3200 & $4 / 7 / 10$ & $\begin{array}{l}\text { Depth to water }=8.32 \mathrm{ft} \text {. Depth of } 4 \text {-in. well } \\
=54.50 \mathrm{ft} \text {. Sample collected by using } \\
\text { low-flow bladder pump after purgiing of } \\
5.5 \mathrm{~L} \text {. Pump intake positioned at } 35 \mathrm{ft} \text {. }\end{array}$ \\
\hline $4 / 7 / 10$ & $11: 43$ & MRMW1SB-W-29979 & Water & MW & MW1S & $11-51$ & 3200 & $4 / 7 / 10$ & $\begin{array}{l}\text { Low-flow pump } 5 \mathrm{ft} \text { above base of screen. } \\
\text { Depth to water }=11.65 \mathrm{ft} \text {. Depth of } 4 \text {-in. } \\
\text { well }=51.31 \mathrm{ft} \text {. Sample collected by } \\
\text { using low-flow bladder pump after } \\
\text { purgiing of } 6 \mathrm{~L} \text {. Pump intake positioned } \\
\text { at } 46.3 \mathrm{ft} \text {. Aliquots collected for } \\
\text { verification analysis. }\end{array}$ \\
\hline
\end{tabular}


TABLE A.1 (Cont.)

\begin{tabular}{|c|c|c|c|c|c|c|c|c|c|}
\hline $\begin{array}{l}\text { Sample } \\
\text { Date }\end{array}$ & Time & Sample & Medium $^{\mathrm{a}}$ & Type $^{a}$ & Location & $\begin{array}{l}\text { Depth } \\
\text { (ft BGL) }\end{array}$ & $\begin{array}{l}\text { Chain of } \\
\text { Custody }\end{array}$ & $\begin{array}{l}\text { Shipping } \\
\text { Date }\end{array}$ & Sample Description \\
\hline
\end{tabular}

\begin{tabular}{|c|c|c|c|c|c|c|c|c|c|}
\hline & & & & & & & & & for verification analysis. \\
\hline $4 / 7 / 10$ & $12: 57$ & MRQCIR-W-30002 & Water & RI & $\mathrm{QC}$ & - & 3200 & $4 / 7 / 10$ & $\begin{array}{l}\text { Rinsate of decontaminated sampling line } \\
\text { after collection of sample MRMW1SM- } \\
\text { W-29980. }\end{array}$ \\
\hline $4 / 7 / 10$ & $13: 25$ & MRMW1ST-W-29981 & Water & MW & MW1S & $11-51$ & 3200 & $4 / 7 / 10$ & $\begin{array}{l}\text { Low-flow pump } 5 \mathrm{ft} \text { below water level. } \\
\text { Depth to water }=11.65 \mathrm{ft} \text {. Depth of } 4 \text {-in. } \\
\text { well }=51.3 \mathrm{ft} \text {. Sample collected by using } \\
\text { low-flow bladder pump after purgiing of } \\
7 \mathrm{~L} \text {. Pump intake positioned at } 16.6 \mathrm{ft} \text {. } \\
\text { Aliquots collected for verification } \\
\text { analysis. }\end{array}$ \\
\hline $4 / 7 / 10$ & $15: 20$ & MRMW1S3X-W-29982 & Water & MW & MW1S & $11-51$ & 3200 & $4 / 7 / 10$ & $\begin{array}{l}\text { Sample collected after purging of three well } \\
\text { volumes. Depth to water }=11.48 \mathrm{ft} \text {. } \\
\text { Depth of } 4 \text {-in. well }=51.3 \mathrm{ft} \text {. Sample } \\
\text { collected by using low-flow bladder } \\
\text { pump after purgiing of } 80 \text { gal. Pump } \\
\text { intake positioned at } 49 \mathrm{ft} \text {. Aliquots } \\
\text { collected for verification analysis. }\end{array}$ \\
\hline $4 / 7 / 10$ & 15:33 & MRISCH-W-29994 & Water & DW & Isch & - & 3200 & $4 / 7 / 10$ & $\begin{array}{l}\text { Let pump run for } 5 \text { min, then sampled. Co- } \\
\text { op had been using the well in the } \\
\text { previous few days. }\end{array}$ \\
\hline $4 / 7 / 10$ & $16: 00$ & MRQCTB-W-30004 & Water & TB & $\mathrm{QC}$ & - & 3200 & $4 / 7 / 10$ & $\begin{array}{l}\text { Trip blank sent to the AGEM Laboratory for } \\
\text { organic analysis with water samples } \\
\text { listed on COC } 3200 \text { and to Test America } \\
\text { for verification organic analysis with } \\
\text { water samples listed on COC } 3180 \text {. }\end{array}$ \\
\hline
\end{tabular}

July 2010 vegetation sampling

7/27/10 12:40 MR021-B-31920

Veg Branch MR021

$2938 \quad 7 / 28 / 10 \quad$ Juniper near Bin 20 
TABLE A.1 (Cont.)

\begin{tabular}{|c|c|c|c|c|c|c|c|c|c|}
\hline $\begin{array}{l}\text { Sample } \\
\text { Date }\end{array}$ & Time & Sample & Medium ${ }^{a}$ & Type $^{a}$ & Location & $\begin{array}{l}\text { Depth } \\
\text { (ft BGL) }\end{array}$ & $\begin{array}{l}\text { Chain of } \\
\text { Custody }\end{array}$ & $\begin{array}{l}\text { Shipping } \\
\text { Date }\end{array}$ & Sample Description \\
\hline
\end{tabular}

July 2010 vegetation sampling (cont.)

\begin{tabular}{|c|c|c|c|c|c|}
\hline $7 / 27 / 10$ & $12: 48$ & MR023-B-31921 & Veg & Branch & MR023 \\
\hline $7 / 27 / 10$ & $12: 51$ & MR024-B-31922 & Veg & Branch & MR024 \\
\hline $7 / 27 / 10$ & $12: 53$ & MR020-B-31923 & Veg & Branch & MR020 \\
\hline $7 / 27 / 10$ & $12: 56$ & MR019-B-31924 & Veg & Branch & MR019 \\
\hline $7 / 27 / 10$ & 13:02 & MR005A-B-31925 & Veg & Branch & MR005A \\
\hline $7 / 27 / 10$ & 13:06 & MR004-B-31926 & Veg & Branch & MR004 \\
\hline $7 / 27 / 10$ & $13: 10$ & MR003-B-31927 & Veg & Branch & MR003 \\
\hline $7 / 27 / 10$ & $13: 12$ & MR002-B-31928 & Veg & Branch & MR002 \\
\hline $7 / 27 / 10$ & $13: 15$ & MR001-B-31929 & Veg & Branch & MR001 \\
\hline $7 / 27 / 10$ & $13: 21$ & MR013-B-31930 & Veg & Branch & MR013 \\
\hline $7 / 27 / 10$ & $13: 30$ & MR006-B-31931 & Veg & Branch & MR006 \\
\hline $7 / 27 / 10$ & $13: 35$ & MR007-B-31932 & Veg & Branch & MR007 \\
\hline $7 / 27 / 10$ & $13: 40$ & MR008-B-31933 & Veg & Branch & MR008 \\
\hline $7 / 27 / 10$ & $13: 45$ & MR014-B-31934 & Veg & Branch & MR014 \\
\hline $7 / 27 / 10$ & $13: 50$ & MR009-B-31935 & Veg & Branch & MR009 \\
\hline $7 / 27 / 10$ & $13: 52$ & MR010-B-31936 & Veg & Branch & MR010 \\
\hline $7 / 27 / 10$ & $13: 57$ & MR011-B-31937 & Veg & Branch & MR011 \\
\hline $7 / 27 / 10$ & $13: 59$ & MR012-B-31938 & Veg & Branch & MR012 \\
\hline $7 / 27 / 10$ & $14: 05$ & MR016-B-31939 & Veg & Branch & MR016 \\
\hline $7 / 27 / 10$ & $14: 05$ & MR017-B-31940 & Veg & Branch & MR017 \\
\hline
\end{tabular}

\begin{tabular}{|c|c|c|}
\hline - & 2938 & $7 / 28 / 10$ \\
\hline - & 2938 & $7 / 28 / 10$ \\
\hline - & 2938 & $7 / 28 / 10$ \\
\hline - & 2938 & $7 / 28 / 10$ \\
\hline - & 2938 & $7 / 28 / 10$ \\
\hline - & 2938 & $7 / 28 / 10$ \\
\hline - & 2938 & $7 / 28 / 10$ \\
\hline- & 2938 & $7 / 28 / 10$ \\
\hline - & 2938 & $7 / 28 / 10$ \\
\hline - & 2938 & $7 / 28 / 10$ \\
\hline- & 2938 & $7 / 28 / 10$ \\
\hline - & 2938 & $7 / 28 / 10$ \\
\hline - & 2938 & $7 / 28 / 10$ \\
\hline- & 2938 & $7 / 28 / 10$ \\
\hline - & 2940 & $7 / 28 / 10$ \\
\hline - & 2940 & $7 / 28 / 10$ \\
\hline- & 2940 & $7 / 28 / 10$ \\
\hline- & 2940 & $7 / 28 / 10$ \\
\hline - & 2940 & $7 / 28 / 10$ \\
\hline - & 2940 & $7 / 28 / 10$ \\
\hline
\end{tabular}

Mulberry near Bin 8. Hackberry gone near Bin 20.

First row of black walnuts over electric fence, southeast of site.

Large sugar maple near house at 104 Roxanna St., close to hydrant.

Large silver maple near pumphouse.

Willow west of the MR005 maple, on north side of creek.

Willow close to creek; still has flagging.

Large willow north side of creek; has

flagging. Multistem. Dying top.

Large willow on north side of creek near bend. Steep bank, eroded.

Large willow near old garbage bonfire.

Headed over railroad tracks to agricultural field access. Large willow in ditch near culvert; has flag.

Large cottonwood; has no flag. North side of creek. Weeds very high. Flooding area.

Large willow has no flag. North side of creek near MW8S. Agricultural field is in corn this year, planted right up to woods at edge of creek.

Large cottonwood next to small willow; no

Large multistem willow northeast of creek.

Three-stem willow east of creek; has flag.

Large elm in thicket east of creek; has flag and stake.

Large multistem Osage orange in thicket; has stake.

Large recumbent willow across creek; from south to north branches. Buried in weeds on north bank.

Two-stemmed willow buried in weeds.

Very small willow on north side slope; has flag. 
TABLE A.1 (Cont.)

\begin{tabular}{|c|c|c|c|c|c|c|c|c|c|}
\hline $\begin{array}{l}\text { Sample } \\
\text { Date }\end{array}$ & Time & Sample & Medium ${ }^{\mathrm{a}}$ & Type $^{\mathrm{a}}$ & Location & $\begin{array}{l}\text { Depth } \\
\text { (ft BGL) }\end{array}$ & $\begin{array}{l}\text { Chain of } \\
\text { Custody }\end{array}$ & $\begin{array}{l}\text { Shipping } \\
\text { Date }\end{array}$ & Sample Description \\
\hline \multicolumn{10}{|c|}{ July 2010 vegetation sampling (cont.) } \\
\hline $7 / 27 / 10$ & $14: 12$ & MR018-B-31941 & Veg & Branch & MR018 & - & 2940 & $7 / 28 / 10$ & $\begin{array}{l}\text { Large multistem Osage orange north side } \\
\text { of creek at bend; has flag. Buried in } \\
\text { thicket. }\end{array}$ \\
\hline $7 / 28 / 10$ & $7: 40$ & MR025-B-31942 & Veg & Branch & MR025 & - & 2940 & $7 / 28 / 10$ & $\begin{array}{l}\text { Large group of mulberries (four trees). } \\
\text { Branch sample from center, largest } \\
\text { trunk, on fencerow west of grain bins. }\end{array}$ \\
\hline $7 / 28 / 10$ & $7: 45$ & MR026-B-31943 & Veg & Branch & MR026 & - & 2940 & $7 / 28 / 10$ & $\begin{array}{l}\text { Large solitary pear tree east of grain bins in } \\
\text { field off Kansas St. }\end{array}$ \\
\hline $7 / 28 / 10$ & $7: 50$ & MR027-B-31944 & Veg & Branch & MR027 & - & 2940 & $7 / 28 / 10$ & $\begin{array}{l}\text { Large white ash in front of old wood-sided } \\
\text { house. }\end{array}$ \\
\hline $7 / 28 / 10$ & $7: 55$ & MR028-B-31945 & Veg & Branch & MR028 & - & 2940 & $7 / 28 / 10$ & $\begin{array}{l}\text { Large black walnut, northeast corner of } \\
\text { Downing and Harrison Sts. }\end{array}$ \\
\hline $7 / 28 / 10$ & $7: 57$ & MR029-B-31946 & Veg & Branch & MR029 & - & 2940 & $7 / 28 / 10$ & $\begin{array}{l}\text { Large green ash on southwest corner of } \\
\text { Downing and Elliot Sts., opposite church } \\
\text { and park. }\end{array}$ \\
\hline $7 / 28 / 10$ & $8: 00$ & MR030-B-31947 & Veg & Branch & MR030 & - & 2940 & $7 / 28 / 10$ & $\begin{array}{l}\text { Large silver maple on south side of } \\
\text { Roxanna; end of Downing St. house } \\
\text { \# 305. }\end{array}$ \\
\hline $7 / 28 / 10$ & $8: 10$ & MR031-B-31948 & Veg & Branch & MR031 & - & 2940 & $7 / 28 / 10$ & $\begin{array}{l}\text { Large black walnut on southwest corner of } \\
\text { Roxanna and Walnut. House \# } 206 \text {. }\end{array}$ \\
\hline $7 / 28 / 10$ & $8: 15$ & MR032-B-31949 & Veg & Branch & MR032 & - & 2940 & $7 / 28 / 10$ & $\begin{array}{l}\text { Large hackberry on west side Walnut St., } \\
\text { south of Elliot St., just south of House } \\
\text { \# } 204 \text {. }\end{array}$ \\
\hline $7 / 28 / 10$ & $8: 18$ & MR033-B-31950 & Veg & Branch & MR033 & - & 2942 & $7 / 28 / 10$ & $\begin{array}{l}\text { Large pin oak in front yard of House \# } 200 \\
\text { on Virginia St., east of Miami St. }\end{array}$ \\
\hline $7 / 28 / 10$ & $8: 25$ & MR034-B-31951 & Veg & Branch & MR034 & - & 2942 & $7 / 28 / 10$ & $\begin{array}{l}\text { Large sugar maple on northwest corner } \\
\text { Roxanna and Miami. House \# } 108 .\end{array}$ \\
\hline $7 / 28 / 10$ & $8: 30$ & MR035-B-31952 & Veg & Branch & MR035 & - & 2942 & $7 / 28 / 10$ & $\begin{array}{l}\text { American elm on fence line east of swale, } \\
\text { south of Roxanna St, east of Miami, } \\
\text { west of House } \# 203 \text {. }\end{array}$ \\
\hline $7 / 28 / 10$ & $8: 35$ & MR036-B-31953 & Veg & Branch & MR036 & - & 2942 & $7 / 28 / 10$ & $\begin{array}{l}\text { Green ash in southeast corner of lot behind } \\
\text { Grace Church. Border of woods on } \\
\text { railroad track. }\end{array}$ \\
\hline $7 / 28 / 10$ & $8: 40$ & MR037-B-31954 & Veg & Branch & MR037 & - & 2942 & $7 / 28 / 10$ & $\begin{array}{l}\text { Austrian pine behind (south of) church; } \\
\text { second tree from east. }\end{array}$ \\
\hline $7 / 28 / 10$ & $8: 45$ & MR038-B-31955 & Veg & Branch & MR038 & - & 2942 & $7 / 28 / 10$ & $\begin{array}{l}\text { Large silver maple on east edge of creek, } \\
\text { south side of railroad track, west of } \\
\text { black tank. }\end{array}$ \\
\hline $7 / 28 / 10$ & $8: 50$ & MR039-B-31956 & Veg & Branch & MR039 & - & 2942 & $7 / 28 / 10$ & $\begin{array}{l}\text { Medium hackberry, edge of thicket, east } \\
\text { side of creek, south of railroad tracks. }\end{array}$ \\
\hline
\end{tabular}


TABLE A.1 (Cont.)

\begin{tabular}{|c|c|c|c|c|c|c|c|c|c|}
\hline $\begin{array}{l}\text { Sample } \\
\text { Date }\end{array}$ & Time & Sample & Medium $^{\mathrm{a}}$ & Type ${ }^{a}$ & Location & $\begin{array}{c}\text { Depth } \\
\text { (ft BGL) }\end{array}$ & $\begin{array}{l}\text { Chain of } \\
\text { Custody }\end{array}$ & $\begin{array}{l}\text { Shipping } \\
\text { Date }\end{array}$ & Sample Description \\
\hline
\end{tabular}

July 2010 vegetation sampling (cont.)

$\begin{array}{llllll}7 / 28 / 10 & 8: 55 & \text { MR040-B-31957 } & \text { Veg } & \text { Branch } & \text { MR040 } \\ 7 / 28 / 10 & 9: 05 & \text { MR041-B-31958 } & \text { Veg } & \text { Branch } & \text { MR041 } \\ 7 / 28 / 10 & 9: 10 & \text { MR042-B-31959 } & \text { Veg } & \text { Branch } & \text { MR042 } \\ 7 / 28 / 10 & 9: 20 & \text { MR043-B-31960 } & \text { Veg } & \text { Branch } & \text { MR043 } \\ 7 / 28 / 10 & 9: 23 & \text { MR044-B-31961 } & \text { Veg } & \text { Branch } & \text { MR044 }\end{array}$

August 2010 indoor air sampling

$\begin{array}{lll}8 / 12 / 10 & 15: 07 & \text { 104 Roxanna St., first floor } \\ 8 / 12 / 10 & 15: 11 & \text { 104 Roxanna St., basement } \\ 8 / 12 / 10 & 15: 29 & \text { 102 Virginia St., first floor } \\ 8 / 12 / 10 & 15: 33 & \text { 102 Virginia St., basement } \\ 8 / 12 / 10 & 13: 04 & \text { 202 Roxanna St., first floor } \\ 8 / 12 / 10 & 13: 06 & \text { 202 Roxanna St., basement } \\ 8 / 12 / 10 & 13: 17 & \text { 203 Roxanna St., first floor } \\ 8 / 12 / 10 & 17: 16 & \begin{array}{l}\text { 107 Roxanna St., main } \\ \text { room }\end{array} \\ 8 / 12 / 10 & 14: 34 & \text { 96 Virginia St., office } \\ 8 / 12 / 10 & 14: 47 & \text { Ambient air, MW1 } \\ 8 / 12 / 10 & 17: 11 & \text { 107 Roxanna St., office } \\ 8 / 12 / 10 & 17: 14 & \begin{array}{l}\text { 107 Roxanna St., back } \\ \text { hallway }\end{array} \\ 8 / 12 / 10 & 15: 39 & \text { 108 Roxanna St., first floor }\end{array}$

Air

$\begin{array}{lllll}\text { Air } & 104 \text { Roxanna St. } & - & \text { TA-1 } & 8 / 12 / 10 \\ \text { Air } & 104 \text { Roxanna St. } & - & \text { TA-1 } & 8 / 12 / 10 \\ \text { Air } & 102 \text { Virginia St. } & - & \text { TA-1 } & 8 / 12 / 10 \\ \text { Air } & 102 \text { Virginia St. } & - & \text { TA-1 } & 8 / 12 / 10 \\ \text { Air } & 202 \text { Roxanna St. } & - & \text { TA-2 } & 8 / 12 / 10 \\ \text { Air } & 202 \text { Roxanna St. } & - & \text { TA-2 } & 8 / 12 / 10 \\ \text { Air } & 203 \text { Roxanna St. } & - & \text { TA-2 } & 8 / 12 / 10 \\ \text { Air } & 107 \text { Roxanna St. } & - & \text { TA-2 } & 8 / 12 / 10 \\ \text { Air } & 96 \text { Virginia St. } & - & \text { TA-3 } & 8 / 12 / 10 \\ \text { Air } & \text { Ambient air } & - & \text { TA-3 } & 8 / 12 / 10 \\ \text { Air } & 107 \text { Roxanna St. } & - & \text { TA-3 } & 8 / 12 / 10 \\ \text { Air } & \text { 107 Roxanna St. } & - & \text { TA-3 } & 8 / 12 / 10 \\ \text { Air } & \text { 108 Roxanna St. } & - & \text { TA-4 } & 8 / 12 / 10\end{array}$

Beginning pressure -28 in. Hg. Ending pressure 3 in. $\mathrm{Hg}$.

Beginning pressure -28 in. Hg. Ending pressure 0 in. $\mathrm{Hg}$.

Beginning pressure -29 in. Hg. Ending pressure 8 in. $\mathrm{Hg}$.

Beginning pressure -28 in. Hg. Ending pressure 6 in. $\mathrm{Hg}$.

Beginning pressure -29 in. Hg. Ending pressure 6 in. $\mathrm{Hg}$.

Beginning pressure $-30 \mathrm{in.} \mathrm{Hg}$. Ending pressure $10 \mathrm{in.} \mathrm{Hg}$.

Beginning pressure -27 in. $\mathrm{Hg}$. Ending pressure 6 in. $\mathrm{Hg}$.

Beginning pressure -28 in. Hg. Ending pressure 7 in. $\mathrm{Hg}$.

Beginning pressure -28 in. Hg. Ending pressure 4 in. $\mathrm{Hg}$.

Beginning pressure -28 in. $\mathrm{Hg}$. Ending pressure $7 \mathrm{in.} \mathrm{Hg}$.

Beginning pressure -28 in. Hg. Ending pressure 6 in. $\mathrm{Hg}$.

Beginning pressure -28 in. Hg. Ending pressure 8 in. $\mathrm{Hg}$.

Beginning pressure -28 in. $\mathrm{Hg}$. Ending pressure $7 \mathrm{in.} \mathrm{Hg}$. 
TABLE A.1 (Cont.)

\begin{tabular}{|c|c|c|c|c|c|c|c|c|c|}
\hline $\begin{array}{l}\text { Sample } \\
\text { Date }\end{array}$ & Time & Sample & Medium ${ }^{a}$ & Type $^{a}$ & Location & $\begin{array}{l}\text { Depth } \\
\text { (ft BGL) }\end{array}$ & $\begin{array}{l}\text { Chain of } \\
\text { Custody }\end{array}$ & $\begin{array}{l}\text { Shipping } \\
\text { Date }\end{array}$ & Sample Description \\
\hline
\end{tabular}

August 2010 indoor air sampling (cont.)

September 2010 groundwater, surface water, and sediment sampling
8/12/10 15:43 108 Roxanna St., basement

8/12/10 14:51 106 Virginia St., first floor

8/12/10 17:03 105 Roxanna St., first floor
108 Roxanna St.

106 Virginia St.

105 Roxanna St.

$\begin{array}{lll}\text { 9/22/10 } & 8: 10 & \text { MRSM4-W-30008 } \\ \text { 9/22/10 } & 8: 11 & \text { MRSM4-S-30008 } \\ \text { 9/22/10 } & 8: 15 & \text { MRSM3-W-30007 } \\ \text { 9/22/10 } & 8: 16 & \text { MRSM3-S-30007 } \\ \text { 9/22/10 } & 8: 21 & \text { MRSM2-W-30006 } \\ \text { 9/22/10 } & 8: 22 & \text { MRSM2-S-30006 } \\ \text { 9/22/10 } & 8: 27 & \text { MRSM1-W-30005 } \\ \text { 9/22/10 } & 8: 28 & \text { MRSM1-S-30005 } \\ \text { 9/22/10 } & 8: 39 & \text { MRSMB-W-30009 } \\ 9 / 22 / 10 & 8: 40 & \text { MRSMB-S-30009 }\end{array}$

Water

Soil

Water

Soil

Wate

Soil

Wate

Soil

Wate

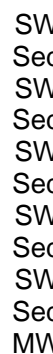

Sed

SW

Sed

SW

Sed

SW

Sed

Sed

MW

SM4
SM4
SM3
SM3
SM2
SM2
SM1
SM1
SMB
SMB
MW6S

\section{$-$}

- 2690

- 2691

- $\quad 2690$

- $\quad 2690$

- $\quad 2691$

$\begin{array}{cr}- & 2690 \\ 10-25 & 2691\end{array}$

$\begin{array}{lll}\text { 9/22/10 } & 10: 43 & \text { MRSTONE-W-3002 } \\ \text { 9/22/10 } & 10: 55 & \text { MRRILLINGER-W-300 } \\ \text { 9/22/10 } & 11: 27 & \text { MRTD12-W-30025 } \\ \text { 9/22/10 } & 11: 55 & \text { MRISCH-W-30022 } \\ & & \\ \text { 9/22/10 } & 11: 59 & \text { MRMW1D-W-30011 } \\ & & \end{array}$

9/22/10 13:30 MRMW4S-W-30014

Water

Water
MW
MW

MW4S

\section{Stone}

Water DW Rillinger

Water DW

Water

DW

Isch

MW1D

MW4S
9/22/10 13:31 MRMW4SDUP-W-30027
- 2691
$8 / 12 / 10$

$8 / 12 / 10$

$8 / 12 / 10$
Beginning pressure -28 in. Hg. Ending pressure $7 \mathrm{in.} \mathrm{Hg}$.

Beginning pressure -28 in. Hg. Ending pressure 8 in. $\mathrm{Hg}$.

Beginning pressure -28 in. $\mathrm{Hg}$. Ending pressure 7 in. $\mathrm{Hg}$.

$9 / 23 / 10$

$9 / 23 / 10$

$9 / 23 / 10$

$9 / 23 / 10$

$9 / 23 / 10$

$9 / 23 / 10$

$9 / 23 / 10$

$9 / 23 / 10$

$9 / 23 / 10$

$9 / 23 / 10$

$9 / 23 / 10$

$$
\text { (23/10 }
$$

\section{$9 / 23 / 10$}

$9 / 23 / 10$

$9 / 23 / 10$

27-67

$$
-
$$

63-88

$17-47$

2691

$9 / 23 / 10$

$17-47$
Water sample from Terrapin Creek. Sediment sample from Terrapin Creek.

Water sample from Terrapin Creek.

Sediment sample from Terrapin Creek.

Water sample from Terrapin Creek.

Sediment sample from Terrapin Creek.

Water sample from Terrapin Creek.

Sediment sample from Terrapin Creek.

Water sample from Terrapin Creek.

Sediment sample from Terrapin Creek.

Depth to water $=5.53 \mathrm{ft}$. Depth of 4 -in. well $=26.9 \mathrm{ft}$. Sample collected by using lowflow bladder pump after purgiing of $8.75 \mathrm{~L}$. Pump intake positioned at $17.5 \mathrm{ft}$.

Purged 5 gal by using bailer, then sampled. Let pump run for $5 \mathrm{~min}$, then sampled.

Collected from Grimm well overflow before the catch pond.

Let pump run for $5 \mathrm{~min}$, then sampled. Well had not been used in a while. Listed on COC as "not received."

Depth to water $=27.92 \mathrm{ft}$. Depth of 4-in. well $=89 \mathrm{ft}$. Sample collected by using low-flow bladder pump after purgiing of $9 \mathrm{~L}$. Pump intake positioned at $75.5 \mathrm{ft}$.

Depth to water $=33.15 \mathrm{ft}$. Depth of 4-in

well $=47.8 \mathrm{ft}$. Sample collected by using low-flow bladder pump after purgiing of $6 \mathrm{~L}$. Pump intake positioned at $32 \mathrm{ft}$.

Replicate of sample MRMW4S-W-30014. 
TABLE A.1 (Cont.)

\begin{tabular}{|c|c|c|c|c|c|c|c|c|c|}
\hline $\begin{array}{l}\text { Sample } \\
\text { Date }\end{array}$ & Time & Sample & Medium $^{\mathrm{a}}$ & Type $^{a}$ & Location & $\begin{array}{c}\text { Depth } \\
\text { (ft BGL) }\end{array}$ & $\begin{array}{l}\text { Chain of } \\
\text { Custody }\end{array}$ & $\begin{array}{l}\text { Shipping } \\
\text { Date }\end{array}$ & Sample Description \\
\hline \multicolumn{10}{|c|}{ September 2010 groundwater, surface water, and sediment sampling (cont.) } \\
\hline 9/22/10 & $13: 36$ & MRMW8S-W-30018 & Water & MW & MW8S & $10-25$ & 2691 & $9 / 23 / 10$ & $\begin{array}{l}\text { Depth to water }=2.53 \mathrm{ft} \text {. Depth of } 4 \text {-in. well } \\
=26.8 \mathrm{ft} \text {. Sample collected by using low- } \\
\text { flow bladder pump after purgiing of } 9 \mathrm{~L} \text {. } \\
\text { Pump intake positioned at } 17.5 \mathrm{ft} \text {. }\end{array}$ \\
\hline 9/22/10 & 13:37 & MRMW8SDUP-W-30026 & Water & MW & MW8S & $10-25$ & 2715 & 9/23/10 & Replicate of sample MRMW8S-W-30018. \\
\hline $9 / 22 / 10$ & $14: 16$ & MRMW5S-W-30015 & Water & MW & MW5S & $15-55$ & 2691 & $9 / 23 / 10$ & $\begin{array}{l}\text { Depth to water }=19.25 \mathrm{ft} \text {. Depth of } 4 \text {-in. } \\
\text { well }=55 \mathrm{ft} \text {. Sample collected by using } \\
\text { low-flow bladder pump after purging of } \\
6.5 \mathrm{~L} \text {. Pump intake positioned at } 35 \mathrm{ft} \text {. }\end{array}$ \\
\hline 9/22/10 & $14: 25$ & MRQCIR-W-30028 & Water & $\mathrm{RI}$ & $\mathrm{QC}$ & - & 2715 & $9 / 23 / 10$ & $\begin{array}{l}\text { Rinsate of decontaminated sampling line } \\
\text { after collection of sample MRMW5S-W- } \\
30015 \text {. }\end{array}$ \\
\hline 9/22/10 & $14: 50$ & MRMW10S-W-30020 & Water & MW & MW10S & $30-45$ & 2715 & $9 / 23 / 10$ & $\begin{array}{l}\text { Depth to water }=11.69 \mathrm{ft} \text {. Depth of } 2 \text {-in. } \\
\text { well }=49.7 \mathrm{ft} \text {. Sample collected by using } \\
\text { low-flow bladder pump after purgiing of } \\
11 \mathrm{~L} \text {. Pump intake positioned at } 37.5 \mathrm{ft} \text {. }\end{array}$ \\
\hline 9/22/10 & $15: 42$ & MRMW2S-W-30012 & Water & MW & MW2S & $13-53$ & 2691 & $9 / 23 / 10$ & $\begin{array}{l}\text { Depth to water }=31.25 \mathrm{ft} \text {. Depth of } 4 \text {-in. } \\
\text { well }=53 \mathrm{ft} \text {. Sample collected by using } \\
\text { low-flow bladder pump after purgiing of } \\
7 \mathrm{~L} \text {. Pump intake positioned at } 33 \mathrm{ft} \text {. }\end{array}$ \\
\hline $9 / 22 / 10$ & $15: 45$ & MRQCIR-W-30029 & Water & RI & $\mathrm{QC}$ & - & 2715 & $9 / 23 / 10$ & $\begin{array}{l}\text { Rinsate of decontaminated sampling line } \\
\text { after collection of sample MRMW10S- } \\
\text { W-30020. }\end{array}$ \\
\hline 9/22/10 & $16: 25$ & MRMW1S-W-30010 & Water & MW & MW1S & $11-51$ & 2691 & $9 / 23 / 10$ & $\begin{array}{l}\text { Depth to water = } 19.89 \mathrm{ft} \text {. Depth of } 4 \text {-in. } \\
\text { well }=54 \mathrm{ft} \text {. Sample collected by using } \\
\text { low-flow bladder pump after purgiing of } \\
10 \mathrm{~L} . \text { Pump intake positioned at } 31 \mathrm{ft} \text {. }\end{array}$ \\
\hline $9 / 22 / 10$ & $17: 06$ & MRMW9S-W-30019 & Water & MW & MW9S & $38.83-53.83$ & 2691 & $9 / 23 / 10$ & $\begin{array}{l}\text { Depth to water }=21.15 \mathrm{ft} \text {. Depth of } 2 \text {-in. } \\
\text { well }=53.83 \mathrm{ft} \text {. Sample collected by } \\
\text { using low-flow bladder pump after } \\
\text { purgiing of } 7 \mathrm{~L} \text {. Pump intake positioned } \\
\text { at } 46.33 \mathrm{ft} \text {. }\end{array}$ \\
\hline 9/23/10 & $11: 54$ & MRMW3S-W-30013 & Water & MW & MW3S & $18-48$ & 2691 & $9 / 23 / 10$ & $\begin{array}{l}\text { Depth to water }=23.73 \mathrm{ft} \text {. Depth of } 4 \text {-in. } \\
\text { well }=47.8 \mathrm{ft} \text {. Sample collected by using } \\
\text { low-flow bladder pump after purgiing of } \\
7 \mathrm{~L} \text {. Pump intake positioned at } 33 \mathrm{ft} \text {. }\end{array}$ \\
\hline 9/23/10 & $12: 30$ & MRQCIR-W-30030 & Water & $\mathrm{RI}$ & $\mathrm{QC}$ & - & 2715 & $9 / 23 / 10$ & $\begin{array}{l}\text { Rinsate of decontaminated sampling line } \\
\text { after collection of sample MRMW3S-W- } \\
30013 \text {. }\end{array}$ \\
\hline
\end{tabular}


TABLE A.1 (Cont.)

\begin{tabular}{|c|c|c|c|c|c|c|c|c|c|}
\hline $\begin{array}{l}\text { Sample } \\
\text { Date }\end{array}$ & Time & Sample & Medium $^{\mathrm{a}}$ & Type $^{a}$ & Location & $\begin{array}{l}\text { Depth } \\
\text { (ft BGL) }\end{array}$ & $\begin{array}{l}\text { Chain of } \\
\text { Custody }\end{array}$ & $\begin{array}{l}\text { Shipping } \\
\text { Date }\end{array}$ & Sample Description \\
\hline \multicolumn{10}{|c|}{ September 2010 groundwater, surface water, and sediment sampling (cont.) } \\
\hline 9/23/10 & 13:06 & MRMW7S-W-30017 & Water & MW & MW7S & $20-45$ & 2691 & $9 / 23 / 10$ & $\begin{array}{l}\text { Depth to water }=14.15 \mathrm{ft} \text {. Depth of } 4 \text {-in. } \\
\text { well }=45 \mathrm{ft} \text {. Sample collected by using } \\
\text { low-flow bladder pump after purgiing of } \\
6 \mathrm{~L} \text {. Pump intake positioned at } 32.5 \mathrm{ft} \text {. }\end{array}$ \\
\hline 9/23/10 & $14: 00$ & MRQCTB-W-30032 & Water & TB & QC & - & 2715 & $9 / 23 / 10$ & $\begin{array}{l}\text { Trip blank sent to the AGEM Laboratory for } \\
\text { organic analysis with water samples } \\
\text { listed on COCs } 2691 \text { and } 2715 \text { and to } \\
\text { Test America for verification organic } \\
\text { analysis with water samples listed on } \\
\text { COC } 2174 \text {. }\end{array}$ \\
\hline 9/23/10 & $14: 03$ & MRMW11S-W-30021 & Water & MW & MW11S & 53-68 & 2715 & $9 / 23 / 10$ & $\begin{array}{l}\text { Depth to water }=34.75 \mathrm{ft} \text {. Depth of } 2 \text {-in. } \\
\text { well }=72.7 \mathrm{ft} \text {. Sample collected by using } \\
\text { low-flow bladder pump after purgiing of } \\
7 \mathrm{~L} \text {. Pump intake positioned at } 60.5 \mathrm{ft} \text {. }\end{array}$ \\
\hline 9/23/10 & $14: 05$ & MRDIH2O-W-30031 & Water & FB & QC & - & 2715 & $9 / 23 / 10$ & $\begin{array}{l}\text { Blank of water used for equipment } \\
\text { decontamination. }\end{array}$ \\
\hline
\end{tabular}

a Medium and sample type abbreviations: DW, domestic well; MW, monitoring well; RI, equipment rinsate; SW, surface water; sed, sediment; TB, trip blank; veg, vegetation. 
Appendix B:

Indoor Air Sampling Plan 
Plan for Indoor Air and Ambient Air Sampling at Morrill, Kansas

March 11, 2010

\section{Revised Work Plan: Indoor Air and Ambient Air Sampling near the Former CCC/USDA Grain Storage Facility in Morrill, Kansas}

The Commodity Credit Corporation of the U.S. Department of Agriculture (CCC/USDA) operated a grain storage facility in the northwestern section of Morrill, Kansas, from 1950 to 1971. The property continued to be used for grain storage after 1971. Sampling by the Kansas Department of Health and Environment (KDHE) in 1985 resulted in the detection of carbon tetrachloride in one public well. Subsequent investigations conducted by and for the KDHE confirmed the presence of carbon tetrachloride (KDHE 1989; GeoCore 1994, 1996).

On behalf of the CCC/USDA, in 2003 Argonne National Laboratory began a series of investigations at Morrill and established a monitoring program to characterize and track the contamination (Argonne 2004, 2005a,b, 2006, 2007a,b,c,d, 2008a,b, 2009). Automatic, continuous monitoring of groundwater levels began in 2004 and is ongoing at seven locations. The results have consistently indicated groundwater flow toward the south-southeast from the former CCC/USDA property. Sitewide periodic sampling of groundwater, surface water, streambed sediment, and vegetation, with analysis for volatile organic compounds (VOCs), began in 2005-2007. At present, the sampling is twice yearly, as approved by the KDHE (2007a).

This document presents a plan for collecting indoor air samples in homes located along and adjacent to the defined extent of carbon tetrachloride contamination. The plan was requested by the KDHE (2009). Ambient air samples to represent the conditions along this pathway will also be taken.

\section{Project Objective}

The purpose of the proposed work is to satisfy KDHE requirements and to collect additional data for assessing the risk to human health due to the potential upward migration of carbon tetrachloride and its primary degradation product (chloroform) into homes located in close proximity to the former grain storage facility and along and within $100 \mathrm{ft}$ laterally from the currently defined plume emanating from the former Morrill facility. Investigation of the indoor air environment was not a defined objective during Argonne's previous investigations at Morrill, as they predated the more recent regulatory guidance (KDHE 2007b) regarding the potential health risks associated with the vapor contaminant pathway.

Proposed Work: Collection of Indoor Air and Ambient Air Samples

Sampling Procedure. Indoor air samples will be collected at the designated homes. In addition, representative ambient air samples will be collected. All collection protocols will be in compliance with the KDHE (2007b) vapor intrusion guidance and KDHE standard operating procedure (SOP) BER-33. Dr. Blayne Hartman (Hartman Environmental Geoscience, Solana Beach, California), a recognized expert in the field of vapor intrusion, will advise Argonne staff as to specific protocols prior to the sampling event. He will also collaborate with Argonne and 
the $\mathrm{CCC} / \mathrm{USDA}$, as well as the KDHE, in interpretation of the results of the vapor intrusion investigation.

Figure 1 illustrates the current interpreted configuration of the carbon tetrachloride plume (derived from a series of monitoring events spanning the period April 2008 to September 2009) at and emanating from the former CCC/USDA facility. Figure 2 provides a detailed view, with the addresses of the homes to be potentially sampled.

Because the plume boundary between the $0-\mu \mathrm{g} / \mathrm{L}$ and $25-\mu \mathrm{g} / \mathrm{L}$ contours (Figure 2 ) is not well defined, the sampling will be done in two phases. Phase 1 will include the 12 homes that fall laterally within $100 \mathrm{ft}$ of the $25-\mu \mathrm{g} / \mathrm{L}$ contour. These 12 homes are identified in Figure 3 . The addresses are summarized in the top section of Table 1 . Phase 2 will address any additional houses selected, on the basis of the Phase 1 results, from the list in the bottom section of Table 1.

Table 2 summarizes the locations within the monitoring well network, the analytical results for the April and September 2009 sampling events, and the measured depths to groundwater during these events. The homes identified in Table 1 for potential sampling are located directly downgradient from the former facility (on Hanson Street), south and east to Roxanna, Walnut, and Virginia Streets. The plume, as currently constrained, trends to the southsoutheast from the former facility, across agricultural land and along the western edge of the Morrill community. The plume continues to Terrapin Creek, again across agricultural lands, with no residences.

Sample Location. Samplers will be placed in the basement and on the first floor of each residence, in a location of common occupancy (as allowed by the residents). Samples will be collected as close to the center of the room as possible, away from heating system registers, and at a height of 3-7 ft above the floor (in the breathing zone). Samples will be collected in Summa canisters, individually certified clean, for a period of $24 \mathrm{hr}$. Meteorological conditions will be noted and recorded. Page 1 of the KDHE Field Data Air Sampling Form (in SOP BER-33) will be completed.

Ambient Air Samples. To be representative of the site, ambient air samples will be collected in an upwind location, away from obvious sources of VOCs, and over the same collection period as the indoor air samples. Additional canisters will be placed to test ambient air conditions at several points along the lateral extent of the contamination.

Duplicate Air Samples. Duplicate air samples will be collected at the rate of at least one duplicate per 20 primary samples.

Sample Analysis. Air samples will be shipped to a laboratory certified for method TO-15 analysis and analyzed for carbon tetrachloride and chloroform by method TO-15, at a detection level at or below the allowable risk-based indoor air levels for each compound.

The $\mathrm{CCC} / \mathrm{USDA}$ intends to offer radon sampling to the residents of homes potentially affected by carbon tetrachloride, to be conducted at the same time. The radon kits used, as approved for sampling in Hanover, Kansas (KDHE 2010), will be obtained from the county office. The kits (charcoal canisters or pouches) will be used as directed and will be left in place for 3-4 days, then returned (after exposure) to National Radon Program Services at Kansas State 

March 11, 2010

University (133 Ward Hall, Manhattan, KS 66506-2508). The results will be reported to the KDHE when available.

Report Deliverable

A report containing all of the data, all relevant quality assurance/quality control information, and an interpretation of the vapor intrusion risk will be submitted following completion of the fieldwork and analytical reviews.

Schedule of Fieldwork

After approval of this plan, the KDHE will have the opportunity to inform the community of the sampling plan. Argonne will make further contact, through its community relations staff, to coordinate the sampling effort with the aim of minimizing any disruption to the residents and the community.

The work is tentatively scheduled for mid March 2010. The KDHE will be informed as to the finalized dates when residences are available for testing, to allow the agency to arrange oversight and the retrieval of duplicate samples.

\section{References}

Argonne, 2004, Final Phase I-Phase II Interim Report: Expedited Site Characterization, Morrill, Kansas, ANL/ER/TR-04/001, prepared for the Commodity Credit Corporation, U.S. Department of Agriculture, Washington, D.C., by Argonne National Laboratory, Argonne, Illinois, December.

Argonne, 2005a, Final Report, Monitoring Well Installation and Sampling, 2004, at Morrill, Kansas, ANL/ER/TR-04/010, prepared for the Commodity Credit Corporation, U.S. Department of Agriculture, Washington, D.C., by Argonne National Laboratory, Argonne, Illinois, November.

Argonne, 2005b, Final Work Plan: Groundwater Monitoring at Morrill, Kansas, ANL/ER/TR05/003, prepared for the Commodity Credit Corporation, U.S. Department of Agriculture, Washington, D.C., by Argonne National Laboratory, Argonne, Illinois, August.

Argonne, 2006, September 2006 Monitoring Results for Morrill, Kansas, ANL/EVS/AGEM/CHRON-1016, prepared for the Commodity Credit Corporation, U.S. Department of Agriculture, Washington, D.C., by Argonne National Laboratory, Argonne, Illinois, December.

Argonne, 2007a, Final Report: Groundwater Monitoring at Morrill, Kansas, in September 2005 and March 2006, with Expansion of the Monitoring Network in January 2006,

ANL/EVS/AGEM/TR-06-09, prepared for the Commodity Credit Corporation, U.S. Department of Agriculture, Washington, D.C., by Argonne National Laboratory, Argonne, Illinois, June.

Argonne, 2007b, Reconnaissance Visit along Terrapin Creek, South of the Former CCC/USDA Grain Bin Site and the Exiting Co-op in Morrill, Kansas, January 2007, 
Plan for Indoor Air and Ambient Air Sampling at Morrill, Kansas

March 11, 2010

ANL/EVS/AGEM/CHRON-1042, prepared for the Commodity Credit Corporation,

U.S. Department of Agriculture, Washington, D.C., by Argonne National Laboratory, Argonne, Illinois, May 3.

Argonne, 2007c, March-April 2007 Monitoring Results for Morrill, Kansas, ANL/EVS/AGEM/TR-07-06, prepared for the Commodity Credit Corporation, U.S. Department of Agriculture, by Argonne National Laboratory, Argonne, Illinois, May.

Argonne, $2007 \mathrm{~d}$, Proposed Addendum to the Groundwater Monitoring Plan for Morrill, Kansas, ANL/EVS/AGEM/CHRON-1042, prepared for the Commodity Credit Corporation,

U.S. Department of Agriculture, Washington, D.C., by Argonne National Laboratory, Argonne, Illinois, May 3.

Argonne, 2008a, October 2007 Monitoring Results for Morrill, Kansas, ANL/EVS/AGEM/TR08-02, prepared for the Commodity Credit Corporation, U.S. Department of Agriculture, Washington, D.C., by Argonne National Laboratory, Argonne, Illinois, March.

Argonne, 2008b, April 2008 Monitoring Results for Morrill, Kansas, ANL/EVS/AGEM/TR08-15, prepared for the Commodity Credit Corporation, U.S. Department of Agriculture, Washington, D.C., by Argonne National Laboratory, Argonne, Illinois, July.

Argonne, 2009, October 2008 Monitoring Results for Morrill, Kansas, ANL/EVS/AGEM/TR09-03, prepared for the Commodity Credit Corporation, U.S. Department of Agriculture, Washington, D.C., by Argonne National Laboratory, Argonne, Illinois, March.

GeoCore, 1994, Preliminary Report: Phase I Sampling: Brown County Groundwater Investigation, prepared by GeoCore Services, Inc., Salina, Kansas, for the Bureau of Environmental Remediation, Kansas Department of Health and Environment, July 19.

GeoCore, 1996, Environmental Site Investigation Report: Phase II Drilling \& Sampling: Morrill Public Water Supply Well \#5, Morrill, Kansas, prepared by GeoCore Services, Inc., Salina, Kansas, for the Bureau of Environmental Remediation, Kansas Department of Health and Environment (draft issued August 31, 1995; revised February 29, 1996).

KDHE, 1989, Preliminary Assessment of the Morrill Public Water Supply Well \#5, Morrill, Kansas (Site Identification Number: KS D981710288), completed by R. Bean and J. Alldritt, Bureau of Environmental Remediation, Kansas Department of Health and Environment, June 6.

KDHE, 2007a, letter from C. Carey (Bureau of Environmental Remediation, Kansas Department of Health and Environment, Topeka, Kansas) to C. Roe (Commodity Credit Corporation, U.S. Department of Agriculture, Washington, D.C.) regarding March-April 2007 Monitoring Results for Morrill, Kansas, August 1.

KDHE, 2007b, Kansas Vapor Intrusion Guidance: Chemical Vapor Intrusion and Residential Indoor Air, Bureau of Environmental Remediation, Kansas Department of Health and Environment, Topeka, Kansas, June (http://www.kdheks.gov/ber/download/ Ks_VI_Guidance.pdf).

KDHE, 2009, letter from E. Finzer (Bureau of Environmental Remediation, Kansas Department of Health and Environment, Topeka, Kansas) to C. Roe (Commodity Credit Corporation, U.S. Department of Agriculture, Washington, D.C.) regarding vapor intrusion prioritization, November 9. 
Plan for Indoor Air and Ambient Air Sampling at Morrill, Kansas

March 11, 2010

KDHE, 2010, electronic mail message from C. Carey (Bureau of Environmental Remediation, Kansas Department of Health and Environment, Topeka, Kansas) to L. LaFreniere (Argonne National Laboratory, Argonne, Illinois), January 21. 
Plan for Indoor Air and Ambient Air Sampling at Morrill, Kansas March 11, 2010

TABLE 1 Contact information for the Morrill residences to be sampled.

\begin{tabular}{|c|c|c|c|c|}
\hline No. & Name & Street Address & City, State, Zip & Telephone \\
\hline \multicolumn{5}{|c|}{ Residences Selected for Phase 1 Sampling } \\
\hline & David Frey & $566246 \mathrm{Hwy}$. & Morrill, KS 66515 & $785-285-1734$ \\
\hline & Larry Allen & 90 Roxanna Street & Morrill, KS 66515 & None \\
\hline & Gruber, Charles & 104 Roxanna Street & Morrill, KS 66515-9702 & $785-459-2231$ \\
\hline & Wetzel, Jim & 105 Roxanna Street & Morrill, KS 66515 & None \\
\hline & Kellenberger, Art & 104 Roxanna Street & Morrill, KS 66515-9702 & $785-285-0399$ \\
\hline & Plooger, Miles & 106 Roxanna Street & Morrill, KS 66515-9702 & $785-459-2543$ \\
\hline & $\begin{array}{l}\text { The Ayore Church, } \\
\text { Casey McNerney, } \\
\text { pastor }\end{array}$ & 107 Roxanna Street & Morrill, KS 66515 & $785-459-2445$ \\
\hline & Gruber, Todd and Jana & 108 Roxanna Street & Morrill, KS 66515-9702 & $785-459-2454$ \\
\hline & $\begin{array}{l}\text { Sailors, William and } \\
\text { Linda }\end{array}$ & 202 Roxanna Street & Morrill, KS 66515-9465 & $785-459-2422$ \\
\hline & Carver, Eldon & 203 Roxanna Street & Morrill, KS 66515 & $785-459-2408$ \\
\hline & $\begin{array}{l}\text { Rahe Welding and } \\
\text { Repairs (Bill Rahe) }\end{array}$ & 96 Virginia Street & Morrill, KS 66515-9714 & $785-459-2525$ \\
\hline & Minard, Shane & 102 Virginia Street & Morrill, KS 66515-9713 & $785-459-2245$ \\
\hline & Frye, Fred and Sherry & 106 Virginia Street & Morrill, KS 66515 & $785-459-2823$ \\
\hline \multicolumn{5}{|c|}{ Residences to be Considered for Phase 2 Sampling, on the Basis of Phase 1 Results } \\
\hline & Anderson, Daniel & 204 Roxanna Street & Morill, KS 66515-9465 & $785-991-1618$ \\
\hline & Information pending ${ }^{a}$ & 205 Roxanna Street & Morrill, KS 66515 & None \\
\hline & Edwards, Aurora & 206 Roxanna Street & Morrill, KS 66515-9484 & $785-459-2815$ \\
\hline & $\begin{array}{l}\text { Manning, David and } \\
\text { Ruth }\end{array}$ & 207 Roxanna Street & Morrill, KS 66515-9465 & $785-459-2827$ \\
\hline & $\begin{array}{l}\text { McNerney, Casey and } \\
\text { Karen }\end{array}$ & 302 Roxanna Street & Morrill, KS 66515-9724 & $785-288-8255$ \\
\hline & Backer, Mark and Janice & 303 Roxanna Street & Morrill, KS 66515-9707 & $785-459-2840$ \\
\hline & Grimm, Randy & 304 Roxanna Street & Morrill, KS 66515 & $785-459-2445$ \\
\hline & Stover, Larry and Judy & 200 Virginia Street & Morrill, KS 66515 & $785-459-2868$ \\
\hline & Babcock, Lyle & 202 Virginia Street & Morrill, KS 66515 & $785-548-5320$ \\
\hline & Information pending ${ }^{\mathrm{b}}$ & 303 Virginia Street & Morrill, KS 66515 & None \\
\hline & $\begin{array}{l}\text { Rilinger, Lawrence and } \\
\text { Debbie }\end{array}$ & 106 Walnut Street & Morrill, KS 66515-9703 & $785-459-2417$ \\
\hline & Sperline, Shani & 202 Walnut Street & Morrill, KS 66515-9721 & $785-285-1800$ \\
\hline
\end{tabular}




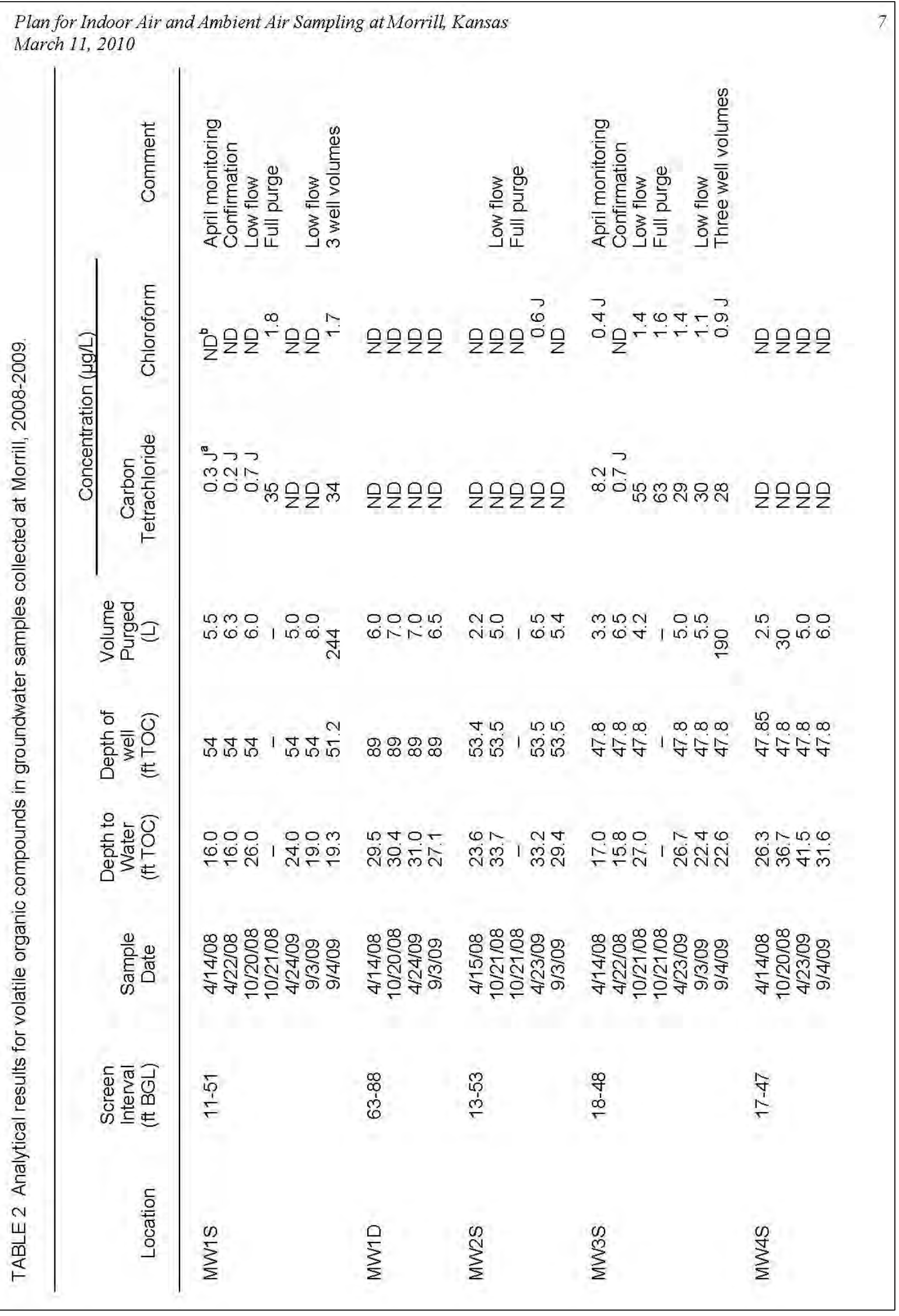




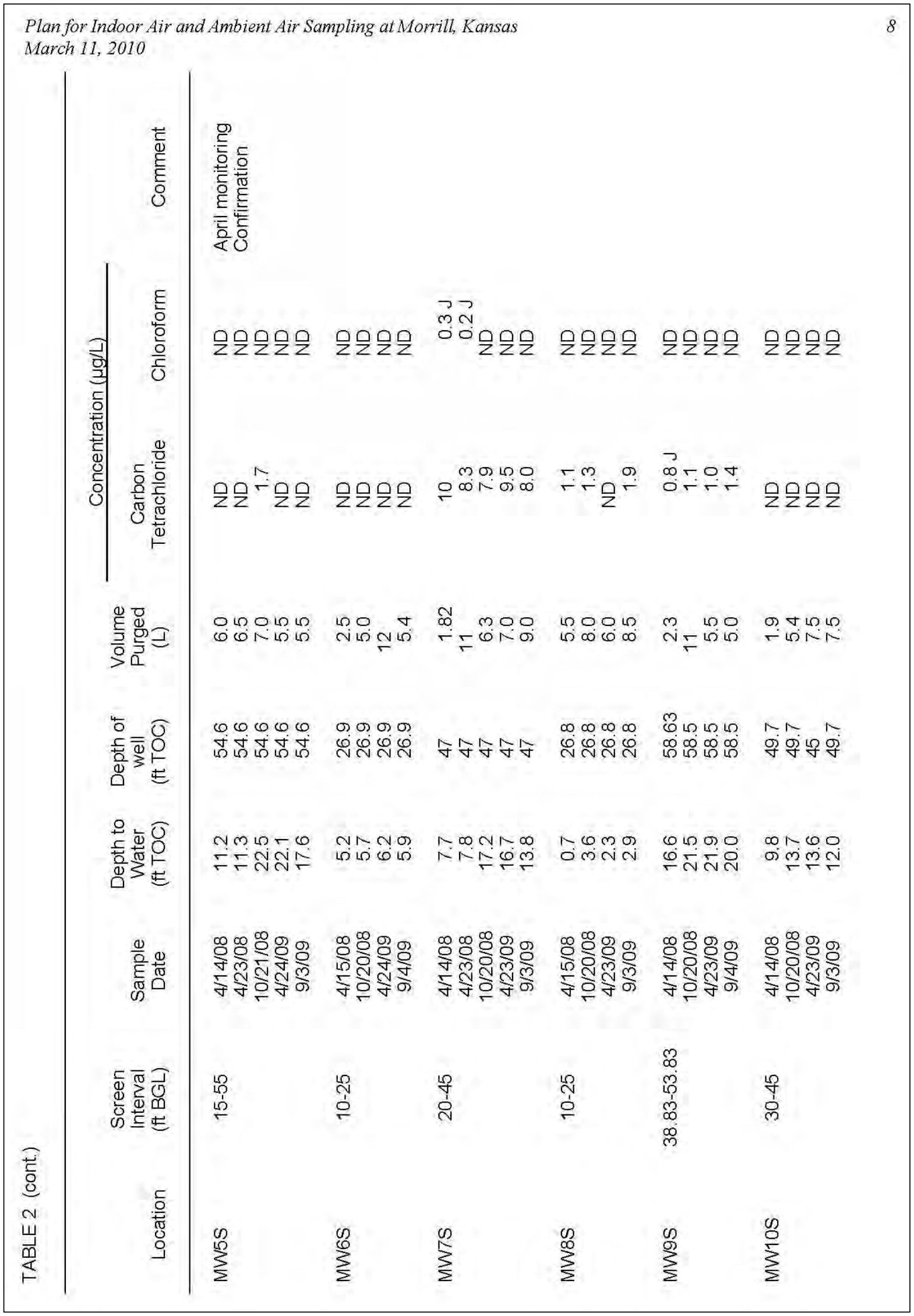




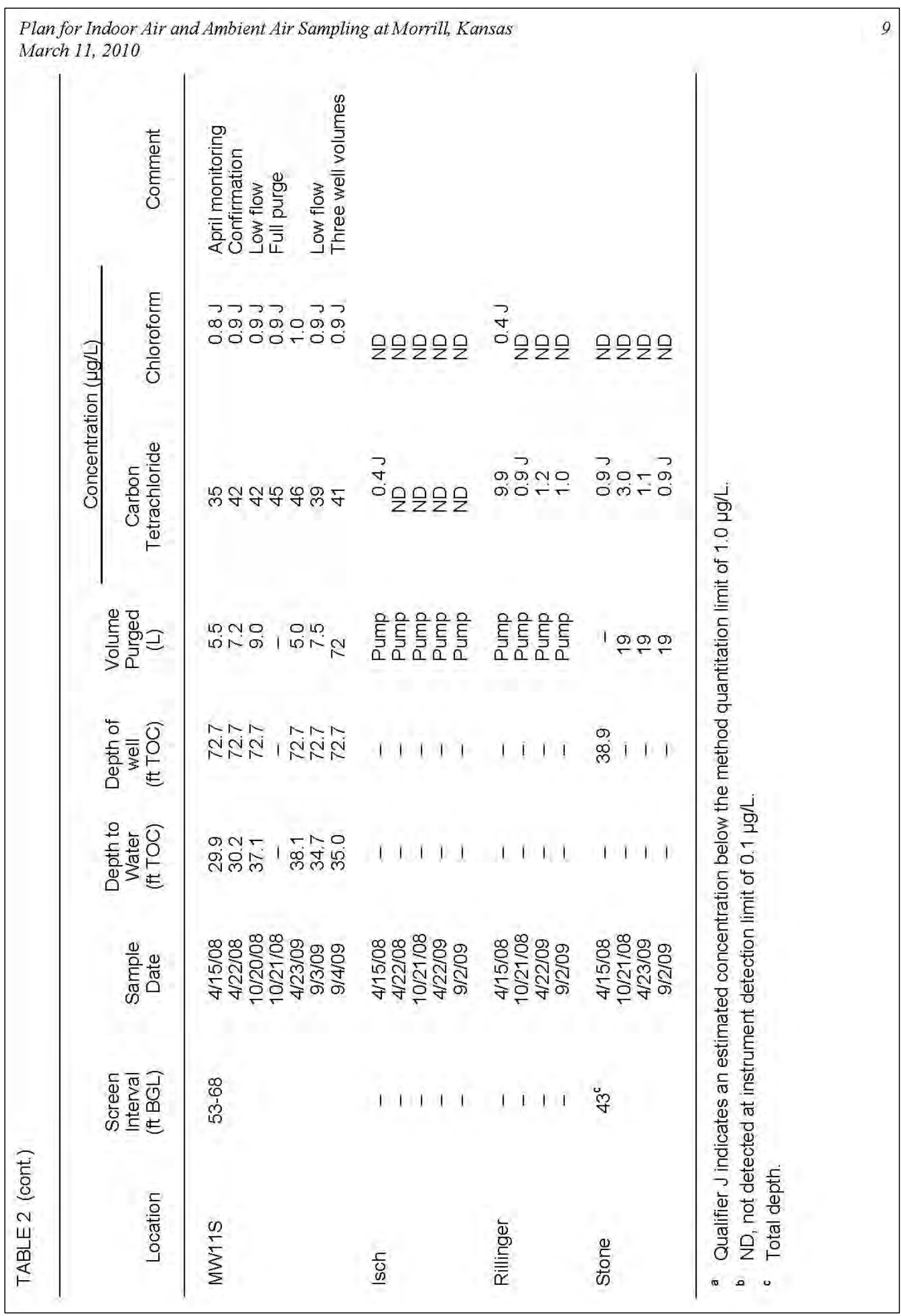




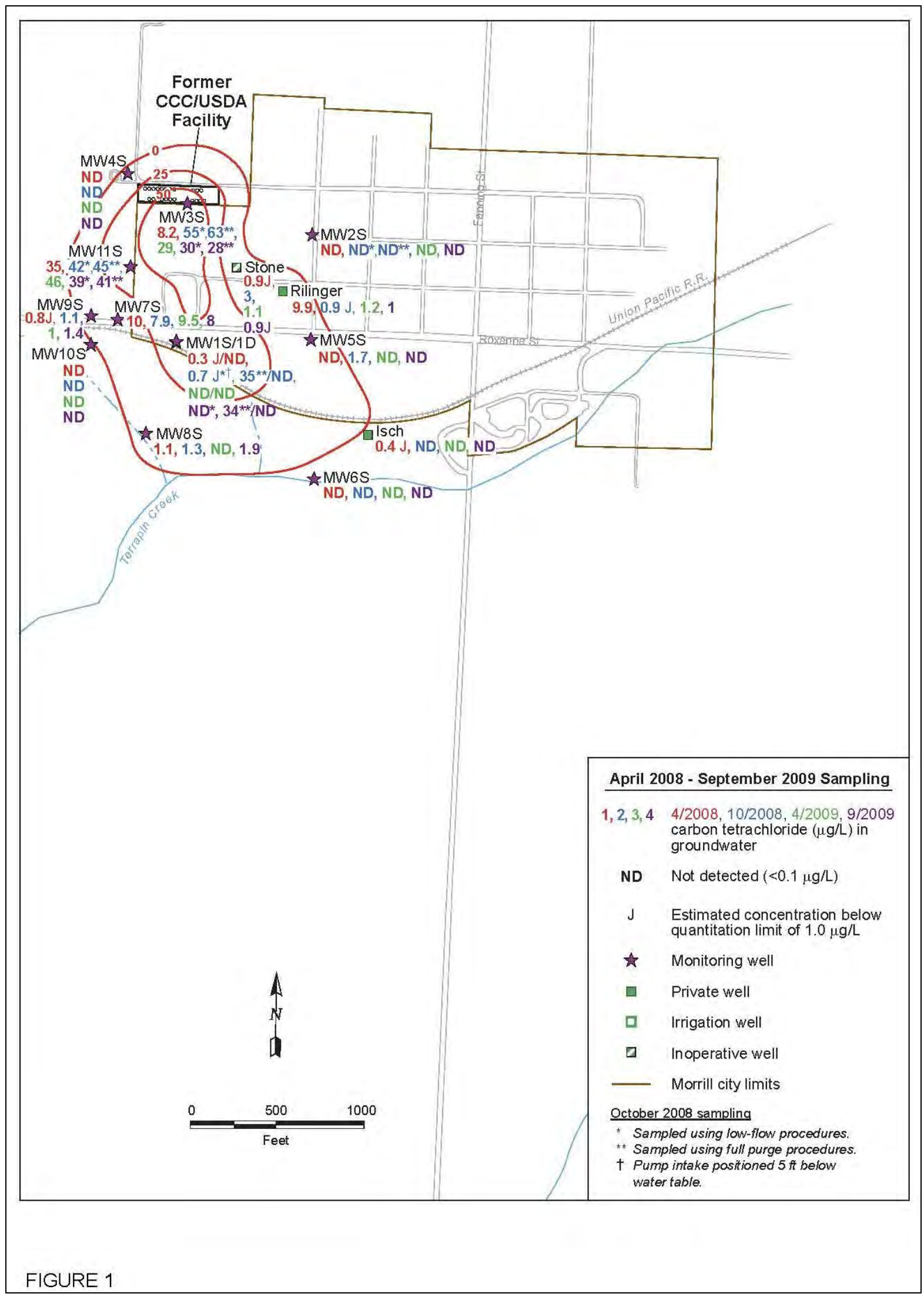




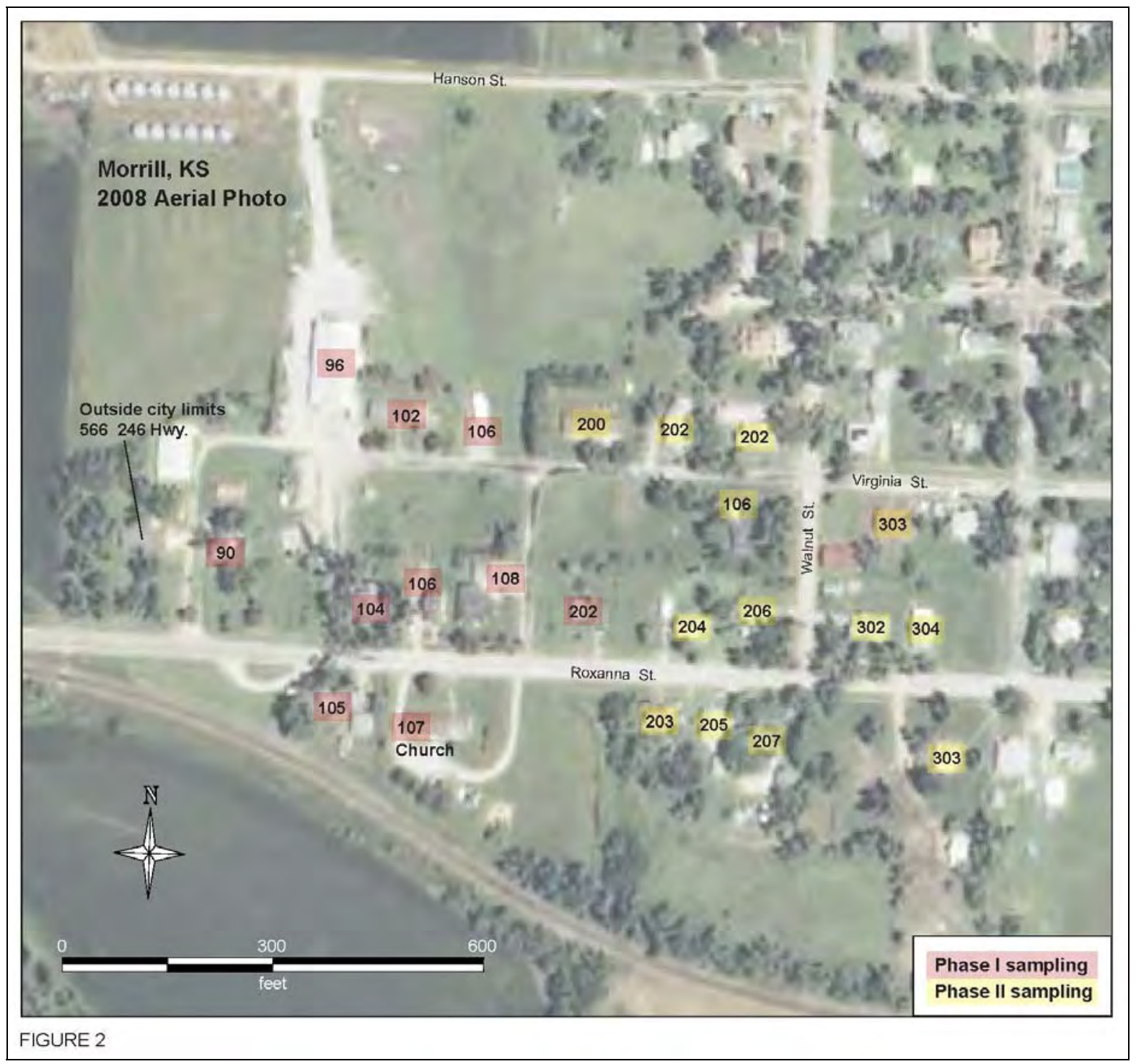




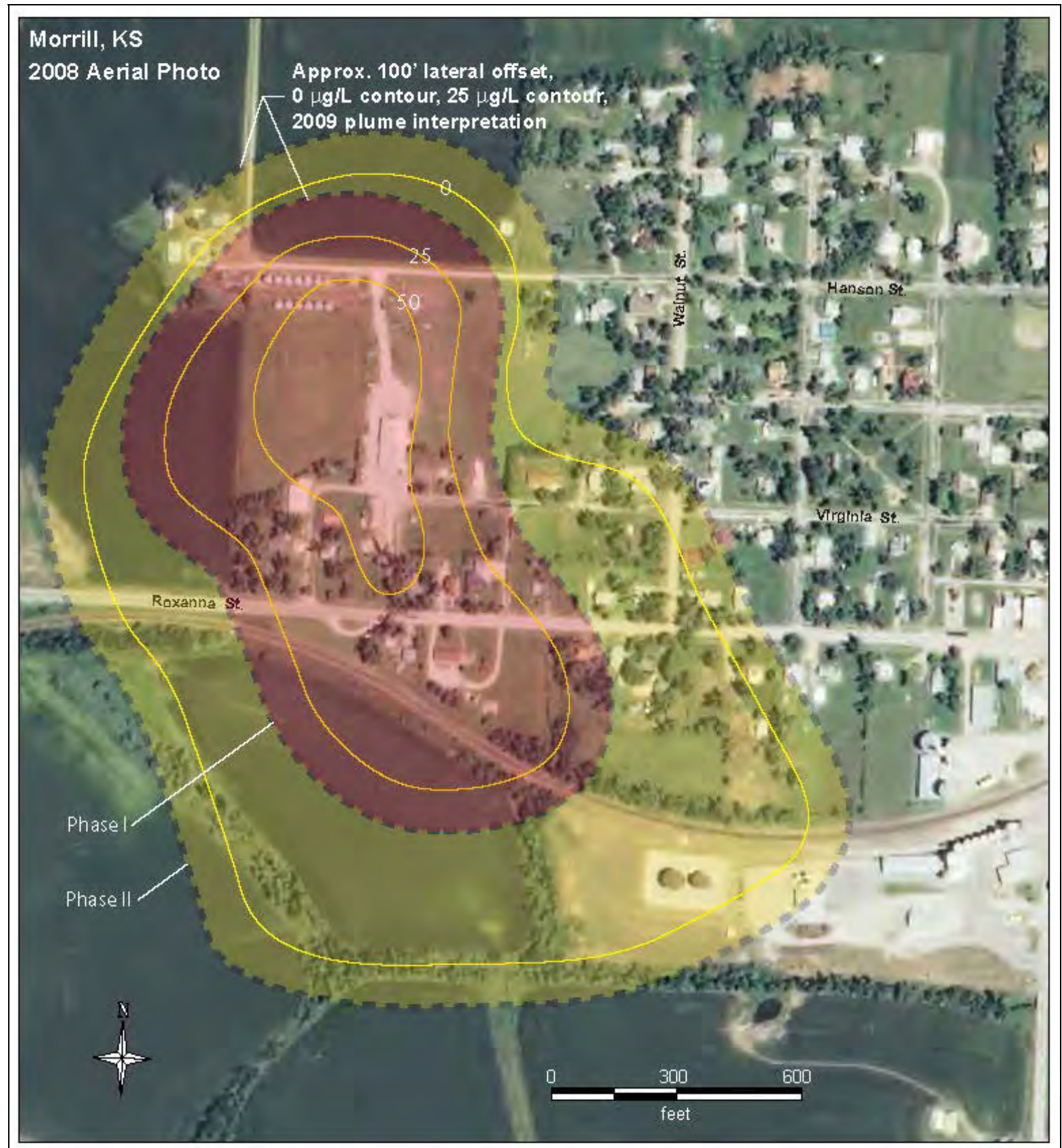

FIGURE 3 
Appendix C:

Results from AGEM Laboratory for Dual Analyses of Samples Collected at Morrill in $\mathbf{2 0 1 0}$ and for Quality Control Samples 
TABLE C.1 Analytical results from the AGEM Laboratory for quality control samples collected to monitor sample collection and handling activities at Morrill in 2010.

\begin{tabular}{|c|c|c|c|c|c|}
\hline \multirow[b]{2}{*}{$\begin{array}{l}\text { Sample } \\
\text { Date }\end{array}$} & \multirow[b]{2}{*}{ Sample } & \multirow[b]{2}{*}{ Type } & \multicolumn{3}{|c|}{ Concentration $(\mu \mathrm{g} / \mathrm{L})$} \\
\hline & & & $\begin{array}{c}\text { Carbon } \\
\text { Tetrachloride }\end{array}$ & Chloroform & $\begin{array}{c}\text { Methylene } \\
\text { Chloride }\end{array}$ \\
\hline $4 / 6 / 10$ & MRQCIR-W-30000 & Equipment rinsate & $\mathrm{ND}^{\mathrm{a}}$ & ND & ND \\
\hline $4 / 6 / 10$ & MRQCTB-W-30003 & Trip blank & ND & ND & ND \\
\hline $4 / 7 / 10$ & MRQCIR-W-30001 & Equipment rinsate & ND & ND & ND \\
\hline $4 / 7 / 10$ & MRQCIR-W-30002 & Equipment rinsate & ND & ND & ND \\
\hline $4 / 7 / 10$ & MRQCTB-W-30004 & Trip blank & ND & ND & ND \\
\hline $9 / 22 / 10$ & MRQCIR-W-30028 & Equipment rinsate & ND & ND & ND \\
\hline 9/22/10 & MRQCIR-W-30029 & Equipment rinsate & ND & ND & ND \\
\hline $9 / 23 / 10$ & MRDIH2O-W-30031 & Field blank & ND & ND & ND \\
\hline 9/23/10 & MRQCIR-W-30030 & Equipment rinsate & ND & ND & ND \\
\hline 9/23/10 & MRQCTB-W-30032 & Trip blank & ND & ND & ND \\
\hline
\end{tabular}

a ND, contaminant not detected at an instrument detection limit of $0.1 \mu \mathrm{g} / \mathrm{L}$. 
TABLE C.2 Analytical results from the AGEM Laboratory for dual analyses of samples collected at Morrill in 2010.

\begin{tabular}{|c|c|c|c|c|c|c|c|}
\hline \multirow[b]{2}{*}{$\begin{array}{c}\text { Sample } \\
\text { Date }\end{array}$} & \multirow[b]{2}{*}{ Location } & \multirow[b]{2}{*}{ Sample } & \multirow[b]{2}{*}{$\begin{array}{l}\text { Analysis } \\
\text { Type }\end{array}$} & \multirow[b]{2}{*}{$\begin{array}{l}\text { Sample } \\
\text { Medium }\end{array}$} & \multicolumn{3}{|c|}{ Concentration ( $\mu \mathrm{g} / \mathrm{kg}$ in soil; $\mu \mathrm{g} / \mathrm{L}$ in water) } \\
\hline & & & & & $\begin{array}{c}\text { Carbon } \\
\text { Tetrachloride }\end{array}$ & Chloroform & $\begin{array}{c}\text { Methylene } \\
\text { Chloride }\end{array}$ \\
\hline $4 / 6 / 10$ & SMB & MRSMB-S-29978 & Primary sample & Soil & $N D^{a}$ & ND & ND \\
\hline $4 / 6 / 10$ & MW3S & MRMW3S-W-29985 & Primary sample & Water & 3.5 & $0.2 \mathrm{Jb}^{\mathrm{b}}$ & ND \\
\hline $4 / 6 / 10$ & MW3S & MRMW3S-W-29985DUP & Duplicate analysis & Water & 3.1 & ND & ND \\
\hline $4 / 6 / 10$ & MW9S & MRMW9S-W-29991 & Primary sample & Water & 1.9 & ND & ND \\
\hline $4 / 6 / 10$ & MW9S & MRMW9SDUP-W-29998 & Replicate sample & Water & 1.8 & ND & ND \\
\hline $9 / 22 / 10$ & MW8S & MRMW8S-W-30018 & Primary sample & Water & 1.6 & ND & ND \\
\hline $9 / 22 / 10$ & MW8S & MRMW8S-W-30018DUP & Duplicate analysis & Water & 1.6 & ND & ND \\
\hline $9 / 22 / 10$ & MW8S & MRMW8SDUP-W-30026 & Replicate sample & Water & 1.7 & ND & ND \\
\hline $9 / 22 / 10$ & SMB & MRSMB-S-30009 & Primary sample & Soil & ND & ND & ND \\
\hline $9 / 22 / 10$ & $\mathrm{SMB}$ & MRSMB-S-30009DUP & Duplicate analysis & Soil & ND & ND & ND \\
\hline
\end{tabular}

a ND, contaminant not detected at an instrument detection limit of $0.1 \mu \mathrm{g} / \mathrm{L}$ for water analyses or $1.0 \mu \mathrm{g} / \mathrm{kg}$ for soil analyses.

b Qualifier J indicates an estimated concentration below the purge-and-trap method quantitation limit of $1.0 \mu \mathrm{g} / \mathrm{L}$. 
TABLE C.3 Analytical results from TestAmerica and the AGEM Laboratory for groundwater samples collected at Morrill in 2010 and submitted for verification analysis.

\begin{tabular}{|c|c|c|c|c|c|}
\hline \multirow[b]{3}{*}{$\begin{array}{l}\text { Sample } \\
\text { Date }\end{array}$} & \multirow[b]{3}{*}{ Sample } & \multicolumn{4}{|c|}{ Concentration $(\mu \mathrm{g} / \mathrm{L})$} \\
\hline & & \multicolumn{2}{|c|}{ TestAmerica } & \multicolumn{2}{|c|}{ AGEM Laboratory } \\
\hline & & $\begin{array}{c}\text { Carbon } \\
\text { Tetrachloride }\end{array}$ & Chloroform & $\begin{array}{c}\text { Carbon } \\
\text { Tetrachloride }\end{array}$ & Chloroform \\
\hline $4 / 7 / 10$ & MRMW4S-W-29986 & $N D^{a}$ & ND & ND & ND \\
\hline $4 / 7 / 10$ & MRMW7S-W-29989 & 13 & 0.52 & 15 & $0.4 \mathrm{~J}^{\mathrm{b}}$ \\
\hline $4 / 7 / 10$ & MRMW10S-W-29992 & ND & $0.34 \mathrm{~J}$ & ND & ND \\
\hline $4 / 7 / 10$ & MRMW1SB-W-29979 & ND & ND & ND & ND \\
\hline 4/7/10 & MRMW1SM-W-29980 & ND & ND & ND & ND \\
\hline $4 / 7 / 10$ & MRMW1ST-W-29981 & ND & ND & ND & ND \\
\hline $4 / 7 / 10$ & MRMW1SX-W-29982 & 18 & 1.1 & 21 & 1.2 \\
\hline $4 / 7 / 10$ & MRQCTB-W-30004 & ND & ND & ND & ND \\
\hline 9/23/10 & MRMW3S-W-30013 & 30 & 1.2 & 47 & 1.6 \\
\hline 9/23/10 & MRMW6S-W-30016 & ND & ND & ND & ND \\
\hline 9/23/10 & MRMW7S-W-30017 & 4.7 & ND & 6.6 & ND \\
\hline 9/23/10 & MRMW11S-W-30021 & 19 & 0.85 & 28 & 1.0 \\
\hline 9/23/10 & MRISCH-W-30022 & ND & ND & ND & ND \\
\hline 9/23/10 & MRQCTB-W-30032 & ND & ND & ND & ND \\
\hline
\end{tabular}

a ND, contaminant not detected at an instrument detection limit of $0.1 \mu \mathrm{g} / \mathrm{L}$.

b Qualifier J indicates an estimated concentration below the method quantitation limit of $0.5 \mu \mathrm{g} / \mathrm{L}$ in analyses at TestAmerica or $1.0 \mu \mathrm{g} / \mathrm{L}$ in analyses at the AGEM Laboratory. 
Supplement 1:

Waste Characterization Data 
October 14, 2010

Mr. Travis Kamler

TCW Construction Inc

$141 \mathrm{M}$ Street

Lincoln, NE 68508

RE: Project: Kansas Waste Water

Pace Project No.: 6086606

Dear Mr. Kamler:

Enclosed are the analytical results for sample(s) received by the laboratory on October 01, 2010.

The results relate only to the samples included in this report. Results reported herein conform to the most current NELAC standards, where applicable, unless otherwise narrated in the body of the report.

If you have any questions concerning this report, please feel free to contact me.

Sincerely,

$$
\text { sudy sigoon }
$$

Trudy Gipson

trudy.gipson@pacelabs.com

Project Manager

Enclosures

cc: Mr. David Surgnier 


\section{CERTIFICATIONS}

Project:

Kansas Waste Water

Pace Project No.: 6086606

Kansas Certification IDs

9608 Loiret Boulevard, Lenexa, KS 66219

A2LA Certification \#: 2456.01

Arkansas Certification \#: 05-008-0

Illinois Certification \#: 001191

lowa Certification \#: 118

Kansas/NELAP Certification \#: E-10116

Louisiana Certification \#: 03055

Nevada Certification \#: KS000212008A

Oklahoma Certification \#: 9205/9935

Texas Certification \#: T104704407-08-TX

Utah Certification \#: 9135995665

This report shall not be reproduced, except in full, without the written consent of Pace Analytical Services, Inc. 
SAMPLE SUMMARY

\begin{tabular}{|c|c|c|c|c|}
\hline Project: & Kansas Waste Water & & & \\
\hline Lab ID & Sample ID & Matrix & Date Collected & Date Received \\
\hline 6086606001 & BAPURGE-W-930101 & Water & 09/30/10 09:00 & 10/01/10 09:15 \\
\hline 6086606002 & CNPURGE-W-930102 & Water & 09/30/10 10:00 & 10/01/10 09:15 \\
\hline 6086606003 & EVPURGE-W-930103 & Water & 09/30/10 11:32 & 10/01/10 09:15 \\
\hline 6086606004 & MRPURGE-W-930104 & Water & 09/30/10 13:42 & 10/01/10 09:15 \\
\hline
\end{tabular}


SAMPLE ANALYTE COUNT

Project: Kansas Waste Water

Pace Project No.: 6086606

\begin{tabular}{|c|c|c|c|c|}
\hline Lab ID & Sample ID & Method & Analysts & $\begin{array}{l}\text { Analytes } \\
\text { Reported }\end{array}$ \\
\hline \multirow[t]{3}{*}{6086606001} & BAPURGE-W-930101 & EPA 504.1 & NAW & 1 \\
\hline & & EPA 5030B/8260 & HMW & 70 \\
\hline & & EPA 300.0 & RAB & 1 \\
\hline \multirow[t]{3}{*}{6086606002} & CNPURGE-W-930102 & EPA 504.1 & NAW & 1 \\
\hline & & EPA $5030 B / 8260$ & HMW & 70 \\
\hline & & EPA 300.0 & $\mathrm{RAB}$ & 1 \\
\hline \multirow[t]{3}{*}{6086606003} & EVPURGE-W-930103 & EPA 504.1 & NAW & 1 \\
\hline & & EPA 5030B/8260 & HMW & 70 \\
\hline & & EPA 300.0 & $\mathrm{RAB}$ & 1 \\
\hline \multirow[t]{3}{*}{6086606004} & MRPURGE-W-930104 & EPA 504.1 & NAW & 1 \\
\hline & & EPA 5030B/8260 & HMW & 70 \\
\hline & & EPA 300.0 & $\mathrm{RAB}$ & 1 \\
\hline
\end{tabular}




\section{ANALYTICAL RESULTS}

Project: Kansas Waste Water

Pace Project No.: 6086606

Sample: BAPURGE-W-930101 Lab ID: 6086606001 Collected: 09/30/10 09:00 Received: 10/01/10 09:15 Matrix: Water

\begin{tabular}{|c|c|}
\hline Parameters & Results \\
\hline
\end{tabular}

504 GCS EDB and DBCP

1,2-Dibromoethane (EDB)

\section{MSV}

Acetone

Benzene

Bromobenzene

Bromochloromethane

Bromodichloromethane

Bromoform

Bromomethane

2-Butanone (MEK)

n-Butylbenzene

sec-Butylbenzene

tert-Butylbenzene

Carbon disulfide

Carbon tetrachloride

Chlorobenzene

Chloroethane

Chloroform

Chloromethane

2-Chlorotoluene

4-Chlorotoluene

1,2-Dibromo-3-chloropropane

Dibromochloromethane

1,2-Dibromoethane (EDB)

Dibromomethane

1,2-Dichlorobenzene

1,3-Dichlorobenzene

1,4-Dichlorobenzene

Dichlorodifluoromethane

1,1-Dichloroethane

1,2-Dichloroethane

1,2-Dichloroethene (Total)

1,1-Dichloroethene

cis-1,2-Dichloroethene

trans-1,2-Dichloroethene

1,2-Dichloropropane

1,3-Dichloropropane

2,2-Dichloropropane

1,1-Dichloropropene

cis-1,3-Dichloropropene

trans-1,3-Dichloropropene

Ethylbenzene

Hexachloro-1,3-butadiene

2-Hexanone

Isopropylbenzene (Cumene)

p-Isopropyltoluene

Date: 10/14/2010 11:06 AM

Analytical Method: EPA 504.1 Preparation Method: EPA 504.1

$$
\begin{array}{llllllll}
\text { ND ug/L } & 0.029 & 1 & 10 / 07 / 10 & 00: 00 & 10 / 07 / 10 & 21: 23 & 106-93-4
\end{array}
$$

Analytical Method: EPA 5030B/8260

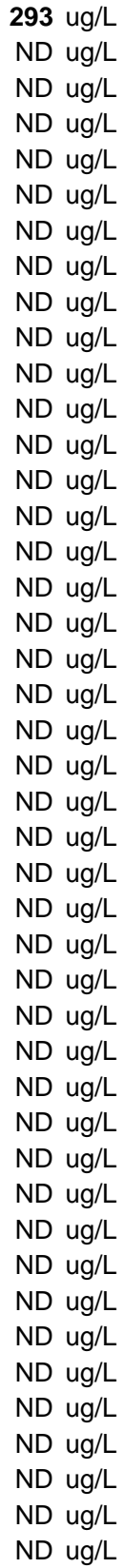

\begin{tabular}{rr}
10.0 & 1 \\
1.0 & 1 \\
1.0 & 1 \\
1.0 & 1 \\
1.0 & 1 \\
1.0 & 1 \\
1.0 & 1 \\
10.0 & 1 \\
1.0 & 1 \\
1.0 & 1 \\
1.0 & 1 \\
5.0 & 1 \\
1.0 & 1 \\
1.0 & 1 \\
1.0 & 1 \\
1.0 & 1 \\
1.0 & 1 \\
1.0 & 1 \\
1.0 & 1 \\
2.5 & 1 \\
1.0 & 1 \\
1.0 & 1 \\
1.0 & 1 \\
1.0 & 1 \\
1.0 & 1 \\
1.0 & 1 \\
1.0 & 1 \\
1.0 & 1 \\
1.0 & 1 \\
1.0 & 1 \\
1.0 & 1 \\
1.0 & 1 \\
1.0 & 1 \\
1.0 & 1 \\
1.0 & 1 \\
1.0 & 1 \\
1.0 & 1 \\
1.0 & 1 \\
1.0 & 1 \\
1.0 & 1 \\
1.0 & 1 \\
10.0 & 1 \\
1.0 & 1 \\
1.0 & 1 \\
& \\
\hline
\end{tabular}

$10 / 03 / 10$ 12:46 67-64-1 $10 / 03 / 1012: 46 \quad 71-43-2$ $10 / 03 / 1012: 46 \quad 108-86-1$ $10 / 03 / 1012: 46 \quad 74-97-5$ $10 / 03 / 1012: 46 \quad 75-27-4$ $10 / 03 / 1012: 46 \quad 75-25-2$ $10 / 03 / 1012: 46 \quad 74-83-9$ $10 / 03 / 1012: 46 \quad 78-93-3$ $10 / 03 / 1012: 46 \quad 104-51-8$ $10 / 03 / 1012: 46 \quad 135-98-8$ $10 / 03 / 1012: 46$ 98-06-6 $10 / 03 / 1012: 46 \quad 75-15-0$ $10 / 03 / 1012: 46 \quad 56-23-5$ $10 / 03 / 1012: 46 \quad 108-90-7$ $10 / 03 / 1012: 46 \quad 75-00-3$ 10/03/10 12:46 67-66-3 10/03/10 12:46 74-87-3 $10 / 03 / 1012: 46 \quad 95-49-8$ $10 / 03 / 10$ 12:46 106-43-4 $10 / 03 / 1012: 46 \quad 96-12-8$ $10 / 03 / 10 \quad 12: 46 \quad 124-48-1$ $10 / 03 / 10$ 12:46 106-93-4 10/03/10 12:46 74-95-3 10/03/10 12:46 95-50-1 10/03/10 12:46 541-73-1 10/03/10 12:46 106-46-7 10/03/10 12:46 75-71-8 $10 / 03 / 10$ 12:46 75-34-3 $10 / 03 / 10 \quad 12: 46 \quad 107-06-2$ 10/03/10 12:46 540-59-0 $10 / 03 / 10$ 12:46 75-35-4 $10 / 03 / 1012: 46 \quad 156-59-2$ $10 / 03 / 1012: 46 \quad 156-60-5$ 10/03/10 12:46 78-87-5 $10 / 03 / 10 \quad 12: 46 \quad 142-28-9$ $10 / 03 / 1012: 46 \quad 594-20-7$ 10/03/10 12:46 563-58-6 10/03/10 12:46 10061-01-5 10/03/10 12:46 10061-02-6 $10 / 03 / 10 \quad 12: 46 \quad 100-41-4$ 10/03/10 12:46 87-68-3 10/03/10 12:46 591-78-6 $10 / 03 / 1012: 46 \quad 98-82-8$ $10 / 03 / 10$ 12:46 99-87-6

This report shall not be reproduced, except in full, without the written consent of Pace Analytical Services, Inc. 


\section{ANALYTICAL RESULTS}

Project: Kansas Waste Water

Pace Project No.: 6086606

Sample: BAPURGE-W-930101 Lab ID: 6086606001 $\quad$ Collected: 09/30/10 09:00 Received: 10/01/10 09:15 Matrix: Water

Parameters $\longrightarrow$ Results $\longrightarrow$ Units $\longrightarrow$ Report Limit $\longrightarrow$ DF

8260 MSV

Methylene chloride

4-Methyl-2-pentanone (MIBK)

Methyl-tert-butyl ether

Naphthalene

n-Propylbenzene

Styrene

1,1,1,2-Tetrachloroethane

1,1,2,2-Tetrachloroethane

Tetrachloroethene

Toluene

1,2,3-Trichlorobenzene

1,2,4-Trichlorobenzene

1,1,1-Trichloroethane

1,1,2-Trichloroethane

Trichloroethene

Trichlorofluoromethane

1,2,3-Trichloropropane

1,2,4-Trimethylbenzene

1,3,5-Trimethylbenzene

Vinyl chloride

Xylene (Total)

4-Bromofluorobenzene (S)

Dibromofluoromethane (S)

1,2-Dichloroethane-d4 (S)

Toluene-d8 (S)

Preservation $\mathrm{pH}$

\subsection{IC Anions}

Nitrate as $\mathrm{N}$
Analytical Method: EPA 5030B/8260

\begin{tabular}{|c|c|c|c|c|}
\hline ND ug/L & 1.0 & 1 & $10 / 03 / 1012: 46$ & $75-09-2$ \\
\hline ND ug/L & 10.0 & 1 & $10 / 03 / 1012: 46$ & $108-10-1$ \\
\hline ND ug/L & 1.0 & 1 & $10 / 03 / 1012: 46$ & $1634-04-4$ \\
\hline ND ug/L & 10.0 & 1 & $10 / 03 / 1012: 46$ & $91-20-3$ \\
\hline ND ug/L & 1.0 & 1 & $10 / 03 / 1012: 46$ & $103-65-1$ \\
\hline ND ug/L & 1.0 & 1 & $10 / 03 / 1012: 46$ & $100-42-5$ \\
\hline ND ug/L & 1.0 & 1 & $10 / 03 / 1012: 46$ & $630-20-6$ \\
\hline ND ug/L & 1.0 & 1 & $10 / 03 / 1012: 46$ & $79-34-5$ \\
\hline ND ug/L & 1.0 & 1 & $10 / 03 / 1012: 46$ & $127-18-4$ \\
\hline ND ug/L & 1.0 & 1 & $10 / 03 / 1012: 46$ & $108-88-3$ \\
\hline ND ug/L & 1.0 & 1 & $10 / 03 / 1012: 46$ & $87-61-6$ \\
\hline ND ug/L & 1.0 & 1 & $10 / 03 / 1012: 46$ & $120-82-1$ \\
\hline ND ug/L & 1.0 & 1 & $10 / 03 / 1012: 46$ & $71-55-6$ \\
\hline ND ug/L & 1.0 & 1 & $10 / 03 / 1012: 46$ & $79-00-5$ \\
\hline ND ug/L & 1.0 & 1 & $10 / 03 / 1012: 46$ & $79-01-6$ \\
\hline ND ug/L & 1.0 & 1 & $10 / 03 / 1012: 46$ & $75-69-4$ \\
\hline ND ug/L & 2.5 & 1 & $10 / 03 / 1012: 46$ & $96-18-4$ \\
\hline ND ug/L & 1.0 & 1 & $10 / 03 / 1012: 46$ & $95-63-6$ \\
\hline ND ug/L & 1.0 & 1 & $10 / 03 / 1012: 46$ & $108-67-8$ \\
\hline ND ug/L & 1.0 & 1 & $10 / 03 / 1012: 46$ & $75-01-4$ \\
\hline ND ug/L & 3.0 & 1 & $10 / 03 / 1012: 46$ & $1330-20-7$ \\
\hline $93 \%$ & $87-113$ & 1 & $10 / 03 / 1012: 46$ & $460-00-4$ \\
\hline $103 \%$ & $86-112$ & 1 & $10 / 03 / 1012: 46$ & $1868-53-7$ \\
\hline $98 \%$ & $82-119$ & 1 & $10 / 03 / 1012: 46$ & $17060-07-0$ \\
\hline $102 \%$ & $90-110$ & 1 & $10 / 03 / 1012: 46$ & $2037-26-5$ \\
\hline 7.0 & 0.10 & 1 & $10 / 03 / 1012: 46$ & \\
\hline
\end{tabular}

Analytical Method: EPA 300.0

$2.7 \mathrm{mg} / \mathrm{L}$

$0.10 \quad 1$

10/01/10 18:59 14797-55-8

This report shall not be reproduced, except in full, 


\section{ANALYTICAL RESULTS}

Project: Kansas Waste Water

Pace Project No.: 6086606

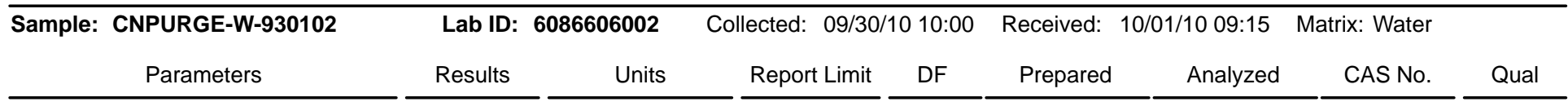

504 GCS EDB and DBCP

1,2-Dibromoethane (EDB)

\section{MSV}

Acetone

Benzene

Bromobenzene

Bromochloromethane

Bromodichloromethane

Bromoform

Bromomethane

2-Butanone (MEK)

n-Butylbenzene

sec-Butylbenzene

tert-Butylbenzene

Carbon disulfide

Carbon tetrachloride

Chlorobenzene

Chloroethane

Chloroform

Chloromethane

2-Chlorotoluene

4-Chlorotoluene

1,2-Dibromo-3-chloropropane

Dibromochloromethane

1,2-Dibromoethane (EDB)

Dibromomethane

1,2-Dichlorobenzene

1,3-Dichlorobenzene

1,4-Dichlorobenzene

Dichlorodifluoromethane

1,1-Dichloroethane

1,2-Dichloroethane

1,2-Dichloroethene (Total)

1,1-Dichloroethene

cis-1,2-Dichloroethene

trans-1,2-Dichloroethene

1,2-Dichloropropane

1,3-Dichloropropane

2,2-Dichloropropane

1,1-Dichloropropene

cis-1,3-Dichloropropene

trans-1,3-Dichloropropene

Ethylbenzene

Hexachloro-1,3-butadiene

2-Hexanone

Isopropylbenzene (Cumene)

p-Isopropyltoluene

Date: 10/14/2010 11:06 AM

Analytical Method: EPA 504.1 Preparation Method: EPA 504.1

$$
\begin{array}{llllllll}
\text { ND ug/L } & 0.029 & 1 & 10 / 07 / 10 & 00: 00 & 10 / 07 / 10 & 21: 34 & 106-93-4
\end{array}
$$

Analytical Method: EPA 5030B/8260

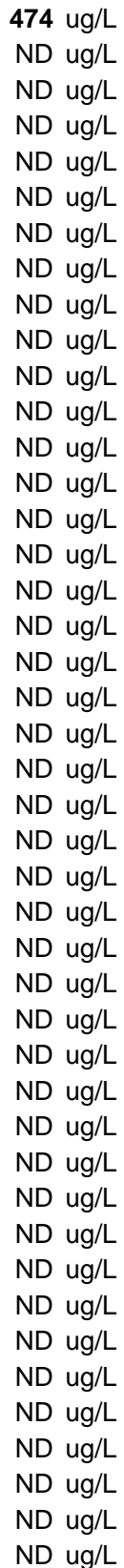

$474 \mathrm{ug} / \mathrm{L}$

ND ug/L

ND ug/L

ug/L

ND ug/L

ND ug/L

ND ug/L

ND ug/L

$N D$ ug/L

$N D$ ug/L

ND ug/L

ND $u g / L$

ug/L

ND ug/L

$\mathrm{ND}$ ug/L

ug/L

ND ug/L

ND ug/L

ND ug/L

ND ug/L

ND ug/L

D ug/L

ND ug/L

ND ug/L

ND ug/L

ND ug/L

$\mathrm{ND}$ ug/L

ND ug/L

ND ug/L

ND ug/L

ND ug/L

ND ug/L

ND ug/L

ND ug/L

$\begin{array}{rr}10.0 & 1 \\ 1.0 & 1 \\ 1.0 & 1 \\ 1.0 & 1 \\ 1.0 & 1 \\ 1.0 & 1 \\ 1.0 & 1 \\ 10.0 & 1 \\ 1.0 & 1 \\ 1.0 & 1 \\ 1.0 & 1 \\ 5.0 & 1 \\ 1.0 & 1 \\ 1.0 & 1 \\ 1.0 & 1 \\ 1.0 & 1 \\ 1.0 & 1 \\ 1.0 & 1 \\ 1.0 & 1 \\ 2.5 & 1 \\ 1.0 & 1 \\ 1.0 & 1 \\ 1.0 & 1 \\ 1.0 & 1 \\ 1.0 & 1 \\ 1.0 & 1 \\ 1.0 & 1 \\ 1.0 & 1 \\ 1.0 & 1 \\ 1.0 & 1 \\ 1.0 & 1 \\ 1.0 & 1 \\ 1.0 & 1 \\ 1.0 & 1 \\ 1.0 & 1 \\ 1.0 & 1 \\ 1.0 & 1 \\ 1.0 & 1 \\ 1.0 & 1 \\ 1.0 & 1 \\ 1.0 & 1 \\ 10.0 & 1 \\ 1.0 & 1 \\ 1.0 & 1 \\ & \end{array}$

10/03/10 13:01 67-64-1

10/03/10 13:01 71-43-2

10/03/10 13:01 108-86-1

10/03/10 13:01 74-97-5

10/03/10 13:01 75-27-4

10/03/10 13:01 75-25-2

10/03/10 13:01 74-83-9

10/03/10 13:01 78-93-3

10/03/10 13:01 104-51-8

10/03/10 13:01 135-98-8

10/03/10 13:01 98-06-6

10/03/10 13:01 75-15-0

10/03/10 13:01 56-23-5

10/03/10 13:01 108-90-7

10/03/10 13:01 75-00-3

10/03/10 13:01 67-66-3

10/03/10 13:01 74-87-3

10/03/10 13:01 95-49-8

10/03/10 13:01 106-43-4

10/03/10 13:01 96-12-8

10/03/10 13:01 124-48-1

$10 / 03 / 10$ 13:01 106-93-4

10/03/10 13:01 74-95-3

10/03/10 13:01 95-50-1

10/03/10 13:01 541-73-1

$10 / 03 / 10$ 13:01 106-46-7

10/03/10 13:01 75-71-8

10/03/10 13:01 75-34-3

10/03/10 13:01 107-06-2

10/03/10 13:01 540-59-0

10/03/10 13:01 75-35-4

10/03/10 13:01 156-59-2

10/03/10 13:01 156-60-5

10/03/10 13:01 78-87-5

10/03/10 13:01 142-28-9

10/03/10 13:01 594-20-7

10/03/10 13:01 563-58-6

10/03/10 13:01 10061-01-5

10/03/10 13:01 10061-02-6

10/03/10 13:01 100-41-4

10/03/10 13:01 87-68-3

10/03/10 13:01 591-78-6

10/03/10 13:01 98-82-8

10/03/10 13:01 99-87-6
L3

L3

Page 7 of 19

This report shall not be reproduced, except in full,

without the written consent of Pace Analytical Services, Inc. 


\section{ANALYTICAL RESULTS}

Project: Kansas Waste Water

Pace Project No.: 6086606

Sample: CNPURGE-W-930102 Lab ID: $6086606002 \quad$ Collected: 09/30/10 10:00 Received: 10/01/10 09:15 Matrix: Water

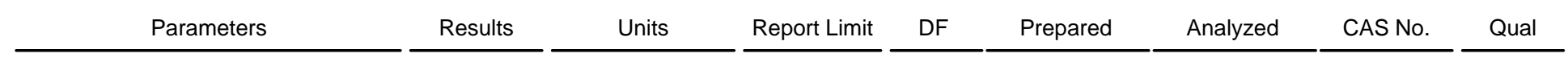

8260 MSV

Methylene chloride

4-Methyl-2-pentanone (MIBK)

Methyl-tert-butyl ether

Naphthalene

n-Propylbenzene

Styrene

1,1,1,2-Tetrachloroethane

1,1,2,2-Tetrachloroethane

Tetrachloroethene

Toluene

1,2,3-Trichlorobenzene

1,2,4-Trichlorobenzene

1,1,1-Trichloroethane

1,1,2-Trichloroethane

Trichloroethene

Trichlorofluoromethane

1,2,3-Trichloropropane

1,2,4-Trimethylbenzene

1,3,5-Trimethylbenzene

Vinyl chloride

Xylene (Total)

4-Bromofluorobenzene (S)

Dibromofluoromethane (S)

1,2-Dichloroethane-d4 (S)

Toluene-d8 (S)

Preservation $\mathrm{pH}$

\subsection{IC Anions}

Nitrate as $\mathrm{N}$
Analytical Method: EPA 5030B/8260

$\begin{array}{lrrll}\text { ND ug/L } & 1.0 & 1 & 10 / 03 / 1013: 01 & 75-09-2 \\ \text { ND ug/L } & 10.0 & 1 & 10 / 03 / 1013: 01 & 108-10-1 \\ \text { ND ug/L } & 1.0 & 1 & 10 / 03 / 1013: 01 & 1634-04-4 \\ \text { ND ug/L } & 10.0 & 1 & 10 / 03 / 1013: 01 & 91-20-3 \\ \text { ND ug/L } & 1.0 & 1 & 10 / 03 / 1013: 01 & 103-65-1 \\ \text { ND ug/L } & 1.0 & 1 & 10 / 03 / 1013: 01 & 100-42-5 \\ \text { ND ug/L } & 1.0 & 1 & 10 / 03 / 1013: 01 & 630-20-6 \\ \text { ND ug/L } & 1.0 & 1 & 10 / 03 / 1013: 01 & 79-34-5 \\ \text { ND ug/L } & 1.0 & 1 & 10 / 03 / 1013: 01 & 127-18-4 \\ \text { ND ug/L } & 1.0 & 1 & 10 / 03 / 1013: 01 & 108-88-3 \\ \text { ND ug/L } & 1.0 & 1 & 10 / 03 / 1013: 01 & 87-61-6 \\ \text { ND ug/L } & 1.0 & 1 & 10 / 03 / 1013: 01 & 120-82-1 \\ \text { ND ug/L } & 1.0 & 1 & 10 / 03 / 1013: 01 & 71-55-6 \\ \text { ND ug/L } & 1.0 & 1 & 10 / 03 / 1013: 01 & 79-00-5 \\ \text { ND ug/L } & 1.0 & 1 & 10 / 03 / 1013: 01 & 79-01-6 \\ \text { ND ug/L } & 1.0 & 1 & 10 / 03 / 1013: 01 & 75-69-4 \\ \text { ND ug/L } & 2.5 & 1 & 10 / 03 / 1013: 01 & 96-18-4 \\ \text { ND ug/L } & 1.0 & 1 & 10 / 03 / 1013: 01 & 95-63-6 \\ \text { ND ug/L } & 1.0 & 1 & 10 / 03 / 1013: 01 & 108-67-8 \\ \text { ND ug/L } & 1.0 & 1 & 10 / 03 / 1013: 01 & 75-01-4 \\ \text { ND ug/L } & 3.0 & 1 & 10 / 03 / 1013: 01 & 1330-20-7 \\ 96 \% & 87-113 & 1 & 10 / 03 / 1013: 01 & 460-00-4 \\ 105 \% & 86-112 & 1 & 10 / 03 / 1013: 01 & 1868-53-7 \\ 100 \% & 82-119 & 1 & 10 / 03 / 1013: 01 & 17060-07-0 \\ 101 \% & 90-110 & 1 & 10 / 03 / 1013: 01 & 2037-26-5 \\ 7.0 & 0.10 & 1 & 10 / 03 / 1013: 01 & \end{array}$

Analytical Method: EPA 300.0

$1.7 \mathrm{mg} / \mathrm{L}$

$0.10 \quad 1$

10/01/10 19:15 14797-55-8

This report shall not be reproduced, except in full, without the written consent of Pace Analytical Services, Inc. 


\section{ANALYTICAL RESULTS}

Project: Kansas Waste Water

Pace Project No.: 6086606

Sample: EVPURGE-W-930103 Lab ID: 6086606003 Collected: 09/30/10 11:32 Received: 10/01/10 09:15 Matrix: Water

\begin{tabular}{|c|c|}
\hline Parameters & Results \\
\hline
\end{tabular}

504 GCS EDB and DBCP

1,2-Dibromoethane (EDB)

\section{MSV}

Acetone

Benzene

Bromobenzene

Bromochloromethane

Bromodichloromethane

Bromoform

Bromomethane

2-Butanone (MEK)

n-Butylbenzene

sec-Butylbenzene

tert-Butylbenzene

Carbon disulfide

Carbon tetrachloride

Chlorobenzene

Chloroethane

Chloroform

Chloromethane

2-Chlorotoluene

4-Chlorotoluene

1,2-Dibromo-3-chloropropane

Dibromochloromethane

1,2-Dibromoethane (EDB)

Dibromomethane

1,2-Dichlorobenzene

1,3-Dichlorobenzene

1,4-Dichlorobenzene

Dichlorodifluoromethane

1,1-Dichloroethane

1,2-Dichloroethane

1,2-Dichloroethene (Total)

1,1-Dichloroethene

cis-1,2-Dichloroethene

trans-1,2-Dichloroethene

1,2-Dichloropropane

1,3-Dichloropropane

2,2-Dichloropropane

1,1-Dichloropropene

cis-1,3-Dichloropropene

trans-1,3-Dichloropropene

Ethylbenzene

Hexachloro-1,3-butadiene

2-Hexanone

Isopropylbenzene (Cumene)

p-Isopropyltoluene

Date: 10/14/2010 11:06 AM
Analytical Method: EPA 504.1 Preparation Method: EPA 504.1

$$
\begin{array}{llllllll}
\text { ND ug/L } & 0.029 & 1 & 10 / 07 / 10 & 00: 00 & 10 / 07 / 10 & 21: 44 & 106-93-4
\end{array}
$$

Analytical Method: EPA 5030B/8260

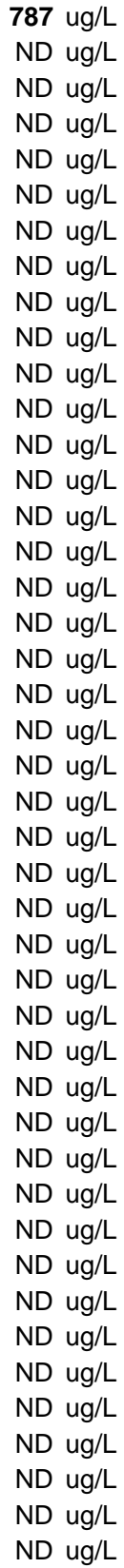

\begin{tabular}{rr}
10.0 & 1 \\
1.0 & 1 \\
1.0 & 1 \\
1.0 & 1 \\
1.0 & 1 \\
1.0 & 1 \\
1.0 & 1 \\
10.0 & 1 \\
1.0 & 1 \\
1.0 & 1 \\
1.0 & 1 \\
5.0 & 1 \\
1.0 & 1 \\
1.0 & 1 \\
1.0 & 1 \\
1.0 & 1 \\
1.0 & 1 \\
1.0 & 1 \\
1.0 & 1 \\
2.5 & 1 \\
1.0 & 1 \\
1.0 & 1 \\
1.0 & 1 \\
1.0 & 1 \\
1.0 & 1 \\
1.0 & 1 \\
1.0 & 1 \\
1.0 & 1 \\
1.0 & 1 \\
1.0 & 1 \\
1.0 & 1 \\
1.0 & 1 \\
1.0 & 1 \\
1.0 & 1 \\
1.0 & 1 \\
1.0 & 1 \\
1.0 & 1 \\
1.0 & 1 \\
1.0 & 1 \\
1.0 & 1 \\
1.0 & 1 \\
10.0 & 1 \\
1.0 & 1 \\
1.0 & 1 \\
& \\
\hline
\end{tabular}

10/03/10 13:16 67-64-1

$10 / 03 / 1013: 16 \quad 71-43-2$

$10 / 03 / 1013: 16 \quad 108-86-1$

10/03/10 13:16 74-97-5

$10 / 03 / 10$ 13:16 75-27-4

$10 / 03 / 10$ 13:16 75-25-2

10/03/10 13:16 74-83-9

10/03/10 13:16 78-93-3

$10 / 03 / 1013: 16 \quad 104-51-8$

10/03/10 13:16 135-98-8

10/03/10 13:16 98-06-6

10/03/10 13:16 75-15-0

$10 / 03 / 1013: 16 \quad 56-23-5$

10/03/10 13:16 108-90-7

10/03/10 13:16 75-00-3

10/03/10 13:16 67-66-3

10/03/10 13:16 74-87-3

10/03/10 13:16 95-49-8

10/03/10 13:16 106-43-4

10/03/10 13:16 96-12-8

10/03/10 13:16 124-48-1

$10 / 03 / 1013: 16 \quad 106-93-4$

10/03/10 13:16 74-95-3

10/03/10 13:16 95-50-1

10/03/10 13:16 541-73-1

10/03/10 13:16 106-46-7

10/03/10 13:16 75-71-8

10/03/10 13:16 75-34-3

$10 / 03 / 1013: 16 \quad 107-06-2$

10/03/10 13:16 540-59-0

10/03/10 13:16 75-35-4

10/03/10 13:16 156-59-2

$10 / 03 / 1013: 16 \quad 156-60-5$

10/03/10 13:16 78-87-5

10/03/10 13:16 142-28-9

10/03/10 13:16 594-20-7

10/03/10 13:16 563-58-6

10/03/10 13:16 10061-01-5

10/03/10 13:16 10061-02-6

$10 / 03 / 1013: 16 \quad 100-41-4$

10/03/10 13:16 87-68-3

10/03/10 13:16 591-78-6

10/03/10 13:16 98-82-8

10/03/10 13:16 99-87-6
E,P2

L3

L3

\section{REPORT OF LABORATORY ANALYSIS}

This report shall not be reproduced, except in full,

without the written consent of Pace Analytical Services, Inc. 


\section{ANALYTICAL RESULTS}

Project: Kansas Waste Water

Pace Project No.: 6086606

Sample: EVPURGE-W-930103 Lab ID: $6086606003 \quad$ Collected: 09/30/10 11:32 Received: 10/01/10 09:15 Matrix: Water

Parameters $\longrightarrow$ Results $\longrightarrow$ Units $\longrightarrow$ Report Limit $\longrightarrow$ DF Prepared $\longrightarrow$ Analyzed CAS No.

8260 MSV

Methylene chloride

4-Methyl-2-pentanone (MIBK)

Methyl-tert-butyl ether

Naphthalene

n-Propylbenzene

Styrene

1,1,1,2-Tetrachloroethane

1,1,2,2-Tetrachloroethane

Tetrachloroethene

Toluene

1,2,3-Trichlorobenzene

1,2,4-Trichlorobenzene

1,1,1-Trichloroethane

1,1,2-Trichloroethane

Trichloroethene

Trichlorofluoromethane

1,2,3-Trichloropropane

1,2,4-Trimethylbenzene

1,3,5-Trimethylbenzene

Vinyl chloride

Xylene (Total)

4-Bromofluorobenzene (S)

Dibromofluoromethane (S)

1,2-Dichloroethane-d4 (S)

Toluene-d8 (S)

Preservation $\mathrm{pH}$

\subsection{IC Anions}

Nitrate as $\mathrm{N}$
Analytical Method: EPA 5030B/8260

\begin{tabular}{|c|c|c|c|c|}
\hline ND ug/L & 1.0 & 1 & $10 / 03 / 1013: 16$ & $75-09-2$ \\
\hline ND ug/L & 10.0 & 1 & $10 / 03 / 1013: 16$ & $108-10-1$ \\
\hline ND ug/L & 1.0 & 1 & $10 / 03 / 1013: 16$ & $1634-04-4$ \\
\hline ND ug/L & 10.0 & 1 & 10/03/10 13:16 & $91-20-3$ \\
\hline ND ug/L & 1.0 & 1 & $10 / 03 / 1013: 16$ & $103-65-1$ \\
\hline ND ug/L & 1.0 & 1 & $10 / 03 / 1013: 16$ & $100-42-5$ \\
\hline ND ug/L & 1.0 & 1 & 10/03/10 13:16 & $630-20-6$ \\
\hline ND ug/L & 1.0 & 1 & $10 / 03 / 1013: 16$ & $79-34-5$ \\
\hline ND ug/L & 1.0 & 1 & $10 / 03 / 1013: 16$ & $127-18-4$ \\
\hline ND ug/L & 1.0 & 1 & 10/03/10 13:16 & $108-88-3$ \\
\hline ND ug/L & 1.0 & 1 & $10 / 03 / 1013: 16$ & $87-61-6$ \\
\hline ND ug/L & 1.0 & 1 & $10 / 03 / 1013: 16$ & $120-82-1$ \\
\hline ND ug/L & 1.0 & 1 & 10/03/10 13:16 & $71-55-6$ \\
\hline ND ug/L & 1.0 & 1 & $10 / 03 / 1013: 16$ & $79-00-5$ \\
\hline ND ug/L & 1.0 & 1 & $10 / 03 / 1013: 16$ & $79-01-6$ \\
\hline ND ug/L & 1.0 & 1 & 10/03/10 13:16 & $75-69-4$ \\
\hline ND ug/L & 2.5 & 1 & $10 / 03 / 1013: 16$ & $96-18-4$ \\
\hline ND ug/L & 1.0 & 1 & $10 / 03 / 1013: 16$ & $95-63-6$ \\
\hline ND ug/L & 1.0 & 1 & $10 / 03 / 1013: 16$ & $108-67-8$ \\
\hline ND ug/L & 1.0 & 1 & $10 / 03 / 1013: 16$ & $75-01-4$ \\
\hline ND ug/L & 3.0 & 1 & 10/03/10 13:16 & $1330-20-7$ \\
\hline $96 \%$ & $87-113$ & 1 & 10/03/10 13:16 & $460-00-4$ \\
\hline $98 \%$ & $86-112$ & 1 & $10 / 03 / 1013: 16$ & $1868-53-7$ \\
\hline $92 \%$ & $82-119$ & 1 & $10 / 03 / 1013: 16$ & $17060-07-0$ \\
\hline $100 \%$ & $90-110$ & 1 & 10/03/10 13:16 & $2037-26-5$ \\
\hline 7.0 & 0.10 & 1 & $10 / 03 / 1013: 16$ & \\
\hline
\end{tabular}

Analytical Method: EPA 300.0

$2.0 \mathrm{mg} / \mathrm{L}$

$0.10 \quad 1$

10/01/10 19:32 14797-55-8

This report shall not be reproduced, except in full, without the written consent of Pace Analytical Services, Inc. 


\section{ANALYTICAL RESULTS}

Project: Kansas Waste Water

Pace Project No.: 6086606

Sample: MRPURGE-W-930104 Lab ID: 6086606004 Collected: 09/30/10 13:42 Received: 10/01/10 09:15 Matrix: Water

\begin{tabular}{|c|c|}
\hline Parameters & Results \\
\hline
\end{tabular}

504 GCS EDB and DBCP

1,2-Dibromoethane (EDB)

\section{MSV}

Acetone

Benzene

Bromobenzene

Bromochloromethane

Bromodichloromethane

Bromoform

Bromomethane

2-Butanone (MEK)

n-Butylbenzene

sec-Butylbenzene

tert-Butylbenzene

Carbon disulfide

Carbon tetrachloride

Chlorobenzene

Chloroethane

Chloroform

Chloromethane

2-Chlorotoluene

4-Chlorotoluene

1,2-Dibromo-3-chloropropane

Dibromochloromethane

1,2-Dibromoethane (EDB)

Dibromomethane

1,2-Dichlorobenzene

1,3-Dichlorobenzene

1,4-Dichlorobenzene

Dichlorodifluoromethane

1,1-Dichloroethane

1,2-Dichloroethane

1,2-Dichloroethene (Total)

1,1-Dichloroethene

cis-1,2-Dichloroethene

trans-1,2-Dichloroethene

1,2-Dichloropropane

1,3-Dichloropropane

2,2-Dichloropropane

1,1-Dichloropropene

cis-1,3-Dichloropropene

trans-1,3-Dichloropropene

Ethylbenzene

Hexachloro-1,3-butadiene

2-Hexanone

Isopropylbenzene (Cumene)

p-Isopropyltoluene

Date: 10/14/2010 11:06 AM

Analytical Method: EPA 504.1 Preparation Method: EPA 504.1

$$
\begin{array}{llllllll}
\text { ND ug/L } & 0.029 & 1 & 10 / 07 / 10 & 00: 00 & 10 / 07 / 10 & 21: 55 & 106-93-4
\end{array}
$$

Analytical Method: EPA 5030B/8260

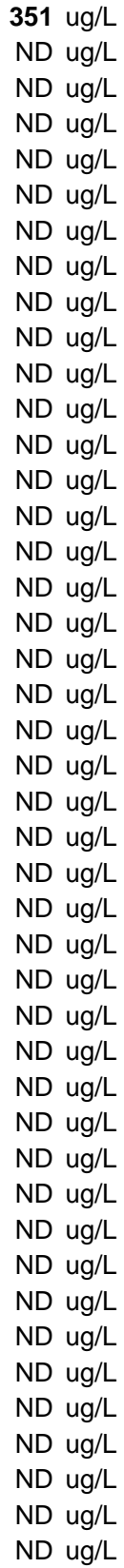

$10.0 \quad 1$

$1.0 \quad 1$

$1.0 \quad 1$

$1.0 \quad 1$

$1.0 \quad 1$

1.01

1.01

10.01

$1.0 \quad 1$

$1.0 \quad 1$

$1.0 \quad 1$

5.01

$1.0 \quad 1$

$1.0 \quad 1$

$1.0 \quad 1$

$1.0 \quad 1$

$1.0 \quad 1$

$1.0 \quad 1$

$1.0 \quad 1$

2.51

$1.0 \quad 1$

$1.0 \quad 1$

$1.0 \quad 1$

$1.0 \quad 1$

$1.0 \quad 1$

1.01

$1.0 \quad 1$

$1.0 \quad 1$

1.01

$1.0 \quad 1$

$1.0 \quad 1$

$1.0 \quad 1$

$1.0 \quad 1$

$1.0 \quad 1$

$1.0 \quad 1$

$1.0 \quad 1$

$1.0 \quad 1$

$1.0 \quad 1$

$1.0 \quad 1$

1.01

1.01

$10.0 \quad 1$

$1.0 \quad 1$

1.01
10/03/10 13:31 67-64-1

10/03/10 13:31 71-43-2

10/03/10 13:31 108-86-1

10/03/10 13:31 74-97-5

10/03/10 13:31 75-27-4

10/03/10 13:31 75-25-2

10/03/10 13:31 74-83-9

10/03/10 13:31 78-93-3

10/03/10 13:31 104-51-8

$10 / 03 / 1013: 31 \quad 135-98-8$

10/03/10 13:31 98-06-6

10/03/10 13:31 75-15-0

10/03/10 13:31 56-23-5

10/03/10 13:31 108-90-7

10/03/10 13:31 75-00-3

10/03/10 13:31 67-66-3

10/03/10 13:31 74-87-3

10/03/10 13:31 95-49-8

$10 / 03 / 10$ 13:31 106-43-4

10/03/10 13:31 96-12-8

10/03/10 13:31 124-48-1

10/03/10 13:31 106-93-4

10/03/10 13:31 74-95-3

10/03/10 13:31 95-50-1

10/03/10 13:31 541-73-1

10/03/10 13:31 106-46-7

10/03/10 13:31 75-71-8

10/03/10 13:31 75-34-3

10/03/10 13:31 107-06-2

10/03/10 13:31 540-59-0

10/03/10 13:31 75-35-4

10/03/10 13:31 156-59-2

10/03/10 13:31 156-60-5

10/03/10 13:31 78-87-5

10/03/10 13:31 142-28-9

$10 / 03 / 1013: 31 \quad 594-20-7$

10/03/10 13:31 563-58-6

10/03/10 13:31 10061-01-5

10/03/10 13:31 10061-02-6

$10 / 03 / 1013: 31 \quad 100-41-4$

10/03/10 13:31 87-68-3

10/03/10 13:31 591-78-6

$10 / 03 / 1013: 31 \quad 98-82-8$

$10 / 03 / 1013: 31 \quad 99-87-6$
L3

L3

L3

Page 11 of 19

\section{REPORT OF LABORATORY ANALYSIS}

This report shall not be reproduced, except in full,

without the written consent of Pace Analytical Services, Inc.
3 


\section{ANALYTICAL RESULTS}

Project: Kansas Waste Water

Pace Project No.: 6086606

Sample: MRPURGE-W-930104 Lab ID: 6086606004 Collected: 09/30/10 13:42 $\quad$ Received: 10/01/10 09:15 Matrix: Water

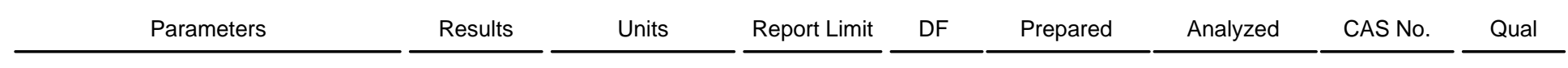

8260 MSV

Methylene chloride

4-Methyl-2-pentanone (MIBK)

Methyl-tert-butyl ether

Naphthalene

n-Propylbenzene

Styrene

1,1,1,2-Tetrachloroethane

1,1,2,2-Tetrachloroethane

Tetrachloroethene

Toluene

1,2,3-Trichlorobenzene

1,2,4-Trichlorobenzene

1,1,1-Trichloroethane

1,1,2-Trichloroethane

Trichloroethene

Trichlorofluoromethane

1,2,3-Trichloropropane

1,2,4-Trimethylbenzene

1,3,5-Trimethylbenzene

Vinyl chloride

Xylene (Total)

4-Bromofluorobenzene (S)

Dibromofluoromethane (S)

1,2-Dichloroethane-d4 (S)

Toluene-d8 (S)

Preservation $\mathrm{pH}$

\subsection{IC Anions}

Nitrate as $\mathrm{N}$

Analytical Method: EPA 5030B/8260

\begin{abstract}
ND ug/L
ND ug/L

ND ug/L

ND ug/L

ND ug/L

ND ug/L

ND ug/L

ND ug/L

ND ug/L

ND ug/L

ND ug/L

ND ug/L

ND ug/L

ND ug/L

ND ug/L

ND ug/L

ND ug/L

ND ug/L

ND ug/L

ND ug/L

ND ug/L

$95 \%$

$105 \%$

$98 \%$

$103 \%$
\end{abstract}

7.0

Analytical Method: EPA 300.0

$0.99 \mathrm{mg} / \mathrm{L}$

$\begin{array}{rr}1.0 & 1 \\ 10.0 & 1 \\ 1.0 & 1 \\ 10.0 & 1 \\ 1.0 & 1 \\ 1.0 & 1 \\ 1.0 & 1 \\ 1.0 & 1 \\ 1.0 & 1 \\ 1.0 & 1 \\ 1.0 & 1 \\ 1.0 & 1 \\ 1.0 & 1 \\ 1.0 & 1 \\ 1.0 & 1 \\ 1.0 & 1 \\ 2.5 & 1 \\ 1.0 & 1 \\ 1.0 & 1 \\ 1.0 & 1 \\ 3.0 & 1 \\ 87-113 & 1 \\ 86-112 & 1 \\ 82-119 & 1 \\ 90-110 & 1 \\ 0.10 & 1\end{array}$

$0.10 \quad 1$
10/03/10 13:31 75-09-2

10/03/10 13:31 108-10-1

10/03/10 13:31 1634-04-4

10/03/10 13:31 91-20-3

10/03/10 13:31 103-65-1

10/03/10 13:31 100-42-5

10/03/10 13:31 630-20-6

10/03/10 13:31 79-34-5

10/03/10 13:31 127-18-4

$10 / 03 / 10$ 13:31 108-88-3

10/03/10 13:31 87-61-6

$10 / 03 / 1013: 31 \quad 120-82-1$

10/03/10 13:31 71-55-6

10/03/10 13:31 79-00-5

10/03/10 13:31 79-01-6

10/03/10 13:31 75-69-4

10/03/10 13:31 96-18-4

10/03/10 13:31 95-63-6

10/03/10 13:31 108-67-8

10/03/10 13:31 75-01-4

10/03/10 13:31 1330-20-7

10/03/10 13:31 460-00-4

10/03/10 13:31 1868-53-7

10/03/10 13:31 17060-07-0

10/03/10 13:31 2037-26-5

10/03/10 13:31

10/01/10 19:48 14797-55-8

This report shall not be reproduced, except in full, without the written consent of Pace Analytical Services, Inc. 


\section{QUALITY CONTROL DATA}

Project: Kansas Waste Water

Pace Project No.: 6086606

\begin{tabular}{llll}
\hline QC Batch: & OEXT/25923 & Analysis Method: & EPA 504.1 \\
QC Batch Method: & EPA 504.1 & Analysis Description: & GCS 504 EDB DBCP
\end{tabular}

Associated Lab Samples: $\quad$ 6086606001, 6086606002, 6086606003, 6086606004

METHOD BLANK: $713551 \quad$ Matrix: Water

Associated Lab Samples: $\quad$ 6086606001, 6086606002, 6086606003, 6086606004

$\frac{\text { Parameter }}{1,2 \text {-Dibromoethane (EDB) }} \frac{\text { Units }}{\mathrm{ug} / \mathrm{L}} \frac{\text { Result }}{\mathrm{ND}} \frac{\begin{array}{c}\text { Blank } \\ \text { Limit }\end{array}}{0.030} \frac{\text { Analyzed }}{10 / 07 / 1020: 50} \stackrel{\text { Qualifiers }}{-}$

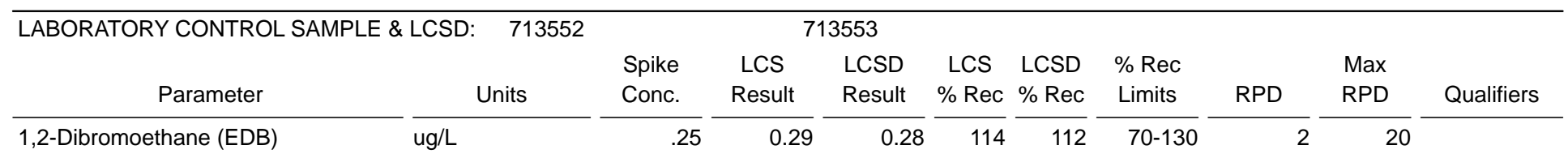




\section{QUALITY CONTROL DATA}

Project: Kansas Waste Water

Pace Project No.: 6086606

\begin{tabular}{llll}
\hline QC Batch: & MSV/32160 & Analysis Method: & EPA 5030B/8260 \\
QC Batch Method: & EPA 5030B/8260 & Analysis Description: & 8260 MSV Water 7 day
\end{tabular}

Associated Lab Samples: $\quad 6086606001,6086606002,6086606003,6086606004$

\begin{tabular}{|c|c|c|c|c|c|}
\hline \multirow{2}{*}{$\begin{array}{l}\text { METHOD BLANK: } 711291 \\
\text { Associated Lab Samples: }\end{array}$} & \multicolumn{4}{|c|}{ Matrix: Water } & \multirow[b]{3}{*}{ Qualifiers } \\
\hline & 001,6086606 & 506003,6086 & 06004 & & \\
\hline Parameter & Units & $\begin{array}{l}\text { Blank } \\
\text { Result }\end{array}$ & $\begin{array}{l}\text { Reporting } \\
\text { Limit }\end{array}$ & Analyzed & \\
\hline 1,1,1,2-Tetrachloroethane & $u g / L$ & ND & 1.0 & $10 / 03 / 1011: 44$ & \\
\hline 1,1,1-Trichloroethane & $\mathrm{ug} / \mathrm{L}$ & ND & 1.0 & $10 / 03 / 1011: 44$ & \\
\hline 1,1,2,2-Tetrachloroethane & $u g / L$ & ND & 1.0 & $10 / 03 / 1011: 44$ & \\
\hline 1,1,2-Trichloroethane & $u g / L$ & ND & 1.0 & $10 / 03 / 1011: 44$ & \\
\hline 1,1-Dichloroethane & $u g / L$ & ND & 1.0 & $10 / 03 / 1011: 44$ & \\
\hline 1,1-Dichloroethene & $\mathrm{ug} / \mathrm{L}$ & ND & 1.0 & $10 / 03 / 1011: 44$ & \\
\hline 1,1-Dichloropropene & $u g / L$ & ND & 1.0 & $10 / 03 / 1011: 44$ & \\
\hline 1,2,3-Trichlorobenzene & $\mathrm{ug} / \mathrm{L}$ & ND & 1.0 & $10 / 03 / 1011: 44$ & \\
\hline 1,2,3-Trichloropropane & $\mathrm{ug} / \mathrm{L}$ & ND & 2.5 & $10 / 03 / 1011: 44$ & \\
\hline 1,2,4-Trichlorobenzene & $u g / L$ & ND & 1.0 & $10 / 03 / 1011: 44$ & \\
\hline 1,2,4-Trimethylbenzene & $\mathrm{ug} / \mathrm{L}$ & ND & 1.0 & $10 / 03 / 1011: 44$ & \\
\hline 1,2-Dibromo-3-chloropropane & $\mathrm{ug} / \mathrm{L}$ & ND & 2.5 & $10 / 03 / 1011: 44$ & \\
\hline 1,2-Dibromoethane (EDB) & ug/L & ND & 1.0 & $10 / 03 / 1011: 44$ & \\
\hline 1,2-Dichlorobenzene & $u g / L$ & ND & 1.0 & $10 / 03 / 1011: 44$ & \\
\hline 1,2-Dichloroethane & $\mathrm{ug} / \mathrm{L}$ & ND & 1.0 & $10 / 03 / 1011: 44$ & \\
\hline 1,2-Dichloroethene (Total) & $\mathrm{ug} / \mathrm{L}$ & ND & 1.0 & $10 / 03 / 1011: 44$ & \\
\hline 1,2-Dichloropropane & $u g / L$ & ND & 1.0 & $10 / 03 / 1011: 44$ & \\
\hline 1,3,5-Trimethylbenzene & $u g / L$ & ND & 1.0 & $10 / 03 / 1011: 44$ & \\
\hline 1,3-Dichlorobenzene & $\mathrm{ug} / \mathrm{L}$ & ND & 1.0 & $10 / 03 / 1011: 44$ & \\
\hline 1,3-Dichloropropane & $u g / L$ & ND & 1.0 & $10 / 03 / 1011: 44$ & \\
\hline 1,4-Dichlorobenzene & ug/L & ND & 1.0 & $10 / 03 / 1011: 44$ & \\
\hline 2,2-Dichloropropane & $\mathrm{ug} / \mathrm{L}$ & ND & 1.0 & $10 / 03 / 1011: 44$ & \\
\hline 2-Butanone (MEK) & $u g / L$ & ND & 10.0 & $10 / 03 / 1011: 44$ & \\
\hline 2-Chlorotoluene & $\mathrm{ug} / \mathrm{L}$ & ND & 1.0 & $10 / 03 / 1011: 44$ & \\
\hline 2-Hexanone & $\mathrm{ug} / \mathrm{L}$ & ND & 10.0 & $10 / 03 / 1011: 44$ & \\
\hline 4-Chlorotoluene & $u g / L$ & ND & 1.0 & $10 / 03 / 1011: 44$ & \\
\hline 4-Methyl-2-pentanone (MIBK) & $\mathrm{ug} / \mathrm{L}$ & ND & 10.0 & $10 / 03 / 1011: 44$ & \\
\hline Acetone & $\mathrm{ug} / \mathrm{L}$ & ND & 10.0 & $10 / 03 / 1011: 44$ & \\
\hline Benzene & ug/L & ND & 1.0 & $10 / 03 / 1011: 44$ & \\
\hline Bromobenzene & $u g / L$ & ND & 1.0 & $10 / 03 / 1011: 44$ & \\
\hline Bromochloromethane & $\mathrm{ug} / \mathrm{L}$ & ND & 1.0 & $10 / 03 / 1011: 44$ & \\
\hline Bromodichloromethane & $\mathrm{ug} / \mathrm{L}$ & ND & 1.0 & $10 / 03 / 1011: 44$ & \\
\hline Bromoform & $u g / L$ & ND & 1.0 & $10 / 03 / 1011: 44$ & \\
\hline Bromomethane & $\mathrm{ug} / \mathrm{L}$ & ND & 1.0 & $10 / 03 / 1011: 44$ & \\
\hline Carbon disulfide & $\mathrm{ug} / \mathrm{L}$ & ND & 5.0 & $10 / 03 / 1011: 44$ & \\
\hline Carbon tetrachloride & $u g / L$ & ND & 1.0 & $10 / 03 / 1011: 44$ & \\
\hline Chlorobenzene & $\mathrm{ug} / \mathrm{L}$ & ND & 1.0 & $10 / 03 / 1011: 44$ & \\
\hline Chloroethane & $\mathrm{ug} / \mathrm{L}$ & ND & 1.0 & $10 / 03 / 1011: 44$ & \\
\hline Chloroform & $u g / L$ & ND & 1.0 & $10 / 03 / 1011: 44$ & \\
\hline Chloromethane & $\mathrm{ug} / \mathrm{L}$ & ND & 1.0 & $10 / 03 / 1011: 44$ & \\
\hline cis-1,2-Dichloroethene & $\mathrm{ug} / \mathrm{L}$ & ND & 1.0 & $10 / 03 / 1011: 44$ & \\
\hline cis-1,3-Dichloropropene & $u g / L$ & ND & 1.0 & $10 / 03 / 1011: 44$ & \\
\hline Dibromochloromethane & $\mathrm{ug} / \mathrm{L}$ & ND & 1.0 & $10 / 03 / 1011: 44$ & \\
\hline
\end{tabular}

Date: 10/14/2010 11:06 AM

REPORT OF LABORATORY ANALYSIS

Page 14 of 19
This report shall not be reproduced, except in full, without the written consent of Pace Analytical Services, Inc.. 


\section{QUALITY CONTROL DATA}

Project: Kansas Waste Water

Pace Project No.: 6086606

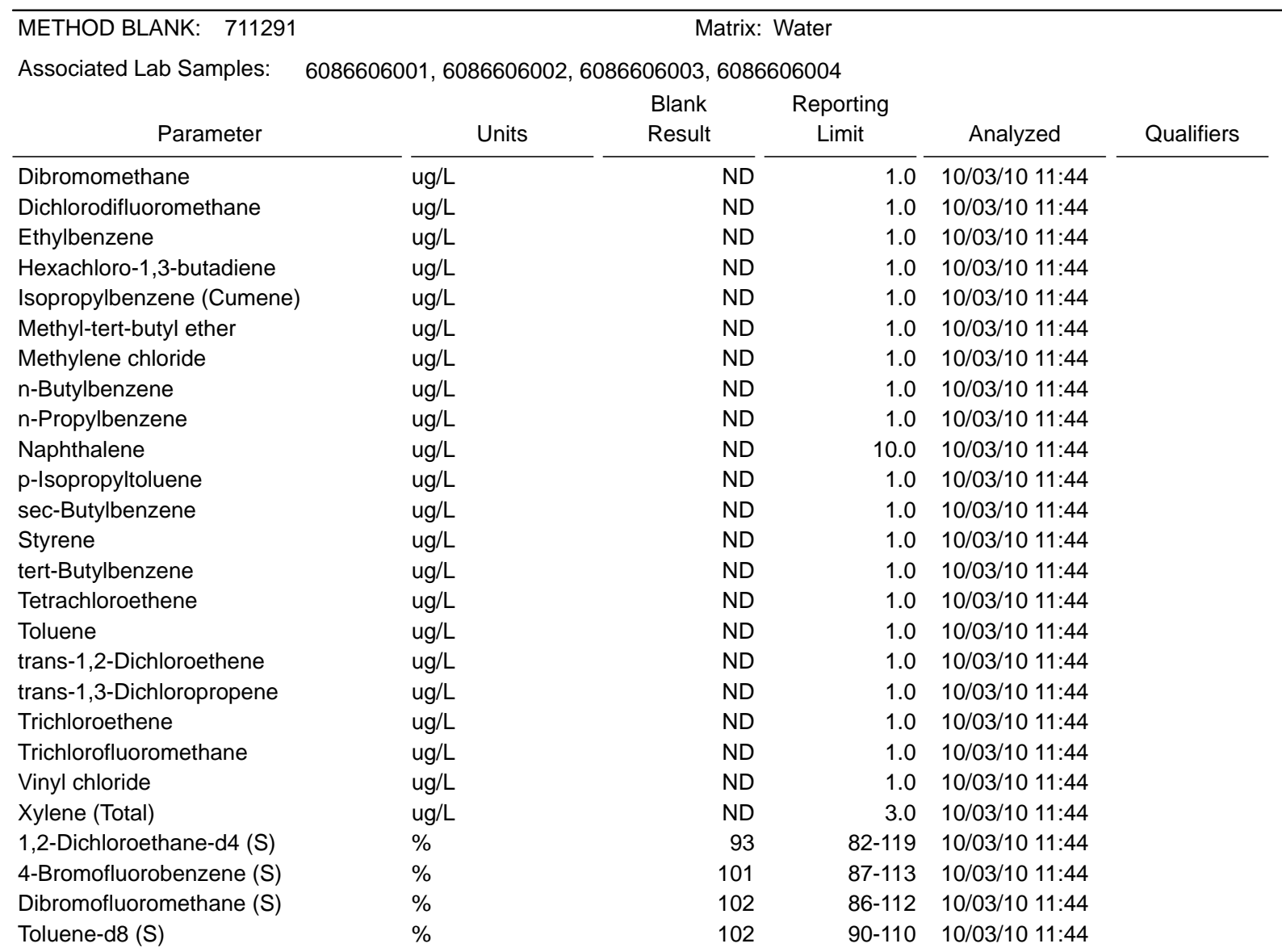

\begin{tabular}{|c|c|c|c|c|c|c|}
\hline \multirow{2}{*}{$\begin{array}{c}\text { LABORATORY CONTROL SAMPLE: } \\
\text { Parameter }\end{array}$} & \multicolumn{6}{|l|}{711292} \\
\hline & Units & $\begin{array}{l}\text { Spike } \\
\text { Conc. }\end{array}$ & $\begin{array}{l}\text { LCS } \\
\text { Result }\end{array}$ & $\begin{array}{c}\text { LCS } \\
\% \operatorname{Rec}\end{array}$ & $\begin{array}{l}\% \text { Rec } \\
\text { Limits }\end{array}$ & Qualifiers \\
\hline $1,1,1,2$-Tetrachloroethane & $u g / L$ & 20 & 22.0 & 110 & $79-116$ & \\
\hline 1,1,1-Trichloroethane & $\mathrm{ug} / \mathrm{L}$ & 20 & 21.2 & 106 & $77-113$ & \\
\hline 1,1,2,2-Tetrachloroethane & $u g / L$ & 20 & 18.9 & 94 & $68-122$ & \\
\hline 1,1,2-Trichloroethane & $u g / L$ & 20 & 20.9 & 104 & $82-117$ & \\
\hline 1,1-Dichloroethane & $u g / L$ & 20 & 20.7 & 103 & $67-122$ & \\
\hline 1,1-Dichloroethene & $u g / L$ & 20 & 23.7 & 118 & $70-119$ & \\
\hline 1,1-Dichloropropene & $u g / L$ & 20 & 21.1 & 106 & $81-115$ & \\
\hline 1,2,3-Trichlorobenzene & ug/L & 20 & 19.9 & 99 & $66-135$ & \\
\hline 1,2,3-Trichloropropane & $u g / L$ & 20 & 18.4 & 92 & $76-126$ & \\
\hline 1,2,4-Trichlorobenzene & $u g / L$ & 20 & 19.7 & 99 & $66-126$ & \\
\hline 1,2,4-Trimethylbenzene & ug/L & 20 & 19.8 & 99 & $78-115$ & \\
\hline 1,2-Dibromo-3-chloropropane & $u g / L$ & 20 & 24.1 & 121 & $58-147$ & \\
\hline 1,2-Dibromoethane (EDB) & $u g / L$ & 20 & 21.2 & 106 & $84-121$ & \\
\hline 1,2-Dichlorobenzene & ug/L & 20 & 20.9 & 105 & $79-116$ & \\
\hline 1,2-Dichloroethane & ug/L & 20 & 19.2 & 96 & 74-119 & \\
\hline
\end{tabular}




\section{QUALITY CONTROL DATA}

Project: Kansas Waste Water

Pace Project No.: 6086606

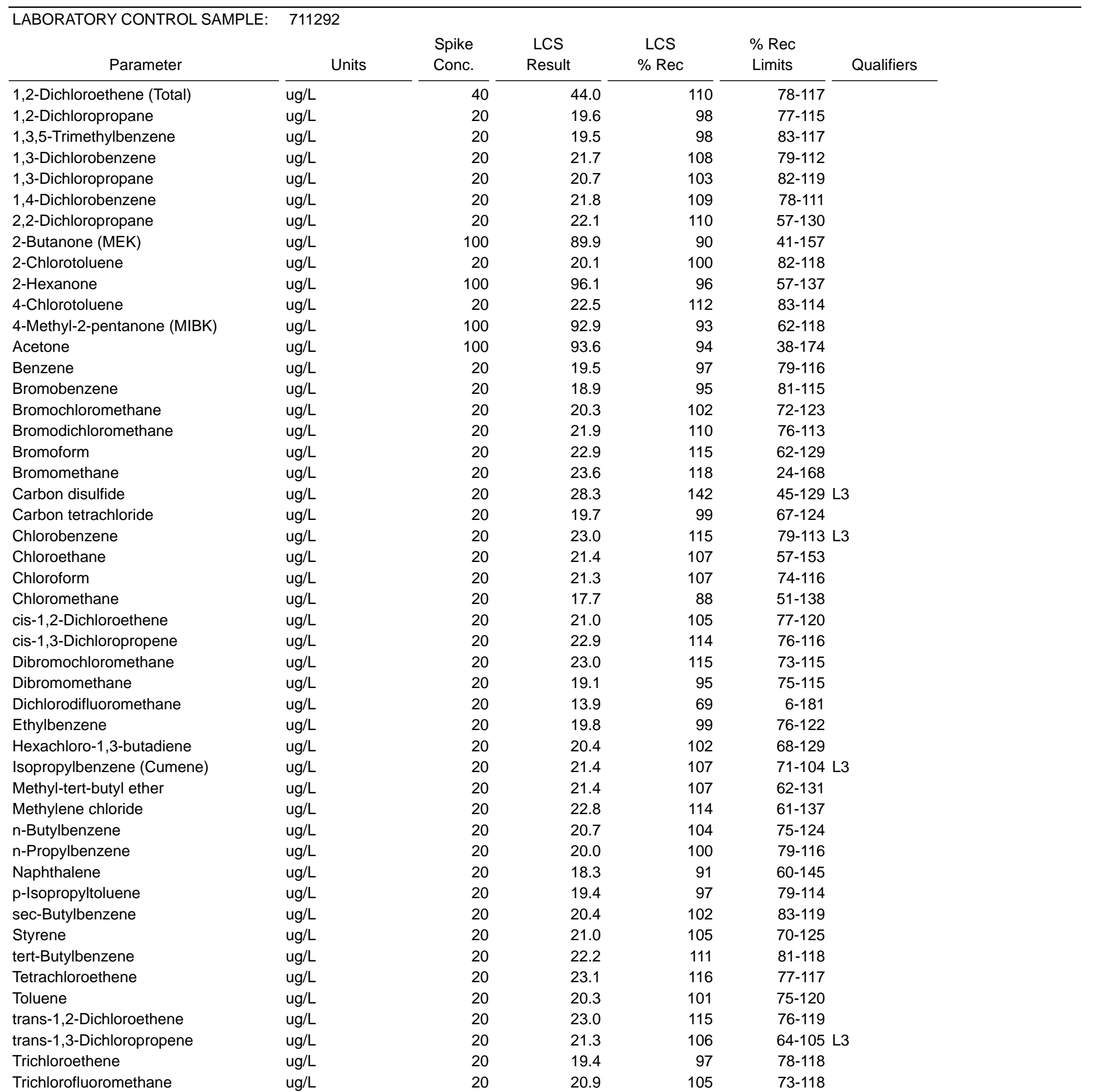

This report shall not be reproduced, except in full, without the written consent of Pace Analytical Services, Inc. 


\section{QUALITY CONTROL DATA}

Project: Kansas Waste Water

Pace Project No.: 6086606

\begin{tabular}{|c|c|c|c|c|c|c|}
\hline LABORATORY CONTROL SAMPLE: & 711292 & & & & & \\
\hline Parameter & Units & $\begin{array}{l}\text { Spike } \\
\text { Conc. }\end{array}$ & $\begin{array}{l}\text { LCS } \\
\text { Result }\end{array}$ & $\begin{array}{l}\text { LCS } \\
\% \operatorname{Rec}\end{array}$ & $\begin{array}{l}\% \operatorname{Rec} \\
\text { Limits }\end{array}$ & Qualifiers \\
\hline Vinyl chloride & $\mathrm{ug} / \mathrm{L}$ & 20 & 22.6 & 113 & $60-122$ & \\
\hline Xylene (Total) & $u g / L$ & 60 & 61.1 & 102 & $74-124$ & \\
\hline 1,2-Dichloroethane-d4 (S) & $\%$ & & & 89 & $82-119$ & \\
\hline 4-Bromofluorobenzene (S) & $\%$ & & & 97 & $87-113$ & \\
\hline Dibromofluoromethane (S) & $\%$ & & & 105 & $86-112$ & \\
\hline Toluene-d8 (S) & $\%$ & & & 101 & $90-110$ & \\
\hline
\end{tabular}

This report shall not be reproduced, except in full, without the written consent of Pace Analytical Services, Inc.. 


\section{QUALITY CONTROL DATA}

Project: Kansas Waste Water

Pace Project No.: 6086606

\begin{tabular}{llll}
\hline QC Batch: & WETA/14191 & Analysis Method: & EPA 300.0 \\
QC Batch Method: & EPA 300.0 & Analysis Description: & 300.0 IC Anions
\end{tabular}

Associated Lab Samples: $\quad 6086606001,6086606002,6086606003,6086606004$

METHOD BLANK: $710224 \quad$ Matrix: Water

Associated Lab Samples: $\quad 6086606001,6086606002,6086606003,6086606004$

$\frac{\text { Parameter }}{\text { Nitrate as } \mathrm{N}} \frac{\text { Units }}{\mathrm{mg} / \mathrm{L}} \frac{\text { Result }}{\mathrm{ND}} \frac{\begin{array}{c}\text { Blank } \\ \text { Limit }\end{array}}{0.10} \frac{\text { Analyzed }}{10 / 01 / 1018: 26} \frac{\text { Qualifiers }}{-}$

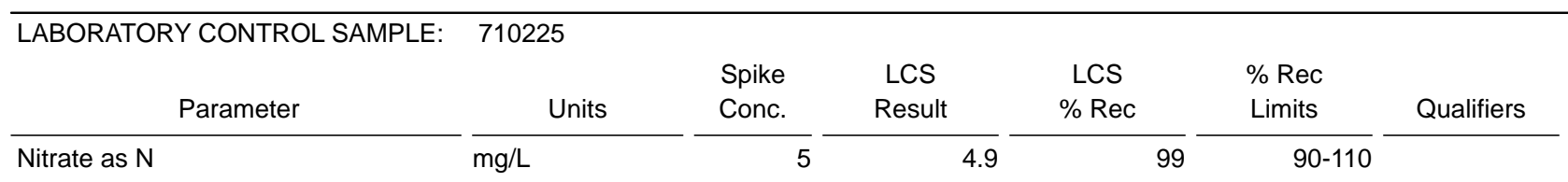

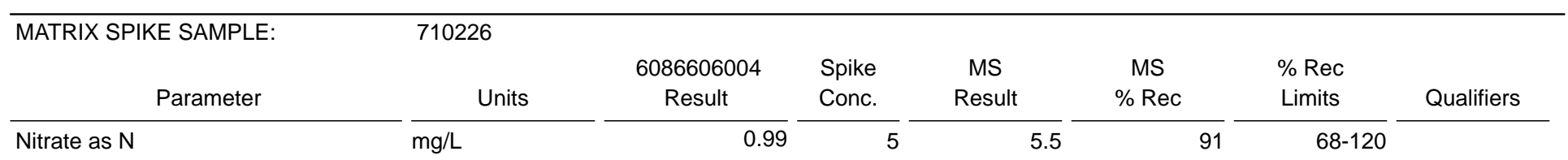

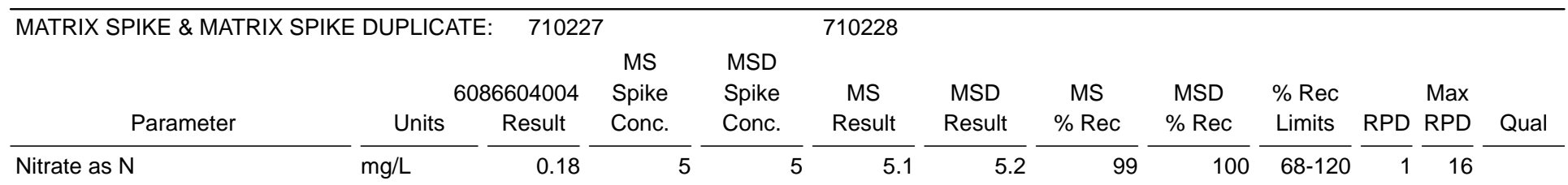




\section{QUALIFIERS}

Project: Kansas Waste Water

Pace Project No.: 6086606

\section{DEFINITIONS}

DF - Dilution Factor, if reported, represents the factor applied to the reported data due to changes in sample preparation, dilution of the sample aliquot, or moisture content.

ND - Not Detected at or above adjusted reporting limit.

$\mathrm{J}$ - Estimated concentration above the adjusted method detection limit and below the adjusted reporting limit.

MDL - Adjusted Method Detection Limit.

$S$ - Surrogate

1,2-Diphenylhydrazine (8270 listed analyte) decomposes to Azobenzene.

Consistent with EPA guidelines, unrounded data are displayed and have been used to calculate \% recovery and RPD values.

LCS(D) - Laboratory Control Sample (Duplicate)

MS(D) - Matrix Spike (Duplicate)

DUP - Sample Duplicate

RPD - Relative Percent Difference

$\mathrm{U}$ - Indicates the compound was analyzed for, but not detected.

N-Nitrosodiphenylamine decomposes and cannot be separated from Diphenylamine using Method 8270. The result reported for each analyte is a combined concentration.

Pace Analytical is NELAP accredited. Contact your Pace PM for the current list of accredited analytes.

\section{BATCH QUALIFIERS}

Batch: MSV/32160

[M5] A matrix spike/matrix spike duplicate was not performed for this batch due to insufficient sample volume.

\section{ANALYTE QUALIFIERS}

E Analyte concentration exceeded the calibration range. The reported result is estimated.

L3 Analyte recovery in the laboratory control sample (LCS) exceeded QC limits. Analyte presence below reporting limits in associated samples. Results unaffected by high bias.

P2 Re-extraction or re-analysis could not be performed due to insufficient sample amount. 


\section{Sample Condition Upon Receipt}

D Pace Analytical
Client Name: TCW
Project \# Co 86606 courier: $\triangle$ Feg Ex $\square$ Uups $\square$ USPS $\square$ client $\square$ Commercial $\square$ pace $\square$ other Tracking \#: 871795231713

Custody Seal on Cooler/Box Present:

Packing Material: $\square$ Bubble Wrapap Thermometer Used:

Cooler Temperature: 2,2

Temperature should be above freezing to $6^{\circ} \mathrm{C}$

Chain of Custody present

Chain of Custody filled out:

Chain of Custody relinquished:

Sampler name \& signature on COC:

Samples arrived within holding time:

Short Hold Time analyses ( $<72 \mathrm{hr})$ :

Rush Turn Around Time requested:

Sufficient volume:

Correct containers used:

-Pace containers used:

Containers intact:

Unpreserved $5035 \mathrm{~A}$ soils frozen w/in $48 \mathrm{hrs}$ ?

Filtered volume received for dissolved tests

Sample labels match COC:

-Includes date/time/ID/analyses Matrix

All containers needing preservation have been checked.

All containers needing preservation are found to be in compliance with EPA recommendation.

Exceptions VOA Coliform, TOC, O\&G, WR-DRO (water),

Phenolics

Trip Blank present:

Pace Trip Blank lot \# (if purchased):

Headspace in VOA vials $(>6 \mathrm{~mm})$ :

Project sampled in USDA Regulated Area:

Client Notification/ Resolution:

Person Contacted:

Pace Shipping Label Used?

$\triangle$ Yes Nolv seals intact: $\square$ Yes

$\triangle$ Yes

$$
\text { bolilo }
$$
Seals in $\square$ Bubble Bags $\square$ Foam Done $\square$ ther

Comments:

DYes $\square$ No $\square$ N/A 5 .

No

No

No

Optional
Proj. Due Date:
Proj. Name:

Type of Ice: WeP Blue None $\square$ Samples on ice, cooling process has begun

Date and Initipłs of person examining contents: Io 17 te by

Gyes $\square$ No $\square$ N/A 1.

Dyes $\square$ No $\square \mathrm{N} / \mathrm{A} 2$.

Dyps $\square$ No $\square \mathrm{N} / \mathrm{A} 3$.

UYes $\square$ No $\square$ N/A 4.

DYes $\square$ No $\square$ N/A 6 . NOJ3

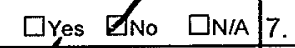

Zyes $\square$ No $\square$ N/A 8

Lres $\square$ No $\square$ N/A 9.

पYes D'No $\square \mathrm{N} / \mathrm{A}$

$\not$ Yres $\square$ No $\square$ N/A 10

$\square$ Yes $\square$ No $\swarrow_{\mathrm{N} / \mathrm{A}} 11$

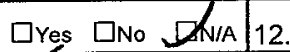

DYes $\square$ No $\square$ N/A 13.

WT

पYes $\square_{\text {No }} Z_{N / \mathrm{A}} 14$.

ares $\square_{\text {No }}$ Dhia

DYes DNg initial when Lot \# of added

\begin{tabular}{l|l}
$\square$ Yes $\square$ Ng & completed $\_$preservative \\
$\square$ Yes $\not$ NNo $\square$ N/A & 15.
\end{tabular}

ares Dtwo aN/A 16.

$\square$ Yes $\square$ No D/N/A 17 17. List State:

Copy COC to Client?

$Y,(N)$

Field Data Required?

$Y / N$

Date/Time:

Comments/Resolution:

Project Manager Review:

Date: $10-1-10$

Note: Whenever there is a discrepancy affecting North Carolina compliance samples, a copy of this form will be sent to the North Carolina DEHNR Certification Office ( i.e out of hold, incorrect preservative, out of temp, incorrect containers) 
Section A

Required Client Information:

Company: TCW Construation

Address: $141 \mathrm{~m}$ Street Lincoln NE 68508

Email To: Phone: 4167255 Fax:

Requested Due Date/TAT:
Section C

Section B

Required Project Information:

Invoice Information:

Report To: Kamlen Q taw construction,com Attention: Travis Kandem Copy To:sargnier@prodigunet

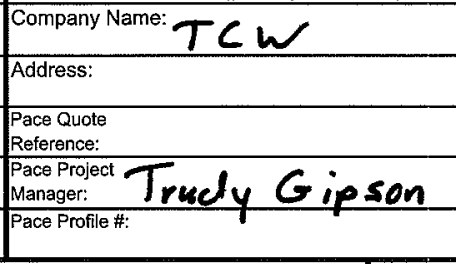

\section{Project Name: Kansas Waste Water}

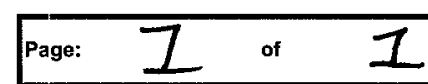

1272142 REGULATORY AGENCY

$\Gamma$ NPDES $\varnothing$ GROUND WATER $\Gamma$ DRINKING WATER

Purchase Order No:

$\Gamma$ OTHER

(2.

$\Gamma$ ust

Site Location

Requested Analysis Filtered (Y/N)

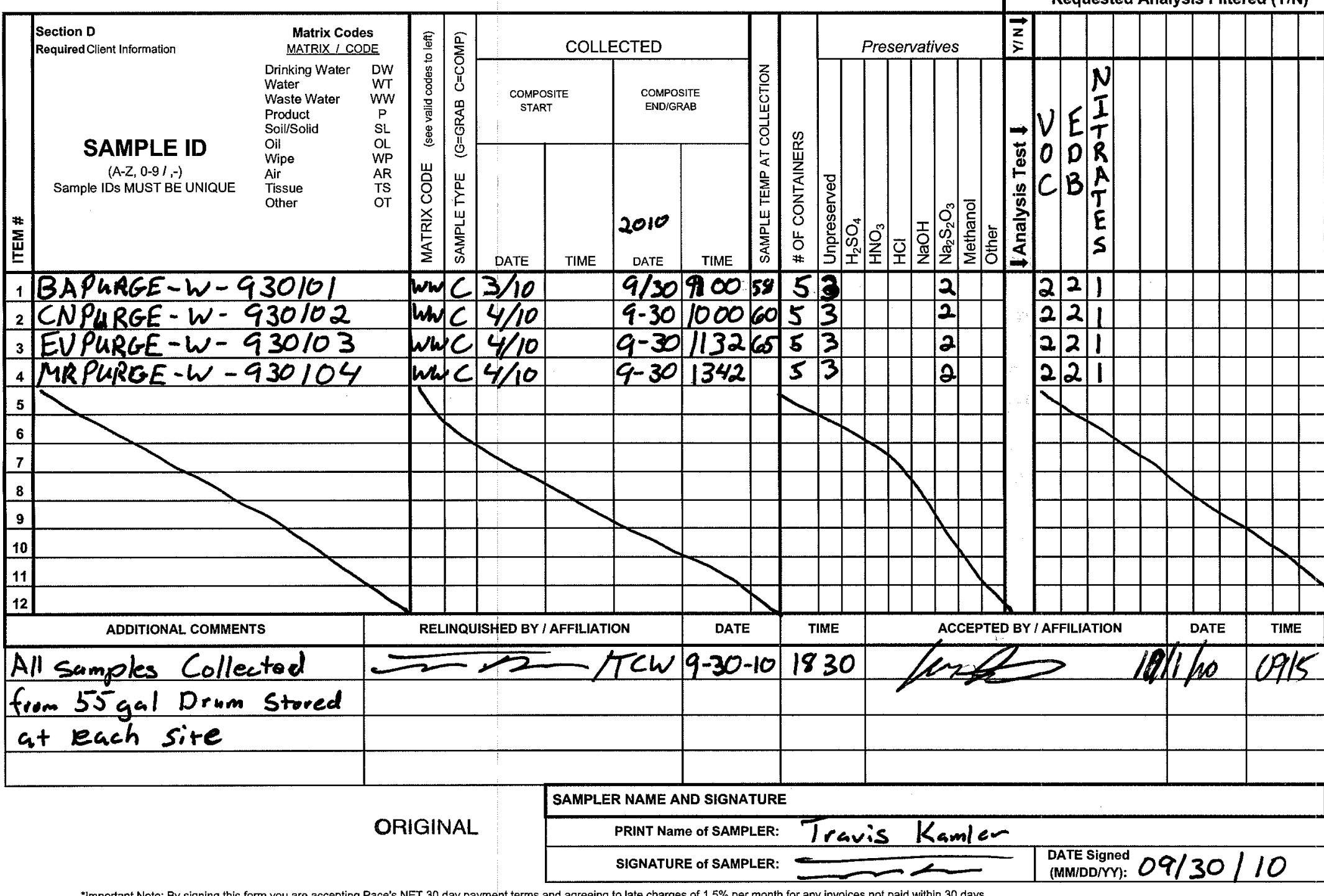

Ceorlebole

$+$


www.pacelabs.com

\section{Sold To:}

Mr. Travis Kamler TCW Construction Inc $141 \mathrm{M}$ Street Lincoln, NE 68508 402-475-5030
Invoice Number: 106081867

Date: $10 / 14 / 2010$

Total Amount Due: $\$ 592.00$

\section{Please Remit To:}

Pace Analytical Services, Inc.

P.O. Box 684056

Milwaukee, WI 53268-4056

\section{Client Number/Client ID 60-508440 / TCW Const}

Client Project: Kansas Waste Water Pace Project No: 6086606

Report Sent To: Mr. David Surgnier,

Comments:

o

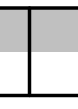

\section{Pace Project Mgr}

Trudy Gipson

\begin{tabular}{|c|c|}
\hline Terms & Page \\
Net 30 Days & \\
\hline
\end{tabular}

Client Name: TCW Construction Inc

Sample Received: 10/1/2010

Mr. Travis Kamler, TCW Construction Inc

\begin{tabular}{|c|c|c|c|c|c|}
\hline \multicolumn{6}{|c|}{ ANALYTICAL CHARGES } \\
\hline Quantity Unit & Description & Method & Matrix & Price & Total \\
\hline $4 \mathrm{Ea}$ & 300.0 IC Anions-Nitrate & EPA 300.0 & Water & $\$ 18.00$ & $\$ 72.00$ \\
\hline $4 \mathrm{Ea}$ & 504 GCS EDB DBCP & EPA 504.1 & Water & $\$ 60.00$ & $\$ 240.00$ \\
\hline \multirow[t]{2}{*}{$4 \mathrm{Ea}$} & 8260 VOC by GC/MS-Full Scan & EPA 5030B/8260 & Water & $\$ 70.00$ & $\$ 280.00$ \\
\hline & & & & Analytical Subtotal & $\$ 592.00$ \\
\hline & & er of Charges 12 & & Total Invoice Amount & $\$ 592.00$ \\
\hline
\end{tabular}

Samples Received for analysis:

\begin{tabular}{lll} 
Lab ID & Client Sample ID & Received \\
\hline 6086606001 & BAPURGE-W-930101 & $10 / 1 / 2010$ 9:15:00 \\
6086606002 & CNPURGE-W-930102 & $10 / 1 / 20109: 15: 00$ \\
6086606003 & EVPURGE-W-930103 & $10 / 1 / 20109: 15: 00$ \\
6086606004 & MRPURGE-W-930104 & $10 / 1 / 20109: 15: 00$
\end{tabular}

If you have any questions or to pay by credit card, please contact Trudy Gipson at Pace.

Phone: 1(913)563-1405 Email: trudy.gipson@pacelabs.com

\section{${ }^{*} 1.5 \%$ MONTHLY FINANCE CHARGE ASSESSED AFTER 30 DAYS OR TERMS OF CONTRACT. PLEASE REFERENCE THE INVOICE NUMBER ON ALL REMITTANCE ADVICE.}

Page 1 of 1

Please complete and return copy of invoice with your payment.

\section{INVOICE TOTAL}

\section{$\$ 592.00$}

\section{Amount Paid: $\quad \$$}

Check No: 


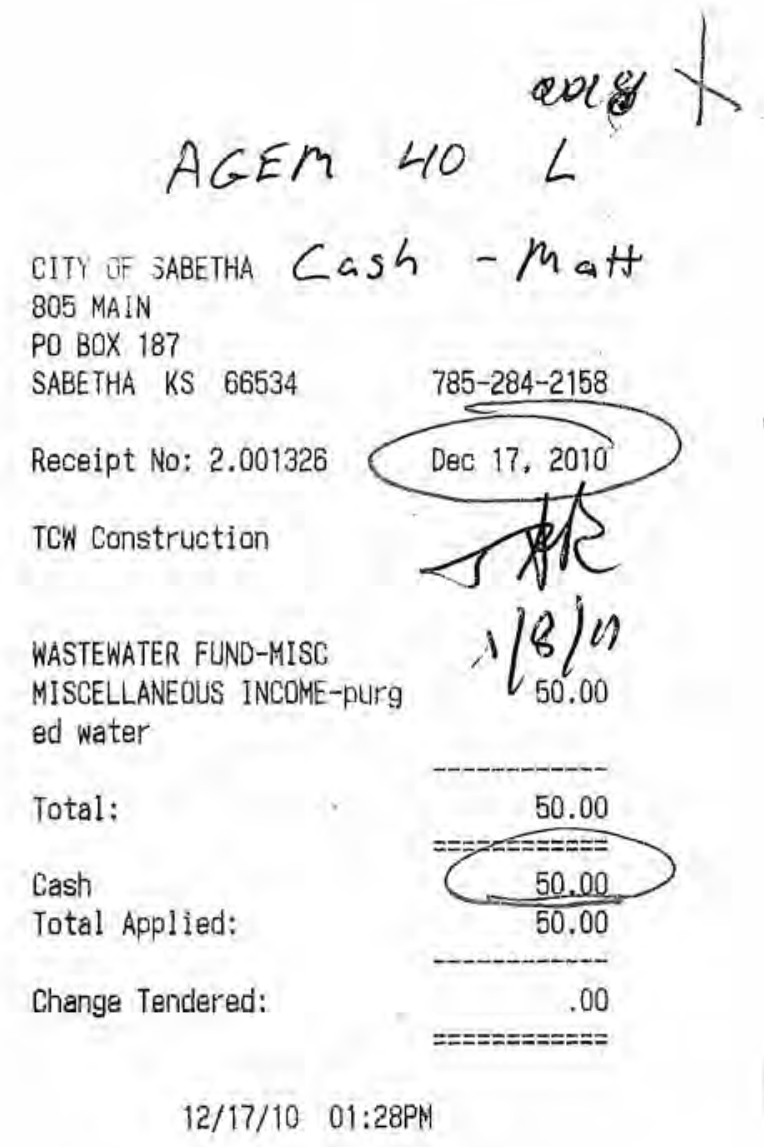


Supplement 2:

Automatically Recorded Water Level Data 
TABLE S2.1 Automatically recorded water levels at Morrill, October 2009 - October 2010

\begin{tabular}{|c|c|c|c|c|c|c|c|c|}
\hline \multirow[b]{2}{*}{ Date } & \multirow[b]{2}{*}{ Time } & \multicolumn{6}{|c|}{ Depth to Water (ft TOC) } & \multirow[b]{2}{*}{ MW8s } \\
\hline & & MW1s & MW2s & MW3s & MW4s & MW6s & MW7s & \\
\hline 10/1/09 & $0: 00$ & 24.314 & 32.810 & 25.145 & 34.236 & 5.699 & 15.305 & \\
\hline $10 / 1 / 09$ & $4: 00$ & 24.321 & 32.788 & 25.076 & 34.216 & 5.707 & 15.271 & \\
\hline 10/1/09 & 8:00 & 24.294 & 32.807 & 25.076 & 34.197 & 5.747 & 15.277 & \\
\hline 10/1/09 & $12: 00$ & 24.286 & 32.823 & 25.078 & 34.181 & 5.705 & 15.290 & \\
\hline 10/1/09 & $16: 00$ & 24.182 & 32.898 & 25.217 & 34.144 & 5.647 & 15.375 & \\
\hline 10/1/09 & $20: 00$ & 24.113 & 32.965 & 25.356 & 34.146 & 5.460 & 15.459 & \\
\hline 10/2/09 & $0: 00$ & 24.175 & 32.963 & 25.345 & 34.220 & 5.365 & 15.450 & \\
\hline 10/2/09 & $4: 00$ & 24.254 & 32.951 & 25.301 & 34.202 & 5.367 & 15.420 & \\
\hline 10/2/09 & 8:00 & 24.271 & 32.964 & 25.302 & 34.230 & 5.381 & 15.431 & \\
\hline 10/2/09 & $12: 00$ & 24.289 & 32.991 & 25.335 & 34.236 & 5.443 & 15.461 & \\
\hline 10/2/09 & $16: 00$ & 24.276 & 33.020 & 25.370 & 34.204 & 5.439 & 15.494 & \\
\hline 10/2/09 & $20: 00$ & 24.227 & 33.066 & 25.455 & 34.248 & 5.321 & 15.537 & \\
\hline 10/3/09 & $0: 00$ & 24.259 & 33.093 & 25.491 & 34.273 & 5.272 & 15.556 & \\
\hline 10/3/09 & $4: 00$ & 24.262 & 33.121 & 25.534 & 34.269 & 5.212 & 15.581 & \\
\hline 10/3/09 & 8:00 & 24.277 & 33.153 & 25.583 & 34.285 & 5.148 & 15.611 & \\
\hline 10/3/09 & $12: 00$ & 24.319 & 33.186 & 25.638 & 34.324 & 5.234 & 15.643 & \\
\hline 10/3/09 & $16: 00$ & 24.378 & 33.191 & 25.636 & 34.405 & 5.255 & 15.643 & \\
\hline 10/3/09 & $20: 00$ & 24.354 & 33.225 & 25.680 & 34.414 & 5.102 & 15.676 & \\
\hline $10 / 4 / 09$ & $0: 00$ & 24.331 & 33.271 & 25.760 & 34.412 & 5.117 & 15.723 & \\
\hline $10 / 4 / 09$ & $4: 00$ & 24.374 & 33.284 & 25.777 & 34.493 & 5.146 & 15.728 & \\
\hline 10/4/09 & 8:00 & 24.403 & 33.308 & 25.808 & 34.514 & 5.125 & 15.749 & \\
\hline $10 / 4 / 09$ & $12: 00$ & 24.456 & 33.344 & 25.852 & 34.551 & 5.173 & 15.775 & \\
\hline $10 / 4 / 09$ & $16: 00$ & 24.560 & 33.321 & 25.784 & 34.597 & 5.395 & 15.738 & \\
\hline $10 / 4 / 09$ & $20: 00$ & 24.522 & 33.349 & 25.813 & 34.622 & 5.276 & 15.771 & \\
\hline 10/5/09 & 0:00 & 24.495 & 33.380 & 25.850 & 34.671 & 5.179 & 15.795 & \\
\hline 10/5/09 & $4: 00$ & 24.540 & 33.376 & 25.821 & 34.680 & 5.197 & 15.773 & \\
\hline 10/5/09 & 8:00 & 24.542 & 33.398 & 25.828 & 34.708 & 5.139 & 15.771 & \\
\hline $10 / 5 / 09$ & $12: 00$ & 24.646 & 33.379 & 25.770 & 34.719 & 5.337 & 15.752 & \\
\hline 10/5/09 & $16: 00$ & 24.718 & 33.350 & 25.673 & 34.745 & 5.654 & 15.710 & \\
\hline 10/5/09 & $20: 00$ & 24.656 & 33.364 & 25.660 & 34.694 & 5.636 & 15.719 & \\
\hline 10/6/09 & 0:00 & 24.644 & 33.370 & 25.634 & 34.696 & 5.712 & 15.710 & \\
\hline 10/6/09 & $4: 00$ & 24.594 & 33.397 & 25.677 & 34.668 & 5.658 & 15.745 & \\
\hline 10/6/09 & $8: 00$ & 24.537 & 33.449 & 25.755 & 34.678 & 5.514 & 15.803 & \\
\hline $10 / 6 / 09$ & $12: 00$ & 24.525 & 33.504 & 25.848 & 34.654 & 5.498 & 15.855 & \\
\hline 10/6/09 & $16: 00$ & 24.552 & 33.529 & 25.886 & 34.696 & 5.523 & 15.879 & \\
\hline 10/6/09 & $20: 00$ & 24.490 & 33.584 & 25.986 & 34.675 & 5.300 & 15.940 & \\
\hline 10/7/09 & $0: 00$ & 24.515 & 33.615 & 26.029 & 34.749 & 5.141 & 15.960 & \\
\hline 10/7/09 & $4: 00$ & 24.560 & 33.630 & 26.047 & 34.777 & 5.023 & 15.970 & \\
\hline 10/7/09 & $8: 00$ & 24.599 & 33.646 & 26.068 & 34.809 & 5.256 & 15.979 & \\
\hline $10 / 7 / 09$ & $12: 00$ & 24.709 & 33.644 & 26.049 & 34.823 & 5.463 & 15.966 & \\
\hline $10 / 7 / 09$ & $16: 00$ & 24.773 & 33.631 & 25.994 & 34.844 & 5.710 & 15.944 & \\
\hline $10 / 7 / 09$ & $20: 00$ & 24.704 & 33.659 & 26.009 & 34.867 & 5.598 & 15.953 & \\
\hline $10 / 8 / 09$ & $0: 00$ & 24.711 & 33.682 & 26.038 & 34.895 & 5.576 & 15.992 & \\
\hline $10 / 8 / 09$ & $4: 00$ & 24.735 & 33.689 & 26.028 & 34.892 & 5.596 & 15.985 & \\
\hline $10 / 8 / 09$ & $8: 00$ & 24.716 & 33.713 & 26.052 & 34.902 & 5.567 & 16.009 & \\
\hline $10 / 8 / 09$ & $12: 00$ & 24.713 & 33.740 & 26.087 & 34.897 & 5.489 & 16.024 & \\
\hline $10 / 8 / 09$ & $16: 00$ & 24.693 & 33.776 & 26.146 & 34.934 & 5.454 & 16.061 & \\
\hline 10/8/09 & $20: 00$ & 24.704 & 33.798 & 26.176 & 34.950 & 5.390 & 16.083 & \\
\hline
\end{tabular}


TABLE S2.1 (Cont.)

\begin{tabular}{|c|c|c|c|c|c|c|c|c|}
\hline \multirow[b]{2}{*}{ Date } & \multirow[b]{2}{*}{ Time } & \multicolumn{6}{|c|}{ Depth to Water (ft TOC) } & \multirow[b]{2}{*}{ MW8s } \\
\hline & & MW1s & MW2s & MW3s & MW4s & MW6s & MW7s & \\
\hline $10 / 9 / 09$ & $0: 00$ & 24.728 & 33.815 & 26.197 & 34.991 & 5.394 & 16.092 & \\
\hline $10 / 9 / 09$ & $4: 00$ & 24.713 & 33.842 & 26.239 & 34.982 & 5.322 & 16.115 & \\
\hline $10 / 9 / 09$ & 8:00 & 24.728 & 33.872 & 26.280 & 35.026 & 5.245 & 16.148 & \\
\hline $10 / 9 / 09$ & $12: 00$ & 24.805 & 33.902 & 26.327 & 35.056 & 5.213 & 16.172 & \\
\hline $10 / 9 / 09$ & $16: 00$ & 24.870 & 33.904 & 26.310 & 35.061 & 5.365 & 16.169 & \\
\hline 10/9/09 & $20: 00$ & 24.803 & 33.943 & 26.371 & 35.112 & 5.210 & 16.211 & \\
\hline $10 / 10 / 09$ & $0: 00$ & 24.798 & 33.968 & 26.415 & 35.149 & 5.217 & 16.232 & \\
\hline $10 / 10 / 09$ & $4: 00$ & 24.790 & 34.016 & 26.493 & 35.156 & 5.355 & 16.280 & \\
\hline $10 / 10 / 09$ & 8:00 & 24.783 & 34.063 & 26.584 & 35.183 & 5.192 & 16.330 & \\
\hline $10 / 10 / 09$ & $12: 00$ & 24.802 & 34.101 & 26.652 & 35.262 & 5.222 & 16.365 & \\
\hline $10 / 10 / 09$ & $16: 00$ & 24.907 & 34.078 & 26.606 & 35.320 & 5.317 & 16.334 & \\
\hline $10 / 10 / 09$ & $20: 00$ & 24.932 & 34.098 & 26.608 & 35.350 & 5.262 & 16.345 & \\
\hline 10/11/09 & $0: 00$ & 24.937 & 34.121 & 26.640 & 35.393 & 5.224 & 16.365 & \\
\hline $10 / 11 / 09$ & $4: 00$ & 24.977 & 34.127 & 26.636 & 35.423 & 5.179 & 16.364 & \\
\hline $10 / 11 / 09$ & $8: 00$ & 24.972 & 34.156 & 26.675 & 35.412 & 5.232 & 16.393 & \\
\hline 10/11/09 & $12: 00$ & 25.014 & 34.149 & 26.651 & 35.451 & 5.249 & 16.380 & \\
\hline $10 / 11 / 09$ & $16: 00$ & 25.093 & 34.116 & 26.565 & 35.502 & 5.385 & 16.328 & \\
\hline $10 / 11 / 09$ & $20: 00$ & 25.058 & 34.138 & 26.568 & 35.518 & 5.385 & 16.343 & \\
\hline $10 / 12 / 09$ & $0: 00$ & 25.066 & 34.140 & 26.548 & 35.509 & 5.467 & 16.339 & \\
\hline $10 / 12 / 09$ & $4: 00$ & 25.088 & 34.131 & 26.509 & 35.550 & 5.470 & 16.321 & \\
\hline $10 / 12 / 09$ & $8: 00$ & 25.044 & 34.174 & 26.554 & 35.495 & 5.457 & 16.364 & \\
\hline $10 / 12 / 09$ & $12: 00$ & 25.024 & 34.200 & 26.593 & 35.509 & 5.521 & 16.391 & \\
\hline $10 / 12 / 09$ & $16: 00$ & 25.044 & 34.218 & 26.610 & 35.532 & 5.655 & 16.408 & \\
\hline $10 / 12 / 09$ & $20: 00$ & 24.976 & 34.274 & 26.708 & 35.530 & 5.486 & 16.464 & \\
\hline 10/13/09 & $0: 00$ & 24.961 & 34.320 & 26.783 & 35.546 & 5.296 & 16.505 & \\
\hline $10 / 13 / 09$ & $4: 00$ & 25.006 & 34.335 & 26.805 & 35.578 & 5.277 & 16.514 & \\
\hline $10 / 13 / 09$ & $8: 00$ & 25.041 & 34.346 & 26.822 & 35.608 & 5.257 & 16.523 & \\
\hline $10 / 13 / 09$ & $12: 00$ & 25.106 & 34.348 & 26.808 & 35.650 & 5.407 & 16.516 & \\
\hline $10 / 13 / 09$ & $16: 00$ & 25.175 & 34.315 & 26.721 & 35.680 & 5.539 & 16.468 & \\
\hline $10 / 13 / 09$ & $20: 00$ & 25.118 & 34.341 & 26.752 & 35.666 & 5.389 & 16.475 & \\
\hline $10 / 14 / 09$ & $0: 00$ & 25.111 & 34.354 & 26.776 & 35.684 & 5.383 & 16.471 & \\
\hline $10 / 14 / 09$ & $4: 00$ & 25.131 & 34.341 & 26.748 & 35.698 & 5.366 & 16.453 & \\
\hline $10 / 14 / 09$ & $8: 00$ & 25.113 & 34.353 & 26.769 & 35.666 & 5.432 & 16.481 & \\
\hline $10 / 14 / 09$ & $12: 00$ & 25.143 & 34.329 & 26.723 & 35.717 & 5.497 & 16.456 & \\
\hline $10 / 14 / 09$ & $16: 00$ & 25.170 & 34.302 & 26.660 & 35.705 & 5.609 & 16.432 & \\
\hline $10 / 14 / 09$ & $20: 00$ & 25.128 & 34.314 & 26.673 & 35.689 & 5.664 & 16.443 & \\
\hline $10 / 15 / 09$ & $0: 00$ & 25.103 & 34.327 & 26.678 & 35.675 & 5.644 & 16.453 & \\
\hline $10 / 15 / 09$ & $4: 00$ & 25.093 & 34.325 & 26.676 & 35.652 & 5.675 & 16.455 & \\
\hline $10 / 15 / 09$ & $8: 00$ & 25.046 & 34.359 & 26.729 & 35.661 & 5.584 & 16.497 & \\
\hline $10 / 15 / 09$ & $12: 00$ & 25.009 & 34.387 & 26.788 & 35.680 & 5.629 & 16.520 & \\
\hline $10 / 15 / 09$ & $16: 00$ & 25.066 & 34.393 & 26.798 & 35.661 & 5.720 & 16.540 & \\
\hline $10 / 15 / 09$ & $20: 00$ & 25.023 & 34.436 & 26.877 & 35.691 & 5.609 & 16.590 & \\
\hline $10 / 16 / 09$ & $0: 00$ & 25.031 & 34.459 & 26.925 & 35.705 & 5.513 & 16.618 & \\
\hline $10 / 16 / 09$ & $4: 00$ & 25.039 & 34.480 & 26.967 & 35.747 & 5.434 & 16.642 & \\
\hline 10/16/09 & $8: 00$ & 25.036 & 34.518 & 27.038 & 35.728 & 5.332 & 16.687 & \\
\hline 10/16/09 & $12: 00$ & 25.076 & 34.539 & 27.079 & 35.781 & 5.396 & 16.711 & \\
\hline 10/16/09 & $16: 00$ & 25.165 & 34.522 & 27.049 & 35.839 & 5.509 & 16.689 & \\
\hline $10 / 16 / 09$ & $20: 00$ & 25.123 & 34.559 & 27.099 & 35.878 & 5.363 & 16.737 & \\
\hline
\end{tabular}


TABLE S2.1 (Cont.)

\begin{tabular}{|c|c|c|c|c|c|c|c|c|}
\hline \multirow[b]{2}{*}{ Date } & \multirow[b]{2}{*}{ Time } & \multicolumn{6}{|c|}{ Depth to Water (ft TOC) } & \multirow[b]{2}{*}{ MW8s } \\
\hline & & MW1s & MW2s & MW3s & MW4s & MW6s & MW7s & \\
\hline $10 / 17 / 09$ & $0: 00$ & 25.113 & 34.595 & 27.167 & 35.906 & 5.281 & 16.776 & \\
\hline $10 / 17 / 09$ & $4: 00$ & 25.118 & 34.620 & 27.216 & 35.950 & 5.221 & 16.800 & \\
\hline $10 / 17 / 09$ & 8:00 & 25.111 & 34.661 & 27.297 & 35.980 & 5.177 & 16.850 & \\
\hline $10 / 17 / 09$ & $12: 00$ & 25.170 & 34.677 & 27.326 & 36.012 & 5.227 & 16.865 & \\
\hline $10 / 17 / 09$ & $16: 00$ & 25.247 & 34.659 & 27.277 & 36.089 & 5.276 & 16.839 & \\
\hline $10 / 17 / 09$ & $20: 00$ & 25.264 & 34.656 & 27.251 & 36.112 & 5.282 & 16.832 & \\
\hline $10 / 18 / 09$ & $0: 00$ & 25.289 & 34.656 & 27.228 & 36.112 & 5.222 & 16.826 & \\
\hline $10 / 18 / 09$ & $4: 00$ & 25.292 & 34.645 & 27.183 & 36.135 & 5.270 & 16.802 & \\
\hline $10 / 18 / 09$ & 8:00 & 25.329 & 34.642 & 27.156 & 36.142 & 5.328 & 16.800 & \\
\hline $10 / 18 / 09$ & $12: 00$ & 25.393 & 34.627 & 27.107 & 36.179 & 5.459 & 16.776 & \\
\hline 10/18/09 & $16: 00$ & 25.455 & 34.579 & 26.994 & 36.156 & 5.771 & 16.724 & \\
\hline $10 / 18 / 09$ & $20: 00$ & 25.369 & 34.599 & 26.998 & 36.116 & 5.736 & 16.748 & \\
\hline 10/19/09 & $0: 00$ & 25.346 & 34.601 & 26.981 & 36.137 & 5.711 & 16.746 & \\
\hline $10 / 19 / 09$ & $4: 00$ & 25.336 & 34.597 & 26.955 & 36.114 & 5.837 & 16.735 & \\
\hline 10/19/09 & $8: 00$ & 25.290 & 34.632 & 26.999 & 36.093 & 5.816 & 16.774 & \\
\hline 10/19/09 & $12: 00$ & 25.301 & 34.659 & 27.038 & 36.096 & 5.830 & 16.806 & \\
\hline 10/19/09 & $16: 00$ & 25.359 & 34.646 & 26.999 & 36.061 & 5.921 & 16.787 & \\
\hline 10/19/09 & $20: 00$ & 25.287 & 34.674 & 27.043 & 36.100 & 5.811 & 16.823 & \\
\hline $10 / 20 / 09$ & $0: 00$ & 25.264 & 34.708 & 27.097 & 36.070 & 5.764 & 16.858 & \\
\hline $10 / 20 / 09$ & $4: 00$ & 25.304 & 34.702 & 27.075 & 36.086 & 5.735 & 16.843 & \\
\hline $10 / 20 / 09$ & $8: 00$ & 25.302 & 34.719 & 27.097 & 36.109 & 5.793 & 16.862 & \\
\hline $10 / 20 / 09$ & $12: 00$ & 25.312 & & & 36.112 & 5.710 & 16.886 & 3.542 \\
\hline $10 / 20 / 09$ & $16: 00$ & 25.346 & 34.697 & 27.118 & 36.122 & 5.678 & 16.886 & 3.663 \\
\hline $10 / 20 / 09$ & $20: 00$ & 25.301 & 34.728 & 27.174 & 36.113 & 5.673 & 16.928 & 3.532 \\
\hline 10/21/09 & $0: 00$ & 25.279 & 34.766 & 27.242 & 36.119 & 5.661 & 16.969 & 3.436 \\
\hline 10/21/09 & $4: 00$ & 25.319 & 34.772 & 27.245 & 36.157 & 5.680 & 16.967 & 3.459 \\
\hline $10 / 21 / 09$ & $8: 00$ & 25.329 & 34.785 & 27.264 & 36.162 & 5.655 & 16.981 & 3.415 \\
\hline $10 / 21 / 09$ & $12: 00$ & 25.358 & 34.806 & 27.293 & 36.186 & 5.654 & 17.003 & 3.523 \\
\hline $10 / 21 / 09$ & $16: 00$ & 25.391 & 34.793 & 27.274 & 36.201 & 5.661 & 16.995 & 3.654 \\
\hline $10 / 21 / 09$ & $20: 00$ & 25.346 & 34.806 & 27.313 & 36.212 & 5.636 & 16.977 & 3.518 \\
\hline $10 / 22 / 09$ & $0: 00$ & 25.334 & 34.780 & 27.279 & 36.221 & 5.654 & 16.750 & 3.423 \\
\hline $10 / 22 / 09$ & $4: 00$ & 25.237 & 34.686 & 27.211 & 36.227 & 5.617 & 16.165 & 3.277 \\
\hline $10 / 22 / 09$ & 8:00 & 25.044 & 34.573 & 27.118 & 36.240 & 5.576 & 15.697 & 3.154 \\
\hline $10 / 22 / 09$ & $12: 00$ & 24.884 & 34.442 & 27.052 & 36.251 & 5.568 & 15.762 & 3.170 \\
\hline $10 / 22 / 09$ & $16: 00$ & 24.708 & 34.291 & 26.949 & 36.253 & 5.510 & 15.399 & 3.139 \\
\hline $10 / 22 / 09$ & $20: 00$ & 24.442 & 34.146 & 26.808 & 36.203 & 5.438 & 15.093 & 2.849 \\
\hline $10 / 23 / 09$ & $0: 00$ & 24.186 & 33.989 & 26.726 & 36.215 & 5.452 & 15.048 & 2.810 \\
\hline $10 / 23 / 09$ & $4: 00$ & 24.012 & 33.849 & 26.630 & 36.193 & 5.508 & 15.252 & 2.802 \\
\hline $10 / 23 / 09$ & $8: 00$ & 23.853 & 33.769 & 26.572 & 36.166 & 5.518 & 15.433 & 2.759 \\
\hline $10 / 23 / 09$ & $12: 00$ & 23.781 & 33.693 & 26.501 & 36.128 & 5.516 & 15.563 & 2.782 \\
\hline $10 / 23 / 09$ & $16: 00$ & 23.740 & 33.618 & 26.409 & 36.161 & 5.557 & 15.660 & 2.845 \\
\hline $10 / 23 / 09$ & $20: 00$ & 23.685 & 33.559 & 26.355 & 36.125 & 5.567 & 15.765 & 2.723 \\
\hline $10 / 24 / 09$ & $0: 00$ & 23.702 & 33.490 & 26.262 & 36.168 & 5.569 & 15.817 & 2.690 \\
\hline $10 / 24 / 09$ & $4: 00$ & 23.709 & 33.437 & 26.199 & 36.180 & 5.573 & 15.864 & 2.690 \\
\hline $10 / 24 / 09$ & $8: 00$ & 23.720 & 33.381 & 26.130 & 36.177 & 5.522 & 15.901 & 2.723 \\
\hline $10 / 24 / 09$ & $12: 00$ & 23.770 & 33.341 & 26.087 & 36.184 & 5.534 & 15.938 & 2.913 \\
\hline $10 / 24 / 09$ & $16: 00$ & 23.839 & 33.265 & 25.955 & 36.217 & 5.567 & 15.916 & 3.043 \\
\hline $10 / 24 / 09$ & $20: 00$ & 23.752 & 33.261 & 25.965 & 36.177 & 5.577 & 15.975 & 2.967 \\
\hline
\end{tabular}


TABLE S2.1 (Cont.)

\begin{tabular}{|c|c|c|c|c|c|c|c|c|}
\hline \multirow[b]{2}{*}{ Date } & \multirow[b]{2}{*}{ Time } & \multicolumn{6}{|c|}{ Depth to Water (ft TOC) } & \multirow[b]{2}{*}{ MW8s } \\
\hline & & MW1s & MW2s & MW3s & MW4s & MW6s & MW7s & \\
\hline $10 / 25 / 09$ & $0: 00$ & 23.740 & 33.252 & 25.977 & 36.173 & 5.544 & 16.031 & 2.837 \\
\hline $10 / 25 / 09$ & $4: 00$ & 23.742 & 33.240 & 25.989 & 36.145 & 5.627 & 16.072 & 2.767 \\
\hline $10 / 25 / 09$ & 8:00 & 23.752 & 33.244 & 26.036 & 36.170 & 5.583 & 16.122 & 2.765 \\
\hline $10 / 25 / 09$ & $12: 00$ & 23.772 & 33.261 & 26.112 & 36.150 & 5.620 & 16.187 & 2.880 \\
\hline $10 / 25 / 09$ & $16: 00$ & 23.824 & 33.252 & 26.123 & 36.193 & 5.592 & 16.212 & 2.996 \\
\hline $10 / 25 / 09$ & $20: 00$ & 23.767 & 33.309 & 26.260 & 36.186 & 5.641 & 16.310 & 2.831 \\
\hline $10 / 26 / 09$ & $0: 00$ & 23.812 & 33.315 & 26.311 & 36.216 & 5.625 & 16.347 & 2.773 \\
\hline $10 / 26 / 09$ & $4: 00$ & 23.857 & 33.332 & 26.351 & 36.258 & 5.643 & 16.386 & 2.714 \\
\hline $10 / 26 / 09$ & 8:00 & 23.899 & 33.332 & 26.365 & 36.295 & 5.610 & 16.404 & 2.751 \\
\hline $10 / 26 / 09$ & $12: 00$ & 24.003 & 33.328 & 26.370 & 36.350 & 5.655 & 16.423 & 2.928 \\
\hline $10 / 26 / 09$ & $16: 00$ & 24.107 & 33.284 & 26.277 & 36.372 & 5.634 & 16.378 & 3.100 \\
\hline $10 / 26 / 09$ & $20: 00$ & 24.087 & 33.284 & 26.267 & 36.383 & 5.612 & 16.393 & 2.947 \\
\hline 10/27/09 & $0: 00$ & 24.115 & 33.267 & 26.233 & 36.394 & 5.573 & 16.380 & 2.865 \\
\hline $10 / 27 / 09$ & $4: 00$ & 24.152 & 33.248 & 26.177 & 36.393 & 5.597 & 16.360 & 2.889 \\
\hline $10 / 27 / 09$ & $8: 00$ & 24.167 & 33.242 & 26.141 & 36.383 & 5.601 & 16.347 & 2.881 \\
\hline $10 / 27 / 09$ & $12: 00$ & 24.231 & 33.225 & 26.089 & 36.383 & 5.589 & 16.328 & 3.091 \\
\hline $10 / 27 / 09$ & $16: 00$ & 24.286 & 33.181 & 25.982 & 36.361 & 5.653 & 16.271 & 3.134 \\
\hline $10 / 27 / 09$ & $20: 00$ & 24.194 & 33.210 & 26.011 & 36.307 & 5.595 & 16.306 & 3.005 \\
\hline $10 / 28 / 09$ & $0: 00$ & 24.167 & 33.240 & 26.063 & 36.288 & 5.574 & 16.345 & 2.954 \\
\hline $10 / 28 / 09$ & $4: 00$ & 24.162 & 33.255 & 26.072 & 36.237 & 5.580 & 16.364 & 2.952 \\
\hline $10 / 28 / 09$ & $8: 00$ & 24.162 & 33.278 & 26.106 & 36.252 & 5.597 & 16.391 & 2.911 \\
\hline $10 / 28 / 09$ & $12: 00$ & 24.216 & 33.291 & 26.126 & 36.246 & 5.570 & 16.401 & 3.046 \\
\hline $10 / 28 / 09$ & $16: 00$ & 24.242 & 33.301 & 26.126 & 36.230 & 5.591 & 16.414 & 3.222 \\
\hline $10 / 28 / 09$ & $20: 00$ & 24.204 & 33.340 & 26.194 & 36.231 & 5.630 & 16.462 & 3.117 \\
\hline $10 / 29 / 09$ & $0: 00$ & 24.224 & 33.366 & 26.236 & 36.235 & 5.578 & 16.488 & 3.048 \\
\hline $10 / 29 / 09$ & $4: 00$ & 24.284 & 33.330 & 26.165 & 36.258 & 5.562 & 16.388 & 3.105 \\
\hline $10 / 29 / 09$ & $8: 00$ & 24.254 & 33.326 & 26.177 & 36.206 & 5.605 & 16.174 & 3.101 \\
\hline $10 / 29 / 09$ & $12: 00$ & 24.209 & 33.238 & 26.075 & 36.235 & 5.444 & 15.597 & 3.126 \\
\hline $10 / 29 / 09$ & $16: 00$ & 24.085 & 33.133 & 25.946 & 36.219 & 5.458 & 15.370 & 3.021 \\
\hline $10 / 29 / 09$ & $20: 00$ & 23.869 & 33.061 & 25.875 & 36.172 & 5.438 & 15.251 & 2.947 \\
\hline 10/30/09 & $0: 00$ & 23.712 & 32.980 & 25.790 & 36.171 & 5.502 & 15.357 & 2.949 \\
\hline $10 / 30 / 09$ & $4: 00$ & 23.561 & 32.948 & 25.792 & 36.135 & 5.522 & 15.481 & 2.753 \\
\hline 10/30/09 & $8: 00$ & 23.464 & 32.913 & 25.785 & 36.089 & 5.520 & 15.568 & 2.535 \\
\hline $10 / 30 / 09$ & $12: 00$ & 23.420 & 32.891 & 25.790 & 36.107 & 5.514 & 15.661 & 2.545 \\
\hline $10 / 30 / 09$ & $16: 00$ & 23.400 & 32.866 & 25.780 & 36.092 & 5.522 & 15.734 & 2.570 \\
\hline $10 / 30 / 09$ & $20: 00$ & 23.333 & 32.889 & 25.863 & 36.055 & 5.588 & 15.847 & 2.435 \\
\hline $10 / 31 / 09$ & 0:00 & 23.360 & 32.872 & 25.872 & 36.109 & 5.565 & 15.899 & 2.398 \\
\hline $10 / 31 / 09$ & $4: 00$ & 23.368 & 32.879 & 25.909 & 36.117 & 5.566 & 15.970 & 2.378 \\
\hline $10 / 31 / 09$ & $8: 00$ & 23.345 & 32.917 & 26.009 & 36.133 & 5.569 & 16.067 & 2.400 \\
\hline $10 / 31 / 09$ & $12: 00$ & 23.447 & 32.919 & 26.046 & 36.181 & 5.592 & 16.118 & 2.583 \\
\hline $10 / 31 / 09$ & $16: 00$ & 23.559 & 32.883 & 26.000 & 36.256 & 5.621 & 16.115 & 2.767 \\
\hline $10 / 31 / 09$ & $20: 00$ & 23.544 & 32.883 & 26.004 & 36.272 & 5.580 & 16.146 & 2.610 \\
\hline 11/1/09 & $0: 00$ & 23.589 & 32.869 & 25.988 & 36.311 & 5.565 & 16.156 & 2.543 \\
\hline $11 / 1 / 09$ & $4: 00$ & 23.636 & 32.850 & 25.951 & 36.301 & 5.544 & 16.156 & 2.551 \\
\hline 11/1/09 & $8: 00$ & 23.663 & 32.841 & 25.934 & 36.334 & 5.547 & 16.167 & 2.545 \\
\hline 11/1/09 & $12: 00$ & 23.728 & 32.839 & 25.924 & 36.345 & 5.647 & 16.174 & 2.722 \\
\hline 11/1/09 & $16: 00$ & 23.783 & 32.816 & 25.872 & 36.363 & 5.614 & 16.158 & 2.845 \\
\hline $11 / 1 / 09$ & $20: 00$ & 23.693 & 32.854 & 25.941 & 36.343 & 5.593 & 16.219 & 2.657 \\
\hline
\end{tabular}


TABLE S2.1 (Cont.)

\begin{tabular}{|c|c|c|c|c|c|c|c|c|}
\hline \multirow[b]{2}{*}{ Date } & \multirow[b]{2}{*}{ Time } & \multicolumn{6}{|c|}{ Depth to Water (ft TOC) } & \multirow[b]{2}{*}{ MW8s } \\
\hline & & MW1s & MW2s & MW3s & MW4s & MW6s & MW7s & \\
\hline $11 / 2 / 09$ & $0: 00$ & 23.673 & 32.889 & 26.004 & 36.320 & 5.596 & 16.264 & 2.543 \\
\hline $11 / 2 / 09$ & $4: 00$ & 23.666 & 32.923 & 26.072 & 36.359 & 5.610 & 16.312 & 2.573 \\
\hline $11 / 2 / 09$ & 8:00 & 23.649 & 32.984 & 26.199 & 36.355 & 5.612 & 16.395 & 2.481 \\
\hline $11 / 2 / 09$ & $12: 00$ & 23.711 & 33.021 & 26.280 & 36.405 & 5.589 & 16.436 & 2.618 \\
\hline $11 / 2 / 09$ & $16: 00$ & 23.802 & 33.019 & 26.282 & 36.456 & 5.573 & 16.444 & 2.816 \\
\hline $11 / 2 / 09$ & $20: 00$ & 23.825 & 33.045 & 26.319 & 36.483 & 5.622 & 16.477 & 2.653 \\
\hline $11 / 3 / 09$ & $0: 00$ & 23.874 & 33.053 & 26.324 & 36.521 & 5.596 & 16.486 & 2.550 \\
\hline $11 / 3 / 09$ & $4: 00$ & 23.922 & 33.055 & 26.314 & 36.547 & 5.633 & 16.492 & 2.591 \\
\hline $11 / 3 / 09$ & 8:00 & 23.961 & 33.055 & 26.294 & 36.566 & 5.620 & 16.496 & 2.573 \\
\hline $11 / 3 / 09$ & $12: 00$ & 24.086 & 33.036 & 26.236 & 36.600 & 5.602 & 16.464 & 2.820 \\
\hline $11 / 3 / 09$ & $16: 00$ & 24.162 & 32.977 & 26.092 & 36.625 & 5.587 & 16.382 & 2.959 \\
\hline $11 / 3 / 09$ & $20: 00$ & 24.105 & 32.998 & 26.100 & 36.599 & 5.554 & 16.403 & 2.902 \\
\hline $11 / 4 / 09$ & $0: 00$ & 24.066 & 33.030 & 26.131 & 36.575 & 5.587 & 16.434 & 2.755 \\
\hline $11 / 4 / 09$ & $4: 00$ & 24.046 & 33.061 & 26.180 & 36.560 & 5.570 & 16.473 & 2.716 \\
\hline $11 / 4 / 09$ & 8:00 & 24.009 & 33.118 & 26.275 & 36.546 & 5.574 & 16.536 & 2.695 \\
\hline $11 / 4 / 09$ & $12: 00$ & 24.048 & 33.162 & 26.358 & 36.559 & 5.620 & 16.585 & 2.809 \\
\hline $11 / 4 / 09$ & $16: 00$ & 24.132 & 33.164 & 26.348 & 36.590 & 5.570 & 16.579 & 2.971 \\
\hline $11 / 4 / 09$ & $20: 00$ & 24.098 & 33.189 & 26.382 & 36.597 & 5.583 & 16.607 & 2.793 \\
\hline $11 / 5 / 09$ & $0: 00$ & 24.123 & 33.206 & 26.399 & 36.617 & 5.595 & 16.618 & 2.744 \\
\hline $11 / 5 / 09$ & $4: 00$ & 24.175 & 33.214 & 26.399 & 36.607 & 5.581 & 16.620 & 2.677 \\
\hline $11 / 5 / 09$ & 8:00 & 24.227 & 33.210 & 26.370 & 36.618 & 5.617 & 16.611 & 2.671 \\
\hline $11 / 5 / 09$ & $12: 00$ & 24.324 & 33.202 & 26.329 & 36.659 & 5.616 & 16.589 & 2.903 \\
\hline $11 / 5 / 09$ & $16: 00$ & 24.410 & 33.151 & 26.202 & 36.663 & 5.607 & 16.510 & 3.146 \\
\hline $11 / 5 / 09$ & $20: 00$ & 24.334 & 33.164 & 26.202 & 36.643 & 5.543 & 16.520 & 3.028 \\
\hline $11 / 6 / 09$ & 0:00 & 24.346 & 33.155 & 26.161 & 36.608 & 5.543 & 16.499 & 3.010 \\
\hline $11 / 6 / 09$ & $4: 00$ & 24.349 & 33.155 & 26.132 & 36.582 & 5.516 & 16.488 & 3.067 \\
\hline $11 / 6 / 09$ & 8:00 & 24.334 & 33.160 & 26.117 & 36.553 & 5.597 & 16.483 & 3.093 \\
\hline $11 / 6 / 09$ & $12: 00$ & 24.371 & 33.168 & 26.112 & 36.560 & 5.512 & 16.484 & 3.070 \\
\hline $11 / 6 / 09$ & $16: 00$ & 24.416 & 33.143 & 26.044 & 36.531 & 5.529 & 16.443 & 3.095 \\
\hline $11 / 6 / 09$ & $20: 00$ & 24.311 & 33.189 & 26.100 & 36.473 & 5.560 & 16.494 & 2.934 \\
\hline $11 / 7 / 09$ & 0:00 & 24.279 & 33.214 & 26.129 & 36.450 & 5.560 & 16.505 & 2.878 \\
\hline $11 / 7 / 09$ & $4: 00$ & 24.290 & 33.238 & 26.165 & 36.442 & 5.582 & 16.540 & 2.831 \\
\hline $11 / 7 / 09$ & 8:00 & 24.254 & 33.286 & 26.253 & 36.434 & 5.539 & 16.594 & 2.795 \\
\hline $11 / 7 / 09$ & $12: 00$ & 24.299 & 33.330 & 26.336 & 36.433 & 5.547 & 16.644 & 2.887 \\
\hline $11 / 7 / 09$ & $16: 00$ & 24.386 & 33.338 & 26.333 & 36.465 & 5.575 & 16.641 & 3.081 \\
\hline $11 / 7 / 09$ & $20: 00$ & 24.322 & 33.380 & 26.399 & 36.466 & 5.541 & 16.682 & 2.919 \\
\hline $11 / 8 / 09$ & $0: 00$ & 24.347 & 33.395 & 26.416 & 36.456 & 5.553 & 16.682 & 2.850 \\
\hline $11 / 8 / 09$ & $4: 00$ & 24.396 & 33.416 & 26.433 & 36.493 & 5.584 & 16.702 & 2.827 \\
\hline $11 / 8 / 09$ & 8:00 & 24.429 & 33.439 & 26.463 & 36.476 & 5.608 & 16.721 & 2.819 \\
\hline $11 / 8 / 09$ & $12: 00$ & 24.498 & 33.449 & 26.468 & 36.526 & 5.604 & 16.724 & 2.976 \\
\hline $11 / 8 / 09$ & $16: 00$ & 24.530 & 33.468 & 26.473 & 36.503 & 5.547 & 16.735 & 3.126 \\
\hline $11 / 8 / 09$ & $20: 00$ & 24.453 & 33.540 & 26.595 & 36.527 & 5.578 & 16.812 & 2.983 \\
\hline $11 / 9 / 09$ & $0: 00$ & 24.433 & 33.603 & 26.704 & 36.556 & 5.621 & 16.871 & 2.907 \\
\hline $11 / 9 / 09$ & $4: 00$ & 24.453 & 33.645 & 26.775 & 36.567 & 5.611 & 16.910 & 2.827 \\
\hline $11 / 9 / 09$ & $8: 00$ & 24.463 & 33.695 & 26.858 & 36.719 & 5.578 & 16.949 & 2.866 \\
\hline $11 / 9 / 09$ & $12: 00$ & 24.563 & 33.718 & 26.889 & 36.762 & 5.637 & 16.964 & 2.938 \\
\hline $11 / 9 / 09$ & $16: 00$ & 24.662 & 33.720 & 26.866 & 36.786 & 5.596 & 16.953 & 3.146 \\
\hline $11 / 9 / 09$ & $20: 00$ & 24.642 & 33.762 & 26.921 & 36.805 & 5.554 & 16.990 & 3.017 \\
\hline
\end{tabular}


TABLE S2.1 (Cont.)

\begin{tabular}{|c|c|c|c|c|c|c|c|c|}
\hline \multirow[b]{2}{*}{ Date } & \multirow[b]{2}{*}{ Time } & \multicolumn{6}{|c|}{ Depth to Water (ft TOC) } & \multirow[b]{2}{*}{ MW8s } \\
\hline & & MW1s & MW2s & MW3s & MW4s & MW6s & MW7s & \\
\hline $11 / 10 / 09$ & $0: 00$ & 24.679 & 33.779 & 26.936 & 36.809 & 5.637 & 16.999 & 2.952 \\
\hline $11 / 10 / 09$ & $4: 00$ & 24.706 & 33.804 & 26.962 & 36.821 & 5.600 & 17.018 & 2.892 \\
\hline $11 / 10 / 09$ & 8:00 & 24.714 & 33.840 & 27.004 & 36.872 & 5.618 & 17.044 & 2.882 \\
\hline $11 / 10 / 09$ & $12: 00$ & 24.761 & 33.878 & 27.058 & 36.892 & 5.639 & 17.075 & 2.943 \\
\hline $11 / 10 / 09$ & $16: 00$ & 24.845 & 33.867 & 27.014 & 36.900 & 5.608 & 17.049 & 3.223 \\
\hline $11 / 10 / 09$ & $20: 00$ & 24.804 & 33.899 & 27.046 & 36.911 & 5.635 & 17.079 & 3.052 \\
\hline $11 / 11 / 09$ & $0: 00$ & 24.806 & 33.920 & 27.065 & 36.936 & 5.651 & 17.087 & 2.919 \\
\hline $11 / 11 / 09$ & $4: 00$ & 24.841 & 33.930 & 27.060 & 36.983 & 5.655 & 17.092 & 2.952 \\
\hline $11 / 11 / 09$ & 8:00 & 24.853 & 33.960 & 27.089 & 36.959 & 5.663 & 17.111 & 2.954 \\
\hline $11 / 11 / 09$ & $12: 00$ & 24.932 & 33.970 & 27.082 & 37.010 & 5.625 & 17.111 & 3.113 \\
\hline 11/11/09 & $16: 00$ & 25.027 & 33.930 & 26.984 & 36.993 & 5.653 & 17.049 & 3.236 \\
\hline $11 / 11 / 09$ & $20: 00$ & 24.972 & 33.951 & 26.985 & 37.013 & 5.669 & 17.061 & 3.140 \\
\hline $11 / 12 / 09$ & $0: 00$ & 24.965 & 33.957 & 26.968 & 36.983 & 5.612 & 17.059 & 3.080 \\
\hline $11 / 12 / 09$ & $4: 00$ & 24.995 & 33.947 & 26.921 & 37.012 & 5.572 & 17.035 & 3.091 \\
\hline $11 / 12 / 09$ & $8: 00$ & 25.002 & 33.936 & 26.870 & 36.969 & 5.655 & 17.016 & 3.146 \\
\hline $11 / 12 / 09$ & $12: 00$ & 25.054 & 33.928 & 26.831 & 36.957 & 5.659 & 16.997 & 3.321 \\
\hline $11 / 12 / 09$ & $16: 00$ & 25.094 & 33.894 & 26.738 & 36.938 & 5.639 & 16.953 & 3.276 \\
\hline $11 / 12 / 09$ & $20: 00$ & 25.024 & 33.918 & 26.753 & 36.898 & 5.621 & 16.970 & 3.207 \\
\hline $11 / 13 / 09$ & $0: 00$ & 24.987 & 33.936 & 26.766 & 36.844 & 5.613 & 16.981 & 3.174 \\
\hline $11 / 13 / 09$ & $4: 00$ & 24.999 & 33.926 & 26.726 & 36.867 & 5.568 & 16.962 & 3.213 \\
\hline $11 / 13 / 09$ & $8: 00$ & 24.960 & 33.951 & 26.748 & 36.824 & 5.615 & 16.984 & 3.219 \\
\hline $11 / 13 / 09$ & $12: 00$ & 24.987 & 33.972 & 26.770 & 36.812 & 5.593 & 16.994 & 3.301 \\
\hline $11 / 13 / 09$ & $16: 00$ & 25.007 & 33.975 & 26.760 & 36.812 & 5.617 & 16.999 & 3.305 \\
\hline $11 / 13 / 09$ & $20: 00$ & 24.943 & 34.029 & 26.846 & 36.787 & 5.605 & 17.057 & 3.182 \\
\hline $11 / 14 / 09$ & $0: 00$ & 24.876 & 34.101 & 26.972 & 36.771 & 5.646 & 17.133 & 3.064 \\
\hline $11 / 14 / 09$ & $4: 00$ & 24.878 & 34.144 & 27.050 & 36.756 & 5.613 & 17.176 & 3.076 \\
\hline $11 / 14 / 09$ & $8: 00$ & 24.893 & 34.186 & 27.133 & 36.820 & 5.630 & 17.215 & 3.041 \\
\hline $11 / 14 / 09$ & $12: 00$ & 24.935 & 34.230 & 27.214 & 36.859 & 5.673 & 17.254 & 3.098 \\
\hline $11 / 14 / 09$ & $16: 00$ & 25.015 & 34.241 & 27.224 & 36.902 & 5.629 & 17.254 & 3.260 \\
\hline $11 / 14 / 09$ & $20: 00$ & 24.997 & 34.285 & 27.299 & 36.934 & 5.675 & 17.293 & 3.086 \\
\hline $11 / 15 / 09$ & $0: 00$ & 25.007 & 34.331 & 27.377 & 36.958 & 5.673 & 17.337 & 3.043 \\
\hline $11 / 15 / 09$ & $4: 00$ & 25.051 & 34.346 & 27.402 & 37.001 & 5.646 & 17.345 & 2.965 \\
\hline $11 / 15 / 09$ & 8:00 & 25.077 & 34.382 & 27.455 & 37.003 & 5.693 & 17.378 & 3.158 \\
\hline $11 / 15 / 09$ & $12: 00$ & 25.129 & 34.407 & 27.487 & 37.069 & 5.660 & 17.393 & 3.188 \\
\hline $11 / 15 / 09$ & $16: 00$ & 25.223 & 34.386 & 27.448 & 37.128 & 5.666 & 17.354 & 3.256 \\
\hline $11 / 15 / 09$ & $20: 00$ & 25.181 & 34.445 & 27.531 & 37.123 & 5.646 & 17.419 & 3.145 \\
\hline 11/16/09 & $0: 00$ & 25.206 & 34.463 & 27.553 & 37.162 & 5.635 & 17.430 & 3.160 \\
\hline $11 / 16 / 09$ & $4: 00$ & 25.225 & 34.466 & 27.545 & 37.163 & 5.641 & 17.427 & 3.151 \\
\hline 11/16/09 & $8: 00$ & 25.235 & 34.472 & 27.553 & 37.188 & 5.635 & 17.414 & 3.131 \\
\hline 11/16/09 & $12: 00$ & 25.243 & 34.461 & 27.538 & 37.236 & 5.668 & 17.391 & 3.139 \\
\hline $11 / 16 / 09$ & $16: 00$ & 25.258 & 34.453 & 27.511 & 37.245 & 5.618 & 17.336 & 3.146 \\
\hline 11/16/09 & $20: 00$ & 25.208 & 34.474 & 27.568 & 37.255 & 5.616 & 17.209 & 3.046 \\
\hline 11/17/09 & $0: 00$ & 25.163 & 34.466 & 27.577 & 37.274 & 5.579 & 17.003 & 2.952 \\
\hline 11/17/09 & $4: 00$ & 25.081 & 34.419 & 27.540 & 37.286 & 5.654 & 16.676 & 2.827 \\
\hline 11/17/09 & $8: 00$ & 24.923 & 34.371 & 27.497 & 37.285 & 5.585 & 16.410 & 2.704 \\
\hline 11/17/09 & $12: 00$ & 24.761 & 34.315 & 27.458 & 37.294 & 5.513 & 16.260 & 2.608 \\
\hline 11/17/09 & $16: 00$ & 24.536 & 34.195 & 27.212 & 37.303 & 5.484 & 16.002 & 2.537 \\
\hline $11 / 17 / 09$ & $20: 00$ & 24.297 & 34.090 & 27.095 & 37.293 & 5.383 & 15.834 & 2.386 \\
\hline
\end{tabular}


TABLE S2.1 (Cont.)

\begin{tabular}{|c|c|c|c|c|c|c|c|c|}
\hline \multirow[b]{2}{*}{ Date } & \multirow[b]{2}{*}{ Time } & \multicolumn{6}{|c|}{ Depth to Water (ft TOC) } & \multirow[b]{2}{*}{ MW8s } \\
\hline & & MW1s & MW2s & MW3s & MW4s & MW6s & MW7s & \\
\hline $11 / 18 / 09$ & $0: 00$ & 24.149 & 33.956 & 27.005 & 37.310 & 5.476 & 15.808 & 2.353 \\
\hline $11 / 18 / 09$ & $4: 00$ & 23.988 & 33.847 & 26.840 & 37.301 & 5.484 & 15.877 & 2.324 \\
\hline $11 / 18 / 09$ & 8:00 & 23.876 & 33.742 & 26.623 & 37.261 & 5.515 & 15.948 & 1.882 \\
\hline $11 / 18 / 09$ & $12: 00$ & 23.779 & 33.645 & 26.436 & 37.250 & 5.573 & 15.988 & 1.743 \\
\hline $11 / 18 / 09$ & $16: 00$ & 23.672 & 33.521 & 25.776 & 37.255 & 5.365 & 15.730 & 1.443 \\
\hline $11 / 18 / 09$ & 20:00 & 23.387 & 33.410 & 25.626 & 37.256 & 5.202 & 15.500 & 1.081 \\
\hline $11 / 19 / 09$ & $0: 00$ & 23.141 & 33.299 & 25.511 & 37.242 & 5.338 & 15.474 & 0.972 \\
\hline $11 / 19 / 09$ & $4: 00$ & 23.018 & 33.186 & 25.364 & 37.231 & 5.393 & 15.480 & 0.950 \\
\hline 11/19/09 & $8: 00$ & 22.869 & 33.091 & 25.232 & 37.183 & 5.457 & 15.480 & 0.942 \\
\hline $11 / 19 / 09$ & $12: 00$ & 22.794 & 33.009 & 25.113 & 37.212 & 5.538 & 15.472 & 1.008 \\
\hline 11/19/09 & $16: 00$ & 22.633 & 32.879 & 24.889 & 37.215 & 5.435 & 15.121 & 0.840 \\
\hline 11/19/09 & 20:00 & 22.334 & 32.789 & 24.772 & 37.181 & 5.261 & 15.030 & 0.677 \\
\hline $11 / 20 / 09$ & $0: 00$ & 22.210 & 32.682 & 24.619 & 37.152 & 5.393 & 15.017 & 0.689 \\
\hline $11 / 20 / 09$ & $4: 00$ & 22.153 & 32.577 & 24.441 & 37.172 & 5.523 & 14.985 & 0.741 \\
\hline $11 / 20 / 09$ & $8: 00$ & 22.055 & 32.495 & 24.302 & 37.156 & 5.562 & 14.954 & 0.763 \\
\hline $11 / 20 / 09$ & $12: 00$ & 22.140 & 32.424 & 24.166 & 37.145 & 5.587 & 14.991 & 0.914 \\
\hline $11 / 20 / 09$ & $16: 00$ & 22.182 & 32.325 & 23.969 & 37.138 & 5.599 & 14.911 & 1.068 \\
\hline $11 / 20 / 09$ & 20:00 & 22.098 & 32.249 & 23.820 & 37.079 & 5.628 & 14.905 & 0.953 \\
\hline $11 / 21 / 09$ & 0:00 & 22.093 & 32.172 & 23.679 & 37.049 & 5.620 & 14.904 & 0.921 \\
\hline $11 / 21 / 09$ & $4: 00$ & 22.112 & 32.094 & 23.528 & 37.045 & 5.597 & 14.892 & 0.931 \\
\hline $11 / 21 / 09$ & $8: 00$ & 22.095 & 32.042 & 23.426 & 36.998 & 5.605 & 14.909 & 0.959 \\
\hline $11 / 21 / 09$ & $12: 00$ & 22.149 & 31.972 & 23.314 & 36.977 & 5.605 & 14.904 & 1.135 \\
\hline $11 / 21 / 09$ & $16: 00$ & 22.211 & 31.886 & 23.158 & 36.935 & 5.568 & 14.855 & 1.242 \\
\hline $11 / 21 / 09$ & 20:00 & 22.157 & 31.857 & 23.146 & 36.844 & 5.584 & 14.887 & 1.229 \\
\hline $11 / 22 / 09$ & $0: 00$ & 22.162 & 31.815 & 23.097 & 36.809 & 5.620 & 14.902 & 1.236 \\
\hline $11 / 22 / 09$ & $4: 00$ & 22.139 & 31.788 & 23.087 & 36.771 & 5.619 & 14.933 & 1.127 \\
\hline $11 / 22 / 09$ & $8: 00$ & 22.139 & 31.756 & 23.060 & 36.761 & 5.553 & 14.950 & 1.062 \\
\hline $11 / 22 / 09$ & $12: 00$ & 22.179 & 31.737 & 23.077 & 36.737 & 5.603 & 14.980 & 1.198 \\
\hline $11 / 22 / 09$ & $16: 00$ & 22.256 & 31.676 & 22.994 & 36.716 & 5.586 & 14.941 & 1.236 \\
\hline $11 / 22 / 09$ & 20:00 & 22.211 & 31.669 & 23.035 & 36.632 & 5.613 & 14.984 & 1.140 \\
\hline $11 / 23 / 09$ & $0: 00$ & 22.206 & 31.651 & 23.045 & 36.603 & 5.601 & 14.991 & 1.086 \\
\hline $11 / 23 / 09$ & $4: 00$ & 22.258 & 31.623 & 23.050 & 36.612 & 5.650 & 14.999 & 1.133 \\
\hline $11 / 23 / 09$ & $8: 00$ & 22.276 & 31.615 & 23.092 & 36.575 & 5.648 & 15.017 & 1.144 \\
\hline $11 / 23 / 09$ & $12: 00$ & 22.306 & 31.608 & 23.142 & 36.552 & 5.665 & 15.036 & 1.240 \\
\hline $11 / 23 / 09$ & $16: 00$ & 22.363 & 31.579 & 23.137 & 36.499 & 5.607 & 15.017 & 1.317 \\
\hline $11 / 23 / 09$ & 20:00 & 22.345 & 31.579 & 23.210 & 36.464 & 5.609 & 15.047 & 1.242 \\
\hline $11 / 24 / 09$ & $0: 00$ & 22.385 & 31.541 & 23.220 & 36.490 & 5.629 & 15.017 & 1.272 \\
\hline $11 / 24 / 09$ & $4: 00$ & 22.333 & 31.493 & 23.207 & 36.429 & 5.367 & 14.462 & 1.066 \\
\hline $11 / 24 / 09$ & $8: 00$ & 21.929 & 31.421 & 22.965 & 36.365 & 4.908 & 14.239 & 0.680 \\
\hline $11 / 24 / 09$ & $12: 00$ & 21.425 & 31.313 & 22.531 & 36.362 & 4.619 & 14.027 & 0.215 \\
\hline $11 / 24 / 09$ & $16: 00$ & 21.120 & 31.178 & 22.436 & 36.301 & 4.807 & 13.698 & 0.254 \\
\hline $11 / 24 / 09$ & 20:00 & 20.936 & 31.084 & 22.311 & 36.310 & 4.996 & 13.507 & 0.368 \\
\hline $11 / 25 / 09$ & $0: 00$ & 20.843 & 30.975 & 22.150 & 36.299 & 5.211 & 13.499 & 0.409 \\
\hline $11 / 25 / 09$ & $4: 00$ & 20.785 & 30.868 & 21.984 & 36.243 & 5.378 & 13.479 & 0.521 \\
\hline $11 / 25 / 09$ & $8: 00$ & 20.760 & 30.801 & 21.918 & 36.215 & 5.441 & 13.523 & 0.551 \\
\hline $11 / 25 / 09$ & $12: 00$ & 20.799 & 30.708 & 21.809 & 36.187 & 5.530 & 13.527 & 0.650 \\
\hline 11/25/09 & $16: 00$ & 20.814 & 30.622 & 21.697 & 36.153 & 5.542 & 13.520 & 0.803 \\
\hline $11 / 25 / 09$ & $20: 00$ & 20.774 & 30.561 & 21.650 & 36.138 & 5.536 & 13.555 & 0.705 \\
\hline
\end{tabular}


TABLE S2.1 (Cont.)

\begin{tabular}{|c|c|c|c|c|c|c|c|c|}
\hline \multirow[b]{2}{*}{ Date } & \multirow[b]{2}{*}{ Time } & \multicolumn{6}{|c|}{ Depth to Water (ft TOC) } & \multirow[b]{2}{*}{ MW8s } \\
\hline & & MW1s & MW2s & MW3s & MW4s & MW6s & MW7s & \\
\hline $11 / 26 / 09$ & $0: 00$ & 20.766 & 30.498 & 21.592 & 36.083 & 5.536 & 13.570 & 0.652 \\
\hline $11 / 26 / 09$ & $4: 00$ & 20.776 & 30.438 & 21.546 & 36.089 & 5.610 & 13.600 & 0.687 \\
\hline $11 / 26 / 09$ & 8:00 & 20.826 & 30.385 & 21.500 & 36.065 & 5.579 & 13.637 & 0.670 \\
\hline $11 / 26 / 09$ & $12: 00$ & 20.892 & 30.338 & 21.465 & 36.014 & 5.618 & 13.672 & 0.742 \\
\hline $11 / 26 / 09$ & $16: 00$ & 20.976 & 30.248 & 21.353 & 36.013 & 5.556 & 13.628 & 1.008 \\
\hline $11 / 26 / 09$ & $20: 00$ & 20.969 & 30.193 & 21.307 & 35.966 & 5.521 & 13.637 & 0.919 \\
\hline $11 / 27 / 09$ & $0: 00$ & 21.004 & 30.124 & 21.236 & 35.968 & 5.583 & 13.618 & 0.903 \\
\hline $11 / 27 / 09$ & $4: 00$ & 21.051 & 30.057 & 21.156 & 35.912 & 5.563 & 13.585 & 0.964 \\
\hline $11 / 27 / 09$ & 8:00 & 21.066 & 30.011 & 21.124 & 35.821 & 5.567 & 13.577 & 1.031 \\
\hline $11 / 27 / 09$ & $12: 00$ & 21.101 & 29.983 & 21.124 & 35.796 & 5.525 & 13.602 & 1.068 \\
\hline 11/27/09 & $16: 00$ & 21.158 & 29.916 & 21.061 & 35.755 & 5.540 & 13.561 & 1.044 \\
\hline $11 / 27 / 09$ & $20: 00$ & 21.085 & 29.902 & 21.081 & 35.694 & 5.537 & 13.585 & 0.968 \\
\hline $11 / 28 / 09$ & $0: 00$ & 21.085 & 29.878 & 21.104 & 35.635 & 5.494 & 13.600 & 0.899 \\
\hline $11 / 28 / 09$ & $4: 00$ & 21.083 & 29.864 & 21.141 & 35.582 & 5.531 & 13.620 & 0.893 \\
\hline $11 / 28 / 09$ & $8: 00$ & 21.070 & 29.862 & 21.197 & 35.528 & 5.544 & 13.659 & 0.916 \\
\hline $11 / 28 / 09$ & $12: 00$ & 21.145 & 29.847 & 21.236 & 35.497 & 5.511 & 13.676 & 1.063 \\
\hline $11 / 28 / 09$ & $16: 00$ & 21.204 & 29.807 & 21.236 & 35.462 & 5.560 & 13.656 & 1.226 \\
\hline $11 / 28 / 09$ & $20: 00$ & 21.145 & 29.830 & 21.333 & 35.408 & 5.513 & 13.709 & 0.967 \\
\hline $11 / 29 / 09$ & $0: 00$ & 21.142 & 29.851 & 21.435 & 35.363 & 5.545 & 13.765 & 0.969 \\
\hline $11 / 29 / 09$ & $4: 00$ & 21.157 & 29.870 & 21.550 & 35.314 & 5.570 & 13.812 & 1.001 \\
\hline $11 / 29 / 09$ & $8: 00$ & 21.157 & 29.914 & 21.703 & 35.318 & 5.587 & 13.891 & 1.050 \\
\hline $11 / 29 / 09$ & $12: 00$ & 21.192 & 29.960 & 21.866 & 35.294 & 5.577 & 13.969 & 1.059 \\
\hline $11 / 29 / 09$ & $16: 00$ & 21.298 & 29.939 & 21.912 & 35.330 & 5.545 & 13.958 & 1.182 \\
\hline $11 / 29 / 09$ & $20: 00$ & 21.289 & 29.981 & 22.050 & 35.316 & 5.557 & 14.018 & 1.032 \\
\hline $11 / 30 / 09$ & $0: 00$ & 21.345 & 29.991 & 22.145 & 35.329 & 5.599 & 14.042 & 1.053 \\
\hline $11 / 30 / 09$ & $4: 00$ & 21.398 & 29.993 & 22.230 & 35.298 & 5.551 & 14.055 & 0.970 \\
\hline $11 / 30 / 09$ & $8: 00$ & 21.470 & 29.983 & 22.289 & 35.337 & 5.564 & 14.046 & 1.068 \\
\hline $11 / 30 / 09$ & $12: 00$ & 21.562 & 29.989 & 22.378 & 35.329 & 5.557 & 14.053 & 1.195 \\
\hline $11 / 30 / 09$ & $16: 00$ & 21.628 & 29.956 & 22.390 & 35.319 & 5.555 & 14.012 & 1.209 \\
\hline $11 / 30 / 09$ & $20: 00$ & 21.608 & 29.954 & 22.439 & 35.295 & 5.533 & 14.007 & 1.146 \\
\hline 12/1/09 & $0: 00$ & 21.613 & 29.951 & 22.490 & 35.242 & 5.529 & 14.001 & 1.113 \\
\hline 12/1/09 & $4: 00$ & 21.631 & 29.933 & 22.504 & 35.246 & 5.570 & 13.975 & 1.126 \\
\hline 12/1/09 & 8:00 & 21.636 & 29.928 & 22.538 & 35.196 & 5.566 & 13.962 & 1.134 \\
\hline 12/1/09 & $12: 00$ & 21.668 & 29.945 & 22.606 & 35.161 & 5.539 & 13.975 & 1.277 \\
\hline 12/1/09 & $16: 00$ & 21.725 & 29.933 & 22.628 & 35.117 & 5.619 & 13.953 & 1.220 \\
\hline 12/1/09 & $20: 00$ & 21.628 & 29.983 & 22.752 & 35.109 & 5.580 & 14.010 & 1.108 \\
\hline 12/2/09 & $0: 00$ & 21.606 & 30.048 & 22.908 & 35.065 & 5.565 & 14.083 & 1.167 \\
\hline $12 / 2 / 09$ & $4: 00$ & 21.618 & 30.084 & 23.019 & 35.064 & 5.569 & 14.129 & 1.178 \\
\hline $12 / 2 / 09$ & 8:00 & 21.643 & 30.113 & 23.105 & 35.074 & 5.547 & 14.161 & 1.165 \\
\hline $12 / 2 / 09$ & $12: 00$ & 21.730 & 30.132 & 23.163 & 35.094 & 5.553 & 14.168 & 1.278 \\
\hline $12 / 2 / 09$ & $16: 00$ & 21.780 & 30.140 & 23.194 & 35.050 & 5.574 & 14.166 & 1.359 \\
\hline $12 / 2 / 09$ & $20: 00$ & 21.725 & 30.208 & 23.330 & 35.068 & 5.538 & 14.241 & 1.231 \\
\hline 12/3/09 & $0: 00$ & 21.760 & 30.247 & 23.423 & 35.078 & 5.536 & 14.282 & 1.262 \\
\hline 12/3/09 & $4: 00$ & 21.770 & 30.298 & 23.528 & 35.078 & 5.569 & 14.317 & 1.125 \\
\hline 12/3/09 & $8: 00$ & 21.825 & 30.329 & 23.598 & 35.098 & 5.538 & 14.354 & 1.361 \\
\hline $12 / 3 / 09$ & $12: 00$ & 21.884 & 30.382 & 23.693 & 35.113 & 5.585 & 14.406 & 1.453 \\
\hline 12/3/09 & $16: 00$ & 21.959 & 30.396 & 23.732 & 35.146 & 5.586 & 14.410 & 1.477 \\
\hline $12 / 3 / 09$ & $20: 00$ & 21.951 & 30.447 & 23.824 & 35.151 & 5.546 & 14.456 & 1.463 \\
\hline
\end{tabular}


TABLE S2.1 (Cont.)

\begin{tabular}{|c|c|c|c|c|c|c|c|c|}
\hline \multirow[b]{2}{*}{ Date } & \multirow[b]{2}{*}{ Time } & \multicolumn{6}{|c|}{ Depth to Water (ft TOC) } & \multirow[b]{2}{*}{ MW8s } \\
\hline & & MW1s & MW2s & MW3s & MW4s & MW6s & MW7s & \\
\hline $12 / 4 / 09$ & $0: 00$ & 21.991 & 30.478 & 23.880 & 35.181 & 5.610 & 14.478 & 1.506 \\
\hline $12 / 4 / 09$ & $4: 00$ & 22.038 & 30.499 & 23.914 & 35.166 & 5.590 & 14.490 & 1.526 \\
\hline $12 / 4 / 09$ & 8:00 & 22.095 & 30.506 & 23.921 & 35.210 & 5.616 & 14.484 & 1.559 \\
\hline $12 / 4 / 09$ & $12: 00$ & 22.165 & 30.520 & 23.938 & 35.229 & 5.583 & 14.486 & 1.625 \\
\hline $12 / 4 / 09$ & $16: 00$ & 22.244 & 30.499 & 23.887 & 35.231 & 5.567 & 14.438 & 1.656 \\
\hline $12 / 4 / 09$ & $20: 00$ & 22.214 & 30.510 & 23.885 & 35.224 & 5.577 & 14.428 & 1.597 \\
\hline $12 / 5 / 09$ & $0: 00$ & 22.227 & 30.512 & 23.877 & 35.206 & 5.559 & 14.417 & 1.625 \\
\hline $12 / 5 / 09$ & $4: 00$ & 22.254 & 30.512 & 23.863 & 35.157 & 5.568 & 14.401 & 1.672 \\
\hline $12 / 5 / 09$ & 8:00 & 22.241 & 30.531 & 23.875 & 35.152 & 5.595 & 14.406 & 1.695 \\
\hline $12 / 5 / 09$ & $12: 00$ & 22.269 & 30.564 & 23.921 & 35.138 & 5.571 & 14.434 & 1.755 \\
\hline $12 / 5 / 09$ & $16: 00$ & 22.313 & 30.556 & 23.897 & 35.088 & 5.556 & 14.406 & 1.774 \\
\hline $12 / 5 / 09$ & $20: 00$ & 22.219 & 30.627 & 24.011 & 35.048 & 5.571 & 14.484 & 1.691 \\
\hline 12/6/09 & $0: 00$ & 22.219 & 30.672 & 24.096 & 35.087 & 5.571 & 14.533 & 1.700 \\
\hline $12 / 6 / 09$ & $4: 00$ & 22.237 & 30.718 & 24.177 & 35.088 & 5.587 & 14.577 & 1.704 \\
\hline 12/6/09 & 8:00 & 22.271 & 30.749 & 24.228 & 35.099 & 5.558 & 14.601 & 1.730 \\
\hline 12/6/09 & $12: 00$ & 22.313 & 30.783 & 24.289 & 35.120 & 5.550 & 14.629 & 1.788 \\
\hline $12 / 6 / 09$ & $16: 00$ & 22.403 & 30.749 & 24.199 & 35.126 & 5.606 & 14.566 & 1.789 \\
\hline 12/6/09 & $20: 00$ & 22.380 & 30.797 & 24.272 & 35.125 & 5.583 & 14.616 & 1.766 \\
\hline 12/7/09 & $0: 00$ & 22.351 & 30.854 & 24.364 & 35.092 & 5.571 & 14.668 & 1.773 \\
\hline 12/7/09 & $4: 00$ & 22.328 & 30.934 & 24.501 & 35.121 & 5.630 & 14.754 & 1.776 \\
\hline 12/7/09 & $8: 00$ & 22.321 & 30.957 & 24.544 & 35.153 & 5.601 & 14.728 & 1.774 \\
\hline 12/7/09 & $12: 00$ & 22.420 & 31.007 & 24.622 & 35.182 & 5.609 & 14.821 & 1.834 \\
\hline 12/7/09 & $16: 00$ & 22.524 & 31.007 & 24.603 & 35.193 & 5.587 & 14.796 & 1.895 \\
\hline 12/7/09 & $20: 00$ & 22.557 & 31.026 & 24.610 & 35.236 & 5.614 & 14.806 & 1.883 \\
\hline 12/8/09 & $0: 00$ & 22.614 & 31.022 & 24.576 & 35.251 & 5.601 & 14.782 & 1.926 \\
\hline $12 / 8 / 09$ & $4: 00$ & 22.673 & 31.009 & 24.518 & 35.237 & 5.584 & 14.739 & 1.937 \\
\hline $12 / 8 / 09$ & 8:00 & 22.760 & 30.942 & 24.369 & 35.262 & 5.551 & 14.635 & 1.933 \\
\hline $12 / 8 / 09$ & $12: 00$ & 22.802 & 30.886 & 24.220 & 35.240 & 5.591 & 14.549 & 1.958 \\
\hline 12/8/09 & $16: 00$ & 22.817 & 30.827 & 24.072 & 35.189 & 5.521 & 14.453 & 1.924 \\
\hline 12/8/09 & $20: 00$ & 22.705 & 30.846 & 24.064 & 35.089 & 5.510 & 14.456 & 1.894 \\
\hline $12 / 9 / 09$ & $0: 00$ & 22.611 & 30.881 & 24.108 & 35.016 & 5.516 & 14.490 & 1.887 \\
\hline $12 / 9 / 09$ & $4: 00$ & 22.470 & 31.016 & 24.345 & 34.907 & 5.593 & 14.646 & 1.830 \\
\hline $12 / 9 / 09$ & 8:00 & 22.452 & 31.091 & 24.510 & 34.941 & 5.572 & 14.742 & 1.847 \\
\hline $12 / 9 / 09$ & $12: 00$ & 22.467 & 31.171 & 24.681 & 34.964 & 5.587 & 14.843 & 1.890 \\
\hline 12/9/09 & $16: 00$ & 22.549 & 31.207 & 24.744 & 34.993 & 5.572 & 14.874 & 1.903 \\
\hline 12/9/09 & $20: 00$ & 22.569 & 31.255 & 24.824 & 35.023 & 5.615 & 14.925 & 1.914 \\
\hline 12/10/09 & $0: 00$ & 22.619 & 31.282 & 24.863 & 35.063 & 5.574 & 14.923 & 1.930 \\
\hline $12 / 10 / 09$ & $4: 00$ & 22.698 & 31.310 & 24.890 & 35.088 & 5.578 & 14.965 & 1.930 \\
\hline $12 / 10 / 09$ & $8: 00$ & 22.738 & 31.341 & 24.932 & 35.118 & 5.594 & 14.990 & 1.946 \\
\hline $12 / 10 / 09$ & $12: 00$ & 22.777 & 31.387 & 25.000 & 35.144 & 5.568 & 15.036 & 1.957 \\
\hline $12 / 10 / 09$ & $16: 00$ & 22.832 & 31.404 & 25.005 & 35.153 & 5.629 & 15.029 & 1.975 \\
\hline $12 / 10 / 09$ & $20: 00$ & 22.865 & 31.436 & 25.039 & 35.212 & 5.615 & 15.064 & 1.984 \\
\hline $12 / 11 / 09$ & $0: 00$ & 22.894 & 31.461 & 25.063 & 35.253 & 5.561 & 15.077 & 2.006 \\
\hline $12 / 11 / 09$ & $4: 00$ & 22.902 & 31.501 & 25.109 & 35.266 & 5.617 & 15.116 & 2.026 \\
\hline $12 / 11 / 09$ & $8: 00$ & 22.936 & 31.524 & 25.131 & 35.300 & 5.658 & 15.125 & 2.027 \\
\hline $12 / 11 / 09$ & $12: 00$ & 23.008 & 31.549 & 25.151 & 35.327 & 5.615 & 15.138 & 2.056 \\
\hline $12 / 11 / 09$ & $16: 00$ & 23.073 & 31.547 & 25.105 & 35.356 & 5.638 & 15.111 & 2.079 \\
\hline $12 / 11 / 09$ & $20: 00$ & 23.048 & 31.574 & 25.129 & 35.337 & 5.584 & 15.129 & 2.085 \\
\hline
\end{tabular}


TABLE S2.1 (Cont.)

\begin{tabular}{|c|c|c|c|c|c|c|c|c|}
\hline \multirow[b]{2}{*}{ Date } & \multirow[b]{2}{*}{ Time } & \multicolumn{6}{|c|}{ Depth to Water (ft TOC) } & \multirow[b]{2}{*}{ MW8s } \\
\hline & & MW1s & MW2s & MW3s & MW4s & MW6s & MW7s & \\
\hline $12 / 12 / 09$ & $0: 00$ & 23.083 & 31.585 & 25.114 & 35.378 & 5.625 & 15.122 & 2.106 \\
\hline $12 / 12 / 09$ & $4: 00$ & 23.107 & 31.597 & 25.112 & 35.352 & 5.613 & 15.127 & 2.129 \\
\hline $12 / 12 / 09$ & 8:00 & 23.140 & 31.595 & 25.073 & 35.390 & 5.608 & 15.098 & 2.116 \\
\hline $12 / 12 / 09$ & $12: 00$ & 23.185 & 31.591 & 25.031 & 35.364 & 5.580 & 15.073 & 2.132 \\
\hline $12 / 12 / 09$ & $16: 00$ & 23.212 & 31.577 & 24.966 & 35.373 & 5.561 & 15.031 & 2.129 \\
\hline $12 / 12 / 09$ & $20: 00$ & 23.167 & 31.610 & 24.997 & 35.340 & 5.584 & 15.053 & 2.125 \\
\hline $12 / 13 / 09$ & $0: 00$ & 23.140 & 31.637 & 25.024 & 35.323 & 5.594 & 15.079 & 2.124 \\
\hline $12 / 13 / 09$ & $4: 00$ & 23.098 & 31.677 & 25.085 & 35.300 & 5.583 & 15.125 & 2.104 \\
\hline $12 / 13 / 09$ & 8:00 & 23.098 & 31.717 & 25.148 & 35.296 & 5.635 & 15.166 & 2.098 \\
\hline $12 / 13 / 09$ & $12: 00$ & 23.157 & 31.745 & 25.187 & 35.325 & 5.596 & 15.187 & 2.118 \\
\hline $12 / 13 / 09$ & $16: 00$ & 23.222 & 31.719 & 25.109 & 35.335 & 5.586 & 15.134 & 2.176 \\
\hline $12 / 13 / 09$ & $20: 00$ & 23.237 & 31.724 & 25.080 & 35.292 & 5.590 & 15.120 & 2.179 \\
\hline $12 / 14 / 09$ & $0: 00$ & 23.269 & 31.722 & 25.046 & 35.312 & 5.627 & 15.098 & 2.159 \\
\hline $12 / 14 / 09$ & $4: 00$ & 23.220 & 31.755 & 25.078 & 35.239 & 5.604 & 15.121 & 2.114 \\
\hline $12 / 14 / 09$ & $8: 00$ & 23.083 & 31.879 & 25.312 & 35.232 & 5.612 & 15.285 & 2.103 \\
\hline $12 / 14 / 09$ & $12: 00$ & 23.053 & 31.984 & 25.516 & 35.261 & 5.626 & 15.404 & 2.084 \\
\hline $12 / 14 / 09$ & $16: 00$ & 23.120 & 32.032 & 25.599 & 35.311 & 5.647 & 15.454 & 2.098 \\
\hline $12 / 14 / 09$ & $20: 00$ & 23.177 & 32.084 & 25.682 & 35.376 & 5.633 & 15.501 & 2.117 \\
\hline $12 / 15 / 09$ & $0: 00$ & 23.232 & 32.143 & 25.775 & 35.425 & 5.649 & 15.556 & 2.113 \\
\hline $12 / 15 / 09$ & $4: 00$ & 23.291 & 32.188 & 25.845 & 35.461 & 5.684 & 15.595 & 2.137 \\
\hline $12 / 15 / 09$ & $8: 00$ & 23.349 & 32.227 & 25.889 & 35.540 & 5.676 & 15.627 & 2.142 \\
\hline $12 / 15 / 09$ & $12: 00$ & 23.433 & 32.269 & 25.947 & 35.611 & 5.678 & 15.662 & 2.153 \\
\hline $12 / 15 / 09$ & $16: 00$ & 23.532 & 32.263 & 25.901 & 35.677 & 5.637 & 15.629 & 2.217 \\
\hline $12 / 15 / 09$ & $20: 00$ & 23.542 & 32.284 & 25.904 & 35.712 & 5.653 & 15.636 & 2.239 \\
\hline $12 / 16 / 09$ & $0: 00$ & 23.592 & 32.293 & 25.884 & 35.754 & 5.606 & 15.623 & 2.270 \\
\hline $12 / 16 / 09$ & $4: 00$ & 23.631 & 32.286 & 25.833 & 35.781 & 5.630 & 15.594 & 2.338 \\
\hline $12 / 16 / 09$ & $8: 00$ & 23.679 & 32.278 & 25.777 & 35.787 & 5.649 & 15.564 & 2.313 \\
\hline $12 / 16 / 09$ & $12: 00$ & 23.696 & 32.297 & 25.777 & 35.791 & 5.657 & 15.571 & 2.318 \\
\hline $12 / 16 / 09$ & $16: 00$ & 23.738 & 32.284 & 25.716 & 35.797 & 5.630 & 15.529 & 2.326 \\
\hline $12 / 16 / 09$ & $20: 00$ & 23.674 & 32.316 & 25.740 & 35.778 & 5.612 & 15.558 & 2.321 \\
\hline $12 / 17 / 09$ & $0: 00$ & 23.693 & 32.316 & 25.706 & 35.785 & 5.645 & 15.536 & 2.343 \\
\hline $12 / 17 / 09$ & $4: 00$ & 23.686 & 32.322 & 25.691 & 35.768 & 5.632 & 15.532 & 2.363 \\
\hline 12/17/09 & $8: 00$ & 23.669 & 32.339 & 25.689 & 35.741 & 5.622 & 15.542 & 2.349 \\
\hline $12 / 17 / 09$ & $12: 00$ & 23.676 & 32.362 & 25.713 & 35.699 & 5.647 & 15.558 & 2.353 \\
\hline $12 / 17 / 09$ & $16: 00$ & 23.698 & 32.364 & 25.691 & 35.722 & 5.620 & 15.547 & 2.346 \\
\hline 12/17/09 & $20: 00$ & 23.641 & 32.406 & 25.753 & 35.704 & 5.603 & 15.595 & 2.303 \\
\hline 12/18/09 & $0: 00$ & 23.639 & 32.425 & 25.772 & 35.703 & 5.583 & 15.608 & 2.313 \\
\hline $12 / 18 / 09$ & $4: 00$ & 23.654 & 32.435 & 25.777 & 35.706 & 5.620 & 15.612 & 2.342 \\
\hline $12 / 18 / 09$ & $8: 00$ & 23.651 & 32.463 & 25.811 & 35.705 & 5.613 & 15.636 & 2.340 \\
\hline $12 / 18 / 09$ & $12: 00$ & 23.691 & 32.473 & 25.821 & 35.711 & 5.644 & 15.640 & 2.365 \\
\hline 12/18/09 & $16: 00$ & 23.713 & 32.484 & 25.816 & 35.706 & 5.630 & 15.638 & 2.376 \\
\hline $12 / 18 / 09$ & $20: 00$ & 23.681 & 32.515 & 25.865 & 35.689 & 5.616 & 15.673 & 2.347 \\
\hline $12 / 19 / 09$ & $0: 00$ & 23.654 & 32.555 & 25.926 & 35.718 & 5.632 & 15.701 & 2.319 \\
\hline $12 / 19 / 09$ & $4: 00$ & 23.689 & 32.578 & 25.962 & 35.742 & 5.632 & 15.729 & 2.320 \\
\hline 12/19/09 & $8: 00$ & 23.664 & 32.624 & 26.038 & 35.744 & 5.628 & 15.783 & 2.319 \\
\hline $12 / 19 / 09$ & $12: 00$ & 23.689 & 32.660 & 26.094 & 35.739 & 5.646 & 15.815 & 2.330 \\
\hline 12/19/09 & $16: 00$ & 23.758 & 32.660 & 26.075 & 35.798 & 5.597 & 15.796 & 2.372 \\
\hline $12 / 19 / 09$ & $20: 00$ & 23.768 & 32.685 & 26.106 & 35.819 & 5.632 & 15.819 & 2.365 \\
\hline
\end{tabular}


TABLE S2.1 (Cont.)

\begin{tabular}{|c|c|c|c|c|c|c|c|c|}
\hline \multirow[b]{2}{*}{ Date } & \multirow[b]{2}{*}{ Time } & \multicolumn{6}{|c|}{ Depth to Water (ft TOC) } & \multirow[b]{2}{*}{ MW8s } \\
\hline & & MW1s & MW2s & MW3s & MW4s & MW6s & MW7s & \\
\hline $12 / 20 / 09$ & $0: 00$ & 23.820 & 32.683 & 26.075 & 35.813 & 5.629 & 15.804 & 2.393 \\
\hline $12 / 20 / 09$ & $4: 00$ & 23.833 & 32.691 & 26.067 & 35.849 & 5.619 & 15.802 & 2.393 \\
\hline $12 / 20 / 09$ & 8:00 & 23.868 & 32.681 & 26.020 & 35.863 & 5.615 & 15.765 & 2.418 \\
\hline $12 / 20 / 09$ & $12: 00$ & 23.848 & 32.717 & 26.067 & 35.851 & 5.582 & 15.807 & 2.400 \\
\hline $12 / 20 / 09$ & $16: 00$ & 23.863 & 32.740 & 26.089 & 35.854 & 5.642 & 15.824 & 2.398 \\
\hline $12 / 20 / 09$ & $20: 00$ & 23.808 & 32.782 & 26.172 & 35.876 & 5.625 & 15.867 & 2.378 \\
\hline $12 / 21 / 09$ & $0: 00$ & 23.806 & 32.807 & 26.211 & 35.895 & 5.609 & 15.882 & 2.377 \\
\hline $12 / 21 / 09$ & $4: 00$ & 23.815 & 32.817 & 26.220 & 35.919 & 5.615 & 15.882 & 2.389 \\
\hline $12 / 21 / 09$ & 8:00 & 23.810 & 32.817 & 26.203 & 35.932 & 5.576 & 15.861 & 2.420 \\
\hline $12 / 21 / 09$ & $12: 00$ & 23.875 & 32.817 & 26.191 & 35.923 & 5.636 & 15.858 & 2.426 \\
\hline $12 / 21 / 09$ & $16: 00$ & 23.895 & 32.811 & 26.152 & 35.917 & 5.629 & 15.826 & 2.454 \\
\hline $12 / 21 / 09$ & $20: 00$ & 23.856 & 32.799 & 26.113 & 35.916 & 5.609 & 15.772 & 2.447 \\
\hline $12 / 22 / 09$ & $0: 00$ & 23.786 & 32.809 & 26.133 & 35.946 & 5.594 & 15.765 & 2.448 \\
\hline $12 / 22 / 09$ & $4: 00$ & 23.752 & 32.814 & 26.128 & 35.938 & 5.613 & 15.752 & 2.427 \\
\hline $12 / 22 / 09$ & $8: 00$ & 23.710 & 32.841 & 26.174 & 35.930 & 5.582 & 15.781 & 2.378 \\
\hline $12 / 22 / 09$ & $12: 00$ & 23.697 & 32.875 & 26.233 & 35.904 & 5.609 & 15.819 & 2.389 \\
\hline $12 / 22 / 09$ & $16: 00$ & 23.722 & 32.877 & 26.228 & 35.948 & 5.643 & 15.815 & 2.429 \\
\hline $12 / 22 / 09$ & $20: 00$ & 23.697 & 32.908 & 26.281 & 35.942 & 5.619 & 15.854 & 2.406 \\
\hline $12 / 23 / 09$ & $0: 00$ & 23.729 & 32.912 & 26.284 & 35.971 & 5.615 & 15.856 & 2.425 \\
\hline $12 / 23 / 09$ & $4: 00$ & 23.774 & 32.897 & 26.243 & 35.955 & 5.615 & 15.826 & 2.493 \\
\hline $12 / 23 / 09$ & $8: 00$ & 23.762 & 32.891 & 26.225 & 35.947 & 5.601 & 15.785 & 2.425 \\
\hline $12 / 23 / 09$ & $12: 00$ & 23.690 & 32.874 & 26.211 & 35.951 & 5.466 & 15.609 & 2.316 \\
\hline $12 / 23 / 09$ & $16: 00$ & 23.616 & 32.818 & 26.125 & 35.981 & 5.443 & 15.439 & 2.240 \\
\hline $12 / 23 / 09$ & $20: 00$ & 23.415 & 32.815 & 26.150 & 35.952 & 5.410 & 15.295 & 2.124 \\
\hline $12 / 24 / 09$ & $0: 00$ & 23.259 & 32.801 & 26.150 & 35.928 & 5.429 & 15.174 & 2.055 \\
\hline $12 / 24 / 09$ & $4: 00$ & 23.130 & 32.780 & 26.145 & 35.966 & 5.427 & 15.089 & 2.040 \\
\hline $12 / 24 / 09$ & $8: 00$ & 23.051 & 32.773 & 26.160 & 35.971 & 5.547 & 15.064 & 1.995 \\
\hline $12 / 24 / 09$ & $12: 00$ & 22.989 & 32.749 & 26.135 & 35.984 & 5.522 & 15.051 & 1.974 \\
\hline $12 / 24 / 09$ & $16: 00$ & 22.984 & 32.690 & 26.028 & 36.008 & 5.563 & 15.024 & 1.983 \\
\hline $12 / 24 / 09$ & $20: 00$ & 22.917 & 32.669 & 25.982 & 35.983 & 5.575 & 15.053 & 1.979 \\
\hline $12 / 25 / 09$ & $0: 00$ & 22.952 & 32.579 & 25.804 & 36.002 & 5.503 & 14.970 & 1.996 \\
\hline $12 / 25 / 09$ & $4: 00$ & 22.883 & 32.545 & 25.728 & 35.925 & 5.534 & 14.970 & 1.986 \\
\hline $12 / 25 / 09$ & $8: 00$ & 22.821 & 32.522 & 25.696 & 35.893 & 5.520 & 14.984 & 1.988 \\
\hline $12 / 25 / 09$ & $12: 00$ & 22.791 & 32.503 & 25.670 & 35.899 & 5.524 & 15.003 & 2.005 \\
\hline $12 / 25 / 09$ & $16: 00$ & 22.769 & 32.484 & 25.633 & 35.838 & 5.476 & 15.012 & 1.965 \\
\hline $12 / 25 / 09$ & $20: 00$ & 22.719 & 32.490 & 25.657 & 35.848 & 5.489 & 15.066 & 1.933 \\
\hline $12 / 26 / 09$ & $0: 00$ & 22.702 & 32.499 & 25.684 & 35.821 & 5.526 & 15.120 & 1.897 \\
\hline $12 / 26 / 09$ & $4: 00$ & 22.692 & 32.520 & 25.738 & 35.787 & 5.586 & 15.185 & 1.895 \\
\hline $12 / 26 / 09$ & $8: 00$ & 22.707 & 32.545 & 25.801 & 35.823 & 5.559 & 15.258 & 1.886 \\
\hline $12 / 26 / 09$ & $12: 00$ & 22.714 & 32.576 & 25.877 & 35.842 & 5.542 & 15.336 & 1.893 \\
\hline $12 / 26 / 09$ & $16: 00$ & 22.741 & 32.603 & 25.938 & 35.857 & 5.554 & 15.404 & 1.917 \\
\hline $12 / 26 / 09$ & $20: 00$ & 22.783 & 32.618 & 25.977 & 35.909 & 5.569 & 15.453 & 1.887 \\
\hline $12 / 27 / 09$ & $0: 00$ & 22.855 & 32.612 & 25.967 & 35.953 & 5.633 & 15.473 & 1.911 \\
\hline $12 / 27 / 09$ & $4: 00$ & 22.910 & 32.605 & 25.948 & 35.982 & 5.579 & 15.490 & 1.911 \\
\hline $12 / 27 / 09$ & $8: 00$ & 22.892 & 32.643 & 26.018 & 35.990 & 5.560 & 15.560 & 1.907 \\
\hline $12 / 27 / 09$ & $12: 00$ & 22.910 & 32.689 & 26.111 & 36.031 & 5.622 & 15.640 & 1.905 \\
\hline $12 / 27 / 09$ & $16: 00$ & 22.999 & 32.685 & 26.101 & 36.088 & 5.604 & 15.650 & 1.898 \\
\hline $12 / 27 / 09$ & $20: 00$ & 23.036 & 32.700 & 26.125 & 36.134 & 5.649 & 15.687 & 1.931 \\
\hline
\end{tabular}


TABLE S2.1 (Cont.)

\begin{tabular}{|c|c|c|c|c|c|c|c|c|}
\hline \multirow[b]{2}{*}{ Date } & \multirow[b]{2}{*}{ Time } & \multicolumn{6}{|c|}{ Depth to Water (ft TOC) } & \multirow[b]{2}{*}{ MW8s } \\
\hline & & MW1s & MW2s & MW3s & MW4s & MW6s & MW7s & \\
\hline $12 / 28 / 09$ & $0: 00$ & 23.078 & 32.706 & 26.130 & 36.146 & 5.653 & 15.705 & 1.924 \\
\hline $12 / 28 / 09$ & $4: 00$ & 23.128 & 32.702 & 26.118 & 36.218 & 5.643 & 15.715 & 1.949 \\
\hline $12 / 28 / 09$ & $8: 00$ & 23.148 & 32.706 & 26.113 & 36.242 & 5.647 & 15.731 & 1.943 \\
\hline $12 / 28 / 09$ & $12: 00$ & 23.158 & 32.740 & 26.179 & 36.267 & 5.629 & 15.789 & 1.981 \\
\hline $12 / 28 / 09$ & $16: 00$ & 23.190 & 32.750 & 26.196 & 36.278 & 5.643 & 15.806 & 1.976 \\
\hline $12 / 28 / 09$ & $20: 00$ & 23.178 & 32.784 & 26.262 & 36.328 & 5.666 & 15.858 & 2.002 \\
\hline $12 / 29 / 09$ & $0: 00$ & 23.245 & 32.773 & 26.245 & 36.362 & 5.655 & 15.852 & 2.031 \\
\hline $12 / 29 / 09$ & $4: 00$ & 23.267 & 32.778 & 26.240 & 36.419 & 5.641 & 15.867 & 2.029 \\
\hline $12 / 29 / 09$ & 8:00 & 23.341 & 32.759 & 26.194 & 36.449 & 5.655 & 15.845 & 2.074 \\
\hline $12 / 29 / 09$ & $12: 00$ & 23.386 & 32.752 & 26.170 & 36.471 & 5.609 & 15.839 & 2.098 \\
\hline $12 / 29 / 09$ & $16: 00$ & 23.460 & 32.696 & 26.042 & 36.491 & 5.653 & 15.776 & 2.117 \\
\hline $12 / 29 / 09$ & $20: 00$ & 23.455 & 32.677 & 25.981 & 36.479 & 5.609 & 15.746 & 2.123 \\
\hline $12 / 30 / 09$ & $0: 00$ & 23.456 & 32.660 & 25.935 & 36.460 & 5.653 & 15.728 & 2.129 \\
\hline $12 / 30 / 09$ & $4: 00$ & 23.460 & 32.631 & 25.865 & 36.433 & 5.609 & 15.696 & 2.114 \\
\hline $12 / 30 / 09$ & $8: 00$ & 23.445 & 32.610 & 25.808 & 36.383 & 5.581 & 15.670 & 2.119 \\
\hline $12 / 30 / 09$ & $12: 00$ & 23.431 & 32.608 & 25.801 & 36.351 & 5.610 & 15.674 & 2.144 \\
\hline $12 / 30 / 09$ & $16: 00$ & 23.425 & 32.593 & 25.765 & 36.348 & 5.585 & 15.659 & 2.138 \\
\hline $12 / 30 / 09$ & $20: 00$ & 23.331 & 32.629 & 25.830 & 36.271 & 5.607 & 15.709 & 2.086 \\
\hline $12 / 31 / 09$ & $0: 00$ & 23.296 & 32.683 & 25.943 & 36.248 & 5.609 & 15.798 & 2.066 \\
\hline $12 / 31 / 09$ & $4: 00$ & 23.269 & 32.731 & 26.048 & 36.279 & 5.616 & 15.867 & 2.066 \\
\hline $12 / 31 / 09$ & $8: 00$ & 23.301 & 32.759 & 26.104 & 36.260 & 5.640 & 15.912 & 2.101 \\
\hline $12 / 31 / 09$ & $12: 00$ & 23.321 & 32.826 & 26.248 & 36.287 & 5.576 & 15.999 & 2.098 \\
\hline 12/31/09 & $16: 00$ & 23.410 & 32.828 & 26.255 & 36.358 & 5.638 & 16.005 & 2.102 \\
\hline $12 / 31 / 09$ & $20: 00$ & 23.410 & 32.847 & 26.289 & 36.392 & 5.636 & 16.023 & 2.114 \\
\hline $1 / 1 / 10$ & $0: 00$ & 23.472 & 32.868 & 26.326 & 36.422 & 5.605 & 16.051 & 2.127 \\
\hline $1 / 1 / 10$ & $4: 00$ & 23.512 & 32.876 & 26.335 & 36.421 & 5.636 & 16.068 & 2.122 \\
\hline $1 / 1 / 10$ & $8: 00$ & 23.554 & 32.887 & 26.350 & 36.475 & 5.663 & 16.077 & 2.142 \\
\hline $1 / 1 / 10$ & $12: 00$ & 23.567 & 32.920 & 26.406 & 36.488 & 5.667 & 16.122 & 2.141 \\
\hline $1 / 1 / 10$ & $16: 00$ & 23.626 & 32.918 & 26.389 & 36.504 & 5.661 & 16.111 & 2.170 \\
\hline $1 / 1 / 10$ & $20: 00$ & 23.624 & 32.935 & 26.421 & 36.545 & 5.657 & 16.127 & 2.150 \\
\hline $1 / 2 / 10$ & 0:00 & 23.631 & 32.964 & 26.467 & 36.576 & 5.647 & 16.164 & 2.096 \\
\hline $1 / 2 / 10$ & $4: 00$ & 23.666 & 32.967 & 26.460 & 36.613 & 5.680 & 16.164 & 2.094 \\
\hline $1 / 2 / 10$ & 8:00 & 23.723 & 32.950 & 26.418 & 36.635 & 5.663 & 16.146 & 2.133 \\
\hline $1 / 2 / 10$ & $12: 00$ & 23.763 & 32.958 & 26.411 & 36.655 & 5.669 & 16.142 & 2.153 \\
\hline $1 / 2 / 10$ & $16: 00$ & 23.820 & 32.925 & 26.323 & 36.634 & 5.659 & 16.094 & 2.274 \\
\hline $1 / 2 / 10$ & $20: 00$ & 23.778 & 32.943 & 26.340 & 36.640 & 5.654 & 16.118 & 2.283 \\
\hline $1 / 3 / 10$ & $0: 00$ & 23.768 & 32.959 & 26.357 & 36.609 & 5.642 & 16.133 & 2.285 \\
\hline $1 / 3 / 10$ & $4: 00$ & 23.788 & 32.959 & 26.347 & 36.652 & 5.611 & 16.135 & 2.319 \\
\hline $1 / 3 / 10$ & $8: 00$ & 23.768 & 32.982 & 26.382 & 36.631 & 5.626 & 16.163 & 2.305 \\
\hline $1 / 3 / 10$ & $12: 00$ & 23.758 & 33.013 & 26.445 & 36.612 & 5.611 & 16.203 & 2.264 \\
\hline $1 / 3 / 10$ & $16: 00$ & 23.807 & 33.013 & 26.433 & 36.640 & 5.644 & 16.198 & 2.295 \\
\hline $1 / 3 / 10$ & $20: 00$ & 23.768 & 33.040 & 26.484 & 36.643 & 5.644 & 16.237 & 2.260 \\
\hline $1 / 4 / 10$ & $0: 00$ & 23.800 & 33.057 & 26.508 & 36.663 & 5.652 & 16.248 & 2.247 \\
\hline $1 / 4 / 10$ & $4: 00$ & 23.830 & 33.067 & 26.518 & 36.671 & 5.648 & 16.259 & 2.254 \\
\hline $1 / 4 / 10$ & 8:00 & 23.830 & 33.082 & 26.538 & 36.650 & 5.623 & 16.278 & 2.243 \\
\hline $1 / 4 / 10$ & $12: 00$ & 23.874 & 33.099 & 26.564 & 36.665 & 5.659 & 16.298 & 2.227 \\
\hline $1 / 4 / 10$ & $16: 00$ & 23.944 & 33.076 & 26.506 & 36.713 & 5.646 & 16.259 & 2.310 \\
\hline $1 / 4 / 10$ & $20: 00$ & 23.922 & 33.086 & 26.508 & 36.714 & 5.634 & 16.271 & 2.334 \\
\hline
\end{tabular}


TABLE S2.1 (Cont.)

\begin{tabular}{|c|c|c|c|c|c|c|c|c|}
\hline \multirow[b]{2}{*}{ Date } & \multirow[b]{2}{*}{ Time } & \multicolumn{6}{|c|}{ Depth to Water (ft TOC) } & \multirow[b]{2}{*}{ MW8s } \\
\hline & & MW1s & MW2s & MW3s & MW4s & MW6s & MW7s & \\
\hline $1 / 5 / 10$ & $0: 00$ & 23.934 & 33.095 & 26.516 & 36.721 & 5.601 & 16.280 & 2.352 \\
\hline $1 / 5 / 10$ & $4: 00$ & 23.934 & 33.109 & 26.528 & 36.715 & 5.642 & 16.293 & 2.361 \\
\hline $1 / 5 / 10$ & $8: 00$ & 23.919 & 33.120 & 26.542 & 36.689 & 5.642 & 16.307 & 2.366 \\
\hline $1 / 5 / 10$ & $12: 00$ & 23.971 & 33.126 & 26.547 & 36.698 & 5.636 & 16.311 & 2.377 \\
\hline $1 / 5 / 10$ & $16: 00$ & 24.031 & 33.101 & 26.489 & 36.734 & 5.580 & 16.270 & 2.464 \\
\hline $1 / 5 / 10$ & $20: 00$ & 23.984 & 33.106 & 26.476 & 36.689 & 5.665 & 16.272 & 2.440 \\
\hline $1 / 6 / 10$ & $0: 00$ & 24.001 & 33.109 & 26.462 & 36.710 & 5.661 & 16.268 & 2.452 \\
\hline $1 / 6 / 10$ & $4: 00$ & 24.011 & 33.097 & 26.423 & 36.697 & 5.642 & 16.245 & 2.441 \\
\hline $1 / 6 / 10$ & $8: 00$ & 24.023 & 33.095 & 26.394 & 36.680 & 5.644 & 16.241 & 2.449 \\
\hline $1 / 6 / 10$ & $12: 00$ & 24.004 & 33.108 & 26.406 & 36.661 & 5.665 & 16.254 & 2.459 \\
\hline $1 / 6 / 10$ & $16: 00$ & 24.023 & 33.099 & 26.379 & 36.645 & 5.673 & 16.239 & 2.438 \\
\hline $1 / 6 / 10$ & $20: 00$ & 23.954 & 33.156 & 26.479 & 36.616 & 5.659 & 16.311 & 2.391 \\
\hline $1 / 7 / 10$ & $0: 00$ & 23.929 & 33.194 & 26.560 & 36.610 & 5.674 & 16.363 & 2.383 \\
\hline $1 / 7 / 10$ & $4: 00$ & 23.961 & 33.192 & 26.547 & 36.638 & 5.672 & 16.352 & 2.415 \\
\hline $1 / 7 / 10$ & $8: 00$ & 23.981 & 33.221 & 26.596 & 36.593 & 5.663 & 16.389 & 2.388 \\
\hline $1 / 7 / 10$ & $12: 00$ & 23.966 & 33.278 & 26.706 & 36.631 & 5.689 & 16.456 & 2.414 \\
\hline $1 / 7 / 10$ & $16: 00$ & 24.018 & 33.286 & 26.718 & 36.664 & 5.707 & 16.466 & 2.413 \\
\hline $1 / 7 / 10$ & $20: 00$ & 24.021 & 33.315 & 26.774 & 36.681 & 5.675 & 16.497 & 2.410 \\
\hline $1 / 8 / 10$ & 0:00 & 24.038 & 33.344 & 26.828 & 36.708 & 5.699 & 16.529 & 2.413 \\
\hline $1 / 8 / 10$ & $4: 00$ & 24.068 & 33.362 & 26.852 & 36.735 & 5.708 & 16.549 & 2.404 \\
\hline $1 / 8 / 10$ & $8: 00$ & 24.120 & 33.370 & 26.859 & 36.744 & 5.697 & 16.553 & 2.403 \\
\hline $1 / 8 / 10$ & $12: 00$ & 24.135 & 33.402 & 26.910 & 36.796 & 5.707 & 16.588 & 2.415 \\
\hline $1 / 8 / 10$ & $16: 00$ & 24.222 & 33.385 & 26.859 & 36.802 & 5.701 & 16.557 & 2.482 \\
\hline $1 / 8 / 10$ & $20: 00$ & 24.212 & 33.407 & 26.886 & 36.846 & 5.720 & 16.586 & 2.477 \\
\hline $1 / 9 / 10$ & 0:00 & 24.219 & 33.423 & 26.903 & 36.861 & 5.699 & 16.601 & 2.470 \\
\hline $1 / 9 / 10$ & $4: 00$ & 24.234 & 33.435 & 26.920 & 36.851 & 5.703 & 16.613 & 2.467 \\
\hline $1 / 9 / 10$ & $8: 00$ & 24.244 & 33.452 & 26.938 & 36.897 & 5.693 & 16.624 & 2.464 \\
\hline $1 / 9 / 10$ & $12: 00$ & 24.249 & 33.488 & 26.999 & 36.907 & 5.699 & 16.665 & 2.453 \\
\hline $1 / 9 / 10$ & $16: 00$ & 24.329 & 33.475 & 26.957 & 36.916 & 5.697 & 16.641 & 2.512 \\
\hline $1 / 9 / 10$ & $20: 00$ & 24.299 & 33.488 & 26.962 & 36.947 & 5.681 & 16.655 & 2.529 \\
\hline $1 / 10 / 10$ & $0: 00$ & 24.329 & 33.486 & 26.947 & 36.935 & 5.695 & 16.648 & 2.563 \\
\hline $1 / 10 / 10$ & $4: 00$ & 24.358 & 33.481 & 26.923 & 36.976 & 5.703 & 16.633 & 2.581 \\
\hline $1 / 10 / 10$ & $8: 00$ & 24.398 & 33.456 & 26.856 & 36.984 & 5.672 & 16.600 & 2.597 \\
\hline $1 / 10 / 10$ & $12: 00$ & 24.438 & 33.439 & 26.811 & 36.954 & 5.584 & 16.574 & 2.603 \\
\hline $1 / 10 / 10$ & $16: 00$ & 24.443 & 33.416 & 26.740 & 36.951 & 5.602 & 16.536 & 2.579 \\
\hline $1 / 10 / 10$ & $20: 00$ & 24.391 & 33.441 & 26.772 & 36.918 & 5.654 & 16.566 & 2.584 \\
\hline $1 / 11 / 10$ & $0: 00$ & 24.376 & 33.452 & 26.779 & 36.904 & 5.639 & 16.575 & 2.616 \\
\hline $1 / 11 / 10$ & $4: 00$ & 24.351 & 33.475 & 26.806 & 36.882 & 5.670 & 16.603 & 2.595 \\
\hline $1 / 11 / 10$ & $8: 00$ & 24.304 & 33.521 & 26.889 & 36.835 & 5.648 & 16.659 & 2.551 \\
\hline $1 / 11 / 10$ & $12: 00$ & 24.281 & 33.580 & 27.006 & 36.871 & 5.615 & 16.732 & 2.555 \\
\hline $1 / 11 / 10$ & $16: 00$ & 24.326 & 33.590 & 27.027 & 36.905 & 5.631 & 16.735 & 2.573 \\
\hline $1 / 11 / 10$ & $20: 00$ & 24.321 & 33.628 & 27.096 & 36.918 & 5.675 & 16.776 & 2.577 \\
\hline $1 / 12 / 10$ & $0: 00$ & 24.361 & 33.647 & 27.127 & 36.929 & 5.656 & 16.789 & 2.569 \\
\hline $1 / 12 / 10$ & $4: 00$ & 24.381 & 33.664 & 27.144 & 36.938 & 5.681 & 16.810 & 2.601 \\
\hline $1 / 12 / 10$ & $8: 00$ & 24.438 & 33.660 & 27.127 & 36.967 & 5.695 & 16.804 & 2.630 \\
\hline $1 / 12 / 10$ & $12: 00$ & 24.497 & 33.653 & 27.103 & 37.031 & 5.697 & 16.787 & 2.656 \\
\hline $1 / 12 / 10$ & $16: 00$ & 24.579 & 33.620 & 27.020 & 37.008 & 5.664 & 16.737 & 2.670 \\
\hline $1 / 12 / 10$ & $20: 00$ & 24.574 & 33.607 & 26.974 & 36.995 & 5.697 & 16.717 & 2.674 \\
\hline
\end{tabular}


TABLE S2.1 (Cont.)

\begin{tabular}{|c|c|c|c|c|c|c|c|c|}
\hline \multirow[b]{2}{*}{ Date } & \multirow[b]{2}{*}{ Time } & \multicolumn{6}{|c|}{ Depth to Water (ft TOC) } & \multirow[b]{2}{*}{ MW8s } \\
\hline & & MW1s & MW2s & MW3s & MW4s & MW6s & MW7s & \\
\hline $1 / 13 / 10$ & $0: 00$ & 24.577 & 33.597 & 26.930 & 37.019 & 5.670 & 16.702 & 2.684 \\
\hline $1 / 13 / 10$ & $4: 00$ & 24.570 & 33.588 & 26.896 & 37.002 & 5.650 & 16.687 & 2.722 \\
\hline $1 / 13 / 10$ & 8:00 & 24.545 & 33.588 & 26.876 & 36.975 & 5.670 & 16.681 & 2.711 \\
\hline $1 / 13 / 10$ & $12: 00$ & 24.542 & 33.601 & 26.886 & 36.957 & 5.641 & 16.693 & 2.720 \\
\hline $1 / 13 / 10$ & $16: 00$ & 24.585 & 33.561 & 26.796 & 36.959 & 5.557 & 16.642 & 2.708 \\
\hline $1 / 13 / 10$ & $20: 00$ & 24.510 & 33.595 & 26.854 & 36.910 & 5.526 & 16.676 & 2.660 \\
\hline $1 / 14 / 10$ & $0: 00$ & 24.487 & 33.611 & 26.881 & 36.898 & 5.631 & 16.689 & 2.661 \\
\hline $1 / 14 / 10$ & $4: 00$ & 24.468 & 33.622 & 26.901 & 36.890 & 5.554 & 16.704 & 2.664 \\
\hline $1 / 14 / 10$ & 8:00 & 24.428 & 33.662 & 26.976 & 36.867 & 5.592 & 16.756 & 2.637 \\
\hline $1 / 14 / 10$ & $12: 00$ & 24.403 & 33.714 & 27.084 & 36.836 & 5.589 & 16.815 & 2.637 \\
\hline $1 / 14 / 10$ & $16: 00$ & 24.453 & 33.725 & 27.103 & 36.885 & 5.557 & 16.821 & 2.632 \\
\hline $1 / 14 / 10$ & $20: 00$ & 24.415 & 33.758 & 27.183 & 36.873 & 5.594 & 16.826 & 2.627 \\
\hline $1 / 15 / 10$ & $0: 00$ & 24.415 & 33.779 & 27.233 & 36.935 & 5.608 & 16.852 & 2.641 \\
\hline $1 / 15 / 10$ & $4: 00$ & 24.413 & 33.802 & 27.284 & 36.960 & 5.633 & 16.882 & 2.634 \\
\hline $1 / 15 / 10$ & 8:00 & 24.456 & 33.798 & 27.279 & 37.009 & 5.629 & 16.873 & 2.652 \\
\hline $1 / 15 / 10$ & $12: 00$ & 24.433 & 33.844 & 27.364 & 37.033 & 5.604 & 16.916 & 2.642 \\
\hline $1 / 15 / 10$ & $16: 00$ & 24.495 & 33.819 & 27.311 & 37.059 & 5.559 & 16.871 & 2.680 \\
\hline $1 / 15 / 10$ & $20: 00$ & 24.500 & 33.821 & 27.305 & 37.049 & 5.614 & 16.875 & 2.686 \\
\hline $1 / 16 / 10$ & $0: 00$ & 24.511 & 33.821 & 27.298 & 37.068 & 5.585 & 16.875 & 2.697 \\
\hline $1 / 16 / 10$ & $4: 00$ & 24.511 & 33.823 & 27.291 & 37.103 & 5.656 & 16.875 & 2.686 \\
\hline $1 / 16 / 10$ & 8:00 & 24.530 & 33.804 & 27.249 & 37.096 & 5.608 & 16.851 & 2.712 \\
\hline $1 / 16 / 10$ & $12: 00$ & 24.515 & 33.832 & 27.286 & 37.125 & 5.614 & 16.880 & 2.694 \\
\hline $1 / 16 / 10$ & $16: 00$ & 24.575 & 33.779 & 27.181 & 37.111 & 5.627 & 16.823 & 2.723 \\
\hline $1 / 16 / 10$ & $20: 00$ & 24.528 & 33.792 & 27.188 & 37.091 & 5.627 & 16.830 & 2.704 \\
\hline $1 / 17 / 10$ & 0:00 & 24.523 & 33.779 & 27.152 & 37.111 & 5.600 & 16.808 & 2.712 \\
\hline $1 / 17 / 10$ & $4: 00$ & 24.503 & 33.784 & 27.157 & 37.072 & 5.589 & 16.814 & 2.725 \\
\hline $1 / 17 / 10$ & 8:00 & 24.498 & 33.775 & 27.135 & 37.092 & 5.649 & 16.802 & 2.751 \\
\hline $1 / 17 / 10$ & $12: 00$ & 24.463 & 33.803 & 27.174 & 37.042 & 5.577 & 16.834 & 2.733 \\
\hline $1 / 17 / 10$ & $16: 00$ & 24.473 & 33.792 & 27.150 & 37.061 & 5.608 & 16.804 & 2.762 \\
\hline $1 / 17 / 10$ & $20: 00$ & 24.382 & 33.834 & 27.220 & 37.043 & 5.590 & 16.836 & 2.695 \\
\hline $1 / 18 / 10$ & 0:00 & 24.357 & 33.849 & 27.252 & 37.031 & 5.589 & 16.845 & 2.682 \\
\hline $1 / 18 / 10$ & $4: 00$ & 24.342 & 33.853 & 27.262 & 37.071 & 5.602 & 16.834 & 2.682 \\
\hline $1 / 18 / 10$ & 8:00 & 24.308 & 33.866 & 27.281 & 37.069 & 5.563 & 16.827 & 2.671 \\
\hline $1 / 18 / 10$ & $12: 00$ & 24.290 & 33.878 & 27.308 & 37.089 & 5.639 & 16.830 & 2.664 \\
\hline $1 / 18 / 10$ & $16: 00$ & 24.315 & 33.859 & 27.266 & 37.095 & 5.662 & 16.786 & 2.692 \\
\hline $1 / 18 / 10$ & $20: 00$ & 24.268 & 33.880 & 27.303 & 37.097 & 5.672 & 16.793 & 2.675 \\
\hline $1 / 19 / 10$ & $0: 00$ & 24.254 & 33.891 & 27.320 & 37.110 & 5.721 & 16.795 & 2.672 \\
\hline $1 / 19 / 10$ & $4: 00$ & 24.256 & 33.878 & 27.296 & 37.100 & 5.721 & 16.780 & 2.690 \\
\hline $1 / 19 / 10$ & $8: 00$ & 24.244 & 33.872 & 27.281 & 37.116 & 5.715 & 16.773 & 2.709 \\
\hline $1 / 19 / 10$ & $12: 00$ & 24.224 & 33.874 & 27.276 & 37.113 & 5.693 & 16.773 & 2.720 \\
\hline $1 / 19 / 10$ & $16: 00$ & 24.261 & 33.834 & 27.198 & 37.123 & 5.717 & 16.717 & 2.740 \\
\hline $1 / 19 / 10$ & $20: 00$ & 24.202 & 33.826 & 27.172 & 37.134 & 5.705 & 16.687 & 2.731 \\
\hline $1 / 20 / 10$ & $0: 00$ & 24.147 & 33.798 & 27.118 & 37.115 & 5.684 & 16.622 & 2.714 \\
\hline $1 / 20 / 10$ & $4: 00$ & 23.817 & 33.716 & 27.003 & 37.140 & 5.519 & 16.252 & 2.442 \\
\hline $1 / 20 / 10$ & $8: 00$ & 23.449 & 33.649 & 26.943 & 37.055 & 5.295 & 15.929 & 2.325 \\
\hline $1 / 20 / 10$ & $12: 00$ & 23.168 & 33.599 & 26.928 & 37.014 & 5.350 & 15.704 & 2.186 \\
\hline $1 / 20 / 10$ & $16: 00$ & 22.945 & 33.498 & 26.819 & 37.041 & 5.369 & 15.437 & 2.104 \\
\hline $1 / 20 / 10$ & $20: 00$ & 22.615 & 33.414 & 26.733 & 37.021 & 5.381 & 15.296 & 1.989 \\
\hline
\end{tabular}


TABLE S2.1 (Cont.)

\begin{tabular}{|c|c|c|c|c|c|c|c|c|}
\hline \multirow[b]{2}{*}{ Date } & \multirow[b]{2}{*}{ Time } & \multicolumn{6}{|c|}{ Depth to Water (ft TOC) } & \multirow[b]{2}{*}{ MW8s } \\
\hline & & MW1s & MW2s & MW3s & MW4s & MW6s & MW7s & \\
\hline $1 / 21 / 10$ & $0: 00$ & 22.397 & 33.290 & 26.541 & 36.929 & 5.322 & 15.207 & 1.934 \\
\hline $1 / 21 / 10$ & 4:00 & 22.198 & 33.181 & 26.390 & 36.916 & 5.365 & 15.142 & 1.849 \\
\hline $1 / 21 / 10$ & $8: 00$ & 22.067 & 33.078 & 26.251 & 36.876 & 5.391 & 15.098 & 1.725 \\
\hline $1 / 21 / 10$ & $12: 00$ & 21.942 & 32.980 & 26.107 & 36.891 & 5.488 & 15.053 & 1.663 \\
\hline $1 / 21 / 10$ & $16: 00$ & 21.890 & 32.860 & 25.886 & 36.887 & 5.515 & 15.023 & 1.509 \\
\hline $1 / 21 / 10$ & $20: 00$ & 21.600 & 32.801 & 25.760 & 36.862 & 5.451 & 14.917 & 1.192 \\
\hline $1 / 22 / 10$ & $0: 00$ & 21.385 & 32.700 & 25.551 & 36.834 & 5.445 & 14.531 & 1.054 \\
\hline $1 / 22 / 10$ & $4: 00$ & 21.295 & 32.560 & 25.267 & 36.854 & 5.449 & 14.412 & 1.025 \\
\hline $1 / 22 / 10$ & $8: 00$ & 21.201 & 32.442 & 25.032 & 36.865 & 5.552 & 14.303 & 0.979 \\
\hline $1 / 22 / 10$ & $12: 00$ & 21.109 & 32.322 & 24.799 & 36.855 & 5.484 & 14.286 & 0.952 \\
\hline $1 / 22 / 10$ & $16: 00$ & 21.126 & 32.152 & 24.464 & 36.843 & 5.515 & 14.163 & 0.859 \\
\hline $1 / 22 / 10$ & $20: 00$ & 20.749 & 32.033 & 24.114 & 36.787 & 5.241 & 13.816 & 0.496 \\
\hline $1 / 23 / 10$ & 0:00 & 20.210 & 31.884 & 23.075 & 36.717 & 4.849 & 13.326 & 0.307 \\
\hline $1 / 23 / 10$ & $4: 00$ & 19.656 & 31.680 & 21.664 & 36.670 & 3.775 & 12.720 & 0.133 \\
\hline $1 / 23 / 10$ & $8: 00$ & 19.043 & 31.370 & 18.920 & 36.621 & 1.975 & 11.875 & -0.007 \\
\hline $1 / 23 / 10$ & $12: 00$ & 18.457 & 31.025 & 16.992 & 36.532 & 1.943 & 11.054 & -0.115 \\
\hline $1 / 23 / 10$ & $16: 00$ & 17.966 & 30.549 & 15.825 & 36.420 & 2.129 & 10.393 & -0.251 \\
\hline $1 / 23 / 10$ & $20: 00$ & 17.469 & 30.082 & 15.283 & 36.261 & 2.936 & 9.904 & -0.150 \\
\hline $1 / 24 / 10$ & 0:00 & 17.182 & 29.531 & 15.015 & 36.163 & 3.747 & 9.519 & -0.078 \\
\hline $1 / 24 / 10$ & $4: 00$ & 16.972 & 29.033 & 14.712 & 36.012 & 4.175 & 9.264 & -0.074 \\
\hline $1 / 24 / 10$ & $8: 00$ & 16.762 & 28.624 & 14.491 & 35.848 & 4.497 & 9.096 & -0.073 \\
\hline $1 / 24 / 10$ & $12: 00$ & 16.663 & 28.204 & 14.310 & 35.637 & 4.687 & 8.921 & -0.053 \\
\hline $1 / 24 / 10$ & $16: 00$ & 16.568 & 27.755 & 14.124 & 35.465 & 4.880 & 8.836 & -0.058 \\
\hline $1 / 24 / 10$ & $20: 00$ & 16.447 & 27.241 & 14.013 & 35.251 & 5.128 & 8.815 & -0.017 \\
\hline $1 / 25 / 10$ & 0:00 & 16.378 & 26.684 & 13.948 & 35.016 & 5.287 & 8.776 & 0.035 \\
\hline $1 / 25 / 10$ & $4: 00$ & 16.317 & 26.239 & 13.923 & 34.793 & 5.346 & 8.769 & 0.092 \\
\hline $1 / 25 / 10$ & 8:00 & 16.263 & 25.876 & 13.935 & 34.582 & 5.416 & 8.800 & 0.133 \\
\hline $1 / 25 / 10$ & $12: 00$ & 16.209 & 25.576 & 13.984 & 34.390 & 5.439 & 8.886 & 0.175 \\
\hline $1 / 25 / 10$ & $16: 00$ & 16.235 & 25.255 & 13.994 & 34.218 & 5.470 & 8.930 & 0.242 \\
\hline $1 / 25 / 10$ & 20:00 & 16.255 & 25.003 & 14.045 & 34.058 & 5.515 & 9.008 & 0.266 \\
\hline $1 / 26 / 10$ & 0:00 & 16.248 & 24.784 & 14.091 & 33.882 & 5.490 & 9.086 & 0.275 \\
\hline $1 / 26 / 10$ & $4: 00$ & 16.258 & 24.583 & 14.128 & 33.741 & 5.482 & 9.138 & 0.315 \\
\hline $1 / 26 / 10$ & 8:00 & 16.301 & 24.425 & 14.179 & 33.587 & 5.482 & 9.205 & 0.358 \\
\hline $1 / 26 / 10$ & $12: 00$ & 16.378 & 24.300 & 14.225 & 33.450 & 5.531 & 9.287 & 0.403 \\
\hline $1 / 26 / 10$ & $16: 00$ & 16.502 & 24.096 & 14.203 & 33.291 & 5.509 & 9.259 & 0.423 \\
\hline $1 / 26 / 10$ & $20: 00$ & 16.500 & 23.972 & 14.217 & 33.147 & 5.495 & 9.285 & 0.418 \\
\hline $1 / 27 / 10$ & 0:00 & 16.547 & 23.842 & 14.225 & 32.961 & 5.525 & 9.283 & 0.434 \\
\hline $1 / 27 / 10$ & $4: 00$ & 16.573 & 23.722 & 14.232 & 32.815 & 5.492 & 9.290 & 0.450 \\
\hline $1 / 27 / 10$ & 8:00 & 16.605 & 23.609 & 14.237 & 32.600 & 5.502 & 9.287 & 0.479 \\
\hline $1 / 27 / 10$ & $12: 00$ & 16.607 & 23.557 & 14.273 & 32.442 & 5.517 & 9.333 & 0.503 \\
\hline $1 / 27 / 10$ & $16: 00$ & 16.635 & 23.479 & 14.297 & 32.250 & 5.540 & 9.348 & 0.505 \\
\hline $1 / 27 / 10$ & $20: 00$ & 16.548 & 23.489 & 14.380 & 32.039 & 5.530 & 9.417 & 0.495 \\
\hline $1 / 28 / 10$ & $0: 00$ & 16.605 & 23.452 & 14.424 & 31.911 & 5.528 & 9.452 & 0.529 \\
\hline $1 / 28 / 10$ & $4: 00$ & 16.640 & 23.418 & 14.480 & 31.748 & 5.488 & 9.489 & 0.535 \\
\hline $1 / 28 / 10$ & $8: 00$ & 16.687 & 23.376 & 14.521 & 31.603 & 5.538 & 9.510 & 0.554 \\
\hline $1 / 28 / 10$ & $12: 00$ & 16.762 & 23.353 & 14.562 & 31.468 & 5.569 & 9.545 & 0.599 \\
\hline $1 / 28 / 10$ & $16: 00$ & 16.861 & 23.231 & 14.552 & 31.314 & 5.557 & 9.480 & 0.579 \\
\hline $1 / 28 / 10$ & $20: 00$ & 16.866 & 23.212 & 14.586 & 31.176 & 5.569 & 9.508 & 0.576 \\
\hline
\end{tabular}


TABLE S2.1 (Cont.)

\begin{tabular}{|c|c|c|c|c|c|c|c|c|}
\hline \multirow[b]{2}{*}{ Date } & \multirow[b]{2}{*}{ Time } & \multicolumn{6}{|c|}{ Depth to Water (ft TOC) } & \multirow[b]{2}{*}{ MW8s } \\
\hline & & MW1s & MW2s & MW3s & MW4s & MW6s & MW7s & \\
\hline $1 / 29 / 10$ & $0: 00$ & 16.893 & 23.175 & 14.613 & 31.029 & 5.565 & 9.506 & 0.588 \\
\hline $1 / 29 / 10$ & $4: 00$ & 16.926 & 23.118 & 14.627 & 30.869 & 5.567 & 9.498 & 0.600 \\
\hline $1 / 29 / 10$ & 8:00 & 16.946 & 23.061 & 14.634 & 30.687 & 5.555 & 9.480 & 0.608 \\
\hline $1 / 29 / 10$ & $12: 00$ & 16.968 & 23.044 & 14.658 & 30.508 & 5.536 & 9.491 & 0.633 \\
\hline $1 / 29 / 10$ & $16: 00$ & 17.025 & 22.965 & 14.656 & 30.347 & 5.535 & 9.439 & 0.622 \\
\hline $1 / 29 / 10$ & $20: 00$ & 16.990 & 22.960 & 14.685 & 30.226 & 5.523 & 9.456 & 0.606 \\
\hline $1 / 30 / 10$ & $0: 00$ & 16.995 & 22.950 & 14.714 & 30.090 & 5.502 & 9.465 & 0.614 \\
\hline $1 / 30 / 10$ & $4: 00$ & 16.985 & 22.946 & 14.750 & 29.951 & 5.537 & 9.484 & 0.631 \\
\hline $1 / 30 / 10$ & 8:00 & 16.987 & 22.950 & 14.794 & 29.818 & 5.555 & 9.504 & 0.626 \\
\hline $1 / 30 / 10$ & $12: 00$ & 17.035 & 22.979 & 14.836 & 29.705 & 5.552 & 9.545 & 0.722 \\
\hline $1 / 30 / 10$ & $16: 00$ & 17.106 & 22.929 & 14.850 & 29.616 & 5.497 & 9.506 & 0.669 \\
\hline $1 / 30 / 10$ & $20: 00$ & 17.050 & 22.958 & 14.896 & 29.465 & 5.548 & 9.549 & 0.665 \\
\hline $1 / 31 / 10$ & $0: 00$ & 17.055 & 22.975 & 14.943 & 29.368 & 5.533 & 9.569 & 0.679 \\
\hline $1 / 31 / 10$ & $4: 00$ & 17.087 & 22.984 & 14.974 & 29.293 & 5.503 & 9.582 & 0.686 \\
\hline $1 / 31 / 10$ & 8:00 & 17.104 & 23.005 & 15.018 & 29.230 & 5.517 & 9.604 & 0.697 \\
\hline $1 / 31 / 10$ & $12: 00$ & 17.154 & 23.044 & 15.069 & 29.155 & 5.558 & 9.645 & 0.705 \\
\hline $1 / 31 / 10$ & $16: 00$ & 17.243 & 23.017 & 15.082 & 29.106 & 5.544 & 9.608 & 0.698 \\
\hline $1 / 31 / 10$ & $20: 00$ & 17.216 & 23.030 & 15.111 & 29.038 & 5.556 & 9.586 & 0.702 \\
\hline $2 / 1 / 10$ & $0: 00$ & 17.231 & 23.034 & 15.135 & 28.977 & 5.521 & 9.560 & 0.706 \\
\hline $2 / 1 / 10$ & $4: 00$ & 17.273 & 23.024 & 15.150 & 28.894 & 5.507 & 9.541 & 0.701 \\
\hline $2 / 1 / 10$ & 8:00 & 17.292 & 23.021 & 15.167 & 28.826 & 5.488 & 9.537 & 0.706 \\
\hline $2 / 1 / 10$ & $12: 00$ & 17.302 & 23.040 & 15.198 & 28.756 & 5.563 & 9.554 & 0.711 \\
\hline $2 / 1 / 10$ & $16: 00$ & 17.330 & 23.026 & 15.210 & 28.712 & 5.513 & 9.534 & 0.713 \\
\hline $2 / 1 / 10$ & $20: 00$ & 17.292 & 23.074 & 15.252 & 28.637 & 5.517 & 9.580 & 0.724 \\
\hline $2 / 2 / 10$ & 0:00 & 17.310 & 23.109 & 15.288 & 28.585 & 5.515 & 9.610 & 0.730 \\
\hline $2 / 2 / 10$ & $4: 00$ & 17.302 & 23.149 & 15.327 & 28.540 & 5.540 & 9.632 & 0.710 \\
\hline $2 / 2 / 10$ & 8:00 & 17.307 & 23.217 & 15.381 & 28.460 & 5.554 & 9.703 & 0.730 \\
\hline $2 / 2 / 10$ & $12: 00$ & 17.350 & 23.271 & 15.427 & 28.449 & 5.587 & 9.746 & 0.760 \\
\hline $2 / 2 / 10$ & $16: 00$ & 17.449 & 23.267 & 15.447 & 28.455 & 5.546 & 9.729 & 0.755 \\
\hline $2 / 2 / 10$ & $20: 00$ & 17.431 & 23.316 & 15.493 & 28.430 & 5.583 & 9.761 & 0.764 \\
\hline $2 / 3 / 10$ & 0:00 & 17.446 & 23.353 & 15.532 & 28.387 & 5.570 & 9.785 & 0.766 \\
\hline $2 / 3 / 10$ & $4: 00$ & 17.508 & 23.361 & 15.564 & 28.373 & 5.602 & 9.775 & 0.771 \\
\hline $2 / 3 / 10$ & 8:00 & 17.501 & 23.403 & 15.625 & 28.370 & 5.604 & 9.809 & 0.783 \\
\hline $2 / 3 / 10$ & $12: 00$ & 17.617 & 23.405 & 15.647 & 28.374 & 5.616 & 9.801 & 0.773 \\
\hline $2 / 3 / 10$ & $16: 00$ & 17.674 & 23.420 & 15.690 & 28.354 & 5.625 & 9.800 & 0.771 \\
\hline $2 / 3 / 10$ & $20: 00$ & 17.625 & 23.456 & 15.756 & 28.326 & 5.604 & 9.811 & 0.777 \\
\hline $2 / 4 / 10$ & $0: 00$ & 17.674 & 23.475 & 15.803 & 28.316 & 5.561 & 9.824 & 0.770 \\
\hline $2 / 4 / 10$ & $4: 00$ & 17.682 & 23.510 & 15.854 & 28.292 & 5.558 & 9.842 & 0.779 \\
\hline $2 / 4 / 10$ & $8: 00$ & 17.724 & 23.508 & 15.874 & 28.282 & 5.606 & 9.820 & 0.773 \\
\hline $2 / 4 / 10$ & $12: 00$ & 17.744 & 23.529 & 15.915 & 28.271 & 5.620 & 9.827 & 0.773 \\
\hline $2 / 4 / 10$ & $16: 00$ & 17.799 & 23.500 & 15.944 & 28.256 & 5.620 & 9.777 & 0.761 \\
\hline $2 / 4 / 10$ & $20: 00$ & 17.753 & 23.538 & 16.032 & 28.220 & 5.561 & 9.790 & 0.763 \\
\hline $2 / 5 / 10$ & $0: 00$ & 17.753 & 23.554 & 16.126 & 28.195 & 5.501 & 9.787 & 0.770 \\
\hline $2 / 5 / 10$ & $4: 00$ & 17.769 & 23.565 & 16.193 & 28.147 & 5.488 & 9.779 & 0.771 \\
\hline $2 / 5 / 10$ & $8: 00$ & 17.771 & 23.580 & 16.239 & 28.153 & 5.500 & 9.777 & 0.774 \\
\hline $2 / 5 / 10$ & $12: 00$ & 17.758 & 23.626 & 16.316 & 28.122 & 5.456 & 9.811 & 0.782 \\
\hline $2 / 5 / 10$ & $16: 00$ & 17.771 & 23.655 & 16.387 & 28.106 & 5.433 & 9.828 & 0.785 \\
\hline $2 / 5 / 10$ & $20: 00$ & 17.726 & 23.743 & 16.514 & 28.082 & 5.511 & 9.894 & 0.802 \\
\hline
\end{tabular}


TABLE S2.1 (Cont.)

\begin{tabular}{|c|c|c|c|c|c|c|c|c|}
\hline \multirow[b]{2}{*}{ Date } & \multirow[b]{2}{*}{ Time } & \multicolumn{6}{|c|}{ Depth to Water (ft TOC) } & \multirow[b]{2}{*}{ MW8s } \\
\hline & & MW1s & MW2s & MW3s & MW4s & MW6s & MW7s & \\
\hline $2 / 6 / 10$ & $0: 00$ & 17.746 & 23.800 & 16.604 & 28.070 & 5.542 & 9.926 & 0.808 \\
\hline $2 / 6 / 10$ & $4: 00$ & 17.791 & 23.836 & 16.670 & 28.109 & 5.536 & 9.948 & 0.812 \\
\hline $2 / 6 / 10$ & $8: 00$ & 17.808 & 23.888 & 16.752 & 28.110 & 5.581 & 9.983 & 0.818 \\
\hline $2 / 6 / 10$ & $12: 00$ & 17.858 & 23.928 & 16.821 & 28.105 & 5.589 & 10.015 & 0.820 \\
\hline $2 / 6 / 10$ & $16: 00$ & 17.935 & 23.930 & 16.841 & 28.148 & 5.530 & 9.998 & 0.811 \\
\hline $2 / 6 / 10$ & $20: 00$ & 17.947 & 23.951 & 16.891 & 28.159 & 5.575 & 9.995 & 0.811 \\
\hline $2 / 7 / 10$ & $0: 00$ & 17.974 & 23.964 & 16.925 & 28.142 & 5.550 & 9.986 & 0.808 \\
\hline $2 / 7 / 10$ & $4: 00$ & 17.984 & 23.968 & 16.950 & 28.189 & 5.515 & 9.969 & 0.807 \\
\hline $2 / 7 / 10$ & $8: 00$ & 18.004 & 23.991 & 16.989 & 28.145 & 5.542 & 9.976 & 0.811 \\
\hline $2 / 7 / 10$ & $12: 00$ & 18.041 & 24.010 & 17.023 & 28.181 & 5.557 & 9.997 & 0.810 \\
\hline $2 / 7 / 10$ & $16: 00$ & 18.083 & 23.993 & 17.018 & 28.190 & 5.530 & 9.967 & 0.794 \\
\hline $2 / 7 / 10$ & $20: 00$ & 18.019 & 24.027 & 17.067 & 28.173 & 5.635 & 9.948 & 0.790 \\
\hline $2 / 8 / 10$ & 0:00 & 18.016 & 24.033 & 17.099 & 28.182 & 5.604 & 9.898 & 0.795 \\
\hline $2 / 8 / 10$ & $4: 00$ & 18.002 & 24.054 & 17.143 & 28.171 & 5.585 & 9.887 & 0.801 \\
\hline $2 / 8 / 10$ & $8: 00$ & 17.977 & 24.100 & 17.216 & 28.158 & 5.625 & 9.932 & 0.811 \\
\hline $2 / 8 / 10$ & $12: 00$ & 17.940 & 24.174 & 17.318 & 28.133 & 5.606 & 10.019 & 0.828 \\
\hline $2 / 8 / 10$ & $16: 00$ & 17.982 & 24.197 & 17.360 & 28.186 & 5.606 & 10.047 & 0.828 \\
\hline $2 / 8 / 10$ & $20: 00$ & 17.985 & 24.234 & 17.411 & 28.196 & 5.606 & 10.088 & 0.831 \\
\hline $2 / 9 / 10$ & 0:00 & 18.046 & 24.222 & 17.411 & 28.217 & 5.608 & 10.075 & 0.825 \\
\hline $2 / 9 / 10$ & $4: 00$ & 18.109 & 24.211 & 17.409 & 28.225 & 5.612 & 10.058 & 0.819 \\
\hline $2 / 9 / 10$ & $8: 00$ & 18.094 & 24.262 & 17.469 & 28.220 & 5.610 & 10.106 & 0.827 \\
\hline $2 / 9 / 10$ & $12: 00$ & 18.121 & 24.323 & 17.537 & 28.194 & 5.598 & 10.156 & 0.834 \\
\hline $2 / 9 / 10$ & $16: 00$ & 18.138 & 24.379 & 17.606 & 28.235 & 5.596 & 10.214 & 0.838 \\
\hline $2 / 9 / 10$ & $20: 00$ & 18.138 & 24.428 & 17.664 & 28.252 & 5.623 & 10.260 & 0.846 \\
\hline $2 / 10 / 10$ & 0:00 & 18.143 & 24.478 & 17.728 & 28.271 & 5.641 & 10.294 & 0.852 \\
\hline $2 / 10 / 10$ & $4: 00$ & 18.215 & 24.493 & 17.754 & 28.272 & 5.639 & 10.314 & 0.850 \\
\hline $2 / 10 / 10$ & $8: 00$ & 18.255 & 24.516 & 17.786 & 28.294 & 5.643 & 10.331 & 0.849 \\
\hline $2 / 10 / 10$ & $12: 00$ & 18.317 & 24.554 & 17.830 & 28.335 & 5.623 & 10.363 & 0.851 \\
\hline $2 / 10 / 10$ & $16: 00$ & 18.418 & 24.522 & 17.800 & 28.374 & 5.633 & 10.320 & 0.833 \\
\hline $2 / 10 / 10$ & $20: 00$ & 18.386 & 24.547 & 17.822 & 28.373 & 5.637 & 10.331 & 0.834 \\
\hline $2 / 11 / 10$ & $0: 00$ & 18.399 & 24.572 & 17.847 & 28.351 & 5.611 & 10.320 & 0.835 \\
\hline $2 / 11 / 10$ & $4: 00$ & 18.444 & 24.568 & 17.839 & 28.360 & 5.637 & 10.311 & 0.831 \\
\hline $2 / 11 / 10$ & $8: 00$ & 18.449 & 24.598 & 17.861 & 28.382 & 5.594 & 10.324 & 0.833 \\
\hline $2 / 11 / 10$ & $12: 00$ & 18.466 & 24.627 & 17.886 & 28.381 & 5.610 & 10.340 & 0.836 \\
\hline $2 / 11 / 10$ & $16: 00$ & 18.486 & 24.614 & 17.873 & 28.363 & 5.581 & 10.289 & 0.828 \\
\hline $2 / 11 / 10$ & $20: 00$ & 18.448 & 24.690 & 17.942 & 28.362 & 5.604 & 10.363 & 0.842 \\
\hline $2 / 12 / 10$ & $0: 00$ & 18.470 & 24.709 & 17.968 & 28.352 & 5.569 & 10.361 & 0.842 \\
\hline $2 / 12 / 10$ & $4: 00$ & 18.486 & 24.730 & 17.990 & 28.390 & 5.620 & 10.359 & 0.844 \\
\hline $2 / 12 / 10$ & $8: 00$ & 18.481 & 24.774 & 18.034 & 28.383 & 5.604 & 10.394 & 0.850 \\
\hline $2 / 12 / 10$ & $12: 00$ & 18.503 & 24.837 & 18.095 & 28.357 & 5.574 & 10.445 & 0.860 \\
\hline $2 / 12 / 10$ & $16: 00$ & 18.575 & 24.826 & 18.092 & 28.376 & 5.588 & 10.422 & 0.846 \\
\hline $2 / 12 / 10$ & $20: 00$ & 18.567 & 24.868 & 18.134 & 28.413 & 5.607 & 10.431 & 0.852 \\
\hline $2 / 13 / 10$ & $0: 00$ & 18.582 & 24.898 & 18.158 & 28.422 & 5.584 & 10.428 & 0.855 \\
\hline $2 / 13 / 10$ & $4: 00$ & 18.617 & 24.896 & 18.158 & 28.404 & 5.588 & 10.405 & 0.853 \\
\hline $2 / 13 / 10$ & 8:00 & 18.642 & 24.910 & 18.165 & 28.416 & 5.578 & 10.400 & 0.851 \\
\hline $2 / 13 / 10$ & $12: 00$ & 18.666 & 24.946 & 18.192 & 28.450 & 5.602 & 10.422 & 0.854 \\
\hline $2 / 13 / 10$ & $16: 00$ & 18.669 & 24.948 & 18.190 & 28.455 & 5.573 & 10.396 & 0.841 \\
\hline $2 / 13 / 10$ & $20: 00$ & 18.644 & 25.017 & 18.248 & 28.415 & 5.578 & 10.428 & 0.855 \\
\hline
\end{tabular}


TABLE S2.1 (Cont.)

\begin{tabular}{|c|c|c|c|c|c|c|c|c|}
\hline \multirow[b]{2}{*}{ Date } & \multirow[b]{2}{*}{ Time } & \multicolumn{6}{|c|}{ Depth to Water (ft TOC) } & \multirow[b]{2}{*}{ MW8s } \\
\hline & & MW1s & MW2s & MW3s & MW4s & MW6s & MW7s & \\
\hline 2/14/10 & $0: 00$ & 18.632 & 25.074 & 18.304 & 28.448 & 5.607 & 10.452 & 0.867 \\
\hline $2 / 14 / 10$ & $4: 00$ & 18.609 & 25.145 & 18.380 & 28.463 & 5.637 & 10.513 & 0.877 \\
\hline $2 / 14 / 10$ & 8:00 & 18.632 & 25.156 & 18.404 & 28.503 & 5.639 & 10.511 & 0.872 \\
\hline $2 / 14 / 10$ & $12: 00$ & 18.729 & 25.166 & 18.409 & 28.494 & 5.598 & 10.525 & 0.873 \\
\hline $2 / 14 / 10$ & $16: 00$ & 18.741 & 25.211 & 18.448 & 28.525 & 5.615 & 10.564 & 0.880 \\
\hline $2 / 14 / 10$ & 20:00 & 18.739 & 25.257 & 18.499 & 28.550 & 5.615 & 10.612 & 0.885 \\
\hline $2 / 15 / 10$ & $0: 00$ & 18.808 & 25.252 & 18.499 & 28.587 & 5.640 & 10.604 & 0.877 \\
\hline $2 / 15 / 10$ & $4: 00$ & 18.857 & 25.252 & 18.496 & 28.600 & 5.615 & 10.595 & 0.871 \\
\hline 2/15/10 & $8: 00$ & 18.843 & 25.307 & 18.540 & 28.587 & 5.646 & 10.642 & 0.877 \\
\hline $2 / 15 / 10$ & $12: 00$ & 18.872 & 25.364 & 18.601 & 28.581 & 5.635 & 10.699 & 0.882 \\
\hline $2 / 15 / 10$ & $16: 00$ & 18.927 & 25.372 & 18.618 & 28.641 & 5.615 & 10.707 & 0.879 \\
\hline $2 / 15 / 10$ & 20:00 & 18.922 & 25.406 & 18.657 & 28.657 & 5.662 & 10.738 & 0.882 \\
\hline $2 / 16 / 10$ & 0:00 & 18.959 & 25.435 & 18.689 & 28.683 & 5.621 & 10.753 & 0.889 \\
\hline 2/16/10 & $4: 00$ & 18.967 & 25.471 & 18.732 & 28.674 & 5.623 & 10.773 & 0.893 \\
\hline 2/16/10 & 8:00 & 19.001 & 25.519 & 18.788 & 28.710 & 5.627 & 10.827 & 0.902 \\
\hline $2 / 16 / 10$ & $12: 00$ & 19.044 & 25.565 & 18.849 & 28.744 & 5.660 & 10.872 & 0.904 \\
\hline $2 / 16 / 10$ & $16: 00$ & 19.123 & 25.555 & 18.851 & 28.783 & 5.633 & 10.853 & 0.893 \\
\hline 2/16/10 & 20:00 & 19.056 & 25.580 & 18.881 & 28.807 & 5.644 & 10.608 & 0.891 \\
\hline $2 / 17 / 10$ & 0:00 & 19.002 & 25.607 & 18.912 & 28.825 & 5.626 & 10.458 & 0.893 \\
\hline $2 / 17 / 10$ & $4: 00$ & 18.995 & 25.628 & 18.942 & 28.847 & 5.638 & 10.459 & 0.897 \\
\hline $2 / 17 / 10$ & $8: 00$ & 18.950 & 25.660 & 18.976 & 28.854 & 5.627 & 10.517 & 0.903 \\
\hline $2 / 17 / 10$ & $12: 00$ & 19.042 & 25.702 & 19.022 & 28.844 & 5.644 & 10.656 & 0.903 \\
\hline $2 / 17 / 10$ & $16: 00$ & 19.126 & 25.693 & 19.024 & 28.886 & 5.630 & 10.684 & 0.886 \\
\hline 2/17/10 & 20:00 & 19.077 & 25.735 & 19.066 & 28.898 & 5.628 & 10.725 & 0.889 \\
\hline $2 / 18 / 10$ & 0:00 & 19.074 & 25.756 & 19.095 & 28.920 & 5.646 & 10.729 & 0.892 \\
\hline 2/18/10 & $4: 00$ & 19.121 & 25.779 & 19.126 & 28.905 & 5.615 & 10.766 & 0.897 \\
\hline $2 / 18 / 10$ & 8:00 & 19.136 & 25.811 & 19.160 & 28.942 & 5.648 & 10.801 & 0.902 \\
\hline 2/18/10 & $12: 00$ & 19.191 & 25.836 & 19.192 & 28.960 & 5.658 & 10.829 & 0.900 \\
\hline $2 / 18 / 10$ & $16: 00$ & 19.295 & 25.802 & 19.158 & 28.979 & 5.636 & 10.768 & 0.867 \\
\hline 2/18/10 & $20: 00$ & 19.206 & 25.853 & 19.163 & 28.967 & 5.582 & 10.668 & 0.867 \\
\hline $2 / 19 / 10$ & 0:00 & 19.176 & 25.888 & 19.165 & 28.985 & 5.636 & 10.621 & 0.878 \\
\hline $2 / 19 / 10$ & $4: 00$ & 19.206 & 25.893 & 19.187 & 28.996 & 5.599 & 10.593 & 0.867 \\
\hline $2 / 19 / 10$ & $8: 00$ & 19.223 & 25.901 & 19.194 & 29.002 & 5.640 & 10.586 & 0.861 \\
\hline $2 / 19 / 10$ & $12: 00$ & 19.228 & 25.916 & 19.214 & 29.013 & 5.609 & 10.621 & 0.864 \\
\hline $2 / 19 / 10$ & $16: 00$ & 19.260 & 25.924 & 19.221 & 28.992 & 5.576 & 10.647 & 0.868 \\
\hline $2 / 19 / 10$ & $20: 00$ & 19.206 & 25.987 & 19.289 & 29.007 & 5.623 & 10.725 & 0.882 \\
\hline $2 / 20 / 10$ & 0:00 & 19.215 & 26.023 & 19.340 & 29.025 & 5.626 & 10.772 & 0.889 \\
\hline 2/20/10 & $4: 00$ & 19.237 & 26.058 & 19.389 & 29.050 & 5.667 & 10.812 & 0.899 \\
\hline 2/20/10 & 8:00 & 19.235 & 26.113 & 19.450 & 29.042 & 5.632 & 10.876 & 0.908 \\
\hline 2/20/10 & $12: 00$ & 19.302 & 26.111 & 19.464 & 29.066 & 5.654 & 10.877 & 0.905 \\
\hline 2/20/10 & $16: 00$ & 19.357 & 26.113 & 19.467 & 29.108 & 5.658 & 10.879 & 0.900 \\
\hline $2 / 20 / 10$ & $20: 00$ & 19.366 & 26.140 & 19.498 & 29.093 & 5.673 & 10.903 & 0.907 \\
\hline $2 / 21 / 10$ & $0: 00$ & 19.396 & 26.155 & 19.525 & 29.115 & 5.651 & 10.920 & 0.908 \\
\hline $2 / 21 / 10$ & $4: 00$ & 19.433 & 26.155 & 19.530 & 29.152 & 5.622 & 10.911 & 0.904 \\
\hline $2 / 21 / 10$ & $8: 00$ & 19.436 & 26.182 & 19.559 & 29.148 & 5.680 & 10.935 & 0.910 \\
\hline $2 / 21 / 10$ & $12: 00$ & 19.463 & 26.189 & 19.576 & 29.143 & 5.622 & 10.933 & 0.907 \\
\hline $2 / 21 / 10$ & $16: 00$ & 19.493 & 26.195 & 19.588 & 29.175 & 5.626 & 10.929 & 0.904 \\
\hline 2/21/10 & $20: 00$ & 19.453 & 26.260 & 19.666 & 29.177 & 5.612 & 10.993 & 0.915 \\
\hline
\end{tabular}


TABLE S2.1 (Cont.)

\begin{tabular}{|c|c|c|c|c|c|c|c|c|}
\hline \multirow[b]{2}{*}{ Date } & \multirow[b]{2}{*}{ Time } & \multicolumn{6}{|c|}{ Depth to Water (ft TOC) } & \multirow[b]{2}{*}{ MW8s } \\
\hline & & MW1s & MW2s & MW3s & MW4s & MW6s & MW7s & \\
\hline $2 / 22 / 10$ & $0: 00$ & 19.453 & 26.306 & 19.732 & 29.148 & 5.605 & 11.033 & 0.923 \\
\hline $2 / 22 / 10$ & $4: 00$ & 19.495 & 26.317 & 19.761 & 29.184 & 5.643 & 11.043 & 0.922 \\
\hline $2 / 22 / 10$ & 8:00 & 19.488 & 26.346 & 19.800 & 29.223 & 5.630 & 11.046 & 0.927 \\
\hline $2 / 22 / 10$ & $12: 00$ & 19.542 & 26.388 & 19.858 & 29.215 & 5.677 & 11.102 & 0.930 \\
\hline $2 / 22 / 10$ & $16: 00$ & 19.610 & 26.382 & 19.863 & 29.239 & 5.673 & 11.087 & 0.925 \\
\hline $2 / 22 / 10$ & $20: 00$ & 19.582 & 26.430 & 19.924 & 29.246 & 5.647 & 11.128 & 0.935 \\
\hline $2 / 23 / 10$ & $0: 00$ & 19.600 & 26.445 & 19.958 & 29.299 & 5.671 & 11.136 & 0.932 \\
\hline $2 / 23 / 10$ & $4: 00$ & 19.642 & 26.455 & 19.975 & 29.316 & 5.661 & 11.139 & 0.931 \\
\hline $2 / 23 / 10$ & 8:00 & 19.644 & 26.478 & 20.002 & 29.316 & 5.653 & 11.136 & 0.933 \\
\hline $2 / 23 / 10$ & $12: 00$ & 19.661 & 26.564 & 20.108 & 29.306 & 5.649 & 11.229 & 0.950 \\
\hline $2 / 23 / 10$ & $16: 00$ & 19.706 & 26.579 & 20.145 & 29.365 & 5.657 & 11.242 & 0.948 \\
\hline $2 / 23 / 10$ & $20: 00$ & 19.684 & 26.629 & 20.213 & 29.385 & 5.678 & 11.288 & 0.955 \\
\hline $2 / 24 / 10$ & $0: 00$ & 19.701 & 26.663 & 20.272 & 29.422 & 5.671 & 11.316 & 0.958 \\
\hline $2 / 24 / 10$ & $4: 00$ & 19.736 & 26.678 & 20.306 & 29.427 & 5.675 & 11.327 & 0.956 \\
\hline $2 / 24 / 10$ & 8:00 & 19.753 & 26.713 & 20.354 & 29.456 & 5.678 & 11.355 & 0.963 \\
\hline $2 / 24 / 10$ & $12: 00$ & 19.823 & 26.747 & 20.405 & 29.514 & 5.632 & 11.383 & 0.963 \\
\hline $2 / 24 / 10$ & $16: 00$ & 19.907 & 26.726 & 20.391 & 29.539 & 5.694 & 11.346 & 0.952 \\
\hline $2 / 24 / 10$ & $20: 00$ & 19.882 & 26.743 & 20.408 & 29.554 & 5.657 & 11.346 & 0.953 \\
\hline $2 / 25 / 10$ & $0: 00$ & 19.875 & 26.772 & 20.447 & 29.549 & 5.671 & 11.368 & 0.956 \\
\hline $2 / 25 / 10$ & $4: 00$ & 19.894 & 26.783 & 20.461 & 29.562 & 5.634 & 11.364 & 0.955 \\
\hline $2 / 25 / 10$ & 8:00 & 19.912 & 26.810 & 20.493 & 29.600 & 5.678 & 11.381 & 0.961 \\
\hline $2 / 25 / 10$ & $12: 00$ & 19.961 & 26.837 & 20.527 & 29.618 & 5.663 & 11.399 & 0.962 \\
\hline $2 / 25 / 10$ & $16: 00$ & 20.004 & 26.836 & 20.519 & 29.638 & 5.680 & 11.383 & 0.955 \\
\hline $2 / 25 / 10$ & $20: 00$ & 19.961 & 26.883 & 20.573 & 29.621 & 5.657 & 11.420 & 0.960 \\
\hline $2 / 26 / 10$ & $0: 00$ & 19.944 & 26.921 & 20.626 & 29.666 & 5.664 & 11.457 & 0.968 \\
\hline $2 / 26 / 10$ & $4: 00$ & 19.964 & 26.940 & 20.655 & 29.660 & 5.653 & 11.470 & 0.971 \\
\hline $2 / 26 / 10$ & 8:00 & 19.994 & 26.961 & 20.680 & 29.675 & 5.680 & 11.481 & 0.972 \\
\hline $2 / 26 / 10$ & $12: 00$ & 20.046 & 26.993 & 20.721 & 29.695 & 5.659 & 11.503 & 0.973 \\
\hline $2 / 26 / 10$ & $16: 00$ & 20.110 & 26.982 & 20.709 & 29.743 & 5.633 & 11.474 & 0.964 \\
\hline $2 / 26 / 10$ & $20: 00$ & 20.058 & 27.010 & 20.741 & 29.756 & 5.598 & 11.455 & 0.962 \\
\hline $2 / 27 / 10$ & $0: 00$ & 20.036 & 27.037 & 20.775 & 29.739 & 5.610 & 11.455 & 0.969 \\
\hline $2 / 27 / 10$ & $4: 00$ & 20.039 & 27.062 & 20.806 & 29.772 & 5.649 & 11.466 & 0.971 \\
\hline $2 / 27 / 10$ & $8: 00$ & 20.016 & 27.104 & 20.860 & 29.756 & 5.629 & 11.500 & 0.981 \\
\hline $2 / 27 / 10$ & $12: 00$ & 20.066 & 27.134 & 20.899 & 29.813 & 5.614 & 11.520 & 0.980 \\
\hline $2 / 27 / 10$ & $16: 00$ & 20.108 & 27.129 & 20.901 & 29.833 & 5.602 & 11.496 & 0.969 \\
\hline $2 / 27 / 10$ & $20: 00$ & 20.056 & 27.159 & 20.930 & 29.851 & 5.641 & 11.446 & 0.970 \\
\hline $2 / 28 / 10$ & $0: 00$ & 20.039 & 27.177 & 20.955 & 29.839 & 5.661 & 11.414 & 0.975 \\
\hline $2 / 28 / 10$ & $4: 00$ & 20.046 & 27.190 & 20.972 & 29.886 & 5.674 & 11.383 & 0.975 \\
\hline $2 / 28 / 10$ & $8: 00$ & 20.027 & 27.234 & 21.023 & 29.863 & 5.655 & 11.398 & 0.983 \\
\hline $2 / 28 / 10$ & $12: 00$ & 20.086 & 27.251 & 21.047 & 29.917 & 5.690 & 11.411 & 0.982 \\
\hline $2 / 28 / 10$ & $16: 00$ & 20.133 & 27.249 & 21.042 & 29.942 & 5.668 & 11.416 & 0.974 \\
\hline $2 / 28 / 10$ & $20: 00$ & 20.076 & 27.284 & 21.079 & 29.945 & 5.719 & 11.412 & 0.977 \\
\hline $3 / 1 / 10$ & $0: 00$ & 20.046 & 27.341 & 21.142 & 29.959 & 5.703 & 11.433 & 0.987 \\
\hline $3 / 1 / 10$ & $4: 00$ & 20.064 & 27.358 & 21.176 & 29.991 & 5.697 & 11.429 & 0.987 \\
\hline $3 / 1 / 10$ & $8: 00$ & 20.069 & 27.383 & 21.205 & 29.983 & 5.674 & 11.446 & 0.989 \\
\hline $3 / 1 / 10$ & $12: 00$ & 20.093 & 27.432 & 21.264 & 30.006 & 5.695 & 11.511 & 0.995 \\
\hline $3 / 1 / 10$ & $16: 00$ & 20.160 & 27.417 & 21.249 & 30.062 & 5.664 & 11.479 & 0.982 \\
\hline $3 / 1 / 10$ & $20: 00$ & 20.116 & 27.442 & 21.276 & 30.085 & 5.685 & 11.401 & 0.972 \\
\hline
\end{tabular}


TABLE S2.1 (Cont.)

\begin{tabular}{|c|c|c|c|c|c|c|c|c|}
\hline \multirow[b]{2}{*}{ Date } & \multirow[b]{2}{*}{ Time } & \multicolumn{6}{|c|}{ Depth to Water (ft TOC) } & \multirow[b]{2}{*}{ MW8s } \\
\hline & & MW1s & MW2s & MW3s & MW4s & MW6s & MW7s & \\
\hline $3 / 2 / 10$ & $0: 00$ & 20.101 & 27.446 & 21.273 & 30.115 & 5.689 & 11.340 & 0.977 \\
\hline $3 / 2 / 10$ & $4: 00$ & 20.104 & 27.444 & 21.259 & 30.135 & 5.676 & 11.308 & 0.976 \\
\hline $3 / 2 / 10$ & 8:00 & 20.096 & 27.454 & 21.269 & 30.145 & 5.670 & 11.297 & 0.979 \\
\hline $3 / 2 / 10$ & $12: 00$ & 20.124 & 27.467 & 21.286 & 30.132 & 5.683 & 11.312 & 0.980 \\
\hline $3 / 2 / 10$ & $16: 00$ & 20.188 & 27.436 & 21.244 & 30.146 & 5.683 & 11.260 & 0.960 \\
\hline $3 / 2 / 10$ & $20: 00$ & 20.084 & 27.463 & 21.249 & 30.159 & 5.662 & 11.175 & 0.955 \\
\hline $3 / 3 / 10$ & $0: 00$ & 20.042 & 27.476 & 21.234 & 30.190 & 5.625 & 11.136 & 0.963 \\
\hline $3 / 3 / 10$ & $4: 00$ & 20.022 & 27.484 & 21.237 & 30.181 & 5.648 & 11.130 & 0.967 \\
\hline $3 / 3 / 10$ & 8:00 & 20.004 & 27.505 & 21.261 & 30.203 & 5.621 & 11.150 & 0.973 \\
\hline $3 / 3 / 10$ & $12: 00$ & 20.042 & 27.537 & 21.308 & 30.212 & 5.615 & 11.206 & 0.979 \\
\hline $3 / 3 / 10$ & $16: 00$ & 20.101 & 27.514 & 21.276 & 30.205 & 5.695 & 11.169 & 0.931 \\
\hline $3 / 3 / 10$ & $20: 00$ & 20.014 & 27.511 & 21.227 & 30.220 & 5.633 & 11.119 & 0.898 \\
\hline 3/4/10 & $0: 00$ & 19.960 & 27.518 & 21.176 & 30.251 & 5.627 & 10.928 & 0.896 \\
\hline 3/4/10 & $4: 00$ & 19.962 & 27.495 & 21.122 & 30.255 & 5.658 & 10.900 & 0.889 \\
\hline $3 / 4 / 10$ & 8:00 & 19.930 & 27.511 & 21.130 & 30.236 & 5.632 & 10.924 & 0.896 \\
\hline 3/4/10 & $12: 00$ & 19.972 & 27.513 & 21.132 & 30.291 & 5.656 & 10.955 & 0.897 \\
\hline 3/4/10 & $16: 00$ & 20.016 & 27.461 & 21.055 & 30.297 & 5.665 & 10.829 & 0.678 \\
\hline 3/4/10 & $20: 00$ & 19.858 & 27.440 & 20.921 & 30.292 & 5.394 & 10.504 & 0.450 \\
\hline $3 / 5 / 10$ & $0: 00$ & 19.729 & 27.427 & 20.765 & 30.296 & 5.487 & 10.376 & 0.607 \\
\hline $3 / 5 / 10$ & $4: 00$ & 19.694 & 27.383 & 20.566 & 30.267 & 5.564 & 10.335 & 0.663 \\
\hline $3 / 5 / 10$ & 8:00 & 19.657 & 27.364 & 20.442 & 30.288 & 5.603 & 10.352 & 0.704 \\
\hline $3 / 5 / 10$ & $12: 00$ & 19.682 & 27.320 & 20.335 & 30.296 & 5.660 & 10.346 & 0.705 \\
\hline $3 / 5 / 10$ & $16: 00$ & 19.724 & 27.251 & 20.123 & 30.272 & 5.448 & 10.298 & 0.434 \\
\hline $3 / 5 / 10$ & $20: 00$ & 19.582 & 27.202 & 19.781 & 30.259 & 5.028 & 9.938 & 0.450 \\
\hline 3/6/10 & 0:00 & 19.456 & 27.152 & 19.511 & 30.234 & 5.247 & 9.886 & 0.569 \\
\hline 3/6/10 & $4: 00$ & 19.431 & 27.052 & 19.241 & 30.196 & 5.438 & 9.856 & 0.664 \\
\hline $3 / 6 / 10$ & 8:00 & 19.359 & 26.988 & 19.053 & 30.197 & 5.568 & 9.874 & 0.636 \\
\hline $3 / 6 / 10$ & $12: 00$ & 19.334 & 26.924 & 18.783 & 30.146 & 5.465 & 9.856 & 0.581 \\
\hline 3/6/10 & $16: 00$ & 19.302 & 26.791 & 18.141 & 30.125 & 4.865 & 9.534 & 0.444 \\
\hline 3/6/10 & $20: 00$ & 19.128 & 26.634 & 17.293 & 30.094 & 4.562 & 9.336 & 0.421 \\
\hline 3/7/10 & 0:00 & 18.905 & 26.485 & 16.598 & 30.036 & 4.816 & 9.235 & 0.421 \\
\hline 3/7/10 & $4: 00$ & 18.783 & 26.329 & 16.203 & 29.966 & 5.201 & 9.219 & 0.467 \\
\hline 3/7/10 & 8:00 & 18.659 & 26.193 & 15.921 & 29.861 & 5.447 & 9.180 & 0.507 \\
\hline 3/7/10 & $12: 00$ & 18.574 & 26.071 & 15.807 & 29.790 & 5.535 & 9.165 & 0.529 \\
\hline 3/7/10 & $16: 00$ & 18.525 & 25.914 & 15.663 & 29.687 & 5.405 & 8.998 & 0.530 \\
\hline 3/7/10 & $20: 00$ & 18.406 & 25.759 & 15.529 & 29.547 & 5.393 & 8.888 & 0.527 \\
\hline 3/8/10 & $0: 00$ & 18.299 & 25.601 & 15.436 & 29.424 & 5.498 & 8.855 & 0.542 \\
\hline 3/8/10 & $4: 00$ & 18.239 & 25.441 & 15.357 & 29.271 & 5.570 & 8.851 & 0.554 \\
\hline 3/8/10 & $8: 00$ & 18.170 & 25.313 & 15.319 & 29.139 & 5.624 & 8.860 & 0.568 \\
\hline $3 / 8 / 10$ & $12: 00$ & 18.148 & 25.173 & 15.263 & 29.038 & 5.587 & 8.803 & 0.566 \\
\hline 3/8/10 & $16: 00$ & 18.135 & 24.984 & 15.110 & 28.902 & 5.319 & 8.552 & 0.546 \\
\hline 3/8/10 & $20: 00$ & 17.942 & 24.849 & 14.943 & 28.737 & 5.220 & 8.470 & 0.518 \\
\hline 3/9/10 & $0: 00$ & 17.746 & 24.591 & 14.296 & 28.621 & 5.035 & 8.407 & 0.098 \\
\hline $3 / 9 / 10$ & $4: 00$ & 17.349 & 24.285 & 13.748 & 28.382 & 4.802 & 8.073 & -0.015 \\
\hline 3/9/10 & $8: 00$ & 16.910 & 23.997 & 13.304 & 28.094 & 4.888 & 7.764 & 0.037 \\
\hline 3/9/10 & $12: 00$ & 16.585 & 23.733 & 13.027 & 27.919 & 5.045 & 7.549 & 0.112 \\
\hline 3/9/10 & $16: 00$ & 16.342 & 23.454 & 12.785 & 27.676 & 5.241 & 7.341 & 0.155 \\
\hline $3 / 9 / 10$ & $20: 00$ & 16.084 & 23.223 & 12.631 & 27.461 & 5.391 & 7.250 & 0.164 \\
\hline
\end{tabular}


TABLE S2.1 (Cont.)

\begin{tabular}{|c|c|c|c|c|c|c|c|c|}
\hline \multirow[b]{2}{*}{ Date } & \multirow[b]{2}{*}{ Time } & \multicolumn{6}{|c|}{ Depth to Water (ft TOC) } & \multirow[b]{2}{*}{ MW8s } \\
\hline & & MW1s & MW2s & MW3s & MW4s & MW6s & MW7s & \\
\hline $3 / 10 / 10$ & $0: 00$ & 15.866 & 23.011 & 12.563 & 27.284 & 5.459 & 7.064 & 0.194 \\
\hline $3 / 10 / 10$ & $4: 00$ & 15.702 & 22.832 & 12.559 & 27.060 & 5.468 & 6.937 & 0.236 \\
\hline $3 / 10 / 10$ & 8:00 & 15.590 & 22.671 & 12.561 & 26.905 & 5.548 & 6.906 & 0.266 \\
\hline $3 / 10 / 10$ & $12: 00$ & 15.527 & 22.524 & 12.581 & 26.756 & 5.536 & 6.889 & 0.289 \\
\hline $3 / 10 / 10$ & $16: 00$ & 15.502 & 22.362 & 12.547 & 26.642 & 5.513 & 6.811 & 0.304 \\
\hline $3 / 10 / 10$ & $20: 00$ & 15.455 & 22.234 & 12.529 & 26.477 & 5.538 & 6.781 & 0.318 \\
\hline $3 / 11 / 10$ & $0: 00$ & 15.418 & 22.115 & 12.513 & 26.371 & 5.496 & 6.748 & 0.320 \\
\hline $3 / 11 / 10$ & $4: 00$ & 15.321 & 22.019 & 12.465 & 26.183 & 5.470 & 6.724 & 0.257 \\
\hline $3 / 11 / 10$ & 8:00 & 14.869 & 21.840 & 11.949 & 26.078 & 4.670 & 6.609 & -0.059 \\
\hline $3 / 11 / 10$ & $12: 00$ & 14.536 & 21.651 & 11.780 & 25.829 & 4.590 & 6.375 & -0.059 \\
\hline $3 / 11 / 10$ & $16: 00$ & 14.343 & 21.449 & 11.690 & 25.585 & 4.802 & 6.163 & -0.021 \\
\hline $3 / 11 / 10$ & $20: 00$ & 14.198 & 21.296 & 11.664 & 25.349 & 5.002 & 5.888 & 0.058 \\
\hline $3 / 12 / 10$ & $0: 00$ & 14.137 & 21.147 & 11.636 & 25.183 & 5.204 & 5.845 & 0.095 \\
\hline $3 / 12 / 10$ & $4: 00$ & 14.105 & 20.998 & 11.595 & 25.027 & 5.322 & 5.804 & 0.126 \\
\hline $3 / 12 / 10$ & 8:00 & 14.036 & 20.925 & 11.621 & 24.832 & 5.394 & 5.799 & 0.154 \\
\hline $3 / 12 / 10$ & $12: 00$ & 13.976 & 20.885 & 11.673 & 24.729 & 5.427 & 5.817 & 0.126 \\
\hline $3 / 12 / 10$ & $16: 00$ & 13.939 & 20.841 & 11.710 & 24.612 & 5.421 & 5.823 & 0.144 \\
\hline $3 / 12 / 10$ & $20: 00$ & 13.912 & 20.805 & 11.734 & 24.481 & 5.489 & 5.864 & 0.171 \\
\hline $3 / 13 / 10$ & $0: 00$ & 13.939 & 20.746 & 11.746 & 24.425 & 5.516 & 5.888 & 0.190 \\
\hline 3/13/10 & $4: 00$ & 13.969 & 20.668 & 11.736 & 24.303 & 5.538 & 5.882 & 0.203 \\
\hline $3 / 13 / 10$ & 8:00 & 13.957 & 20.652 & 11.780 & 24.232 & 5.507 & 5.933 & 0.225 \\
\hline 3/13/10 & $12: 00$ & 13.984 & 20.620 & 11.808 & 24.169 & 5.538 & 5.972 & 0.243 \\
\hline $3 / 13 / 10$ & $16: 00$ & 14.029 & 20.553 & 11.772 & 24.130 & 5.515 & 5.973 & 0.256 \\
\hline $3 / 13 / 10$ & $20: 00$ & 13.993 & 20.566 & 11.824 & 24.071 & 5.511 & 6.064 & 0.283 \\
\hline $3 / 14 / 10$ & 0:00 & 14.013 & 20.570 & 11.882 & 24.012 & 5.524 & 6.142 & 0.296 \\
\hline $3 / 14 / 10$ & $4: 00$ & 14.054 & 20.561 & 11.932 & 23.968 & 5.551 & 6.194 & 0.307 \\
\hline $3 / 14 / 10$ & 8:00 & 14.073 & 20.587 & 12.013 & 23.905 & 5.539 & 6.278 & 0.322 \\
\hline $3 / 14 / 10$ & $12: 00$ & 14.105 & 20.624 & 12.098 & 23.930 & 5.538 & 6.369 & 0.334 \\
\hline $3 / 14 / 10$ & $16: 00$ & 14.197 & 20.601 & 12.122 & 23.926 & 5.561 & 6.388 & 0.333 \\
\hline $3 / 14 / 10$ & $20: 00$ & 14.199 & 20.637 & 12.199 & 23.885 & 5.532 & 6.464 & 0.354 \\
\hline $3 / 15 / 10$ & 0:00 & 14.239 & 20.675 & 12.278 & 23.902 & 5.572 & 6.538 & 0.369 \\
\hline $3 / 15 / 10$ & $4: 00$ & 14.309 & 20.667 & 12.319 & 23.922 & 5.559 & 6.562 & 0.373 \\
\hline $3 / 15 / 10$ & 8:00 & 14.348 & 20.696 & 12.397 & 23.943 & 5.594 & 6.618 & 0.390 \\
\hline 3/15/10 & $12: 00$ & 14.409 & 20.719 & 12.462 & 23.967 & 5.594 & 6.666 & 0.395 \\
\hline $3 / 15 / 10$ & $16: 00$ & 14.500 & 20.698 & 12.486 & 23.968 & 5.603 & 6.664 & 0.400 \\
\hline $3 / 15 / 10$ & $20: 00$ & 14.513 & 20.724 & 12.552 & 23.944 & 5.607 & 6.707 & 0.413 \\
\hline 3/16/10 & $0: 00$ & 14.543 & 20.753 & 12.616 & 23.986 & 5.590 & 6.757 & 0.427 \\
\hline $3 / 16 / 10$ & $4: 00$ & 14.595 & 20.750 & 12.660 & 23.990 & 5.598 & 6.770 & 0.433 \\
\hline $3 / 16 / 10$ & $8: 00$ & 14.633 & 20.759 & 12.712 & 23.970 & 5.584 & 6.791 & 0.441 \\
\hline $3 / 16 / 10$ & $12: 00$ & 14.680 & 20.771 & 12.759 & 24.005 & 5.598 & 6.813 & 0.446 \\
\hline $3 / 16 / 10$ & $16: 00$ & 14.747 & 20.740 & 12.775 & 24.020 & 5.580 & 6.787 & 0.445 \\
\hline $3 / 16 / 10$ & $20: 00$ & 14.757 & 20.746 & 12.818 & 24.010 & 5.587 & 6.802 & 0.454 \\
\hline 3/17/10 & $0: 00$ & 14.779 & 20.765 & 12.864 & 24.021 & 5.537 & 6.828 & 0.462 \\
\hline 3/17/10 & $4: 00$ & 14.809 & 20.761 & 12.897 & 24.025 & 5.570 & 6.830 & 0.464 \\
\hline 3/17/10 & $8: 00$ & 14.802 & 20.803 & 12.965 & 24.021 & 5.525 & 6.878 & 0.475 \\
\hline $3 / 17 / 10$ & $12: 00$ & 14.835 & 20.820 & 13.009 & 24.020 & 5.551 & 6.891 & 0.477 \\
\hline $3 / 17 / 10$ & $16: 00$ & 14.934 & 20.801 & 13.033 & 24.083 & 5.543 & 6.895 & 0.479 \\
\hline $3 / 17 / 10$ & $20: 00$ & 14.917 & 20.807 & 13.069 & 24.043 & 5.558 & 6.904 & 0.487 \\
\hline
\end{tabular}


TABLE S2.1 (Cont.)

\begin{tabular}{|c|c|c|c|c|c|c|c|c|}
\hline \multirow[b]{2}{*}{ Date } & \multirow[b]{2}{*}{ Time } & \multicolumn{6}{|c|}{ Depth to Water (ft TOC) } & \multirow[b]{2}{*}{ MW8s } \\
\hline & & MW1s & MW2s & MW3s & MW4s & MW6s & MW7s & \\
\hline $3 / 18 / 10$ & 0:00 & 14.922 & 20.824 & 13.113 & 24.051 & 5.566 & 6.930 & 0.493 \\
\hline $3 / 18 / 10$ & 4:00 & 14.955 & 20.826 & 13.145 & 24.051 & 5.547 & 6.930 & 0.496 \\
\hline $3 / 18 / 10$ & $8: 00$ & 14.970 & 20.853 & 13.193 & 24.075 & 5.516 & 6.962 & 0.503 \\
\hline $3 / 18 / 10$ & $12: 00$ & 15.056 & 20.849 & 13.217 & 24.093 & 5.510 & 6.958 & 0.505 \\
\hline $3 / 18 / 10$ & $16: 00$ & 15.133 & 20.801 & 13.209 & 24.088 & 5.538 & 6.910 & 0.499 \\
\hline $3 / 18 / 10$ & 20:00 & 15.076 & 20.820 & 13.251 & 24.064 & 5.523 & 6.934 & 0.510 \\
\hline $3 / 19 / 10$ & $0: 00$ & 15.049 & 20.864 & 13.303 & 24.070 & 5.507 & 6.982 & 0.519 \\
\hline $3 / 19 / 10$ & $4: 00$ & 15.042 & 20.912 & 13.365 & 24.062 & 5.509 & 7.041 & 0.528 \\
\hline $3 / 19 / 10$ & $8: 00$ & 15.070 & 20.948 & 13.415 & 24.078 & 5.507 & 7.075 & 0.535 \\
\hline $3 / 19 / 10$ & $12: 00$ & 15.107 & 21.013 & 13.491 & 24.103 & 5.505 & 7.136 & 0.545 \\
\hline $3 / 19 / 10$ & $16: 00$ & 15.122 & 21.067 & 13.560 & 24.092 & 5.540 & 7.199 & 0.555 \\
\hline $3 / 19 / 10$ & $20: 00$ & 15.120 & 21.126 & 13.637 & 24.118 & 5.492 & 7.262 & 0.565 \\
\hline $3 / 20 / 10$ & $0: 00$ & 15.164 & 21.183 & 13.700 & 24.177 & 5.492 & 7.316 & 0.566 \\
\hline $3 / 20 / 10$ & 4:00 & 15.261 & 21.189 & 13.730 & 24.206 & 5.542 & 7.326 & 0.561 \\
\hline $3 / 20 / 10$ & $8: 00$ & 15.276 & 21.256 & 13.804 & 24.220 & 5.589 & 7.381 & 0.573 \\
\hline $3 / 20 / 10$ & $12: 00$ & 15.364 & 21.279 & 13.851 & 24.306 & 5.614 & 7.394 & 0.570 \\
\hline $3 / 20 / 10$ & $16: 00$ & 15.431 & 21.282 & 13.884 & 24.338 & 5.594 & 7.385 & 0.566 \\
\hline $3 / 20 / 10$ & $20: 00$ & 15.431 & 21.294 & 13.920 & 24.341 & 5.577 & 7.376 & 0.561 \\
\hline $3 / 21 / 10$ & $0: 00$ & 15.448 & 21.304 & 13.955 & 24.397 & 5.604 & 7.359 & 0.556 \\
\hline $3 / 21 / 10$ & $4: 00$ & 15.478 & 21.301 & 13.976 & 24.428 & 5.590 & 7.329 & 0.549 \\
\hline $3 / 21 / 10$ & $8: 00$ & 15.485 & 21.325 & 14.013 & 24.434 & 5.590 & 7.329 & 0.550 \\
\hline $3 / 21 / 10$ & $12: 00$ & 15.508 & 21.359 & 14.053 & 24.434 & 5.554 & 7.341 & 0.550 \\
\hline $3 / 21 / 10$ & $16: 00$ & 15.543 & 21.321 & 14.051 & 24.449 & 5.373 & 7.266 & 0.452 \\
\hline $3 / 21 / 10$ & $20: 00$ & 15.297 & 21.263 & 13.978 & 24.490 & 4.924 & 7.080 & 0.311 \\
\hline $3 / 22 / 10$ & $0: 00$ & 15.099 & 21.236 & 13.959 & 24.501 & 5.093 & 6.928 & 0.350 \\
\hline $3 / 22 / 10$ & $4: 00$ & 15.022 & 21.214 & 13.953 & 24.514 & 5.320 & 6.846 & 0.380 \\
\hline $3 / 22 / 10$ & $8: 00$ & 14.954 & 21.231 & 13.974 & 24.501 & 5.418 & 6.843 & 0.409 \\
\hline $3 / 22 / 10$ & $12: 00$ & 15.019 & 21.210 & 13.968 & 24.491 & 5.491 & 6.806 & 0.415 \\
\hline $3 / 22 / 10$ & $16: 00$ & 15.016 & 21.143 & 13.760 & 24.514 & 5.266 & 6.676 & 0.345 \\
\hline $3 / 22 / 10$ & $20: 00$ & 14.849 & 21.118 & 13.484 & 24.488 & 5.283 & 6.555 & 0.349 \\
\hline $3 / 23 / 10$ & $0: 00$ & 14.732 & 21.118 & 13.461 & 24.468 & 5.419 & 6.540 & 0.388 \\
\hline $3 / 23 / 10$ & $4: 00$ & 14.706 & 21.107 & 13.484 & 24.452 & 5.466 & 6.536 & 0.404 \\
\hline $3 / 23 / 10$ & $8: 00$ & 14.670 & 21.141 & 13.543 & 24.389 & 5.473 & 6.590 & 0.427 \\
\hline $3 / 23 / 10$ & $12: 00$ & 14.726 & 21.164 & 13.566 & 24.386 & 5.472 & 6.624 & 0.418 \\
\hline $3 / 23 / 10$ & $16: 00$ & 14.791 & 21.126 & 13.287 & 24.386 & 5.483 & 6.553 & 0.393 \\
\hline $3 / 23 / 10$ & $20: 00$ & 14.714 & 21.168 & 13.261 & 24.380 & 5.479 & 6.623 & 0.414 \\
\hline $3 / 24 / 10$ & $0: 00$ & 14.717 & 21.199 & 13.346 & 24.374 & 5.489 & 6.702 & 0.446 \\
\hline $3 / 24 / 10$ & $4: 00$ & 14.779 & 21.187 & 13.378 & 24.369 & 5.487 & 6.722 & 0.459 \\
\hline $3 / 24 / 10$ & $8: 00$ & 14.789 & 21.214 & 13.424 & 24.330 & 5.497 & 6.763 & 0.467 \\
\hline $3 / 24 / 10$ & $12: 00$ & 14.668 & 21.070 & 12.939 & 24.340 & 4.749 & 6.738 & 0.002 \\
\hline $3 / 24 / 10$ & $16: 00$ & 13.356 & 20.295 & 11.139 & 24.325 & 1.091 & 5.891 & -0.323 \\
\hline $3 / 24 / 10$ & 20:00 & 12.513 & 19.136 & 9.634 & 23.997 & 1.208 & 5.056 & -0.334 \\
\hline $3 / 25 / 10$ & $0: 00$ & 12.185 & 18.360 & 9.500 & 23.209 & 1.808 & 4.535 & -0.082 \\
\hline $3 / 25 / 10$ & $4: 00$ & 12.047 & 17.902 & 9.527 & 22.652 & 2.958 & 4.249 & -0.058 \\
\hline $3 / 25 / 10$ & 8:00 & 11.933 & 17.659 & 9.595 & 22.153 & 3.993 & 4.197 & -0.056 \\
\hline $3 / 25 / 10$ & $12: 00$ & 11.922 & 17.482 & 9.612 & 21.720 & 4.591 & 4.194 & -0.019 \\
\hline $3 / 25 / 10$ & $16: 00$ & 11.933 & 17.340 & 9.591 & 21.385 & 4.910 & 4.203 & 0.042 \\
\hline $3 / 25 / 10$ & $20: 00$ & 11.920 & 17.262 & 9.582 & 21.086 & 5.129 & 4.279 & 0.089 \\
\hline
\end{tabular}


TABLE S2.1 (Cont.)

\begin{tabular}{|c|c|c|c|c|c|c|c|c|}
\hline \multirow[b]{2}{*}{ Date } & \multirow[b]{2}{*}{ Time } & \multicolumn{6}{|c|}{ Depth to Water (ft TOC) } & \multirow[b]{2}{*}{ MW8s } \\
\hline & & MW1s & MW2s & MW3s & MW4s & MW6s & MW7s & \\
\hline $3 / 26 / 10$ & $0: 00$ & 11.910 & 17.208 & 9.569 & 20.818 & 5.209 & 4.359 & 0.117 \\
\hline $3 / 26 / 10$ & $4: 00$ & 11.949 & 17.147 & 9.541 & 20.565 & 5.213 & 4.420 & 0.138 \\
\hline $3 / 26 / 10$ & 8:00 & 11.990 & 17.109 & 9.516 & 20.340 & 5.292 & 4.487 & 0.158 \\
\hline $3 / 26 / 10$ & $12: 00$ & 12.102 & 17.059 & 9.476 & 20.194 & 5.290 & 4.530 & 0.160 \\
\hline $3 / 26 / 10$ & $16: 00$ & 12.212 & 16.924 & 9.346 & 20.010 & 5.306 & 4.470 & 0.170 \\
\hline $3 / 26 / 10$ & $20: 00$ & 12.188 & 16.914 & 9.327 & 19.898 & 5.290 & 4.552 & 0.200 \\
\hline $3 / 27 / 10$ & $0: 00$ & 12.172 & 16.962 & 9.376 & 19.748 & 5.318 & 4.680 & 0.219 \\
\hline $3 / 27 / 10$ & $4: 00$ & 12.259 & 16.954 & 9.364 & 19.703 & 5.321 & 4.740 & 0.225 \\
\hline $3 / 27 / 10$ & 8:00 & 12.282 & 16.947 & 9.359 & 19.628 & 5.342 & 4.786 & 0.232 \\
\hline $3 / 27 / 10$ & $12: 00$ & 12.293 & 16.962 & 9.391 & 19.576 & 5.350 & 4.857 & 0.191 \\
\hline $3 / 27 / 10$ & $16: 00$ & 11.949 & 16.727 & 8.894 & 19.546 & 3.944 & 4.814 & -0.061 \\
\hline $3 / 27 / 10$ & $20: 00$ & 11.720 & 16.670 & 8.878 & 19.293 & 4.381 & 4.738 & -0.057 \\
\hline $3 / 28 / 10$ & $0: 00$ & 11.632 & 16.637 & 8.883 & 19.160 & 4.637 & 4.691 & -0.021 \\
\hline $3 / 28 / 10$ & $4: 00$ & 11.618 & 16.607 & 8.897 & 18.987 & 4.869 & 4.370 & 0.036 \\
\hline $3 / 28 / 10$ & 8:00 & 11.614 & 16.643 & 8.960 & 18.907 & 5.038 & 4.417 & 0.073 \\
\hline $3 / 28 / 10$ & $12: 00$ & 11.693 & 16.666 & 9.004 & 18.823 & 5.119 & 4.456 & 0.105 \\
\hline $3 / 28 / 10$ & $16: 00$ & 11.795 & 16.632 & 8.979 & 18.730 & 5.177 & 4.457 & 0.133 \\
\hline $3 / 28 / 10$ & $20: 00$ & 11.819 & 16.651 & 9.004 & 18.683 & 5.227 & 4.528 & 0.166 \\
\hline $3 / 29 / 10$ & $0: 00$ & 11.861 & 16.674 & 9.031 & 18.661 & 5.224 & 4.597 & 0.187 \\
\hline $3 / 29 / 10$ & $4: 00$ & 11.918 & 16.685 & 9.042 & 18.639 & 5.255 & 4.643 & 0.197 \\
\hline $3 / 29 / 10$ & 8:00 & 11.966 & 16.708 & 9.070 & 18.614 & 5.264 & 4.690 & 0.209 \\
\hline 3/29/10 & $12: 00$ & 12.115 & 16.704 & 9.066 & 18.631 & 5.292 & 4.721 & 0.212 \\
\hline $3 / 29 / 10$ & $16: 00$ & 12.213 & 16.636 & 9.001 & 18.617 & 5.282 & 4.691 & 0.226 \\
\hline $3 / 29 / 10$ & $20: 00$ & 12.198 & 16.651 & 9.019 & 18.584 & 5.286 & 4.747 & 0.255 \\
\hline $3 / 30 / 10$ & $0: 00$ & 12.223 & 16.685 & 9.055 & 18.613 & 5.297 & 4.821 & 0.267 \\
\hline $3 / 30 / 10$ & $4: 00$ & 12.264 & 16.695 & 9.068 & 18.642 & 5.311 & 4.859 & 0.271 \\
\hline $3 / 30 / 10$ & 8:00 & 12.292 & 16.754 & 9.128 & 18.670 & 5.317 & 4.931 & 0.285 \\
\hline $3 / 30 / 10$ & $12: 00$ & 12.389 & 16.768 & 9.149 & 18.704 & 5.322 & 4.970 & 0.294 \\
\hline $3 / 30 / 10$ & $16: 00$ & 12.479 & 16.743 & 9.139 & 18.710 & 5.369 & 4.972 & 0.310 \\
\hline $3 / 30 / 10$ & $20: 00$ & 12.474 & 16.766 & 9.178 & 18.714 & 5.303 & 5.026 & 0.329 \\
\hline $3 / 31 / 10$ & $0: 00$ & 12.502 & 16.812 & 9.235 & 18.780 & 5.303 & 5.098 & 0.343 \\
\hline $3 / 31 / 10$ & $4: 00$ & 12.559 & 16.827 & 9.268 & 18.791 & 5.316 & 5.128 & 0.347 \\
\hline $3 / 31 / 10$ & 8:00 & 12.530 & 16.951 & 9.408 & 18.790 & 5.318 & 5.265 & 0.367 \\
\hline $3 / 31 / 10$ & $12: 00$ & 12.610 & 17.081 & 9.544 & 18.894 & 5.314 & 5.392 & 0.381 \\
\hline $3 / 31 / 10$ & $16: 00$ & 12.729 & 17.111 & 9.600 & 18.983 & 5.338 & 5.423 & 0.389 \\
\hline $3 / 31 / 10$ & $20: 00$ & 12.765 & 17.151 & 9.677 & 19.077 & 5.295 & 5.479 & 0.398 \\
\hline $4 / 1 / 10$ & $0: 00$ & 12.802 & 17.205 & 9.765 & 19.126 & 5.310 & 5.538 & 0.405 \\
\hline $4 / 1 / 10$ & $4: 00$ & 12.902 & 17.212 & 9.809 & 19.215 & 5.349 & 5.542 & 0.402 \\
\hline $4 / 1 / 10$ & $8: 00$ & 12.941 & 17.258 & 9.892 & 19.258 & 5.324 & 5.587 & 0.406 \\
\hline $4 / 1 / 10$ & $12: 00$ & 13.031 & 17.310 & 9.974 & 19.377 & 5.341 & 5.631 & 0.410 \\
\hline $4 / 1 / 10$ & $16: 00$ & 13.127 & 17.289 & 9.988 & 19.433 & 5.314 & 5.620 & 0.416 \\
\hline $4 / 1 / 10$ & $20: 00$ & 13.087 & 17.354 & 10.093 & 19.469 & 5.341 & 5.691 & 0.434 \\
\hline $4 / 2 / 10$ & $0: 00$ & 13.144 & 17.382 & 10.149 & 19.565 & 5.349 & 5.717 & 0.434 \\
\hline $4 / 2 / 10$ & $4: 00$ & 13.185 & 17.443 & 10.241 & 19.630 & 5.364 & 5.778 & 0.445 \\
\hline $4 / 2 / 10$ & $8: 00$ & 13.192 & 17.522 & 10.356 & 19.716 & 5.374 & 5.850 & 0.454 \\
\hline $4 / 2 / 10$ & $12: 00$ & 13.227 & 17.545 & 10.454 & 19.819 & 5.362 & 5.847 & 0.413 \\
\hline $4 / 2 / 10$ & $16: 00$ & 13.282 & 17.569 & 10.535 & 19.917 & 5.374 & 5.813 & 0.403 \\
\hline $4 / 2 / 10$ & $20: 00$ & 13.235 & 17.669 & 10.674 & 19.976 & 5.374 & 5.917 & 0.432 \\
\hline
\end{tabular}


TABLE S2.1 (Cont.)

\begin{tabular}{|c|c|c|c|c|c|c|c|c|}
\hline \multirow[b]{2}{*}{ Date } & \multirow[b]{2}{*}{ Time } & \multicolumn{6}{|c|}{ Depth to Water (ft TOC) } & \multirow[b]{2}{*}{ MW8s } \\
\hline & & MW1s & MW2s & MW3s & MW4s & MW6s & MW7s & \\
\hline $4 / 3 / 10$ & $0: 00$ & 13.222 & 17.795 & 10.827 & 20.090 & 5.390 & 6.043 & 0.453 \\
\hline $4 / 3 / 10$ & $4: 00$ & 13.315 & 17.833 & 10.912 & 20.209 & 5.459 & 6.077 & 0.456 \\
\hline $4 / 3 / 10$ & 8:00 & 13.372 & 17.898 & 11.017 & 20.316 & 5.393 & 6.135 & 0.464 \\
\hline $4 / 3 / 10$ & $12: 00$ & 13.515 & 17.925 & 11.085 & 20.423 & 5.385 & 6.149 & 0.466 \\
\hline $4 / 3 / 10$ & $16: 00$ & 13.624 & 17.904 & 11.112 & 20.535 & 5.368 & 6.127 & 0.466 \\
\hline $4 / 3 / 10$ & $20: 00$ & 13.631 & 17.938 & 11.182 & 20.584 & 5.385 & 6.155 & 0.476 \\
\hline $4 / 4 / 10$ & $0: 00$ & 13.641 & 17.980 & 11.260 & 20.659 & 5.360 & 6.190 & 0.480 \\
\hline $4 / 4 / 10$ & $4: 00$ & 13.684 & 18.009 & 11.329 & 20.748 & 5.411 & 6.203 & 0.483 \\
\hline $4 / 4 / 10$ & 8:00 & 13.676 & 18.085 & 11.432 & 20.794 & 5.411 & 6.274 & 0.488 \\
\hline $4 / 4 / 10$ & $12: 00$ & 13.791 & 18.102 & 11.478 & 20.911 & 5.372 & 6.281 & 0.485 \\
\hline $4 / 4 / 10$ & $16: 00$ & 13.874 & 18.114 & 11.535 & 20.977 & 5.411 & 6.287 & 0.488 \\
\hline $4 / 4 / 10$ & $20: 00$ & 13.804 & 18.228 & 11.663 & 21.034 & 5.403 & 6.396 & 0.507 \\
\hline $4 / 5 / 10$ & $0: 00$ & 13.807 & 18.314 & 11.763 & 21.124 & 5.404 & 6.473 & 0.514 \\
\hline $4 / 5 / 10$ & $4: 00$ & 13.881 & 18.356 & 11.843 & 21.204 & 5.404 & 6.500 & 0.517 \\
\hline $4 / 5 / 10$ & 8:00 & 13.968 & 18.360 & 11.886 & 21.281 & 5.383 & 6.491 & 0.510 \\
\hline $4 / 5 / 10$ & $12: 00$ & 14.082 & 18.353 & 11.900 & 21.350 & 5.393 & 6.439 & 0.499 \\
\hline $4 / 5 / 10$ & $16: 00$ & 14.212 & 18.255 & 11.851 & 21.412 & 5.369 & 6.331 & 0.478 \\
\hline $4 / 5 / 10$ & $20: 00$ & 14.153 & 18.316 & 11.932 & 21.402 & 5.373 & 6.380 & 0.493 \\
\hline $4 / 6 / 10$ & $0: 00$ & 14.148 & 18.351 & 11.986 & 21.487 & 5.377 & 6.398 & 0.495 \\
\hline $4 / 6 / 10$ & $4: 00$ & 14.198 & 18.312 & 11.990 & 21.512 & 5.358 & 6.346 & 0.488 \\
\hline $4 / 6 / 10$ & $8: 00$ & 14.163 & 18.375 & 12.059 & 21.559 & 5.367 & 6.389 & 0.493 \\
\hline $4 / 6 / 10$ & $12: 00$ & 14.227 & 18.423 & 12.113 & 21.600 & 5.417 & 6.419 & 0.498 \\
\hline $4 / 6 / 10$ & $16: 00$ & 14.215 & & 12.149 & 21.910 & 5.796 & 6.484 & 0.531 \\
\hline $4 / 6 / 10$ & $20: 00$ & 14.134 & 18.589 & 12.288 & 21.754 & 5.470 & 6.612 & 0.546 \\
\hline $4 / 7 / 10$ & $0: 00$ & 14.153 & 18.671 & 12.377 & 21.736 & 5.421 & 6.686 & 0.550 \\
\hline $4 / 7 / 10$ & $4: 00$ & 14.225 & 18.702 & 12.429 & 21.753 & 5.400 & 6.712 & 0.548 \\
\hline $4 / 7 / 10$ & $8: 00$ & 14.246 & 18.797 & 12.531 & 21.758 & 5.377 & 6.782 & 0.561 \\
\hline $4 / 7 / 10$ & $12: 00$ & & 18.906 & 12.632 & 21.789 & 5.394 & 6.864 & 0.565 \\
\hline $4 / 7 / 10$ & $16: 00$ & 15.095 & 19.000 & 12.726 & 21.907 & 5.375 & 6.937 & 0.571 \\
\hline $4 / 7 / 10$ & $20: 00$ & 14.505 & 19.053 & 12.788 & 22.003 & 5.416 & 6.963 & 0.570 \\
\hline $4 / 8 / 10$ & $0: 00$ & 14.487 & 19.118 & 12.854 & 22.056 & 5.417 & 7.004 & 0.570 \\
\hline $4 / 8 / 10$ & $4: 00$ & 14.526 & 19.147 & 12.899 & 22.149 & 5.416 & 7.004 & 0.568 \\
\hline $4 / 8 / 10$ & 8:00 & 14.564 & 19.204 & 12.959 & 22.177 & 5.403 & 7.030 & 0.574 \\
\hline $4 / 8 / 10$ & $12: 00$ & 14.648 & 19.242 & 13.006 & 22.250 & 5.411 & 7.037 & 0.576 \\
\hline $4 / 8 / 10$ & $16: 00$ & 14.745 & 19.240 & 13.017 & 22.293 & 5.423 & 7.007 & 0.571 \\
\hline $4 / 8 / 10$ & $20: 00$ & 14.738 & 19.280 & 13.065 & 22.362 & 5.425 & 7.030 & 0.575 \\
\hline $4 / 9 / 10$ & $0: 00$ & 14.704 & 19.340 & 13.122 & 22.372 & 5.440 & 7.057 & 0.580 \\
\hline $4 / 9 / 10$ & $4: 00$ & 14.739 & 19.380 & 13.168 & 22.441 & 5.400 & 7.070 & 0.581 \\
\hline $4 / 9 / 10$ & 8:00 & 14.754 & 19.445 & 13.227 & 22.487 & 5.429 & 7.106 & 0.584 \\
\hline $4 / 9 / 10$ & $12: 00$ & 14.853 & 19.504 & 13.273 & 22.504 & 5.423 & 7.122 & 0.588 \\
\hline $4 / 9 / 10$ & $16: 00$ & 14.928 & 19.506 & 13.289 & 22.552 & 5.432 & 7.104 & 0.591 \\
\hline $4 / 9 / 10$ & $20: 00$ & 14.901 & 19.557 & 13.340 & 22.602 & 5.436 & 7.145 & 0.596 \\
\hline $4 / 10 / 10$ & $0: 00$ & 14.872 & 19.641 & 13.409 & 22.638 & 5.428 & 7.204 & 0.600 \\
\hline $4 / 10 / 10$ & $4: 00$ & 14.907 & 19.691 & 13.457 & 22.681 & 5.467 & 7.230 & 0.601 \\
\hline $4 / 10 / 10$ & $8: 00$ & 14.927 & 19.771 & 13.523 & 22.715 & 5.436 & 7.280 & 0.607 \\
\hline $4 / 10 / 10$ & $12: 00$ & 15.041 & 19.800 & 13.555 & 22.757 & 5.444 & 7.286 & 0.607 \\
\hline $4 / 10 / 10$ & $16: 00$ & 15.131 & 19.796 & 13.565 & 22.797 & 5.436 & 7.262 & 0.605 \\
\hline $4 / 10 / 10$ & $20: 00$ & 15.076 & 19.871 & 13.630 & 22.865 & 5.432 & 7.301 & 0.611 \\
\hline
\end{tabular}


TABLE S2.1 (Cont.)

\begin{tabular}{|c|c|c|c|c|c|c|c|c|}
\hline \multirow[b]{2}{*}{ Date } & \multirow[b]{2}{*}{ Time } & \multicolumn{6}{|c|}{ Depth to Water (ft TOC) } & \multirow[b]{2}{*}{ MW8s } \\
\hline & & MW1s & MW2s & MW3s & MW4s & MW6s & MW7s & \\
\hline $4 / 11 / 10$ & $0: 00$ & 15.081 & 19.957 & 13.693 & 22.898 & 5.459 & 7.375 & 0.616 \\
\hline $4 / 11 / 10$ & $4: 00$ & 15.117 & 19.999 & 13.736 & 22.918 & 5.451 & 7.394 & 0.614 \\
\hline $4 / 11 / 10$ & $8: 00$ & 15.140 & 20.077 & 13.800 & 22.970 & 5.488 & 7.440 & 0.621 \\
\hline $4 / 11 / 10$ & $12: 00$ & 15.241 & 20.115 & 13.836 & 22.987 & 5.480 & 7.455 & 0.620 \\
\hline 4/11/10 & $16: 00$ & 15.326 & 20.115 & 13.846 & 23.082 & 5.436 & 7.433 & 0.619 \\
\hline $4 / 11 / 10$ & $20: 00$ & 15.313 & 20.174 & 13.887 & 23.125 & 5.453 & 7.464 & 0.621 \\
\hline $4 / 12 / 10$ & $0: 00$ & 15.320 & 20.218 & 13.928 & 23.165 & 5.459 & 7.485 & 0.616 \\
\hline $4 / 12 / 10$ & $4: 00$ & 15.364 & 20.251 & 13.962 & 23.204 & 5.457 & 7.492 & 0.614 \\
\hline $4 / 12 / 10$ & $8: 00$ & 15.386 & 20.310 & 14.006 & 23.233 & 5.464 & 7.518 & 0.620 \\
\hline $4 / 12 / 10$ & $12: 00$ & 15.520 & 20.342 & 14.035 & 23.256 & 5.639 & 7.523 & 0.625 \\
\hline $4 / 12 / 10$ & $16: 00$ & 15.612 & 20.352 & 14.051 & 23.306 & 5.818 & 7.514 & 0.626 \\
\hline $4 / 12 / 10$ & $20: 00$ & 15.624 & 20.411 & 14.092 & 23.351 & 6.040 & 7.549 & 0.630 \\
\hline $4 / 13 / 10$ & $0: 00$ & 15.622 & 20.459 & 14.136 & 23.390 & 6.253 & 7.583 & 0.627 \\
\hline $4 / 13 / 10$ & $4: 00$ & 15.704 & 20.493 & 14.169 & 23.419 & 6.465 & 7.598 & 0.626 \\
\hline $4 / 13 / 10$ & $8: 00$ & 15.736 & 20.579 & 14.233 & 23.426 & 6.666 & 7.655 & 0.637 \\
\hline $4 / 13 / 10$ & $12: 00$ & 15.868 & 20.600 & 14.263 & 23.529 & 6.852 & 7.661 & 0.639 \\
\hline $4 / 13 / 10$ & $16: 00$ & 15.974 & 20.621 & 14.283 & 23.588 & 7.050 & 7.666 & 0.648 \\
\hline $4 / 13 / 10$ & $20: 00$ & 16.005 & 20.665 & 14.319 & 23.608 & 7.315 & 7.704 & 0.651 \\
\hline $4 / 14 / 10$ & 0:00 & 16.017 & 20.745 & 14.378 & 23.630 & 7.683 & 7.763 & 0.652 \\
\hline $4 / 14 / 10$ & $4: 00$ & 16.072 & 20.803 & 14.429 & 23.694 & 8.103 & 7.808 & 0.654 \\
\hline $4 / 14 / 10$ & $8: 00$ & 16.129 & 20.879 & 14.486 & 23.761 & 8.378 & 7.860 & 0.663 \\
\hline $4 / 14 / 10$ & $12: 00$ & 16.211 & 20.908 & 14.521 & 23.832 & 8.151 & 7.876 & 0.667 \\
\hline $4 / 14 / 10$ & $16: 00$ & 16.285 & 20.910 & 14.538 & 23.872 & 7.347 & 7.869 & 0.675 \\
\hline $4 / 14 / 10$ & $20: 00$ & 16.251 & 20.959 & 14.576 & 23.927 & 6.843 & 7.906 & 0.679 \\
\hline $4 / 15 / 10$ & 0:00 & 16.229 & 21.017 & 14.624 & 23.968 & 6.512 & 7.945 & 0.674 \\
\hline $4 / 15 / 10$ & $4: 00$ & 16.239 & 21.047 & 14.657 & 23.972 & 6.295 & 7.960 & 0.667 \\
\hline $4 / 15 / 10$ & $8: 00$ & 16.244 & 21.114 & 14.709 & 24.033 & 6.101 & 8.001 & 0.673 \\
\hline $4 / 15 / 10$ & $12: 00$ & 16.320 & 21.154 & 14.740 & 24.056 & 5.959 & 8.021 & 0.684 \\
\hline $4 / 15 / 10$ & $16: 00$ & 16.383 & 21.154 & 14.757 & 24.092 & 5.866 & 8.004 & 0.691 \\
\hline $4 / 15 / 10$ & $20: 00$ & 16.264 & 21.242 & 14.816 & 24.117 & 5.785 & 8.081 & 0.672 \\
\hline $4 / 16 / 10$ & $0: 00$ & 16.249 & 21.221 & 14.850 & 24.170 & 5.694 & 8.032 & 0.615 \\
\hline $4 / 16 / 10$ & $4: 00$ & 16.141 & 21.152 & 14.865 & 24.178 & 5.585 & 7.808 & 0.560 \\
\hline $4 / 16 / 10$ & $8: 00$ & 15.976 & 21.158 & 14.889 & 24.225 & 5.525 & 7.707 & 0.530 \\
\hline $4 / 16 / 10$ & $12: 00$ & 15.971 & 21.168 & 14.894 & 24.254 & 5.492 & 7.717 & 0.534 \\
\hline $4 / 16 / 10$ & $16: 00$ & 15.974 & 21.173 & 14.895 & 24.305 & 5.442 & 7.735 & 0.549 \\
\hline $4 / 16 / 10$ & $20: 00$ & 15.957 & 21.187 & 14.902 & 24.350 & 5.397 & 7.753 & 0.559 \\
\hline $4 / 17 / 10$ & $0: 00$ & 15.917 & 21.238 & 14.933 & 24.365 & 5.399 & 7.800 & 0.569 \\
\hline $4 / 17 / 10$ & $4: 00$ & 15.936 & 21.261 & 14.956 & 24.391 & 5.383 & 7.813 & 0.574 \\
\hline $4 / 17 / 10$ & $8: 00$ & 15.919 & 21.326 & 14.999 & 24.362 & 5.354 & 7.869 & 0.588 \\
\hline $4 / 17 / 10$ & $12: 00$ & 16.006 & 21.351 & 15.024 & 24.440 & 5.346 & 7.878 & 0.591 \\
\hline $4 / 17 / 10$ & $16: 00$ & 16.076 & 21.341 & 15.024 & 24.440 & 5.334 & 7.859 & 0.592 \\
\hline $4 / 17 / 10$ & $20: 00$ & 16.046 & 21.383 & 15.057 & 24.455 & 5.323 & 7.895 & 0.599 \\
\hline $4 / 18 / 10$ & $0: 00$ & 16.019 & 21.431 & 15.090 & 24.429 & 5.325 & 7.932 & 0.602 \\
\hline $4 / 18 / 10$ & $4: 00$ & 16.036 & 21.469 & 15.125 & 24.471 & 5.288 & 7.958 & 0.605 \\
\hline $4 / 18 / 10$ & $8: 00$ & 16.036 & 21.536 & 15.176 & 24.453 & 5.303 & 8.014 & 0.615 \\
\hline $4 / 18 / 10$ & $12: 00$ & 16.130 & 21.561 & 15.201 & 24.531 & 5.334 & 8.023 & 0.622 \\
\hline $4 / 18 / 10$ & $16: 00$ & 16.212 & 21.555 & 15.210 & 24.564 & 5.288 & 8.010 & 0.623 \\
\hline $4 / 18 / 10$ & $20: 00$ & 16.185 & 21.599 & 15.246 & 24.532 & 5.284 & 8.047 & 0.627 \\
\hline
\end{tabular}


TABLE S2.1 (Cont.)

\begin{tabular}{|c|c|c|c|c|c|c|c|c|}
\hline \multirow[b]{2}{*}{ Date } & \multirow[b]{2}{*}{ Time } & \multicolumn{6}{|c|}{ Depth to Water (ft TOC) } & \multirow[b]{2}{*}{ MW8s } \\
\hline & & MW1s & MW2s & MW3s & MW4s & MW6s & MW7s & \\
\hline $4 / 19 / 10$ & $0: 00$ & 16.170 & 21.647 & 15.282 & 24.575 & 5.288 & 8.079 & 0.628 \\
\hline $4 / 19 / 10$ & $4: 00$ & 16.198 & 21.681 & 15.309 & 24.595 & 5.247 & 8.097 & 0.629 \\
\hline $4 / 19 / 10$ & 8:00 & 16.205 & 21.744 & 15.353 & 24.608 & 5.262 & 8.142 & 0.637 \\
\hline $4 / 19 / 10$ & $12: 00$ & 16.307 & 21.760 & 15.375 & 24.628 & 5.245 & 8.142 & 0.639 \\
\hline $4 / 19 / 10$ & $16: 00$ & 16.377 & 21.760 & 15.386 & 24.652 & 5.250 & 8.119 & 0.645 \\
\hline $4 / 19 / 10$ & $20: 00$ & 16.367 & 21.798 & 15.418 & 24.707 & 5.256 & 8.160 & 0.646 \\
\hline $4 / 20 / 10$ & $0: 00$ & 16.325 & 21.851 & 15.456 & 24.716 & 5.254 & 8.197 & 0.648 \\
\hline $4 / 20 / 10$ & $4: 00$ & 16.352 & 21.878 & 15.479 & 24.697 & 5.283 & 8.209 & 0.645 \\
\hline $4 / 20 / 10$ & 8:00 & 16.372 & 21.922 & 15.521 & 24.735 & 5.258 & 8.233 & 0.649 \\
\hline $4 / 20 / 10$ & $12: 00$ & 16.466 & 21.951 & 15.549 & 24.762 & 5.227 & 8.242 & 0.657 \\
\hline 4/20/10 & $16: 00$ & 16.520 & 21.964 & 15.567 & 24.782 & 5.250 & 8.238 & 0.665 \\
\hline $4 / 20 / 10$ & $20: 00$ & 16.508 & 22.006 & 15.606 & 24.793 & 5.258 & 8.264 & 0.664 \\
\hline $4 / 21 / 10$ & $0: 00$ & 16.511 & 22.042 & 15.651 & 24.809 & 5.252 & 8.287 & 0.659 \\
\hline $4 / 21 / 10$ & $4: 00$ & 16.533 & 22.071 & 15.692 & 24.829 & 5.287 & 8.298 & 0.657 \\
\hline $4 / 21 / 10$ & 8:00 & 16.541 & 22.121 & 15.733 & 24.810 & 5.231 & 8.327 & 0.661 \\
\hline $4 / 21 / 10$ & $12: 00$ & 16.586 & 22.159 & 15.771 & 24.875 & 5.246 & 8.342 & 0.662 \\
\hline $4 / 21 / 10$ & $16: 00$ & 16.657 & 22.170 & 15.791 & 24.901 & 5.217 & 8.331 & 0.668 \\
\hline $4 / 21 / 10$ & $20: 00$ & 16.663 & 22.210 & 15.842 & 24.930 & 5.248 & 8.353 & 0.671 \\
\hline $4 / 22 / 10$ & $0: 00$ & 16.637 & 22.258 & 15.942 & 24.940 & 5.242 & 8.387 & 0.672 \\
\hline $4 / 22 / 10$ & $4: 00$ & 16.670 & 22.281 & 15.986 & 24.968 & 5.256 & 8.392 & 0.670 \\
\hline $4 / 22 / 10$ & 8:00 & 16.687 & 22.333 & 16.050 & 24.953 & 5.211 & 8.420 & 0.676 \\
\hline $4 / 22 / 10$ & $12: 00$ & 16.709 & 22.380 & 16.110 & 25.028 & 5.252 & 8.448 & 0.675 \\
\hline $4 / 22 / 10$ & $16: 00$ & 16.782 & 22.375 & 16.116 & 25.063 & 5.223 & 8.420 & 0.666 \\
\hline $4 / 22 / 10$ & $20: 00$ & 16.802 & 22.400 & 16.156 & 25.081 & 5.223 & 8.420 & 0.665 \\
\hline $4 / 23 / 10$ & 0:00 & 16.797 & 22.442 & 16.209 & 25.098 & 5.232 & 8.442 & 0.670 \\
\hline $4 / 23 / 10$ & $4: 00$ & 16.792 & 22.365 & 16.170 & 25.150 & 5.194 & 8.273 & 0.574 \\
\hline $4 / 23 / 10$ & 8:00 & 16.556 & 22.170 & 16.041 & 25.143 & 5.121 & 7.863 & -0.105 \\
\hline $4 / 23 / 10$ & $12: 00$ & 15.869 & 21.911 & 15.603 & 25.117 & 4.647 & 7.426 & -0.057 \\
\hline $4 / 23 / 10$ & $16: 00$ & 15.532 & 21.788 & 15.512 & 25.111 & 4.898 & 7.328 & 0.016 \\
\hline $4 / 23 / 10$ & $20: 00$ & 15.353 & 21.752 & 15.479 & 25.180 & 4.997 & 7.365 & 0.134 \\
\hline $4 / 24 / 10$ & 0:00 & 15.217 & 21.760 & 15.472 & 25.147 & 5.110 & 7.432 & 0.198 \\
\hline $4 / 24 / 10$ & $4: 00$ & 15.203 & 21.733 & 15.452 & 25.095 & 5.104 & 7.450 & 0.229 \\
\hline $4 / 24 / 10$ & 8:00 & 15.196 & 21.741 & 15.449 & 25.100 & 5.123 & 7.491 & 0.257 \\
\hline $4 / 24 / 10$ & $12: 00$ & 15.251 & 21.741 & 15.451 & 25.037 & 5.117 & 7.517 & 0.283 \\
\hline $4 / 24 / 10$ & $16: 00$ & 15.300 & 21.725 & 15.440 & 25.045 & 5.158 & 7.528 & 0.302 \\
\hline $4 / 24 / 10$ & $20: 00$ & 15.298 & 21.744 & 15.455 & 25.000 & 5.195 & 7.575 & 0.324 \\
\hline $4 / 25 / 10$ & $0: 00$ & 15.298 & 21.792 & 15.484 & 24.993 & 5.144 & 7.649 & 0.347 \\
\hline $4 / 25 / 10$ & $4: 00$ & 15.344 & 21.821 & 15.514 & 24.990 & 5.187 & 7.699 & 0.366 \\
\hline $4 / 25 / 10$ & $8: 00$ & 15.365 & 21.882 & 15.564 & 24.991 & 5.222 & 7.779 & 0.389 \\
\hline $4 / 25 / 10$ & $12: 00$ & 15.420 & 21.924 & 15.611 & 25.056 & 5.173 & 7.837 & 0.401 \\
\hline $4 / 25 / 10$ & $16: 00$ & 15.490 & 21.949 & 15.644 & 25.089 & 5.167 & 7.880 & 0.420 \\
\hline $4 / 25 / 10$ & $20: 00$ & 15.533 & 21.998 & 15.702 & 25.086 & 5.175 & 7.941 & 0.440 \\
\hline $4 / 26 / 10$ & $0: 00$ & 15.595 & 22.037 & 15.749 & 25.130 & 5.163 & 7.997 & 0.449 \\
\hline $4 / 26 / 10$ & $4: 00$ & 15.660 & 22.065 & 15.784 & 25.157 & 5.185 & 8.037 & 0.458 \\
\hline $4 / 26 / 10$ & 8:00 & 15.724 & 22.113 & 15.835 & 25.195 & 5.179 & 8.091 & 0.472 \\
\hline $4 / 26 / 10$ & $12: 00$ & 15.816 & 22.126 & 15.896 & 25.198 & 5.181 & 8.123 & 0.480 \\
\hline $4 / 26 / 10$ & $16: 00$ & 15.898 & 22.115 & 15.940 & 25.256 & 5.151 & 8.125 & 0.488 \\
\hline $4 / 26 / 10$ & $20: 00$ & 15.920 & 22.159 & 16.007 & 25.223 & 5.198 & 8.173 & 0.501 \\
\hline
\end{tabular}


TABLE S2.1 (Cont.)

\begin{tabular}{|c|c|c|c|c|c|c|c|c|}
\hline \multirow[b]{2}{*}{ Date } & \multirow[b]{2}{*}{ Time } & \multicolumn{6}{|c|}{ Depth to Water (ft TOC) } & \multirow[b]{2}{*}{ MW8s } \\
\hline & & MW1s & MW2s & MW3s & MW4s & MW6s & MW7s & \\
\hline $4 / 27 / 10$ & $0: 00$ & 15.968 & 22.174 & 16.049 & 25.236 & 5.182 & 8.197 & 0.507 \\
\hline $4 / 27 / 10$ & $4: 00$ & 15.990 & 22.224 & 16.127 & 25.263 & 5.196 & 8.255 & 0.518 \\
\hline $4 / 27 / 10$ & 8:00 & 16.025 & 22.275 & 16.206 & 25.260 & 5.184 & 8.313 & 0.531 \\
\hline $4 / 27 / 10$ & $12: 00$ & 16.112 & 22.312 & 16.268 & 25.312 & 5.182 & 8.357 & 0.547 \\
\hline $4 / 27 / 10$ & $16: 00$ & 16.194 & 22.304 & 16.273 & 25.291 & 5.190 & 8.345 & 0.552 \\
\hline $4 / 27 / 10$ & $20: 00$ & 16.256 & 22.308 & 16.291 & 25.330 & 5.186 & 8.370 & 0.556 \\
\hline $4 / 28 / 10$ & $0: 00$ & 16.258 & & 16.331 & 25.343 & 5.194 & 8.396 & 0.553 \\
\hline $4 / 28 / 10$ & $4: 00$ & 16.301 & & 16.353 & 25.374 & 5.176 & 8.405 & 0.557 \\
\hline $4 / 28 / 10$ & 8:00 & 16.343 & & 16.381 & 25.374 & 5.178 & 8.414 & 0.563 \\
\hline $4 / 28 / 10$ & $12: 00$ & 16.468 & & 16.368 & 25.380 & 5.188 & 8.392 & 0.566 \\
\hline $4 / 28 / 10$ & $16: 00$ & 16.539 & & 16.337 & 25.370 & 5.341 & 8.364 & 0.604 \\
\hline $4 / 28 / 10$ & $20: 00$ & 16.515 & 22.278 & 16.363 & 24.983 & 5.310 & 8.375 & 0.604 \\
\hline $4 / 29 / 10$ & $0: 00$ & 16.514 & 22.274 & 16.364 & 25.090 & 5.269 & 8.373 & 0.598 \\
\hline $4 / 29 / 10$ & $4: 00$ & 16.550 & 22.249 & 16.357 & 25.175 & 5.230 & 8.349 & 0.591 \\
\hline $4 / 29 / 10$ & 8:00 & 16.530 & 22.278 & 16.401 & 25.225 & 5.246 & 8.379 & 0.601 \\
\hline $4 / 29 / 10$ & $12: 00$ & 16.537 & 22.326 & 16.454 & 25.286 & 5.224 & 8.425 & 0.624 \\
\hline $4 / 29 / 10$ & $16: 00$ & 16.580 & 22.341 & 16.477 & 25.347 & 5.278 & 8.438 & 0.641 \\
\hline 4/29/10 & $20: 00$ & 16.587 & 22.339 & 16.495 & 25.387 & 5.239 & 8.448 & 0.636 \\
\hline $4 / 30 / 10$ & $0: 00$ & 16.589 & 22.364 & 16.522 & 25.406 & 5.230 & 8.472 & 0.633 \\
\hline $4 / 30 / 10$ & $4: 00$ & 16.502 & 22.444 & 16.664 & 25.433 & 5.270 & 8.520 & 0.604 \\
\hline $4 / 30 / 10$ & 8:00 & 16.441 & 22.457 & 16.705 & 25.469 & 5.237 & 8.513 & 0.608 \\
\hline $4 / 30 / 10$ & $12: 00$ & 16.500 & 22.448 & 16.719 & 25.517 & 5.222 & 8.492 & 0.607 \\
\hline $4 / 30 / 10$ & $16: 00$ & 16.572 & 22.457 & 16.744 & 25.557 & 5.251 & 8.516 & 0.620 \\
\hline $4 / 30 / 10$ & $20: 00$ & 16.550 & 22.509 & 16.805 & 25.581 & 5.226 & 8.576 & 0.635 \\
\hline $5 / 1 / 10$ & $0: 00$ & 16.547 & 22.566 & 16.887 & 25.616 & 5.235 & 8.635 & 0.644 \\
\hline $5 / 1 / 10$ & $4: 00$ & 16.587 & 22.606 & 16.931 & 25.654 & 5.282 & 8.674 & 0.650 \\
\hline $5 / 1 / 10$ & 8:00 & 16.632 & 22.648 & 16.986 & 25.696 & 5.270 & 8.711 & 0.658 \\
\hline $5 / 1 / 10$ & $12: 00$ & 16.741 & 22.671 & 17.005 & 25.742 & 5.332 & 8.730 & 0.666 \\
\hline $5 / 1 / 10$ & $16: 00$ & 16.850 & 22.648 & 16.972 & 25.828 & 5.250 & 8.702 & 0.669 \\
\hline $5 / 1 / 10$ & $20: 00$ & 16.841 & 22.681 & 17.009 & 25.827 & 5.256 & 8.735 & 0.671 \\
\hline $5 / 2 / 10$ & 0:00 & 16.868 & 22.702 & 17.031 & 25.862 & 5.254 & 8.746 & 0.666 \\
\hline $5 / 2 / 10$ & $4: 00$ & 16.900 & 22.719 & 17.058 & 25.898 & 5.266 & 8.752 & 0.665 \\
\hline $5 / 2 / 10$ & 8:00 & 16.849 & 22.788 & 17.136 & 25.917 & 5.240 & 8.811 & 0.680 \\
\hline $5 / 2 / 10$ & $12: 00$ & 16.967 & 22.807 & 17.161 & 25.938 & 5.266 & 8.826 & 0.687 \\
\hline $5 / 2 / 10$ & $16: 00$ & 17.025 & 22.828 & 17.171 & 25.948 & 5.266 & 8.836 & 0.704 \\
\hline $5 / 2 / 10$ & $20: 00$ & 17.002 & 22.902 & 17.249 & 25.987 & 5.266 & 8.897 & 0.716 \\
\hline $5 / 3 / 10$ & $0: 00$ & 16.987 & 22.959 & 17.323 & 26.015 & 5.281 & 8.951 & 0.713 \\
\hline $5 / 3 / 10$ & $4: 00$ & 17.010 & 22.994 & 17.359 & 26.035 & 5.289 & 8.967 & 0.712 \\
\hline $5 / 3 / 10$ & 8:00 & 17.049 & 23.067 & 17.439 & 26.060 & 5.320 & 9.038 & 0.722 \\
\hline $5 / 3 / 10$ & $12: 00$ & 17.163 & 23.101 & 17.476 & 26.111 & 5.304 & 9.056 & 0.732 \\
\hline $5 / 3 / 10$ & $16: 00$ & 17.278 & 23.089 & 17.451 & 26.173 & 5.287 & 9.034 & 0.737 \\
\hline $5 / 3 / 10$ & $20: 00$ & 17.271 & 23.141 & 17.481 & 26.197 & 5.318 & 9.062 & 0.742 \\
\hline $5 / 4 / 10$ & $0: 00$ & 17.303 & 23.148 & 17.485 & 26.230 & 5.312 & 9.064 & 0.727 \\
\hline $5 / 4 / 10$ & $4: 00$ & 17.354 & 23.145 & 17.476 & 26.262 & 5.271 & 9.051 & 0.716 \\
\hline $5 / 4 / 10$ & $8: 00$ & 17.367 & 23.183 & 17.504 & 26.278 & 5.302 & 9.075 & 0.723 \\
\hline $5 / 4 / 10$ & $12: 00$ & 17.457 & 23.190 & 17.506 & 26.297 & 5.310 & 9.069 & 0.738 \\
\hline $5 / 4 / 10$ & $16: 00$ & 17.533 & 23.175 & 17.472 & 26.310 & 5.316 & 9.038 & 0.756 \\
\hline $5 / 4 / 10$ & $20: 00$ & 17.524 & 23.227 & 17.506 & 26.297 & 5.481 & 9.077 & 0.774 \\
\hline
\end{tabular}


TABLE S2.1 (Cont.)

\begin{tabular}{|c|c|c|c|c|c|c|c|c|}
\hline \multirow[b]{2}{*}{ Date } & \multirow[b]{2}{*}{ Time } & \multicolumn{6}{|c|}{ Depth to Water (ft TOC) } & \multirow[b]{2}{*}{ MW8s } \\
\hline & & MW1s & MW2s & MW3s & MW4s & MW6s & MW7s & \\
\hline $5 / 5 / 10$ & $0: 00$ & 17.444 & 23.334 & 17.633 & 26.289 & 5.640 & 9.185 & 0.775 \\
\hline $5 / 5 / 10$ & $4: 00$ & 17.524 & 23.357 & 17.664 & 26.339 & 5.830 & 9.201 & 0.759 \\
\hline $5 / 5 / 10$ & $8: 00$ & 17.553 & 23.429 & 17.731 & 26.371 & 5.978 & 9.266 & 0.769 \\
\hline $5 / 5 / 10$ & $12: 00$ & 17.682 & 23.460 & 17.765 & 26.426 & 6.201 & 9.289 & 0.782 \\
\hline $5 / 5 / 10$ & $16: 00$ & 17.744 & 23.467 & 17.764 & 26.470 & 6.176 & 9.291 & 0.794 \\
\hline $5 / 5 / 10$ & $20: 00$ & 17.745 & 23.515 & 17.804 & 26.443 & 6.045 & 9.331 & 0.803 \\
\hline $5 / 6 / 10$ & 0:00 & 17.770 & 23.567 & 17.855 & 26.485 & 6.006 & 9.378 & 0.795 \\
\hline $5 / 6 / 10$ & $4: 00$ & 17.861 & 23.578 & 17.858 & 26.537 & 6.133 & 9.380 & 0.786 \\
\hline $5 / 6 / 10$ & $8: 00$ & 17.941 & 23.605 & 17.867 & 26.585 & 6.327 & 9.394 & 0.780 \\
\hline $5 / 6 / 10$ & $12: 00$ & 18.027 & 23.588 & 17.841 & 26.628 & 6.302 & 9.359 & 0.784 \\
\hline $5 / 6 / 10$ & $16: 00$ & 18.095 & 23.569 & 17.794 & 26.651 & 6.101 & 9.318 & 0.790 \\
\hline $5 / 6 / 10$ & $20: 00$ & 18.070 & 23.599 & 17.809 & 26.626 & 5.963 & 9.339 & 0.800 \\
\hline $5 / 7 / 10$ & 0:00 & 18.114 & 23.559 & 17.762 & 26.665 & 5.859 & 9.285 & 0.772 \\
\hline $5 / 7 / 10$ & $4: 00$ & 17.939 & 23.616 & 17.785 & 26.594 & 5.751 & 9.255 & 0.677 \\
\hline $5 / 7 / 10$ & $8: 00$ & 17.789 & 23.620 & 17.904 & 26.598 & 5.703 & 9.194 & 0.707 \\
\hline $5 / 7 / 10$ & $12: 00$ & 17.724 & 23.656 & 17.968 & 26.638 & 5.623 & 9.283 & 0.718 \\
\hline $5 / 7 / 10$ & $16: 00$ & 17.730 & 23.683 & 18.016 & 26.680 & 5.600 & 9.337 & 0.737 \\
\hline $5 / 7 / 10$ & $20: 00$ & 17.720 & 23.723 & 18.067 & 26.747 & 5.559 & 9.398 & 0.751 \\
\hline $5 / 8 / 10$ & $0: 00$ & 17.683 & 23.771 & 18.124 & 26.777 & 5.526 & 9.452 & 0.758 \\
\hline $5 / 8 / 10$ & $4: 00$ & 17.755 & 23.796 & 18.140 & 26.808 & 5.514 & 9.489 & 0.759 \\
\hline $5 / 8 / 10$ & 8:00 & 17.777 & 23.851 & 18.180 & 26.825 & 5.487 & 9.535 & 0.768 \\
\hline $5 / 8 / 10$ & $12: 00$ & 17.861 & 23.880 & 18.196 & 26.870 & 5.503 & 9.556 & 0.780 \\
\hline $5 / 8 / 10$ & $16: 00$ & 17.968 & 23.855 & 18.153 & 26.920 & 5.491 & 9.524 & 0.778 \\
\hline $5 / 9 / 10$ & $20: 00$ & 17.966 & 23.874 & 18.152 & 26.935 & 5.489 & 9.539 & 0.780 \\
\hline $5 / 9 / 10$ & 0:00 & 17.951 & 23.920 & 18.191 & 26.960 & 5.443 & 9.574 & 0.778 \\
\hline $5 / 9 / 10$ & $4: 00$ & 17.988 & 23.933 & 18.196 & 26.989 & 5.439 & 9.576 & 0.775 \\
\hline $5 / 9 / 10$ & 8:00 & 18.016 & 23.956 & 18.209 & 27.017 & 5.415 & 9.587 & 0.776 \\
\hline $5 / 9 / 10$ & $12: 00$ & 18.052 & 23.964 & 18.207 & 27.031 & 5.402 & 9.584 & 0.779 \\
\hline $5 / 9 / 10$ & $16: 00$ & 18.090 & 23.943 & 18.172 & 27.057 & 5.392 & 9.545 & 0.777 \\
\hline $5 / 9 / 10$ & $20: 00$ & 18.085 & 23.970 & 18.180 & 27.038 & 5.390 & 9.560 & 0.779 \\
\hline $5 / 10 / 10$ & 0:00 & 18.090 & 23.985 & 18.187 & 27.031 & 5.415 & 9.560 & 0.775 \\
\hline $5 / 10 / 10$ & $4: 00$ & 18.119 & 23.985 & 18.172 & 27.023 & 5.372 & 9.547 & 0.771 \\
\hline $5 / 10 / 10$ & 8:00 & 18.162 & 23.964 & 18.141 & 27.038 & 5.386 & 9.504 & 0.759 \\
\hline $5 / 10 / 10$ & $12: 00$ & 18.077 & 23.937 & 18.088 & 26.985 & 5.339 & 9.261 & 0.600 \\
\hline $5 / 10 / 10$ & $16: 00$ & 17.918 & 23.777 & 17.955 & 26.973 & 5.320 & 8.960 & 0.522 \\
\hline $5 / 10 / 10$ & $20: 00$ & 17.769 & 23.704 & 17.909 & 26.966 & 5.283 & 8.930 & 0.520 \\
\hline $5 / 11 / 10$ & 0:00 & 17.493 & 23.746 & 17.963 & 26.870 & 5.291 & 8.935 & 0.515 \\
\hline $5 / 11 / 10$ & $4: 00$ & 17.334 & 23.763 & 18.021 & 26.834 & 5.311 & 8.980 & 0.513 \\
\hline $5 / 11 / 10$ & 8:00 & 17.232 & 23.828 & 18.102 & 26.838 & 5.308 & 9.071 & 0.529 \\
\hline $5 / 11 / 10$ & $12: 00$ & 17.215 & 23.870 & 18.150 & 26.851 & 5.294 & 9.127 & 0.544 \\
\hline $5 / 11 / 10$ & $16: 00$ & 17.284 & 23.836 & 18.119 & 26.914 & 5.313 & 9.103 & 0.544 \\
\hline $5 / 11 / 10$ & $20: 00$ & 17.278 & 23.857 & 18.131 & 26.924 & 5.329 & 9.132 & 0.555 \\
\hline $5 / 12 / 10$ & 0:00 & 17.297 & 23.874 & 18.142 & 26.956 & 5.331 & 9.158 & 0.561 \\
\hline $5 / 12 / 10$ & $4: 00$ & 17.352 & 23.838 & 18.074 & 26.970 & 5.315 & 9.127 & 0.559 \\
\hline $5 / 12 / 10$ & $8: 00$ & 17.339 & 23.886 & 18.121 & 26.968 & 5.325 & 9.166 & 0.573 \\
\hline $5 / 12 / 10$ & $12: 00$ & 17.376 & 23.886 & 18.116 & 26.979 & 5.315 & 9.166 & 0.578 \\
\hline $5 / 12 / 10$ & $16: 00$ & 17.426 & 23.868 & 18.084 & 26.996 & 5.310 & 9.141 & 0.574 \\
\hline $5 / 12 / 10$ & $20: 00$ & 17.409 & 23.909 & 18.123 & 26.977 & 5.305 & 9.178 & 0.586 \\
\hline
\end{tabular}


TABLE S2.1 (Cont.)

\begin{tabular}{|c|c|c|c|c|c|c|c|c|}
\hline \multirow[b]{2}{*}{ Date } & \multirow[b]{2}{*}{ Time } & \multicolumn{6}{|c|}{ Depth to Water (ft TOC) } & \multirow[b]{2}{*}{ MW8s } \\
\hline & & MW1s & MW2s & MW3s & MW4s & MW6s & MW7s & \\
\hline $5 / 13 / 10$ & $0: 00$ & 17.389 & 23.945 & 18.133 & 26.979 & 5.311 & 9.204 & 0.576 \\
\hline $5 / 13 / 10$ & $4: 00$ & 17.317 & 23.905 & 18.126 & 26.958 & 5.292 & 8.961 & 0.419 \\
\hline $5 / 13 / 10$ & 8:00 & 17.106 & 23.907 & 18.153 & 26.960 & 5.218 & 8.872 & 0.362 \\
\hline $5 / 13 / 10$ & $12: 00$ & 17.021 & 23.901 & 18.181 & 26.996 & 5.261 & 8.911 & 0.386 \\
\hline $5 / 13 / 10$ & $16: 00$ & 16.988 & 23.895 & 18.186 & 27.036 & 5.282 & 8.946 & 0.415 \\
\hline $5 / 13 / 10$ & $20: 00$ & 16.966 & 23.916 & 18.200 & 27.033 & 5.303 & 8.989 & 0.442 \\
\hline $5 / 14 / 10$ & $0: 00$ & 16.936 & 23.945 & 18.230 & 27.065 & 5.373 & 9.043 & 0.461 \\
\hline $5 / 14 / 10$ & $4: 00$ & 16.974 & 23.941 & 18.219 & 27.099 & 5.332 & 9.051 & 0.476 \\
\hline $5 / 14 / 10$ & 8:00 & 17.010 & 23.985 & 18.255 & 27.134 & 5.363 & 9.114 & 0.498 \\
\hline $5 / 14 / 10$ & $12: 00$ & 17.095 & 24.012 & 18.272 & 27.164 & 5.410 & 9.149 & 0.520 \\
\hline $5 / 14 / 10$ & $16: 00$ & 17.192 & 23.979 & 18.228 & 27.204 & 5.332 & 9.124 & 0.530 \\
\hline $5 / 14 / 10$ & $20: 00$ & 17.222 & 23.968 & 18.206 & 27.206 & 5.355 & 9.129 & 0.539 \\
\hline $5 / 15 / 10$ & $0: 00$ & 17.202 & 24.004 & 18.245 & 27.204 & 5.344 & 9.180 & 0.550 \\
\hline $5 / 15 / 10$ & $4: 00$ & 17.256 & 24.004 & 18.237 & 27.212 & 5.328 & 9.186 & 0.556 \\
\hline $5 / 15 / 10$ & 8:00 & 17.275 & 24.033 & 18.255 & 27.201 & 5.347 & 9.219 & 0.569 \\
\hline $5 / 15 / 10$ & $12: 00$ & 17.348 & 24.040 & 18.267 & 27.214 & 5.334 & 9.232 & 0.580 \\
\hline $5 / 15 / 10$ & $16: 00$ & 17.418 & 24.019 & 18.238 & 27.225 & 5.324 & 9.210 & 0.594 \\
\hline $5 / 15 / 10$ & $20: 00$ & 17.411 & 24.038 & 18.255 & 27.198 & 5.361 & 9.234 & 0.604 \\
\hline $5 / 16 / 10$ & $0: 00$ & 17.405 & 24.079 & 18.296 & 27.189 & 5.345 & 9.279 & 0.616 \\
\hline $5 / 16 / 10$ & $4: 00$ & 17.465 & 24.071 & 18.287 & 27.183 & 5.316 & 9.275 & 0.616 \\
\hline $5 / 16 / 10$ & 8:00 & 17.481 & 24.096 & 18.306 & 27.170 & 5.332 & 9.299 & 0.625 \\
\hline $5 / 16 / 10$ & $12: 00$ & 17.524 & 24.109 & 18.318 & 27.172 & 5.365 & 9.308 & 0.626 \\
\hline $5 / 16 / 10$ & $16: 00$ & 17.577 & 24.109 & 18.322 & 27.233 & 5.293 & 9.305 & 0.630 \\
\hline $5 / 16 / 10$ & $20: 00$ & 17.557 & 24.119 & 18.339 & 27.206 & 5.297 & 9.297 & 0.613 \\
\hline $5 / 17 / 10$ & $0: 00$ & 17.531 & 24.138 & 18.377 & 27.187 & 5.337 & 9.253 & 0.621 \\
\hline $5 / 17 / 10$ & $4: 00$ & 17.537 & 24.145 & 18.397 & 27.176 & 5.332 & 9.271 & 0.626 \\
\hline $5 / 17 / 10$ & 8:00 & 17.544 & 24.163 & 18.428 & 27.174 & 5.327 & 9.293 & 0.634 \\
\hline $5 / 17 / 10$ & $12: 00$ & 17.565 & 24.191 & 18.462 & 27.180 & 5.374 & 9.325 & 0.641 \\
\hline $5 / 17 / 10$ & $16: 00$ & 17.621 & 24.197 & 18.477 & 27.197 & 5.350 & 9.329 & 0.646 \\
\hline $5 / 17 / 10$ & $20: 00$ & 17.646 & 24.201 & 18.485 & 27.198 & 5.341 & 9.338 & 0.649 \\
\hline $5 / 18 / 10$ & $0: 00$ & 17.641 & 24.212 & 18.506 & 27.208 & 5.339 & 9.358 & 0.653 \\
\hline $5 / 18 / 10$ & $4: 00$ & 17.666 & 24.231 & 18.525 & 27.206 & 5.329 & 9.377 & 0.661 \\
\hline $5 / 18 / 10$ & 8:00 & 17.701 & 24.241 & 18.533 & 27.201 & 5.335 & 9.386 & 0.665 \\
\hline $5 / 18 / 10$ & $12: 00$ & 17.774 & 24.258 & 18.557 & 27.214 & 5.349 & 9.401 & 0.675 \\
\hline $5 / 18 / 10$ & $16: 00$ & 17.850 & 24.235 & 18.519 & 27.220 & 5.334 & 9.373 & 0.684 \\
\hline $5 / 18 / 10$ & $20: 00$ & 17.840 & 24.254 & 18.536 & 27.214 & 5.358 & 9.397 & 0.687 \\
\hline $5 / 19 / 10$ & $0: 00$ & 17.815 & 24.270 & 18.555 & 27.218 & 5.369 & 9.416 & 0.683 \\
\hline $5 / 19 / 10$ & $4: 00$ & 17.816 & 24.304 & 18.582 & 27.203 & 5.336 & 9.444 & 0.691 \\
\hline $5 / 19 / 10$ & $8: 00$ & 17.855 & 24.310 & 18.588 & 27.210 & 5.368 & 9.447 & 0.690 \\
\hline $5 / 19 / 10$ & $12: 00$ & 17.897 & 24.331 & 18.611 & 27.203 & 5.344 & 9.466 & 0.704 \\
\hline $5 / 19 / 10$ & $16: 00$ & 17.937 & 24.331 & 18.605 & 27.206 & 5.385 & 9.460 & 0.708 \\
\hline $5 / 19 / 10$ & $20: 00$ & 17.949 & 24.348 & 18.616 & 27.206 & 5.334 & 9.479 & 0.712 \\
\hline $5 / 20 / 10$ & $0: 00$ & 17.918 & 24.354 & 18.631 & 27.210 & 5.358 & 9.457 & 0.698 \\
\hline $5 / 20 / 10$ & $4: 00$ & 17.962 & 24.317 & 18.614 & 27.204 & 5.291 & 9.394 & 0.661 \\
\hline $5 / 20 / 10$ & $8: 00$ & 17.768 & 24.237 & 18.495 & 27.174 & 4.835 & 9.037 & 0.415 \\
\hline $5 / 20 / 10$ & $12: 00$ & 17.354 & 24.103 & 18.323 & 27.157 & 4.510 & 8.701 & -0.004 \\
\hline $5 / 20 / 10$ & $16: 00$ & 16.903 & 23.972 & 18.228 & 27.138 & 4.720 & 8.502 & 0.045 \\
\hline $5 / 20 / 10$ & $20: 00$ & 16.476 & 23.844 & 18.086 & 27.122 & 4.660 & 8.288 & 0.000 \\
\hline
\end{tabular}


TABLE S2.1 (Cont.)

\begin{tabular}{|c|c|c|c|c|c|c|c|c|}
\hline \multirow[b]{2}{*}{ Date } & \multirow[b]{2}{*}{ Time } & \multicolumn{6}{|c|}{ Depth to Water (ft TOC) } & \multirow[b]{2}{*}{ MW8s } \\
\hline & & MW1s & MW2s & MW3s & MW4s & MW6s & MW7s & \\
\hline $5 / 21 / 10$ & $0: 00$ & 16.261 & 23.750 & 18.034 & 27.096 & 4.876 & 8.286 & 0.105 \\
\hline $5 / 21 / 10$ & $4: 00$ & 16.145 & 23.674 & 17.995 & 27.063 & 5.066 & 8.320 & 0.165 \\
\hline $5 / 21 / 10$ & 8:00 & 16.076 & 23.640 & 17.993 & 27.033 & 5.188 & 8.376 & 0.214 \\
\hline $5 / 21 / 10$ & $12: 00$ & 16.089 & 23.588 & 17.956 & 27.029 & 5.240 & 8.407 & 0.247 \\
\hline $5 / 21 / 10$ & $16: 00$ & 16.091 & 23.525 & 17.898 & 27.017 & 5.254 & 8.376 & 0.280 \\
\hline $5 / 21 / 10$ & $20: 00$ & 16.160 & 23.466 & 17.845 & 27.002 & 5.278 & 8.444 & 0.309 \\
\hline $5 / 22 / 10$ & $0: 00$ & 16.140 & 23.433 & 17.812 & 26.976 & 5.324 & 8.478 & 0.331 \\
\hline $5 / 22 / 10$ & $4: 00$ & 16.189 & 23.380 & 17.763 & 26.945 & 5.306 & 8.487 & 0.350 \\
\hline $5 / 22 / 10$ & 8:00 & 16.232 & 23.336 & 17.725 & 26.909 & 5.318 & 8.498 & 0.364 \\
\hline $5 / 22 / 10$ & $12: 00$ & 16.313 & 23.284 & 17.679 & 26.891 & 5.283 & 8.498 & 0.384 \\
\hline $5 / 22 / 10$ & $16: 00$ & 16.359 & 23.246 & 17.645 & 26.844 & 5.260 & 8.517 & 0.406 \\
\hline $5 / 22 / 10$ & $20: 00$ & 16.343 & 23.244 & 17.649 & 26.802 & 5.287 & 8.567 & 0.425 \\
\hline $5 / 23 / 10$ & $0: 00$ & 16.326 & 23.248 & 17.675 & 26.768 & 5.283 & 8.630 & 0.439 \\
\hline $5 / 23 / 10$ & $4: 00$ & 16.369 & 23.227 & 17.669 & 26.729 & 5.303 & 8.657 & 0.452 \\
\hline $5 / 23 / 10$ & 8:00 & 16.389 & 23.246 & 17.711 & 26.708 & 5.341 & 8.721 & 0.472 \\
\hline $5 / 23 / 10$ & $12: 00$ & 16.454 & 23.254 & 17.739 & 26.692 & 5.328 & 8.775 & 0.494 \\
\hline $5 / 23 / 10$ & $16: 00$ & 16.536 & 23.233 & 17.737 & 26.703 & 5.288 & 8.806 & 0.514 \\
\hline $5 / 23 / 10$ & $20: 00$ & 16.586 & 23.221 & 17.737 & 26.687 & 5.330 & 8.836 & 0.518 \\
\hline $5 / 24 / 10$ & $0: 00$ & 16.608 & 23.231 & 17.766 & 26.665 & 5.323 & 8.886 & 0.530 \\
\hline $5 / 24 / 10$ & $4: 00$ & 16.668 & 23.223 & 17.773 & 26.661 & 5.358 & 8.916 & 0.537 \\
\hline $5 / 24 / 10$ & 8:00 & 16.725 & 23.227 & 17.796 & 26.655 & 5.331 & 8.949 & 0.549 \\
\hline $5 / 24 / 10$ & $12: 00$ & 16.817 & 23.227 & 17.803 & 26.653 & 5.349 & 8.976 & 0.570 \\
\hline $5 / 24 / 10$ & $16: 00$ & 16.904 & 23.200 & 17.781 & 26.672 & 5.358 & 8.983 & 0.589 \\
\hline $5 / 24 / 10$ & $20: 00$ & 16.924 & 23.195 & 17.781 & 26.651 & 5.343 & 9.013 & 0.595 \\
\hline $5 / 25 / 10$ & $0: 00$ & 16.927 & 23.212 & 17.815 & 26.630 & 5.331 & 9.061 & 0.598 \\
\hline $5 / 25 / 10$ & $4: 00$ & 16.982 & 23.198 & 17.817 & 26.626 & 5.327 & 9.072 & 0.598 \\
\hline $5 / 25 / 10$ & 8:00 & 16.962 & 23.254 & 17.890 & 26.600 & 5.366 & 9.152 & 0.616 \\
\hline $5 / 25 / 10$ & $12: 00$ & 17.086 & 23.246 & 17.889 & 26.617 & 5.319 & 9.161 & 0.628 \\
\hline $5 / 25 / 10$ & $16: 00$ & 17.138 & 23.239 & 17.887 & 26.617 & 5.346 & 9.176 & 0.641 \\
\hline $5 / 25 / 10$ & $20: 00$ & 17.173 & 23.250 & 17.893 & 26.604 & 5.364 & 9.200 & 0.647 \\
\hline $5 / 26 / 10$ & $0: 00$ & 17.164 & 23.277 & 17.929 & 26.596 & 5.366 & 9.243 & 0.650 \\
\hline $5 / 26 / 10$ & $4: 00$ & 17.213 & 23.263 & 17.927 & 26.594 & 5.385 & 9.246 & 0.652 \\
\hline $5 / 26 / 10$ & 8:00 & 17.245 & 23.305 & 17.978 & 26.594 & 5.364 & 9.295 & 0.660 \\
\hline $5 / 26 / 10$ & $12: 00$ & 17.342 & 23.317 & 17.981 & 26.585 & 5.370 & 9.300 & 0.673 \\
\hline $5 / 26 / 10$ & $16: 00$ & 17.014 & 23.107 & 17.588 & 26.577 & 4.934 & 8.799 & -0.020 \\
\hline $5 / 26 / 10$ & $20: 00$ & 16.269 & 22.778 & 17.551 & 26.569 & 4.775 & 8.381 & -0.003 \\
\hline $5 / 27 / 10$ & $0: 00$ & 15.923 & 22.611 & 17.542 & 26.533 & 5.078 & 8.386 & 0.158 \\
\hline $5 / 27 / 10$ & $4: 00$ & 15.823 & 22.456 & 17.483 & 26.510 & 5.229 & 8.382 & 0.230 \\
\hline $5 / 27 / 10$ & $8: 00$ & 15.781 & 22.358 & 17.461 & 26.466 & 5.284 & 8.422 & 0.281 \\
\hline $5 / 27 / 10$ & $12: 00$ & 15.840 & 22.255 & 17.411 & 26.453 & 5.334 & 8.431 & 0.320 \\
\hline $5 / 27 / 10$ & $16: 00$ & 15.895 & 22.143 & 17.337 & 26.428 & 5.336 & 8.441 & 0.354 \\
\hline $5 / 27 / 10$ & $20: 00$ & 15.918 & 22.072 & 17.296 & 26.407 & 5.324 & 8.470 & 0.379 \\
\hline $5 / 28 / 10$ & $0: 00$ & 15.890 & 22.036 & 17.301 & 26.358 & 5.373 & 8.524 & 0.402 \\
\hline $5 / 28 / 10$ & $4: 00$ & 15.922 & 21.983 & 17.283 & 26.318 & 5.317 & 8.553 & 0.417 \\
\hline $5 / 28 / 10$ & $8: 00$ & 15.976 & 21.942 & 17.269 & 26.272 & 5.315 & 8.583 & 0.432 \\
\hline $5 / 28 / 10$ & $12: 00$ & 16.084 & 21.910 & 17.251 & 26.243 & 5.334 & 8.602 & 0.456 \\
\hline $5 / 28 / 10$ & $16: 00$ & 16.180 & 21.837 & 17.190 & 26.218 & 5.375 & 8.596 & 0.472 \\
\hline $5 / 28 / 10$ & $20: 00$ & 16.201 & 21.805 & 17.173 & 26.192 & 5.355 & 8.626 & 0.486 \\
\hline
\end{tabular}


TABLE S2.1 (Cont.)

\begin{tabular}{|c|c|c|c|c|c|c|c|c|}
\hline \multirow[b]{2}{*}{ Date } & \multirow[b]{2}{*}{ Time } & \multicolumn{6}{|c|}{ Depth to Water (ft TOC) } & \multirow[b]{2}{*}{ MW8s } \\
\hline & & MW1s & MW2s & MW3s & MW4s & MW6s & MW7s & \\
\hline $5 / 29 / 10$ & $0: 00$ & 16.178 & 21.805 & 17.198 & 26.133 & 5.378 & 8.674 & 0.496 \\
\hline $5 / 29 / 10$ & $4: 00$ & 16.214 & 21.778 & 17.193 & 26.096 & 5.355 & 8.693 & 0.504 \\
\hline $5 / 29 / 10$ & $8: 00$ & 16.246 & 21.767 & 17.202 & 26.066 & 5.353 & 8.713 & 0.513 \\
\hline $5 / 29 / 10$ & $12: 00$ & 16.358 & 21.753 & 17.195 & 26.047 & 5.369 & 8.739 & 0.534 \\
\hline $5 / 29 / 10$ & $16: 00$ & 16.445 & 21.717 & 17.158 & 26.055 & 5.336 & 8.739 & 0.553 \\
\hline $5 / 29 / 10$ & $20: 00$ & 16.455 & 21.715 & 17.160 & 26.005 & 5.347 & 8.771 & 0.566 \\
\hline $5 / 30 / 10$ & $0: 00$ & 16.452 & 21.727 & 17.185 & 25.978 & 5.327 & 8.808 & 0.569 \\
\hline $5 / 30 / 10$ & $4: 00$ & 16.481 & 21.715 & 17.178 & 25.969 & 5.349 & 8.812 & 0.570 \\
\hline $5 / 30 / 10$ & $8: 00$ & 16.505 & 21.763 & 17.237 & 25.917 & 5.359 & 8.884 & 0.589 \\
\hline $5 / 30 / 10$ & $12: 00$ & 16.610 & 21.761 & 17.240 & 25.902 & 5.324 & 8.891 & 0.604 \\
\hline $5 / 30 / 10$ & $16: 00$ & 16.633 & 21.769 & 17.255 & 25.873 & 5.327 & 8.910 & 0.621 \\
\hline $5 / 30 / 10$ & $20: 00$ & 16.237 & 21.440 & 17.014 & 25.854 & 4.708 & 8.464 & 0.157 \\
\hline $5 / 31 / 10$ & $0: 00$ & 15.928 & 21.297 & 17.010 & 25.843 & 5.007 & 8.373 & 0.187 \\
\hline $5 / 31 / 10$ & $4: 00$ & 15.753 & 21.188 & 16.958 & 25.830 & 5.124 & 8.299 & 0.275 \\
\hline $5 / 31 / 10$ & $8: 00$ & 15.676 & 21.137 & 16.924 & 25.811 & 5.256 & 8.323 & 0.328 \\
\hline $5 / 31 / 10$ & $12: 00$ & 15.711 & 21.089 & 16.873 & 25.807 & 5.277 & 8.336 & 0.364 \\
\hline $5 / 31 / 10$ & $16: 00$ & 15.748 & 21.045 & 16.813 & 25.790 & 5.280 & 8.347 & 0.398 \\
\hline $5 / 31 / 10$ & $20: 00$ & 15.773 & 21.011 & 16.778 & 25.782 & 5.284 & 8.371 & 0.423 \\
\hline $6 / 1 / 10$ & $0: 00$ & 15.731 & 21.020 & 16.788 & 25.736 & 5.356 & 8.423 & 0.444 \\
\hline $6 / 1 / 10$ & $4: 00$ & 15.781 & 20.990 & 16.753 & 25.721 & 5.366 & 8.430 & 0.450 \\
\hline $6 / 1 / 10$ & $8: 00$ & 15.824 & 20.990 & 16.751 & 25.681 & 5.381 & 8.459 & 0.461 \\
\hline $6 / 1 / 10$ & $12: 00$ & 15.933 & 20.950 & 16.700 & 25.677 & 5.329 & 8.442 & 0.474 \\
\hline $6 / 1 / 10$ & $16: 00$ & 16.007 & 20.910 & 16.642 & 25.650 & 5.354 & 8.436 & 0.496 \\
\hline $6 / 1 / 10$ & $20: 00$ & 16.018 & 20.883 & 16.609 & 25.614 & 5.343 & 8.440 & 0.505 \\
\hline $6 / 2 / 10$ & 0:00 & 15.928 & 20.961 & 16.717 & 25.589 & 5.340 & 8.514 & 0.483 \\
\hline $6 / 2 / 10$ & $4: 00$ & 15.724 & 20.774 & 16.632 & 25.523 & 5.068 & 8.234 & 0.208 \\
\hline $6 / 2 / 10$ & 8:00 & 14.319 & 19.662 & 15.167 & 25.536 & 2.370 & 7.832 & -0.325 \\
\hline $6 / 2 / 10$ & $12: 00$ & 13.813 & 19.192 & 15.110 & 25.467 & 3.728 & 7.327 & -0.041 \\
\hline $6 / 2 / 10$ & $16: 00$ & 13.736 & 18.889 & 15.021 & 25.414 & 4.404 & 7.324 & 0.097 \\
\hline $6 / 2 / 10$ & $20: 00$ & 13.716 & 18.713 & 14.946 & 25.332 & 4.757 & 7.359 & 0.064 \\
\hline $6 / 3 / 10$ & 0:00 & 13.693 & 18.641 & 14.913 & 25.257 & 4.992 & 7.420 & -0.146 \\
\hline $6 / 3 / 10$ & $4: 00$ & 13.736 & 18.562 & 14.868 & 25.173 & 5.120 & 7.441 & 0.093 \\
\hline $6 / 3 / 10$ & $8: 00$ & 13.806 & 18.514 & 14.838 & 25.112 & 5.208 & 7.465 & 0.211 \\
\hline $6 / 3 / 10$ & $12: 00$ & 13.920 & 18.464 & 14.806 & 25.056 & 5.251 & 7.479 & 0.244 \\
\hline $6 / 3 / 10$ & $16: 00$ & 14.033 & 18.407 & 14.763 & 24.997 & 5.254 & 7.481 & 0.276 \\
\hline $6 / 3 / 10$ & $20: 00$ & 14.070 & 18.382 & 14.737 & 24.913 & 5.278 & 7.517 & 0.308 \\
\hline $6 / 4 / 10$ & 0:00 & 14.093 & 18.390 & 14.738 & 24.859 & 5.268 & 7.569 & 0.328 \\
\hline $6 / 4 / 10$ & $4: 00$ & 14.167 & 18.380 & 14.728 & 24.809 & 5.305 & 7.593 & 0.340 \\
\hline $6 / 4 / 10$ & $8: 00$ & 14.217 & 18.388 & 14.732 & 24.752 & 5.281 & 7.630 & 0.356 \\
\hline $6 / 4 / 10$ & $12: 00$ & 14.282 & 18.409 & 14.728 & 24.708 & 5.241 & 7.639 & 0.381 \\
\hline $6 / 4 / 10$ & $16: 00$ & 14.404 & 18.428 & 14.729 & 24.676 & 5.283 & 7.700 & 0.412 \\
\hline $6 / 4 / 10$ & $20: 00$ & 14.452 & 18.451 & 14.730 & 24.624 & 5.287 & 7.743 & 0.431 \\
\hline $6 / 5 / 10$ & $0: 00$ & 14.466 & 18.491 & 14.742 & 24.596 & 5.328 & 7.788 & 0.438 \\
\hline $6 / 5 / 10$ & $4: 00$ & 14.509 & 18.518 & 14.765 & 24.541 & 5.299 & 7.829 & 0.449 \\
\hline $6 / 5 / 10$ & $8: 00$ & 14.550 & 18.586 & 14.793 & 24.531 & 5.287 & 7.884 & 0.457 \\
\hline $6 / 5 / 10$ & $12: 00$ & 14.665 & 18.590 & 14.790 & 24.525 & 5.291 & 7.892 & 0.467 \\
\hline $6 / 5 / 10$ & $16: 00$ & 14.739 & 18.634 & 14.811 & 24.512 & 5.293 & 7.936 & 0.495 \\
\hline $6 / 5 / 10$ & $20: 00$ & 14.740 & 18.751 & 14.862 & 24.499 & 5.361 & 8.033 & 0.524 \\
\hline
\end{tabular}


TABLE S2.1 (Cont.)

\begin{tabular}{|c|c|c|c|c|c|c|c|c|}
\hline \multirow[b]{2}{*}{ Date } & \multirow[b]{2}{*}{ Time } & \multicolumn{6}{|c|}{ Depth to Water (ft TOC) } & \multirow[b]{2}{*}{ MW8s } \\
\hline & & MW1s & MW2s & MW3s & MW4s & MW6s & MW7s & \\
\hline $6 / 6 / 10$ & $0: 00$ & 14.763 & 18.840 & 14.905 & 24.518 & 5.330 & 8.107 & 0.526 \\
\hline $6 / 6 / 10$ & $4: 00$ & 14.816 & 18.913 & 14.949 & 24.531 & 5.295 & 8.172 & 0.536 \\
\hline $6 / 6 / 10$ & $8: 00$ & 14.899 & 18.980 & 14.991 & 24.543 & 5.314 & 8.231 & 0.542 \\
\hline $6 / 6 / 10$ & $12: 00$ & 15.053 & 18.999 & 15.004 & 24.577 & 5.330 & 8.243 & 0.555 \\
\hline $6 / 6 / 10$ & $16: 00$ & 15.172 & 19.020 & 15.008 & 24.605 & 5.339 & 8.254 & 0.575 \\
\hline $6 / 6 / 10$ & $20: 00$ & 15.224 & 19.052 & 15.016 & 24.586 & 5.349 & 8.284 & 0.580 \\
\hline $6 / 7 / 10$ & $0: 00$ & 15.225 & 19.115 & 15.044 & 24.586 & 5.374 & 8.333 & 0.585 \\
\hline $6 / 7 / 10$ & $4: 00$ & 15.321 & 19.108 & 15.057 & 24.560 & 5.335 & 8.321 & 0.579 \\
\hline $6 / 7 / 10$ & 8:00 & 15.374 & 19.157 & 15.070 & 24.584 & 5.320 & 8.345 & 0.582 \\
\hline $6 / 7 / 10$ & $12: 00$ & 15.432 & 19.196 & 15.097 & 24.567 & 5.310 & 8.365 & 0.594 \\
\hline $6 / 7 / 10$ & $16: 00$ & 15.520 & 19.199 & 15.087 & 24.584 & 5.326 & 8.347 & 0.597 \\
\hline $6 / 7 / 10$ & $20: 00$ & 15.506 & 19.245 & 15.109 & 24.579 & 5.314 & 8.365 & 0.610 \\
\hline $6 / 8 / 10$ & $0: 00$ & 15.570 & 19.270 & 15.116 & 24.592 & 5.345 & 8.380 & 0.605 \\
\hline $6 / 8 / 10$ & $4: 00$ & 15.637 & 19.253 & 15.113 & 24.592 & 5.347 & 8.350 & 0.597 \\
\hline $6 / 8 / 10$ & 8:00 & 15.653 & 19.210 & 15.101 & 24.588 & 5.271 & 8.298 & 0.519 \\
\hline $6 / 8 / 10$ & $12: 00$ & 15.516 & 19.317 & 15.172 & 24.518 & 5.354 & 8.374 & 0.555 \\
\hline $6 / 8 / 10$ & $16: 00$ & 15.581 & 19.342 & 15.167 & 24.560 & 5.372 & 8.348 & 0.578 \\
\hline $6 / 8 / 10$ & $20: 00$ & 15.583 & 19.398 & 15.186 & 24.560 & 5.335 & 8.384 & 0.599 \\
\hline $6 / 9 / 10$ & $0: 00$ & 15.576 & 19.482 & 15.230 & 24.558 & 5.323 & 8.434 & 0.611 \\
\hline $6 / 9 / 10$ & $4: 00$ & 15.614 & 19.529 & 15.250 & 24.569 & 5.321 & 8.447 & 0.613 \\
\hline $6 / 9 / 10$ & 8:00 & 15.644 & 19.610 & 15.288 & 24.579 & 5.345 & 8.493 & 0.623 \\
\hline $6 / 9 / 10$ & $12: 00$ & 15.757 & 19.646 & 15.307 & 24.624 & 5.325 & 8.499 & 0.636 \\
\hline $6 / 9 / 10$ & $16: 00$ & 15.865 & 19.673 & 15.306 & 24.665 & 5.335 & 8.485 & 0.649 \\
\hline $6 / 9 / 10$ & $20: 00$ & 15.875 & 19.726 & 15.324 & 24.672 & 5.347 & 8.512 & 0.654 \\
\hline $6 / 10 / 10$ & $0: 00$ & 15.880 & 19.776 & 15.352 & 24.678 & 5.309 & 8.536 & 0.654 \\
\hline $6 / 10 / 10$ & $4: 00$ & 15.951 & 19.778 & 15.351 & 24.697 & 5.346 & 8.512 & 0.646 \\
\hline $6 / 10 / 10$ & 8:00 & 15.972 & 19.827 & 15.372 & 24.687 & 5.303 & 8.530 & 0.652 \\
\hline $6 / 10 / 10$ & $12: 00$ & 16.007 & 19.860 & 15.396 & 24.674 & 5.315 & 8.538 & 0.661 \\
\hline $6 / 10 / 10$ & $16: 00$ & 16.086 & 19.887 & 15.397 & 24.726 & 5.348 & 8.523 & 0.665 \\
\hline $6 / 10 / 10$ & $20: 00$ & 16.102 & 19.929 & 15.410 & 24.724 & 5.340 & 8.538 & 0.674 \\
\hline $6 / 11 / 10$ & $0: 00$ & 16.106 & 19.978 & 15.426 & 24.735 & 5.315 & 8.556 & 0.669 \\
\hline $6 / 11 / 10$ & $4: 00$ & 16.112 & 20.015 & 15.453 & 24.712 & 5.335 & 8.563 & 0.671 \\
\hline $6 / 11 / 10$ & 8:00 & 16.098 & 20.129 & 15.512 & 24.703 & 5.338 & 8.634 & 0.681 \\
\hline $6 / 11 / 10$ & $12: 00$ & 16.178 & 20.158 & 15.540 & 24.731 & 5.333 & 8.630 & 0.691 \\
\hline $6 / 11 / 10$ & $16: 00$ & 16.227 & 20.221 & 15.567 & 24.752 & 5.336 & 8.664 & 0.706 \\
\hline $6 / 11 / 10$ & $20: 00$ & 16.239 & 20.290 & 15.620 & 24.777 & 5.359 & 8.704 & 0.710 \\
\hline $6 / 12 / 10$ & $0: 00$ & 16.271 & 20.351 & 15.668 & 24.800 & 5.373 & 8.731 & 0.705 \\
\hline $6 / 12 / 10$ & $4: 00$ & 16.304 & 20.400 & 15.707 & 24.817 & 5.373 & 8.749 & 0.705 \\
\hline $6 / 12 / 10$ & $8: 00$ & 16.304 & 20.486 & 15.774 & 24.825 & 5.373 & 8.801 & 0.714 \\
\hline $6 / 12 / 10$ & $12: 00$ & 16.399 & 20.498 & 15.783 & 24.868 & 5.377 & 8.774 & 0.708 \\
\hline $6 / 12 / 10$ & $16: 00$ & 16.453 & 20.584 & 15.835 & 24.901 & 5.352 & 8.820 & 0.730 \\
\hline $6 / 12 / 10$ & $20: 00$ & 16.461 & 20.645 & 15.907 & 24.928 & 5.371 & 8.842 & 0.729 \\
\hline $6 / 13 / 10$ & $0: 00$ & 16.505 & 20.691 & 15.987 & 24.968 & 5.361 & 8.857 & 0.725 \\
\hline $6 / 13 / 10$ & $4: 00$ & 16.543 & 20.725 & 16.030 & 24.983 & 5.369 & 8.857 & 0.723 \\
\hline $6 / 13 / 10$ & $8: 00$ & 16.560 & 20.746 & 16.071 & 25.002 & 5.324 & 8.859 & 0.688 \\
\hline $6 / 13 / 10$ & $12: 00$ & 16.588 & 20.811 & 16.147 & 25.015 & 5.351 & 8.887 & 0.701 \\
\hline $6 / 13 / 10$ & $16: 00$ & 16.652 & 20.817 & 16.142 & 25.044 & 5.351 & 8.855 & 0.716 \\
\hline $6 / 13 / 10$ & $20: 00$ & 16.682 & 20.865 & 16.178 & 25.082 & 5.355 & 8.870 & 0.729 \\
\hline
\end{tabular}


TABLE S2.1 (Cont.)

\begin{tabular}{|c|c|c|c|c|c|c|c|c|}
\hline \multirow[b]{2}{*}{ Date } & \multirow[b]{2}{*}{ Time } & \multicolumn{6}{|c|}{ Depth to Water (ft TOC) } & \multirow[b]{2}{*}{ MW8s } \\
\hline & & MW1s & MW2s & MW3s & MW4s & MW6s & MW7s & \\
\hline $6 / 14 / 10$ & $0: 00$ & 16.648 & 20.920 & 16.231 & 25.080 & 5.367 & 8.890 & 0.718 \\
\hline $6 / 14 / 10$ & $4: 00$ & 16.626 & 20.874 & 16.227 & 25.099 & 5.378 & 8.777 & 0.624 \\
\hline $6 / 14 / 10$ & $8: 00$ & 16.595 & 20.902 & 16.264 & 25.090 & 5.345 & 8.764 & 0.651 \\
\hline $6 / 14 / 10$ & $12: 00$ & 16.613 & 20.954 & 16.301 & 25.105 & 5.349 & 8.781 & 0.679 \\
\hline $6 / 14 / 10$ & $16: 00$ & 16.655 & 20.991 & 16.313 & 25.126 & 5.361 & 8.786 & 0.696 \\
\hline $6 / 14 / 10$ & $20: 00$ & 16.647 & 21.044 & 16.348 & 25.137 & 5.394 & 8.816 & 0.710 \\
\hline $6 / 15 / 10$ & $0: 00$ & 16.625 & 21.109 & 16.410 & 25.153 & 5.367 & 8.853 & 0.719 \\
\hline $6 / 15 / 10$ & $4: 00$ & 16.684 & 21.130 & 16.418 & 25.183 & 5.384 & 8.846 & 0.714 \\
\hline $6 / 15 / 10$ & $8: 00$ & 15.311 & 20.270 & 15.377 & 25.164 & 2.734 & 7.792 & -0.213 \\
\hline $6 / 15 / 10$ & $12: 00$ & 14.356 & 19.796 & 15.096 & 25.149 & 2.493 & 7.649 & -0.212 \\
\hline $6 / 15 / 10$ & $16: 00$ & 14.023 & 19.472 & 14.946 & 25.132 & 3.876 & 7.229 & -0.110 \\
\hline $6 / 15 / 10$ & $20: 00$ & 13.930 & 19.311 & 14.858 & 25.086 & 4.605 & 7.300 & 0.118 \\
\hline $6 / 16 / 10$ & $0: 00$ & 13.866 & 19.262 & 14.819 & 25.004 & 4.906 & 7.383 & 0.196 \\
\hline $6 / 16 / 10$ & $4: 00$ & 13.914 & 19.204 & 14.768 & 24.964 & 5.052 & 7.413 & 0.238 \\
\hline $6 / 16 / 10$ & $8: 00$ & 13.953 & 19.191 & 14.747 & 24.895 & 5.201 & 7.450 & 0.275 \\
\hline $6 / 16 / 10$ & $12: 00$ & 14.074 & 19.174 & 14.722 & 24.863 & 5.277 & 7.487 & 0.320 \\
\hline $6 / 16 / 10$ & $16: 00$ & 14.181 & 19.111 & 14.683 & 24.865 & 5.302 & 7.477 & 0.340 \\
\hline $6 / 16 / 10$ & $20: 00$ & 14.231 & 19.113 & 14.677 & 24.849 & 5.292 & 7.519 & 0.365 \\
\hline $6 / 17 / 10$ & 0:00 & 14.240 & 19.140 & 14.686 & 24.827 & 5.320 & 7.573 & 0.380 \\
\hline $6 / 17 / 10$ & $4: 00$ & 14.347 & 19.094 & 14.662 & 24.819 & 5.325 & 7.551 & 0.388 \\
\hline $6 / 17 / 10$ & $8: 00$ & 14.379 & 19.121 & 14.675 & 24.766 & 5.325 & 7.588 & 0.404 \\
\hline $6 / 17 / 10$ & $12: 00$ & 14.455 & 19.128 & 14.676 & 24.710 & 5.323 & 7.609 & 0.430 \\
\hline $6 / 17 / 10$ & $16: 00$ & 14.542 & 19.147 & 14.679 & 24.665 & 5.312 & 7.643 & 0.465 \\
\hline $6 / 17 / 10$ & $20: 00$ & 14.575 & 19.189 & 14.700 & 24.615 & 5.320 & 7.694 & 0.493 \\
\hline $6 / 18 / 10$ & 0:00 & 14.548 & 19.287 & 14.759 & 24.556 & 5.309 & 7.798 & 0.511 \\
\hline $6 / 18 / 10$ & $4: 00$ & 14.670 & 19.270 & 14.759 & 24.554 & 5.356 & 7.794 & 0.508 \\
\hline $6 / 18 / 10$ & $8: 00$ & 14.741 & 19.287 & 14.774 & 24.535 & 5.319 & 7.818 & 0.513 \\
\hline $6 / 18 / 10$ & $12: 00$ & 14.810 & 19.327 & 14.796 & 24.520 & 5.352 & 7.853 & 0.536 \\
\hline $6 / 18 / 10$ & $16: 00$ & 14.915 & 19.363 & 14.808 & 24.525 & 5.352 & 7.880 & 0.562 \\
\hline $6 / 18 / 10$ & $20: 00$ & 14.960 & 19.409 & 14.828 & 24.516 & 5.335 & 7.922 & 0.576 \\
\hline $6 / 19 / 10$ & $0: 00$ & 14.930 & 19.512 & 14.889 & 24.482 & 5.307 & 8.018 & 0.586 \\
\hline $6 / 19 / 10$ & $4: 00$ & 14.992 & 19.558 & 14.926 & 24.495 & 5.348 & 8.052 & 0.588 \\
\hline $6 / 19 / 10$ & $8: 00$ & 15.057 & 19.617 & 14.968 & 24.506 & 5.354 & 8.104 & 0.589 \\
\hline $6 / 19 / 10$ & $12: 00$ & 15.105 & 19.669 & 15.003 & 24.543 & 5.368 & 8.126 & 0.495 \\
\hline $6 / 19 / 10$ & $16: 00$ & 15.193 & 19.606 & 14.970 & 24.579 & 5.350 & 8.030 & 0.506 \\
\hline $6 / 19 / 10$ & $20: 00$ & 15.184 & 19.678 & 15.030 & 24.548 & 5.329 & 8.086 & 0.558 \\
\hline $6 / 20 / 10$ & $0: 00$ & 15.000 & 19.652 & 14.948 & 24.560 & 4.486 & 8.281 & 0.018 \\
\hline $6 / 20 / 10$ & $4: 00$ & 14.680 & 19.304 & 14.790 & 24.605 & 4.503 & 7.460 & 0.086 \\
\hline $6 / 20 / 10$ & $8: 00$ & 14.420 & 19.380 & 14.805 & 24.552 & 4.952 & 7.541 & 0.255 \\
\hline $6 / 20 / 10$ & $12: 00$ & 14.472 & 19.323 & 14.786 & 24.558 & 5.144 & 7.512 & 0.325 \\
\hline $6 / 20 / 10$ & $16: 00$ & 14.520 & 19.323 & 14.761 & 24.563 & 5.255 & 7.524 & 0.388 \\
\hline $6 / 20 / 10$ & $20: 00$ & 14.557 & 19.327 & 14.740 & 24.560 & 5.288 & 7.536 & 0.423 \\
\hline $6 / 21 / 10$ & $0: 00$ & 14.260 & 19.283 & 14.471 & 24.510 & 4.118 & 7.831 & -0.025 \\
\hline $6 / 21 / 10$ & $4: 00$ & 13.521 & 18.578 & 13.942 & 24.495 & 2.592 & 6.722 & -0.025 \\
\hline $6 / 21 / 10$ & $8: 00$ & 13.261 & 18.429 & 13.999 & 24.348 & 4.000 & 6.696 & -0.015 \\
\hline $6 / 21 / 10$ & $12: 00$ & 13.299 & 18.290 & 13.909 & 24.340 & 4.682 & 6.614 & 0.016 \\
\hline $6 / 21 / 10$ & $16: 00$ & 13.346 & 18.259 & 13.894 & 24.247 & 5.004 & 6.659 & 0.193 \\
\hline $6 / 21 / 10$ & $20: 00$ & 13.403 & 18.265 & 13.879 & 24.194 & 5.152 & 6.711 & 0.258 \\
\hline
\end{tabular}


TABLE S2.1 (Cont.)

\begin{tabular}{|c|c|c|c|c|c|c|c|c|}
\hline \multirow[b]{2}{*}{ Date } & \multirow[b]{2}{*}{ Time } & \multicolumn{6}{|c|}{ Depth to Water (ft TOC) } & \multirow[b]{2}{*}{ MW8s } \\
\hline & & MW1s & MW2s & MW3s & MW4s & MW6s & MW7s & \\
\hline $6 / 22 / 10$ & $0: 00$ & 13.422 & 18.309 & 13.904 & 24.129 & 5.216 & 6.802 & 0.294 \\
\hline $6 / 22 / 10$ & $4: 00$ & 13.457 & 18.345 & 13.914 & 24.100 & 5.198 & 6.861 & 0.314 \\
\hline $6 / 22 / 10$ & 8:00 & 13.520 & 18.401 & 13.957 & 24.049 & 5.227 & 6.958 & 0.343 \\
\hline $6 / 22 / 10$ & $12: 00$ & 13.637 & 18.401 & 13.951 & 24.060 & 5.260 & 6.943 & 0.369 \\
\hline $6 / 22 / 10$ & $16: 00$ & 13.787 & 18.426 & 13.952 & 24.051 & 5.289 & 7.014 & 0.417 \\
\hline $6 / 22 / 10$ & $20: 00$ & 13.854 & 18.479 & 13.973 & 24.018 & 5.254 & 7.079 & 0.451 \\
\hline $6 / 23 / 10$ & $0: 00$ & 13.872 & 18.552 & 14.022 & 23.984 & 5.291 & 7.166 & 0.464 \\
\hline $6 / 23 / 10$ & $4: 00$ & 13.992 & 18.544 & 14.020 & 23.992 & 5.279 & 7.171 & 0.461 \\
\hline $6 / 23 / 10$ & 8:00 & 13.999 & 18.649 & 14.092 & 23.953 & 5.314 & 7.279 & 0.477 \\
\hline $6 / 23 / 10$ & $12: 00$ & 14.109 & 18.693 & 14.118 & 23.961 & 5.310 & 7.322 & 0.498 \\
\hline $6 / 23 / 10$ & $16: 00$ & 14.169 & 18.789 & 14.170 & 23.973 & 5.291 & 7.407 & 0.532 \\
\hline $6 / 23 / 10$ & $20: 00$ & 14.241 & 18.848 & 14.210 & 23.997 & 5.322 & 7.467 & 0.555 \\
\hline $6 / 24 / 10$ & $0: 00$ & 14.269 & 18.930 & 14.266 & 24.003 & 5.307 & 7.545 & 0.558 \\
\hline $6 / 24 / 10$ & $4: 00$ & 14.360 & 18.963 & 14.298 & 24.030 & 5.336 & 7.580 & 0.556 \\
\hline $6 / 24 / 10$ & 8:00 & 14.453 & 19.014 & 14.336 & 24.051 & 5.328 & 7.624 & 0.561 \\
\hline $6 / 24 / 10$ & $12: 00$ & 14.583 & 19.050 & 14.360 & 24.085 & 5.309 & 7.651 & 0.574 \\
\hline $6 / 24 / 10$ & $16: 00$ & 14.692 & 19.062 & 14.367 & 24.100 & 5.305 & 7.649 & 0.595 \\
\hline $6 / 24 / 10$ & $20: 00$ & 14.748 & 19.089 & 14.381 & 24.108 & 5.329 & 7.665 & 0.606 \\
\hline $6 / 25 / 10$ & $0: 00$ & 14.736 & 19.142 & 14.417 & 24.102 & 5.343 & 7.708 & 0.604 \\
\hline $6 / 25 / 10$ & $4: 00$ & 14.785 & 19.167 & 14.443 & 24.100 & 5.308 & 7.721 & 0.601 \\
\hline $6 / 25 / 10$ & 8:00 & 14.847 & 19.209 & 14.471 & 24.110 & 5.320 & 7.747 & 0.600 \\
\hline $6 / 25 / 10$ & $12: 00$ & 14.950 & 19.238 & 14.490 & 24.133 & 5.316 & 7.757 & 0.618 \\
\hline $6 / 25 / 10$ & $16: 00$ & 15.037 & 19.257 & 14.491 & 24.152 & 5.310 & 7.755 & 0.636 \\
\hline $6 / 25 / 10$ & $20: 00$ & 15.074 & 19.297 & 14.506 & 24.148 & 5.368 & 7.767 & 0.644 \\
\hline $6 / 26 / 10$ & $0: 00$ & 15.047 & 19.369 & 14.550 & 24.140 & 5.318 & 7.825 & 0.646 \\
\hline 6/26/10 & $4: 00$ & 15.048 & 19.404 & 14.576 & 24.131 & 5.318 & 7.821 & 0.640 \\
\hline $6 / 26 / 10$ & 8:00 & 15.137 & 19.459 & 14.616 & 24.131 & 5.316 & 7.877 & 0.641 \\
\hline $6 / 26 / 10$ & $12: 00$ & 15.226 & 19.501 & 14.639 & 24.194 & 5.293 & 7.890 & 0.658 \\
\hline $6 / 26 / 10$ & $16: 00$ & 15.318 & 19.518 & 14.644 & 24.222 & 5.335 & 7.881 & 0.675 \\
\hline $6 / 26 / 10$ & $20: 00$ & 15.360 & 19.547 & 14.654 & 24.224 & 5.291 & 7.890 & 0.682 \\
\hline $6 / 27 / 10$ & $0: 00$ & 15.335 & 19.627 & 14.693 & 24.218 & 5.318 & 7.936 & 0.675 \\
\hline $6 / 27 / 10$ & $4: 00$ & 15.388 & 19.637 & 14.705 & 24.226 & 5.329 & 7.925 & 0.667 \\
\hline $6 / 27 / 10$ & 8:00 & 15.353 & 19.736 & 14.766 & 24.218 & 5.316 & 7.992 & 0.673 \\
\hline $6 / 27 / 10$ & $12: 00$ & 15.400 & 19.822 & 14.818 & 24.234 & 5.335 & 8.044 & 0.693 \\
\hline $6 / 27 / 10$ & $16: 00$ & 15.472 & 19.891 & 14.852 & 24.270 & 5.314 & 8.079 & 0.706 \\
\hline $6 / 27 / 10$ & $20: 00$ & 15.516 & 19.963 & 14.891 & 24.299 & 5.339 & 8.120 & 0.718 \\
\hline $6 / 28 / 10$ & $0: 00$ & 15.504 & 20.053 & 14.947 & 24.308 & 5.322 & 8.182 & 0.712 \\
\hline $6 / 28 / 10$ & $4: 00$ & 15.576 & 20.095 & 14.979 & 24.331 & 5.343 & 8.198 & 0.704 \\
\hline $6 / 28 / 10$ & $8: 00$ & 15.613 & 20.177 & 15.032 & 24.350 & 5.327 & 8.250 & 0.709 \\
\hline $6 / 28 / 10$ & $12: 00$ & 15.784 & 20.225 & 15.064 & 24.413 & 5.449 & 8.265 & 0.732 \\
\hline $6 / 28 / 10$ & $16: 00$ & 15.896 & 20.303 & 15.093 & 24.461 & 5.616 & 8.293 & 0.757 \\
\hline $6 / 28 / 10$ & $20: 00$ & 15.960 & 20.368 & 15.132 & 24.489 & 5.877 & 8.332 & 0.759 \\
\hline $6 / 29 / 10$ & $0: 00$ & 15.924 & 20.458 & 15.190 & 24.504 & 5.857 & 8.392 & 0.747 \\
\hline $6 / 29 / 10$ & $4: 00$ & 15.981 & 20.525 & 15.240 & 24.544 & 5.830 & 8.423 & 0.743 \\
\hline $6 / 29 / 10$ & $8: 00$ & 16.043 & 20.582 & 15.280 & 24.590 & 5.731 & 8.453 & 0.741 \\
\hline $6 / 29 / 10$ & $12: 00$ & 16.165 & 20.649 & 15.325 & 24.651 & 5.764 & 8.484 & 0.770 \\
\hline $6 / 29 / 10$ & $16: 00$ & 16.283 & 20.693 & 15.350 & 24.695 & 5.896 & 8.493 & 0.793 \\
\hline $6 / 29 / 10$ & $20: 00$ & 16.337 & 20.748 & 15.383 & 24.735 & 5.982 & 8.514 & 0.794 \\
\hline
\end{tabular}


TABLE S2.1 (Cont.)

\begin{tabular}{|c|c|c|c|c|c|c|c|c|}
\hline \multirow[b]{2}{*}{ Date } & \multirow[b]{2}{*}{ Time } & \multicolumn{6}{|c|}{ Depth to Water (ft TOC) } & \multirow[b]{2}{*}{ MW8s } \\
\hline & & MW1s & MW2s & MW3s & MW4s & MW6s & MW7s & \\
\hline $6 / 30 / 10$ & $0: 00$ & 16.362 & 20.808 & 15.424 & 24.760 & 6.024 & 8.551 & 0.777 \\
\hline $6 / 30 / 10$ & $4: 00$ & 16.436 & 20.855 & 15.460 & 24.802 & 6.191 & 8.566 & 0.769 \\
\hline 6/30/10 & 8:00 & 16.492 & 20.926 & 15.519 & 24.821 & 6.444 & 8.611 & 0.772 \\
\hline 6/30/10 & $12: 00$ & 16.609 & 20.964 & 15.551 & 24.853 & 6.583 & 8.616 & 0.792 \\
\hline $6 / 30 / 10$ & $16: 00$ & 16.756 & 20.995 & 15.573 & 24.933 & 6.696 & 8.614 & 0.817 \\
\hline $6 / 30 / 10$ & $20: 00$ & 16.809 & 21.056 & 15.615 & 24.964 & 6.890 & 8.642 & 0.821 \\
\hline $7 / 1 / 10$ & $0: 00$ & 16.803 & 21.138 & 15.692 & 24.981 & 7.086 & 8.692 & 0.807 \\
\hline $7 / 1 / 10$ & $4: 00$ & 16.813 & 21.178 & 15.729 & 25.021 & 7.134 & 8.687 & 0.792 \\
\hline $7 / 1 / 10$ & 8:00 & 16.866 & 21.232 & 15.785 & 25.050 & 6.831 & 8.727 & 0.789 \\
\hline $7 / 1 / 10$ & $12: 00$ & 16.991 & 21.279 & 15.821 & 25.099 & 6.750 & 8.735 & 0.822 \\
\hline $7 / 1 / 10$ & $16: 00$ & 17.100 & 21.312 & 15.866 & 25.162 & 6.903 & 8.731 & 0.847 \\
\hline $7 / 1 / 10$ & $20: 00$ & 17.146 & 21.369 & 15.958 & 25.208 & 7.096 & 8.755 & 0.852 \\
\hline $7 / 2 / 10$ & $0: 00$ & 17.131 & 21.434 & 16.035 & 25.215 & 7.284 & 8.789 & 0.828 \\
\hline $7 / 2 / 10$ & $4: 00$ & 17.206 & 21.465 & 16.077 & 25.246 & 7.472 & 8.789 & 0.814 \\
\hline $7 / 2 / 10$ & 8:00 & 17.267 & 21.511 & 16.136 & 25.271 & 7.750 & 8.803 & 0.810 \\
\hline $7 / 2 / 10$ & $12: 00$ & 17.371 & 21.543 & 16.164 & 25.313 & 8.106 & 8.798 & 0.840 \\
\hline $7 / 2 / 10$ & $16: 00$ & 17.465 & 21.579 & 16.184 & 25.351 & 8.380 & 8.806 & 0.870 \\
\hline $7 / 2 / 10$ & $20: 00$ & 17.513 & 21.631 & 16.220 & 25.400 & 8.587 & 8.820 & 0.874 \\
\hline $7 / 3 / 10$ & $0: 00$ & 17.478 & 21.707 & 16.298 & 25.410 & 8.876 & 8.850 & 0.853 \\
\hline $7 / 3 / 10$ & $4: 00$ & 17.567 & 21.726 & 16.322 & 25.448 & 9.194 & 8.850 & 0.834 \\
\hline $7 / 3 / 10$ & 8:00 & 17.600 & 21.784 & 16.386 & 25.467 & 9.565 & 8.878 & 0.836 \\
\hline $7 / 3 / 10$ & $12: 00$ & 17.687 & 21.826 & 16.429 & 25.494 & 9.881 & 8.889 & 0.868 \\
\hline $7 / 3 / 10$ & $16: 00$ & 17.761 & 21.864 & 16.451 & 25.519 & 10.066 & 8.892 & 0.890 \\
\hline $7 / 3 / 10$ & $20: 00$ & 17.781 & 21.923 & 16.499 & 25.557 & 10.227 & 8.918 & 0.878 \\
\hline $7 / 4 / 10$ & 0:00 & 17.751 & 21.973 & 16.557 & 25.576 & 10.178 & 8.935 & 0.856 \\
\hline $7 / 4 / 10$ & $4: 00$ & 17.737 & 22.011 & 16.598 & 25.625 & 9.238 & 8.927 & 0.845 \\
\hline $7 / 4 / 10$ & 8:00 & 17.730 & 22.061 & 16.656 & 25.633 & 8.057 & 8.959 & 0.843 \\
\hline $7 / 4 / 10$ & $12: 00$ & 17.698 & 22.110 & 16.701 & 25.649 & 7.160 & 8.959 & 0.847 \\
\hline $7 / 4 / 10$ & $16: 00$ & 17.660 & 22.124 & 16.750 & 25.661 & 6.732 & 8.957 & 0.719 \\
\hline $7 / 4 / 10$ & $20: 00$ & 17.458 & 21.929 & 16.699 & 25.690 & 6.425 & 8.680 & 0.433 \\
\hline $7 / 5 / 10$ & 0:00 & 17.102 & 21.738 & 16.581 & 25.698 & 6.195 & 8.062 & 0.463 \\
\hline $7 / 5 / 10$ & $4: 00$ & 16.926 & 21.711 & 16.566 & 25.696 & 6.012 & 7.988 & 0.577 \\
\hline $7 / 5 / 10$ & 8:00 & 16.817 & 21.744 & 16.564 & 25.690 & 5.886 & 8.049 & 0.634 \\
\hline $7 / 5 / 10$ & $12: 00$ & 16.753 & 21.784 & 16.571 & 25.671 & 5.801 & 8.079 & 0.663 \\
\hline $7 / 5 / 10$ & $16: 00$ & 16.728 & 21.761 & 16.538 & 25.667 & 5.659 & 8.055 & 0.610 \\
\hline $7 / 5 / 10$ & $20: 00$ & 16.654 & 21.784 & 16.567 & 25.663 & 5.609 & 8.030 & 0.653 \\
\hline $7 / 6 / 10$ & $0: 00$ & 16.594 & 21.839 & 16.603 & 25.644 & 5.543 & 8.086 & 0.676 \\
\hline $7 / 6 / 10$ & $4: 00$ & 16.585 & 21.889 & 16.631 & 25.629 & 5.487 & 8.125 & 0.690 \\
\hline $7 / 6 / 10$ & $8: 00$ & 16.589 & 21.944 & 16.670 & 25.631 & 5.473 & 8.173 & 0.698 \\
\hline $7 / 6 / 10$ & $12: 00$ & 16.647 & 21.990 & 16.691 & 25.652 & 5.442 & 8.206 & 0.722 \\
\hline $7 / 6 / 10$ & $16: 00$ & 16.700 & 22.026 & 16.697 & 25.671 & 5.411 & 8.235 & 0.747 \\
\hline $7 / 6 / 10$ & $20: 00$ & 16.714 & 22.082 & 16.723 & 25.665 & 5.385 & 8.277 & 0.757 \\
\hline $7 / 7 / 10$ & $0: 00$ & 16.694 & 22.141 & 16.775 & 25.660 & 5.372 & 8.326 & 0.753 \\
\hline $7 / 7 / 10$ & $4: 00$ & 16.735 & 22.168 & 16.798 & 25.673 & 5.360 & 8.343 & 0.747 \\
\hline $7 / 7 / 10$ & $8: 00$ & 16.760 & 22.217 & 16.839 & 25.681 & 5.357 & 8.378 & 0.747 \\
\hline $7 / 7 / 10$ & $12: 00$ & 16.769 & 22.275 & 16.891 & 25.688 & 5.351 & 8.424 & 0.757 \\
\hline $7 / 7 / 10$ & $16: 00$ & 16.866 & 22.292 & 16.881 & 25.738 & 5.299 & 8.420 & 0.760 \\
\hline $7 / 7 / 10$ & $20: 00$ & 16.869 & 22.345 & 16.922 & 25.728 & 5.322 & 8.457 & 0.773 \\
\hline
\end{tabular}


TABLE S2.1 (Cont.)

\begin{tabular}{|c|c|c|c|c|c|c|c|c|}
\hline \multirow[b]{2}{*}{ Date } & \multirow[b]{2}{*}{ Time } & \multicolumn{6}{|c|}{ Depth to Water (ft TOC) } & \multirow[b]{2}{*}{ MW8s } \\
\hline & & MW1s & MW2s & MW3s & MW4s & MW6s & MW7s & \\
\hline $7 / 8 / 10$ & $0: 00$ & 16.864 & 22.397 & 16.974 & 25.730 & 5.259 & 8.491 & 0.765 \\
\hline $7 / 8 / 10$ & $4: 00$ & 16.899 & 22.435 & 17.001 & 25.743 & 5.257 & 8.511 & 0.761 \\
\hline $7 / 8 / 10$ & 8:00 & 16.905 & 22.490 & 17.050 & 25.747 & 5.257 & 8.547 & 0.765 \\
\hline $7 / 8 / 10$ & $12: 00$ & 16.959 & 22.538 & 17.087 & 25.772 & 5.240 & 8.575 & 0.779 \\
\hline $7 / 8 / 10$ & $16: 00$ & 17.028 & 22.565 & 17.087 & 25.808 & 5.236 & 8.580 & 0.796 \\
\hline $7 / 8 / 10$ & $20: 00$ & 17.073 & 22.611 & 17.111 & 25.818 & 5.330 & 8.606 & 0.803 \\
\hline $7 / 9 / 10$ & $0: 00$ & 17.105 & 22.670 & 17.163 & 25.820 & 5.500 & 8.649 & 0.796 \\
\hline $7 / 9 / 10$ & $4: 00$ & 17.177 & 22.695 & 17.177 & 25.845 & 5.687 & 8.655 & 0.785 \\
\hline $7 / 9 / 10$ & 8:00 & 17.222 & 22.758 & 17.224 & 25.854 & 5.918 & 8.690 & 0.789 \\
\hline $7 / 9 / 10$ & $12: 00$ & 17.293 & 22.792 & 17.248 & 25.875 & 5.908 & 8.710 & 0.816 \\
\hline $7 / 9 / 10$ & $16: 00$ & 17.353 & 22.815 & 17.242 & 25.911 & 5.823 & 8.712 & 0.847 \\
\hline $7 / 9 / 10$ & $20: 00$ & 17.324 & 22.861 & 17.258 & 25.947 & 5.659 & 8.695 & 0.845 \\
\hline $7 / 10 / 10$ & $0: 00$ & 17.326 & 22.909 & 17.296 & 25.938 & 5.564 & 8.751 & 0.824 \\
\hline $7 / 10 / 10$ & $4: 00$ & 17.370 & 22.941 & 17.319 & 25.942 & 5.496 & 8.769 & 0.814 \\
\hline $7 / 10 / 10$ & 8:00 & 17.393 & 22.987 & 17.348 & 25.944 & 5.407 & 8.788 & 0.812 \\
\hline $7 / 10 / 10$ & $12: 00$ & 17.461 & 23.014 & 17.362 & 25.968 & 5.415 & 8.790 & 0.845 \\
\hline $7 / 10 / 10$ & $16: 00$ & 17.513 & 23.039 & 17.352 & 25.993 & 5.415 & 8.790 & 0.882 \\
\hline $7 / 10 / 10$ & $20: 00$ & 17.525 & 23.077 & 17.346 & 25.993 & 5.382 & 8.797 & 0.876 \\
\hline $7 / 11 / 10$ & $0: 00$ & 17.498 & 23.128 & 17.384 & 25.974 & 5.341 & 8.823 & 0.847 \\
\hline $7 / 11 / 10$ & $4: 00$ & 17.525 & 23.134 & 17.365 & 25.986 & 5.304 & 8.794 & 0.827 \\
\hline $7 / 11 / 10$ & $8: 00$ & 17.424 & 23.044 & 17.308 & 25.974 & 5.248 & 8.439 & 0.573 \\
\hline $7 / 11 / 10$ & $12: 00$ & 17.287 & 22.981 & 17.309 & 25.967 & 5.226 & 8.309 & 0.656 \\
\hline $7 / 11 / 10$ & $16: 00$ & 17.241 & 22.968 & 17.296 & 25.986 & 5.205 & 8.301 & 0.722 \\
\hline $7 / 11 / 10$ & $20: 00$ & 17.182 & 22.979 & 17.291 & 25.999 & 5.234 & 8.320 & 0.733 \\
\hline $7 / 12 / 10$ & $0: 00$ & 17.093 & 23.050 & 17.351 & 25.963 & 5.257 & 8.387 & 0.751 \\
\hline $7 / 12 / 10$ & $4: 00$ & 17.123 & 23.050 & 17.325 & 25.965 & 5.211 & 8.381 & 0.749 \\
\hline $7 / 12 / 10$ & 8:00 & 17.128 & 23.092 & 17.339 & 25.959 & 5.203 & 8.407 & 0.751 \\
\hline $7 / 12 / 10$ & $12: 00$ & 17.147 & 23.157 & 17.388 & 25.953 & 5.191 & 8.457 & 0.772 \\
\hline $7 / 12 / 10$ & $16: 00$ & 17.203 & 23.151 & 17.357 & 25.980 & 5.162 & 8.442 & 0.774 \\
\hline $7 / 12 / 10$ & $20: 00$ & 17.212 & 23.174 & 17.364 & 25.974 & 5.174 & 8.468 & 0.776 \\
\hline $7 / 13 / 10$ & $0: 00$ & 17.180 & 23.216 & 17.391 & 25.976 & 5.176 & 8.492 & 0.776 \\
\hline $7 / 13 / 10$ & $4: 00$ & 17.225 & 23.239 & 17.403 & 26.005 & 5.182 & 8.515 & 0.772 \\
\hline $7 / 13 / 10$ & 8:00 & 17.237 & 23.288 & 17.435 & 26.022 & 5.167 & 8.554 & 0.774 \\
\hline $7 / 13 / 10$ & $12: 00$ & 17.298 & 23.314 & 17.442 & 26.037 & 5.183 & 8.563 & 0.801 \\
\hline $7 / 13 / 10$ & $16: 00$ & 17.370 & 23.355 & 17.443 & 26.087 & 5.218 & 8.593 & 0.839 \\
\hline $7 / 13 / 10$ & $20: 00$ & 17.391 & 23.384 & 17.452 & 26.071 & 5.174 & 8.622 & 0.847 \\
\hline $7 / 14 / 10$ & $0: 00$ & 17.380 & 23.437 & 17.494 & 26.064 & 5.202 & 8.667 & 0.829 \\
\hline $7 / 14 / 10$ & $4: 00$ & 17.410 & 23.470 & 17.513 & 26.073 & 5.174 & 8.691 & 0.819 \\
\hline $7 / 14 / 10$ & $8: 00$ & 17.395 & 23.516 & 17.547 & 26.075 & 5.215 & 8.714 & 0.815 \\
\hline $7 / 14 / 10$ & $12: 00$ & 17.489 & 23.558 & 17.566 & 26.106 & 5.210 & 8.749 & 0.858 \\
\hline $7 / 14 / 10$ & $16: 00$ & 17.539 & 23.594 & 17.579 & 26.148 & 5.218 & 8.775 & 0.903 \\
\hline $7 / 14 / 10$ & $20: 00$ & 17.552 & 23.626 & 17.574 & 26.155 & 5.218 & 8.788 & 0.906 \\
\hline $7 / 15 / 10$ & $0: 00$ & 17.424 & 23.714 & 17.652 & 26.108 & 5.228 & 8.749 & 0.678 \\
\hline $7 / 15 / 10$ & $4: 00$ & 17.339 & 23.630 & 17.644 & 26.150 & 5.202 & 8.410 & 0.690 \\
\hline $7 / 15 / 10$ & 8:00 & 17.295 & 23.619 & 17.639 & 26.174 & 5.283 & 8.412 & 0.733 \\
\hline $7 / 15 / 10$ & $12: 00$ & 17.319 & 23.636 & 17.640 & 26.211 & 5.235 & 8.438 & 0.782 \\
\hline $7 / 15 / 10$ & $16: 00$ & 17.379 & 23.644 & 17.616 & 26.249 & 5.199 & 8.457 & 0.832 \\
\hline $7 / 15 / 10$ & $20: 00$ & 17.415 & 23.657 & 17.586 & 26.264 & 5.199 & 8.462 & 0.841 \\
\hline
\end{tabular}


TABLE S2.1 (Cont.)

\begin{tabular}{|c|c|c|c|c|c|c|c|c|}
\hline \multirow[b]{2}{*}{ Date } & \multirow[b]{2}{*}{ Time } & \multicolumn{6}{|c|}{ Depth to Water (ft TOC) } & \multirow[b]{2}{*}{ MW8s } \\
\hline & & MW1s & MW2s & MW3s & MW4s & MW6s & MW7s & \\
\hline $7 / 16 / 10$ & $0: 00$ & 17.340 & 23.707 & 17.610 & 26.252 & 5.218 & 8.515 & 0.828 \\
\hline $7 / 16 / 10$ & $4: 00$ & 17.394 & 23.718 & 17.596 & 26.264 & 5.288 & 8.518 & 0.816 \\
\hline $7 / 16 / 10$ & $8: 00$ & 17.432 & 23.739 & 17.600 & 26.270 & 5.195 & 8.537 & 0.810 \\
\hline $7 / 16 / 10$ & $12: 00$ & 17.491 & 23.760 & 17.593 & 26.281 & 5.241 & 8.550 & 0.837 \\
\hline $7 / 16 / 10$ & $16: 00$ & 17.553 & 23.835 & 17.568 & 26.312 & 5.259 & 8.553 & 0.877 \\
\hline $7 / 16 / 10$ & $20: 00$ & 17.539 & 23.846 & 17.579 & 26.287 & 5.214 & 8.589 & 0.879 \\
\hline $7 / 17 / 10$ & $0: 00$ & 17.524 & 23.867 & 17.596 & 26.277 & 5.235 & 8.611 & 0.851 \\
\hline $7 / 17 / 10$ & $4: 00$ & 17.536 & 23.892 & 17.609 & 26.266 & 5.234 & 8.635 & 0.839 \\
\hline $7 / 17 / 10$ & 8:00 & 17.589 & 23.908 & 17.606 & 26.267 & 5.247 & 8.646 & 0.832 \\
\hline $7 / 17 / 10$ & $12: 00$ & 17.673 & 23.936 & 17.611 & 26.281 & 5.397 & 8.667 & 0.875 \\
\hline $7 / 17 / 10$ & $16: 00$ & 17.766 & 23.951 & 17.596 & 26.315 & 5.593 & 8.681 & 0.918 \\
\hline $7 / 17 / 10$ & $20: 00$ & 17.804 & 23.972 & 17.582 & 26.321 & 5.812 & 8.672 & 0.916 \\
\hline $7 / 18 / 10$ & $0: 00$ & 17.814 & 24.024 & 17.618 & 26.294 & 5.950 & 8.737 & 0.885 \\
\hline $7 / 18 / 10$ & $4: 00$ & 17.888 & 24.039 & 17.604 & 26.319 & 6.113 & 8.741 & 0.861 \\
\hline $7 / 18 / 10$ & 8:00 & 17.859 & 24.119 & 17.681 & 26.296 & 6.350 & 8.798 & 0.865 \\
\hline $7 / 18 / 10$ & $12: 00$ & 17.924 & 24.117 & 17.672 & 26.334 & 6.302 & 8.812 & 0.865 \\
\hline $7 / 18 / 10$ & $16: 00$ & 17.971 & 24.142 & 17.670 & 26.357 & 6.092 & 8.832 & 0.911 \\
\hline $7 / 18 / 10$ & $20: 00$ & 18.003 & 24.171 & 17.677 & 26.382 & 5.941 & 8.860 & 0.926 \\
\hline $7 / 19 / 10$ & $0: 00$ & 17.998 & 24.213 & 17.707 & 26.388 & 5.849 & 8.893 & 0.897 \\
\hline $7 / 19 / 10$ & $4: 00$ & 18.107 & 24.199 & 17.668 & 26.422 & 5.948 & 8.869 & 0.870 \\
\hline $7 / 19 / 10$ & 8:00 & 18.135 & 24.238 & 17.685 & 26.418 & 6.226 & 8.879 & 0.864 \\
\hline $7 / 19 / 10$ & $12: 00$ & 18.191 & 24.283 & 17.714 & 26.435 & 6.556 & 8.930 & 0.884 \\
\hline $7 / 19 / 10$ & $16: 00$ & 18.296 & 24.295 & 17.696 & 26.483 & 6.841 & 8.930 & 0.933 \\
\hline $7 / 19 / 10$ & $20: 00$ & 18.353 & 24.324 & 17.707 & 26.514 & 7.121 & 8.960 & 0.955 \\
\hline $7 / 20 / 10$ & $0: 00$ & 18.340 & 24.373 & 17.738 & 26.500 & 7.308 & 8.999 & 0.920 \\
\hline $7 / 20 / 10$ & $4: 00$ & 18.320 & 24.459 & 17.812 & 26.479 & 7.521 & 9.075 & 0.920 \\
\hline $7 / 20 / 10$ & 8:00 & 18.407 & 24.455 & 17.801 & 26.531 & 7.772 & 9.053 & 0.899 \\
\hline $7 / 20 / 10$ & $12: 00$ & 18.450 & 24.534 & 17.860 & 26.563 & 8.020 & 9.114 & 0.924 \\
\hline $7 / 20 / 10$ & $16: 00$ & 18.561 & 24.530 & 17.849 & 26.647 & 8.282 & 9.111 & 0.938 \\
\hline $7 / 20 / 10$ & $20: 00$ & 18.598 & 24.583 & 17.884 & 26.706 & 8.505 & 9.157 & 0.950 \\
\hline $7 / 21 / 10$ & $0: 00$ & 18.643 & 24.614 & 17.912 & 26.731 & 8.745 & 9.182 & 0.926 \\
\hline $7 / 21 / 10$ & $4: 00$ & 18.691 & 24.654 & 17.938 & 26.758 & 9.006 & 9.214 & 0.919 \\
\hline $7 / 21 / 10$ & 8:00 & 18.737 & 24.698 & 17.970 & 26.794 & 9.316 & 9.248 & 0.915 \\
\hline $7 / 21 / 10$ & $12: 00$ & 18.782 & 24.734 & 18.004 & 26.832 & 9.689 & 9.277 & 0.909 \\
\hline $7 / 21 / 10$ & $16: 00$ & 18.918 & 24.717 & 17.969 & 26.895 & 9.957 & 9.249 & 0.935 \\
\hline $7 / 21 / 10$ & $20: 00$ & 18.985 & 24.732 & 17.952 & 26.927 & 10.118 & 9.253 & 0.961 \\
\hline $7 / 22 / 10$ & $0: 00$ & 19.012 & 24.763 & 17.970 & 26.948 & 10.244 & 9.272 & 0.937 \\
\hline $7 / 22 / 10$ & $4: 00$ & 19.069 & 24.774 & 17.960 & 26.962 & 10.408 & 9.268 & 0.923 \\
\hline $7 / 22 / 10$ & $8: 00$ & 19.076 & 24.837 & 17.996 & 26.967 & 10.670 & 9.305 & 0.926 \\
\hline $7 / 22 / 10$ & $12: 00$ & 19.164 & 24.849 & 17.993 & 27.002 & 10.961 & 9.310 & 0.990 \\
\hline $7 / 22 / 10$ & $16: 00$ & 19.234 & 24.893 & 17.999 & 27.059 & 11.120 & 9.335 & 1.055 \\
\hline $7 / 22 / 10$ & $20: 00$ & 19.258 & 25.019 & 18.016 & 27.076 & 11.109 & 9.363 & 1.088 \\
\hline $7 / 23 / 10$ & $0: 00$ & 19.236 & 25.051 & 18.060 & 27.097 & 11.243 & 9.401 & 1.084 \\
\hline $7 / 23 / 10$ & $4: 00$ & 19.287 & 25.032 & 18.080 & 27.120 & 11.530 & 9.413 & 1.026 \\
\hline $7 / 23 / 10$ & $8: 00$ & 19.301 & 25.076 & 18.128 & 27.133 & 11.793 & 9.457 & 1.016 \\
\hline $7 / 23 / 10$ & $12: 00$ & 19.362 & 25.101 & 18.155 & 27.158 & 12.030 & 9.489 & 1.077 \\
\hline $7 / 23 / 10$ & $16: 00$ & 19.451 & 25.109 & 18.155 & 27.219 & 12.211 & 9.502 & 1.141 \\
\hline $7 / 23 / 10$ & $20: 00$ & 19.493 & 25.137 & 18.160 & 27.253 & 12.246 & 9.532 & 1.238 \\
\hline
\end{tabular}


TABLE S2.1 (Cont.)

\begin{tabular}{|c|c|c|c|c|c|c|c|c|}
\hline \multirow[b]{2}{*}{ Date } & \multirow[b]{2}{*}{ Time } & \multicolumn{6}{|c|}{ Depth to Water (ft TOC) } & \multirow[b]{2}{*}{ MW8s } \\
\hline & & MW1s & MW2s & MW3s & MW4s & MW6s & MW7s & \\
\hline $7 / 24 / 10$ & 0:00 & 19.494 & 25.185 & 18.211 & 27.263 & 12.282 & 9.576 & 1.249 \\
\hline $7 / 24 / 10$ & 4:00 & 19.544 & 25.179 & 18.211 & 27.301 & 12.429 & 9.567 & 1.230 \\
\hline $7 / 24 / 10$ & $8: 00$ & 19.568 & 25.225 & 18.262 & 27.322 & 12.624 & 9.621 & 1.109 \\
\hline $7 / 24 / 10$ & $12: 00$ & 19.616 & 25.311 & 18.332 & 27.349 & 12.837 & 9.680 & 1.126 \\
\hline $7 / 24 / 10$ & $16: 00$ & 19.632 & 25.321 & 18.348 & 27.450 & 13.016 & 9.637 & 0.960 \\
\hline $7 / 24 / 10$ & 20:00 & 19.657 & 25.298 & 18.351 & 27.473 & 13.030 & 9.623 & 0.993 \\
\hline $7 / 25 / 10$ & $0: 00$ & 19.543 & 25.374 & 18.431 & 27.486 & 12.608 & 9.691 & 1.005 \\
\hline $7 / 25 / 10$ & $4: 00$ & 19.560 & 25.332 & 18.409 & 27.547 & 11.336 & 9.630 & 0.992 \\
\hline $7 / 25 / 10$ & $8: 00$ & 19.522 & 25.382 & 18.449 & 27.562 & 9.860 & 9.678 & 1.001 \\
\hline $7 / 25 / 10$ & $12: 00$ & 19.538 & 25.401 & 18.473 & 27.606 & 8.608 & 9.689 & 1.039 \\
\hline $7 / 25 / 10$ & $16: 00$ & 19.558 & 25.399 & 18.463 & 27.654 & 7.600 & 9.684 & 1.090 \\
\hline $7 / 25 / 10$ & $20: 00$ & 19.523 & 25.420 & 18.457 & 27.665 & 7.134 & 9.678 & 1.104 \\
\hline $7 / 26 / 10$ & $0: 00$ & 19.454 & 25.471 & 18.501 & 27.656 & 6.788 & 9.723 & 1.071 \\
\hline $7 / 26 / 10$ & $4: 00$ & 19.470 & 25.458 & 18.486 & 27.673 & 6.551 & 9.695 & 1.039 \\
\hline $7 / 26 / 10$ & $8: 00$ & 19.440 & 25.504 & 18.520 & 27.665 & 6.392 & 9.725 & 1.031 \\
\hline $7 / 26 / 10$ & $12: 00$ & 19.520 & 25.513 & 18.524 & 27.688 & 6.440 & 9.721 & 1.063 \\
\hline $7 / 26 / 10$ & $16: 00$ & 19.596 & 25.508 & 18.507 & 27.722 & 6.709 & 9.712 & 1.126 \\
\hline $7 / 26 / 10$ & $20: 00$ & 19.593 & 25.544 & 18.520 & 27.745 & 7.056 & 9.747 & 1.163 \\
\hline $7 / 27 / 10$ & $0: 00$ & 19.559 & 25.596 & 18.563 & 27.726 & 7.439 & 9.782 & 1.139 \\
\hline $7 / 27 / 10$ & $4: 00$ & 19.596 & 25.617 & 18.577 & 27.724 & 7.897 & 9.789 & 1.081 \\
\hline $7 / 27 / 10$ & $8: 00$ & 19.620 & 25.655 & 18.605 & 27.722 & 8.335 & 9.812 & 1.059 \\
\hline $7 / 27 / 10$ & $12: 00$ & 19.713 & 25.680 & 18.623 & 27.753 & 8.748 & 9.819 & 1.106 \\
\hline $7 / 27 / 10$ & $16: 00$ & 19.777 & 25.695 & 18.626 & 27.799 & 9.132 & 9.838 & 1.181 \\
\hline $7 / 27 / 10$ & $20: 00$ & 19.812 & 25.720 & 18.635 & 27.808 & 9.472 & 9.860 & 1.269 \\
\hline $7 / 28 / 10$ & $0: 00$ & 19.778 & 25.783 & 18.694 & 27.808 & 9.761 & 9.912 & 1.289 \\
\hline $7 / 28 / 10$ & $4: 00$ & 19.817 & 25.808 & 18.715 & 27.823 & 9.986 & 9.927 & 1.240 \\
\hline $7 / 28 / 10$ & $8: 00$ & 19.844 & 25.850 & 18.762 & 27.850 & 10.157 & 9.958 & 1.138 \\
\hline $7 / 28 / 10$ & $12: 00$ & 19.924 & 25.878 & 18.790 & 27.886 & 10.317 & 9.977 & 1.177 \\
\hline $7 / 28 / 10$ & $16: 00$ & 20.010 & 25.903 & 18.807 & 27.951 & 10.513 & 10.003 & 1.287 \\
\hline $7 / 28 / 10$ & $20: 00$ & 20.015 & 25.941 & 18.836 & 27.982 & 10.750 & 10.035 & 1.391 \\
\hline $7 / 29 / 10$ & $0: 00$ & 20.005 & 26.010 & 18.906 & 27.995 & 11.021 & 10.091 & 1.411 \\
\hline $7 / 29 / 10$ & $4: 00$ & 20.064 & 26.027 & 18.931 & 28.041 & 11.302 & 10.103 & 1.393 \\
\hline $7 / 29 / 10$ & $8: 00$ & 20.117 & 26.050 & 18.955 & 28.073 & 11.590 & 10.118 & 1.241 \\
\hline $7 / 29 / 10$ & $12: 00$ & 20.214 & 26.065 & 18.970 & 28.117 & 11.891 & 10.131 & 1.235 \\
\hline $7 / 29 / 10$ & $16: 00$ & 20.297 & 26.073 & 18.966 & 28.147 & 12.198 & 10.142 & 1.339 \\
\hline $7 / 29 / 10$ & $20: 00$ & 20.335 & 26.077 & 18.960 & 28.181 & 12.470 & 10.124 & 1.185 \\
\hline $7 / 30 / 10$ & $0: 00$ & 20.305 & 26.121 & 19.002 & 28.182 & 12.595 & 10.151 & 1.157 \\
\hline $7 / 30 / 10$ & $4: 00$ & 20.360 & 26.121 & 18.992 & 28.207 & 12.581 & 10.131 & 1.134 \\
\hline $7 / 30 / 10$ & $8: 00$ & 20.384 & 26.144 & 19.002 & 28.208 & 12.572 & 10.137 & 1.120 \\
\hline $7 / 30 / 10$ & $12: 00$ & 20.378 & 26.203 & 19.041 & 28.202 & 12.696 & 10.183 & 1.134 \\
\hline $7 / 30 / 10$ & $16: 00$ & 20.459 & 26.174 & 19.022 & 28.241 & 12.853 & 10.124 & 1.113 \\
\hline $7 / 30 / 10$ & 20:00 & 20.469 & 26.209 & 19.053 & 28.243 & 12.993 & 10.152 & 1.146 \\
\hline $7 / 31 / 10$ & $0: 00$ & 20.433 & 26.258 & 19.096 & 28.266 & 13.038 & 10.187 & 1.135 \\
\hline $7 / 31 / 10$ & $4: 00$ & 20.439 & 26.293 & 19.133 & 28.290 & 12.950 & 10.205 & 1.123 \\
\hline $7 / 31 / 10$ & $8: 00$ & 20.432 & 26.344 & 19.182 & 28.310 & 12.820 & 10.241 & 1.121 \\
\hline $7 / 31 / 10$ & $12: 00$ & 20.479 & 26.377 & 19.218 & 28.353 & 12.795 & 10.267 & 1.158 \\
\hline $7 / 31 / 10$ & $16: 00$ & 20.560 & 26.394 & 19.230 & 28.386 & 12.937 & 10.283 & 1.231 \\
\hline $7 / 31 / 10$ & $20: 00$ & 20.603 & 26.413 & 19.243 & 28.424 & 13.073 & 10.302 & 1.316 \\
\hline
\end{tabular}


TABLE S2.1 (Cont.)

\begin{tabular}{|c|c|c|c|c|c|c|c|c|}
\hline \multirow[b]{2}{*}{ Date } & \multirow[b]{2}{*}{ Time } & \multicolumn{6}{|c|}{ Depth to Water (ft TOC) } & \multirow[b]{2}{*}{ MW8s } \\
\hline & & MW1s & MW2s & MW3s & MW4s & MW6s & MW7s & \\
\hline $8 / 1 / 10$ & $0: 00$ & 20.533 & 26.463 & 19.290 & 28.449 & 13.063 & 10.330 & 1.335 \\
\hline $8 / 1 / 10$ & $4: 00$ & 20.524 & 26.480 & 19.311 & 28.477 & 12.171 & 10.346 & 1.222 \\
\hline $8 / 1 / 10$ & $8: 00$ & 20.477 & 26.524 & 19.358 & 28.496 & 10.806 & 10.380 & 1.183 \\
\hline $8 / 1 / 10$ & $12: 00$ & 20.497 & 26.539 & 19.375 & 28.540 & 9.407 & 10.389 & 1.209 \\
\hline $8 / 1 / 10$ & $16: 00$ & 20.505 & 26.552 & 19.386 & 28.572 & 8.329 & 10.402 & 1.309 \\
\hline 8/1/10 & $20: 00$ & 20.474 & 26.568 & 19.395 & 28.576 & 7.478 & 10.415 & 1.424 \\
\hline $8 / 2 / 10$ & $0: 00$ & 20.410 & 26.604 & 19.436 & 28.574 & 7.099 & 10.440 & 1.448 \\
\hline $8 / 2 / 10$ & $4: 00$ & 20.434 & 26.627 & 19.449 & 28.580 & 6.936 & 10.450 & 1.454 \\
\hline $8 / 2 / 10$ & $8: 00$ & 20.457 & 26.648 & 19.467 & 28.588 & 7.043 & 10.454 & 1.316 \\
\hline $8 / 2 / 10$ & $12: 00$ & 20.495 & 26.682 & 19.507 & 28.599 & 7.309 & 10.480 & 1.297 \\
\hline $8 / 2 / 10$ & $16: 00$ & 20.561 & 26.701 & 19.512 & 28.620 & 7.686 & 10.495 & 1.425 \\
\hline $8 / 2 / 10$ & $20: 00$ & 20.571 & 26.740 & 19.542 & 28.629 & 8.210 & 10.532 & 1.553 \\
\hline $8 / 3 / 10$ & $0: 00$ & 20.551 & 26.780 & 19.587 & 28.637 & 8.651 & 10.568 & 1.581 \\
\hline $8 / 3 / 10$ & $4: 00$ & 20.590 & 26.803 & 19.615 & 28.655 & 9.160 & 10.582 & 1.600 \\
\hline $8 / 3 / 10$ & $8: 00$ & 20.586 & 26.860 & 19.677 & 28.670 & 9.814 & 10.634 & 1.614 \\
\hline 8/3/10 & $12: 00$ & 20.682 & 26.898 & 19.722 & 28.702 & 10.486 & 10.666 & 1.681 \\
\hline $8 / 3 / 10$ & $16: 00$ & 20.768 & 26.908 & 19.733 & 28.748 & 10.966 & 10.677 & 1.755 \\
\hline $8 / 3 / 10$ & $20: 00$ & 20.802 & 26.927 & 19.758 & 28.779 & 11.223 & 10.701 & 1.802 \\
\hline $8 / 4 / 10$ & 0:00 & 20.807 & 26.967 & 19.800 & 28.801 & 11.464 & 10.738 & 1.791 \\
\hline $8 / 4 / 10$ & $4: 00$ & 20.819 & 27.024 & 19.857 & 28.836 & 11.814 & 10.783 & 1.779 \\
\hline $8 / 4 / 10$ & $8: 00$ & 20.887 & 27.030 & 19.890 & 28.868 & 12.191 & 10.788 & 1.770 \\
\hline $8 / 4 / 10$ & $12: 00$ & 20.867 & 27.106 & 19.963 & 28.899 & 12.568 & 10.815 & 1.811 \\
\hline $8 / 4 / 10$ & $16: 00$ & 21.002 & 27.160 & 19.986 & 28.963 & 12.956 & 10.855 & 1.819 \\
\hline $8 / 4 / 10$ & $20: 00$ & 21.053 & 27.167 & 20.008 & 28.994 & 13.308 & 10.880 & 1.854 \\
\hline $8 / 5 / 10$ & 0:00 & 21.048 & 27.206 & 20.068 & 29.017 & 13.596 & 10.920 & 1.837 \\
\hline $8 / 5 / 10$ & $4: 00$ & 21.104 & 27.217 & 20.096 & 29.057 & 13.817 & 10.933 & 1.806 \\
\hline $8 / 5 / 10$ & $8: 00$ & 21.102 & 27.267 & 20.162 & 29.076 & 14.002 & 10.980 & 1.736 \\
\hline $8 / 5 / 10$ & $12: 00$ & 21.196 & 27.288 & 20.196 & 29.129 & 14.155 & 11.004 & 1.648 \\
\hline $8 / 5 / 10$ & $16: 00$ & 21.288 & 27.282 & 20.192 & 29.171 & 14.198 & 11.005 & 1.785 \\
\hline $8 / 5 / 10$ & $20: 00$ & 21.312 & 27.316 & 20.213 & 29.208 & 14.142 & 11.037 & 1.868 \\
\hline $8 / 6 / 10$ & $0: 00$ & 21.290 & 27.370 & 20.277 & 29.221 & 14.130 & 11.085 & 1.878 \\
\hline $8 / 6 / 10$ & $4: 00$ & 21.323 & 27.389 & 20.307 & 29.251 & 14.237 & 11.102 & 1.872 \\
\hline $8 / 6 / 10$ & $8: 00$ & 21.340 & 27.441 & 20.368 & 29.272 & 14.381 & 11.147 & 1.874 \\
\hline $8 / 6 / 10$ & $12: 00$ & 21.419 & 27.471 & 20.402 & 29.318 & 14.517 & 11.167 & 1.915 \\
\hline $8 / 6 / 10$ & $16: 00$ & 21.516 & 27.467 & 20.397 & 29.364 & 14.626 & 11.170 & 1.972 \\
\hline $8 / 6 / 10$ & $20: 00$ & 21.588 & 27.488 & 20.407 & 29.383 & 14.734 & 11.195 & 2.012 \\
\hline $8 / 7 / 10$ & $0: 00$ & 21.525 & 27.546 & 20.463 & 29.391 & 14.859 & 11.243 & 2.006 \\
\hline $8 / 7 / 10$ & $4: 00$ & 21.581 & 27.548 & 20.471 & 29.423 & 14.993 & 11.234 & 1.990 \\
\hline $8 / 7 / 10$ & $8: 00$ & 21.754 & 27.588 & 20.503 & 29.440 & 15.125 & 11.265 & 1.983 \\
\hline $8 / 7 / 10$ & $12: 00$ & 21.833 & 27.609 & 20.522 & 29.469 & 15.253 & 11.284 & 2.022 \\
\hline $8 / 7 / 10$ & $16: 00$ & 21.824 & 27.620 & 20.517 & 29.510 & 15.333 & 11.297 & 2.077 \\
\hline $8 / 7 / 10$ & $20: 00$ & 21.864 & 27.647 & 20.527 & 29.516 & 15.263 & 11.323 & 2.115 \\
\hline $8 / 8 / 10$ & $0: 00$ & 21.831 & 27.714 & 20.586 & 29.524 & 15.240 & 11.365 & 2.111 \\
\hline $8 / 8 / 10$ & $4: 00$ & 21.824 & 27.737 & 20.607 & 29.543 & 15.168 & 11.389 & 2.099 \\
\hline $8 / 8 / 10$ & $8: 00$ & 21.835 & 27.761 & 20.630 & 29.564 & 14.995 & 11.401 & 2.086 \\
\hline 8/8/10 & $12: 00$ & 21.788 & 27.798 & 20.666 & 29.597 & 13.907 & 11.436 & 2.133 \\
\hline 8/8/10 & $16: 00$ & 21.776 & 27.811 & 20.666 & 29.665 & 12.380 & 11.445 & 2.186 \\
\hline $8 / 8 / 10$ & $20: 00$ & 21.737 & 27.870 & 20.701 & 29.671 & 10.967 & 11.479 & 2.225 \\
\hline
\end{tabular}


TABLE S2.1 (Cont.)

\begin{tabular}{|c|c|c|c|c|c|c|c|c|}
\hline \multirow[b]{2}{*}{ Date } & \multirow[b]{2}{*}{ Time } & \multicolumn{6}{|c|}{ Depth to Water (ft TOC) } & \multirow[b]{2}{*}{ MW8s } \\
\hline & & MW1s & MW2s & MW3s & MW4s & MW6s & MW7s & \\
\hline $8 / 9 / 10$ & $0: 00$ & 21.858 & 27.929 & 20.759 & 29.688 & 9.864 & 11.525 & 2.229 \\
\hline $8 / 9 / 10$ & $4: 00$ & 21.814 & 27.964 & 20.793 & 29.698 & 9.510 & 11.551 & 2.226 \\
\hline $8 / 9 / 10$ & 8:00 & 21.735 & 28.025 & 20.859 & 29.693 & 9.807 & 11.599 & 2.222 \\
\hline $8 / 9 / 10$ & $12: 00$ & 21.768 & 28.138 & 20.906 & 29.737 & 10.320 & 11.623 & 2.253 \\
\hline $8 / 9 / 10$ & $16: 00$ & 21.846 & 28.216 & 20.910 & 29.806 & 10.802 & 11.638 & 2.304 \\
\hline $8 / 9 / 10$ & $20: 00$ & 21.842 & 28.271 & 20.947 & 29.827 & 11.297 & 11.677 & 2.342 \\
\hline $8 / 10 / 10$ & $0: 00$ & 21.978 & 28.308 & 21.016 & 29.842 & 11.768 & 11.727 & 2.340 \\
\hline $8 / 10 / 10$ & $4: 00$ & 21.961 & 28.306 & 21.035 & 29.867 & 12.084 & 11.738 & 2.326 \\
\hline $8 / 10 / 10$ & 8:00 & 21.995 & 28.321 & 21.068 & 29.900 & 12.279 & 11.759 & 2.314 \\
\hline $8 / 10 / 10$ & $12: 00$ & 22.022 & 28.346 & 21.103 & 29.938 & 12.421 & 11.791 & 2.358 \\
\hline $8 / 10 / 10$ & $16: 00$ & 22.065 & 28.338 & 21.096 & 29.970 & 12.543 & 11.802 & 2.407 \\
\hline $8 / 10 / 10$ & $20: 00$ & 22.038 & 28.377 & 21.133 & 29.981 & 12.743 & 11.850 & 2.439 \\
\hline $8 / 11 / 10$ & $0: 00$ & 22.028 & 28.411 & 21.181 & 30.012 & 13.017 & 11.876 & 2.433 \\
\hline $8 / 11 / 10$ & $4: 00$ & 22.038 & 28.443 & 21.218 & 30.029 & 13.284 & 11.907 & 2.425 \\
\hline $8 / 11 / 10$ & 8:00 & 22.043 & 28.483 & 21.264 & 30.048 & 13.503 & 11.945 & 2.416 \\
\hline $8 / 11 / 10$ & $12: 00$ & 22.140 & 28.510 & 21.291 & 30.094 & 13.682 & 11.965 & 2.449 \\
\hline $8 / 11 / 10$ & $16: 00$ & 22.226 & 28.512 & 21.291 & 30.140 & 13.834 & 11.980 & 2.492 \\
\hline $8 / 11 / 10$ & $20: 00$ & 22.234 & 28.541 & 21.318 & 30.147 & 13.966 & 12.013 & 2.520 \\
\hline $8 / 12 / 10$ & $0: 00$ & 22.215 & 28.586 & 21.367 & 30.174 & 14.075 & 12.047 & 2.508 \\
\hline $8 / 12 / 10$ & $4: 00$ & 22.244 & 28.602 & 21.386 & 30.193 & 14.131 & 12.056 & 2.493 \\
\hline $8 / 12 / 10$ & 8:00 & 22.309 & 28.631 & 21.421 & 30.218 & 14.131 & 12.076 & 2.479 \\
\hline $8 / 12 / 10$ & $12: 00$ & 22.390 & 28.649 & 21.428 & 30.250 & 14.090 & 12.087 & 2.517 \\
\hline $8 / 12 / 10$ & $16: 00$ & 22.469 & 28.644 & 21.416 & 30.284 & 14.049 & 12.089 & 2.566 \\
\hline $8 / 12 / 10$ & $20: 00$ & 22.425 & 28.674 & 21.428 & 30.279 & 14.040 & 12.108 & 2.594 \\
\hline $8 / 13 / 10$ & $0: 00$ & 22.432 & 28.717 & 21.473 & 30.296 & 14.090 & 12.141 & 2.586 \\
\hline $8 / 13 / 10$ & $4: 00$ & 22.442 & 28.751 & 21.507 & 30.307 & 14.222 & 12.168 & 2.578 \\
\hline $8 / 13 / 10$ & 8:00 & 22.460 & 28.777 & 21.530 & 30.327 & 14.385 & 12.184 & 2.575 \\
\hline $8 / 13 / 10$ & $12: 00$ & 22.509 & 28.816 & 21.570 & 30.346 & 14.591 & 12.219 & 2.612 \\
\hline $8 / 13 / 10$ & $16: 00$ & 22.532 & 28.820 & 21.572 & 30.382 & 14.275 & 12.223 & 2.626 \\
\hline $8 / 13 / 10$ & $20: 00$ & 22.468 & 28.888 & 21.633 & 30.376 & 13.870 & 12.277 & 2.624 \\
\hline $8 / 14 / 10$ & $0: 00$ & 22.443 & 28.959 & 21.722 & 30.411 & 13.820 & 12.337 & 2.615 \\
\hline $8 / 14 / 10$ & $4: 00$ & 22.473 & 29.001 & 21.776 & 30.458 & 13.966 & 12.369 & 2.603 \\
\hline $8 / 14 / 10$ & 8:00 & 22.507 & 29.041 & 21.823 & 30.515 & 14.168 & 12.399 & 2.591 \\
\hline $8 / 14 / 10$ & $12: 00$ & 22.569 & 29.085 & 21.879 & 30.578 & 14.246 & 12.429 & 2.599 \\
\hline $8 / 14 / 10$ & $16: 00$ & 22.633 & 29.119 & 21.911 & 30.630 & 14.316 & 12.461 & 2.642 \\
\hline $8 / 14 / 10$ & $20: 00$ & 22.621 & 29.163 & 21.961 & 30.659 & 14.454 & 12.500 & 2.668 \\
\hline $8 / 15 / 10$ & $0: 00$ & 22.599 & 29.232 & 22.055 & 30.694 & 14.491 & 12.554 & 2.662 \\
\hline $8 / 15 / 10$ & $4: 00$ & 22.555 & 29.285 & 22.120 & 30.738 & 13.712 & 12.591 & 2.651 \\
\hline $8 / 15 / 10$ & $8: 00$ & 22.549 & 29.314 & 22.159 & 30.803 & 12.318 & 12.608 & 2.641 \\
\hline $8 / 15 / 10$ & $12: 00$ & 22.569 & 29.339 & 22.193 & 30.853 & 10.978 & 12.628 & 2.672 \\
\hline $8 / 15 / 10$ & $16: 00$ & 22.810 & 29.353 & 22.194 & 30.891 & 9.719 & 12.641 & 2.710 \\
\hline $8 / 15 / 10$ & $20: 00$ & 22.877 & 29.360 & 22.201 & 31.013 & 8.705 & 12.648 & 2.729 \\
\hline $8 / 16 / 10$ & $0: 00$ & 22.720 & 29.413 & 22.272 & 31.023 & 7.910 & 12.686 & 2.722 \\
\hline $8 / 16 / 10$ & $4: 00$ & 22.680 & 29.442 & 22.309 & 31.021 & 7.516 & 12.706 & 2.714 \\
\hline $8 / 16 / 10$ & 8:00 & 22.658 & 29.471 & 22.343 & 31.000 & 7.638 & 12.717 & 2.704 \\
\hline $8 / 16 / 10$ & $12: 00$ & 22.706 & 29.502 & 22.345 & 31.026 & 8.050 & 12.723 & 2.732 \\
\hline $8 / 16 / 10$ & $16: 00$ & 22.758 & 29.517 & 22.304 & 31.053 & 8.612 & 12.716 & 2.769 \\
\hline $8 / 16 / 10$ & $20: 00$ & 22.926 & 29.542 & 22.294 & 31.055 & 9.192 & 12.721 & 2.781 \\
\hline
\end{tabular}


TABLE S2.1 (Cont.)

\begin{tabular}{|c|c|c|c|c|c|c|c|c|}
\hline \multirow[b]{2}{*}{ Date } & \multirow[b]{2}{*}{ Time } & \multicolumn{6}{|c|}{ Depth to Water (ft TOC) } & \multirow[b]{2}{*}{ MW8s } \\
\hline & & MW1s & MW2s & MW3s & MW4s & MW6s & MW7s & \\
\hline $8 / 17 / 10$ & $0: 00$ & 22.951 & 29.624 & 22.396 & 31.042 & 9.861 & 12.785 & 2.777 \\
\hline $8 / 17 / 10$ & $4: 00$ & 23.040 & 29.629 & 22.377 & 31.078 & 10.560 & 12.765 & 2.759 \\
\hline $8 / 17 / 10$ & 8:00 & 23.075 & 29.662 & 22.408 & 31.072 & 11.182 & 12.791 & 2.749 \\
\hline $8 / 17 / 10$ & $12: 00$ & 23.090 & 29.704 & 22.462 & 31.099 & 11.700 & 12.819 & 2.740 \\
\hline $8 / 17 / 10$ & $16: 00$ & 23.135 & 29.721 & 22.458 & 31.118 & 12.040 & 12.825 & 2.730 \\
\hline $8 / 17 / 10$ & $20: 00$ & 23.164 & 29.733 & 22.460 & 31.137 & 12.340 & 12.821 & 2.718 \\
\hline $8 / 18 / 10$ & $0: 00$ & 23.127 & 29.786 & 22.525 & 31.148 & 12.670 & 12.860 & 2.710 \\
\hline $8 / 18 / 10$ & $4: 00$ & 23.117 & 29.807 & 22.538 & 31.175 & 12.993 & 12.860 & 2.701 \\
\hline $8 / 18 / 10$ & 8:00 & 23.127 & 29.843 & 22.570 & 31.198 & 13.323 & 12.892 & 2.693 \\
\hline $8 / 18 / 10$ & $12: 00$ & 23.140 & 29.874 & 22.594 & 31.233 & 13.681 & 12.909 & 2.687 \\
\hline $8 / 18 / 10$ & $16: 00$ & 23.239 & 29.882 & 22.554 & 31.326 & 14.052 & 12.897 & 2.723 \\
\hline $8 / 18 / 10$ & $20: 00$ & 23.231 & 29.908 & 22.550 & 31.337 & 14.346 & 12.910 & 2.758 \\
\hline $8 / 19 / 10$ & $0: 00$ & 23.177 & 29.989 & 22.618 & 31.332 & 14.542 & 12.951 & 2.754 \\
\hline $8 / 19 / 10$ & $4: 00$ & 23.221 & 30.002 & 22.611 & 31.350 & 14.779 & 12.947 & 2.745 \\
\hline $8 / 19 / 10$ & 8:00 & 23.232 & 30.038 & 22.640 & 31.356 & 15.018 & 12.970 & 2.738 \\
\hline $8 / 19 / 10$ & $12: 00$ & 23.301 & 30.065 & 22.657 & 31.373 & 15.216 & 12.986 & 2.767 \\
\hline $8 / 19 / 10$ & $16: 00$ & 23.375 & 30.063 & 22.619 & 31.400 & 15.313 & 12.990 & 2.814 \\
\hline $8 / 19 / 10$ & $20: 00$ & 23.375 & 30.092 & 22.633 & 31.415 & 15.370 & 13.018 & 2.844 \\
\hline $8 / 20 / 10$ & $0: 00$ & 23.346 & 30.132 & 22.682 & 31.417 & 15.482 & 13.044 & 2.841 \\
\hline $8 / 20 / 10$ & $4: 00$ & 23.378 & 30.147 & 22.680 & 31.432 & 15.585 & 13.047 & 2.835 \\
\hline $8 / 20 / 10$ & 8:00 & 23.392 & 30.185 & 22.713 & 31.438 & 15.698 & 13.072 & 2.833 \\
\hline $8 / 20 / 10$ & $12: 00$ & 23.422 & 30.212 & 22.748 & 31.453 & 15.836 & 13.088 & 2.831 \\
\hline $8 / 20 / 10$ & $16: 00$ & 23.440 & 30.218 & 22.741 & 31.474 & 15.964 & 13.070 & 2.812 \\
\hline $8 / 20 / 10$ & $20: 00$ & 23.462 & 30.243 & 22.765 & 31.478 & 15.967 & 13.111 & 2.810 \\
\hline $8 / 21 / 10$ & $0: 00$ & 23.432 & 30.290 & 22.852 & 31.503 & 15.904 & 13.159 & 2.803 \\
\hline $8 / 21 / 10$ & $4: 00$ & 23.430 & 30.332 & 22.908 & 31.541 & 15.916 & 13.191 & 2.795 \\
\hline $8 / 21 / 10$ & 8:00 & 23.447 & 30.384 & 22.987 & 31.574 & 15.967 & 13.243 & 2.789 \\
\hline $8 / 21 / 10$ & $12: 00$ & 23.499 & 30.435 & 23.069 & 31.629 & 15.971 & 13.293 & 2.813 \\
\hline $8 / 21 / 10$ & $16: 00$ & 23.549 & 30.456 & 23.082 & 31.680 & 15.990 & 13.306 & 2.858 \\
\hline $8 / 21 / 10$ & $20: 00$ & 23.601 & 30.489 & 23.109 & 31.738 & 16.079 & 13.343 & 2.886 \\
\hline $8 / 22 / 10$ & $0: 00$ & 23.561 & 30.537 & 23.174 & 31.779 & 16.095 & 13.382 & 2.876 \\
\hline $8 / 22 / 10$ & $4: 00$ & 23.556 & 30.556 & 23.184 & 31.821 & 15.195 & 13.384 & 2.862 \\
\hline $8 / 22 / 10$ & 8:00 & 23.506 & 30.605 & 23.240 & 31.850 & 13.705 & 13.419 & 2.857 \\
\hline $8 / 22 / 10$ & $12: 00$ & 23.536 & 30.634 & 23.272 & 31.894 & 12.205 & 13.439 & 2.880 \\
\hline $8 / 22 / 10$ & $16: 00$ & 23.569 & 30.638 & 23.230 & 31.943 & 10.930 & 13.436 & 2.924 \\
\hline $8 / 22 / 10$ & $20: 00$ & 23.519 & 30.655 & 23.223 & 31.970 & 9.766 & 13.443 & 2.941 \\
\hline $8 / 23 / 10$ & $0: 00$ & 23.425 & 30.680 & 23.255 & 31.980 & 8.828 & 13.458 & 2.930 \\
\hline $8 / 23 / 10$ & $4: 00$ & 23.395 & 30.672 & 23.260 & 31.995 & 8.073 & 13.456 & 2.920 \\
\hline $8 / 23 / 10$ & $8: 00$ & 23.383 & 30.668 & 23.284 & 32.007 & 7.536 & 13.473 & 2.912 \\
\hline $8 / 23 / 10$ & $12: 00$ & 23.445 & 30.649 & 23.282 & 32.033 & 7.525 & 13.475 & 2.938 \\
\hline $8 / 23 / 10$ & $16: 00$ & 23.465 & 30.636 & 23.267 & 32.037 & 7.888 & 13.481 & 2.975 \\
\hline $8 / 23 / 10$ & $20: 00$ & 23.478 & 30.638 & 23.274 & 32.058 & 8.469 & 13.512 & 2.991 \\
\hline $8 / 24 / 10$ & $0: 00$ & 23.425 & 30.676 & 23.335 & 32.060 & 9.069 & 13.545 & 2.981 \\
\hline $8 / 24 / 10$ & $4: 00$ & 23.378 & 30.735 & 23.450 & 32.075 & 9.685 & 13.606 & 2.973 \\
\hline $8 / 24 / 10$ & 8:00 & 23.413 & 30.772 & 23.503 & 32.121 & 10.250 & 13.636 & 2.965 \\
\hline $8 / 24 / 10$ & $12: 00$ & 23.482 & 30.804 & 23.540 & 32.167 & 10.689 & 13.655 & 2.969 \\
\hline $8 / 24 / 10$ & $16: 00$ & 23.576 & 30.812 & 23.526 & 32.228 & 11.055 & 13.662 & 3.010 \\
\hline $8 / 24 / 10$ & $20: 00$ & 23.604 & 30.844 & 23.537 & 32.274 & 11.403 & 13.690 & 3.030 \\
\hline
\end{tabular}


TABLE S2.1 (Cont.)

\begin{tabular}{|c|c|c|c|c|c|c|c|c|}
\hline \multirow[b]{2}{*}{ Date } & \multirow[b]{2}{*}{ Time } & \multicolumn{6}{|c|}{ Depth to Water (ft TOC) } & \multirow[b]{2}{*}{ MW8s } \\
\hline & & MW1s & MW2s & MW3s & MW4s & MW6s & MW7s & \\
\hline $8 / 25 / 10$ & $0: 00$ & 23.606 & 30.888 & 23.579 & 32.308 & 11.787 & 13.718 & 3.022 \\
\hline $8 / 25 / 10$ & $4: 00$ & 23.653 & 30.911 & 23.589 & 32.339 & 12.195 & 13.726 & 3.011 \\
\hline $8 / 25 / 10$ & $8: 00$ & 23.673 & 30.953 & 23.637 & 32.365 & 12.514 & 13.759 & 3.005 \\
\hline $8 / 25 / 10$ & $12: 00$ & 23.716 & 30.964 & 23.643 & 32.409 & 12.831 & 13.733 & 3.036 \\
\hline $8 / 25 / 10$ & $16: 00$ & 23.884 & 30.957 & 23.593 & 32.451 & 13.198 & 13.774 & 3.074 \\
\hline $8 / 25 / 10$ & $20: 00$ & 23.897 & 30.976 & 23.602 & 32.489 & 13.583 & 13.794 & 3.090 \\
\hline $8 / 26 / 10$ & $0: 00$ & 23.807 & 31.008 & 23.631 & 32.512 & 13.285 & 13.813 & 3.072 \\
\hline $8 / 26 / 10$ & $4: 00$ & 23.783 & 31.029 & 23.645 & 32.533 & 12.164 & 13.818 & 3.061 \\
\hline $8 / 26 / 10$ & $8: 00$ & 23.738 & 31.064 & 23.680 & 32.550 & 10.921 & 13.844 & 3.053 \\
\hline $8 / 26 / 10$ & $12: 00$ & 23.776 & 31.087 & 23.684 & 32.579 & 9.711 & 13.854 & 3.089 \\
\hline $8 / 26 / 10$ & $16: 00$ & 23.837 & 31.092 & 23.633 & 32.603 & 8.881 & 13.846 & 3.128 \\
\hline $8 / 26 / 10$ & $20: 00$ & 23.832 & 31.110 & 23.613 & 32.604 & 8.714 & 13.854 & 3.146 \\
\hline $8 / 27 / 10$ & 0:00 & 23.798 & 31.157 & 23.652 & 32.615 & 9.095 & 13.878 & 3.132 \\
\hline $8 / 27 / 10$ & $4: 00$ & 23.803 & 31.176 & 23.657 & 32.626 & 9.722 & 13.874 & 3.119 \\
\hline $8 / 27 / 10$ & $8: 00$ & 23.810 & 31.201 & 23.692 & 32.626 & 10.389 & 13.908 & 3.111 \\
\hline $8 / 27 / 10$ & $12: 00$ & 23.877 & 31.197 & 23.686 & 32.653 & 10.919 & 13.887 & 3.140 \\
\hline $8 / 27 / 10$ & $16: 00$ & 23.937 & 31.176 & 23.626 & 32.674 & 11.350 & 13.891 & 3.178 \\
\hline $8 / 27 / 10$ & $20: 00$ & 23.999 & 31.184 & 23.621 & 32.676 & 11.792 & 13.926 & 3.194 \\
\hline $8 / 28 / 10$ & 0:00 & 23.944 & 31.226 & 23.682 & 32.672 & 12.211 & 13.963 & 3.180 \\
\hline $8 / 28 / 10$ & $4: 00$ & 23.964 & 31.245 & 23.689 & 32.686 & 12.503 & 13.965 & 3.169 \\
\hline $8 / 28 / 10$ & $8: 00$ & 23.911 & 31.278 & 23.721 & 32.688 & 12.062 & 13.987 & 3.157 \\
\hline $8 / 28 / 10$ & $12: 00$ & 23.959 & 31.295 & 23.724 & 32.720 & 11.019 & 13.995 & 3.192 \\
\hline $8 / 28 / 10$ & $16: 00$ & 23.986 & 31.297 & 23.682 & 32.728 & 9.853 & 13.993 & 3.230 \\
\hline $8 / 28 / 10$ & $20: 00$ & 23.910 & 31.327 & 23.697 & 32.729 & 8.833 & 14.017 & 3.248 \\
\hline $8 / 29 / 10$ & 0:00 & 23.797 & 31.373 & 23.767 & 32.733 & 8.025 & 14.047 & 3.230 \\
\hline $8 / 29 / 10$ & $4: 00$ & 23.763 & 31.396 & 23.777 & 32.741 & 7.425 & 14.053 & 3.216 \\
\hline $8 / 29 / 10$ & $8: 00$ & 23.734 & 31.430 & 23.818 & 32.739 & 7.110 & 14.073 & 3.185 \\
\hline $8 / 29 / 10$ & $12: 00$ & 23.776 & 31.444 & 23.828 & 32.760 & 6.840 & 14.080 & 3.234 \\
\hline $8 / 29 / 10$ & $16: 00$ & 23.800 & 31.440 & 23.797 & 32.777 & 6.638 & 14.080 & 3.319 \\
\hline $8 / 29 / 10$ & $20: 00$ & 23.733 & 31.467 & 23.821 & 32.764 & 6.525 & 14.108 & 3.330 \\
\hline $8 / 30 / 10$ & $0: 00$ & 23.684 & 31.503 & 23.892 & 32.765 & 6.479 & 14.145 & 3.270 \\
\hline $8 / 30 / 10$ & $4: 00$ & 23.709 & 31.505 & 23.921 & 32.783 & 6.684 & 14.164 & 3.246 \\
\hline $8 / 30 / 10$ & $8: 00$ & 23.753 & 31.511 & 23.963 & 32.796 & 7.046 & 14.188 & 3.236 \\
\hline $8 / 30 / 10$ & $12: 00$ & 23.871 & 31.501 & 23.955 & 32.838 & 7.483 & 14.193 & 3.285 \\
\hline $8 / 30 / 10$ & $16: 00$ & 23.980 & 31.461 & 23.901 & 32.880 & 8.087 & 14.180 & 3.368 \\
\hline $8 / 30 / 10$ & $20: 00$ & 23.969 & 31.449 & 23.888 & 32.890 & 8.678 & 14.184 & 3.338 \\
\hline $8 / 31 / 10$ & $0: 00$ & 23.957 & 31.461 & 23.933 & 32.895 & 9.212 & 14.225 & 3.301 \\
\hline $8 / 31 / 10$ & $4: 00$ & 24.001 & 31.453 & 23.941 & 32.908 & 9.832 & 14.236 & 3.315 \\
\hline $8 / 31 / 10$ & $8: 00$ & 24.067 & 31.451 & 23.965 & 32.920 & 10.483 & 14.255 & 3.311 \\
\hline $8 / 31 / 10$ & $12: 00$ & 24.146 & 31.442 & 23.972 & 32.947 & 11.101 & 14.273 & 3.366 \\
\hline $8 / 31 / 10$ & $16: 00$ & 24.225 & 31.434 & 23.948 & 32.971 & 11.674 & 14.284 & 3.410 \\
\hline $8 / 31 / 10$ & $20: 00$ & 24.166 & 31.449 & 23.957 & 32.994 & 11.730 & 14.308 & 3.402 \\
\hline $9 / 1 / 10$ & $0: 00$ & 23.940 & 31.486 & 24.092 & 32.989 & 10.891 & 14.277 & 3.253 \\
\hline $9 / 1 / 10$ & $4: 00$ & 23.913 & 31.415 & 24.031 & 33.029 & 9.725 & 14.006 & 3.202 \\
\hline $9 / 1 / 10$ & $8: 00$ & 23.820 & 31.358 & 24.013 & 33.038 & 8.690 & 14.017 & 3.192 \\
\hline $9 / 1 / 10$ & $12: 00$ & 23.684 & 31.341 & 24.079 & 33.023 & 7.876 & 14.091 & 3.101 \\
\hline $9 / 1 / 10$ & $16: 00$ & 23.567 & 31.136 & 23.799 & 33.072 & 7.266 & 13.216 & 2.099 \\
\hline $9 / 1 / 10$ & $20: 00$ & 23.298 & 31.019 & 23.769 & 33.042 & 6.937 & 13.417 & 1.937 \\
\hline
\end{tabular}


TABLE S2.1 (Cont.)

\begin{tabular}{|c|c|c|c|c|c|c|c|c|}
\hline \multirow[b]{2}{*}{ Date } & \multirow[b]{2}{*}{ Time } & \multicolumn{6}{|c|}{ Depth to Water (ft TOC) } & \multirow[b]{2}{*}{ MW8s } \\
\hline & & MW1s & MW2s & MW3s & MW4s & MW6s & MW7s & \\
\hline $9 / 2 / 10$ & $0: 00$ & 23.124 & 30.922 & 23.736 & 33.031 & 6.652 & 13.511 & 1.882 \\
\hline $9 / 2 / 10$ & 4:00 & 23.014 & 30.836 & 23.692 & 33.025 & 6.437 & 13.552 & 1.905 \\
\hline 9/2/10 & $8: 00$ & 22.895 & 30.802 & 23.707 & 32.985 & 6.275 & 13.619 & 1.967 \\
\hline 9/2/10 & $12: 00$ & 22.813 & 30.777 & 23.733 & 32.977 & 6.153 & 13.671 & 2.060 \\
\hline 9/2/10 & $16: 00$ & 22.724 & 30.777 & 23.787 & 32.989 & 6.062 & 13.738 & 2.145 \\
\hline 9/2/10 & $20: 00$ & 22.719 & 30.762 & 23.799 & 33.004 & 5.982 & 13.761 & 2.184 \\
\hline $9 / 3 / 10$ & $0: 00$ & 22.629 & 30.793 & 23.875 & 33.000 & 5.904 & 13.837 & 2.205 \\
\hline $9 / 3 / 10$ & $4: 00$ & 22.638 & 30.791 & 23.885 & 33.027 & 5.856 & 13.861 & 2.246 \\
\hline 9/3/10 & $8: 00$ & 22.650 & 30.793 & 23.882 & 33.042 & 5.811 & 13.870 & 2.285 \\
\hline $9 / 3 / 10$ & $12: 00$ & 22.702 & 30.796 & 23.875 & 33.046 & 5.751 & 13.881 & 2.341 \\
\hline 9/3/10 & $16: 00$ & 22.756 & 30.779 & 23.811 & 33.059 & 5.704 & 13.866 & 2.458 \\
\hline 9/3/10 & $20: 00$ & 22.734 & 30.789 & 23.787 & 33.046 & 5.683 & 13.876 & 2.513 \\
\hline 9/4/10 & 0:00 & 22.672 & 30.810 & 23.804 & 33.038 & 5.680 & 13.902 & 2.507 \\
\hline $9 / 4 / 10$ & $4: 00$ & 22.667 & 30.825 & 23.807 & 33.036 & 5.648 & 13.909 & 2.513 \\
\hline 9/4/10 & $8: 00$ & 22.665 & 30.842 & 23.812 & 33.025 & 5.633 & 13.916 & 2.521 \\
\hline 9/4/10 & $12: 00$ & 22.727 & 30.835 & 23.768 & 33.046 & 5.586 & 13.887 & 2.577 \\
\hline 9/4/10 & $16: 00$ & 22.823 & 30.789 & 23.653 & 33.044 & 5.577 & 13.840 & 2.597 \\
\hline 9/4/10 & $20: 00$ & 22.780 & 30.781 & 23.617 & 33.012 & 5.565 & 13.837 & 2.636 \\
\hline 9/5/10 & 0:00 & 22.720 & 30.770 & 23.617 & 32.974 & 5.551 & 13.844 & 2.648 \\
\hline $9 / 5 / 10$ & $4: 00$ & 22.718 & 30.745 & 23.585 & 32.947 & 5.530 & 13.831 & 2.687 \\
\hline 9/5/10 & $8: 00$ & 22.733 & 30.728 & 23.551 & 32.920 & 5.522 & 13.818 & 2.689 \\
\hline 9/5/10 & $12: 00$ & 22.765 & 30.724 & 23.529 & 32.913 & 5.507 & 13.818 & 2.725 \\
\hline 9/5/10 & $16: 00$ & 22.772 & 30.712 & 23.475 & 32.870 & 5.495 & 13.805 & 2.752 \\
\hline 9/5/10 & $20: 00$ & 22.738 & 30.720 & 23.471 & 32.829 & 5.509 & 13.809 & 2.774 \\
\hline 9/6/10 & 0:00 & 22.696 & 30.743 & 23.495 & 32.802 & 5.478 & 13.831 & 2.781 \\
\hline 9/6/10 & $4: 00$ & 22.713 & 30.745 & 23.480 & 32.783 & 5.449 & 13.824 & 2.792 \\
\hline 9/6/10 & $8: 00$ & 22.703 & 30.766 & 23.493 & 32.750 & 5.480 & 13.837 & 2.743 \\
\hline 9/6/10 & $12: 00$ & 22.722 & 30.791 & 23.509 & 32.716 & 5.437 & 13.854 & 2.778 \\
\hline 9/6/10 & $16: 00$ & 22.708 & 30.840 & 23.573 & 32.716 & 5.453 & 13.907 & 2.801 \\
\hline 9/6/10 & $20: 00$ & 22.547 & 30.957 & 23.806 & 32.708 & 5.459 & 14.041 & 2.796 \\
\hline 9/7/10 & $0: 00$ & 22.559 & 31.011 & 23.911 & 32.754 & 5.445 & 14.093 & 2.833 \\
\hline 9/7/10 & $4: 00$ & 22.617 & 31.043 & 23.944 & 32.806 & 5.449 & 14.112 & 2.831 \\
\hline 9/7/10 & $8: 00$ & 22.657 & 31.089 & 23.992 & 32.852 & 5.453 & 14.145 & 2.833 \\
\hline 9/7/10 & $12: 00$ & 22.746 & 31.129 & 24.029 & 32.908 & 5.447 & 14.167 & 2.862 \\
\hline 9/7/10 & $16: 00$ & 22.857 & 31.134 & 23.985 & 32.950 & 5.468 & 14.154 & 2.896 \\
\hline 9/7/10 & $20: 00$ & 22.856 & 31.163 & 23.980 & 32.964 & 5.439 & 14.171 & 2.918 \\
\hline 9/8/10 & $0: 00$ & 22.852 & 31.197 & 23.995 & 32.977 & 5.463 & 14.184 & 2.910 \\
\hline 9/8/10 & $4: 00$ & 22.873 & 31.217 & 23.992 & 32.990 & 5.410 & 14.171 & 2.902 \\
\hline $9 / 8 / 10$ & $8: 00$ & 22.883 & 31.259 & 24.024 & 32.998 & 5.442 & 14.210 & 2.900 \\
\hline 9/8/10 & $12: 00$ & 22.964 & 31.274 & 24.002 & 33.017 & 5.455 & 14.199 & 2.924 \\
\hline 9/8/10 & $16: 00$ & 23.031 & 31.272 & 23.937 & 33.038 & 5.440 & 14.174 & 2.955 \\
\hline 9/8/10 & $20: 00$ & 23.024 & 31.289 & 23.907 & 33.034 & 5.414 & 14.171 & 2.971 \\
\hline $9 / 9 / 10$ & $0: 00$ & 22.999 & 31.325 & 23.937 & 33.021 & 5.368 & 14.197 & 2.969 \\
\hline 9/9/10 & $4: 00$ & 23.034 & 31.327 & 23.900 & 33.021 & 5.428 & 14.173 & 2.961 \\
\hline 9/9/10 & 8:00 & 23.022 & 31.354 & 23.907 & 32.992 & 5.480 & 14.184 & 2.953 \\
\hline 9/9/10 & $12: 00$ & 23.057 & 31.362 & 23.890 & 33.002 & 5.389 & 14.171 & 2.944 \\
\hline 9/9/10 & $16: 00$ & 23.083 & 31.358 & 23.841 & 32.992 & 5.405 & 14.141 & 2.948 \\
\hline $9 / 9 / 10$ & $20: 00$ & 23.052 & 31.388 & 23.864 & 32.987 & 5.397 & 14.165 & 2.954 \\
\hline
\end{tabular}


TABLE S2.1 (Cont.)

\begin{tabular}{|c|c|c|c|c|c|c|c|c|}
\hline \multirow[b]{2}{*}{ Date } & \multirow[b]{2}{*}{ Time } & \multicolumn{6}{|c|}{ Depth to Water (ft TOC) } & \multirow[b]{2}{*}{ MW8s } \\
\hline & & MW1s & MW2s & MW3s & MW4s & MW6s & MW7s & \\
\hline $9 / 10 / 10$ & $0: 00$ & 23.029 & 31.419 & 23.898 & 32.981 & 5.411 & 14.191 & 2.950 \\
\hline $9 / 10 / 10$ & $4: 00$ & 23.051 & 31.427 & 23.888 & 32.989 & 5.327 & 14.187 & 2.938 \\
\hline $9 / 10 / 10$ & 8:00 & 23.059 & 31.442 & 23.890 & 32.985 & 5.366 & 14.186 & 2.931 \\
\hline $9 / 10 / 10$ & $12: 00$ & 23.057 & 31.463 & 23.900 & 32.977 & 5.409 & 14.197 & 2.927 \\
\hline $9 / 10 / 10$ & $16: 00$ & 23.111 & 31.469 & 23.883 & 32.979 & 5.399 & 14.187 & 2.923 \\
\hline $9 / 10 / 10$ & $20: 00$ & 23.049 & 31.516 & 23.961 & 32.950 & 5.403 & 14.238 & 2.938 \\
\hline $9 / 11 / 10$ & $0: 00$ & 22.997 & 31.568 & 24.059 & 32.948 & 5.380 & 14.290 & 2.939 \\
\hline $9 / 11 / 10$ & $4: 00$ & 22.995 & 31.612 & 24.151 & 32.983 & 5.401 & 14.330 & 2.935 \\
\hline $9 / 11 / 10$ & 8:00 & 22.961 & 31.663 & 24.234 & 33.004 & 5.359 & 14.379 & 2.937 \\
\hline $9 / 11 / 10$ & $12: 00$ & 23.065 & 31.677 & 24.248 & 33.059 & 5.357 & 14.385 & 2.956 \\
\hline $9 / 11 / 10$ & $16: 00$ & 23.161 & 31.681 & 24.219 & 33.103 & 5.427 & 14.381 & 2.996 \\
\hline $9 / 11 / 10$ & $20: 00$ & 23.159 & 31.707 & 24.238 & 33.153 & 5.407 & 14.407 & 3.022 \\
\hline $9 / 12 / 10$ & $0: 00$ & 23.139 & 31.738 & 24.271 & 33.156 & 5.380 & 14.426 & 3.016 \\
\hline $9 / 12 / 10$ & $4: 00$ & 23.168 & 31.759 & 24.280 & 33.168 & 5.367 & 14.435 & 3.014 \\
\hline $9 / 12 / 10$ & 8:00 & 23.174 & 31.789 & 24.310 & 33.177 & 5.421 & 14.457 & 3.014 \\
\hline $9 / 12 / 10$ & $12: 00$ & 23.245 & 31.803 & 24.315 & 33.202 & 5.433 & 14.463 & 3.044 \\
\hline $9 / 12 / 10$ & $16: 00$ & 23.340 & 31.797 & 24.254 & 33.242 & 5.440 & 14.444 & 3.083 \\
\hline $9 / 12 / 10$ & $20: 00$ & 23.300 & 31.818 & 24.263 & 33.240 & 5.415 & 14.468 & 3.107 \\
\hline $9 / 13 / 10$ & $0: 00$ & 23.268 & 31.849 & 24.300 & 33.248 & 5.396 & 14.488 & 3.103 \\
\hline $9 / 13 / 10$ & $4: 00$ & 23.270 & 31.866 & 24.307 & 33.263 & 5.372 & 14.487 & 3.097 \\
\hline $9 / 13 / 10$ & 8:00 & 23.275 & 31.904 & 24.359 & 33.265 & 5.392 & 14.520 & 3.094 \\
\hline $9 / 13 / 10$ & $12: 00$ & 23.362 & 31.904 & 24.332 & 33.290 & 5.379 & 14.507 & 3.107 \\
\hline $9 / 13 / 10$ & $16: 00$ & 23.446 & 31.881 & 24.246 & 33.317 & 5.371 & 14.468 & 3.141 \\
\hline $9 / 13 / 10$ & $20: 00$ & 23.221 & 31.904 & 24.241 & 33.242 & 5.357 & 14.403 & 2.703 \\
\hline $9 / 14 / 10$ & $0: 00$ & 22.891 & 31.744 & 23.965 & 33.278 & 5.340 & 13.230 & 1.843 \\
\hline $9 / 14 / 10$ & $4: 00$ & 22.664 & 31.514 & 23.817 & 33.294 & 5.291 & 13.130 & 1.661 \\
\hline $9 / 14 / 10$ & 8:00 & 22.336 & 31.363 & 23.699 & 33.255 & 5.313 & 13.227 & 1.606 \\
\hline $9 / 14 / 10$ & $12: 00$ & 22.197 & 31.227 & 23.491 & 33.259 & 5.350 & 13.377 & 1.582 \\
\hline $9 / 14 / 10$ & $16: 00$ & 22.173 & 31.086 & 23.161 & 33.261 & 5.353 & 13.444 & 1.596 \\
\hline $9 / 14 / 10$ & $20: 00$ & 22.079 & 30.990 & 22.931 & 33.248 & 5.357 & 13.526 & 1.699 \\
\hline $9 / 15 / 10$ & $0: 00$ & 22.001 & 30.912 & 22.770 & 33.229 & 5.348 & 13.599 & 1.808 \\
\hline $9 / 15 / 10$ & $4: 00$ & 21.976 & 30.833 & 22.594 & 33.212 & 5.383 & 13.623 & 1.871 \\
\hline $9 / 15 / 10$ & 8:00 & 21.994 & 30.746 & 22.423 & 33.227 & 5.373 & 13.610 & 1.890 \\
\hline $9 / 15 / 10$ & $12: 00$ & 21.943 & 30.706 & 22.325 & 33.143 & 5.399 & 13.647 & 1.747 \\
\hline $9 / 15 / 10$ & $16: 00$ & 21.977 & 30.622 & 22.208 & 33.145 & 5.387 & 13.617 & 1.744 \\
\hline $9 / 15 / 10$ & $20: 00$ & 21.952 & 30.578 & 22.157 & 33.112 & 5.371 & 13.630 & 1.849 \\
\hline $9 / 16 / 10$ & $0: 00$ & 21.881 & 30.571 & 22.201 & 33.082 & 5.389 & 13.682 & 1.919 \\
\hline $9 / 16 / 10$ & $4: 00$ & 21.840 & 30.573 & 22.272 & 33.063 & 5.408 & 13.734 & 1.968 \\
\hline $9 / 16 / 10$ & $8: 00$ & 21.843 & 30.571 & 22.330 & 33.061 & 5.368 & 13.769 & 2.011 \\
\hline $9 / 16 / 10$ & $12: 00$ & 21.859 & 30.571 & 22.411 & 33.074 & 5.397 & 13.805 & 2.056 \\
\hline $9 / 16 / 10$ & $16: 00$ & 21.915 & 30.544 & 22.399 & 33.086 & 5.384 & 13.799 & 2.103 \\
\hline $9 / 16 / 10$ & $20: 00$ & 21.960 & 30.508 & 22.381 & 33.093 & 5.383 & 13.779 & 2.135 \\
\hline $9 / 17 / 10$ & $0: 00$ & 21.941 & 30.514 & 22.440 & 33.078 & 5.397 & 13.801 & 2.154 \\
\hline $9 / 17 / 10$ & $4: 00$ & 21.978 & 30.504 & 22.467 & 33.080 & 5.362 & 13.797 & 2.166 \\
\hline $9 / 17 / 10$ & $8: 00$ & 21.955 & 30.518 & 22.541 & 33.061 & 5.409 & 13.820 & 2.184 \\
\hline $9 / 17 / 10$ & $12: 00$ & 22.045 & 30.512 & 22.574 & 33.071 & 5.382 & 13.814 & 2.205 \\
\hline 9/17/10 & $16: 00$ & 22.105 & 30.487 & 22.556 & 33.067 & 5.399 & 13.790 & 2.268 \\
\hline $9 / 17 / 10$ & $20: 00$ & 22.067 & 30.508 & 22.624 & 33.082 & 5.418 & 13.816 & 2.315 \\
\hline
\end{tabular}


TABLE S2.1 (Cont.)

\begin{tabular}{|c|c|c|c|c|c|c|c|c|}
\hline \multirow[b]{2}{*}{ Date } & \multirow[b]{2}{*}{ Time } & \multicolumn{6}{|c|}{ Depth to Water (ft TOC) } & \multirow[b]{2}{*}{ MW8s } \\
\hline & & MW1s & MW2s & MW3s & MW4s & MW6s & MW7s & \\
\hline $9 / 18 / 10$ & $0: 00$ & 22.027 & 30.543 & 22.727 & 33.059 & 5.380 & 13.853 & 2.329 \\
\hline $9 / 18 / 10$ & $4: 00$ & 22.047 & 30.550 & 22.777 & 33.051 & 5.374 & 13.859 & 2.335 \\
\hline $9 / 18 / 10$ & $8: 00$ & 22.028 & 30.594 & 22.899 & 33.038 & 5.354 & 13.902 & 2.347 \\
\hline $9 / 18 / 10$ & $12: 00$ & 22.069 & 30.613 & 22.972 & 33.059 & 5.390 & 13.918 & 2.358 \\
\hline $9 / 18 / 10$ & $16: 00$ & 22.122 & 30.608 & 22.983 & 33.080 & 5.394 & 13.910 & 2.388 \\
\hline $9 / 18 / 10$ & $20: 00$ & 22.125 & 30.627 & 23.027 & 33.068 & 5.396 & 13.928 & 2.412 \\
\hline 9/19/10 & $0: 00$ & 22.139 & 30.650 & 23.080 & 33.078 & 5.425 & 13.946 & 2.420 \\
\hline 9/19/10 & $4: 00$ & 22.160 & 30.663 & 23.113 & 33.076 & 5.425 & 13.952 & 2.424 \\
\hline 9/19/10 & 8:00 & 22.162 & 30.699 & 23.181 & 33.078 & 5.448 & 13.976 & 2.426 \\
\hline $9 / 19 / 10$ & $12: 00$ & 22.214 & 30.715 & 23.220 & 33.091 & 5.417 & 13.983 & 2.428 \\
\hline 9/19/10 & $16: 00$ & 22.283 & 30.703 & 23.185 & 33.107 & 5.506 & 13.940 & 2.443 \\
\hline 9/19/10 & $20: 00$ & 22.281 & 30.728 & 23.214 & 33.109 & 5.419 & 13.966 & 2.469 \\
\hline 9/20/10 & $0: 00$ & 22.291 & 30.743 & 23.234 & 33.111 & 5.400 & 13.965 & 2.467 \\
\hline 9/20/10 & $4: 00$ & 22.340 & 30.736 & 23.207 & 33.111 & 5.371 & 13.933 & 2.460 \\
\hline 9/20/10 & 8:00 & 22.329 & 30.766 & 23.249 & 33.080 & 5.408 & 13.952 & 2.462 \\
\hline $9 / 20 / 10$ & $12: 00$ & 22.390 & 30.776 & 23.251 & 33.084 & 5.417 & 13.942 & 2.489 \\
\hline 9/20/10 & $16: 00$ & 22.448 & 30.764 & 23.195 & 33.080 & 5.452 & 13.900 & 2.540 \\
\hline 9/20/10 & $20: 00$ & 22.418 & 30.783 & 23.205 & 33.053 & 5.429 & 13.918 & 2.572 \\
\hline $9 / 21 / 10$ & $0: 00$ & 22.378 & 30.820 & 23.273 & 33.042 & 5.439 & 13.953 & 2.582 \\
\hline $9 / 21 / 10$ & $4: 00$ & 22.378 & 30.843 & 23.310 & 33.044 & 5.425 & 13.965 & 2.584 \\
\hline $9 / 21 / 10$ & 8:00 & 22.366 & 30.881 & 23.364 & 33.030 & 5.429 & 13.994 & 2.586 \\
\hline $9 / 21 / 10$ & $12: 00$ & 22.445 & 30.898 & 23.377 & 33.044 & 5.433 & 13.989 & 2.606 \\
\hline $9 / 21 / 10$ & $16: 00$ & 22.450 & 30.919 & 23.398 & 33.051 & 5.464 & 14.004 & 2.637 \\
\hline $9 / 21 / 10$ & $20: 00$ & 22.415 & 30.965 & 23.455 & 33.063 & 5.430 & 14.048 & 2.643 \\
\hline $9 / 22 / 10$ & $0: 00$ & 22.410 & 30.992 & 23.527 & 33.057 & 5.412 & 14.063 & 2.637 \\
\hline $9 / 22 / 10$ & $4: 00$ & 22.433 & 31.024 & 23.586 & 33.069 & 5.443 & 14.086 & 2.635 \\
\hline $9 / 22 / 10$ & 8:00 & 22.438 & 31.064 & 23.644 & 33.076 & 5.420 & 14.117 & 2.635 \\
\hline $9 / 22 / 10$ & $12: 00$ & 22.505 & 31.085 & 23.663 & 33.109 & 5.433 & 14.124 & 2.648 \\
\hline $9 / 22 / 10$ & $16: 00$ & & 31.035 & 23.600 & 32.985 & 5.465 & 14.087 & 2.666 \\
\hline $9 / 22 / 10$ & $20: 00$ & 22.345 & 31.033 & 23.606 & 32.937 & 5.441 & 14.091 & 2.681 \\
\hline $9 / 23 / 10$ & $0: 00$ & 22.406 & 31.071 & 23.669 & 32.939 & 5.402 & 14.135 & 2.682 \\
\hline $9 / 23 / 10$ & $4: 00$ & 22.470 & 31.074 & 23.662 & 32.956 & 5.431 & 14.115 & 2.680 \\
\hline $9 / 23 / 10$ & 8:00 & 22.542 & 31.092 & 23.671 & 32.965 & 5.397 & 14.135 & 2.678 \\
\hline $9 / 23 / 10$ & $12: 00$ & 22.602 & 31.112 & & 32.977 & 5.864 & 14.139 & 2.692 \\
\hline $9 / 23 / 10$ & $16: 00$ & 22.619 & 31.108 & 23.669 & 32.981 & 5.591 & 14.118 & 2.680 \\
\hline $9 / 23 / 10$ & $20: 00$ & 22.575 & 31.123 & 23.727 & 32.963 & 5.605 & 14.031 & 2.649 \\
\hline $9 / 24 / 10$ & $0: 00$ & 22.416 & 31.125 & 23.825 & 32.981 & 5.572 & 13.645 & 2.547 \\
\hline $9 / 24 / 10$ & $4: 00$ & 22.316 & 31.110 & 23.891 & 33.030 & 5.580 & 13.829 & 2.527 \\
\hline $9 / 24 / 10$ & $8: 00$ & 22.282 & 31.118 & 23.959 & 33.084 & 5.533 & 13.940 & 2.521 \\
\hline $9 / 24 / 10$ & $12: 00$ & 22.344 & 31.114 & 23.991 & 33.168 & 5.539 & 14.001 & 2.531 \\
\hline $9 / 24 / 10$ & $16: 00$ & 22.423 & 31.087 & 23.947 & 33.232 & 5.545 & 14.024 & 2.561 \\
\hline $9 / 24 / 10$ & $20: 00$ & 22.388 & 31.095 & 23.954 & 33.253 & 5.527 & 14.066 & 2.584 \\
\hline $9 / 25 / 10$ & $0: 00$ & 22.378 & 31.104 & 23.978 & 33.293 & 5.479 & 14.096 & 2.576 \\
\hline $9 / 25 / 10$ & $4: 00$ & 22.422 & 31.100 & 23.957 & 33.303 & 5.502 & 14.100 & 2.569 \\
\hline $9 / 25 / 10$ & $8: 00$ & 22.483 & 31.076 & 23.903 & 33.345 & 5.477 & 14.072 & 2.557 \\
\hline $9 / 25 / 10$ & $12: 00$ & 22.470 & 31.104 & 23.918 & 33.331 & 5.500 & 14.111 & 2.557 \\
\hline $9 / 25 / 10$ & $16: 00$ & 22.438 & 31.137 & 23.973 & 33.322 & 5.504 & 14.152 & 2.565 \\
\hline $9 / 25 / 10$ & $20: 00$ & 22.433 & 31.154 & 23.999 & 33.347 & 5.510 & 14.173 & 2.565 \\
\hline
\end{tabular}


TABLE S2.1 (Cont.)

\begin{tabular}{|c|c|c|c|c|c|c|c|c|}
\hline \multirow[b]{2}{*}{ Date } & \multirow[b]{2}{*}{ Time } & \multicolumn{6}{|c|}{ Depth to Water (ft TOC) } & \multirow[b]{2}{*}{ MW8s } \\
\hline & & MW1s & MW2s & MW3s & MW4s & MW6s & MW7s & \\
\hline 9/26/10 & $0: 00$ & 22.438 & 31.177 & 24.030 & 33.364 & 5.509 & 14.197 & 2.561 \\
\hline $9 / 26 / 10$ & $4: 00$ & 22.470 & 31.194 & 24.044 & 33.387 & 5.511 & 14.212 & 2.557 \\
\hline $9 / 26 / 10$ & $8: 00$ & 22.452 & 31.223 & 24.079 & 33.404 & 5.485 & 14.219 & 2.556 \\
\hline $9 / 26 / 10$ & $12: 00$ & 22.507 & 31.249 & 24.101 & 33.423 & 5.479 & 14.254 & 2.556 \\
\hline $9 / 26 / 10$ & $16: 00$ & 22.634 & 31.223 & 24.011 & 33.465 & 5.522 & 14.202 & 2.570 \\
\hline 9/26/10 & $20: 00$ & 22.602 & 31.241 & 24.008 & 33.461 & 5.518 & 14.213 & 2.593 \\
\hline $9 / 27 / 10$ & $0: 00$ & 22.592 & 31.255 & 24.011 & 33.448 & 5.501 & 14.223 & 2.589 \\
\hline $9 / 27 / 10$ & 4:00 & 22.612 & 31.261 & 23.998 & 33.444 & 5.474 & 14.219 & 2.582 \\
\hline $9 / 27 / 10$ & 8:00 & 22.605 & 31.288 & 24.020 & 33.431 & 5.477 & 14.241 & 2.582 \\
\hline $9 / 27 / 10$ & $12: 00$ & 22.686 & 31.293 & 23.998 & 33.440 & 5.459 & 14.232 & 2.593 \\
\hline $9 / 27 / 10$ & $16: 00$ & 22.746 & 31.289 & 23.948 & 33.423 & 5.513 & 14.210 & 2.629 \\
\hline 9/27/10 & $20: 00$ & 22.704 & 31.302 & 23.935 & 33.393 & 5.495 & 14.213 & 2.649 \\
\hline $9 / 28 / 10$ & $0: 00$ & 22.684 & 31.323 & 23.952 & 33.362 & 5.492 & 14.228 & 2.649 \\
\hline $9 / 28 / 10$ & $4: 00$ & 22.648 & 31.354 & 23.998 & 33.353 & 5.589 & 14.262 & 2.651 \\
\hline 9/28/10 & 8:00 & 22.624 & 31.393 & 24.067 & 33.353 & 5.493 & 14.299 & 2.649 \\
\hline $9 / 28 / 10$ & $12: 00$ & 22.676 & 31.421 & 24.101 & 33.373 & 5.507 & 14.316 & 2.663 \\
\hline $9 / 28 / 10$ & $16: 00$ & 22.775 & 31.412 & 24.045 & 33.416 & 5.511 & 14.293 & 2.694 \\
\hline $9 / 28 / 10$ & $20: 00$ & 22.713 & 31.440 & 24.064 & 33.408 & 5.519 & 14.312 & 2.716 \\
\hline $9 / 29 / 10$ & $0: 00$ & 22.701 & 31.461 & 24.089 & 33.394 & 5.511 & 14.325 & 2.708 \\
\hline $9 / 29 / 10$ & $4: 00$ & 22.751 & 31.475 & 24.091 & 33.399 & 5.488 & 14.334 & 2.700 \\
\hline 9/29/10 & 8:00 & 22.734 & 31.511 & 24.135 & 33.398 & 5.509 & 14.362 & 2.698 \\
\hline $9 / 29 / 10$ & $12: 00$ & 22.840 & 31.524 & 24.130 & 33.434 & 5.484 & 14.362 & 2.718 \\
\hline 9/29/10 & $16: 00$ & 22.882 & 31.538 & 24.121 & 33.463 & 5.502 & 14.371 & 2.756 \\
\hline 9/29/10 & $20: 00$ & 22.808 & 31.585 & 24.186 & 33.429 & 5.502 & 14.416 & 2.777 \\
\hline 9/30/10 & 0:00 & 22.743 & 31.650 & 24.311 & 33.425 & 5.519 & 14.477 & 2.783 \\
\hline 9/30/10 & $4: 00$ & 22.758 & 31.687 & 24.371 & 33.467 & 5.539 & 14.512 & 2.782 \\
\hline 9/30/10 & $8: 00$ & 22.783 & 31.729 & 24.437 & 33.513 & 5.542 & 14.546 & 2.782 \\
\hline $9 / 30 / 10$ & $12: 00$ & 22.877 & 31.755 & 24.466 & 33.593 & 5.552 & 14.561 & 2.796 \\
\hline 9/30/10 & $16: 00$ & 22.977 & 31.763 & 24.432 & 33.677 & 5.551 & 14.555 & 2.821 \\
\hline 9/30/10 & $20: 00$ & 22.952 & 31.792 & 24.461 & 33.685 & 5.506 & 14.581 & 2.839 \\
\hline $10 / 1 / 10$ & 0:00 & 22.942 & 31.828 & 24.503 & 33.690 & 5.496 & 14.607 & 2.835 \\
\hline $10 / 1 / 10$ & $4: 00$ & 22.982 & 31.849 & 24.516 & 33.719 & 5.531 & 14.613 & 2.829 \\
\hline $10 / 1 / 10$ & $8: 00$ & 22.997 & 31.885 & 24.547 & 33.740 & 5.539 & 14.633 & 2.825 \\
\hline $10 / 1 / 10$ & $12: 00$ & & 31.897 & 24.541 & 33.795 & 5.533 & & \\
\hline
\end{tabular}


Supplement 3:

Sample Documentation from TestAmerica Laboratories, Inc., for Groundwater Verification Samples 


\section{TestAmerica}

THE LEADER IN ENVIRONMENTAL TESTING

TestAmerica Laboratories, Inc.

April 30, 2010

Mr. Clyde Dennis

Argonne National Laboratory

9700 S. Cass Avenue

Building 203, Office B149

Argonne, IL 60439

Re: Laboratory Project No. 21005

Case: MORRILL; SDG: 136730

Dear Mr. Dennis:

Enclosed are analytical results for samples that were received by TestAmerica Burlington on April $8^{\text {th }}, 2010$. Laboratory identification numbers were assigned, and designated as follows:

$\begin{array}{llll}\text { Lab ID } & \text { Client } & \text { Sample ID } & \text { Sample } \\ & \underline{\text { Sample }} & \underline{\text { Matrix }}\end{array}$

Received: 04/08/10 ETR No: 136730

$\begin{array}{llll}825418 & \text { MRMW4S-W-29986 } & 04 / 06 / 10 & \text { WATER } \\ 825419 & \text { MRMW7S-W-29989 } & 04 / 06 / 10 & \text { WATER } \\ 825420 & \text { MRMW10S-W-29992 } & 04 / 06 / 10 & \text { WATER } \\ 825421 & \text { MRMW1SB-W-29979 } & 04 / 07 / 10 & \text { WATER } \\ 825422 & \text { MRMW1SM-W-29980 } & 04 / 07 / 10 & \text { WATER } \\ 825423 & \text { MRMW1ST-W-29981 } & 04 / 07 / 10 & \text { WATER } \\ 825424 & \text { MRMW1SX-W-29982 } & 04 / 07 / 10 & \text { WATER } \\ 825425 & \text { MRQCTB-W-30004 } & 04 / 07 / 10 & \text { WATER } \\ 825426 & \text { VHBLK01 } & 04 / 08 / 10 & \text { WATER }\end{array}$

Documentation of the condition of the samples at the time of their receipt and any exception to the laboratory's Sample Acceptance Policy is documented in the Sample Handling section of this submittal. The samples, as received, were not acid preserved. On that basis, the laboratory did provide for the analytical work to be performed within seven days of sample collection.

In order to accommodate field length limitations in processing the data summary forms, the laboratory did, in certain instances, abbreviate the sample identifier. The electronically formatted data provides for the full sample identifier. 


\section{TestAmerica}

THE LEADER IN ENVIRONMENTAL TESTING

\section{SOM01.2 Volatile Organics (Trace Level Water)}

A storage blank was prepared for volatile organics analysis, and stored in association with the storage of the samples. That storage blank, identified as VHBLK01, was carried through the holding period with the samples, and analyzed.

Each of the analyses associated with the sample set exhibited an acceptable internal standard performance, and there was an acceptable recovery of each deuterated monitoring compound (DMC) in each analysis. Matrix spike and matrix spike duplicate analyses were not performed on samples in this sample set. Trace concentrations of acetone and 2-butanone were identified in the analysis of the method blank associated with the analytical work. The concentration of each compound in that analysis was below the established reporting limit, and the analysis did meet the technical acceptance criteria for a compliant method blank analysis. A trace concentration of acetone was identified in the analysis of the storage blank associated with the sample set. The concentration of acetone in that analysis was below the established reporting limit, and the analysis did meet the technical acceptance criteria for a compliant storage blank analysis. Present in the method blank and storage blank analyses was a non-target constituent that represented a compound that is related to the DMC formulation. The fact that the presence of this compound is not within the laboratory's control is at issue. The derived results for that compound have been qualified with an " $X$ " qualifier to reflect the source of the contamination.

The responses for each of the target analytes met the relative standard deviation criterion in the initial calibration. The response for each target analyte met the percent difference criterion in the continuing calibration check acquisition. The response for each target analyte met the 50.0 percent difference criterion in the closing calibration check acquisition.

The primary quantitation mass for methylcyclohexane that is specified in the Statement of Work is mass 83 . The laboratory did identify a contribution to mass 83 from 1,2-dichloropropane- $d_{6}$, one of the deuterated monitoring compounds (DMCs). The laboratory did change the primary quantitation mass assignment to mass 55 for the quantification of methylcyclohexane.

Manual integration was employed in deriving certain of the analytical results. The values that have been derived from manual integration are qualified on the quantitation reports. Extracted ion current profiles for each manual integration are included in the data package, and further documented in the Sample Preparation section of this submittal.

Any reference within this report to Severn Trent Laboratories, Inc. or STL, should be understood to refer to TestAmerica Laboratories, Inc. (formerly known as Severn Trent Laboratories, Inc.) The analytical results associated with the samples presented in this test report were generated under a quality system that adheres to requirements specified in the NELAC standard. Release of the data in this test report and any associated electronic deliverables is authorized by the Laboratory Director's designee as verified by the following signature. 


\section{TestAmerica \\ THE LEADER IN ENVIRONMENTAL TESTING}

If there are any questions regarding this submittal, please contact me at 802 660-1990.

Sincerely,

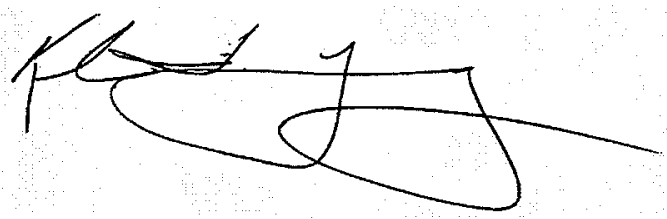

Kirk F. Young

Project Manager

KFY/hsf

Enclosure 


\section{TestAmerica Burlington Data Qualifier Definitions}

\section{Organic}

$\mathrm{U}$ : Compound analyzed but not detected at a concentration above the reporting limit.

$\mathrm{J}: \quad$ Estimated value.

$\mathrm{N}$ : Indicates presumptive evidence of a compound. This flag is used only for tentatively identified compounds (TICs) where the identification of a compound is based on a mass spectral library search.

P: $\quad$ SW-846: The relative percent difference for detected concentrations between two GC columns is greater than $40 \%$. Unless otherwise specified the higher of the two values is reported on the Form I.

CLP SOW: Greater than $25 \%$ difference for detected concentrations between two GC columns. Unless otherwise specified the lower of the two values is reported on the Form I.

C: Pesticide result whose identification has been confirmed by GC/MS.

B: Analyte is found in the sample and the associated method blank. The flag is used for tentatively identified compounds as well as positively identified compounds.

E: Compounds whose concentrations exceed the upper limit of the calibration range of the instrument for that specific analysis.

D: Concentrations identified from analysis of the sample at a secondary dilution.

A: Tentatively identified compound is a suspected aldol condensation product.

$X, Y, Z$ : Laboratory defined flags that may be used alone or combined, as needed. If used, the description of the flag is defined in the project narrative.

\section{Inorganic/Metals}

E: Reported value is estimated due to the presence of interference.

$\mathrm{N}$ : Matrix spike sample recovery is not within control limits.

* Duplicate sample analysis is not within control limits.

B: The result reported is less than the reporting limit but greater than the instrument detection limit.

U: $\quad$ Analyte was analyzed for but not detected above the reporting limit.

Method Codes:

P ICP-AES

MS ICP-MS

CV Cold Vapor AA

AS Semi-Automated Spectrophotometric 


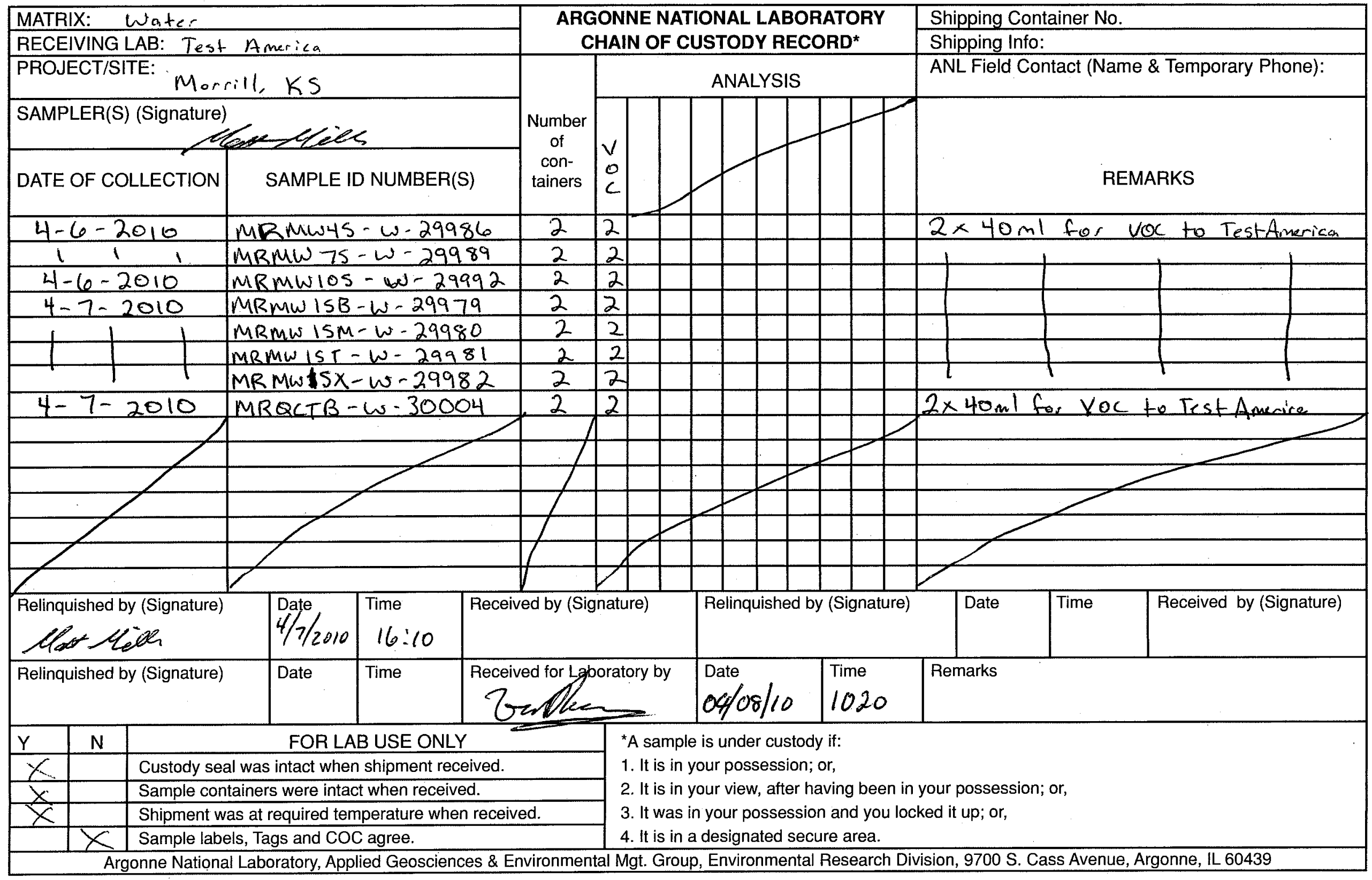




\section{TestAmerica}

THE LEADER IN ENVIRONMENTAL TESTING

\section{Sample Data Summary - SOM01.2 Volatiles - Trace}


$1 A$ - FORM I VOA-I

VOLATILE ORGANICS ANALYSIS DATA SHEET
EPA SAMPLE NO
Lab Name: TESTAMERICA BURLINGTON

Lab Code: STLV Case No.: MORRILI Mod. Ref No.:
Contract: $8 \mathrm{E}-00302$

SDG NO.: 136730
Matrix: (SOIL/SED/WATER) water

Sample wt/vol: 25.0

$(\mathrm{g} / \mathrm{mL}) \mathrm{mL}$

Level: (TRACE/LOW/MED) TRACE

$\%$ Moisture: not dec.

GC Column: DB-624

Soil Extract Volume:

ID $: 0.53$

(mm)

(UI)

$(\mathrm{mL})$

Purge Volume: 25.0
Lab Sample ID: 825420

Lab File ID: 825420

Date Received: 04/08/2010

Date Analyzed: 04/10/2010

Dilution Factor: 1.0

Soil Aliquot Volume:

$(\mathrm{mL})$

\begin{tabular}{|c|c|c|c|}
\hline CAS NO. & COMPOUND & $\begin{array}{l}\text { CONCENTRATION UNITS: } \\
(\mathrm{ug} / \mathrm{L} \text { or } \mathrm{ug} / \mathrm{kg}) \mathrm{ug} / \mathrm{L}\end{array}$ & $Q$ \\
\hline$===========$ & 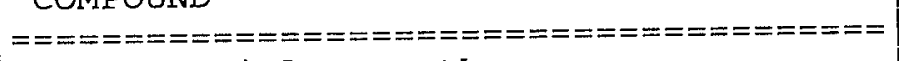 & $====================$ & $=======$ \\
\hline $75-71-8$ & Dichlorodifluoromethane & 0.50 & $\mathrm{U}$ \\
\hline $74-87-3$ & Chloromethane & 0.50 & U \\
\hline $75-01-4$ & Vinyl chloride & 0.50 & $\mathrm{U}$ \\
\hline $74-83-9$ & Bromomethane & 0.50 & $\mathrm{U}$ \\
\hline $75-00-3$ & Chloroethane & 0.50 & $\mathrm{U}$ \\
\hline $75-69-4$ & Trichlorofluoromethane & 0.50 & $\mathrm{U}$ \\
\hline $75-35-4$ & 1,1-Dichloroethene & 0.50 & $\mathrm{U}$ \\
\hline $76-13-1$ & $1,1,2$-Trichloro-1,2,2-trifluoroethane & 0.50 & $\mathrm{U}$ \\
\hline $67-64-1$ & Acetone & 5.0 & $\mathrm{U}$ \\
\hline $75-15-0$ & Carbon disulfide & 0.50 & $\mathrm{U}$ \\
\hline $79-20-9$ & Methyl acetate & 0.50 & $\mathrm{U}$ \\
\hline $75-09-2$ & Methylene chloride & 0.50 & $\mathrm{U}$ \\
\hline $156-60-5$ & trans-1,2-Dichloroethene & 0.50 & $\mathrm{U}$ \\
\hline $1634-04-4$ & Methyl tert-butyl ether & 0.50 & $\mathrm{U}$ \\
\hline $75-34-3$ & 1,1-Dichloroethane & 0.50 & $\mathrm{U}$ \\
\hline $156-59-2$ & cis-1,2-Dichloroethene & 0.50 & $\mathrm{U}$ \\
\hline $78-93-3$ & 2 -Butanone & 5.0 & U \\
\hline $74-97-5$ & Bromochloromethane & 0.50 & $\mathrm{U}$ \\
\hline $67-66-3$ & Chloroform & 0.34 & $\mathrm{~J}$ \\
\hline $71-55-6$ & $1,1,1$-Trichloroethane & 0.50 & $\mathrm{U}$ \\
\hline $110-82-7$ & Cyclohexane & 0.50 & $\mathrm{U}$ \\
\hline $56-23-5$ & Carbon tetrachloride & 0.50 & $\mathrm{U}$ \\
\hline $71-43-2$ & Benzene & 0.50 & $\mathrm{U}$ \\
\hline $107-06-2$ & 1,2-Dichloroethane & 0.50 & $\mathrm{U}$ \\
\hline
\end{tabular}

Report 1,4-Dioxane for Low-Medium VOA analysis only 


\section{$1 B$ - FORM I VOA-2 \\ VOLATILE ORGANICS ANALYSIS DATA SHEET}

Lab Name: TESTAMERICA BURLINGTON

Contract: $8 \mathrm{E}-00302$
EPA SAMPLE NO.

RMW10SW2 9992

Lab Code: STLV Case No.: MORRILL Mod. Ref No.:

SDG No.: 136730

Matrix: (SOIL/SED/WATER) Water

Sample wt/vol: 25.0

$(\mathrm{g} / \mathrm{mL}) \mathrm{mL}$

Level: (TRACE/LOW/MED) TRACE

\% Moisture: not dec.

GC Column: DB-624

ID $: 0.53$

Soil Extract Volume:

Purge Volume: 25.0
Lab Sample ID: 825420

Lab File ID: 825420

Date Received: 04/08/2010

Date Analyzed: 04/10/2010

Dilution Factor: 1.0

Soil Aliquot Volume:

(mL)

\begin{tabular}{|c|c|c|c|}
\hline CAS NO. & COMPOUND & $\begin{array}{l}\text { CONCENTRATION UNITS: } \\
(\mathrm{ug} / \mathrm{L} \text { or } \mathrm{ug} / \mathrm{kg}) \mathrm{ug} / \mathrm{L}\end{array}$ & Q \\
\hline$==========$ & 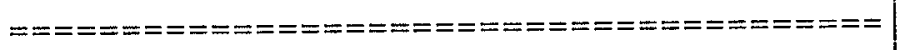 & 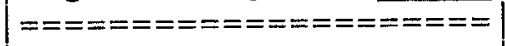 & $========$ \\
\hline $79-01-6$ & Trichloroethene & 0.50 & $U$ \\
\hline $108-87-2$ & Methylcyclohexane & 0.50 & $\mathrm{U}$ \\
\hline $78-87-5$ & 1,2-Dichloropropane & 0.50 & $\mathrm{U}$ \\
\hline $75-27-4$ & Bromodichloromethane & 0.50 & $\mathrm{U}$ \\
\hline $10061-01-5$ & cis-1,3-Dichloropropene & 0.50 & $\mathrm{U}$ \\
\hline $108-10-1$ & 4-Methyl-2-pentanone & 5.0 & $\mathrm{U}$ \\
\hline $108-88-3$ & Toluene & 0.91 & \\
\hline $10061-02-6$ & trans-1,3-Dichloropropene & 0.50 & $\overline{\mathrm{U}}$ \\
\hline $79-00-5$ & $1,1,2$-Trichloroethane & 0.50 & $\mathrm{U}$ \\
\hline $127-18-4$ & Tetrachloroethene & 0.50 & $\mathrm{U}$ \\
\hline $591-78-6$ & 2 -Hexanone & 5.0 & U \\
\hline $124-48-1$ & Dibromochloromethane & 0.50 & U \\
\hline $106-93-4$ & 1,2-Dibromoethane & 0.50 & U \\
\hline $108-90-7$ & Chlorobenzene & 0.50 & $\mathrm{U}$ \\
\hline $100-41-4$ & Ethylbenzene & 0.50 & $\mathrm{U}$ \\
\hline $95-47-6$ & o-Xylene & 0.50 & $\mathrm{U}$ \\
\hline $179601-23-1$ & $\mathrm{~m}, \mathrm{p}$-Xylene & 0.50 & $\mathrm{U}$ \\
\hline $100-42-5$ & Styrene & 0.50 & $\mathrm{U}$ \\
\hline $75-25-2$ & Bromoform & 0.50 & U \\
\hline $98-82-8$ & Isopropylbenzene & 0.50 & $\mathrm{U}$ \\
\hline $79-34-5$ & $1,1,2,2$-Tet rachloroet hane & 0.50 & $\mathrm{U}$ \\
\hline $541-73-1$ & 1,3 -Dichlorobenzene & 0.50 & U \\
\hline $106-46-7$ & 1,4-Dichlorobenzene & 0.50 & U \\
\hline $95-50-1$ & 1,2-Dichlorobenzene & 0.50 & $\mathrm{U}$ \\
\hline $96-12-8$ & 1,2-Dibromo-3-chloropropane & 0.50 & $\mathrm{U}$ \\
\hline $120-82-1$ & $1,2,4$-Trichlorobenzene & 0.50 & $\mathrm{U}$ \\
\hline $87-61-6$ & $1,2,3$-Trichlorobenzene & 0.50 & $\mathrm{U}$ \\
\hline
\end{tabular}


IJ - FORM I VOA-TIC

VOLATILE ORGANICS ANALYSIS DATA SHEET

TENTATIVELY IDENTIFIED COMPOUNDS
EPA SAMPLE NO.

RMW10SW29992
Lab Name: TESTAMERICA BURLINGTON

Lab Code: STLV Case No.: MORRILL

Matrix: (SOIL/SED/WATER) Water

Sample wt/vol: $25.0 \quad(\mathrm{~g} / \mathrm{mL}) \mathrm{mL}$

Level: (TRACE or LOW/MED) TRACE

\% Moisture: not dec.

GC Column: DB-624 ID: 0.53

Soil Extract Volume:

CONCENTRATION UNITS: (ug/L or $\mathrm{ug} / \mathrm{kg}$ ) $\mathrm{ug} / \mathrm{L}$
Contract: $8 \mathrm{E}-00302$

Mod. Ref No.:

SDG No.: 136730
Lab Sample ID: 825420

Lab File ID: 825420

Date Received: 04/08/2010

Date Analyzed: 04/10/2010

(mm) Dilution Factor: 1.0

(uL) Soil Aliquot Volume:

(UL)

Purge Volume: 25.0

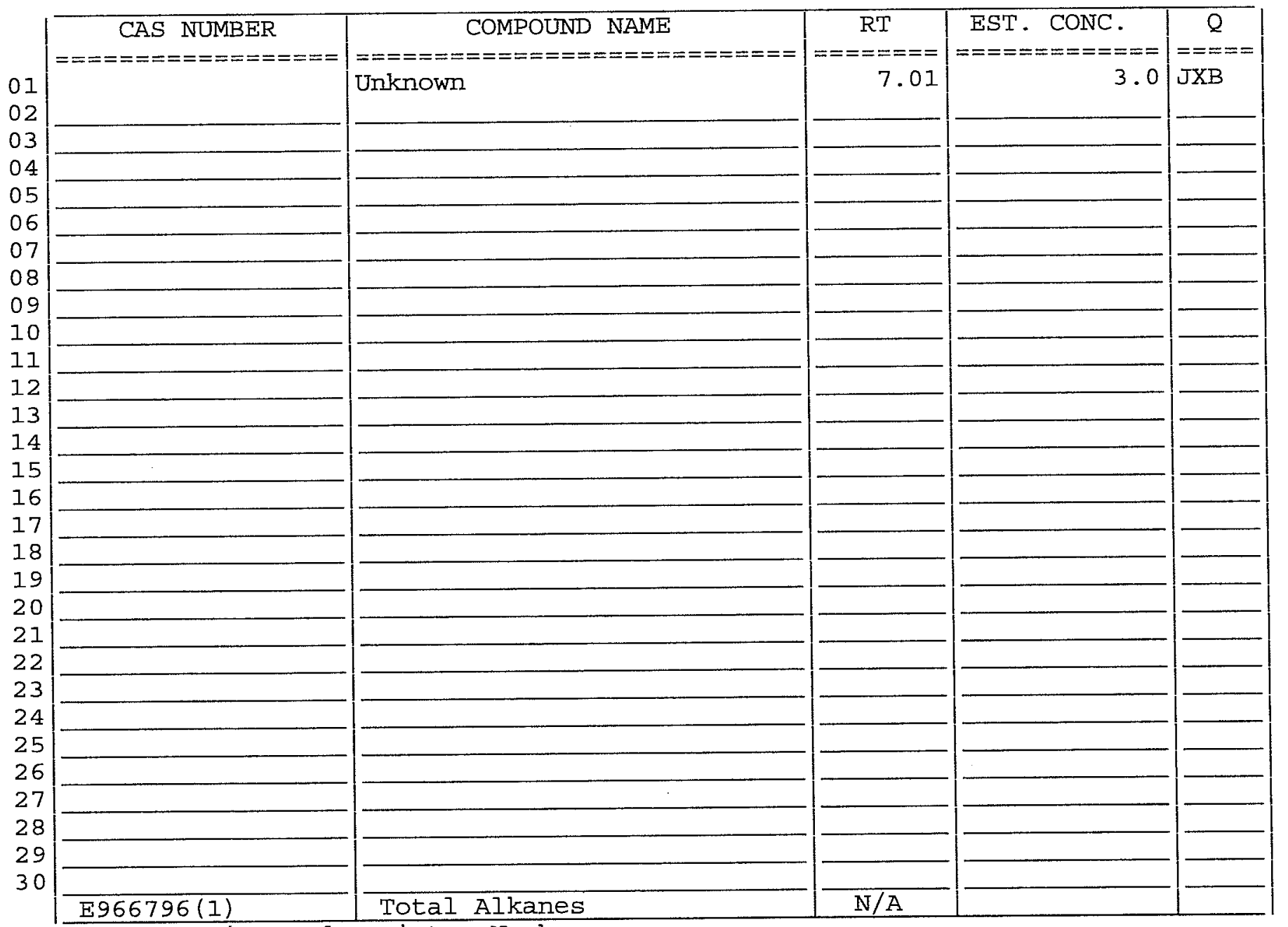

(1) EPA-designated Registry Number. 
$1 A$ - FORM I VOA-1

VOLATILE ORGANICS ANALYSIS DATA SHEET

EPA SAMPLE NO.

RMW1SBW29979

Lab Name: TESTAMERICA BURLINGTON

Contract: $8 \mathrm{E}-00302$

Lab Code: STLV Case No.: MORRILL Mod. Ref No.: $\quad$ SDG No.: 136730

Matrix: (SOIL/SED/WATER) Water

Lab Sample ID: 825421

Sample wt/vol: $25.0 \quad(\mathrm{~g} / \mathrm{mL}) \mathrm{mL}$

Lab File ID: 825421

Level: (TRACE/LOW/MED) TRACE

Date Received: 04/08/2010

\%oisture: not dec.

Date Analyzed: 04/10/2010

GC Column: DB-624 ID: 0.53 (mm) Dilution Factor: 1.0

Soil Extract Volume:

(uL) Soil Aliquot Volume:

Purge Volume: 25.0

(mL)

\begin{tabular}{|c|c|c|c|}
\hline CAS NO. & COMPOUND & $\begin{array}{l}\text { CONCENTRATION UNITS: } \\
(\mathrm{ug} / \mathrm{L} \text { or } \mathrm{ug} / \mathrm{kg}) \mathrm{ug} / \mathrm{L}\end{array}$ & $\mathrm{Q}$ \\
\hline$===========$ & 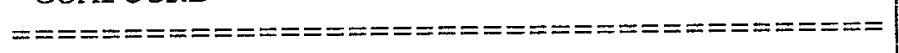 & $=====0============ \pm=$ & $=======$ \\
\hline $75-71-8$ & Dichlorodifluoromethane & 0.50 & $\mathrm{U}$ \\
\hline $74-87-3$ & Chloromethane & 0.50 & $\mathrm{U}$ \\
\hline $75-01-4$ & Vinyl chloride & 0.50 & $\mathrm{U}$ \\
\hline $74-83-9$ & Bromomethane & 0.50 & $\mathrm{U}$ \\
\hline $75-00-3$ & Chloroethane & 0.50 & $\mathrm{U}$ \\
\hline $75-69-4$ & Trichlorofluoromethane & 0.50 & $\mathrm{U}$ \\
\hline $75-35-4$ & 1,1 -Dichloroethene & 0.50 & $\mathrm{U}$ \\
\hline $76-13-1$ & $1,1,2$-Trichloro-1,2,2-trifluoroethane & 0.50 & $\mathrm{U}$ \\
\hline $67-64-1$ & Acetone & 5.0 & $\mathrm{U}$ \\
\hline $75-15-0$ & Carbon disulfide & 0.50 & $\mathrm{U}$ \\
\hline $79-20-9$ & Methyl acetate & 0.50 & $\mathrm{U}$ \\
\hline $75-09-2$ & Methylene chloride & 0.50 & $\mathrm{U}$ \\
\hline $156-60-5$ & trans-1,2-Dichloroethene & 0.50 & $\mathrm{U}$ \\
\hline $1634-04-4$ & Methyl tert-butyl ether & 0.50 & $\mathrm{U}$ \\
\hline $75-34-3$ & 1,1-Dichloroethane & 0.50 & $\mathrm{U}$ \\
\hline $156-59-2$ & cis-1,2-Dichloroethene & 0.50 & $\mathrm{U}$ \\
\hline $78-93-3$ & 2-Butanone & 5.0 & $\mathrm{U}$ \\
\hline $74-97-5$ & Bromochloromethane & 0.50 & $\mathrm{U}$ \\
\hline $67-66-3$ & Chloroform & 0.50 & $\mathrm{U}$ \\
\hline $71-55-6$ & 1,1,1-Trichloroethane & 0.50 & $\mathrm{U}$ \\
\hline $110-82-7$ & Cyclohexane & 0.50 & $\mathrm{U}$ \\
\hline $56-23-5$ & Carbon tetrachloride & 0.50 & $\mathrm{U}$ \\
\hline $71-43-2$ & Benzene & 0.50 & $\mathrm{U}$ \\
\hline $107-06-2$ & 1,2-Dichloroethane & 0.50 & $\mathrm{U}$ \\
\hline
\end{tabular}

Report 1,4-Dioxane for Low-Medium VOA analysis only

SOM01.2 
$I B$ - FORM I VOA-2

VOLATILE ORGANICS ANALYSIS DATA SHEET
EPA SAMPLE NO.

RMW1SBW2 9979
Lab Name: TESTAMERICA BURLINGTON

Lab code: STLV Case No.: MORRILI Mod. Ref No.:
Contract: $8 E-00302$

SDG No.: 136730
Matrix: (SOIL/SED/WATER) Water

Sample wt/vol: $25.0 \quad(\mathrm{~g} / \mathrm{mL}) \mathrm{mL}$

Level: (TRACE/LOW/MED) TRACE

\% Moisture: not dec.

GC Column: DB-624

Soil Extract Volume:

ID $: 0.53 \quad(\mathrm{~mm})$

(uL)

(mL)

Purge Volume: 25.0
Lab Sample ID: 825421

Lab File ID: 825421

Date Received: 04/08/2010

Date Analyzed: 04/10/2010

Dilution Factor: 1.0

Soil Aliquot Volume:

(uL)

\begin{tabular}{|c|c|c|c|}
\hline CAS NO. & COMPOUND & $\begin{array}{l}\text { CONCENTRATION UNITS: } \\
(\mathrm{ug} / \mathrm{L} \text { or } \mathrm{ug} / \mathrm{kg}) \quad \mathrm{ug} / \mathrm{L}\end{array}$ & $\mathrm{Q}$ \\
\hline$==========$ & 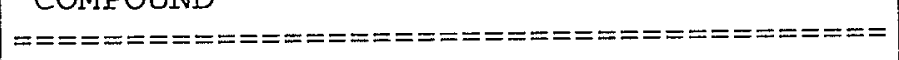 & 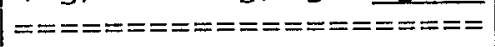 & $=======$ \\
\hline $79-01-6$ & Trichloroethene & 0.50 & $\mathrm{U}$ \\
\hline $108-87-2$ & Methylcyclohexane & 0.50 & $\mathrm{U}$ \\
\hline $78-87-5$ & 1,2-Dichloropropane & 0.50 & $\mathrm{U}$ \\
\hline $75-27-4$ & Bromodichloromethane & 0.50 & $\mathrm{U}$ \\
\hline $10061-01-5$ & Cis-1,3-Dichloropropene & 0.50 & $\mathrm{U}$ \\
\hline $108-10-1$ & 4-Methyl-2-pentanone & 5.0 & $\mathrm{U}$ \\
\hline $108-88-3$ & Toluene & 1.3 & \\
\hline $10061-02-6$ & trans-1,3-Dichloropropene & 0.50 & $\mathrm{U}$ \\
\hline $79-00-5$ & 1,1,2-Trichloroethane & 0.50 & $\mathrm{U}$ \\
\hline $127-18-4$ & Tetrachloroethene & 1.3 & \\
\hline $591-78-6$ & 2 -Hexanone & 5.0 & $\mathrm{U}$ \\
\hline $124-48-1$ & Dibromochloromethane & 0.50 & $\mathrm{U}$ \\
\hline $106-93-4$ & 1,2-Dibromoethane & 0.50 & $\mathrm{U}$ \\
\hline $108-90-7$ & Chlorobenzene & 0.50 & $\mathrm{U}$ \\
\hline $100-41-4$ & Ethylbenzene & 0.50 & $\mathrm{U}$ \\
\hline $95-47-6$ & o-Xylene & 0.50 & $\mathrm{U}$ \\
\hline $179601-23-1$ & $\mathrm{~m}, \mathrm{p}$-Xylene & 0.50 & $\mathrm{U}$ \\
\hline $100-42-5$ & Styrene & 0.50 & $\mathrm{U}$ \\
\hline $75-25-2$ & Bromoform & 0.50 & $\mathrm{U}$ \\
\hline $98-82-8$ & Isopropylbenzene & 0.50 & $\mathrm{U}$ \\
\hline $79-34-5$ & $1,1,2,2$-Tetrachloroethane & 0.50 & $\mathrm{U}$ \\
\hline $541-73-1$ & 1,3-Dichlorobenzene & 0.50 & $\mathrm{U}$ \\
\hline $106-46-7$ & 1,4-Dichlorobenzene & 0.50 & $\mathrm{U}$ \\
\hline $95-50-1$ & 1,2 -Dichlorobenzene & 0.50 & $\mathrm{U}$ \\
\hline $96-12-8$ & 1,2-Dibromo-3-chloropropane & 0.50 & $\mathrm{U}$ \\
\hline $120-82-1$ & $1,2,4$-Trichlorobenzene & 0.50 & $\mathrm{U}$ \\
\hline $87-61-6$ & $1,2,3$-Trichlorobenzene & 0.50 & $\mathrm{U}$ \\
\hline
\end{tabular}


$1 \mathrm{~J}-$ FORM I VOA-TIC

VOLATILE ORGANICS ANALYSIS DATA SHEET

TENTATIVELY IDENTIFIED COMPOUNDS
EPA SAMPLE NO.

RMW1SBW29979
Lab Name: TESTAMERICA BURLINGTON

Lab Code: STLV Case No.: MORRILL

Matrix: (SOIL/SED/WATER) Water

Sample wt/vol: $25.0 \quad(\mathrm{~g} / \mathrm{mL}) \mathrm{mL}$

Level: (TRACE or LOW/MED) TRACE

\% Moisture: not dec.

GC Column: $\mathrm{DB}-624$

ID : 0.53

Soil Extract Volume:

CONCENTRATION UNITS: (ug/L or $\mathrm{ug} / \mathrm{kg}$ ) ug/L
Contract : $8 E-00302$

Mod. Ref No.:

SDG No.: 136730
Lab Sample ID: 825421

Lab File ID: 825421

Date Received: 04/08/2010

Date Analyzed: 04/10/2010

(mm) Dilution Factor: 1.0

(uL) Soil Aliquot Volume:

(uL)

Purge Volume: 25.0

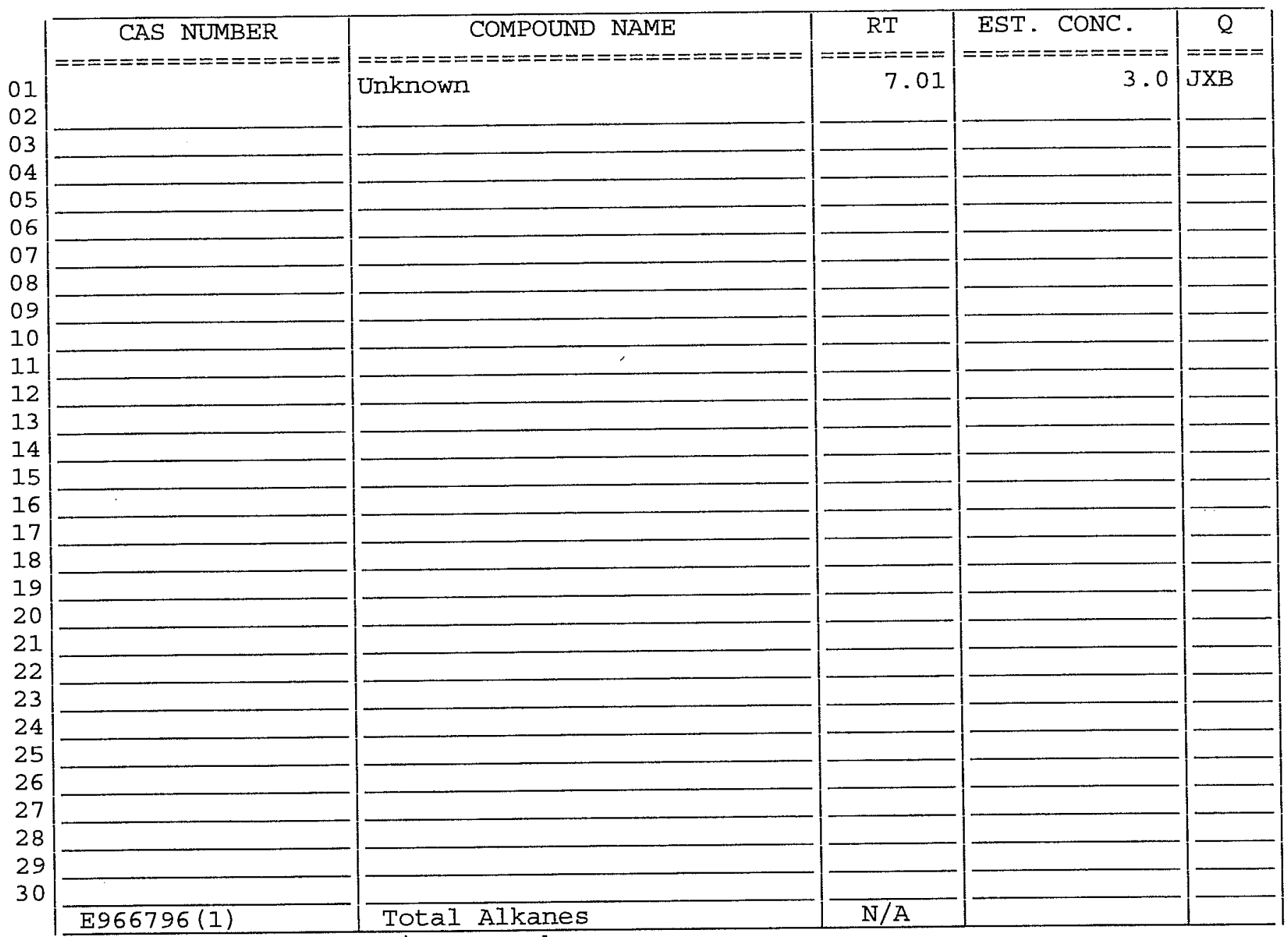

(1) EPA-designated Registry Number. 
$1 A$ - FORM I VOA-I

VOLATILE ORGANICS ANALYSIS DATA SHEET

EPA SAMPLEE NO.

Lab Name: TESTAMERICA BURLINGTON

Contract: $8 \mathrm{E}-00302$

Lab Code: STLV Case No.: MORRILL Mod. Ref No.:

SDG NO.: 136730

Matrix: (SOIL/SED/WATER) Water

Lab sample ID: 825422

Sample wt/vol: $25.0 \quad(\mathrm{~g} / \mathrm{mL}) \mathrm{mL}$

Lab File ID: 825422

Level: (TRACE/LOW/MED) TRACE

Date Received: 04/08/2010

음 Moisture: not dec.

Date Analyzed: 04/10/2010

GC Column: DB-624

ID: 0.53

$(\mathrm{mm})$

Dilution Factor: 1.0

Soil Extract Volume:

(uI) Soil Aliquot Volume:

Purge Volume: 25.0

(m工)

\begin{tabular}{|c|c|c|c|}
\hline $\begin{array}{c}\text { CAS NO. } \\
========== \\
75-71-8 \\
74-87-3 \\
75-01-4 \\
74-83-9 \\
75-00-3 \\
75-69-4 \\
75-35-4 \\
76-13-1 \\
67-64-1 \\
75-15-0 \\
79-20-9 \\
75-09-2 \\
156-60-5 \\
1634-04-4 \\
75-34-3 \\
156-59-2 \\
78-93-3 \\
74-97-5 \\
67-66-3 \\
71-55-6 \\
110-82-7 \\
56-23-5 \\
71-43-2 \\
107-06-2\end{array}$ & $\begin{array}{l}\text { COMPOUND } \\
=========================== \\
\text { Dichlorodifluoromethane } \\
\text { Chloromethane } \\
\text { Vinyl chloride } \\
\text { Bromomethane } \\
\text { Chloroethane } \\
\text { Trichlorofluoromethane } \\
\text { 1,1-Dichloroethene } \\
\text { 1,1,2-Trichloro-1,2,2-trifluoroethane } \\
\text { Acetone } \\
\text { Carbon disulfide } \\
\text { Methyl acetate } \\
\text { Methylene chloride } \\
\text { trans-1,2-Dichloroethene } \\
\text { Methyl tert-butyl ether } \\
1,1-D i c h l o r o e t h a n e \\
\text { cis-1,2-Dichloroethene } \\
2-\text { Butanone } \\
\text { Bromochloromethane } \\
\text { Chloroform } \\
1,1,1-\text { Trichloroethane } \\
\text { Cyclohexane } \\
\text { Carbon tetrachloride } \\
\text { Benzene } \\
\text { 1,2-Dichloroethane }\end{array}$ & 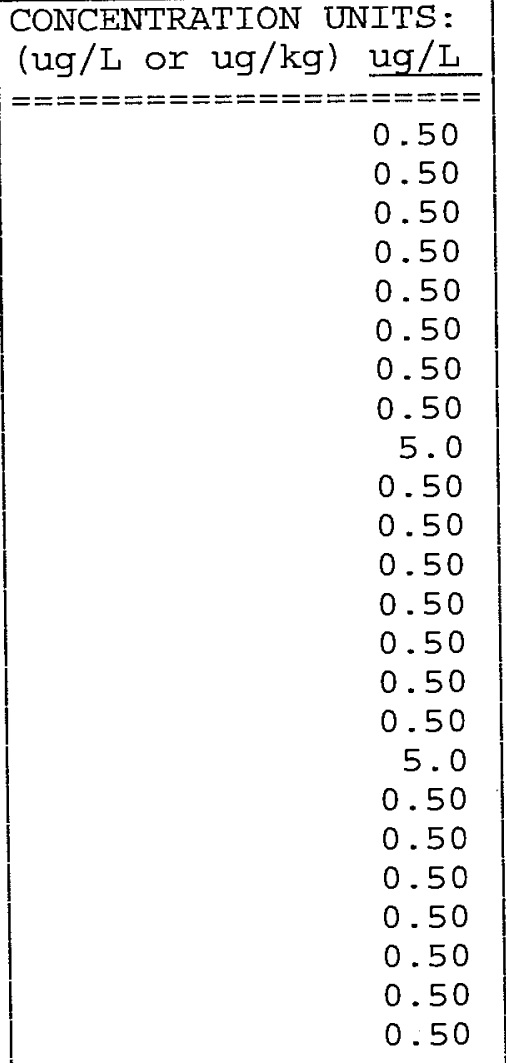 & $\begin{array}{l}========= \\
\mathrm{U} \\
\mathrm{U} \\
\mathrm{U} \\
\mathrm{U} \\
\mathrm{U} \\
\mathrm{U} \\
\mathrm{U} \\
\mathrm{U} \\
\mathrm{U} \\
\mathrm{U} \\
\mathrm{U} \\
\mathrm{U} \\
\mathrm{U} \\
\mathrm{U} \\
\mathrm{U} \\
\mathrm{U} \\
\mathrm{U} \\
\mathrm{U} \\
\mathrm{U} \\
\mathrm{U} \\
\mathrm{U} \\
\mathrm{U} \\
\mathrm{U} \\
\mathrm{U}\end{array}$ \\
\hline
\end{tabular}

Report 1,4-Dioxane for Low-Medium VOA analysis only 
$1 B$ - FORM I VOA-2

VOLATILE ORGANICS ANALYSIS DATA SHEET
EPA SAMPLE NO. RMW1SMW29980
Lab Name: TESTAMERICA BURLINGTON

Lab Code: STLV Case No.: MORRILL Mod. Ref No.:
Contract: 8E-00302

SDG NO.: 136730
Matrix: (SOIL/SED/WATER) Water

Sample wt/vol: $25.0 \quad(\mathrm{~g} / \mathrm{mL}) \mathrm{mL}$

Level: (TRACE/LOW/MED) TRACE

$\%$ Moisture: not dec.

GC Column: DB-624

ID $: 0.53$

Soil Extract Volume:

Purge Volume: 25.0
Lab Sample ID: 825422

Lab File ID: 825422

Date Received: 04/08/2010

Date Analyzed: 04/10/2010

Dilution Factor: 1.0

(uL) Soil Aliquot Volume:

(uL)

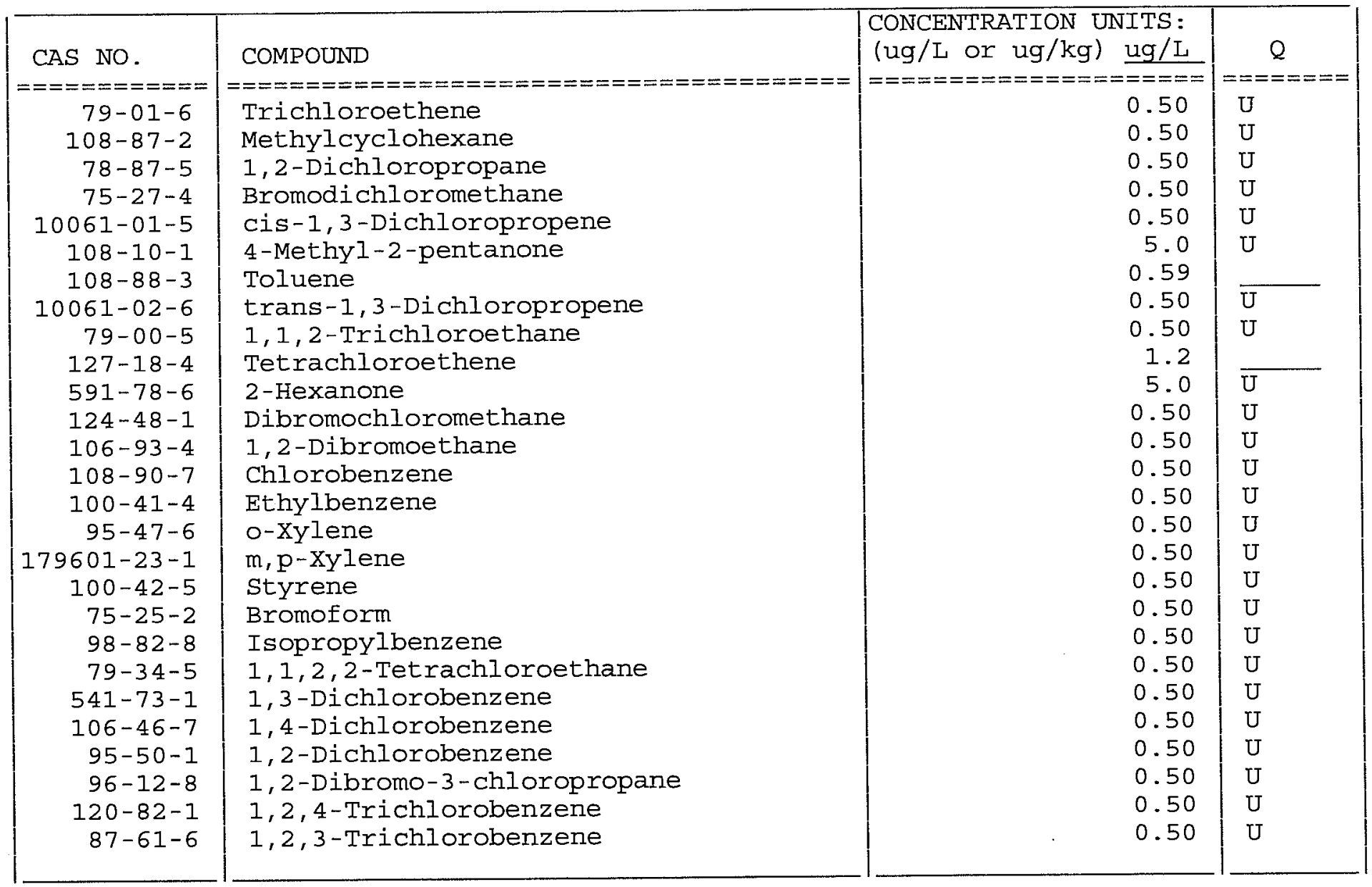


IJ - FORM I VOA-TIC

VOLATILE ORGANICS ANALYSIS DATA SHEET

TENTATIVELY IDENTIFIED COMPOUNDS
EPA SAMPLE NO.

RMWISMW29980
Lab Name: TESTAMERICA BURLINGTON

Lab Code: STLV Case No.: MORRILL

Matrix: (SOIL/SED/WATER) Water

Sample wt/vol: $25.0 \quad(\mathrm{~g} / \mathrm{mL}) \mathrm{mL}$

Level: (TRACE or LOW/MED) TRACE

\% Moisture: not dec.

GC Column: DB-624

ID $: 0.53$

Soil Extract Volume:

CONCENTRATION UNITS: (ug/L or ug/kg) ug/L
Contract: $8 \mathrm{E}-00302$

Mod. Ref No.:

SDG No.: 136730
Lab Sample ID: 825422

Lab File ID: 825422

Date Received: 04/08/2010

Date Analyzed: 04/10/2010

(mm) Dilution Factor: 1.0

(uL) Soil Aliquot Volume:

Purge Volume: 25.0

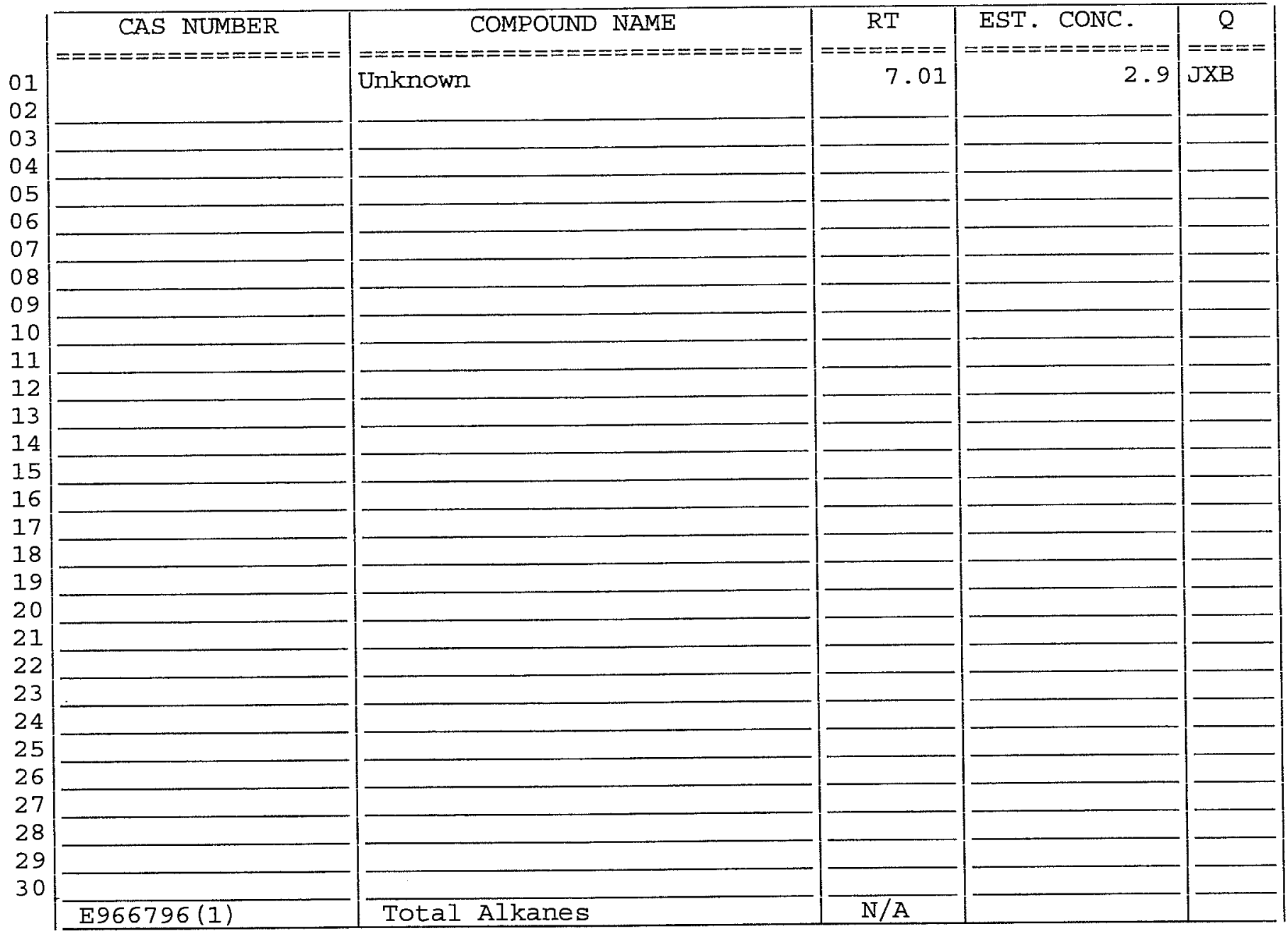

(1) EPA-designated Registry Number. 
$1 A$ - FORM I VOA-1

VOLATILE ORGANICS ANALYSIS DATA SHEET

EPA SAMPLE NO.

Lab Name: TESTAMERICA BURIINGTON

Contract: $8 E-00302$

Lab Code: STIV Case No.: MORRILI Mod. Ref No.:

SDG NO.: 136730

Matrix: (SOIL/SED/WATER) water

Lab Sample ID: 825423

Sample wt/vol: $25.0 \quad(\mathrm{~g} / \mathrm{mL}) \mathrm{mL}$

Lab File ID: 825423

Level: (TRACE/LOW/MED) TRACE

Date Received: 04/08/2010

\% Moisture: not dec.

Date Analyzed: 04/10/2010

GC Column: DB-624 ID: $0.53 \quad(\mathrm{~mm})$ Dilution Factor: 1.0

Soil Extract Volume:

(uI) Soil Aliquot Volume:

Purge Volume: 25.0

(mL)

\begin{tabular}{|c|c|c|c|}
\hline $\begin{array}{c}\text { CAS NO. } \\
========= \\
75-71-8 \\
74-87-3 \\
75-01-4 \\
74-83-9 \\
75-00-3 \\
75-69-4 \\
75-35-4 \\
76-13-1 \\
67-64-1 \\
75-15-0 \\
79-20-9 \\
75-09-2 \\
156-60-5 \\
1634-04-4 \\
75-34-3 \\
156-59-2 \\
78-93-3 \\
74-97-5 \\
67-66-3 \\
71-55-6 \\
110-82-7 \\
56-23-5 \\
71-43-2 \\
107-06-2\end{array}$ & $\begin{array}{l}\text { COMPOUND } \\
=========================== \\
\text { Dichlorodifluoromethane } \\
\text { Chloromethane } \\
\text { Vinyl chloride } \\
\text { Bromomethane } \\
\text { Chloroethane } \\
\text { Trichlorofluoromethane } \\
\text { 1,1-Dichloroethene } \\
\text { 1,1,2-Trichloro-1,2,2-trifluoroethane } \\
\text { Acetone } \\
\text { Carbon disulfide } \\
\text { Methyl acetate } \\
\text { Methylene chloride } \\
\text { trans-1,2-Dichloroethene } \\
\text { Methyl tert-butyl ether } \\
1,1-D i c h l o r o e t h a n e \\
\text { Cis-1,2-Dichloroethene } \\
2-\text { Butanone } \\
\text { Bromochloromethane } \\
\text { Chloroform } \\
1,1,1-\text { Trichloroethane } \\
\text { Cyclohexane } \\
\text { Carbon tetrachloride } \\
\text { Benzene } \\
\text { 1,2-Dichloroethane }\end{array}$ & 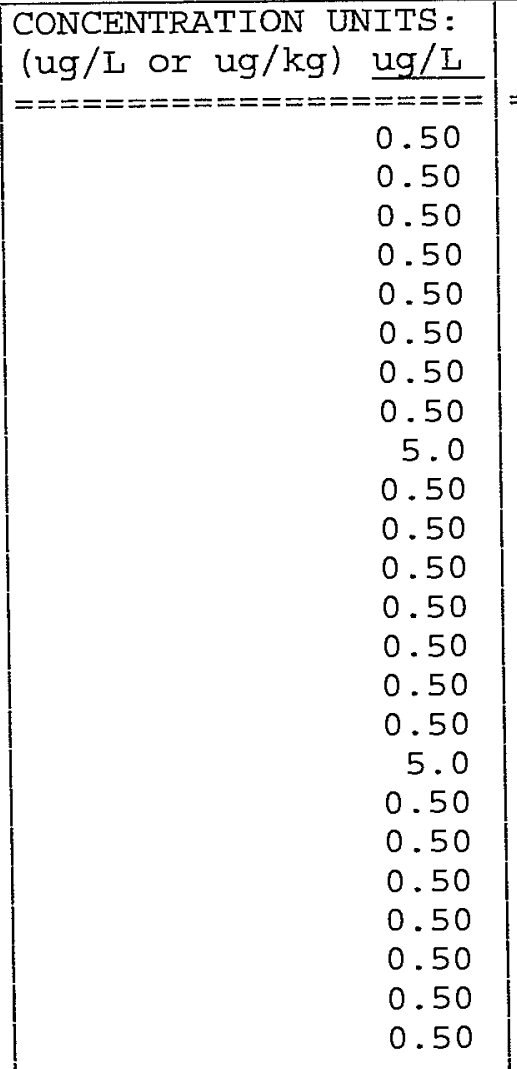 & $\begin{array}{l}======= \\
U \\
U \\
U \\
U \\
U \\
U \\
U \\
U \\
U \\
U \\
U \\
U \\
U \\
U \\
U \\
U \\
U \\
U \\
U \\
U \\
U \\
U \\
U \\
U\end{array}$ \\
\hline
\end{tabular}

Report 1,4-Dioxane for Low-Medium VOA analysis only 
IB - FORM I VOA-2

VOLATILE ORGANICS ANALYSIS DATA SHEET
EPA SAMPLE NO.
Lab Name: TESTAMERICA BURLINGTON

Lab Code: STLV Case No.: MORRILL Mod. Ref No.:
Contract: $8 E-00302$

SDG NO.: 136730
Matrix: (SOIL/SED/WATER) Water

Sample wt/vol: 25.0

$(g / m L) \quad m L$

Level: (TRACE/LOW/MED) TRACE

\% Moisture: not dec.

GC Column: DB-624

ID $: 0.53$

(mm)

(uL)

(m工)

Purge Volume: 25.0
Lab Sample ID: 825423

Lab File ID: 825423

Date Received: 04/08/2010

Date Analyzed: 04/10/2010

Dilution Factor: 1.0

Soil Aliquot Volume:

(uL)

\begin{tabular}{|c|c|c|c|}
\hline CAS NO. & COMPOUND & $\begin{array}{l}\text { CONCENTRATION UNITS: } \\
(\mathrm{ug} / \mathrm{L} \text { or } \mathrm{ug} / \mathrm{kg}) \mathrm{ug} / \mathrm{L} \\
\end{array}$ & Q \\
\hline$==========$ & 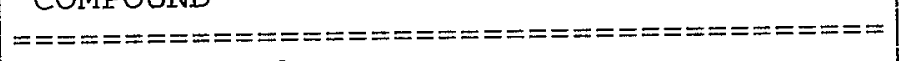 & $====================$ & $=======$ \\
\hline $79-01-6$ & Trichloroethene & 0.50 & $\mathrm{U}$ \\
\hline $108-87-2$ & Methylcyclohexane & 0.50 & $\mathrm{U}$ \\
\hline $78-87-5$ & 1,2-Dichloropropane & 0.50 & $\mathrm{U}$ \\
\hline $75-27-4$ & Bromodichloromethane & 0.50 & $\mathrm{U}$ \\
\hline $10061-01-5$ & cis-1,3-Dichloropropene & 0.50 & $\mathrm{U}$ \\
\hline $108-10-1$ & 4-Methyl-2-pentanone & 5.0 & $\mathrm{U}$ \\
\hline $108-88-3$ & Toluene & 0.51 & \\
\hline $10061-02-6$ & trans-1,3-Dichloropropene & 0.50 & $\overline{\mathrm{U}}$ \\
\hline $79-00-5$ & $1,1,2$-Trichloroethane & 0.50 & U \\
\hline $127-18-4$ & Tetrachloroethene & 0.92 & \\
\hline $591-78-6$ & 2 -Hexanone & 5.0 & $\overline{\mathrm{U}}$ \\
\hline $124-48-1$ & Dibromochloromethane & 0.50 & $\mathrm{U}$ \\
\hline $106-93-4$ & 1,2-Dibromoethane & 0.50 & $\mathrm{U}$ \\
\hline $108-90-7$ & Chlorobenzene & 0.50 & $\mathrm{U}$ \\
\hline $100-41-4$ & Ethylbenzene & 0.50 & $\mathrm{U}$ \\
\hline $95-47-6$ & o-Xylene & 0.50 & $\mathrm{U}$ \\
\hline $179601-23-1$ & $\mathrm{~m}, \mathrm{p}$-XYlene & 0.50 & $\mathrm{U}$ \\
\hline $100-42-5$ & styrene & 0.50 & $\mathrm{U}$ \\
\hline $75-25-2$ & Bromoform & 0.50 & $\mathrm{U}$ \\
\hline $98-82-8$ & Isopropylbenzene & 0.50 & $\mathrm{U}$ \\
\hline $79-34-5$ & $1,1,2,2$-Tetrachloroethane & 0.50 & $\mathrm{U}$ \\
\hline $541-73-1$ & 1,3-Dichlorobenzene & 0.50 & $\mathrm{U}$ \\
\hline $106-46-7$ & 1,4 -Dichlorobenzene & 0.50 & U \\
\hline $95-50-1$ & 1,2 -Dichlorobenzene & 0.50 & $\mathrm{U}$ \\
\hline $96-12-8$ & 1,2 -Dibromo-3-chloropropane & 0.50 & $\mathrm{U}$ \\
\hline $120-82-1$ & $1,2,4$-Trichlorobenzene & 0.50 & U \\
\hline $87-61-6$ & $1,2,3$-Trichlorobenzene & 0.50 & $\mathrm{U}$ \\
\hline
\end{tabular}


IJ - FORM I VOA-TIC

VOLATILE ORGANICS ANALYSIS DATA SHEET TENTATIVELY IDENTIFIED COMPOUNDS
EPA SAMPLE NO. RMW1STW29981
Lab Name: TESTAMERICA BURLINGTON

Lab Code: STLV Case No.: MORRILI

Matrix: (SOIL/SED/WATER) Water

Sample wt/vol: $25.0 \quad(\mathrm{~g} / \mathrm{mL}) \mathrm{mL}$

Level: (TRACE or LOW/MED) TRACE

\% Moisture: not dec.

GC Column: DB-624

$I D: 0.53$

Soil Extract Volume:

CONCENTRATION UNITS: (ug/L or $\mathrm{ug} / \mathrm{kg}$ ) ug/L
Contract: $8 \mathrm{E}-00302$

Mod. Ref No.:

SDG NO.: 136730

Lab Sample ID: 825423

Lab File ID: 825423

Date Received: 04/08/2010

Date Analyzed: 04/10/2010

(mm) Dilution Factor: 1.0

(uL) Soil Aliquot Volume:

(uL)

Purge Volume: 25.0

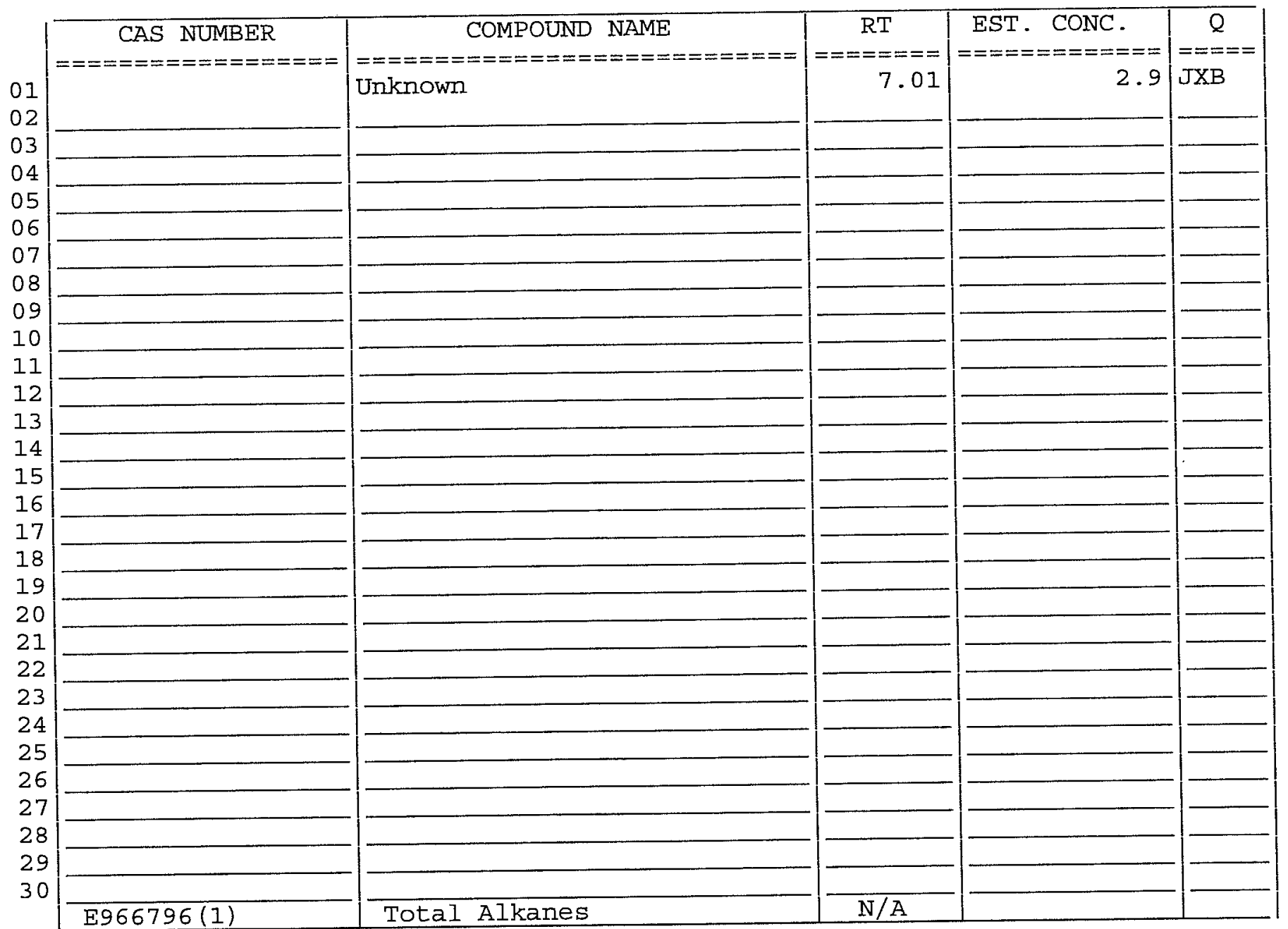

(1) EPA-designated Registry Number. 
Lab Name: TESTAMERICA BURLINGTON

Lab Code: STLV Case No.: MORRILL Mod. ReI No.:
Contract: $8 \mathrm{E}-00302$

Lab Sample ID: 825424

Lab File ID: 825424

Date Received: 04/08/2010

Date Analyzed: 04/10/2010

\% Moisture: not dec.

GC Column: DB-624 ID: 0.53 (mm) Dilution Factor: 1.0

Soil Extract Volume:

(uL) Soil Aliquot Volume:

(uL)

$(\mathrm{mL})$

Purge Volume: 25.0

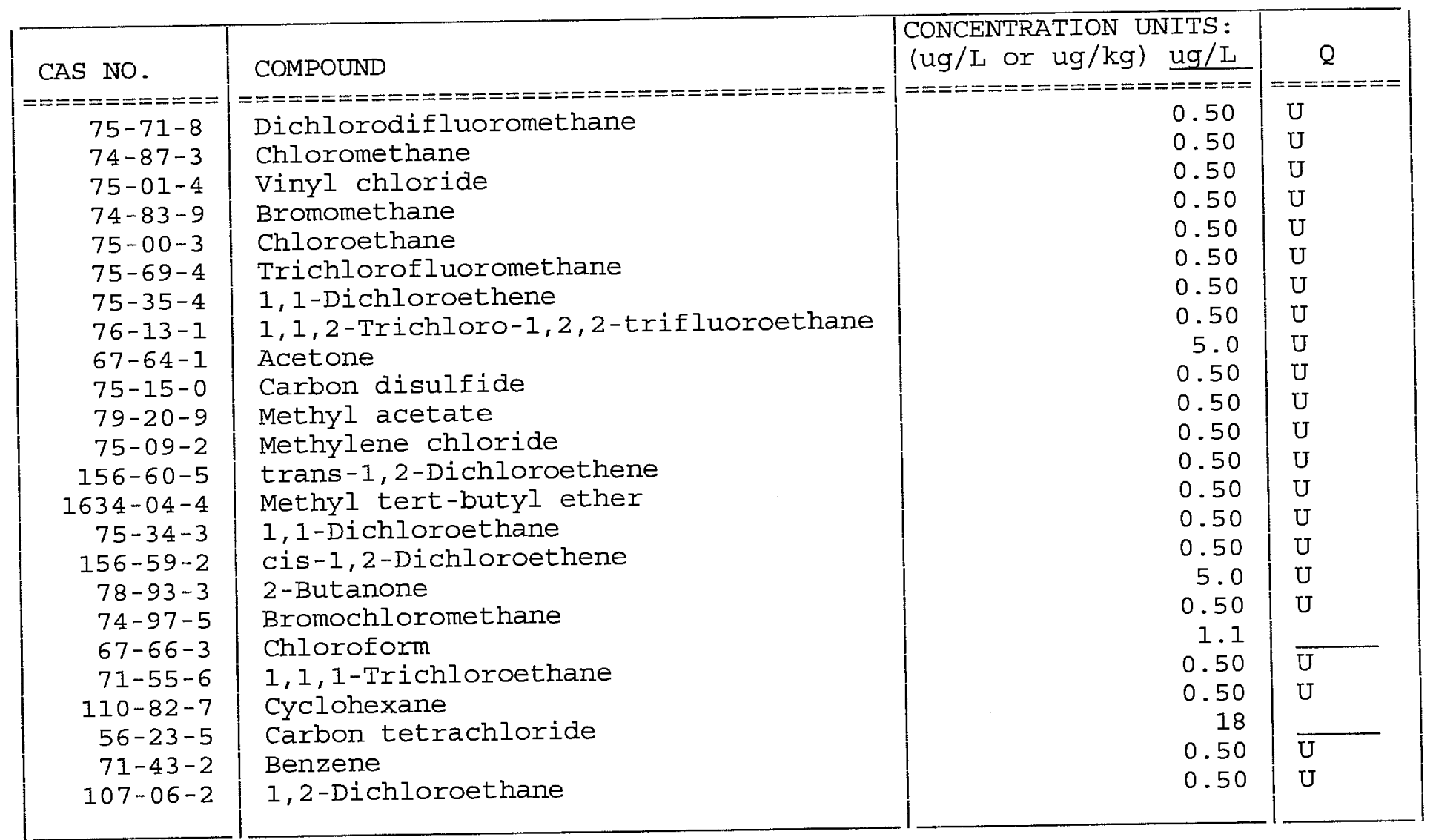

Report 1,4-Dioxane for Low-Medium VOA analysis only 
Lab Name: TESTAMERICA BURLINGTON

Contract: $8 \mathrm{E}-00302$

Lab Code: STLV Case No.: MORRILL Mod. Ref No.:

SDG NO.: 136730

Matrix: (SOIL/SED/WATER) water

Sample wt/vol: $25.0(\mathrm{~g} / \mathrm{mL}) \mathrm{mL}$

Level : (TRACE/LOW/MED) TRACE

\% Moisture: not dec.

GC Column: DB-624

ID : 0.53

Soil Extract Volume:

Purge Volume: 25.0
Lab Sample ID: 825424

Lab File ID: 825424

Date Received: 04/08/2010

Date Analyzed: 04/10/2010

Dilution Factor: 1.0

(uL) Soil Aliquot Volume:

(uL)

\begin{tabular}{|c|c|c|c|}
\hline $\begin{array}{l}\text { CAS NO. } \\
========== \\
79-01-6 \\
108-87-2 \\
78-87-5 \\
75-27-4 \\
10061-01-5 \\
108-10-1 \\
108-88-3 \\
10061-02-6 \\
79-00-5 \\
127-18-4 \\
591-78-6 \\
124-48-1 \\
106-93-4 \\
108-90-7 \\
100-41-4 \\
95-47-6 \\
17601-23-1 \\
100-42-5 \\
75-25-2 \\
98-82-8 \\
79-34-5 \\
541-73-1 \\
106-46-7 \\
95-50-1 \\
96-12-8 \\
120-82-1 \\
87-61-6\end{array}$ & 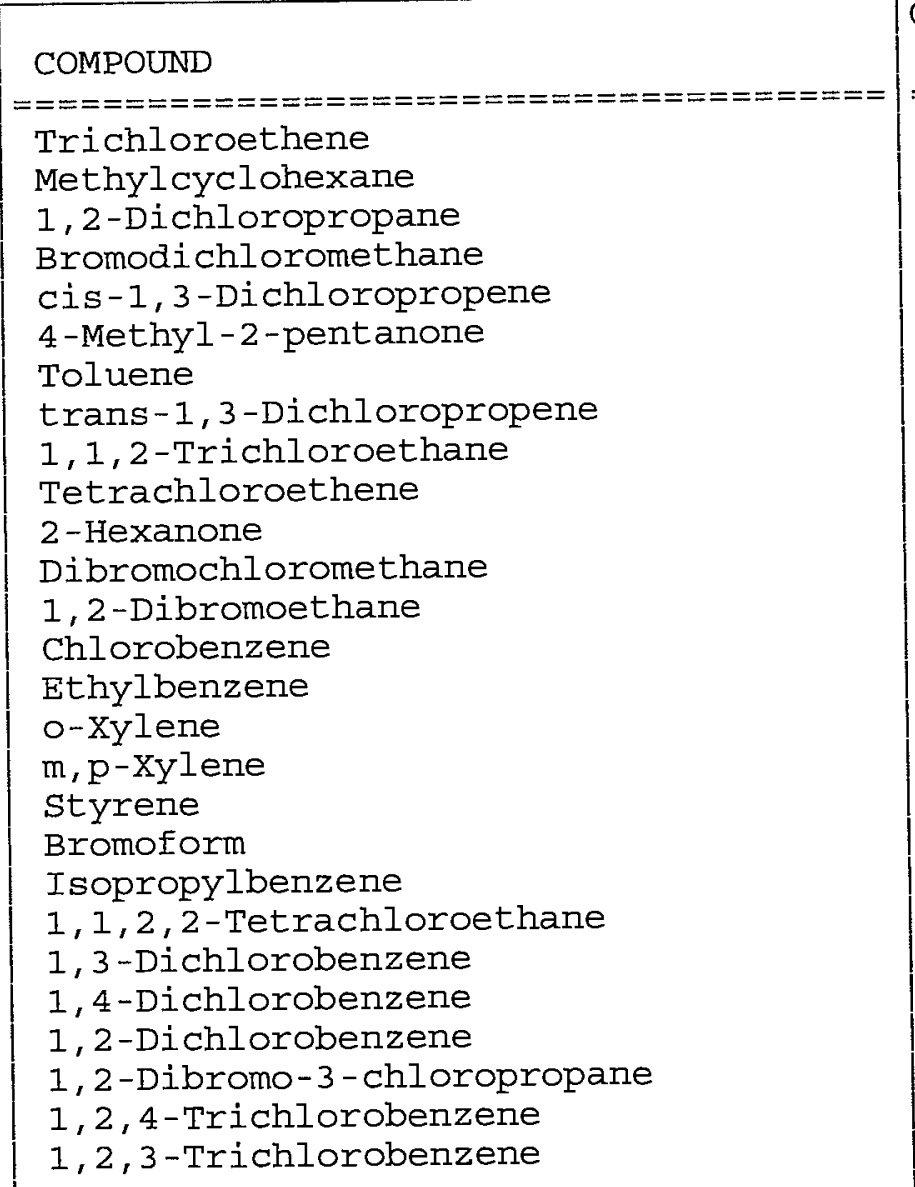 & $\begin{array}{c}\text { CONCENTRATION UNITS: } \\
\text { (ug/L or ug/kg) } \\
=================== \\
\text { ug } / \mathrm{L} \\
0.50 \\
0.50 \\
0.50 \\
0.50 \\
0.50 \\
5.0 \\
0.50 \\
0.50 \\
0.50 \\
0.50 \\
5.0 \\
0.50 \\
0.50 \\
0.50 \\
0.50 \\
0.50 \\
0.50 \\
0.50 \\
0.50 \\
0.50 \\
0.50 \\
0.50 \\
0.50 \\
0.50 \\
0.50 \\
0.50 \\
0.50\end{array}$ & $\begin{array}{l}U \\
U \\
U \\
U \\
U \\
U \\
U \\
U \\
U \\
U \\
U \\
U \\
U \\
U \\
U \\
U \\
U \\
U \\
U\end{array}$ \\
\hline
\end{tabular}


IJ - FORM I VOA-TIC

VOLATILE ORGANICS ANALYSIS DATA SHEET

TENTATIVELY IDENTIFIED COMPOUNDS
EPA SAMPLE NO.

RMW1SXW29982
Lab Name: TESTAMERICA BURLINGTON

Lab Code: STLV Case No.: MORRILL

Matrix: (SOIL/SED/WATER) water

Sample wt/vol: $25.0(\mathrm{~g} / \mathrm{mL}) \mathrm{mL}$

Level: (TRACE or LOW/MED) TRACE

\% Moisture: not dec.

GC Column: DB-624

ID : 0.53

Soil Extract Volume:

CONCENTRATION UNITS: (ug/L or ug/kg) ug/L
Contract: $8 \mathrm{E}-00302$

Mod. Ref No. :

SDG NO. : 136730
Lab Sample ID: 825424

Lab File ID: 825424

Date Received: 04/08/2010

Date Analyzed: 04/10/2010

(mm) Dilution Factor: 1.0

(uL) Soil Aliquot Volume:

Purge Volume: 25.0

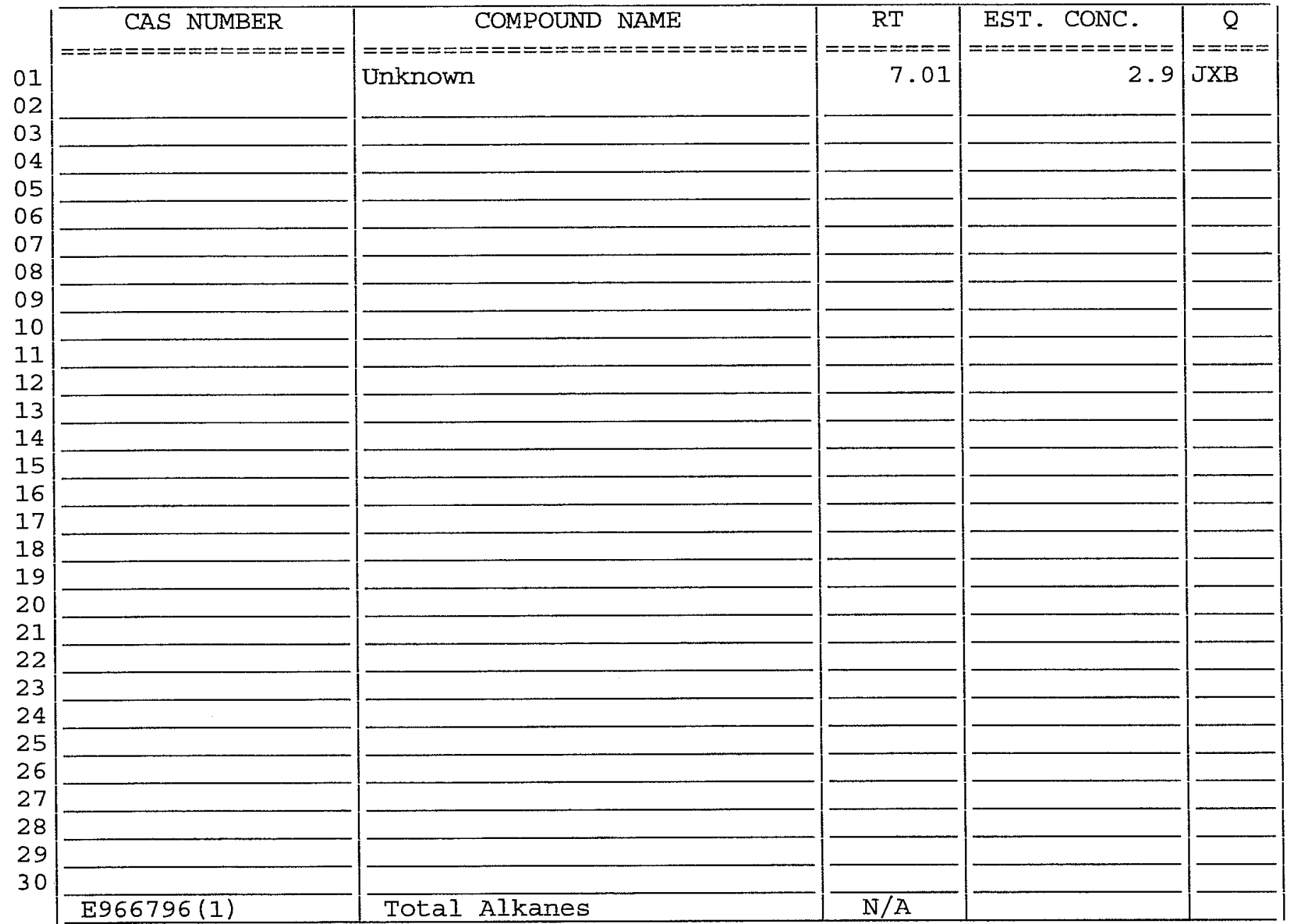

(1) EPA-designated Registry Number. 
$1 A$ - FORM I VOA-1

VOLATILE ORGANICS ANALYSIS DATA SHEET
EPA SAMPLE NO. RMW4SW29986
Lab Name: TESTAMERICA BURLINGTON

Lab Code: STLV Case No.: MORRILL

Matrix: (SOIL/SED/WATER) Water

Sample wt/vol: $25.0(\mathrm{~g} / \mathrm{mL}) \mathrm{mL}$

Level: (TRACE/LOW/MED) TRACE

\% Moisture: not dec.

GC Column: DB-624

ID $: 0.53$

Soil Extract Volume:

Purge Volume: 25.0
Contract: $8 \mathrm{E}-00302$
SDG No.: 136730
Lab Sample ID: 825418

Lab File ID: 825418

Date Received: 04/08/2010

Date Analyzed: 04/10/2010

Dilution Factor: 1.0

(uL) Soil Aliquot Volume: (uL)

$(\mathrm{mL})$

\begin{tabular}{|c|c|c|c|}
\hline $\begin{array}{c}\text { CAS NO. } \\
========= \\
75-71-8 \\
74-87-3 \\
75-01-4 \\
74-83-9 \\
75-00-3 \\
75-69-4 \\
75-35-4 \\
76-13-1 \\
67-64-1 \\
75-15-0 \\
79-20-9 \\
75-09-2 \\
156-60-5 \\
1634-04-4 \\
75-34-3 \\
156-59-2 \\
78-93-3 \\
74-97-5 \\
67-66-3 \\
71-55-6 \\
110-82-7 \\
56-23-5 \\
71-43-2 \\
107-06-2\end{array}$ & $\begin{array}{l}\text { COMPOUND } \\
=========================== \\
\text { Dichlorodifluoromethane } \\
\text { Chloromethane } \\
\text { Vinyl chloride } \\
\text { Bromomethane } \\
\text { Chloroethane } \\
\text { Trichlorofluoromethane } \\
1,1-D i c h l o r o e t h e n e \\
1,1,2-\text { Trichloro-1, } 2,2-\text { trifluoroethane } \\
\text { Acetone } \\
\text { Carbon disulfide } \\
\text { Methyl acetate } \\
\text { Methylene chloride } \\
\text { trans-1,2-Dichloroethene } \\
\text { Methyl tert-butyl ether } \\
1,1-D i c h l o r o e t h a n e \\
\text { cis-1,2-Dichloroethene } \\
2-\text { Butanone } \\
\text { Bromochloromethane } \\
\text { Chloroform } \\
1,1,1-\text { Trichloroethane } \\
\text { Cyclohexane } \\
\text { Carbon tetrachloride } \\
\text { Benzene } \\
1,2-D i c h l o r o e t h a n e\end{array}$ & \begin{tabular}{|cc} 
CONCENTRATION UNITS: \\
(ug/L or ug/kg) & ug $/ \mathrm{L}$ \\
$================$ \\
0.50 \\
0.50 \\
0.50 \\
0.50 \\
0.50 \\
0.50 \\
0.50 \\
0.50 \\
5.0 \\
0.50 \\
0.50 \\
0.50 \\
0.50 \\
0.50 \\
0.50 \\
0.50 \\
5.0 \\
0.50 \\
0.50 \\
0.50 \\
0.50 \\
0.50 \\
0.50 \\
0.50
\end{tabular} & $\begin{array}{l}======= \\
U \\
U \\
U \\
U \\
U \\
U \\
U \\
U \\
U \\
U \\
U \\
U \\
U \\
U \\
U \\
U \\
U \\
U \\
U \\
U \\
U \\
U \\
U \\
U\end{array}$ \\
\hline
\end{tabular}

Report 1,4-Dioxane for Low-Medium VOA analysis only 
$1 B$ - FORM I VOA-2

VOLATILE ORGANICS ANALYSIS DATA SHEET
EPA SAMPLE NO.

RMW4SW2 9986

Lab Name: TESTAMERICA BURLINGTON

Contract: $8 E-00302$

Lab Code: STLV Case No.: MORRILL Mod. Ref No.:

SDG NO.: 136730

Matrix: (SOIL/SED/WATER) Water

Sample wt/vol: $25.0 \quad(\mathrm{~g} / \mathrm{mL}) \mathrm{mL}$

Level : (TRACE/LOW/MED) TRACE

\% Moisture: not dec.

GC Column: DB-624 ID : 0.53 (mm)

Soil Extract Volume:

(uL)

Lab Sample ID: 825418

Purge Volume: 25.0

(mL)
Lab File ID: 825418

Date Received: 04/08/2010

Date Analyzed: 04/10/2010

Dilution Factor: 1.0

Soil Aliquot Volume:

(uL)

\begin{tabular}{|c|c|c|c|}
\hline $\begin{array}{l}\text { CAS NO. } \\
========== \\
79-01-6 \\
108-87-2 \\
78-87-5 \\
75-27-4 \\
10061-01-5 \\
108-10-1 \\
108-88-3 \\
10061-02-6 \\
79-00-5 \\
127-18-4 \\
591-78-6 \\
124-48-1 \\
106-93-4 \\
108-90-7 \\
100-41-4 \\
95-47-6 \\
17601-23-1 \\
100-42-5 \\
75-25-2 \\
98-82-8 \\
79-34-5 \\
541-73-1 \\
106-46-7 \\
95-50-1 \\
96-12-8 \\
120-82-1 \\
87-61-6\end{array}$ & 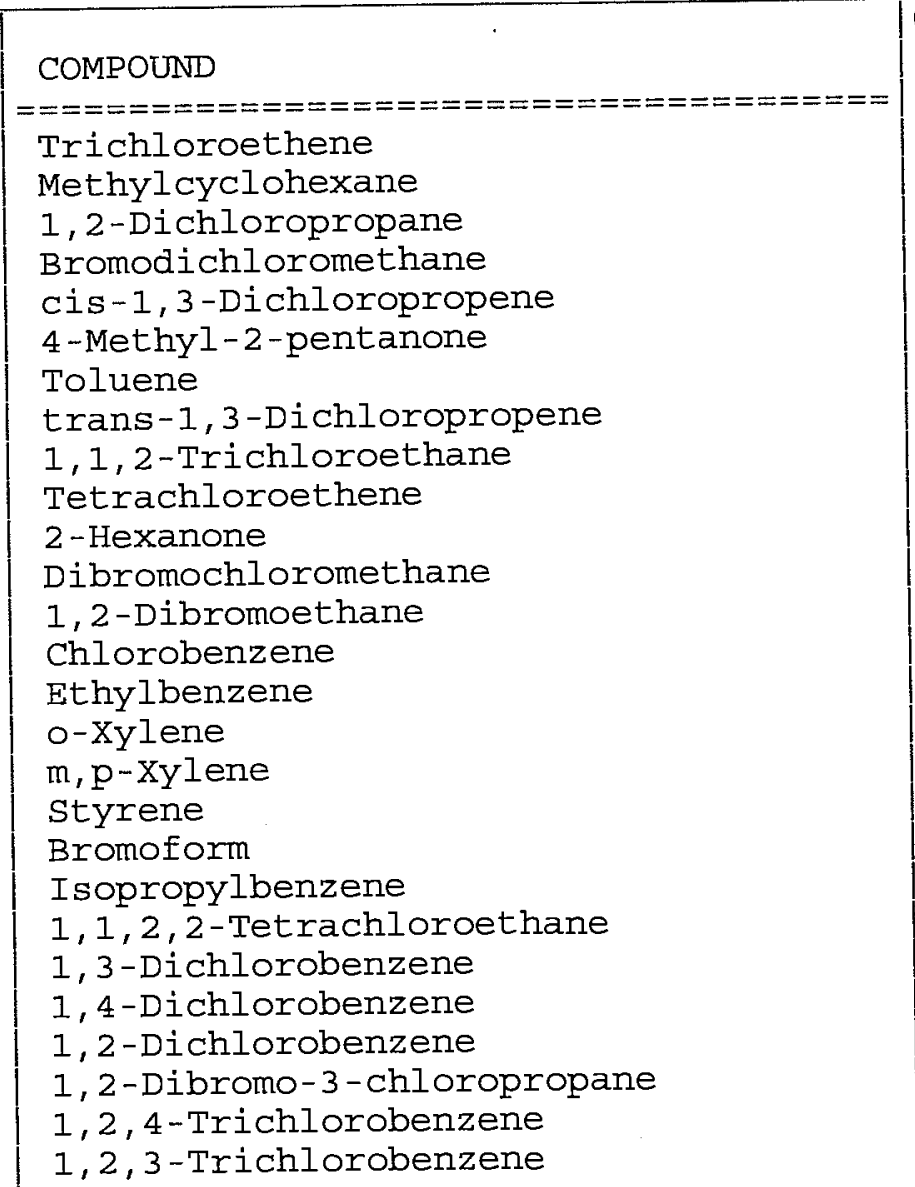 & 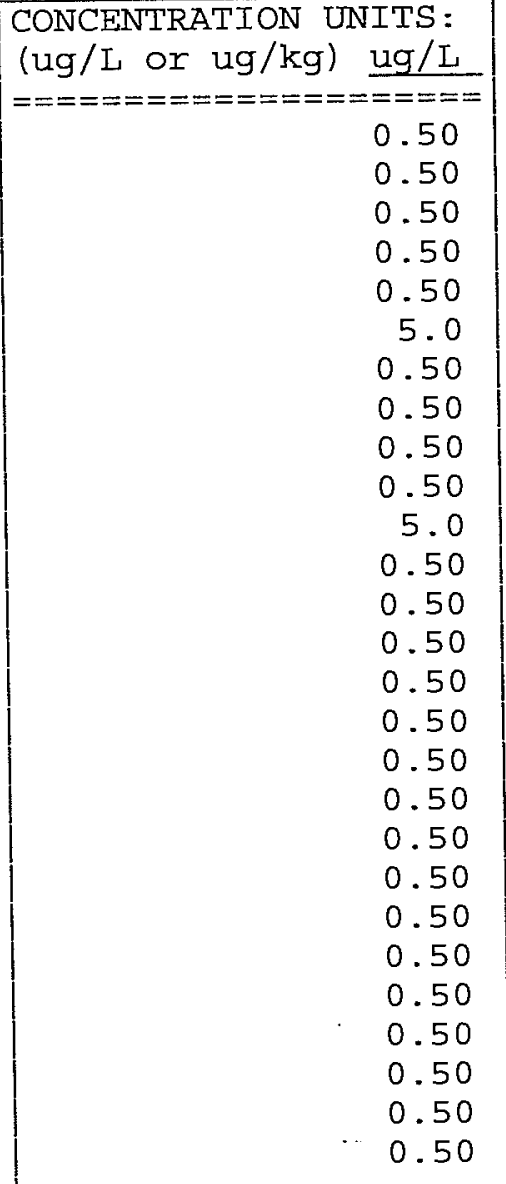 & $\begin{array}{l}========= \\
\mathrm{U} \\
\mathrm{U} \\
\mathrm{U} \\
\mathrm{U} \\
\mathrm{U} \\
\mathrm{U} \\
\mathrm{U} \\
\mathrm{U} \\
\mathrm{U} \\
\mathrm{U} \\
\mathrm{U} \\
\mathrm{U} \\
\mathrm{U} \\
\mathrm{U} \\
\mathrm{U} \\
\mathrm{U} \\
\mathrm{U} \\
\mathrm{U} \\
\mathrm{U} \\
\mathrm{U} \\
\mathrm{U} \\
\mathrm{U} \\
\mathrm{U} \\
\mathrm{U} \\
\mathrm{U} \\
\mathrm{U} \\
\mathrm{U}\end{array}$ \\
\hline
\end{tabular}


IJ - FORM I VOA-TIC

VOLATILE ORGANICS ANALYSIS DATA SHEET

TENTATIVELY IDENTIFIED COMPOUNDS
EPA SAMPLE NO. RMW4SW2 9986
Lab Name: TESTAMERICA BURLINGTON

Lab Code: STLV Case No.: MORRILL

Matrix: (SOIL/SED/WATER) water

Sample wt/vol: $25.0 \quad(\mathrm{~g} / \mathrm{mL}) \mathrm{mL}$

Level: (TRACE or LOW/MED) TRACE

\% Moisture: not dec.

GC Column: DB-624

ID : 0.53

Soil Extract Volume:

CONCENTRATION UNITS: (ug/L or ug/kg) ug/L
Contract: $8 \mathrm{E}-00302$

Mod. Ref No.:

SDG No.: 136730
Lab Sample ID: 825418

Lab File ID: 825418

Date Received: 04/08/2010

Date Analyzed: 04/10/2010

(mm) Dilution Factor: 1.0

(uL) Soil Aliquot Volume:

(uI)

Purge Volume: 25.0

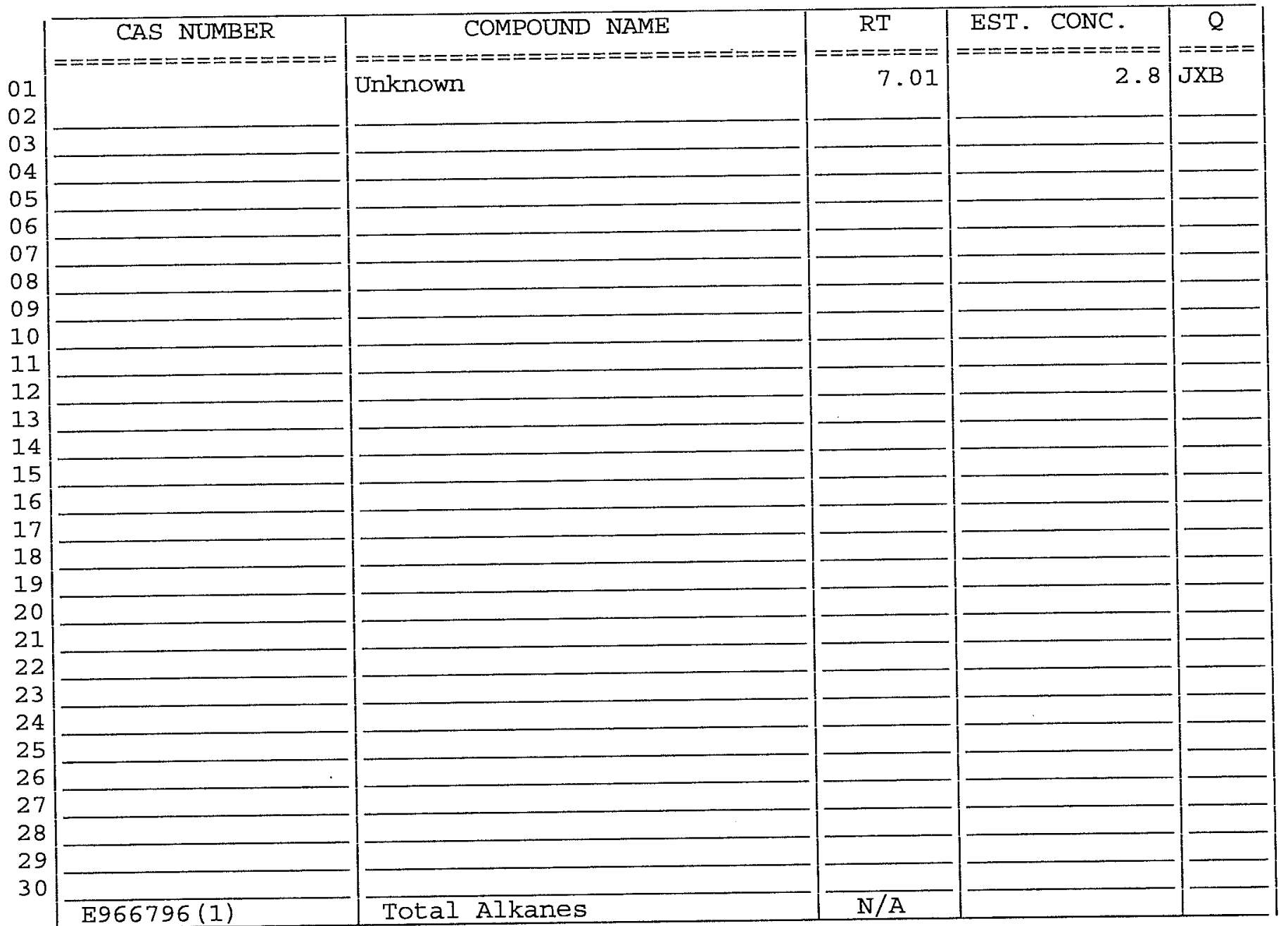

(1) EPA-designated Registry Number. 
$1 A$ - FORM I VOA-1

VOLATILE ORGANICS ANALYSIS DATA SHEET

EPA SAMPLE NO.

Lab Name: TESTAMERICA BURLINGTON

Contract: $8 \mathrm{E}-00302$

Lab Code: STLV Case No.: MORRILI Mod. Ref No.:

SDG NO.: 136730

Matrix: (SOIL/SED/WATER) Water

Lab Sample ID: 825419

Sample wt/vol: 25.0

$(g / m L) \quad m L$

Lab File ID: 825419

Level: (TRACE/LOW/MED) TRACE

Date Received: 04/08/2010

\% Moisture: not dec.

Date Analyzed: 04/10/2010

GC Columin: $\mathrm{DB}-624$

ID $: 0.53$

(mm)

Dilution Factor: 1.0

Soil Extract Volume:

(uL) Soil Aliquot Volume:

(uL)

Purge Volume: 25.0

(mL)

\begin{tabular}{|c|c|c|c|}
\hline $\begin{array}{c}\text { CAS NO. } \\
========= \\
75-71-8 \\
74-87-3 \\
75-01-4 \\
74-83-9 \\
75-00-3 \\
75-69-4 \\
75-35-4 \\
76-13-1 \\
67-64-1 \\
75-15-0 \\
79-20-9 \\
75-09-2 \\
156-60-5 \\
1634-04-4 \\
75-34-3 \\
156-59-2 \\
78-93-3 \\
74-97-5 \\
67-66-3 \\
71-55-6 \\
110-82-7 \\
56-23-5 \\
71-43-2 \\
107-06-2\end{array}$ & $\begin{array}{l}\text { COMPOUND } \\
\text { Dichlorodifluoromethane } \\
\text { Chloromethane } \\
\text { Vinyl chloride } \\
\text { Bromomethane } \\
\text { Chloroethane } \\
\text { Trichlorofluoromethane } \\
1,1-\text { Dichloroethene } \\
1,1,2-\text { Trichloro-1,2,2-trifluoroethane } \\
\text { Acetone } \\
\text { Carbon disulfide } \\
\text { Methyl acetate } \\
\text { Methylene chloride } \\
\text { trans-1,2-Dichloroethene } \\
\text { Methyl tert-butyl ether } \\
1,1-D i c h l o r o e t h a n e \\
\text { Cis-1,2-Dichloroethene } \\
2-\text { Butanone } \\
\text { Bromochloromethane } \\
\text { Chloroform } \\
1,1,1-\text { Trichloroethane } \\
\text { Cyclohexane } \\
\text { Carbon tetrachloride } \\
\text { Benzene } \\
1,2-D i c h l o r o e t h a n e\end{array}$ & \begin{tabular}{|c|c} 
CONCENTRATION UNITS : \\
(ug/L Or ug/kg) & ug $/ \mathrm{L}$ \\
$=================$ \\
0.50 \\
0.50 \\
0.50 \\
0.50 \\
0.50 \\
0.50 \\
0.50 \\
0.50 \\
5.0 \\
0.50 \\
0.50 \\
0.50 \\
0.50 \\
0.50 \\
0.50 \\
0.50 \\
5.0 \\
0.50 \\
0.52 \\
0.50 \\
0.50 \\
13 \\
0.50 \\
0.50
\end{tabular} & $\begin{array}{l}======= \\
U \\
U \\
U \\
U \\
U \\
U \\
U \\
U \\
U \\
U \\
U \\
U \\
U \\
U \\
U \\
U \\
U \\
U\end{array}$ \\
\hline
\end{tabular}

Report 1,4-Dioxane for Low-Medium VOA analysis only 
Lab Name: TESTAMERICA BURLINGTON

Lab Code: STLV Case No.: MORRILL Mod. Ref No.:
Contract: $8 \mathrm{E}-00302$
Matrix: (SOIL/SED/WATER) water

Sample wt/vol: $25.0 \quad(\mathrm{~g} / \mathrm{mL}) \mathrm{mL}$

Level: (TRACE/LOW/MED) TRACE

\% Moisture: not dec.

GC Column: DB-624

ID $: 0.53 \quad(\mathrm{~mm})$

Soil Extract Volume:

(uI)

Lab Sample ID: 825419

Lab File ID: 825419

Date Received: 04/08/2010

Date Analyzed: 04/10/2010

Dilution Factor: 1.0

Soil Aliquot Volume:

(uL)

Purge Volume: 25.0

(mL)

\begin{tabular}{|c|c|c|c|}
\hline CAS NO. & COMPOUND & $\begin{array}{l}\text { CONCENTRATION UNITS: } \\
(\mathrm{ug} / \mathrm{L} \text { or } \mathrm{ug} / \mathrm{kg}) \mathrm{ug} / \mathrm{L}\end{array}$ & $\mathrm{Q}$ \\
\hline$===========$ & 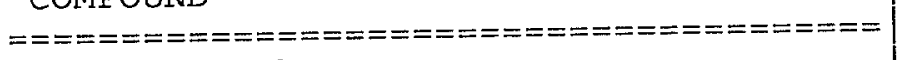 & $====================0$ & $=======$ \\
\hline $79-01-6$ & Trichloroethene & 0.50 & $\mathrm{U}$ \\
\hline $108-87-2$ & Methylcyclohexane & 0.50 & $\mathrm{U}$ \\
\hline $78-87-5$ & 1,2 -Dichloropropane & 0.50 & $\mathrm{U}$ \\
\hline $75-27-4$ & Bromodichloromethane & 0.50 & $\mathrm{U}$ \\
\hline $10061-01-5$ & cis-1,3-Dichloropropene & 0.50 & U \\
\hline $108-10-1$ & 4-Methyl-2-pentanone & 5.0 & $\mathrm{U}$ \\
\hline $108-88-3$ & Toluene & 0.50 & $\mathrm{U}$ \\
\hline $10061-02-6$ & trans-1,3-Dichloropropene & 0.50 & $\mathrm{U}$ \\
\hline $79-00-5$ & $1,1,2$-Trichloroethane & 0.50 & $\mathrm{U}$ \\
\hline $127-18-4$ & Tetrachloroethene & 0.50 & $\mathrm{U}$ \\
\hline $591-78-6$ & 2 -Hexanone & 5.0 & $\mathrm{U}$ \\
\hline $124-48-1$ & Dibromochloromethane & 0.50 & $\mathrm{U}$ \\
\hline $106-93-4$ & 1,2-Dibromoethane & 0.50 & $\mathrm{U}$ \\
\hline $108-90-7$ & Chlorobenzene & 0.50 & $\mathrm{U}$ \\
\hline $100-41-4$ & Ethylbenzene & 0.50 & $\mathrm{U}$ \\
\hline $95-47-6$ & o-Xylene & 0.50 & $\mathrm{U}$ \\
\hline $179601-23-1$ & $\mathrm{~m}, \mathrm{p}$-Xylene & 0.50 & $\mathrm{U}$ \\
\hline $100-42-5$ & Styrene & 0.50 & $\mathrm{U}$ \\
\hline $75-25-2$ & Bromoform & 0.50 & $\mathrm{U}$ \\
\hline $98-82-8$ & Isopropylbenzene & 0.50 & U \\
\hline $79-34-5$ & $1,1,2,2$-Tetrachloroethane & 0.50 & $\mathrm{U}$ \\
\hline $541-73-1$ & 1,3 -Dichlorobenzene & 0.50 & $\mathrm{U}$ \\
\hline $106-46-7$ & 1,4-Dichlorobenzene & 0.50 & $\mathrm{U}$ \\
\hline $95-50-1$ & 1,2 -Dichlorobenzene & 0.50 & $\mathrm{U}$ \\
\hline $96-12-8$ & 1,2-Dibromo-3-chloropropane & 0.50 & $\mathrm{U}$ \\
\hline $120-82-1$ & $1,2,4$-Trichlorobenzene & 0.50 & $U$ \\
\hline $87-61-6$ & $1,2,3$-Trichlorobenzene & 0.50 & $\mathrm{U}$ \\
\hline
\end{tabular}


IJ - FORM I VOA-TIC

VOLATILE ORGANICS ANALYSIS DATA SHEET

TENTATIVELY IDENTIFIED COMPOUNDS
EPA SAMPLE NO. RMW7SW29989
Lab Name: TESTAMERICA BURLINGTON

Lab Code: STLV Case No.: MORRILL

Matrix: (SOIL/SED/WATER) Water

Sample wt/vol: $25.0 \quad(\mathrm{~g} / \mathrm{mL}) \mathrm{mL}$

Level: (TRACE or LOW/MED) TRACE

\% Moisture: not dec.

GC Column: DB-624

ID $: 0.53$

Soil Extract Volume:

CONCENTRATION UNITS: (ug/I or $u g / \mathrm{kg}) \mathrm{ug} / \mathrm{L}$
Contract: $8 \mathrm{E}-00302$

Mod. Ref No.:

SDG NO.: 136730

Lab Sample ID: 825419

Lab File ID: 825419

Date Received: 04/08/2010

Date Analyzed: 04/10/2010

(mm) Dilution Factor: 1.0

(uL) Soil Aliquot Volume:

(UI)

Purge Volume: 25.0

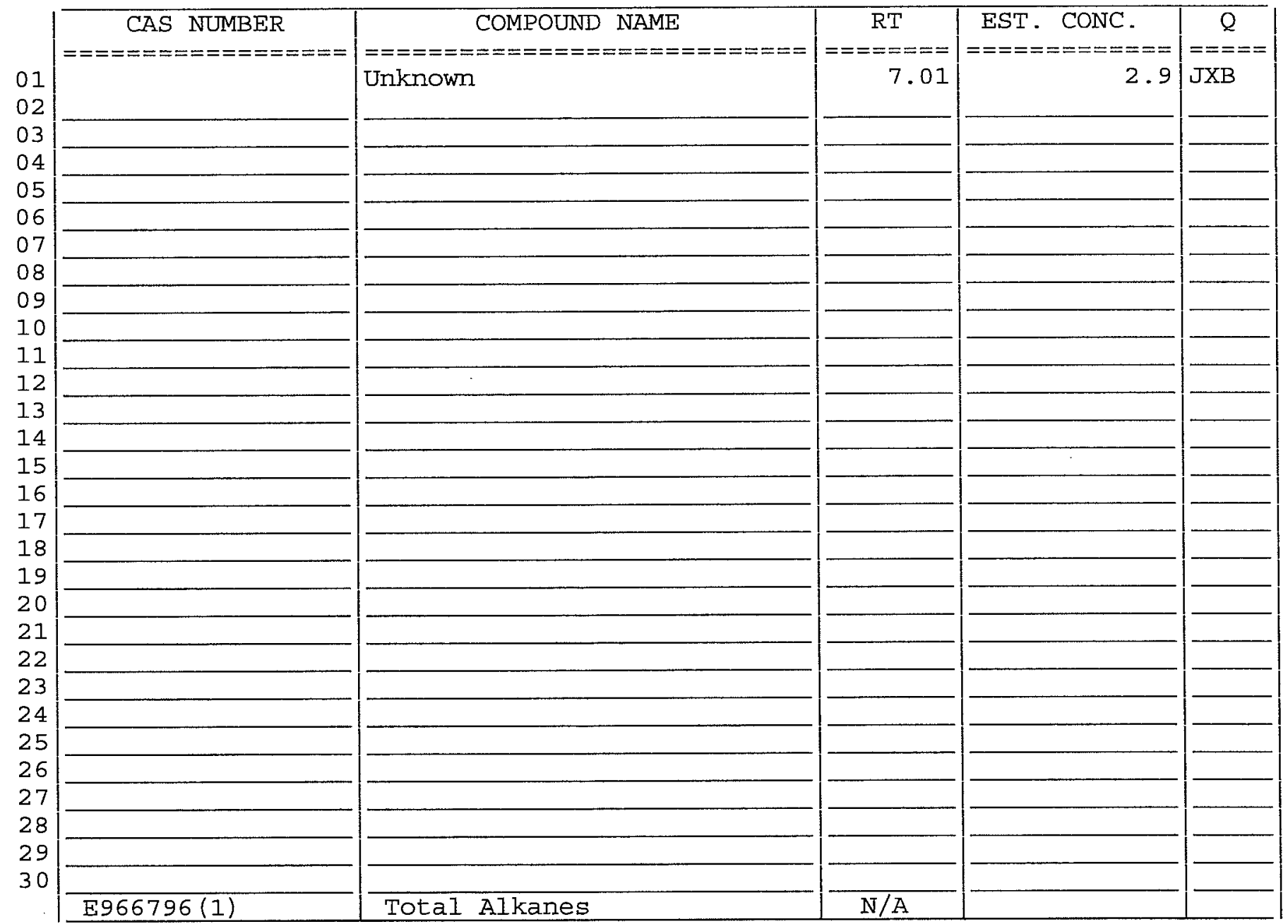

(1) EPA-designated Registry Number. 
$1 A$ - FORM I VOA-1

VOLATILE ORGANICS ANALYSIS DATA SHEET

EPA SAMPLE NO.

Lab Name: TESTAMERICA BURLINGTON

Contract: $8 E-00302$

Lab Code: STLV Case No.: MORRILL Mod. Ref No.:

SDG No. : 136730

Matrix: (SOIL/SED/WATER) Water

Lab Sample ID: 825425

Sample wt/vol: $25.0 \quad(\mathrm{~g} / \mathrm{mL}) \mathrm{mL}$

Lab File ID: 825425

Level : (TRACE/LOW/MED) TRACE

Date Received: 04/08/2010

\% Moisture: not dec.

Date Analyzed: 04/10/2010

GC Column: DB-624

ID $: 0.53 \quad(\mathrm{~mm})$

Dilution Factor: 1.0

Soil Extract Volume:

(uL) Soil Aliquot Volume:

Purge Volume: 25.0

(mL)

\begin{tabular}{|c|c|c|c|}
\hline $\begin{array}{l}\text { CAS NO. } \\
========= \\
75-71-8 \\
74-87-3 \\
75-01-4 \\
74-83-9 \\
75-00-3 \\
75-69-4 \\
75-35-4 \\
76-13-1 \\
67-64-1 \\
75-15-0 \\
79-20-9 \\
75-09-2 \\
156-60-5 \\
1634-04-4 \\
75-34-3 \\
156-59-2 \\
78-93-3 \\
74-97-5 \\
67-66-3 \\
71-55-6 \\
110-82-7 \\
56-23-5 \\
71-43-2 \\
107-06-2\end{array}$ & $\begin{array}{l}\text { COMPOUND } \\
============================= \\
\text { Dichlorodifluoromethane } \\
\text { Chloromethane } \\
\text { Vinyl chloride } \\
\text { Bromomethane } \\
\text { Chloroethane } \\
\text { Trichlorofluoromethane } \\
\text { 1,1-Dichloroethene } \\
\text { 1,1,2-Trichloro-1,2,2-trifluoroethane } \\
\text { Acetone } \\
\text { Carbon disulfide } \\
\text { Methyl acetate } \\
\text { Methylene chloride } \\
\text { trans-1, } 2 \text {-Dichloroethene } \\
\text { Methyl tert-butyl ether } \\
1,1-D i c h l o r o e t h a n e \\
\text { cis-1,2-Dichloroethene } \\
\text { 2-Butanone } \\
\text { Bromochloromethane } \\
\text { Chloroform } \\
1,1,1-\text { Trichloroethane } \\
\text { Cyclohexane } \\
\text { Carbon tetrachloride } \\
\text { Benzene } \\
\text { 1,2-Dichloroethane }\end{array}$ & 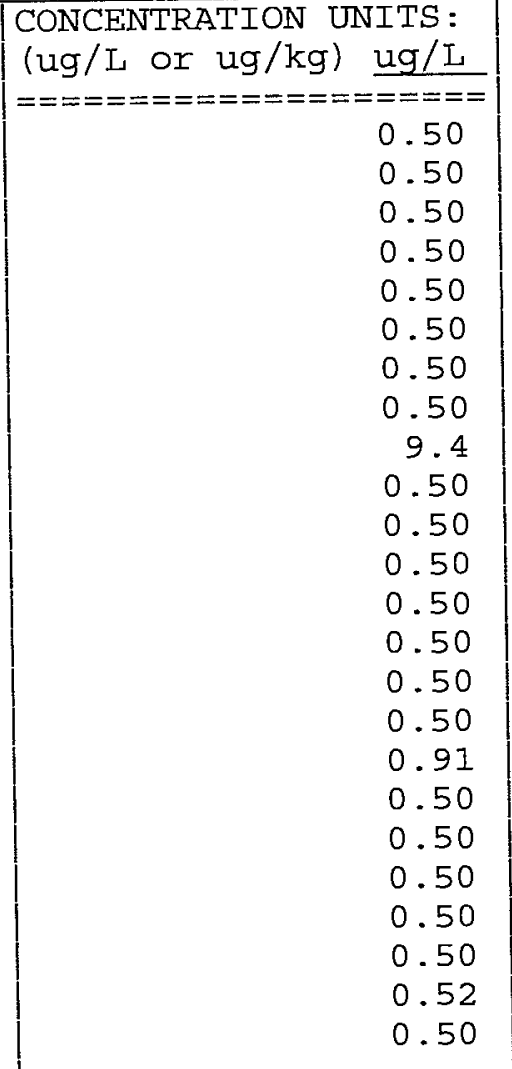 & $\begin{array}{l}======== \\
U \\
U \\
U \\
U \\
U \\
U \\
U \\
U \\
B \\
U \\
U \\
U \\
U \\
U \\
U \\
U \\
J B \\
U \\
U \\
U \\
U \\
U\end{array}$ \\
\hline
\end{tabular}

Report 1,4-Dioxane for Low-Medium VOA analysis only 
IB - FORM I VOA-2

VOLATILE ORGANICS ANALYSIS DATA SHEET
EPA SAMPLE NO.

RQCTBW3 0004
Lab Name: TESTAMERICA BURLINGTON

Lab Code: STLV Case No.: MORRILL

Matrix: (SOIL/SED/WATER) water

Sample wt/vol: 25.0

$(\mathrm{g} / \mathrm{mL}) \mathrm{mL}$

Level: (TRACE/LOW/MED) TRACE

\% Moisture: not dec.

GC Column: DB-624

ID $: 0.53$

Soil Extract Volume:

Purge Volume: 25.0
Contract: $8 \mathrm{E}-00302$

Mod. Ref No.:
Lab Sample ID: 825425

Lab File ID: 825425

Date Received: 04/08/2010

Date Analyzed: 04/10/2010

Dilution Factor: 1.0

(uL) Soil Aliquot Volume:

(uL)

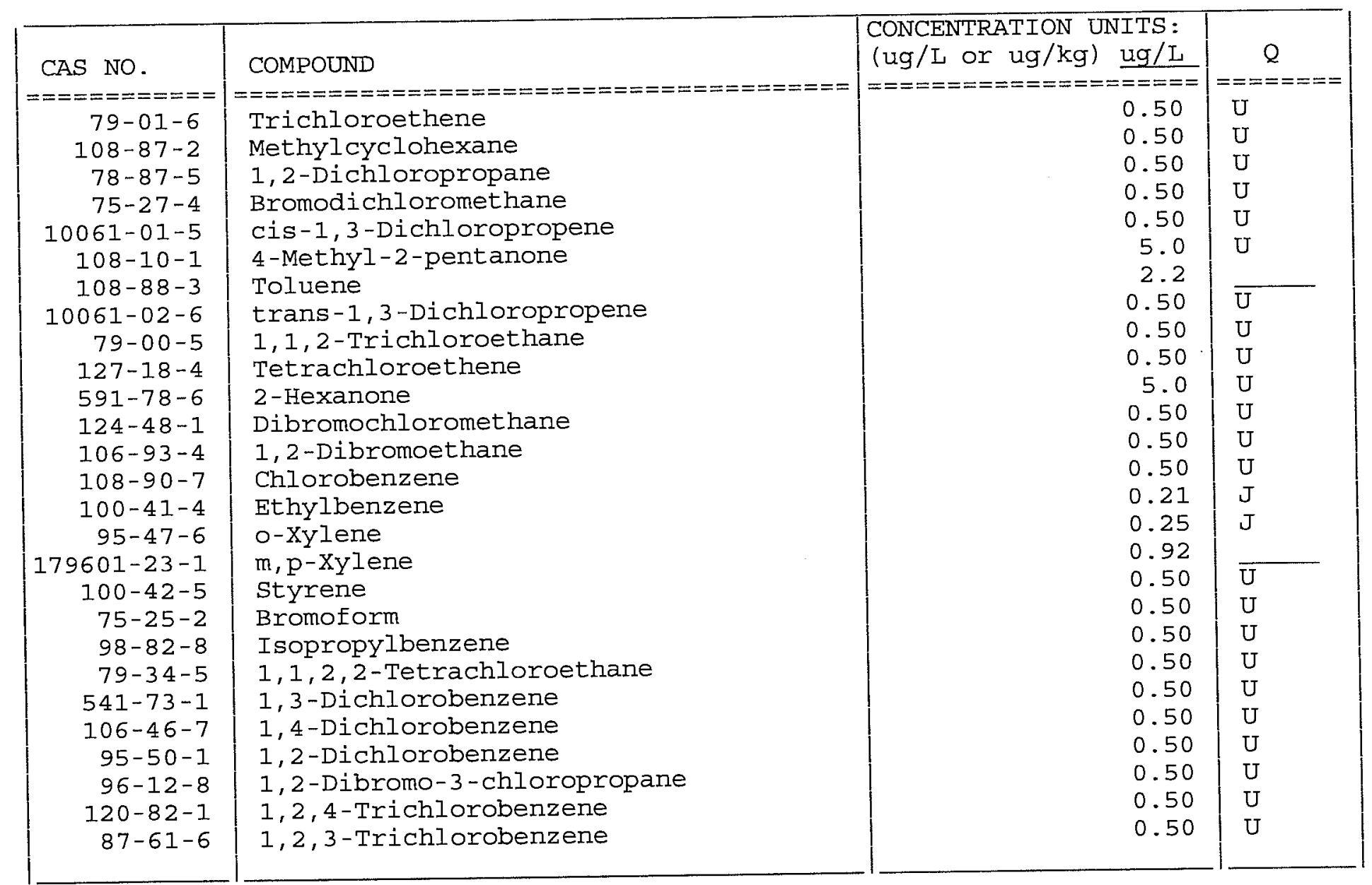


$1 \mathrm{~J}$ - FORM I VOA-TIC

VOL.ATILE ORGANICS ANALYSIS DATA SHEET

TENTATIVELY IDENTIFIED COMPOUNDS
EPA SAMPLE NO. RQCTBW3 0004
Lab Name: TESTAMERICA BURLINGTON

Lab Code: STLV Case No.: MORRILL

Matrix: (SOIL/SED/WATER) water

Sample wt/vol: $25.0(\mathrm{~g} / \mathrm{mL}) \mathrm{mL}$

Level: (TRACE or LOW/MED) TRACE

\% Moisture: not dec.

GC Column: DB-624

$I D: 0.53$

Soil Extract Volume:

CONCENTRATION UNITS: (ug/L or ug/kg) ug/L
Contract: $8 \mathrm{E}-00302$

Mod. Ref No.:

SDG No.: 136730
Lab Sample ID: 825425

Lab File ID: 825425

Date Received: 04/08/2010

Date Analyzed: 04/10/2010

(mm) Dilution Factor: 1.0

(uL) Soil Aliquot Volume:

(uL)

Purge Volume: 25.0

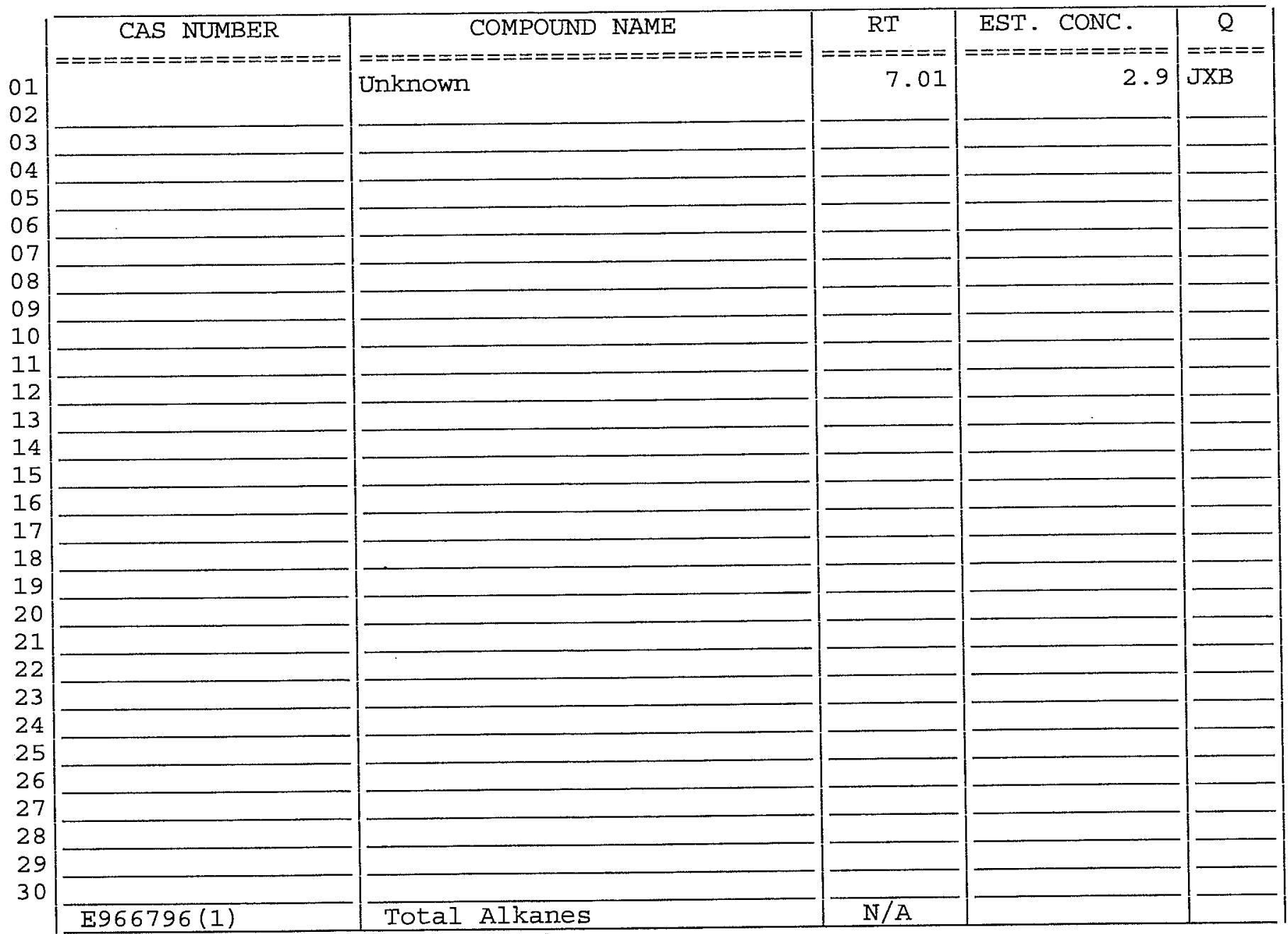

(1) EPA-designated Registry Number. 
$1 A$ - FORM I VOA-1

VOLATILE ORGANICS ANALYSIS DATA SHEET
EPA SAMPLE NO.

VBLKJO
Lab Name: TESTAMERICA BURLINGTON

Lab Code: STLV Case No.: MORRILL

Matrix: (SOIL/SED/WATER) Water

Sample wt/vol: 25.0

$(\mathrm{g} / \mathrm{mL}) \mathrm{mL}$

Level: (TRACE/LOW/MED) TRACE

\% Moisture: not dec.

GC Column: DB-624

ID $: 0.53$

Soil Extract Volume:

Purge Volume: 25.0
Contract : $8 E-00302$

Mod. Ref No.:

SDG No. : 136730

Lab Sample ID: VBLKJO

Lab File ID: JAQB02G

Date Received:

Date Analyzed: 04/10/2010

Dilution Factor: 1.0

(uL) Soil Aliquot Volume:

(uL)

(mL)

\begin{tabular}{|c|c|c|c|}
\hline CAS NO. & COMPOUND & $\begin{array}{l}\text { CONCENTRATION UNITS: } \\
(\mathrm{ug} / \mathrm{L} \text { or } \mathrm{ug} / \mathrm{kg}) \mathrm{ug} / \mathrm{L}\end{array}$ & $\mathrm{Q}$ \\
\hline$===========$ & 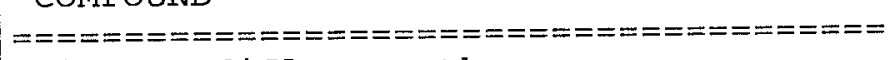 & $========0== \pm========0$ & $=======$ \\
\hline $75-71-8$ & Dichlorodifluoromethane & 0.50 & $U$ \\
\hline $74-87-3$ & Chloromethane & 0.50 & $\mathrm{U}$ \\
\hline $75-01-4$ & Vinyl chloride & 0.50 & $\mathrm{U}$ \\
\hline $74-83-9$ & Bromomethane & 0.50 & $\mathrm{U}$ \\
\hline $75-00-3$ & Chloroethane & 0.50 & $\mathrm{U}$ \\
\hline $75-69-4$ & Trichlorofluoromethane & 0.50 & $\mathrm{U}$ \\
\hline $75-35-4$ & 1,1-Dichloroethene & 0.50 & $\mathrm{U}$ \\
\hline $76-13-1$ & $1,1,2$-Trichloro-1,2,2-trifluoroethane & 0.50 & $\mathrm{U}$ \\
\hline $67-64-1$ & Acetone & 2.6 & $\mathrm{~J}$ \\
\hline $75-15-0$ & Carbon disulfide & 0.50 & $\mathrm{U}$ \\
\hline $79-20-9$ & Methyl acetate & 0.50 & $\mathrm{U}$ \\
\hline $75-09-2$ & Methylene chloride & 0.50 & $\mathrm{U}$ \\
\hline $156-60-5$ & trans-1,2-Dichloroethene & 0.50 & $\mathrm{U}$ \\
\hline $1634-04-4$ & Methyl tert-butyl ether & 0.50 & $\mathrm{U}$ \\
\hline $75-34-3$ & 1,1-Dichloroethane & 0.50 & $\mathrm{U}$ \\
\hline $156-59-2$ & cis-1,2-Dichloroethene & 0.50 & U \\
\hline $78-93-3$ & 2-Butanone & 1.3 & $\mathrm{~J}$ \\
\hline $74-97-5$ & Bromochloromethane & 0.50 & $\mathrm{U}$ \\
\hline $67-66-3$ & Chloroform & 0.50 & $\mathrm{U}$ \\
\hline $71-55-6$ & $1,1,1$-Trichloroethane & 0.50 & $\mathrm{U}$ \\
\hline $110-82-7$ & Cyclohexane & 0.50 & $\mathrm{U}$ \\
\hline $56-23-5$ & Carbon tetrachloride & 0.50 & $\mathrm{U}$ \\
\hline $71-43-2$ & Benzene & 0.50 & U \\
\hline $107-06-2$ & 1,2-Dichloroethane & 0.50 & $\mathrm{U}$ \\
\hline
\end{tabular}

Report 1,4-Dioxane for Low-Medium VOA analysis only 
$1 B$ - FORM I VOA-2

VOLATILE ORGANICS ANALYSIS DATA SHEET
EPA SAMPLE NO.

VBLKJO

Contract: $8 E-00302$

Lab Name: TESTAMERICA BURLINGTON

Lab Code: STLV Case No.: MORRILI Mod. Ref No.:

SDG No.: 136730
Matrix: (SOIL/SED/WATER) water

Sample wt/vol: $25.0 \quad(\mathrm{~g} / \mathrm{mL}) \mathrm{mL}$

Level: (TRACE/LOW/MED) TRACE

\% Moisture: not dec.

GC Column: DB-624

Soil Extract Volume:

ID $: 0.53$

$(\mathrm{mm})$

(uL)

(mL)

Purge Volume: 25.0
Lab Sample ID: VBLKJO

Lab File ID: JAQB02G

Date Received:

Date Analyzed: 04/10/2010

Dilution Factor: 1.0

Soil Aliquot Volume:

(uL)

\section{(mL)}

\begin{tabular}{|c|c|c|c|}
\hline & & CONCENTRATION UNITS : & \\
\hline CAS NO. & COMPOUND & $(u g / L$ or $u g / k g) ~ u g / L$ & Q \\
\hline$====== \pm===$ & 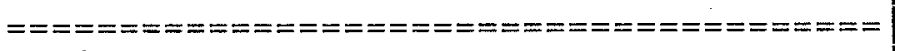 & 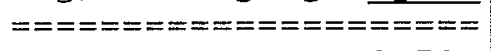 & $=======$ \\
\hline $79-01-6$ & Trichloroethene & 0.50 & $\mathrm{U}$ \\
\hline $108-87-2$ & Methylcyclohexane & 0.50 & $\mathrm{U}$ \\
\hline $78-87-5$ & 1,2 -Dichloropropane & 0.50 & $\mathrm{U}$ \\
\hline $75-27-4$ & Bromodichloromethane & 0.50 & $\mathrm{U}$ \\
\hline $10061-01-5$ & cis-1,3-Dichloropropene & 0.50 & $\mathrm{U}$ \\
\hline $108-10-1$ & 4-Methyl-2-pentanone & 5.0 & $\mathrm{U}$ \\
\hline $108-88-3$ & Toluene & 0.50 & $\mathrm{U}$ \\
\hline $10061-02-6$ & trans-1,3-Dichloropropene & 0.50 & $\mathrm{U}$ \\
\hline $79-00-5$ & $1,1,2-\operatorname{Trichloroethane}$ & 0.50 & $\mathrm{U}$ \\
\hline $127-18-4$ & Tetrachloroethene & 0.50 & $\mathrm{U}$ \\
\hline $591-78-6$ & 2-Hexanone & 5.0 & $\mathrm{U}$ \\
\hline $124-48-1$ & Dibromochloromethane & 0.50 & $\mathrm{U}$ \\
\hline $106-93-4$ & 1,2-Dibromoethane & 0.50 & $\mathrm{U}$ \\
\hline $108-90-7$ & Chlorobenzene & 0.50 & $\mathrm{U}$ \\
\hline $100-41-4$ & Ethylbenzene & 0.50 & $\mathrm{U}$ \\
\hline $95-47-6$ & o-xylene & 0.50 & $\mathrm{U}$ \\
\hline $179601-23-1$ & $\mathrm{~m}, \mathrm{p}$-Xylene & 0.50 & $\mathrm{U}$ \\
\hline $100-42-5$ & styrene & 0.50 & $\mathrm{U}$ \\
\hline $75-25-2$ & Bromoform & 0.50 & $\mathrm{U}$ \\
\hline $98-82-8$ & Isopropylbenzene & 0.50 & $\mathrm{U}$ \\
\hline $79-34-5$ & $1,1,2,2$-Tetrachloroethane & 0.50 & $\mathrm{U}$ \\
\hline $541-73-1$ & 1,3-Dichlorobenzene & 0.50 & $\mathrm{U}$ \\
\hline $106-46-7$ & 1,4-Dichlorobenzene & 0.50 & $\mathrm{U}$ \\
\hline $95-50-1$ & 1,2 -Dichlorobenzene & 0.50 & $\mathrm{U}$ \\
\hline $96-12-8$ & 1,2-Dibromo-3-chloropropane & 0.50 & $\mathrm{U}$ \\
\hline $120-82-1$ & 1,2,4-Trichlorobenzene & 0.50 & $\mathrm{U}$ \\
\hline $87-61-6$ & $1,2,3$-Trichlorobenzene & 0.50 & $\mathrm{U}$ \\
\hline
\end{tabular}


$1 J$ - FORM I VOA-TIC

VOLATILE ORGANICS ANALYSIS DATA SHEET

TENTATIVELY IDENTIFIED COMPOUNDS
EPA SAMPLE NO.

VBLKJO
Lab Name: TESTAMERICA BURIINGTON

Lab Code: STLV Case No.: MORRILL

Matrix: (SOIL/SED/WATER) Water

Sample wt/vol: $25.0(\mathrm{~g} / \mathrm{mL}) \mathrm{mL}$

Level: (TRACE or IOW/MED) TRACE

\% Moisture: not dec.

GC Column: DB-624

ID $: 0.53$

Soil Extract Volume:

CONCENTRATION UNITS: (ug/L or $u g / \mathrm{kg}$ ) ug/L
Contract: $8 \mathrm{E}-00302$

Mod. Ref No.:

SDG No.: 136730
Lab Sample ID: VBLKJO

Lab File ID: JAQBO2G

Date Received:

Date Analyzed: 04/10/2010

(mm) Dilution Factor: 1.0

(uI) Soil Aliquot Volume:

(uL)

Purge Volume: 25.0

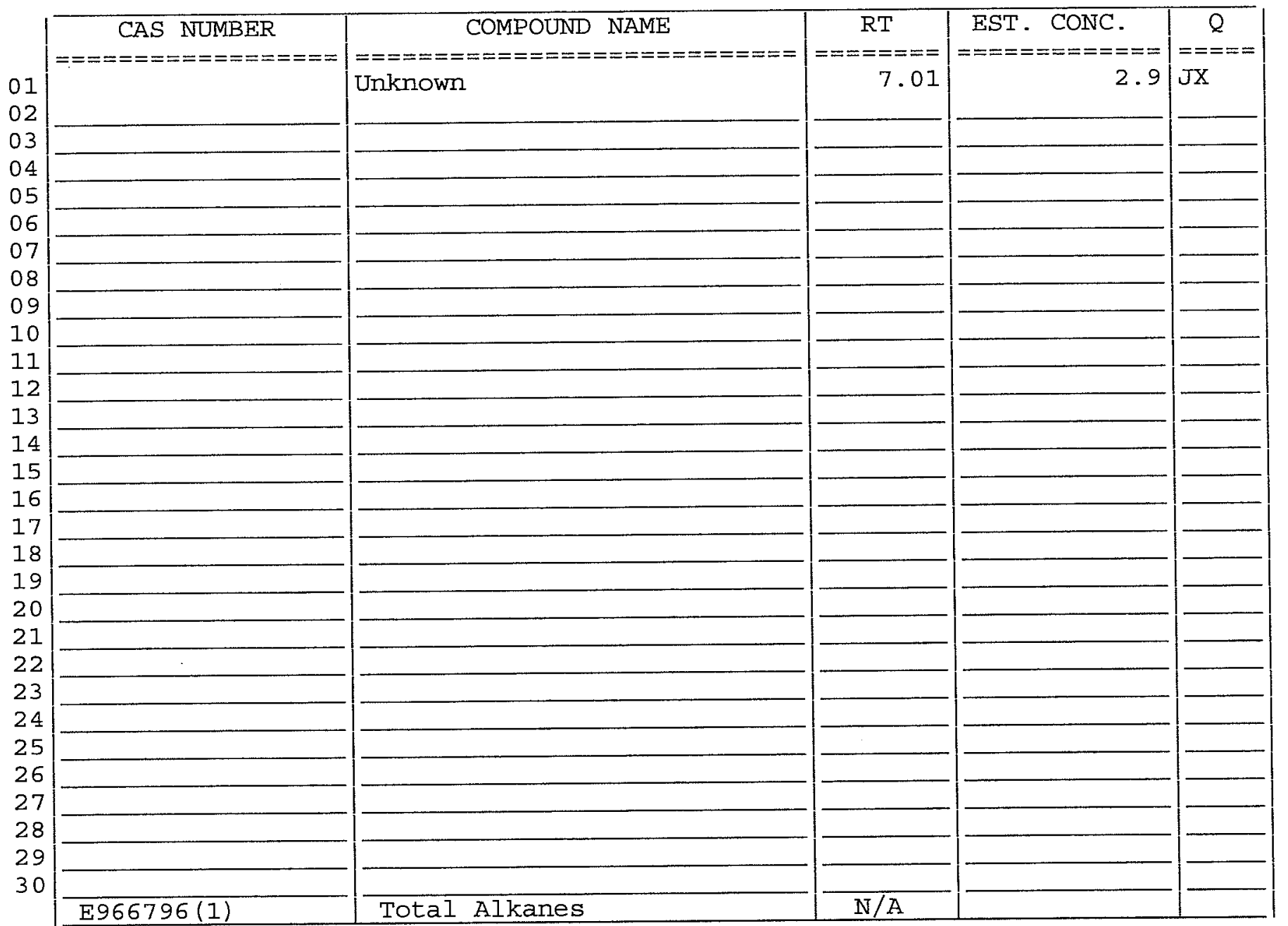

(1) EPA-designated Registry Number. 
$1 A$ - FORM I VOA-1

VOLATILE ORGANECS ANALYSIS DATA SHEET
EPA SAMPIE NO.

VHBLKO1

Contract: $8 E-00302$

Lab Code: STLV Case No.: MORRILI Mod. Ref No.:

SDG NO. : 136730

Matrix: (SOIL/SED/WATER) water

Sample wt/vol: 25.0

$(\mathrm{g} / \mathrm{mL}) \mathrm{mL}$

Level: (TRACE/LOW/MED) TRACE

\% Moisture: not dec.

GC Column: DB-624

ID : 0.53

(mm)

(uL)

Iab Sample ID: 825426

Lab File ID: 825426

Date Received:

Date Analyzed: 04/10/2010

Soil Extract Volume:

Purge Volume: 25.0 (m工)

\begin{tabular}{|c|c|c|c|}
\hline CAS NO. & COMPOUND & $\begin{array}{l}\text { CONCENTRATION UNITS: } \\
(\mathrm{ug} / \mathrm{L} \text { or } \mathrm{ug} / \mathrm{kg}) \mathrm{ug} / \mathrm{L} \\
\end{array}$ & Q \\
\hline$==========$ & 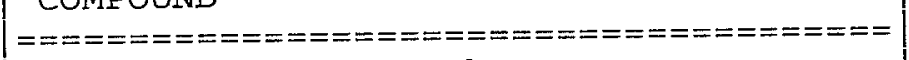 & $===============\equiv====$ & |=====-== \\
\hline $75-71-8$ & Dichlorodifluoromethane & 0.50 & $\mathrm{U}$ \\
\hline $74-87-3$ & Chloromethane & 0.50 & $\mathrm{U}$ \\
\hline $75-01-4$ & Vinyl chloride & 0.50 & $\mathrm{U}$ \\
\hline $74-83-9$ & Bromomethane & 0.50 & $\mathrm{U}$ \\
\hline $75-00-3$ & Chloroethane & 0.50 & $\mathrm{U}$ \\
\hline $75-69-4$ & Trichlorofluoromethane & 0.50 & U \\
\hline $75-35-4$ & 1,1 -Dichloroethene & 0.50 & $\mathrm{U}$ \\
\hline $76-13-1$ & $1,1,2$-Trichloro-1,2,2-trifluoroethane & 0.50 & U \\
\hline $67-64-1$ & Acetone & 1.3 & JB \\
\hline $75-15-0$ & Carbon disulfide & 0.50 & $\mathrm{U}$ \\
\hline $79-20-9$ & Methyl acetate & 0.50 & $\mathrm{U}$ \\
\hline $75-09-2$ & Methylene chloride & 0.50 & $\mathrm{U}$ \\
\hline $156-60-5$ & trans-1,2-Dichloroethene & 0.50 & $\mathrm{U}$ \\
\hline $1634-04-4$ & Methyl tert-butyl ether & 0.50 & $\mathrm{U}$ \\
\hline $75-34-3$ & 1,1-Dichloroethane & 0.50 & $\mathrm{U}$ \\
\hline $156-59-2$ & cis-1,2-Dichloroethene & 0.50 & $\mathrm{U}$ \\
\hline $78-93-3$ & 2 -Butanone & 5.0 & $\mathrm{U}$ \\
\hline $74-97-5$ & Bromochloromethane & 0.50 & $\mathrm{U}$ \\
\hline $67-66-3$ & Chloroform & 0.50 & $\mathrm{U}$ \\
\hline $71-55-6$ & $1,1,1$-Trichloroethane & 0.50 & $\mathrm{U}$ \\
\hline $110-82-7$ & Cyclohexane & 0.50 & $\mathrm{U}$ \\
\hline $56-23-5$ & Carbon tetrachloride & 0.50 & $\mathrm{U}$ \\
\hline $71-43-2$ & Benzene & 0.50 & $\mathrm{U}$ \\
\hline $107-06-2$ & 1,2-Dichloroethane & 0.50 & U \\
\hline
\end{tabular}

Report 1,4-Dioxane for Low-Medium VOA analysis only 
Lab Name: TESTAMERICA BURLINGTON

Lab Code: STLV Case No.: MORRILL

Matrix: (SOIL/SED/WATER) Water

Sample wt/vol: 25.0

$(\mathrm{g} / \mathrm{mL}) \mathrm{mL}$

Level: (TRACE/LOW/MED) TRACE

\% Moisture: not dec.

GC Column: $\mathrm{DB}-624$

ID $: 0.53$

$(\mathrm{mm})$

(uL)

Contract: 8E-00302

Mod. Ref No.:
Soil Extract Volume:

Purge Volume: 25.0
Lab Sample ID: 825426

Lab File ID: 825426

Date Received:

Date Analyzed: 04/10/2010

Dilution Factor: 1.0

Soil Aliquot Volume:

(uL)

(mL)

\begin{tabular}{|c|c|c|c|}
\hline & & CONCENTRATION UNITS: & \\
\hline CAS NO. & COMPOUND & $(u g / L$ or $u g / k g) ~ u g / L$ & Q \\
\hline 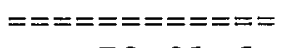 & 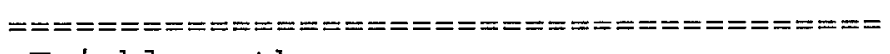 & 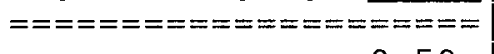 & $=======$ \\
\hline & Trichloroethene & 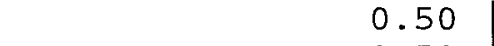 & $\mathrm{U}$ \\
\hline $108-87-2$ & Methylcyclohexane & 0.50 & $\mathrm{U}$ \\
\hline $78-87-5$ & 1,2-Dichloropropane & 0.50 & $\mathrm{U}$ \\
\hline $75-27-4$ & Bromodichloromethane & 0.50 & $\mathrm{U}$ \\
\hline $10061-01-5$ & cis-1,3-Dichloropropene & 0.50 & $\mathrm{U}$ \\
\hline $108-10-1$ & 4-Methyl-2-pentanone & 5.0 & $\mathrm{U}$ \\
\hline $108-88-3$ & Toluene & 0.50 & $\mathrm{U}$ \\
\hline $10061-02-6$ & trans-1,3-Dichloropropene & 0.50 & $\mathrm{U}$ \\
\hline $79-00-5$ & 1,1,2-Trichloroethane & 0.50 & $\mathrm{U}$ \\
\hline $127-18-4$ & Tetrachloroethene & 0.50 & $\mathrm{U}$ \\
\hline $591-78-6$ & 2-Hexanone & 5.0 & $\mathrm{U}$ \\
\hline $124-48-1$ & Dibromochloromethane & 0.50 & $\mathrm{U}$ \\
\hline $106-93-4$ & 1,2-Dibromoethane & 0.50 & $\mathrm{U}$ \\
\hline $108-90-7$ & Chlorobenzene & 0.50 & $\mathrm{U}$ \\
\hline $100-41-4$ & Ethylbenzene & 0.50 & $\mathrm{U}$ \\
\hline $95-47-6$ & o-xylene & 0.50 & $\mathrm{U}$ \\
\hline $179601-23-1$ & $\mathrm{~m}, \mathrm{p}$-xylene & 0.50 & $\mathrm{U}$ \\
\hline $100-42-5$ & Styrene & 0.50 & $\mathrm{U}$ \\
\hline $75-25-2$ & Bromoform & 0.50 & $\mathrm{U}$ \\
\hline $98-82-8$ & Isopropylbenzene & 0.50 & $\mathrm{U}$ \\
\hline $79-34-5$ & $1,1,2,2$-Tetrachloroethane & 0.50 & $\mathrm{U}$ \\
\hline $541-73-1$ & 1,3-Dichlorobenzene & 0.50 & $\mathrm{U}$ \\
\hline $106-46-7$ & I,4-Dichlorobenzene & 0.50 & $\mathrm{U}$ \\
\hline $95-50-1$ & 1,2-Dichlorobenzene & 0.50 & $\mathrm{U}$ \\
\hline $96-12-8$ & 1,2-Dibromo-3-chloropropane & 0.50 & $\mathrm{U}$ \\
\hline $120-82-1$ & $1,2,4$-Trichlorobenzene & 0.50 & $\mathrm{U}$ \\
\hline $87-61-6$ & 1,2,3-Trichlorobenzene & 0.50 & $\mathrm{U}$ \\
\hline
\end{tabular}


IJ - FORM I VOA-TIC

VOLATILE ORGANICS ANALYSIS DATA SHEET TENTATIVELY IDENTIFIED COMPOUNDS
EPA SAMPLE NO.

VHBLKO1
Lab Name: TESTAMERICA BURLINGTON

Lab Code: STLV Case No.: MORRILL

Matrix: (SOIL/SED/WATER) Water

Sample wt/vol: $25.0 \quad(\mathrm{~g} / \mathrm{mL}) \mathrm{mL}$

Level: (TRACE or LOW/MED) TRACE

\% Moisture: not dec.

GC Column: DB-624

ID $: 0.53$

Soil Extract Volume:

CONCENTRATION UNITS: (ug/L or ug/kg) ug/L
Contract: 8E-00302

Mod. Ref No.:

SDG No.: 136730

Lab Sample ID: 825426

Lab File ID: 825426

Date Received:

Date Analyzed: 04/10/2010

(mm) Dilution Factor: 1.0

(uL) Soil Aliquot Volume:

Purge Volume: 25.0

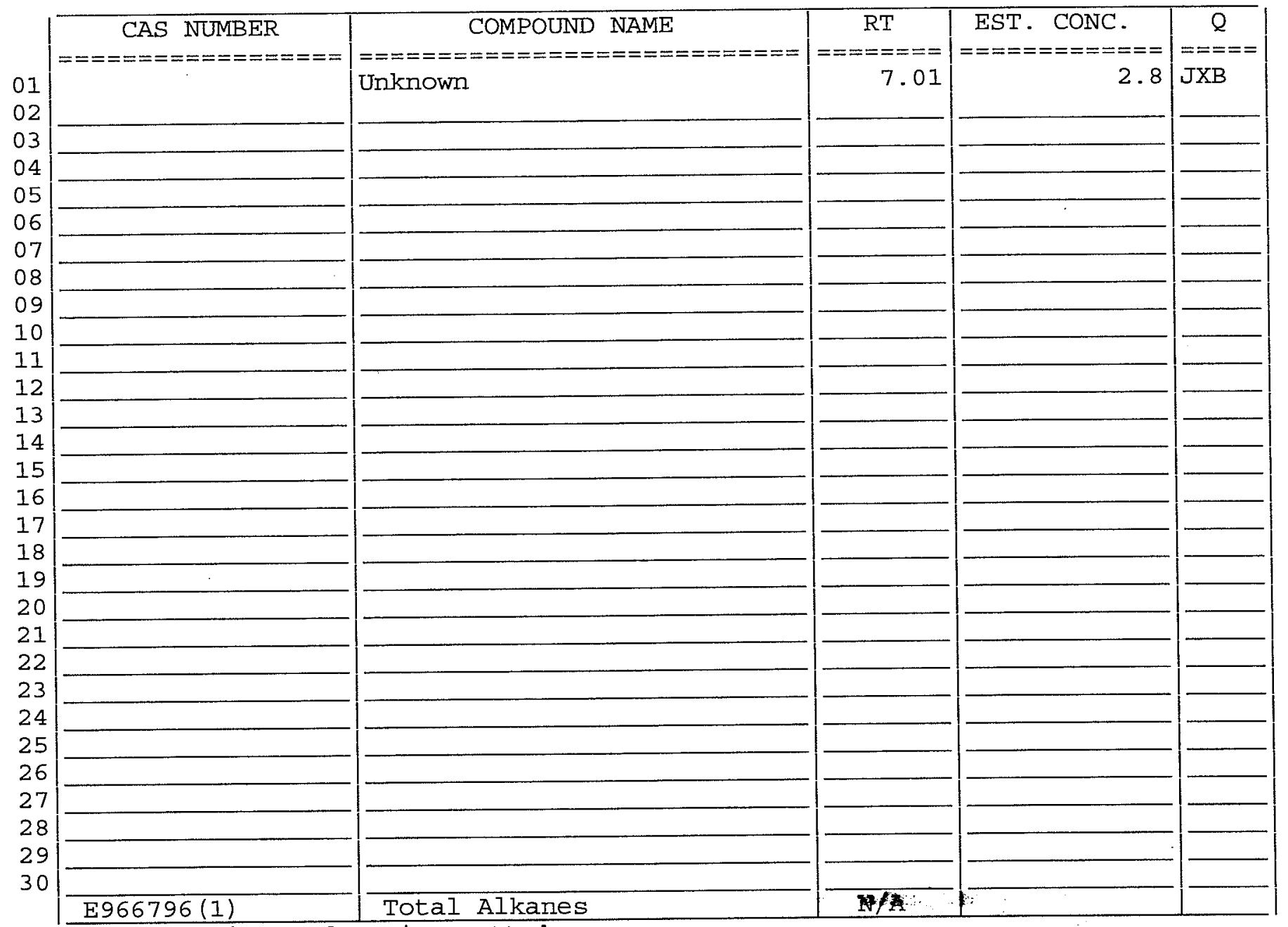

(1) EPA-designated Registry Number. 
$2 A$ - FORM II VOA-1

WATER VOLATILE DEUTERATED MONITORING COMPOUND RECOVERY

Lab Name: TESTAMERICA BURLINGTON

Contract: $8 \mathrm{E}-00302$

Lab Code: STLV Case No.: MORRILL Mod. Ref No.:

SDG No.: 136730

Level: (TRACE or LOW) TRACE

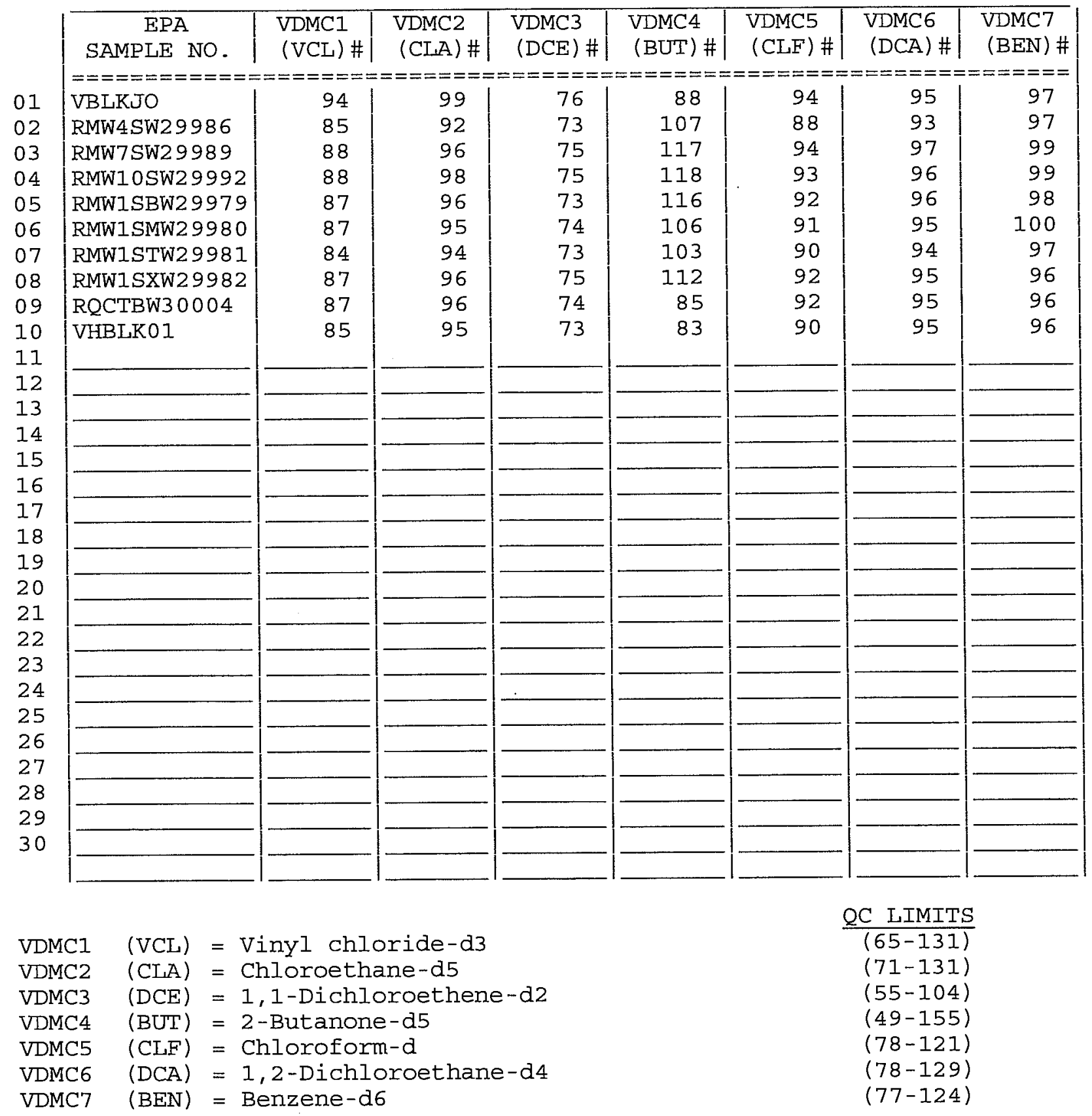

\# Column to be used to flag recovery values

* Values outside of contract required QC limits Page 1 of 2

SOMO1. 2 
$2 B$ - FORM II VOA-2

WATER VOLATILE DEUTERATED MONITORING COMPOUND RECOVERY

Lab Name: TESTAMERICA BURLINGTON

Contract: $8 \mathrm{E}-00302$

Lab Code: STLV Case No.: MORRILL Mod. Ref No.:

SDG No.: 136730

Level: (TRACE or LOW) TRACE

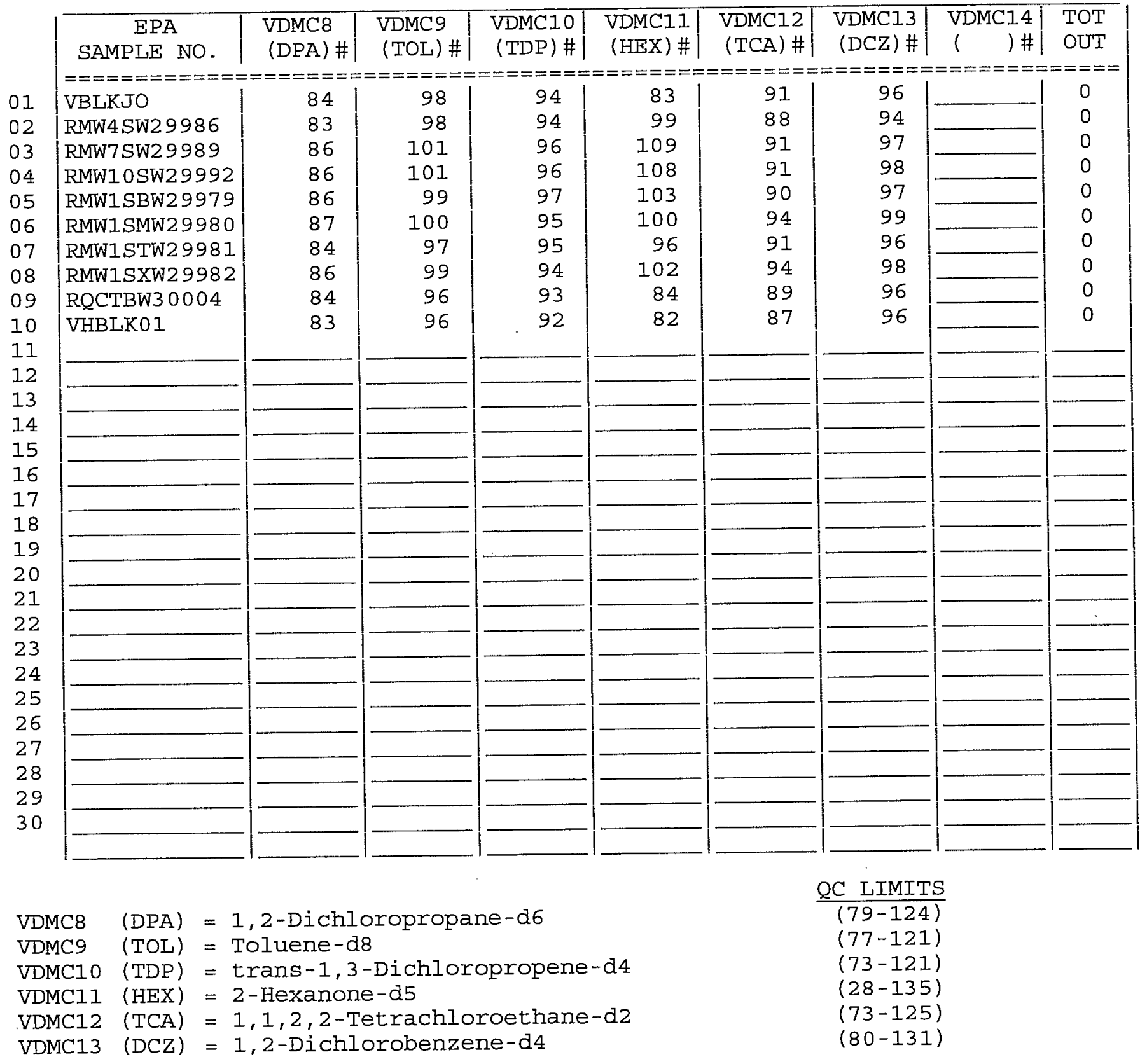

\# Column to be used to flag recovery values

* Values outside of contract required QC limits

Report 1,4-Dioxane-d8 for Low-Medium VOA analysis only Page 2 of 2

SOMO1.2 
$4 A$ - FORM IV VOA

VOLATILE METHOD BL_ANK SUMMARY
EPA SAMPLE NO.

VBLKJO
Lab Name: TESTAMERICA BURLINGTON

Lab Code: STLV Case No.: MORRILL Mod. Ref No.:
Contract : $8 \mathrm{E}-00302$

Lab Sample ID: VBLKJO

SDG NO.: 136730
Lab File ID: JAQBO2G

Instrument ID: J.i

Matrix: (SOIL/SED/WATER) Water

Level: ' (TRACE or LOW/MED) TRACE

GC Column: DB-624
ID $: 0.53$
Date Analyzed: 04/10/2010

Time Analyzed: 1042

(mm) Heated Purge: (Y/N) N

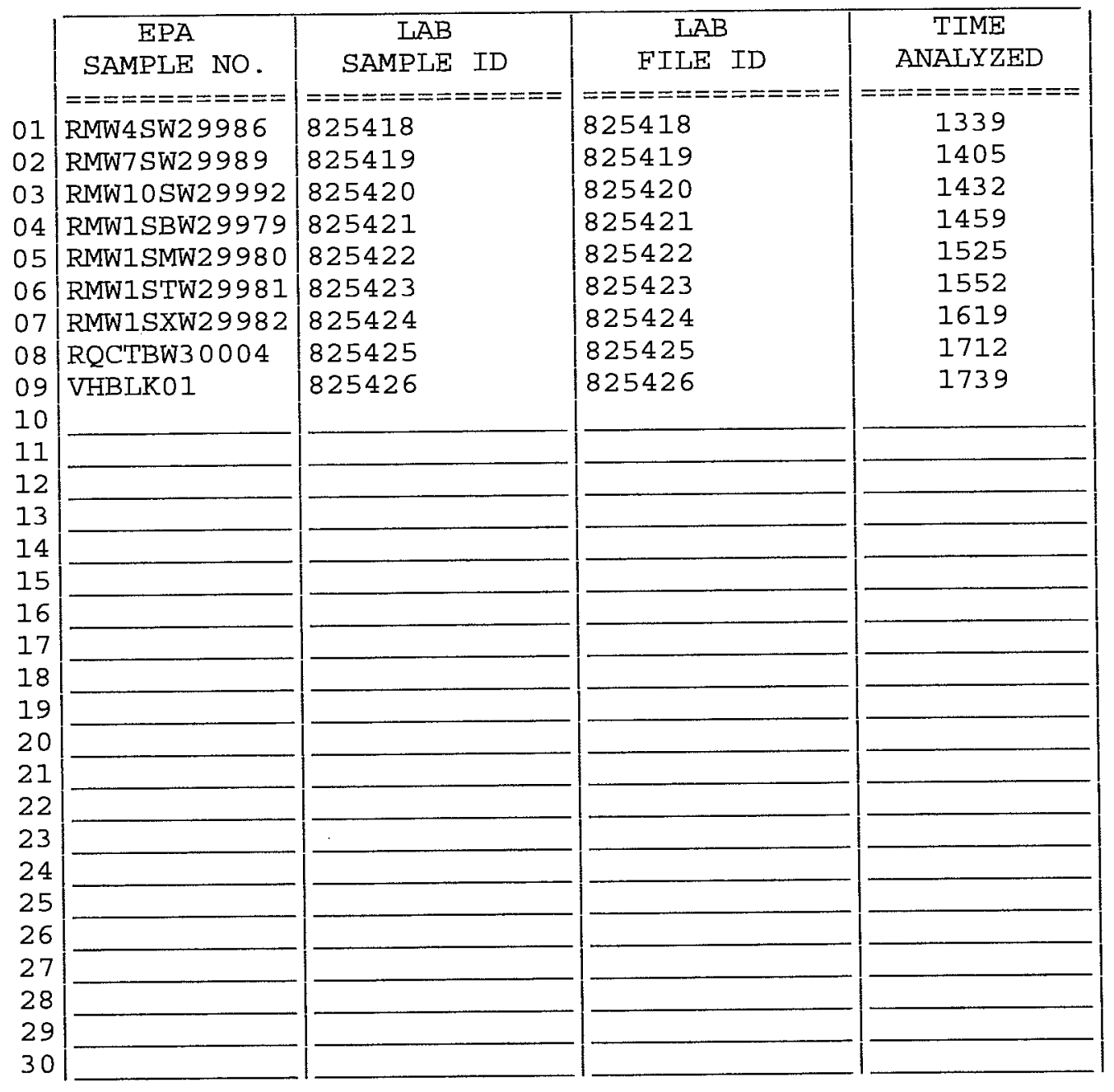

COMMENTS : 
$5 A$ - FORM V VOA

VOLATILE ORGANIC INSTRUMENT

PERFORMANCE CHECK

BROMOFLUOROBENZENE (BFB)
EPA SAMPLE NO.

BFBJG

Contract : $8 \mathrm{E}-00302$

Lab Name: TESTAMERICA BURLINGTON

SDG No.: 136730

Case NO.: MORRILI
Lab File ID: JAQ01PV

Instrument ID: J.i

GC Column: DB-624
Mod. Ref No.:

BFB Injection Date: 04/05/2010

BFB Injection Time: 1223

\begin{tabular}{|c|c|c|}
\hline$==\frac{\mathrm{m} / \mathrm{e}}{50}==$ & 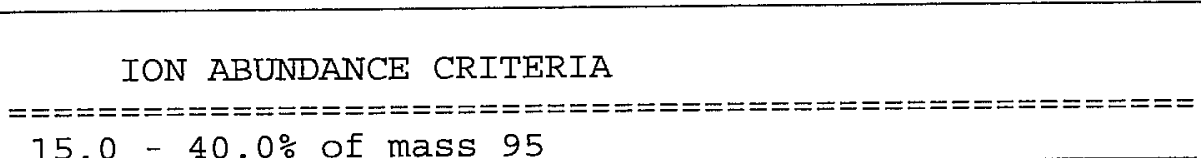 & $\begin{aligned} & \% \text { REIAATIVE } \\
& \text { ABUNDANCE } \\
&============ \\
& 18.0\end{aligned}$ \\
\hline 75 & $30.0-80.0 \%$ of mass 95 & 48.7 \\
\hline 95 & Base Peak, $100 \%$ relative abundance & 100.0 \\
\hline 96 & $5.0-9.0 \%$ of mass 95 & 7.0 \\
\hline 173 & Less than $2.0 \%$ of mass 174 & $(0.0) 1$ \\
\hline 174 & $50.0-120.0 \%$ of mass 95 & 66.9 \\
\hline 175 & $5.0-9.0 \%$ of mass 174 & $6.0(8.9) 1$ \\
\hline 176 & $95.0-101.0 \%$ of mass $\overline{174}$ & $(99.2) 1$ \\
\hline 177 & $5.0-9.0 \%$ of & $4.5(6.8) 2$ \\
\hline
\end{tabular}

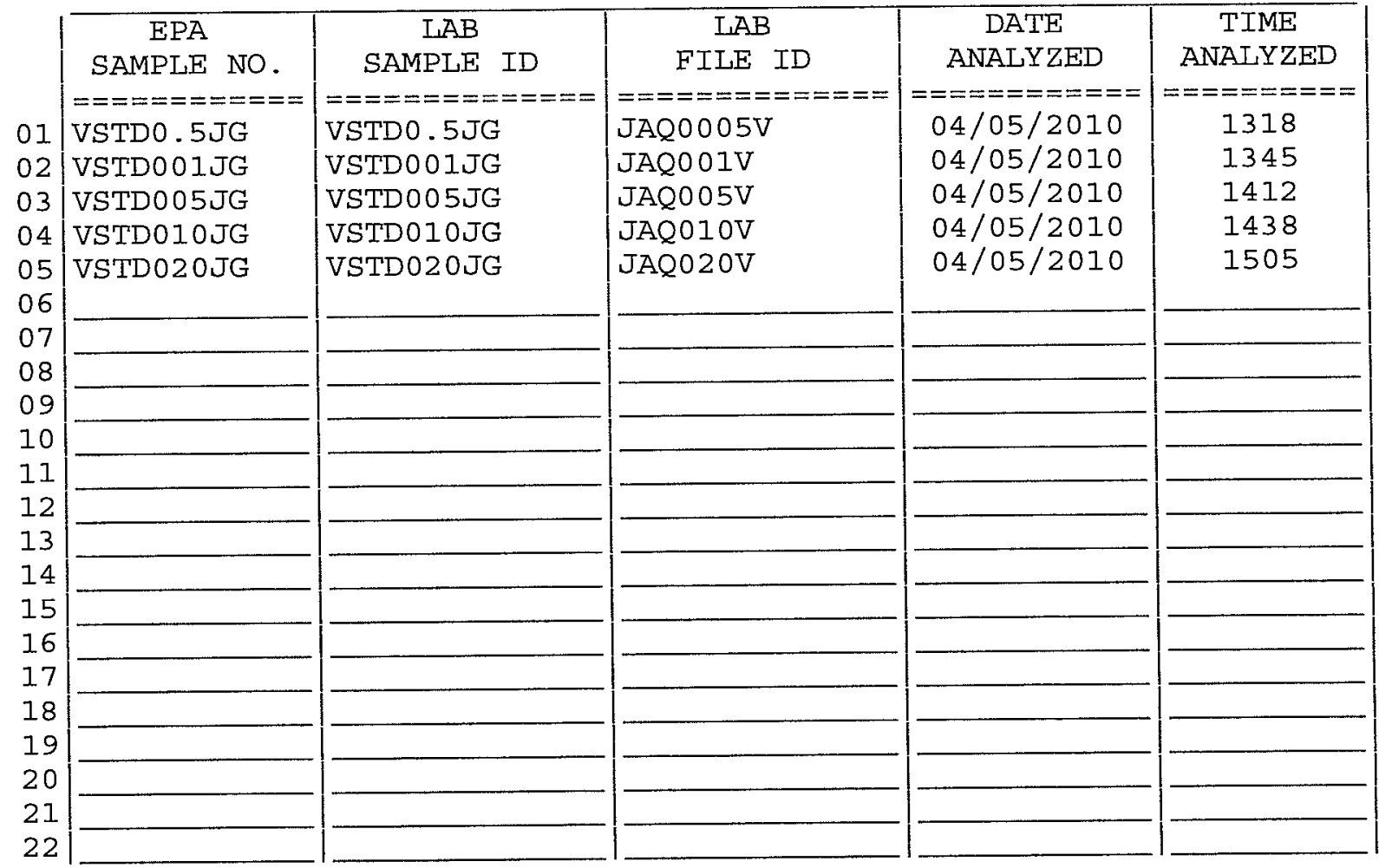


5A - FORM V VOA

VOLATILE ORGANIC INSTRUMENT

PERFORMANCE CHECK

BROMOFLUOROBENZENE (BFB)
EPA SAMPLE NO

BFBJO

Contract: 8E-00302

Lab Name: TESTAMERICA BURLINGTON

SDG NO.: 136730

Case No.: MORRIII
Lab File ID: JAQ16PV

Instrument ID: J.i

GC Column: DB-624
Mod. Ref No.:

BFB Injection Date: 04/10/2010

BFB Injection Time: 0920

\begin{tabular}{|c|c|c|}
\hline$==\begin{array}{c}\mathrm{m} / \mathrm{e} \\
50\end{array}$ & $\begin{array}{l}\text { ION ABUNDANCE CRITERIA } \\
=============================================== \\
15.0-40.0 \% \text { of mass } 95\end{array}$ & $\begin{aligned} & \circ \text { RELATIVE } \\
& \text { ABUNDANCE } \\
&============ \\
& 18.1\end{aligned}$ \\
\hline 75 & $30.0-80.0 \%$ of mass 95 & 48.8 \\
\hline 95 & Base Peak, $100 \%$ relative abundance & 100.0 \\
\hline 96 & $5.0-9.0 \%$ of mass 95 & 6.6 \\
\hline 173 & Less than $2.0 \%$ of mass 174 & $(0.2) 1$ \\
\hline 174 & $50.0-120.0 \%$ of mass 95 & 65.8 \\
\hline 175 & $5.0-9.0 \%$ of mass 174 & $(8.9) 1$ \\
\hline 176 & $95.0-101.0 \%$ of mass $\overline{174}$ & $(97.4) 1$ \\
\hline 177 & $5.0-9.0 \%$ of mass 176 & $(7.7) 2$ \\
\hline
\end{tabular}

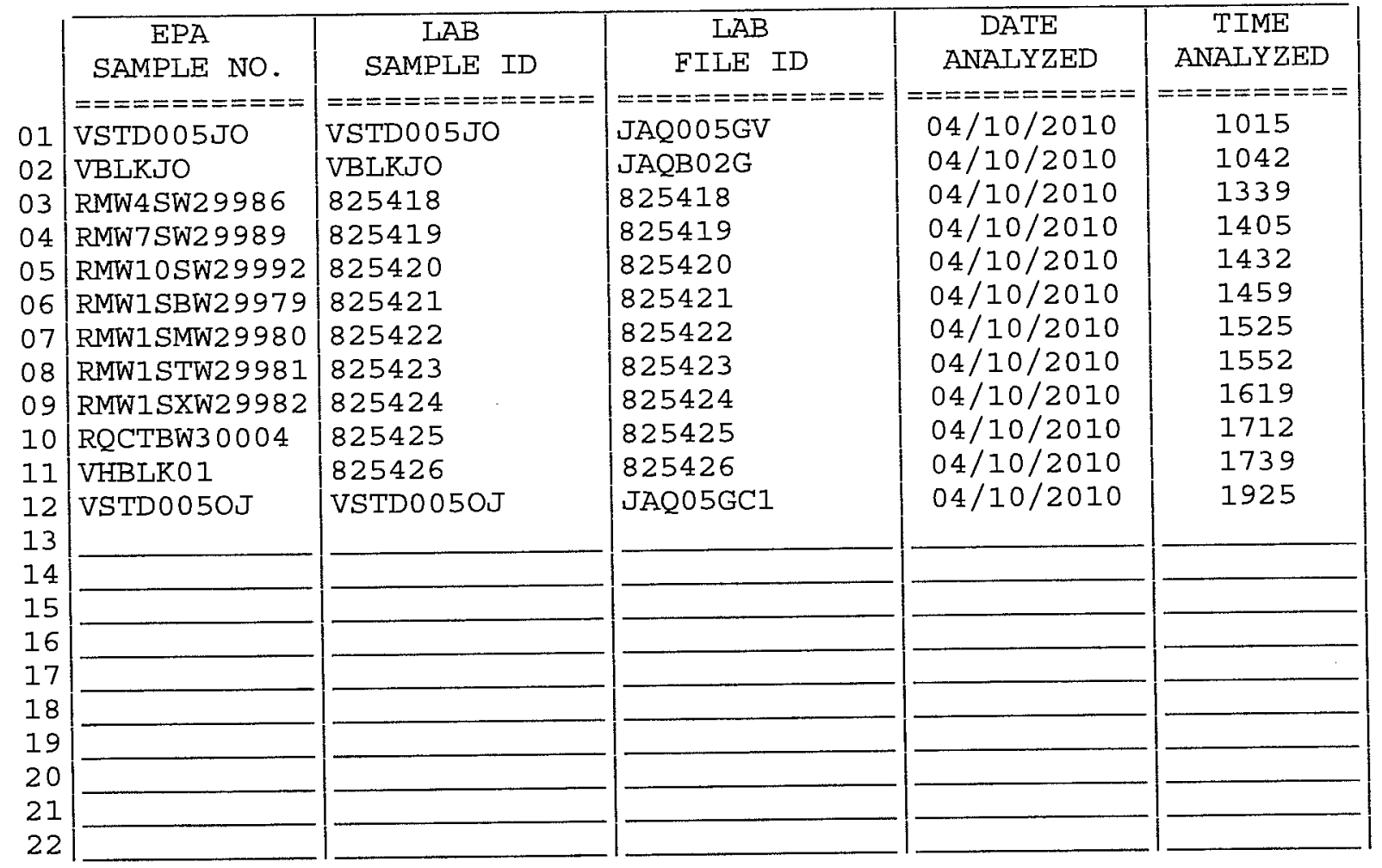


$6 A$ - FORM VI VOA-1

VOLATILE ORGANICS INITIAL CALIBRATION DATA

Lab Name: TESTAMERICA BURLINGTON

Lab Code: STLV Case No.: MORRIIL

Instrument ID: J.i

Heated Purge: $(Y / N) N$

Purge Volume: 25.0

GC Column: $\mathrm{DB}-624$
Contract: $8 \mathrm{E}-00302$

Mod. Ref No.:

SDG No.: 136730

Calibration Date (s): 04/05/2010 04/05/2010

Calibration Time(s): $1318 \quad 1505$

(mI)

ID $: 0.53$ (mm) Length: 75 (m)

\begin{tabular}{|c|c|c|c|c|c|c|c|}
\hline $\begin{array}{l}\text { LAB FILE ID: } \\
\text { RRF5.0 = JAQ005V }\end{array}$ & $\begin{array}{l}5.5=J A S \\
10=J A Q\end{array}$ & $\begin{array}{l}20005 \mathrm{~V} \\
310 \mathrm{~V}\end{array}$ & & $\begin{array}{l}2 F 1.0=J \\
2 F 20=J A\end{array}$ & $\begin{array}{l}\text { JAQ001V } \\
\text { AQ020V }\end{array}$ & & \\
\hline COMPOUND & RRF0. 5 & RRF1 . 0 & RRF5. 0 & RRF10 & RRF20 & $\overline{\mathrm{RRF}}$ & $\because \mathrm{RSD}$ \\
\hline$==2$ & $=======$ & $=======$ & $=======$ & $=======$ & $=======3$ & $=====3$ & $====$ \\
\hline Dichlorodifluoromethane & 0.457 & 0.437 & 0.408 & 0.407 & 0.415 & 0.425 & 5.1 \\
\hline Chloromethane & 0.462 & 0.439 & 0.449 & 0.448 & 0.455 & 0.451 & 1.9 \\
\hline Vinyl chloride & 0.488 & 0.458 & 0.442 & 0.443 & 0.442 & 0.454 & 4.4 \\
\hline Bromomethane & 0.187 & 0.173 & 0.161 & 0.165 & 0.170 & 0.171 & 5.8 \\
\hline Chloroethane & 0.273 & 0.279 & 0.254 & 0.254 & 0.254 & 0.263 & 4.6 \\
\hline Trichlorofluoromethane & 0.524 & 0.522 & 0.507 & 0.501 & 0.502 & 0.511 & 2.2 \\
\hline 1,1-Dichloroethene & 0.320 & 0.313 & 0.300 & 0.296 & 0.301 & 0.306 & 3.3 \\
\hline 1,1,2-Trichloro- & & & & & & & \\
\hline $1,2,2$-trifluoroethane & 0.348 & 0.330 & 0.315 & 0.310 & 0.314 & 0.323 & 4.8 \\
\hline Acetone & 0.016 & 0.015 & 0.013 & 0.013 & 0.013 & 14 & 10.1 \\
\hline Carbon disulfide & 1.128 & 0.982 & 0.959 & 0.931 & 0.933 & .986 & 8.3 \\
\hline Methyl acetate & 0.067 & 0.054 & 0.048 & 0.047 & 0.047 & 0.053 & 16.1 \\
\hline Methylene chloride & 0.262 & 0.252 & 0.250 & 0.242 & 0.246 & 0.250 & 3.0 \\
\hline trans-1,2-Dich & 0.336 & 0.344 & 0.321 & 0.313 & 0.314 & .326 & 4.3 \\
\hline Methyl tert-but & 0.403 & 0.409 & 0.394 & 0.383 & 0.395 & .397 & 2.5 \\
\hline 1,1-Dichloroethane & 0.606 & 0.610 & 0.595 & 0.588 & 0.584 & .597 & 1.9 \\
\hline cis-1,2-Dichloroethene & 0.328 & 0.321 & .316 & 0.305 & 0.308 & 0.316 & 3.0 \\
\hline 2-Butanone & 0.029 & .028 & .027 & 0.027 & 0.028 & 0.028 & 3.2 \\
\hline Bromochloromethane & 0.093 & 0.092 & 0.091 & 0.086 & 0.087 & 0.090 & 3.5 \\
\hline Chloroform & 0.531 & 0.499 & 0.491 & 0.488 & 0.493 & 0.500 & 3.5 \\
\hline $1,1,1$-Trichloroethane & 0.663 & 0.647 & 0.610 & 0.604 & 0.599 & 0.625 & 4.6 \\
\hline Cyclohexane & 0.995 & 0.891 & 0.871 & 0.854 & 0.838 & 0.890 & 7.0 \\
\hline Carbon tetrachloride & 0.554 & 0.529 & 0.509 & 0.510 & 0.507 & 0.522 & 3.8 \\
\hline Benzene & 1.911 & 1.904 & 1.793 & 1.765 & 1.725 & 1.820 & 4.6 \\
\hline 1,2-Dichloroethane & 0.215 & 0.208 & 0.206 & 0.200 & 0.199 & 0.206 & 3.1 \\
\hline Trichloroethene & 0.440 & 0.432 & 0.423 & 0.420 & 0.419 & 0.427 & 2.1 \\
\hline Methylcyclohexane & 0.708 & 0.715 & 0.665 & 0.662 & 0.646 & 0.679 & 4.5 \\
\hline
\end{tabular}

Report 1,4-Dioxane for Low-Medium VOA analysis only 
$6 B$ - FORM VI VOA-2

VOLATILE ORGANICS INITIAL CALIBRATION DATA

Lab Name: TESTAMERICA BURLINGTON

Lab Code: STLV Case No.: MORRILL

Instrument ID: J.i

Heated Purge: $(\mathrm{Y} / \mathrm{N}) \mathrm{N}$

Purge Volume: 25.0

GC Column: DB-624
Contract: 8E-00302

Mod. Ref No.:

SDG NO.: 136730

Calibration Date (s): 04/05/2010 04/05/2010

Calibration Time (s) : $1318 \quad 1505$

(mL)

ID: $0.53(\mathrm{~mm})$ Length: $75 \quad$ (m)

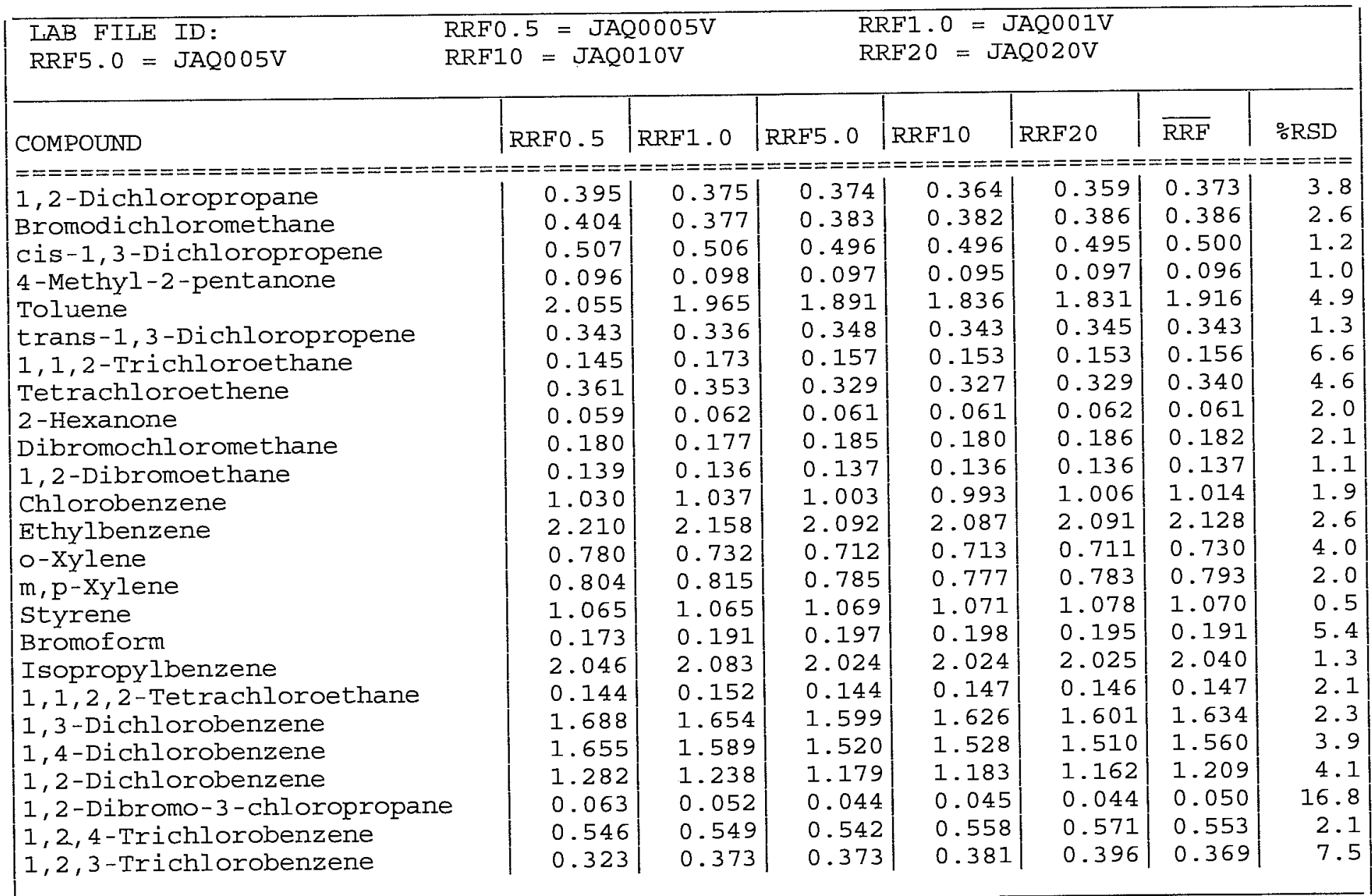


$6 \mathrm{C}-$ FORM VI VOA-3

VOIATILE ORGANICS INITIAL CALIBRATION DATA

Lab Name: TESTAMERICA BURLINGTON

Lab Code: STLV Case No.: MORRILL

Instrument ID: J.i

Heated Purge: $(Y / N) N$

Purge Volume: 25.0

GC Column: DB-624
ID : $0.53 \quad(\mathrm{~mm})$
Contract: $8 \mathrm{E}-00302$

Mod. Ref No.:

SDG No.: 136730

Calibration Date(s): 04/05/2010 04/05/2010

Calibration Time (s): $1318 \quad 1505$

(mL)

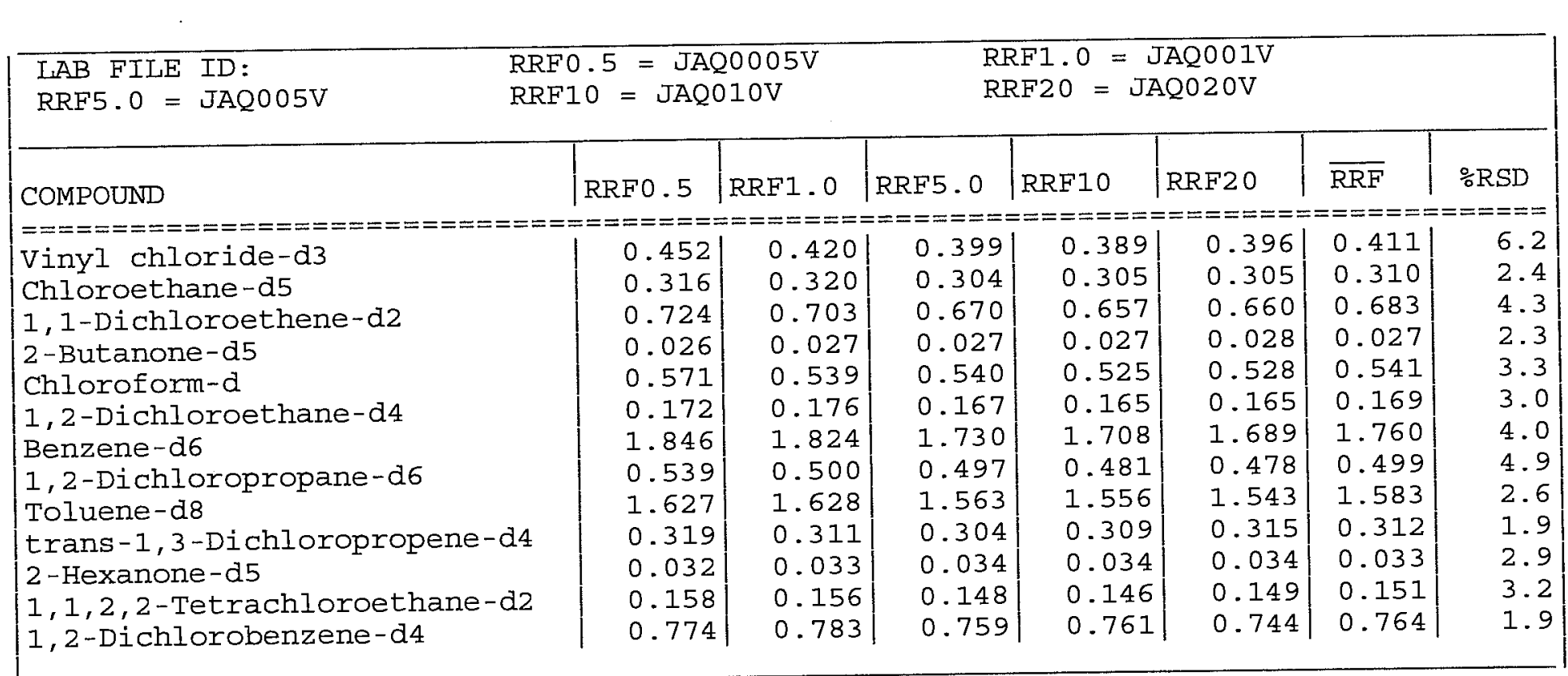

Report 1,4-Dioxane-d8 for Low-Medium VOA analysis only 
$7 A$ - FORM VII VOA-1

VOLATILE CONTINUING CALIBRATION DATA

Lab Name: TESTAMERICA BURLINGTON

Contract : $8 \mathrm{E}-00302$

Lab Code: STLV Case No.: MORRILI Mod. Ref No.:

SDG No. : 136730

Instrument ID: J.i

Calibration Date: 04/10/2010 Time: 1015

Lab File ID: JAQ005GV

Init. Calib. Date (s) : 04/05/2010 04/05/2010

EPA Sample No. (VSTD\#\#\#\#\#) : VSTD005Jo Init. Calib. Time(s): 131811505

Heated Purge: ( $Y / N) N$ GC Column: DB-624 ID: 0.53 (mm) Length: 75 (m)

Purge Volume: 25.0

$(\mathrm{mL})$

\begin{tabular}{|c|c|c|c|c|c|}
\hline COMPOUND & $\overline{\mathrm{RRF}}$ & RRF5. 0 & $\begin{array}{l}\text { MIN } \\
\text { RRF }\end{array}$ & $\div \mathrm{D}$ & $\operatorname{MAX} \div \mathrm{D}$ \\
\hline \multirow{2}{*}{\multicolumn{6}{|c|}{ 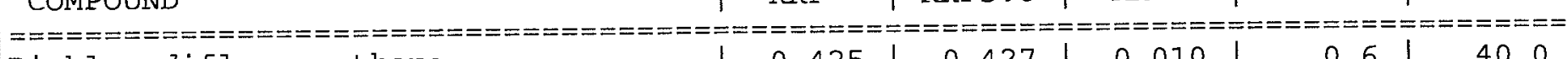 }} \\
\hline Dichlorodifluoromethane & 0.425 & 0.427 & 0.010 & 0.6 & \\
\hline Chloromethane & 0.451 & 0.479 & 0.010 & 6.4 & 40.0 \\
\hline Vinyl chloride & 0.454 & 0.477 & 0.100 & 4.9 & 30.0 \\
\hline Bromomethane & 0.171 & 0.185 & 0.100 & 7.8 & 30.0 \\
\hline Chloroethane & 0.263 & 0.268 & 0.010 & 2.0 & 40.0 \\
\hline Trichlorofluoromethane & 0.511 & 0.523 & 0.010 & 2.2 & 40.0 \\
\hline 1,1-Dichloroethene & 0.306 & 0.305 & 0.100 & -0.2 & 30.0 \\
\hline $1,1,2$-Trichloro-1,2,2-trifluoroethane & 0.323 & 0.333 & 0.010 & . 2.9 & 40.0 \\
\hline Acetone & 0.014 & 0.012 & 0.010 & -15.6 & 40.0 \\
\hline Carbon disulfide & 0.986 & 0.977 & 0.010 & -1.0 & 40.0 \\
\hline Methyl acetate & 0.053 & 0.046 & 0.010 & -12.3 & 40.0 \\
\hline Methylene chloride & 0.250 & 0.240 & 0.010 & -4.2 & 40.0 \\
\hline trans-1,2-Dichloroethene & 0.326 & 0.333 & 0.010 & 2.4 & 40.0 \\
\hline Methyl tert-butyl ether & 0.397 & 0.356 & 0.010 & -10.2 & 40.0 \\
\hline 1,1-Dichloroethane & 0.597 & 0.611 & 0.200 & 2.4 & 30.0 \\
\hline cis-1,2-Dichloroethene & 0.316 & 0.313 & 0.010 & -0.8 & 40.0 \\
\hline 2-Butanone & 0.028 & 0.024 & 0.010 & -13.6 & 40.0 \\
\hline Bromochloromethane & 0.090 & 0.087 & 0.050 & -2.4 & 30.0 \\
\hline Chloroform & 0.500 & 0.509 & 0.200 & 1.7 & 30.0 \\
\hline $1,1,1$-Trichloroethane & 0.625 & 0.644 & 0.100 & 3.1 & 30.0 \\
\hline Cyclohexane & 0.890 & 0.933 & 0.010 & 4.8 & 40.0 \\
\hline Carbon tetrachloride & 0.522 & 0.543 & 0.100 & 4.1 & 30.0 \\
\hline Benzene & 1.820 & 1.838 & 0.400 & 1.0 & 30.0 \\
\hline 1,2-Dichloroethane & 0.206 & 0.203 & 0.100 & -1.3 & 30.0 \\
\hline Trichloroethene & 0.427 & 0.442 & 0.300 & 3.6 & 30.0 \\
\hline Methylcyclohexane & 0.679 & 0.716 & 0.010 & 5.4 & 40.0 \\
\hline
\end{tabular}

Report 1,4-Dioxane for Low-Medium VOA analysis only 
$7 B$ - FORM VII VOA-2

VOLATILE CONTINUING CALIBRATION DATA

Lab Name: TESTAMERICA BURLINGTON

Contract: $8 E-00302$

Lab Code: STLV Case No.: MORRIIL Mod. Ref No.:

SDG No.: 136730

Instrument ID: J.i

Calibration Date: 04/10/2010 Time: 1015

Lab File ID: JAQ005GV

Init. Calib. Date (s): 04/05/2010 04/05/2010

EPA Sample No. (VSTD\#\#\#\#) : VSTD005JO Init. Calib. Time (s): 13181505

Heated Purge: (Y/N)N GC Column: DB-624 ID: 0.53 (mm) Length: 75 (m)

Purge Volume: 25.0 (mL)

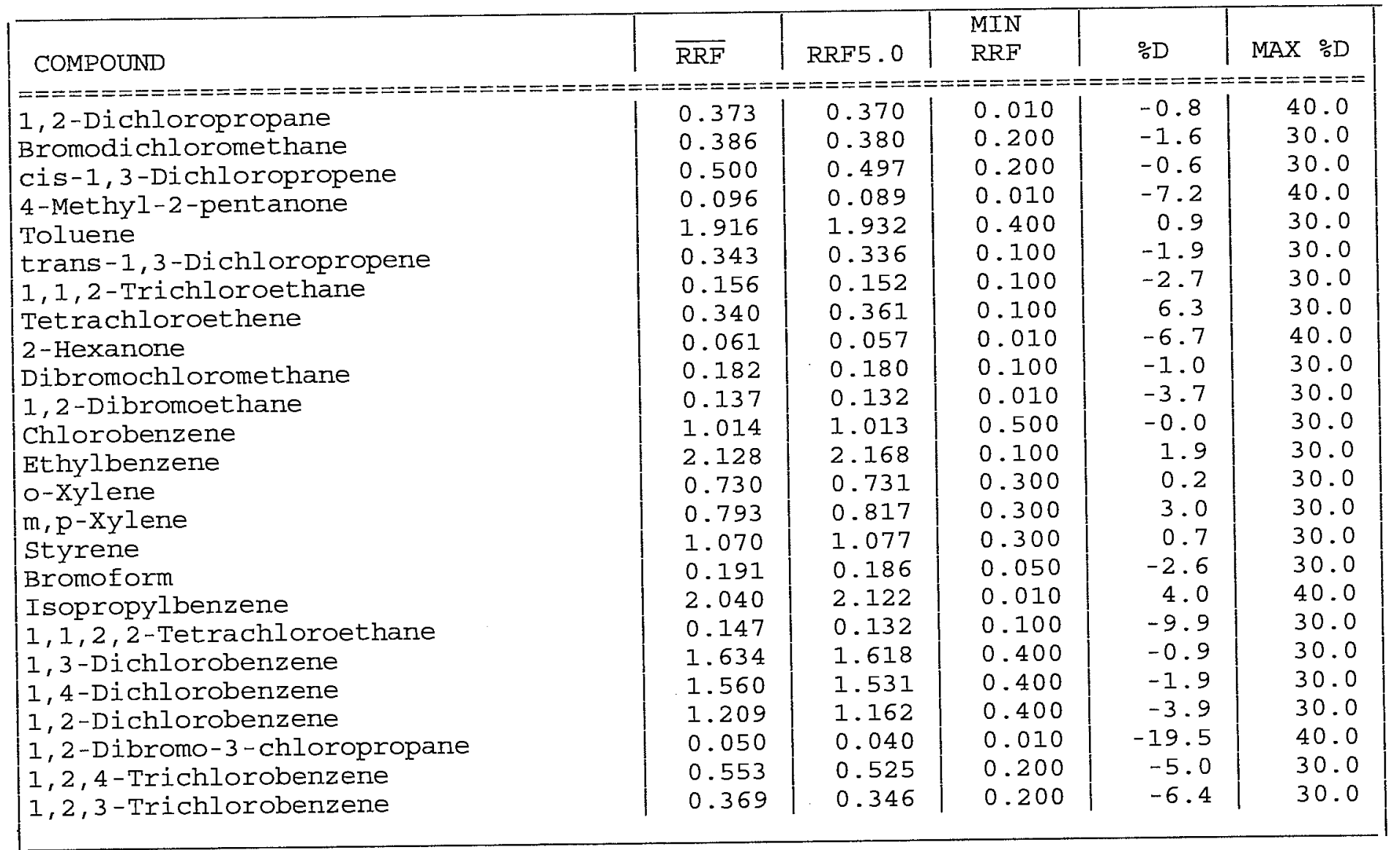

SOMO1. 2 
$7 \mathrm{C}$ - FORM VII VOA-3

VOLATILE CONTINUING CALIBRATION DATA

Lab Name: TESTAMERICA BURLINGTON

Lab Code: STLV Case No.: MORRILI Mod. Ref No.:
Contract: $8 \mathrm{E}-00302$

Calibration Date: 04/10/2010 Time: 1015

Init. Calib. Date(s): 04/05/2010 04/05/2010

Init. Calib. Time(s): $1318 \quad 1505$

Heated Purge: (Y/N)N GC Column: DB-624 ID: 0.53 (mm) Length: 75 (m)

Purge Volume: 25.0 (mL)

\begin{tabular}{|c|c|c|c|c|c|}
\hline COMPOUND & $\overline{\mathrm{RRF}}$ & RRF5. 0 & $\begin{array}{l}\text { MIN } \\
\text { RRF }\end{array}$ & $\because \mathrm{D}$ & MAX $\div D$ \\
\hline \multicolumn{6}{|c|}{ 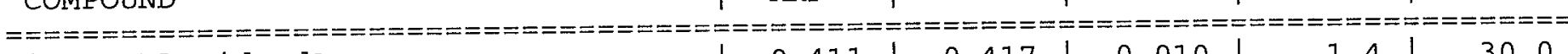 } \\
\hline Vinyl chloride-d3 & 0.411 & 0.417 & 0.010 & 1.4 & 30.0 \\
\hline Chloroethane- $\mathrm{d} 5$ & 0.310 & 0.325 & 0.010 & 4.9 & 40.0 \\
\hline 1,1-Dichloroethene-d2 & 0.683 & 0.704 & 0.010 & 3.1 & 30.0 \\
\hline 2-Butanone-d5 & 0.027 & 0.023 & 0.010 & -14.0 & 40.0 \\
\hline Chloroform-d & 0.541 & 0.533 & 0.010 & -1.5 & 30.0 \\
\hline 1,2-Dichloroethane-d4 & 0.169 & 0.160 & 0.010 & -5.1 & 30.0 \\
\hline Benzene-d6 & 1.760 & 1.784 & 0.400 & 1.4 & 30.0 \\
\hline 1,2-Dichloropropane-d6 & 0.499 & 0.440 & 0.010 & -11.8 & 40.0 \\
\hline Toluene-d8 & 1.583 & 1.623 & 0.010 & 2.5 & 30.0 \\
\hline trans-1,3-Dichloropropene-d4 & 0.312 & 0.304 & 0.010 & -2.4 & 30.0 \\
\hline $2-$ Hexanone-d5 & 0.033 & 0.028 & 0.010 & -16.9 & 40.0 \\
\hline $1,1,2,2$-Tetrachloroethane-d2 & 0.151 & 0.137 & 0.010 & -9.5 & 30.0 \\
\hline 1,2 -Dichlorobenzene-d4 & 0.764 & 0.755 & 0.010 & -1.1 & 30.0 \\
\hline
\end{tabular}

Report 1,4-Dioxane-d8 for Low-Medium VOA analysis only 
$7 A$ - FORM VII VOA-1

VOLATILE CONTINUING CALIBRATION DATA

Lab Name: TESTAMERICA BURLINGTON

Lab Code: STLV Case No.: MORRILL

Instrument ID: J.i

Lab File ID: JAQ05GCl

EPA Sample No. (VSTD\#\#\#\#\#): VSTD0050J
Contract : $8 \mathrm{E}-00302$

Mod. Ref No.:

SDG No.: 136730

Heated Purge: (Y/N)N GC Column: DB-624 ID: 0.53 (mm) Length: 75 (m)

Purge Volume: $25.0 \quad$ (m工)

\begin{tabular}{|c|c|c|c|c|c|}
\hline COMPOUND & $\overline{\mathrm{RRF}}$ & RRF5. 0 & $\begin{array}{l}\text { MIN } \\
\text { RRF }\end{array}$ & $\div D$ & MAX $\div D$ \\
\hline$====================0$ & $=======$ & $==-== \pm=$ & $======$ & -----1 & $========$ \\
\hline Dichlorodifluoromethane & 0.425 & 0.414 & 0.010 & -2.4 & 50.0 \\
\hline Chloromethane & 0.451 & 0.491 & 0.010 & 9.0 & 50.0 \\
\hline vinyl chloride & 0.454 & 0.479 & 0.010 & 5.4 & 50.0 \\
\hline Bromomethane & 0.171 & 0.177 & 0.010 & 3.2 & 50.0 \\
\hline Chloroethane & 0.263 & 0.271 & 0.010 & 3.1 & 50.0 \\
\hline Trichlorofluoromethane & 0.511 & 0.516 & 0.010 & 1.0 & 50.0 \\
\hline 1,1-Dichloroethene & 0.306 & 0.312 & 0.010 & 2.0 & 50.0 \\
\hline $1,1,2$-Trichloro-1,2,2-trifluoroethane & 0.323 & 0.328 & 0.010 & 1.5 & 50.0 \\
\hline Acetone & 0.014 & 0.013 & 0.010 & -11.0 & 50.0 \\
\hline Carbon disulfide & 0.986 & 0.952 & 0.010 & -3.4 & 50.0 \\
\hline Methyl acetate & 0.053 & 0.045 & 0.010 & -14.5 & 50.0 \\
\hline Methylene chloride & 0.250 & 0.247 & 0.010 & -1.2 & 50.0 \\
\hline trans-1,2-Dichloroethene & 0.326 & 0.327 & 0.010 & 0.4 & 50.0 \\
\hline Methyl tert-butyl ether & 0.397 & 0.353 & 0.010 & -11.0 & 50.0 \\
\hline 1,1-Dichloroethane & 0.597 & 0.612 & 0.010 & 2.6 & 50.0 \\
\hline cis-1,2-Dichloroethene & 0.316 & 0.310 & 0.010 & -1.7 & 50.0 \\
\hline 2 -Butanone & 0.028 & 0.025 & 0.010 & -10.1 & 50.0 \\
\hline Bromochloromethane & 0.090 & 0.086 & 0.010 & -3.4 & 50.0 \\
\hline Chloroform & 0.500 & 0.509 & 0.010 & 1.8 & 50.0 \\
\hline $1,1,1$-Trichloroethane & 0.625 & 0.654 & 0.010 & 4.7 & 50.0 \\
\hline Cyclohexane & 0.890 & 0.936 & 0.010 & 5.1 & 50.0 \\
\hline Carbon tetrachloride & 0.522 & 0.549 & 0.010 & 5.2 & 50.0 \\
\hline Benzene & 1.820 & 1.855 & 0.010 & 1.9 & 50.0 \\
\hline 1,2-Dichloroethane & 0.206 & 0.201 & 0.010 & -2.1 & 50.0 \\
\hline Trichloroethene & 0.427 & 0.447 & 0.010 & 4.9 & 50.0 \\
\hline Methylcyclohexane & 0.679 & 0.725 & 0.010 & 6.8 & 50.0 \\
\hline
\end{tabular}

Report 1,4-Dioxane for Low-Medium VOA analysis only 
$7 B$ - FORM VII VOA-2

VOLATILE CONTINUING CALIBRATION DATA

Lab Name: TESTAMERICA BURLINGTON

Lab Code: STLV Case No.: MORRILL
Contract: $8 \mathrm{E}-00302$

Mod. Ref No.:

SDG NO.: 136730
Instrument ID: J.i

Lab File ID: JAQ05GC1

EPA Sample No. (VSTD\#\#\#\#\#) : VSTD0050J

Heated Purge: (Y/N)N GC Column: DB-624

Purge Volume: 25.0
Calibration Date: 04/10/2010 Time: 1925

Init. Calib. Date(s) : 04/05/2010 04/05/2010

Init. Calib. Time(s): $1318 \quad 1505$

ID $: 0.53 \quad(\mathrm{~mm})$ Length: 75

(m)

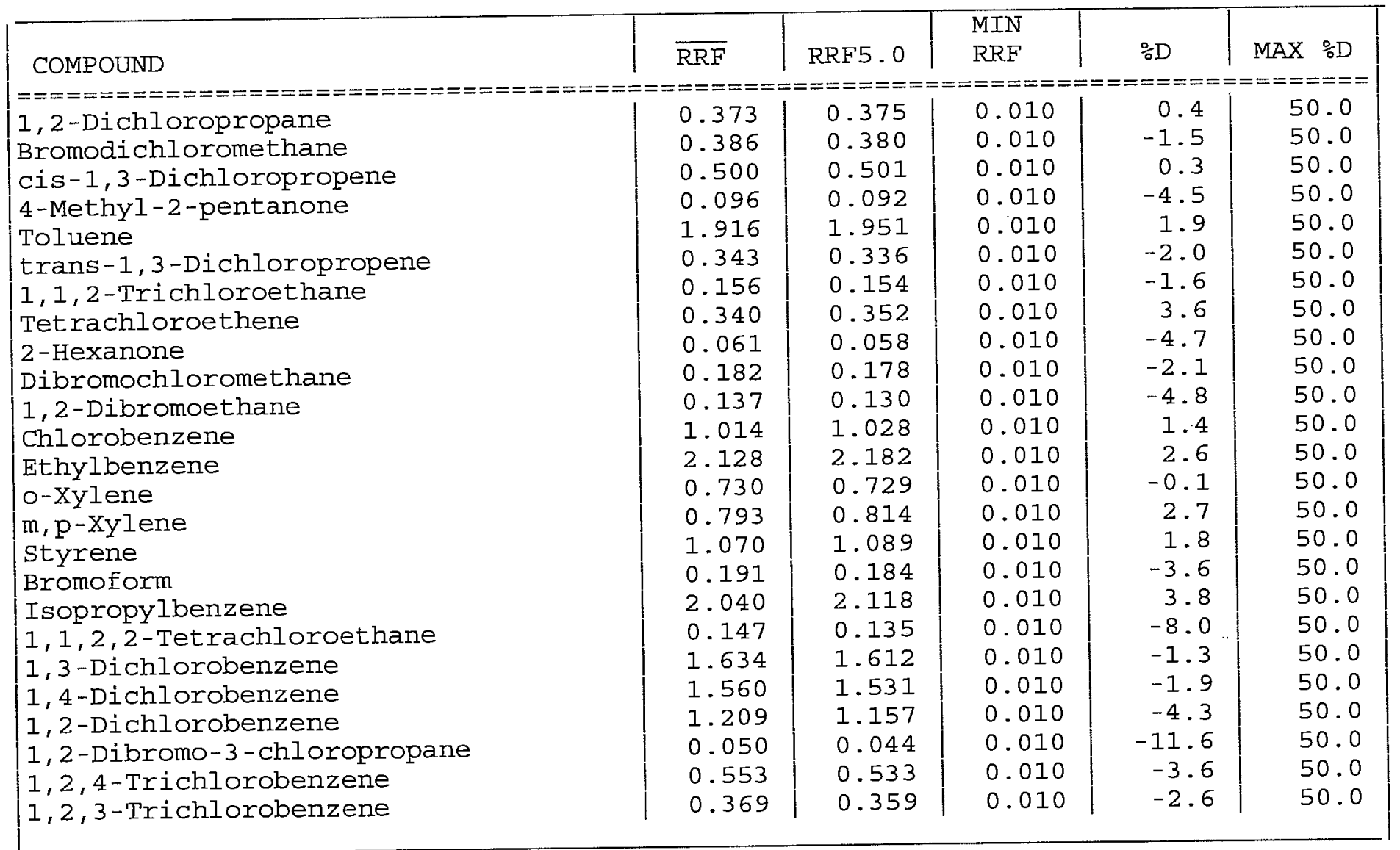


$7 C$ - FORM VII VOA-3

VOLATILE CONTINUING CALIBRATION DATA

Lab Name: TESTAMERICA BURLINGTON

Contract : $8 E-00302$

Lab Code: STLV Case No.: MORRILL Mod. Ref No.:

SDG No.: 136730

Instrument ID: J.i

Calibration Date: 04/10/2010 Time: 1925

Lab File ID: JAQ05GC1

Init. Calib. Date (s) : 04/05/2010 04/05/2010

EPA Sample No. (VSTD\#\#\#\#\#): VSTD0050J Init. Calib. Time(s): $1318 \quad 1505$

Heated Purge: (Y/N)N GC Column: DB-624 ID: 0.53 (mm) Length: 75 (m)

Purge Volume: 25.0 (mL)

\begin{tabular}{|c|c|c|c|c|c|}
\hline COMPOUND & $\overline{\mathrm{RRF}}$ & RRF5. 0 & $\begin{array}{l}\text { MIN } \\
\text { RRF }\end{array}$ & $\% \mathrm{D}$ & MAX $\% D$ \\
\hline \multicolumn{6}{|c|}{ 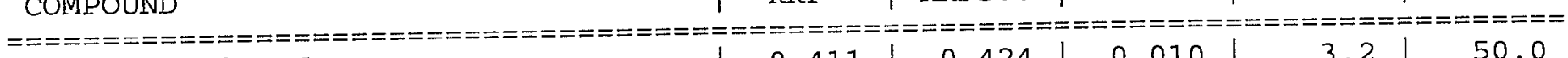 } \\
\hline Vinyl chloride-d3 & 0.411 & 0.424 & 0.010 & 3.2 & 50.0 \\
\hline Chloroethane-d5 & 0.310 & 0.324 & 0.010 & 4.4 & 50.0 \\
\hline 1,1 -Dichloroethene-d2 & 0.683 & 0.696 & 0.010 & 1.9 & 50.0 \\
\hline 2-Butanone-d5 & 0.027 & 0.025 & 0.010 & -8.5 & 50.0 \\
\hline Chloroform-d & 0.541 & 0.543 & 0.010 & 0.3 & 50.0 \\
\hline 1,2 -Dichloroethane-d4 & 0.169 & 0.163 & 0.010 & -3.7 & 50.0 \\
\hline Benzene-d6 & 1.760 & 1.790 & 0.010 & 1.7 & 50.0 \\
\hline 1,2 -Dichloropropane-d6 & 0.499 & 0.498 & 0.010 & -0.1 & 50.0 \\
\hline Toluene-d8 & 1.583 & 1.628 & 0.010 & 2.8 & 50.0 \\
\hline trans-1,3-Dichloropropene-d4 & 0.312 & 0.300 & 0.010 & -3.8 & 50.0 \\
\hline 2-Hexanone-d5 & 0.033 & 0.030 & 0.010 & -10.5 & 50.0 \\
\hline $1,1,2,2$-Tetrachloroethane-d2 & 0.151 & 0.144 & 0.010 & -4.7 & 50.0 \\
\hline 1,2 -Dichlorobenzene-d4 & 0.764 & 0.759 & 0.010 & -0.6 & 50.0 \\
\hline
\end{tabular}

Report 1,4-Dioxane-d8 for Low-Medium VOA analysis only 
$8 A$ - FORM VIII VOA

VOIATILE INTERNAL STANDARD AREA AND RETENTION TIME SUMMARY

Lab Name: TESTAMERICA BURLINGTON

Lab Code: STLV Case No.: MORRILL Mod. Ref No.:
SDG NO. : 136730
GC Column: DB-624 ID: $0.53 \quad$ (mm)

EPA Sample No. (VSTD\#\#\#\#\#) : VSTD005JO

Lab File ID (Standard): JAQ005GV

Instrument ID: J.i
Init. Calib. Date(s): 04/05/2010 04/05/2010

Date Analyzed: 04/10/2010

Time Analyzed: 1015

Heated Purge: $(Y / N)$ N

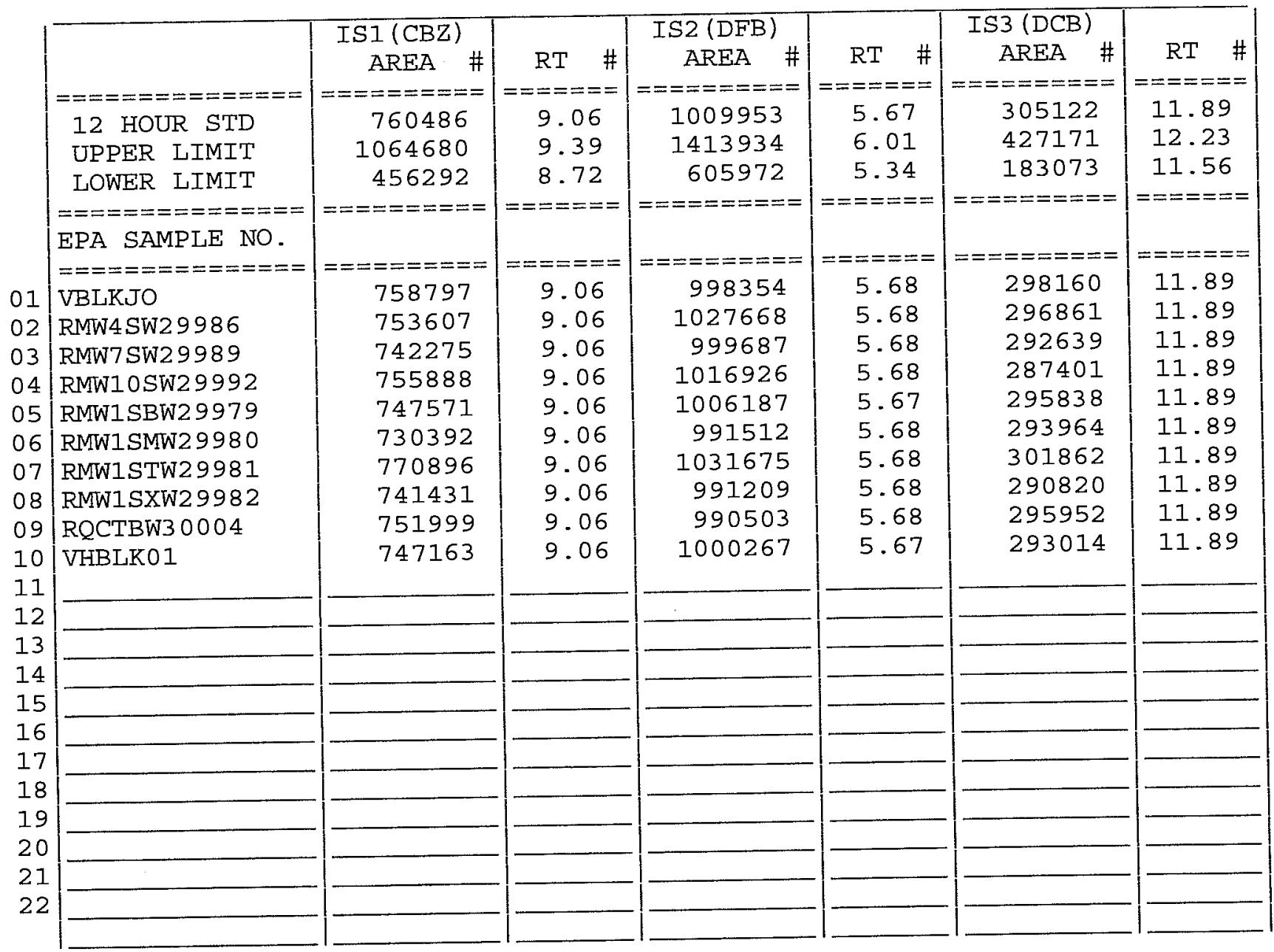

IS1 (CBZ) = Chlorobenzene $-\mathrm{d} 5$

IS2 (DFB) = 1,4-Difluorobenzene

IS3 $(D C B)=1,4-D i c h l o r o b e n z e n e-d 4$

AREA UPPER LIMIT $=200 \%$ (Low-Medium Volatiles) and $140 \%$ (Trace Volatiles) of internal standard area

AREA LOWER LIMIT $=50 \%$ (Low-Medium Volatiles) and $60 \%$ (Trace Volatiles) of internal standard area

RT UPPER LIMIT $=\quad+0.50$ (Low-Medium Volatiles) and +0.33 (Trace Volatiles) minutes of internal standard RT

RT LOWER LIMIT $=-0.50$ (Low-Medium Volatiles) and - 0.33 (Trace Volatiles) minutes of internal standard RT

\# Column used to flag values outside QC limits with an asterisk Page 1 of 1 


\section{TestAmerica}

THE LEADER IN ENVIRONMENTAL TESTING

TestAmerica Laboratories, Inc.

\section{CASE NARRATIVE}

\section{Client: Argonne National Laboratory}

Project: Morrill (200-1702)

Report Number: 200-1702-1

With the exceptions noted as flags or footnotes, standard analytical protocols were followed in the analysis of the samples and no problems were encountered or anomalies observed.

Calculations are performed before rounding to avoid round-off errors in calculated results.

All holding times were met and proper preservation noted for the methods performed on these samples, unless otherwise detailed in the individual sections below.

\section{Receipt}

The samples were received on 9/24/2010. Documentation of the condition of the samples at the time of their receipt and any exception to the laboratory's Sample Acceptance Policy is documented in the Sample Handling section of this submittal. The samples, as received, were not acid preserved. On that basis, the laboratory did provide for the analytical work to be performed within seven days of sample collection.

Sample MRISCH-W-30022 was not listed on the chain-of-custody record that was received with the samples. The sample was logged in for analysis using the information from the label on the sample container(s). Four $20 \mathrm{~mL}$ volumes were received for the referenced sample.

\section{SOM01.2 Volatile Organics (Trace Level Water)}

A storage blank was prepared for volatile organics analysis, and stored in association with the storage of the samples. That storage blank, identified as VHBLK01, was carried through the holding period with the samples, and analyzed.

Sample MRMW3S-W-30013 was analyzed at a dilution based on the results of preliminary screening. An additional, more concentrated analysis was performed on the sample in order to provide for a lower reporting limit for those compounds that were not identified in the primary analysis. An additional, dilution analysis was performed on sample MRMW11S-W-30021 in order to provide for quantification within the range of calibrated instrument response. Both sets of results for the analysis of samples MRMW3S-W-30013 and MRMW11S-W-30021 are included in this submittal. 


\section{TestAmerica}

THE LEADER IN ENVIRONMENTAL. TESTING

With the exception of the more concentrated analysis that was performed on sample MRMW11S-W-30021, each of the analyses associated with the sample set exhibited an acceptable internal standard performance. The referenced analysis did exhibit a low response for 1,4-dichlorobenzene- $d_{6}$. There was an acceptable recovery of each deuterated monitoring compound (DMC) in the analysis of the method blanks associated with the analytical work, and in the analysis of the storage blank associated with the sample set. The analysis of the samples in this sample set did meet the technical acceptance criteria specific to DMC recoveries, although not all DMC recoveries were within the control range in each analysis. The technical acceptance criteria does provide for the recovery of up to three DMCs to fall outside of the control range in the analysis of field samples. Matrix spike and matrix spike duplicate analyses were not performed on samples in this sample set. To varying extent, trace concentrations of acetone, carbon disulfide, and 1,2,3-trichlorobenzene were identified in the analysis of the method blanks associated with the analytical work. The concentration of each compound in each analysis was below the established reporting limit, and each analysis did meet the technical acceptance criteria for a compliant method blank analysis. Trace concentrations of acetone and carbon disulfide were identified in the analysis of the storage blank associated with the sample set. The concentration each compound in that analysis was below the established reporting limit, and the analysis did meet the technical acceptance criteria for a compliant storage blank analysis. Present in the method blank and storage blank analyses was a non-target constituent that represents a compound that is related to the DMC formulation. The fact that the presence of this compound is not within the laboratory's control is at issue. The derived results for that compound have been qualified with an "X" qualifier to reflect the source of the contamination.

With the exception of that for bromomethane, the responses for each of the target analytes met the relative standard deviation criterion in the initial calibration. The relative standard deviation of the responses for bromomethane was 20.2 percent. Although above the 20.0 percent criterion established for that compound, the initial calibration did meet the technical acceptance criteria for a compliant initial calibration. The response for each target analyte met the percent difference criterion in the continuing calibration check acquisition. The response for each target analyte met the 50.0 percent difference criterion in the closing calibration check acquisition.

The primary quantitation mass for methylcyclohexane that is specified in the Statement of Work is mass 83 . The laboratory did identify a contribution to mass 83 from 1,2-dichloropropane- $\mathrm{d}_{6}$, one of the deuterated monitoring compounds (DMCs). The laboratory did change the primary quantitation mass assignment to mass 55 for the quantification of methylcyclohexane.

Manual integration was employed in deriving certain of the analytical results. The values that have been derived from manual integration are qualified on the quantitation reports. Extracted ion current profiles for each manual integration are included in the data package, and further documented in the Sample Preparation section of this submittal.

The analytical results associated with the samples presented in this test report were generated under a quality system that adheres to requirements specified in the NELAC standard. Release of the data in this test report and any associated electronic deliverables is authorized by the 


\section{TestAmerica \\ THE LEADER IN ENVIRONMENTAL TESTING}

Laboratory Director's designee as verified by the following signature.

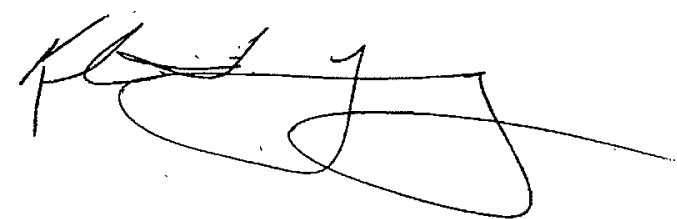

Kirk F. Young

Project Manager 


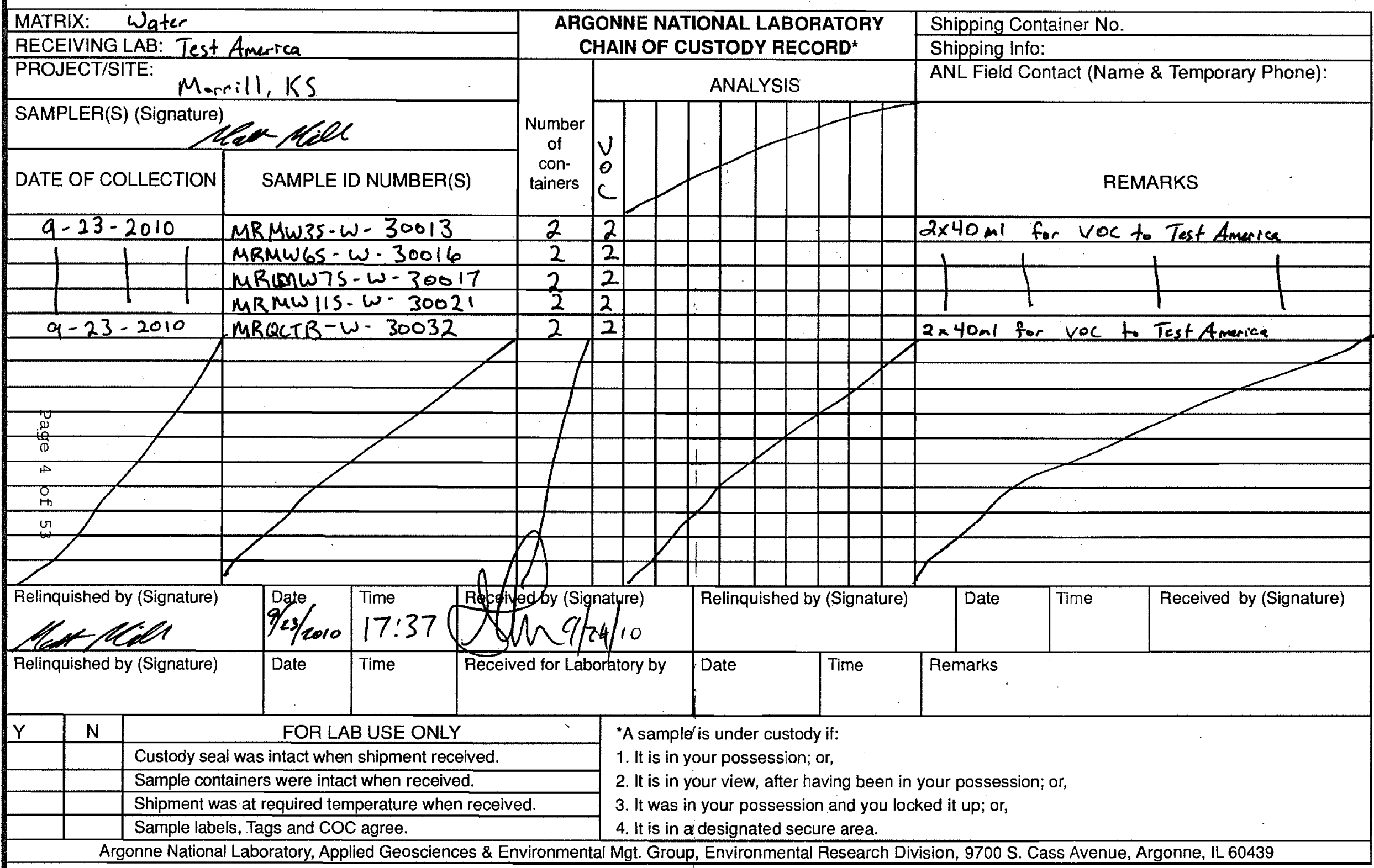




\section{urlington Facility}

ternal Chain of Custody Log (ICOC)

ject Information:

DG-IN NUMBER: $266-17602$

IENT: ARGLAB

Miethod: $\operatorname{SOM} 01 \cdot 2=V 0 A$

amples associated with this Log-in were LAB IDs: $260-1702-1$ thrs $17602-7$

amples associated with this Log-in were placed into storage on

$\frac{89 / 2+100}{(0 a t e)}$
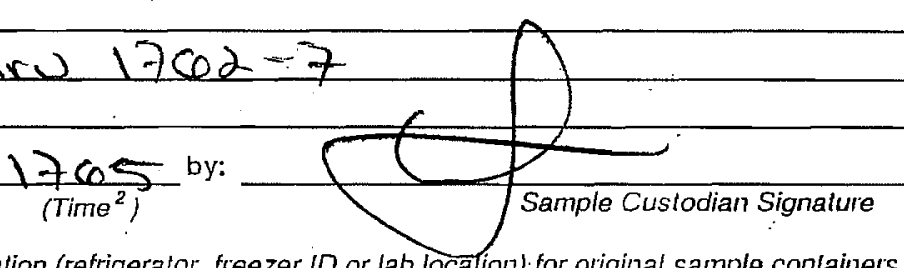

torage Location: $\frac{\text { VOAB, Shelf } 99}{\text { torage Condition: }}$

ternal Transfer: Information

Specify storage location (refrigerator, freezer ID or lab localion). for original sample conlainers Ambient

\begin{tabular}{|c|c|c|c|c|c|c|c|c|c|c|}
\hline \multicolumn{2}{|c|}{ Sample Type } & \multirow{2}{*}{ Lab ID(s) } & \multirow{2}{*}{$\begin{array}{c}\text { Transfer } \\
\text { Date }\end{array}$} & \multirow{2}{*}{$\begin{array}{c}\text { Transfer } \\
\text { Time }^{2}\end{array}$} & \multicolumn{3}{|c|}{ Purpose of Transfer } & \multirow{2}{*}{$\begin{array}{l}\text { Relinquished } \\
\text { By: }\end{array}$} & \multirow{2}{*}{$\begin{array}{c}\text { Received } \\
\text { By: }\end{array}$} & \multirow{2}{*}{$\begin{array}{l}\text { Storage Location } \\
\text { Prepared Sample }\end{array}$} \\
\hline Driginal & Prepared ${ }^{1}$ & & & & Prep & Analysis & Storage & & & \\
\hline レ & & $1-7$ & $9-25 \cdot 10$ & 1140 & 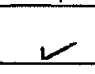 & & & Thomus lachou & Thomus focha & VoA Prep \\
\hline$\kappa$ & & $1 \cdot 7$ & $9-25-10$ & 1215 & & & - & Domex Buth & Them then & VoA Fridge \\
\hline$\frac{\pi}{0}$ & & $u v$ & $q(+8)^{29}$ & 080 & & $r$ & & onduallysy & munail balles & dungess \\
\hline \% & & $4 \quad 1$ & $9(2 \times 110$ & $160 \mathrm{~V}$ & & & r & Mluhal Bellem & Meduad Valler & Arrap \\
\hline u & & & 29 & & & & & & & \\
\hline $\begin{array}{l}O \\
\Pi\end{array}$ & & & & & & & & & & \\
\hline$\stackrel{w}{w}$ & & & & & & & & & & \\
\hline & & & & & & & & & & \\
\hline & & & & & & & & & & \\
\hline & & & & & & & & & & \\
\hline & & & & & & & & & & \\
\hline & & & & & & & & & & \\
\hline & & & & & & & & & & \\
\hline & & & & & & & & & & \\
\hline & & & & & & & & & & \\
\hline & - & & & & & & & & & \\
\hline & & & & & & & & & & \\
\hline
\end{tabular}

'Extract, digestate, or any other prepared sample that is no longer in original sample container

${ }^{2}$ Milliary Time 
$2 A$ - FORM II VOA-1

WATER VOLATILE DEUTERATED MONITORING COMPOUND RECOVERY

Lab Name: TESTAMERICA BURLINGTON

Contract: $8 \mathrm{E}-00302$

Lab Code: STLV_Case No.: MORRIL Mod. Ref No.:

SDG No.: 200-1702

Level: (TRACE or LOW) TRACE

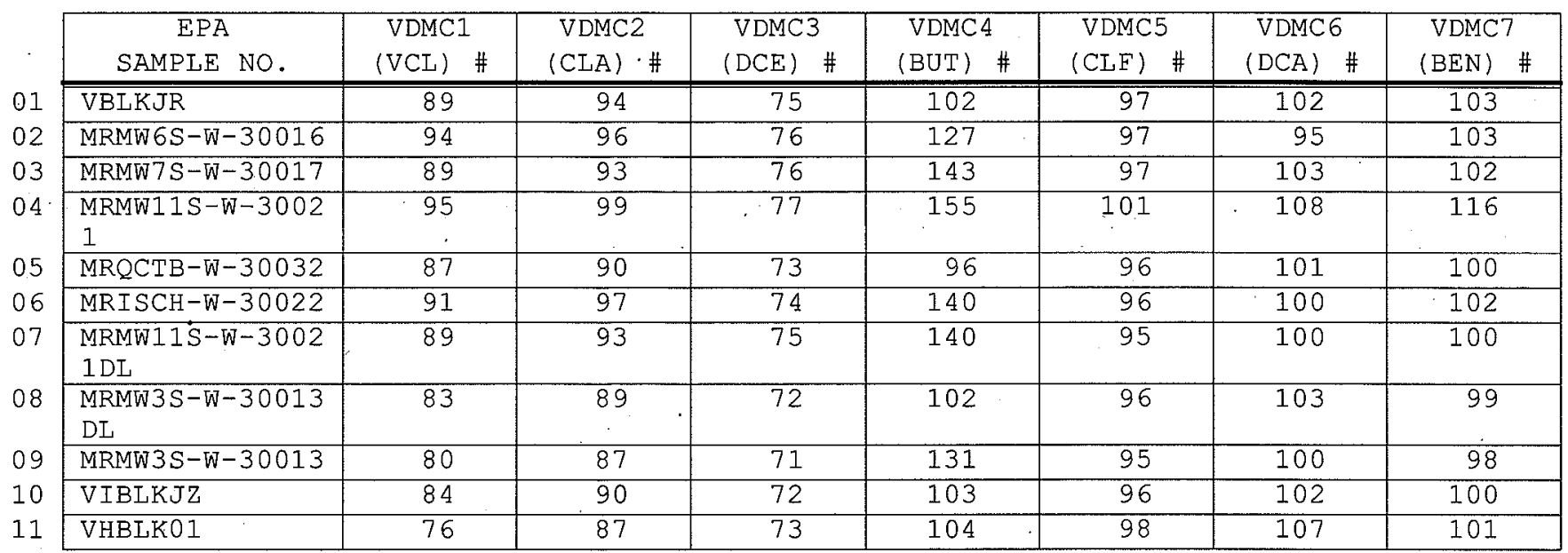

\footnotetext{
VDMC1 $($ VCL $)=$ Vinyl Chloride-d3

VDMC2 (CLA) = Chloroethane-d5

VDMC3 $(\mathrm{DCE})=1,1-\mathrm{Di}$ chloroethene-d2

VDMC4 $\quad(B U T)=2$-Butanone-d5

VDMC5 $(\mathrm{CLF})=$ Chloroform-d

VDMC6 $(D C A)=1,2-$ Dichloroethane-d4

VDMC7 $(B E N)=$ Benzene-d 6
}

\# Column to be used to flag recovery values

* Values outside of contract required QC limits

$$
\begin{aligned}
& \frac{Q C \text { LIMITS }}{(65-131)} \\
& (71-131) \\
& (55-104) \\
& (49-155) \\
& (78-121) \\
& (78-129) \\
& (77-124)
\end{aligned}
$$


$2 B$ - FORM II VOA-2

WATER VOLATILE DEUTERATED MONITORING COMPOUND RECOVERY

Lab Name: TESTAMERICA BURLINGTON

Contract: 8E-00302

Lab Code: STLV Case No.: MORRIL Mod. Ref No.:

SDG No.: 200-1702

Level: (TRACE Or LOW) TRACE

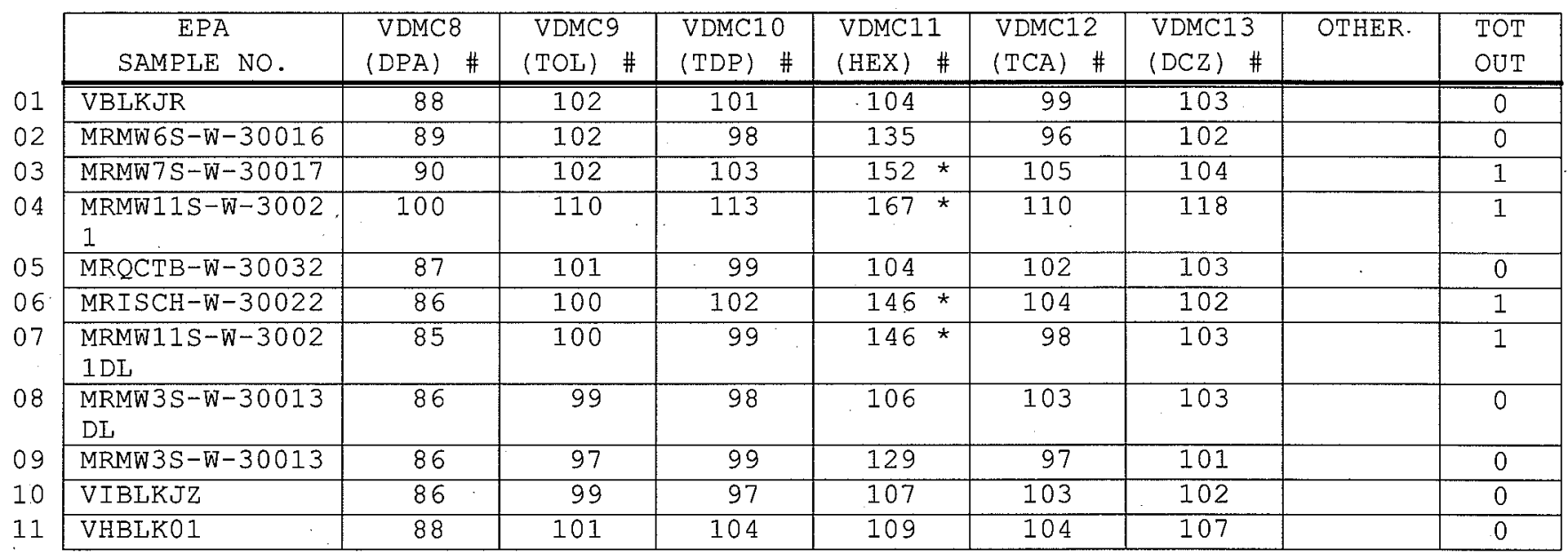

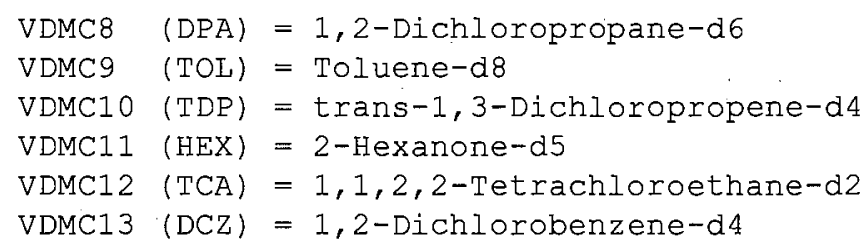

\# Column to be used to flag recovery values

* Values outside of contract required QC limits

Report 1,4-Dioxane-d8 for Low-Medium VOA analysis only

Page 1 of 1
SOM01.2 (4/2007) 
$4 A$ - FORM IV VOA

VOLATILE METHOD BLANK SUMMARY
EPA SAMPLE NO.

VBLKJR
Lab Name: TESTAMERICA BURLINGTON

Lab Code: STIV Case No.: MORRIL Mod. Ref No.:
Contract: $8 \mathrm{E}-00302$

Lab Sample ID: MB 200-7244/5

Date Analyzed: 09/29/2010

Time Analyzed: 0941

Heated Purge: ( $Y / N)$ N SDG No.: 200-1702
Level: (TRACE or I
GC Column: DB-624

ID: 0.20 (mm)

Lab File ID: JBOC05.D

Instrument ID: J.i

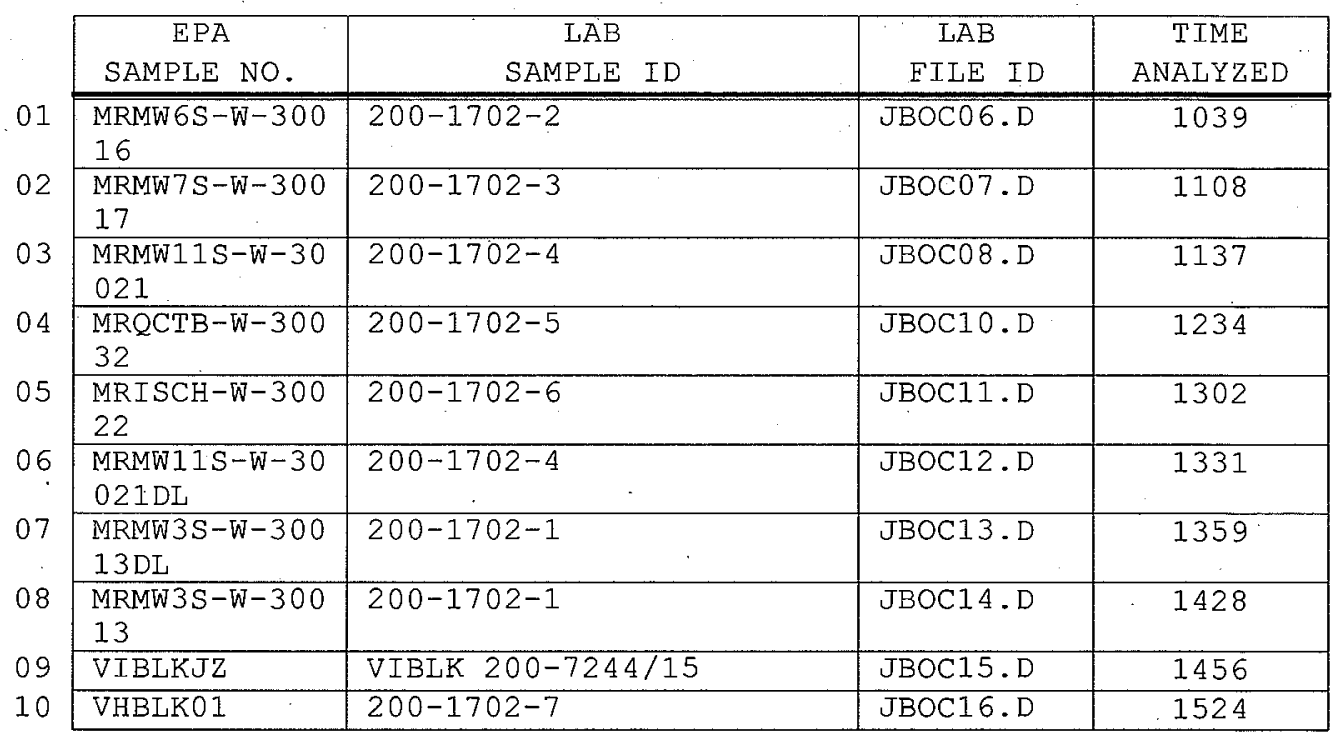

COMMENTS :

Page 1 of 1 
$5 A$ - FORM V VOA

VOLATILE ORGANICS INSTRUMENT

PERFORMANCE CHECK BROMOFLUOROBENZENE
EPA SAMPLE NO.

BEBJO
Lab Name: TESTAMERICA BURLINGTON

Lab Code: STLV

Lab File Id: JBO01.D

Instrument Id: J.i

GC Column: DB-624
Case No.: MORRIL Mod. Ref No.:

Contract: $8 \mathrm{E}-00302$
SDG No.: 200-1702

BFB Injection Date: 09/27/2010

BFB Injection Time: 1523 $(\mathrm{mm})$

\begin{tabular}{|c|c|c|}
\hline $\mathrm{m} / \mathrm{e}$ & ION ABUNDANCE CRITERIA & $\begin{array}{l}\% \text { RELATIVE } \\
\text { ABUNDANCE }\end{array}$ \\
\hline 50 & $15.0-40.0 \%$ of mass 95 & 16.8 \\
\hline 75 & $30.0-80.0 \%$ of mass 95 & 50.8 \\
\hline 95 & Base peak, $100 \%$ relative abundance & 100 \\
\hline 96 & $5.0-9.0 \%$ of mass 95 & 6.3 \\
\hline 173 & Less than $2.0 \%$ of mass 174 & $0.6) 1$ \\
\hline 174 & $50.0-120 \%$ of mass 95 & 88.9 \\
\hline 175 & $5.0-9.0 \%$ of mass 174 & $6.6(7.5) 1$ \\
\hline 176 & $95.0-101 \%$ of mass 174 & $86.9(97.8) 1$ \\
\hline 177 & $5.0-9.0 \%$ of mass 176 & $(6.5) 2$ \\
\hline
\end{tabular}

1 - Value is omass $174 \quad 2$ - Value is omass 176

\begin{tabular}{|c|c|c|c|c|c|}
\hline \multicolumn{1}{|c|}{$\begin{array}{c}\text { EPA } \\
\text { SAMPLE NO. }\end{array}$} & LAB & LAB & DATE & TIME \\
\cline { 2 - 7 } 01 & SAMPLE ID & FILE ID & ANALYZED & ANALYZED \\
\cline { 2 - 7 } 02 & VSTD0.5JO & IC 200-7152/2 & JBO02.D & $09 / 27 / 2010$ & 1544 \\
\cline { 2 - 7 } 03 & VSTD001JO & IC 200-7152/3 & JBO03.D & $09 / 27 / 2010$ & 1611 \\
\cline { 2 - 7 } 04 & VSTD005JO & ICIS 200-7152/4 & JBO04.D & $09 / 27 / 2010$ & 1639 \\
\cline { 2 - 7 } 05 & VSTD010JO & IC 200-7152/5 & JBO05.D & $09 / 27 / 2010$ & 1708 \\
\cline { 2 - 7 } & VSTD020JO & IC 200-7152/6 & JBO06.D & $09 / 27 / 2010$ & 1736 \\
\cline { 2 - 7 }
\end{tabular}


$5 A$ - FORM V VOA

VOLATILE ORGANICS INSTRUMENT

PEREORMANCE CHECK

BROMOELUOROBENZENE (BFB)
EPA SAMPLE NO.

BEBJR
Lab Name: TESTAMERICA BURLINGTON

Lab Code: STLV

Case No.: MORRIL Mod. Ref No.:
Contract: $8 \mathrm{E}-00302$
Lab File Id: JBOC01.D

Instrument Id: J.i

GC Column: DB-624
ID: 0.20 (mm)

\begin{tabular}{|r|l|r|}
\hline $\mathrm{m} / \mathrm{e}$ & ION ABUNDANCE CRITERIA & $\begin{array}{c}\text { \% RELATIVE } \\
\text { ABUNDANCE }\end{array}$ \\
\hline 50 & $15.0-40.0 \%$ of mass 95 & 16.8 \\
\hline 75 & $30.0-80.0 \%$ of mass 95 & 52.6 \\
\hline 95 & Base peak, 100\% relative abundance & 100 \\
\hline 96 & $5.0-9.0 \%$ of mass 95 & 6.4 \\
\hline 173 & Less than 2.0\% of mass 174 & 0.4( \\
\hline 174 & $50.0-120 \%$ of mass 95 & 85.0 \\
\hline 175 & $5.0-9.0 \%$ of mass 174 & $5.9(1$ \\
\hline 176 & $95.0-101 \%$ of mass 174 & $82.9) 1$ \\
\hline 177 & $5.0-9.0 \%$ of mass 176 & $56.6) 1$ \\
\hline
\end{tabular}

1 - Value is omass 174 2 - Value is omass 176

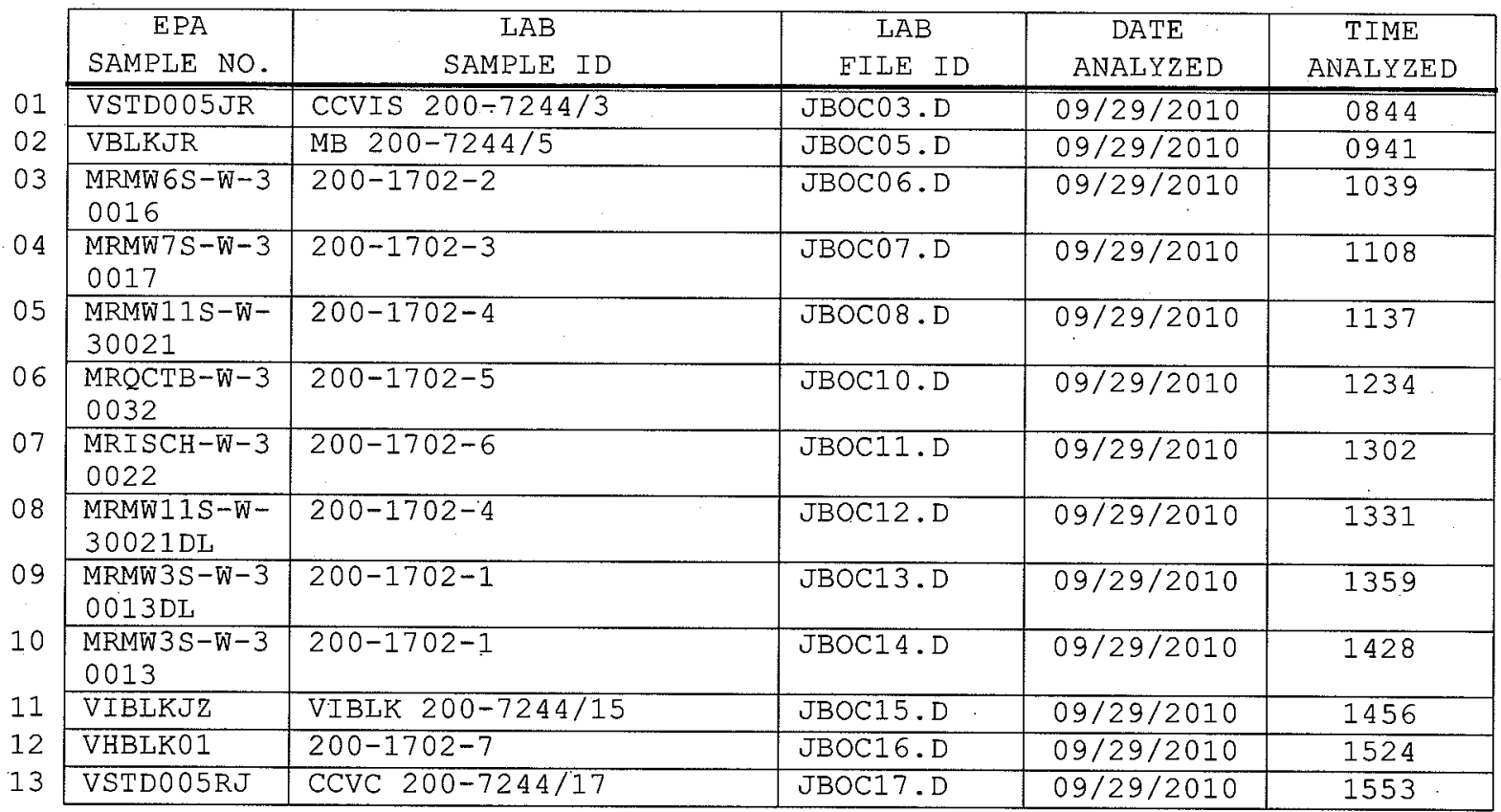


$8 A$ - FORM VIII VOA

- VOLATILE INTERNAL STANDARD AREA AND RETENTION TIME SUMMARY

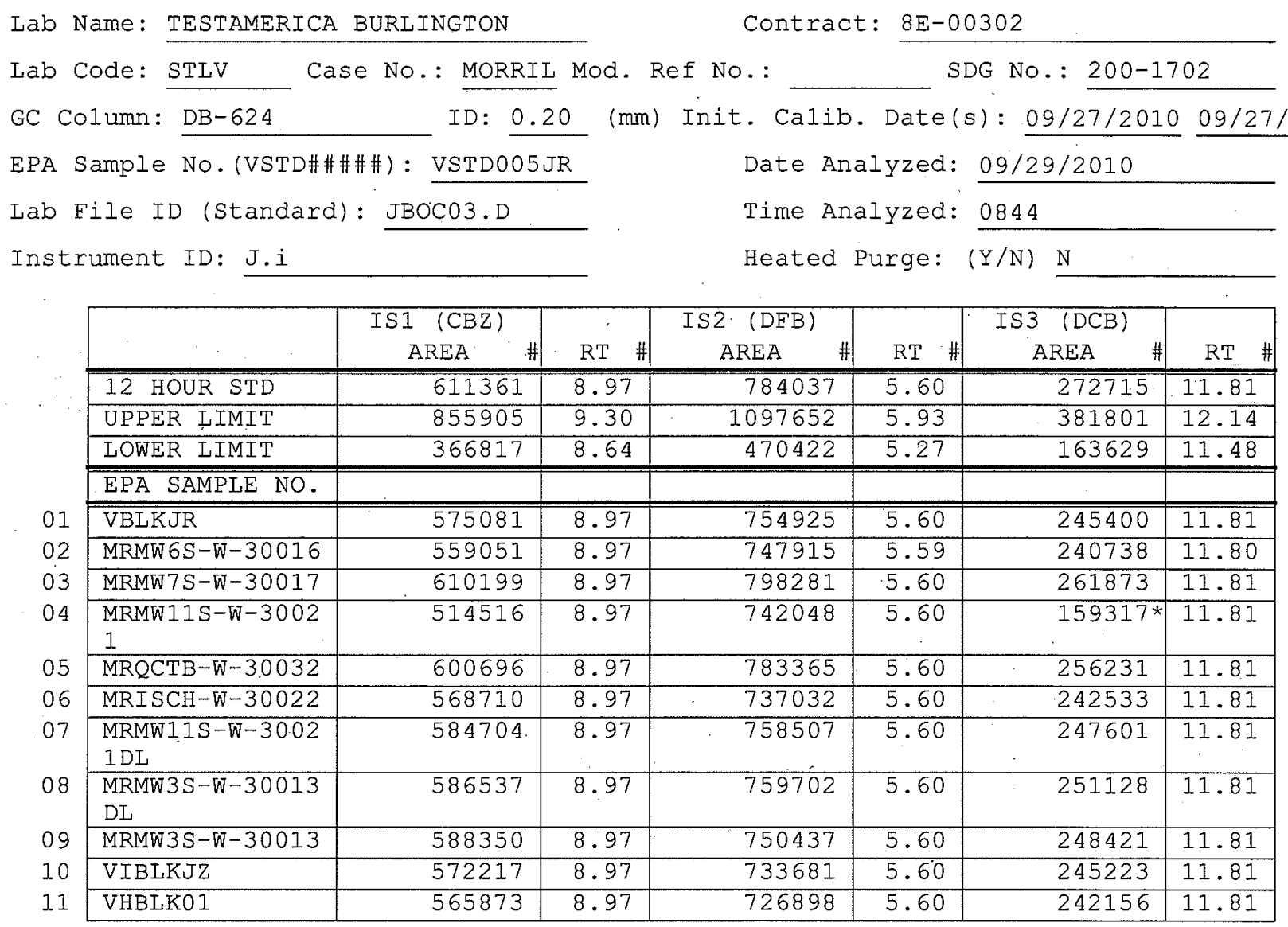

\footnotetext{
IS1 $(\mathrm{CBZ})=$ Chlorobenzene-d5

IS2 $(\mathrm{DFB})=1,4-\mathrm{Difluorobenzene}$

IS3 $(D C B)=1,4$-Dichlorobenzene-d 4
}

AREA UPPER LIMIT $=140 \%$ (Trace Volatiles) of internal standard area AREA LOWER LIMIT $=60 \%$ (Trace Volatiles) of internal standard area RT UPPER LIMIT $=+0.33$ (Trace Volatiles) minutes of internal standard RT RT LOWER LIMIT $=-0.33$ (Trace Volatiles) minutes of internal standard RT \# Column used to flag values outside contract required QC limits with an asterisk. Page 1 of 1 
$1 A$ - FORM I VOA-1

VOLATILE ORGANICS ANALYSIS DATA SHEET
EPA SAMPLE NO.

MRISCH-W-30022
Lab Name: TESTAMERICA BURLINGTON

Lab Code: STLV Case No.: MORRIL Mod. Ref No.:
Contract: $8 \mathrm{E}-00302$

Lab Sample ID: 200-1702-6

Lab File ID: JBOC11.D

Date Received: 09/24/2010

Date Analyzed: 09/29/2010

Dilution Factor: 1.0

Soil Aliquot Volume: (iL) $(\mathrm{mL})$

Purge Volume: 25.0 ID: $0.20 \quad(\mathrm{~mm})$

\begin{tabular}{|c|c|c|c|}
\hline CAS NO. & COMPOUND & $\begin{array}{l}\text { CONCENTRATION UNITS: } \\
(\mathrm{ug} / \mathrm{L} \text { or } \mathrm{ug} / \mathrm{kg}) \mathrm{ug} / \mathrm{I} \\
\end{array}$ & $Q$ \\
\hline $75-71-8$ & Dichlorodifluoromethane & 0.50 & $\mathrm{U}$ \\
\hline $74-87-3$ & Chloromethane & 0.50 & $\mathrm{U}$ \\
\hline $75-01-4$ & Vinyl chloride & 0.50 & $\mathrm{U}$ \\
\hline $74-83-9$ & Bromomethane & 0.50 & $\bar{U}$ \\
\hline $75-00-3$ & Chloroethane & 0.50 & $\mathrm{U}$ \\
\hline $75-69-4$ & Trichlorofluoromethane & 0.50 & $\mathrm{U}$ \\
\hline $75-35-4$ & 1,1-Dichloroethene & 0.50 & $\mathrm{U}$ \\
\hline $76-13-1$ & 1,1,2-Trichloro-1,2,2-trifluoroethane & 0.50 & $\mathrm{U}$ \\
\hline $67-64-1$ & Acetone & 2.9 & $\mathrm{~J} B$ \\
\hline $75-15-0$ & Carbon disulfide & 0.13 & $\mathrm{~J} B$ \\
\hline $79-20-9$ & Methyl acetate & 0.50 & $\mathrm{U}$ \\
\hline $75-09-2$ & Methylene Chloride & 0.50 & $\mathrm{U}$ \\
\hline $156-60-5$ & trans-1,2-Dichloroethene & 0.50 & $\mathrm{U}$ \\
\hline $1634-04-4$ & Methyl tert-butyl ether & 0.50 & $\mathrm{U}$ \\
\hline $75-34-3$ & 1,1-Dichloroethane & 0.50 & $\mathrm{U}$ \\
\hline $156-59-2$ & cis-1,2-Dichloroethene & 0.50 & $\mathrm{U}$ \\
\hline $78-93-3$ & 2-Butanone & 5.0 & $\overline{\mathrm{U}}$ \\
\hline $74-97-5$ & Bromochloromethane & 0.50 & $\bar{U}$ \\
\hline $57-66-3$ & Chloroform & 0.50 & $\mathrm{U}$ \\
\hline $71-55-6$ & $1,1,1$-Trichloroethane & 0.50 & $\mathrm{U}$ \\
\hline $110-82-7$ & Cyclohexane & 0.50 & $\mathrm{U}$ \\
\hline $56-23-5$ & Carbon tetrachloride & 0.50 & $\mathrm{U}$ \\
\hline $71-43-2$ & Benzene & 0.50 & $\mathrm{U}$ \\
\hline $107-06-2$ & 1,2-Dichloroethane & 0.50 & $\mathrm{U}$ \\
\hline
\end{tabular}

Report 1,4-Dioxane for Low-Medium VOA analysis only 
Lab Name: TESTAMERICA BURLINGTON

Lab Code: STLV Case No.: MORRIL Mod. Ref No.:
Contract: $8 \mathrm{E}-00302$ SDG No.: 200-1702
Matrix: (SOIL/SED/WATER) Water

Sample wt/vol: 25.0 $(\mathrm{g} / \mathrm{mL}) \mathrm{mL}$

Level: (TRACE/LOW/MED) TRACE

응 Moisture: not dec.

GC Column: DB-624 ID $: 0.20 \quad(\mathrm{~mm})$

Soil Extract Volume: (uI)

Purge Volume: 25.0 (mL)
Lab Sample ID: 200-1702-6

Lab File ID: JBOC11.D

Date Received: 09/24/2010

Date Analyzed: 09/29/2010

Dilution Factor: 1.0

Soil Aliquot Volume: (uL)

\begin{tabular}{|c|c|c|c|}
\hline CAS NO. & COMPOUND & $\begin{array}{l}\text { CONCENTRATION UNITS: } \\
(\mathrm{ug} / \mathrm{L} \text { or } \mathrm{ug} / \mathrm{kg}) \mathrm{ug} / \mathrm{L}\end{array}$ & $Q$ \\
\hline $79-01-6$ & Trichloroethene & 0.50 & $\bar{U}$ \\
\hline $108-87-2$ & Methylcyclohexane & 0.50 & $\bar{U}$ \\
\hline $78-87-5$ & 1,2-Dichloropropane & 0.50 & $\mathrm{U}$ \\
\hline $75-27-4$ & Bromodichloromethane & 0.50 & $\mathrm{U}$ \\
\hline $10061-01-5$ & cis-1,3-Dichloropropene & 0.50 & $\overline{\mathrm{U}}$ \\
\hline $108-10-1$ & 4-Methyl-2-pentanone & 5.0 & $\mathrm{U}$ \\
\hline $108-88-3$ & Toluene & 0.50 & $\overline{\mathrm{U}}$ \\
\hline $10061-02-6$ & trans-1,3-Dichloropropene & 0.50 & $\mathrm{U}$ \\
\hline $79-00-5$ & 1,1,2-Trichloroethane & 0.50 & $\mathrm{U}$ \\
\hline $127-18-4$ & Tetrachloroethene & 0.50 & $\mathrm{U}$ \\
\hline $591-78-6$ & 2-Hexanone & 5.0 & $\mathrm{U}$ \\
\hline $124-48-1$ & Dibromochloromethane & 0.50 & $\mathrm{U}$ \\
\hline $106-93-4$ & 1,2-Dibromoethane & 0.50 & $\mathrm{U}$ \\
\hline $108-90-7$ & Chlorobenzene & 0.50 & $\mathrm{U}$ \\
\hline $100-41-4$ & Ethylbenzene & 0.50 & $\mathrm{U}$ \\
\hline $95-47-6$ & o-xylene & 0.50 & U. \\
\hline $179601-23-1$ & $\mathrm{~m}, \mathrm{p}$-xylene & 0.50 & $\mathrm{U}$ \\
\hline $100-42-5$ & Styrene & 0.50 & $\mathrm{U}$ \\
\hline $75-25-2$ & Bromoform & 0.50 & $\overline{\mathrm{U}}$ \\
\hline $98-82-8$ & Isopropylbenzene & 0.50 & $\mathrm{U}$ \\
\hline $79-34-5$ & $1,1,2,2$-Tetrachloroethane & 0.50 & $\mathrm{U}$ \\
\hline $541-73-1$ & 1,3-Dichlorobenzene & 0.50 & $\mathrm{U}$ \\
\hline $106-46-7$ & 1,4-Dichlorobenzene & 0.50 & $\mathrm{U}$ \\
\hline $95-50-1$ & 1,2-Dichlorobenzene & 0.50 & $\mathrm{U}$ \\
\hline $96-12-8$ & 1,2-Dibromo-3-Chloropropane & 0.50 & $\mathrm{U}$ \\
\hline $120-82-1$ & 1,2,4-Trichlorobenzene & 0.50 & $\mathrm{U}$ \\
\hline $87-61-6$ & 1,2,3-Trichlorobenzene & 0.50 & $\mathrm{U}$ \\
\hline
\end{tabular}


$1 \mathrm{~J}$ - FORM I VOA-TIC

VOLATILE ORGANICS ANALYSIS DATA SHEET

TENTATIVELY IDENTIFIED COMPOUNDS
EPA SAMPLE NO. MRISCH $-\mathrm{W}-30022$

Lab Name: TESTAMERICA BURLINGTON

Lab Code: STLV Case No.: MORRIL Mod. Ref No.:

Contract: $8 \mathrm{E}-00302$

Matrix: (SOIL/SED/WATER)

Sample wt/vol: 25.0

Level: (TRACE or LOW/MED)

Water

$(\mathrm{g} / \mathrm{mL}) \mathrm{mL}$

TRACE

\% Moisture: not dec.

GC Column: DB-624

Soil Extract Volume: ID $: 0.20 \quad(\mathrm{~mm})$ (uL)

CONCENTRATION UNITS: (ug/L Or ug/kg) ug/L
SDG No.: 200-1702

Lab Sample ID: 200-1702-6

Lab File ID: JBOC11.D

Date Received: 09/24/2010

Date Analyzed: 09/29/2010

Dilution Factor: 1.0

Soil Aliquot Volume:

(uL)

Purge volume: 25:0

$(\mathrm{mL})$

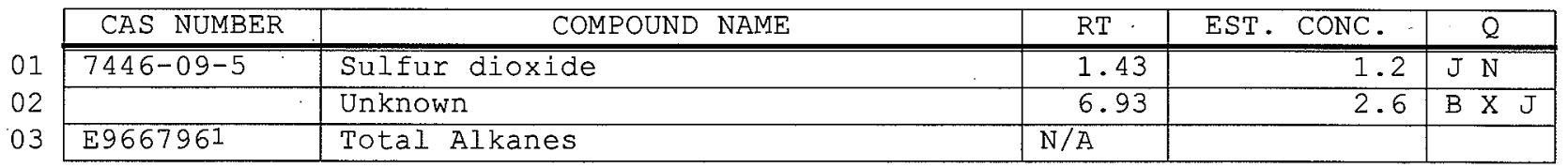

lEPA-designated Registry Number. 
Lab Name: TESTAMERICA BURLINGTON

Lab Code: STLV

Matrix: (SOIL/SED/WATER)

Sample wt/vol: 25.0

Case No.: MORRIL Mod. Ref No.:

Contract: $8 \mathrm{E}-00302$

Level: (TRACE/LOW/MED) TRACE

응 Moisture: not dec.

GC Column: DB-624

ID $: 0.20$

$(\mathrm{mm})$

(uL)
(mL)
SDG No.: 200-1702

Lab Sample ID: 200-1702-4

Lab File ID: JBOC08.D

Date Received: 09/24/2010

Date Analyzed: 09/29/2010

Dilution Factor: 1.0

Soil Aliquot Volume: (UL)

\begin{tabular}{|c|c|c|c|}
\hline CAS NO. & COMPOUND & $\begin{array}{l}\text { CONCENTRATION UNITS: } \\
\text { (ug/L or } u g / k g) u g / I\end{array}$ & $Q$ \\
\hline $75-71-8$ & Dichlorodifluoromethane & 0.50 & $\overline{\mathrm{U}}$ \\
\hline $74-87-3$ & Chloromethane & 0.50 & $\mathrm{U}$ \\
\hline $75-01-4$ & Vinyl chloride & 0.50 & $\mathrm{U}$ \\
\hline $74-83-9$ & Bromomethane & 0.50 & $\mathrm{U}$ \\
\hline $75-00-3$ & Chloroethane & 0.50 & $\mathrm{U}$ \\
\hline $75-69-4$ & Trichlorofluoromethane & 0.50 & $\mathrm{U}$ \\
\hline $75-35-4$ & 1,1-Dichloroethene & 0.50 & $\mathrm{U}$ \\
\hline $76-13-1$ & $1,1,2$-Trichloro-1,2,2-trifluoroethane. & 0.50 & $\mathrm{U}$ \\
\hline $67-64-1$ & Acetone & 2.3 & $\mathrm{~J} B$ \\
\hline $75-15-0$ & Carbon disulfide & 0.24 & $\mathrm{~J} B$ \\
\hline $79-20-9$ & Methyl acetate & 0.50 & $\mathrm{U}$ \\
\hline $75-09-2$ & Methylene Chloride & 0.50 & $\mathrm{U}$ \\
\hline $156-60-5$ & trans-1,2-Dichloroethene & 0.50 & $\mathrm{U}$ \\
\hline $1634-04-4$ & Methyl tert-butyl ether & 0.50 & $\mathrm{U}$ \\
\hline $75-34-3$ & 1,1-Dichloroethane & 0.50 & $\mathrm{U}$ \\
\hline $156-59-2$ & cis-1,2-Dichloroethene & 0.50 & $\mathrm{U}$ \\
\hline $78-93-3$ & 2-Butanone & 5.0 & $\mathrm{U}$ \\
\hline $74-97-5$ & Bromochloromethane & 0.50 & $\mathrm{U}$ \\
\hline $67-66-3$ & Chloroform & 0.85 & \\
\hline $71-55-6$ & 1,1,1-Trichloroethane & 0.50 & $\mathrm{U}$ \\
\hline $110-82-7$ & Cyclohexane & 0.50 & $\mathrm{U}$ \\
\hline $56-23-5$ & Carbon tetrachloride & 25 & $\mathrm{E}$ \\
\hline $71-43-2$ & Benzene & 0.50 & $\mathrm{U}$ \\
\hline $107-06-2$ & 1,2-Dichloroethane & 0.50 & $\mathrm{U}$ \\
\hline
\end{tabular}

Report 1,4-Dioxane for Low-Medium VOA analysis only 
Lab Name: TESTAMERICA BURLINGTON

Làb Code: STLV

Matrix: (SOIL/SED/WATER)

Sample wt/vol: 25.0

Case No.: MORRIL Mod. Ref No.:

Contract: $8 \mathrm{E}-00302$

Level: (TRACE/LOW/MED) TRACE

응 Moisture: not dec.

GC Column: DB-624

ID $: 0.20$ (mm)

Soil Extract Volume:
Water

$(\mathrm{g} / \mathrm{mL}) \mathrm{mI}$
SDG No.: 200-1702

Lab Sample ID: 200-1702-4

Lab File ID: JBOC08.D

Date Received: 09/24/2010

Date Analyzed: 09/29/2010

Dilution Factor: 1.0

Soil Aliquot Volume:

(uL)

(uL)

Purge Volume: 25.0

(mL)

\begin{tabular}{|c|c|c|c|}
\hline CAS NO. & COMPOUND & $\begin{array}{l}\text { CONCEENTRATION UNITS: } \\
(\mathrm{ug} / \mathrm{I} \text { or } u g / \mathrm{kg}) \mathrm{ug} / \mathrm{I}\end{array}$ & $Q$ \\
\hline $79-01-6$ & Trichloroethene & 0.50 & $\overline{\mathrm{U}}$ \\
\hline $108-87-2$ & Methylcyclohexane & 0.50 & $\mathrm{U}$ \\
\hline $78-87-5$ & 1,2-Dichloropropane & 0.50 & $\mathrm{U}$ \\
\hline $75-27-4$ & Bromodichloromethane & 0.50 & $\bar{U}$ \\
\hline $10061-01-5$ & cis-1,3-Dichloropropene & 0.50 & $\bar{U}$ \\
\hline $108-10-1$ & 4-Methyl-2-pentanone & 5.0 & $\bar{U}$ \\
\hline $108-88-3$ & Toluene & 0.50 & $\mathrm{U}$ \\
\hline $10061-0.2-6$ & trans-1,3-Dichloropropene & 0.50 & $\mathrm{U}$ \\
\hline $79-00-5$ & $1,1,2-$ Trichloroethane & 0.50 & $\mathrm{U}$ \\
\hline $127-18-4$ & Tetrachloroethene & 0.50 & $\bar{U}$ \\
\hline $591-78-6$ & 2-Hexanone & 5.0 & $\mathrm{U}$ \\
\hline $124-48-1$ & Dibromochloromethane & 0.5 .0 & $\mathrm{U}$ \\
\hline $106-93-4$ & 1,2-Dibromoethane & 0.50 & $\mathrm{U}$ \\
\hline $108-90-7$ & Chlorobenzene & 0.50 & $\mathrm{U}$ \\
\hline $100-41-4$ & Ethylbenzene & 0.50 & $\mathrm{U}$ \\
\hline $95-47-6$ & o-Xylene & 0.50 & $\bar{U}$ \\
\hline $179601-23-1$ & m,p-xylene & 0.50 & $\bar{U}$ \\
\hline $100-42-5$ & Styrene & 0.50 & $\bar{U}$ \\
\hline $75-25-2$ & Bromoform & 0.50 & $\bar{U}$ \\
\hline $98-82-8$ & Isopropylbenzene. & 0.50 & $\mathrm{U}$ \\
\hline $79-34-5$ & $1,1,2,2-$ Tetrachloroethane & 0.50 & $\mathrm{U}$ \\
\hline $541-73-1$ & 1,3-Dichlorobenzene & 0.50 & $\mathrm{U}$ \\
\hline $106-46-7$ & 1,4-Dichlorobenzene & 0.50 & $\mathrm{U}$ \\
\hline $95-50-1$ & 1,2-Dichlorobenzene & 0.50 & $\mathrm{U}$ \\
\hline $96-12-8$ & 1,2-Dibromo-3-Chloropropane & 0.50 & $\mathrm{U}$ \\
\hline $120-82-1$ & $1,2,4$-Trichlorobenzene & 0.50 & $\mathrm{U}$ \\
\hline $87-61-6$ & $1,2,3$-Trichlorobenzene & 0.50 & $\mathrm{U}$ \\
\hline
\end{tabular}


IJ - FORM I VOA-TIC

VOLATILE ORGANICS ANALYSIS DATA SHEET

TENTATIVELY IDENTIFIED COMPOUNDS
EPA SAMPLE NO.

MRMW11S-W-30021
Lab Name: TESTAMERICA BURLINGTON

Lab Code: STLV

Matrix: (SOIL/SED/WATER)

Sample wt/vol: 25.0

Water

Level: (TRACE or LOW/MED) TRACE

\% Moisture: not dec.

GC Column: DB-624 ID: $0.20 \quad(\mathrm{~mm})$

Soil Extract Volume: (uL)

CONCENTRATION UNITS: (ug/L or $\mathrm{ug} / \mathrm{kg}$ ) ug/L
Contract: $8 \mathrm{E}-00302$ SDG No.: 200-1702

Laḅ Sample ID: 200-1702-4

Lab. File ID: JBOC08.D

Date Received: 09/24/2010

Date Analyzed: 09/29/2010

Dilution Factor: 1.0

Soil Aliquot volume: (ù)

Purge Volume: 25.0 $(\mathrm{mL})$

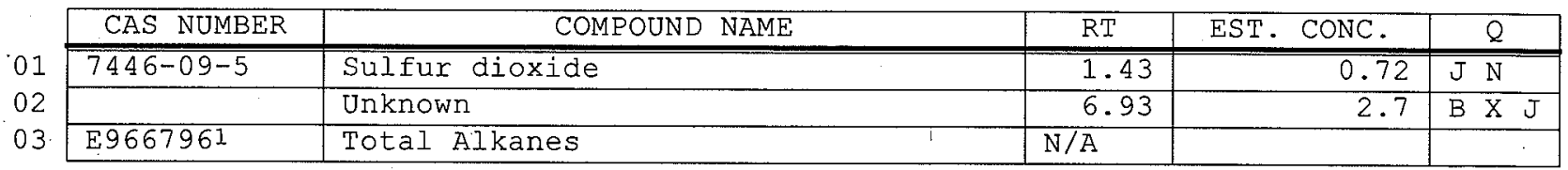

1EPA-designated Registry Number. 
$1 A$ - FORM I VOA-1

VOLATILE ORGANICS ANALYSIS DATA SHEET
EPA SAMPLE NO.

MRMW11S-W-30021DL
Lab Name: TESTAMERICA BURLINGTON

Lab Code: STLV Case No.: MORRIL Mod. Ref No.:
Contract: $8 \mathrm{E}-00302$ SDG NO.: 200-1702
Matrix: (SOIL/SED/WATER) Water

Sample wt/vol: 25.0 $(\mathrm{g} / \mathrm{mL}) \mathrm{mL}$

Level: (TRACE/LOW/MED) TRACE

응 Moisture: not dec.

GC Column: DB-624 ID: $0.20 \quad(\mathrm{~mm})$

Soil Extract Volume: (uL)

Purge Volume: 25.0 (mi)
Lab Sample ID: 200-1702-4

Lab File ID: JBOC12.D

Date Received: 09/24/2010

Date Analyzed: 09/29/2010

Dilution Factor: 1.9

Soil Aliquot Volume:

(uL)

\begin{tabular}{|c|c|c|c|}
\hline CAS NO. & COMPOUND & $\begin{array}{l}\text { CONCENTRATION UNITS: } \\
(\mathrm{ug} / \mathrm{L} \text { or } \mathrm{ug} / \mathrm{kg}) \mathrm{ug} / \mathrm{L}\end{array}$ & $Q$ \\
\hline $75-71-8$ & Dichlorodifluoromethane & 0.95 & $\overline{\mathrm{U}}$ \\
\hline $74-87-3$ & Chloromethane & 0.95 & $\mathrm{U}$ \\
\hline $75-01-4$ & Vinyl chloride & 0.95 & $\mathrm{U}$ \\
\hline $74-83-9$ & Bromomethane & 0.95 & $\mathrm{U}$ \\
\hline $75-00-3$ & Chloroethane & 0.95 & $\mathrm{U}$ \\
\hline $75-69-4$ & Trichlorofluoromethane & 0.95 & $\bar{U}$ \\
\hline $75-35-4$ & 1,1-Dichloroethene & 0.95 & $\overline{\mathrm{U}}$ \\
\hline $76-13-1$ & 1,1,2-Trichloro-1,2,2-trifluoroethane & 0.95 & $\overline{\mathrm{U}}$ \\
\hline $67-64-1$ & Acetone & 4.1 & J B D \\
\hline $75-15-0$ & Carbon disulfide & 0.35 & $J$ B D \\
\hline $79-20-9$ & Methyl acetate & 0.95 & $\mathrm{U}$ \\
\hline $75-09-2$ & Methylene Chloride & 0.95 & $\mathrm{U}$ \\
\hline $156-60-5$ & trans-1,2-Dichloroethene & 0.95 & $\mathrm{U}$ \\
\hline $1634-04-4$ & Methyl tert-butyl ether & 0.95 & $\mathrm{U}$ \\
\hline $75-34-3$ & 1,1-Dichloroethane & 0.95 & $\mathrm{U}$ \\
\hline $156-59-2$ & cis-1,2-Dichloroethene & 0.95 & $\mathrm{U}$ \\
\hline $78-93-3$ & 2-Butanone & 9.5 & $\mathrm{U}$ \\
\hline $74-97-5$ & Bromochloromethane & 0.95 & $\overline{\mathrm{U}}$ \\
\hline $67-66-3$ & Chloroform & 0.77 & J D \\
\hline $71-55-6$ & 1,1,1-Trichloroethane & 0.95 & $\bar{U}$ \\
\hline $110-82-7$ & Cyclohexane & 0.95 & $\mathrm{U}$ \\
\hline $56-23-5$ & Carbon tetrachloride & 19 & $\mathrm{D}$ \\
\hline $71-43-2$ & Benzene & 0.95 & $\mathrm{U}$ \\
\hline $107-06-2$ & 1,2-Dichloroethane & 0.95 & $\mathrm{U}$ \\
\hline
\end{tabular}

Report 1,4-Dioxane for Low-Medium VOA analysis only 
Lab Name: TESTAMERICA BURLINGTON

Lab Code: STLV Case No.: MORRIL Mod. Ref No.:

Contract: $8 \mathrm{E}-00302$

Matrix: (SOIL/SED/WATER) Water

Sample wt/vol: 25.0 $(\mathrm{g} / \mathrm{mL}) \mathrm{mL}$

Level: (TRACE/LOW/MED) TRACE

\% Moisture: not dec.

GC Column: DB-624

ID: 0.20 (mm)

Soil Extract Volume: (uL) Purge Volume: 25.0 (mL)
Lab Sample ID: 200-1702-4

Lab File ID: JBOC12.D

Date Received: 09/24/2010

Date Analyzed: 09/29/2010

Dilution Factor: 1.9

Soil Aliquot. Volume: (uL)

\begin{tabular}{|c|c|c|c|}
\hline CAS NO. & COMPOUND & $\begin{array}{l}\text { CONCENTRATION UNITS: } \\
(\mathrm{ug} / \mathrm{L} \text { or } \mathrm{ug} / \mathrm{kg}) \mathrm{ug} / \mathrm{L}\end{array}$ & $Q$ \\
\hline $79-01-6$ & Trichloroethene & 0.95 & $\mathrm{U}$ \\
\hline $108-87-2$ & Methylcyclohexane & 0.95 & $\mathrm{U}$ \\
\hline $78-87-5$ & 1,2-Dichloropropane & 0.95 & $\bar{U}$ \\
\hline $75-27-4$ & Bromodichloromethane & 0.95 & $\bar{U}$ \\
\hline $10061-01-5$ & cis-1,3-Dichloropropene & 0.95 & $\bar{U}$ \\
\hline $108-10-1$ & 4-Methyl-2-pentanone & 9.5 & $\mathrm{U}$ \\
\hline $108-88-3$ & Toluene & 0.95 & $\mathrm{U}$ \\
\hline $10061-02-6$ & trans-1,3-Dichloropropene & 0.95 & $\mathrm{U}$ \\
\hline $79-00-5$ & $1,1,2$-Trichloroethane & 0.95 & $\mathrm{U}$ \\
\hline $127-18-4$ & Tetrachloroethene & 0.95 & $\mathrm{U}$ \\
\hline $591-78-6$ & 2-Hexanone & 9.5 & $\mathrm{U}$ \\
\hline $124-48-1$ & Dibromochloromethane & 0.95 & $\mathrm{U}$ \\
\hline $106-93-4$ & 1,2-Dibromoethane & 0.95 & $\mathrm{U}$ \\
\hline $108-90-7$ & Chlorobenzene & 0.95 & $\mathrm{U}$ \\
\hline $100-41-4$ & Ethylbenzene & 0.95 & $\overline{\mathrm{U}}$ \\
\hline $95-47-6$ & o-xylene & 0.95 & $\mathrm{U}$ \\
\hline $179601-23-1$ & m,p-xylene & 0.95 & $\mathrm{U}$ \\
\hline $100-42-5$ & Styrene & 0.95 & $\mathrm{U}$ \\
\hline $75-25-2$ & Bromoform & 0.95 & $\mathrm{U}$ \\
\hline $98-82-8$ & Isopropylbenzene & 0.95 & $\mathrm{U}$ \\
\hline $79-34-5$ & $1,1,2,2$-Tetrachloroethane & 0.95 & $\bar{U}$ \\
\hline $541-73-1$ & 1,3-Dichlorobenzene & 0.95 & $\mathrm{U}$ \\
\hline $106-46-7$ & 1,4-Dichlorobenzene & 0.95 & $\mathrm{U}$ \\
\hline $95-50-1$ & 1,2-Dichlorobenzene & 0.95 & $\mathrm{U}$ \\
\hline $96-12-8$ & 1,2-Dibromo-3-Chloropropane & 0.95 & $\mathrm{U}$ \\
\hline $120-82-1$ & $1,2,4$-Trichlorobenzene & 0.95 & $\mathrm{U}$ \\
\hline $87-61-6$ & 1,2,3-Trichlorobenzene & 0.95 & $\mathrm{U}$ \\
\hline
\end{tabular}


$1 \mathrm{~J}$ - FORM I VOA-TIC

VOLATILE ORGANICS ANALYSIS DATA SHEET

TENTATIVELY IDENTIFIED COMPOUNDS
EPA SAMPLE NO.

MRMW11S-W-30021DL

Lab Name: TESTAMERICA BURLINGTON

Contract: $8 \mathrm{E}-00302$

Lab Code: STLV Case No.: MORRIL Mod. Ref No.: SDG No.: 200-1702

Matrix: (SOIL/SED/WATER) Water

Lab Sample ID: 200-1702-4

Sample wt/vol: 25.0 $(\mathrm{g} / \mathrm{mL}) \mathrm{mI}$

Lab File ID: JBOC12.D

Level: (TRACE or LOW/MED) TRACE

\% Moisture: not dec.

GC Column: DB-624 ID: 0.20 (mm)

Date Received: 09/24/2010

Date Analyzed: 09/29/2010

Soil Extract Volume: (uL)

Dilution Factor: 1.9

CONCENTRATION UNITS: (ug/L or ug/kg) ug/L

Soil Aliquot Volume: (uL)

Purge Volume: 25.0 (mL)

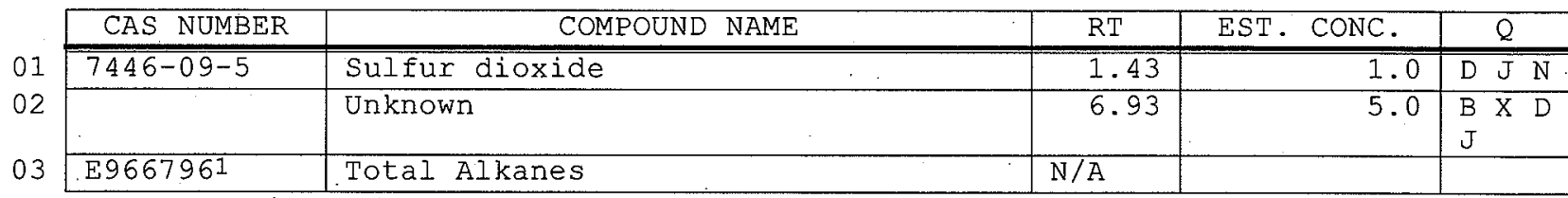

1 EPA-designated Registry Number. 
Lab Name: TESTAMERICA BURLINGTON

Lab Code: STLV Case No.: MORRIL Mod. Ref No.:
Contract: $8 \mathrm{E}-00302$

SDG No.: 200-1702
Matrix: (SOIL/SED/WATER) Water

Sample wt/vol: 25.0 $(\mathrm{g} / \mathrm{mL}) \mathrm{mL}$

Level: (TRACE/LOW/MED) TRACE

o Moisture: not dec.

GC Column: $\mathrm{DB}-624$ ID: 0.20 $(\mathrm{mm})$

Soil Extract Volume: (uI)

Purge Volume: 25.0 (mL)
Lab Sample ID: 200-1702-1

Lab File ID: JBOC14.D

Date Received: 09/24/2010

Date Analyzed: 09/29/2010

Dilution Factor: 1.1

Soil Aliquot volume:

(uL)

\begin{tabular}{|c|c|c|c|}
\hline CAS NO. & COMPOUND & $\begin{array}{l}\text { CONCENTRATION UNITS: } \\
(\mathrm{ug} / \mathrm{L} \text { or } \mathrm{ug} / \mathrm{kg}) \mathrm{ug} / \mathrm{I}\end{array}$ & $Q$ \\
\hline $75-71-8$ & Dichlorodifluoromethane & 0.55 & $\mathrm{U}$ \\
\hline $74-87-3$ & Chloromethane & 0.55 & $\mathrm{U}$ \\
\hline $75-01-4$ & Vinyl chloride & 0.55 & $\mathrm{U}$ \\
\hline $74-83-9$ & Bromomethane & 0.55 & $\mathrm{U}$ \\
\hline $75-00-3$ & Chloroethane & 0.55 & $\mathrm{U}$ \\
\hline $75-69-4$ & Trichlorofluoromethane & 0.55 & $\mathrm{U}$ \\
\hline $75-35-4$ & 1,1-Dichloroethene & 0.55 & $\mathrm{U}$ \\
\hline $76-13-1$ & $1,1,2$-Trichloro-1,2,2-trifluoroethane & 0.55 & $\mathrm{U}$ \\
\hline $67-64-1$ & Acetone & 2.1 & $\mathrm{~J} B$ \\
\hline $75-15-0$ & Carbon disulfide & 0.18 & $J B$ \\
\hline $79-20-9$ & Methyl acetate & 0.55 & $\mathrm{U}$ \\
\hline $75-09-2$ & Methylene Chloride & 0.55 & $\overline{\mathrm{U}}$ \\
\hline $156-60-5$ & trans-1,2-Dichloroethene & 0.55 & $\mathrm{U}$ \\
\hline $1634-04-4$ & Methyl tert-butyl ether & 0.55 & $\dot{U}$ \\
\hline $75-34-3$ & 1,1-Dichloroethane & 0.55 & $\mathrm{U}$ \\
\hline $156-59-2$ & cis-1,2-Dichloroethene & 0.55 & $\mathrm{U}$ \\
\hline $78-93-3$ & 2-Butanone & 5.5 & $\bar{U}$ \\
\hline $74-97-5$ & Bromochloromethané & 0.55 & $\mathrm{U}$ \\
\hline $67-66-3$ & Chloroform & 1.2 & \\
\hline $71-55-6$ & 1,1,1-Trichloroethane & 0.55 & $\mathrm{U}$ \\
\hline $110-82-7$ & Cyclohexane & 0.55 & $\overline{\mathrm{U}}$ \\
\hline $56-23-5$ & Carbon tetrachloride & 32 & E \\
\hline $71-43-2$ & Benzene & 0.55 & $\mathrm{U}$ \\
\hline $107-06-2$ & 1,2-Dichloroethane & 0.55 & $\bar{U}$ \\
\hline
\end{tabular}

Report 1,4-Dioxane for Low-Medium VOA analysis only 
$1 B$ - FORM I VOA-2

VOLATILE ORGANICS ANALYSIS DATA SHEET
EPA SAMPLE NO.

MRMW $3 S-W-30013$
Lab Name: TESTAMERICA BURLINGTON

Lab Code: STLV

Matrix: (SOIL/SED/WATER)

Sample wt/vol: 25:0

water

Level: (TRACE/LOW/MED) TRACE

\% Moisture: not dec.

GC Column: DB-624 ID $: 0.20$ (mm)

Soil Extract Volume: (uL) $(\mathrm{mL})$

Purge Volume: 25.0
Contract: $8 \mathrm{E}-00302$

SDG No.: 200-1702

Lab Sample ID: 200-1702-1

Lab File ID: JBOC14.D

Date Received: 09/24/2010

Date Analyzed: 09/29/2010

Dilution Factor: 1.1

Soil Aliquot volume: (uL)

\begin{tabular}{|c|c|c|c|}
\hline CAS NO. & COMPOUND & $\begin{array}{l}\text { CONCENTRATION UNITS: } \\
(\mathrm{ug} / \mathrm{L} \text { or } u g / \mathrm{kg}) \mathrm{ug} / \mathrm{L}\end{array}$ & $Q$ \\
\hline $79-01-6$ & Trichloroethene & 0.55 & $\overline{\mathrm{U}}$ \\
\hline $108-87-2$ & Methylcyclohexane & 0.55 & $\mathrm{U}$ \\
\hline $78-87-5$ & 1,2-Dichloropropane & 0.55 & $\mathrm{U}$ \\
\hline $75-27-4$ & Bromodichloromethane & 0.55 & $\mathrm{U}$ \\
\hline $10061-01-5$ & cis-1,3-Dichloropropene & 0.55 & $\mathrm{U}$ \\
\hline $108-10-1$ & 4-Methyl-2-pentanone & 5.5 & $\mathrm{U}$ \\
\hline $108-88-3$ & Toluene & 0.55 & $\mathrm{U}$ \\
\hline $10061-02-6$ & trans-1,3-Dichloropropene & 0.55 & $\mathrm{U}$ \\
\hline $79-00-5$ & $1,1,2$-Trichloroethane & 0.55 & $\mathrm{U}$ \\
\hline $127-18-4$ & Tetrachloroethene & 0.55 & $\bar{U}$ \\
\hline $591-78-6$ & 2-Hexanone & 5.5 & $\mathrm{U}$ \\
\hline $124-48-1$ & Dibromochloromethane & 0.55 & $\mathrm{U}$ \\
\hline $106-93-4$ & 1,2-Dibromoethane & 0.55 & $\mathrm{U}$ \\
\hline $108-90-7$ & Chlorobenzene & 0.55 & $\mathrm{U}$ \\
\hline $100-41-4$ & Ethylbenzene & 0.55 & $\mathrm{U}$ \\
\hline $95-47-6$ & o-Xylene & 0.55 & $\mathrm{U}$ \\
\hline $179601-23-1$ & $\mathrm{~m}, \mathrm{p}$-Xylene & 0.55 & $\overline{\mathrm{U}}$ \\
\hline $100-42-5$ & styrene & 0.55 & $\overline{\mathrm{U}}$ \\
\hline $75-25-2$ & Bromoform & 0.55 & $\mathrm{U}$ \\
\hline $98-82-8$ & Isopropylbenzene & 0.55 & $\mathrm{U}$ \\
\hline $79-34-5$ & $1,1,2,2$-Tetrachloroethane & 0.55 & $\overline{\mathrm{U}}$ \\
\hline $541-73-1$ & 1,3-Dichlorobenzene & 0.55 & $\mathrm{U}$ \\
\hline $106-46-7$ & 1,4-Dichlorobenzene & 0.55 & $\bar{U}$ \\
\hline $95-50-1$ & 1,2-Dichlorobenzene & 0.55 & $\bar{U}$ \\
\hline $96-12-8$ & 1,2-Dibromo-3-Chloropropane & 0.55 & $\mathrm{U}$ \\
\hline $120-82-1$ & 1,2,4-Trichlorobenzene & 0.55 & $\mathrm{U}$ \\
\hline $87-61-6$ & 1,2,3-Trichlorobenzene & 0.55 & $\mathrm{U}$ \\
\hline
\end{tabular}


$1 \mathrm{~J}$ - FORM I VOA-TIC

VOLATILE ORGANICS ANALYSIS DATA SHEET

TENTATIVELY IDENTIFIED COMPOUNDS
EPA. SAMPLE NO.

MRMW3S-W-30013

Lab Name: TESTAMERICA BURLINGTON

Contract: $8 \mathrm{E}-00302$

Lab Code: STLV

Case No.: MORRIL Mod. Ref No.: SDG No.: 200-1702

Matrix: (SOIL/SED/WATER)

Sample wt/vol: 25.0

Level: (TRACE or LOW/MED) TRACE

Moisture: not dec.

GC Column: DB-624 ID $: 0.20 \quad(\mathrm{~mm})$

Soil Extract Volume: (uL)

CONCENTRATION UNITS: (ug/L or $\mathrm{ug} / \mathrm{kg}$ ) ug/L
Lab Sample ID: 200-1702-1

Lab File ID: JBOC14.D

Date Received: 09/24/2010

Date Analyzed: 09/29/2010

Dilution Factor: 1.1

Soil Aliquot Volume: (uL)

Purge Volume: 25.0 (mL)

\begin{tabular}{l|l|l|c|r|r|}
\hline \multicolumn{1}{|c|}{ CAS NUMBER } & \multicolumn{1}{|c|}{ COMPOUND NAME } & RT & EST. CONC. & Q \\
\cline { 2 - 6 } 01 & $7446-09-5$ & Sulfur dioxide & 1.43 & 0.71 & $\mathrm{~J} \mathrm{~N}$ \\
\hline & & Unknown & 6.93 & $\mathrm{~B}$ X J \\
\cline { 2 - 6 } 03 & E9667961 & Total Alkanes & N/A & & \\
\cline { 2 - 6 }
\end{tabular}

lEPA-designated Registry Number. 
$1 A$ - FORM I VOA-1

VOLATILE ORGANICS ANALYSIS DATA SHEET
EPA SAMPLE NO.

MRMW3S-W-30013DI
Lab Name: TESTAMERICA BURLINGTON

Lab Code: STLV Case No.: MORRIL Mod. Ref No.:
Contract: $8 \mathrm{E}-00302$ SDG No.: 200-1702
Matrix: (SOIL/SED/WATER) Water

Sample wt/vol: 25.0 $(\mathrm{g} / \mathrm{mL}) \mathrm{mL}$

Level: (TRACE/LOW/MED) TRACE

\% Moisture: not dec.

GC Column: DB-624 ID: 0.20 (mrn)

Soil Extract Volume: (uL)

Purge volume: 25.0 (mL)
Lab Sample ID: 200-1702-1

Lab File ID: JBOC13.D

Date Received: 09/24/2010

Date Analyzed: 09/29/2010

Dilution Factor: 3.0

Soil Aliquot Volume:

\begin{tabular}{|c|c|c|c|}
\hline CAS NO. & COMPOUND & $\begin{array}{l}\text { CONCENTRATION UNITS: } \\
(\mathrm{ug} / \mathrm{L} \text { or } \mathrm{ug} / \mathrm{kg}) \mathrm{ug} / \mathrm{L}\end{array}$ & Q \\
\hline $75-71-8$ & Dichlorodifluoromethane & 1.5 & $\mathrm{U}$ \\
\hline $74-87-3$ & Chloromethane & 1.5 & $\mathrm{U}$ \\
\hline $75-01-4$ & Vinyl chloride & 1.5 & $\mathrm{U}$ \\
\hline $74-83-9$ & Bromomethane & 1.5 & $\mathrm{U}$ \\
\hline $75-00-3$ & Chloroethane & 1.5 & $\mathrm{U}$ \\
\hline $75-69-4$ & Trichlorofluoromethane & 1.5 & $\mathrm{U}$ \\
\hline $75-35-4$ & 1,1-Dichloroethene & 1.5 & $\mathrm{U}$ \\
\hline $76-13-1$ & 1,1,2-Trichloro-1,2,2-trifluoroethane & 1.5 & $\mathrm{U}$ \\
\hline $67-64-1$ & Acetone & 6.9 & $\mathrm{~J} \quad \mathrm{~B} \quad \mathrm{D}$ \\
\hline $75-15-0$ & Carbon disulfide & 0.37 & $\mathrm{~J} B \mathrm{D}$ \\
\hline $79-20-9$ & Methyl acetate & 1.5 & $\mathrm{U}$ \\
\hline $75-09-2$ & Methylene Chloride & 1.5 & $\mathrm{U}$ \\
\hline $156-60-5$ & trans-1,2-Dichloroethene & 1.5 & $\mathrm{U}$ \\
\hline $1634-04-4$ & Methyl tert-butyl ether & 1.5 & $\mathrm{U}$ \\
\hline $75-34-3$ & 1,1-Dichloroethane & 1.5 & $\mathrm{U}$ \\
\hline $156-59-2$ & cis-1,2-Dichloroethene & 1.5 & $\mathrm{U}$ \\
\hline $78-93-3$ & 2-Butánone & 15 & $\mathrm{U}$ \\
\hline $74-97-5$ & Bromochloromethane & 1.5 & $\mathrm{U}$ \\
\hline $67-66-3$ & Chloroform & 1.2 & $J \mathrm{D}$ \\
\hline $71-55-6$ & 1,1,1-Trichloroethane & 1.5 & $\mathrm{U}$ \\
\hline $110-82-7$ & Cyclohexane & 1.5 & $\mathrm{U}$ \\
\hline $56-23-5$ & Carbon tetrachloride & 30 & $\mathrm{D}$ \\
\hline $71-43-2$ & Benzene & 1.5 & $\overline{\mathrm{U}}$ \\
\hline $107-06-2$ & 1,2-Dichloroethane & 1.5 & $\mathrm{U}$ \\
\hline
\end{tabular}

Report 1,4-Dioxane for Low-Medium VOA analysis only 
Iab Name: TESTAMERICA BURLINGTON

Lab Code: STLV

Matrix: (SOIL/SED/WATER)

Sample wt/vol: 25.0

Case No.: MORRII Mod. Ref No..

Contract: 8E-00302

Level: (TRACE/LOW/MED) TRACE

\% Moisture: not dec.

GC Column: DB-624 ID: $0.20 \quad(\mathrm{~mm})$

Soil Extract Volume:

(uI)

Purge Volume: 25.0

(mL)
SDG No.: 200-1702

Lab Sample ID: 200-1702-1

Lab File ID: JBOC13.D

Date Received: 09/24/2010

Date Analyzed: 09/29/2010

Dilution Factor: 3.0

Soil Aliquot volume: (uI)

\begin{tabular}{|c|c|c|c|}
\hline CAS NO. & COMPOUND & $\begin{array}{l}\text { CONCENTRATION UNITS: } \\
(\mathrm{ug} / \mathrm{I} \text { or } u g / \mathrm{kg}) \mathrm{ug} / \mathrm{L}\end{array}$ & $Q$ \\
\hline $79-01-6$ & Trichloroethene & 1.5 & $\overline{\mathrm{U}}$ \\
\hline $108-87-2$ & Methylcyclohexane & 1.5 & $\mathrm{U}$ \\
\hline $78-87-5$ & 1,2-Dichloropropane & 1.5 & $\mathrm{U}$ \\
\hline $75-27-4$ & Bromodichloromethane & 1.5 & $\mathrm{U}$ \\
\hline $10061-01-5$ & cis-1,3-Dichloropropene & 1.5 & $\mathrm{U}$ \\
\hline $108-10-1$ & 4-Methyl-2-pentanone & 15 & $\bar{U}$ \\
\hline $108-88-3$ & Toluene & 1.5 & $\mathrm{U}$ \\
\hline $10061-02-6$ & trans-1,3-Dichloropropene & 1.5 & $\mathrm{U}$ \\
\hline $79-00-5$ & $1,1,2$-Trichloroethane & 1.5 & $\mathrm{U}$ \\
\hline $127-18-4$ & Tetrachloroethene & 1.5 & $\mathrm{U}$ \\
\hline $591-78-6$ & 2-Hexanone & 15 & $\mathrm{U}$ \\
\hline $124-48-1$ & Dibromochloromethane & 1.5 & $\overline{\mathrm{U}}$ \\
\hline $106-93-4$ & 1,2-Dibromoethane & 1.5 & $\bar{U}$ \\
\hline $108-90-7$ & Chlorobenzene & 1.5 & $\bar{U}$ \\
\hline $100-41-4$ & Ethylbenzene & 1.5 & $\mathrm{U}$ \\
\hline $95-47-6$ & o-Xylene & 1.5 & $\overline{\mathrm{U}}$ \\
\hline $179601-23-1$ & $\mathrm{~m}, \mathrm{p}$-Xylene & 1.5 & $\mathrm{U}$ \\
\hline $100-42-5$ & styrene & 1.5 & $\overline{\mathrm{U}}$ \\
\hline $75-25-2$ & Bromoform & 1.5 & $\mathrm{U}$ \\
\hline $98-82-8$ & Isopropylbenzene & 1.5 & $\mathrm{U}$ \\
\hline $79-34-5$ & $1,1,2,2$-Tetrachloroethane & 1.5 & $\mathrm{U}$ \\
\hline $541-73-1$ & 1,3-Dichlorobenzene & 1.5 & $\mathrm{U}$ \\
\hline $106-46-7$ & 1,4-Dichlorobenzene & 1.5 & $\overline{\mathrm{U}}$ \\
\hline $95-50-1$ & 1,2-Dichlorobenzene & 1.5 & $\overline{\mathrm{U}}$ \\
\hline $96-12-8$ & 1,2-Dibromo-3-Chloropropane & 1.5 & $\overline{\mathrm{U}}$ \\
\hline $120-82-1$ & $1,2,4-T r i c h l o r o b e n z e n e$ & 1.5 & $\mathrm{U}$ \\
\hline $87-61-6$ & $1,2,3$-Trichlorobenzene & 1.5 & $\mathrm{U}$ \\
\hline
\end{tabular}


$1 \mathrm{~J}-$ FORM I VOA-TIC

VOLATILE ORGANICS ANALYSIS DATA SHEET

TENTATIVELY IDENTIFIED COMPOUNDS
EPA SAMPLE NO.

MRMW 3S-W-30013DL
Lab Name: TESTAMERICA BURLINGTON

Lab Code: STLV Cas

Matrix: (SOIL/SED/WATER) Water

Sample wt/vol: 25.0

$(\mathrm{g} / \mathrm{mL}) \mathrm{mL}$

Level: (TRACE or LOW/MED) TRACE

\% Moisture: not dec.

GC Column: DB-624 ID $: 0.20$ (mm)

Soil Extract Volume: (uL)

CONCENTRATION UNITS: (ug/L or $\mathrm{ug} / \mathrm{kg}) \mathrm{ug} / \mathrm{L}$
Contract: $8 \mathrm{E}-00302$

SDG No.: 200-1702

Lab Sample ID: 200-1702-1

Lab File ID: JBOC13.D

Date Received: 09/24/2010

Date Analyzed: 09/29/2010

Dilution Factor: 3.0

Soil Aliquot Volume: (uL)

Purge Volume: 25.0 (mL)

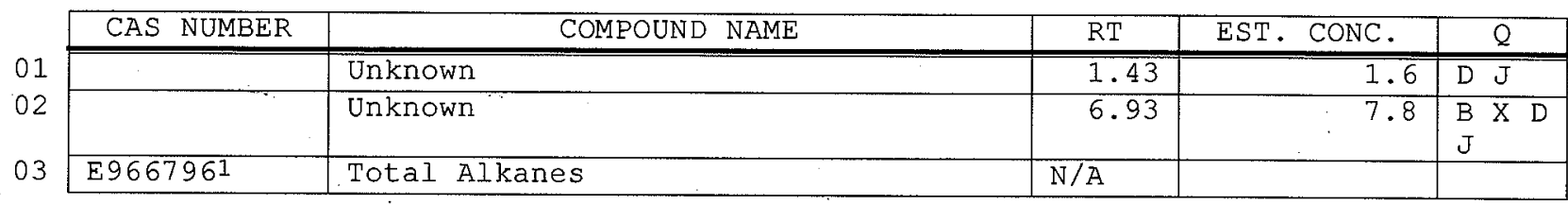

1 EPA-designated Registry Number. 
$1 A$ - FORM I VOA-1

VOLATILE ORGANICS ANALYSIS DATA SHEET
EPA SAMPLE NO.

MRMW6S-W-30016
Lab Name: TESTAMERICA BURLINGTON

Lab Code: STLV

Case No.: MORRIL Mod. Ref No.:
Contract: $8 \mathrm{E}-00302$ SDG No.: 200-1702
Matrix: (SOIL/SED/WATER) Water

Sample wt/vol: 25.0 $(\mathrm{g} / \mathrm{mL}) \mathrm{mL}$

Level: (TRACE/LOW/MED) TRACE

\% Moisture: not dec.

GC Column: DB-624 ID: $0.20 \quad(\mathrm{~mm})$

Soil Extract Volume: (uL)

Purge Volume: 25.0 $(\mathrm{mL})$
Lab Sample ID: 200-1702-2

Lab File ID: JBOC06.D

Date Received: 09/24/2010

Date Analyzed: 09/29/2010

Dilution Factor: 1.0

Soil Aliquot Volume:

(uL)

\begin{tabular}{|c|c|c|c|}
\hline CAS NO. & COMPOUND. & $\begin{array}{l}\text { CONCENTRATION UNITS: } \\
(\mathrm{ug} / \mathrm{L} \text { or } \mathrm{ug} / \mathrm{kg}) \mathrm{ug} / \mathrm{L}\end{array}$ & $Q$ \\
\hline $75-71-8$ & Dichlorodifluoromethane & 0.50 & $\overline{\mathrm{U}}$ \\
\hline $74-87-3$ & Chloromethane & 0.50 & $\mathrm{U}$ \\
\hline $75-01-4$ & Vinyl chloride & 0.50 & $\mathrm{U}$ \\
\hline $74-83-9$ & Bromomethane & 0.060 & $\mathrm{~J}$ \\
\hline $75-00-3$ & Chloroethane & 0.50 & $\mathrm{U}$ \\
\hline $75-69-4$ & Trichlorofluoromethane & 0.50 & $\mathrm{U}$ \\
\hline $75-35-4$ & 1,1-Dichloroethene & 0.50 & $\mathrm{U}$ \\
\hline $76-13-1$ & 1, 1,2-Trichloro-1,2,2-trifluoroethane & 0.50 & $\mathrm{U}$ \\
\hline $67-64-1$ & Acetone & 1.9 & $\mathrm{JB}$ \\
\hline $75-15-0$ & Carbon disulfide & 0.093 & $\mathrm{~J} \mathrm{~B}$ \\
\hline $79-20-9$ & Methyl acetate & 0.50 & $\mathrm{U}$ \\
\hline $75-09-2$ & Methylene Chloride & 0.50 & $\overline{\mathrm{U}}$ \\
\hline $156-60-5$ & trans-1,2-Dichloroethene & 0.50 & $\bar{U}$ \\
\hline $1634-04-4$ & Methyl tert-butyl ether & 0.50 & $\mathrm{U}$ \\
\hline $75-34-3$ & 1,1-Dichloroethane & 0.50 & $\mathrm{U}$ \\
\hline $156-59-2$ & cis-1,2-Dichloroethene & 0.50 & $\mathrm{U}$ \\
\hline $78-93-3$ & 2-Butanone & 5.0 & $\mathrm{U}$ \\
\hline $74-97-5$ & Bromochloromethane & 0.50 & $\mathrm{U}$ \\
\hline $67-66-3$ & Chloroform & 0.50 & $\mathrm{U}$ \\
\hline $71-55-6$ & 1,1,1-Trichloroethane & 0.50 & $\mathrm{U}$ \\
\hline $110-82-7$ & Cyclohexane & 0.50 & $\mathrm{U}$ \\
\hline $56-23-5$ & Carbon tetrachloride & 0.50 & $\mathrm{U}$ \\
\hline $71-43-2$ & Benzene & 0.50 & $\mathrm{U}$ \\
\hline $107-06-2$ & 1,2-Dichloroethane & 0.50 & $\mathrm{U}$ \\
\hline
\end{tabular}

Report 1,4-Dioxane for Low-Medium VOA analysis only 
Lab Name: TESTAMERICA BURLINGTON

Lab Code: STLV

Case No.: MORRIL Mod. Ref No.:
Contract: $8 \mathrm{E}-00302$

SDG NO.: 200-1702
Matrix: (SOIL/SED/WATER) Water

Sample wt/vol: 25.0 $(\mathrm{g} / \mathrm{mL}) \cdot \mathrm{mL}$

Level: (TRACE/LOW/MED) TRACE

\% Moisture: not dec.

GC Column: DB-624 ID: 0.20 (mm)

Sojil Extract Volume:

Purge Volume: 25.0 (mL)
Lab Sample ID: 200-1702-2

Lab File ID: JBOC06.D

Date Received: 09/24/2010

Date Analyzed: 09/29/2010

Dilution Factor: 1.0

Soil Aliquot volume:

\begin{tabular}{|c|c|c|c|}
\hline CAS NO. & COMPOUND & $\begin{array}{l}\text { CONCENTRATION UNITS: } \\
(\mathrm{ug} / \mathrm{L} \text { or } \mathrm{ug} / \mathrm{kg}) \mathrm{ug} / \mathrm{L} \\
\end{array}$ & $Q$ \\
\hline $79-01-6$ & Trichloroethene & 0.50 & $\overline{\mathrm{U}}$ \\
\hline $108-87-2$ & Methylcyclohexane & 0.50 & U \\
\hline $78-87-5$ & 1,2-Dichloropropane & 0.50 & U \\
\hline $75-27-4$ & Bromodichloromethane & 0.50 & U \\
\hline $10061-01-5$ & cis-1,3-Dichloropropene & 0.50 & $\mathrm{U}$ \\
\hline $108-10-1$ & 4-Methyl-2-pentanone & 5.0 & $\bar{U}$ \\
\hline $108-88-3$ & Toluene & 0.039 & $\bar{J}$ \\
\hline $10061-02-6$ & trans-1,3-Dichloropropene & 0.50 & $\mathrm{U}$ \\
\hline $79-00-5$ & $1,1,2$-Trichloroethane & 0.50 & U \\
\hline $127-18-4$ & Tetrachloroethene & 0.50 & $\mathrm{U}$ \\
\hline $591-78-6$ & 2-Hexanone & 5.0 & U \\
\hline $124-48-1$ & Dibromochloromethane & 0.50 & $\mathrm{U}$ \\
\hline $106-93-4$ & 1,2-Dibromoethane & 0.50 & $\mathrm{U}$ \\
\hline $108-90-7$ & Chlorobenzene & 0.50 & $\bar{U}$ \\
\hline $100-41-4$ & Ethylbenzene & 0.50 & U \\
\hline $95-47-6$ & o-xylene & 0.50 & $\mathrm{U}$ \\
\hline $179601-23-1$ & $\mathrm{~m}, \mathrm{p}$-Xylene & 0.50 & $\mathrm{U}$ \\
\hline $100-42-5$ & Styrene & 0.50 & $\mathrm{U}$ \\
\hline $75-25-2$ & Bromoform & 0.50 & $\mathrm{U}$ \\
\hline $98-82-8$ & Isopropylbenzene & 0.50 & $\mathrm{U}$ \\
\hline $79-34-5$ & $1,1,2,2$-Tetrachloroethane & 0.50 & $\mathrm{U}$ \\
\hline $541-73-1$ & 1,3-Dichlorobenzene & 0.50 & U \\
\hline $106-46-7$ & 1,4-Dichlorobenzene & 0.50 & $\mathrm{U}$ \\
\hline $95-50-1$ & 1,2-Dichlorobenzene & 0.50 & $\mathrm{U}$ \\
\hline $96-12-8$ & 1,2-Dibromo-3-Chloropropane & 0.50 & $\mathrm{U}$ \\
\hline $120-82-1$ & $1,2,4$-Trichlorobenzene & 0.50 & U \\
\hline $87-61-6$ & 1,2,3-Trichlorobenzene & 0.50 & U \\
\hline
\end{tabular}


IJ - FORM I VOA-TIC

VOLATILE ORGANICS ANALYSIS DATA SHEET

TENTATIVELY IDENTIFIED COMPOUNDS
EPA SAMPLE NO.

MRMW 6S-W-30016

Lab Name: TESTAMERICA BURLINGTON

Contract: $8 \mathrm{E}-00302$

Lab Code: STLV Case No.: MORRIL Mod. Ref No.: SDG No.: 200-1702

Matrix: (SOIL/SED/WATER) water

Sample wt/vol: 25.0 $(\mathrm{g} / \mathrm{mL}) \mathrm{mI}$

Leve 1: (TRACE or LOW/MED) TRACE

\% Moisture: not dec.

GC Column: DB-624 ID: 0.20 $(\mathrm{mm})$

Soil Extract Volume: (uI)

CONCENTRATION UNITS: (ug/L or $\mathrm{ug} / \mathrm{kg}$ ) ug/L
Lab Sample ID: 200-1702-2

Lab File ID: JBOC06.D

Date Received: 09/24/2010

Date Analyzed: 09/29/2010

Dilution Factor: 1.0

Soil Aliquot Volume: (uL)

Purge Volume: 25.0 $(\mathrm{mL})$

\begin{tabular}{|c|c|c|c|c|c|}
\hline & CAS NUMBER & COMPOUND NAME & RT & EST, CONC. & $Q$ \\
\hline 01 & $7446-09-5$ & Sulfur dioxide & 1.43 & 0.78 & $\mathrm{JN}$ \\
\hline 02 & $420-56-4$ & Trimethylsilyl fluoride & 1.68 & 0.52 & $\mathrm{JN}$ \\
\hline 03 & & Unknown & 6.93 & 2.6 & $\mathrm{~B} \mathrm{X} \mathrm{J}$ \\
\hline 04 & $541-05-9$ & Cyclotrisiloxane, hexamethyl- & 7.88 & 1.1 & $\mathrm{~B} \mathrm{~J} N$ \\
\hline 05 & & Unknown siloxane derivative & 10.72 & 1.5 & $\mathrm{~B} \mathrm{~J}$ \\
\hline 06 & E9667961 & Total Alkanes & $\mathrm{N} / \mathrm{A}$ & & \\
\hline
\end{tabular}

lEPA-designated Registry Number. 
$1 A$ - FORM I VOA-1

VOLATILE ORGANICS ANALYSIS DATA SHEET
EPA SAMPLE NO.

MRMW 7 S-W-30017
Lab Name: TESTAMERICA BURLINGTON

Lab Code: STLV

Case No.

Matrix: (SOIL/SED/WATER) Water

Sample wt/vol: $25: 0$

$(\mathrm{g} / \mathrm{mL}) \mathrm{mL}$

Level: (TRACE/LOW/MED) TRACE

: Moisture: not dec.

GC Column: DB-624

ID $: 0.20$

Soil Extract Volume:

Purge Volume: 25.0

Contract: 8E-00302

SDG No.: 200-1702

Lab Sample ID: 200-1702-3

Lab File ID: JBOC07.D

Date Received: 09/24/2010

Date Analyzed: 09/29/2010

Dilution Factor: 1.0

Soil Aliquot Volume:

(uL)

(mL)

\begin{tabular}{|c|c|c|c|}
\hline CAS NO. & COMPOUND & $\begin{array}{l}\text { CONCENTRATION UNITS: } \\
(\mathrm{ug} / \mathrm{L} \text { or } u g / \mathrm{kg}) \mathrm{ug} / \mathrm{E}\end{array}$ & $Q$ \\
\hline $75-71-8$ & Dichlorodifluoromethane & 0.50 & $\overline{\mathrm{U}}$ \\
\hline $74-87-3$ & Chloromethane & 0.50 & $\overline{\mathrm{U}}$ \\
\hline $75-01-4$ & Vinyl chloride & 0.50 & $\mathrm{U}$ \\
\hline $74-83-9$ & Bromomethane & 0.50 & $\mathrm{U}$ \\
\hline $75-00-3$ & Chloroethane & 0.50 & $\mathrm{U}$ \\
\hline $75-69-4$ & Trichlorofluoromethane & 0.50 & $\mathrm{U}$ \\
\hline $75-35-4$ & 1,1-Dichloroethene & 0.50 & $\overline{\mathrm{U}}$ \\
\hline $76-13-1$ & 1,1,2-Trichloro-1,2,2-trifluoroethane & 0.50 & $\overline{\mathrm{U}}$ \\
\hline $67-64-1$ & Acetone & 2.2 & $\mathrm{~J} B$ \\
\hline $75-15-0$ & Carbon disulfide & 0.11 & $\mathrm{~J} B$ \\
\hline $79-20-9$ & Methyl acetate & 0.50 & $\mathrm{U}$ \\
\hline $75-09-2$ & Methylene Chloride & 0.50 & $\mathrm{U}$ \\
\hline $156-60-5$ & trans-1,2-Dichloroethene & 0.50 & $\overline{\mathrm{U}}$ \\
\hline $1634-04-4$ & Methyl tert-butyl ether & 0.50 & $\mathrm{U}$ \\
\hline $75-34-3$ & 1,1-Dichloroethane & 0.50 & $\mathrm{U}$ \\
\hline $156-59-2$ & cis-1,2-Dichloroethene & 0.50 & $\mathrm{U}$ \\
\hline $78-93-3$ & 2-Butanone & 5.0 & $\mathrm{U}$ \\
\hline $74-97-5$ & Bromochloromethane. & 0.50 & $\mathrm{U}$ \\
\hline $67-66-3$ & Chloroform & 0.50 & $\bar{U}$ \\
\hline $71-55-6$ & $1,1,1$-Trichloroethane & 0.50 & $\bar{U}$ \\
\hline $110-82-7$ & Cyclohexane & 0.50 & $\mathrm{U}$ \\
\hline $56-23-5$ & Carbon tetrachloride & 4.7 & \\
\hline $71-43-2$ & Benzene & 0.50 & $\mathrm{U}$ \\
\hline $107-06-2$ & 1,2-Dichloroethane & 0.50 & $\bar{U}$ \\
\hline
\end{tabular}

Report 1,4-Dioxane for Low-Medium VOA analysis only 
Lab Name: TESTAMERICA BURLINGTON

Lab Code: STLV

Case

Matrix: (SOIL/SED/WATER) Water

Sample wt/vol: 25.0 $(\mathrm{g} / \mathrm{mL}) \mathrm{mL}$

Level: (TRACE/LOW/MED) TRACE

\% Moisture: not dec.

GC Column: DB-624 ID : 0.20 $(\mathrm{mm})$

Soil Extract Volume: (uL) (mL)

Purge Volume: 25.0

Contract: $8 \mathrm{E}-00302$ SDG No.: 200-1702

Lab Sample ID: 200-1702-3

Lab File ID: JBOC07.D

Date Received: 09/24/2010

Date Analyzed: 09/29/2010

Dilution Factor: 1.0

Soil Aliquot Volume: (uL)

\begin{tabular}{|c|c|c|c|}
\hline CAS NO. & COMPOUND & $\begin{array}{l}\text { CONCENTRATION UNITS: } \\
(\mathrm{ug} / \mathrm{L} \text { or } \mathrm{ug} / \mathrm{kg}) \mathrm{ug} / \mathrm{L}\end{array}$ & $Q$ \\
\hline $79-01-6$ & Trichloroethene & 0.50 & $\overline{\mathrm{U}}$ \\
\hline $108-87-2$ & Methylcyclohexane & 0.50 & $\mathrm{U}$ \\
\hline $78-87-5$ & 1,2-Dichloropropane & 0.50 & $\mathrm{U}$ \\
\hline $75-27-4$ & Bromodichloromethane & 0.50 & $\bar{U}$ \\
\hline $10061-01-5$ & cis-1,3-Dichloropropene & 0.50 & $\mathrm{U}$ \\
\hline $108-10-1$ & 4-Methyl-2-pentanone & 5.0 & $\overrightarrow{\mathrm{U}}$ \\
\hline $108-88-3$ & Toluene & 0.062 & $\mathrm{~J}$ \\
\hline $10061-02-6$ & trans-1,3-Dichloropropene & 0.50 & $\mathrm{U}$ \\
\hline $79-00-5$ & $1,1,2-$ Trichloroethane & 0.50 & $\mathrm{U}$ \\
\hline $127-18-4$ & Tetrachloroethene & 0.50 & $\overline{\mathrm{U}}$ \\
\hline $591-78-6$ & 2-Hexanone & 5.0 & $\mathrm{U}$ \\
\hline $124-48-1$ & Dibromochloromethane & 0.50 & $\mathrm{U}$ \\
\hline $106-93-4$ & 1,2-Dibromoethane & 0.50 & $\mathrm{U}$ \\
\hline $108-90-7$ & Chlorobenzene & 0.50 & $\mathrm{U}$ \\
\hline $100-41-4$ & Ethylbenzene & 0.50 & $\mathrm{U}$ \\
\hline $95-47-6$ & o-xylene & 0.50 & $\bar{U}$ \\
\hline $179601-23-1$ & $m, p-x y l e n e$ & 0.50 & $\overline{\mathrm{U}}$ \\
\hline $100-42-5$ & Styrene & 0.50 & $\mathrm{U}$ \\
\hline $75-25-2$ & Bromoform & 0.50 & $\mathrm{U}$ \\
\hline $98-82-8$ & Isopropylbenzene & 0.50 & $\mathrm{U}$ \\
\hline $79-34-5$ & $1,1,2,2-$ Tetrachloroethane & 0.50 & $\bar{U}$ \\
\hline $541-73-1$ & 1,3-Dichlorobenzene & 0.50 & $\mathrm{U}$ \\
\hline $106-46-7$ & 1,4-Dichlorobenzene & 0.50 & $\mathrm{U}$ \\
\hline $95-50-1$ & 1,2-Dichlorobenzene & 0.50 & $\mathrm{U}$ \\
\hline $96-12-8$ & 1,2-Dibromo-3-Chloropropane & 0.50 & $\mathrm{U}$ \\
\hline $120-82-1$ & 1,2,4-Trichlorobenzene & 0.50 & $\mathrm{U}$ \\
\hline $87-61-6$ & 1,2,3-Trichlorobenzene & 0.50 & $\mathrm{U}$ \\
\hline
\end{tabular}


$1 \mathrm{~J}$ - FORM I VOA-TIC

VOLATILE ORGANICS ANALYSIS DATA SHEET

TENTATIVELY IDENTIFIED COMPOUNDS
EPA SAMPLE NO.

MRMW 7 S-W-30017

Lab Name: TESTAMERICA BURLINGTON

Contract: $8 \mathrm{E}-00302$

Lab Code: STLV

Case No.: MORRIL Mod. Ref No.:

SDG No.: 200-1702

Matrix: (SOIL/SED/WATER) Water

Sample wt/vol: 25.0

$(\mathrm{g} / \mathrm{mL}) \mathrm{mL}$

Level: (TRACE or LOW/MED) TRACE

- Moisture: not dec.

GC Column: DB-624

ID $: 0.20$ (mm)

Soil Extract Volume: (uL)

CONCENTRATION UNITS: (ug/L or $\mathrm{ug} / \mathrm{kg}$ ) $\mathrm{ug} / \mathrm{L}$
Lab Sample ID: 200-1702-3

Lab File ID: JBOC07.D

Date Received: 09/24/2010

Date Analyzed: 09/29/2010

Dilution Factor: 1.0

Soil Áliquot volume: (uL)

Purge Volume: 25.0 (mL)

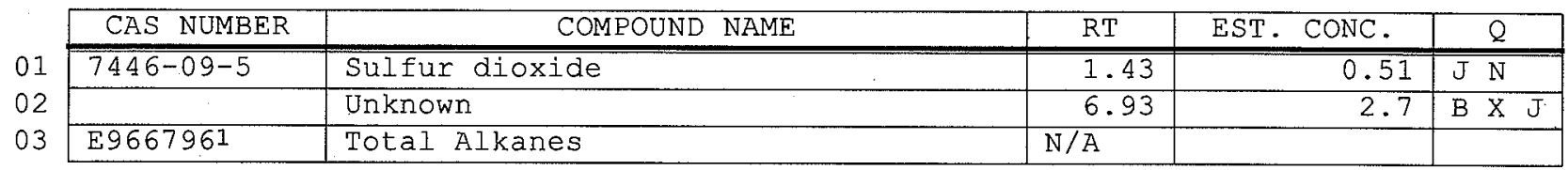

1EPA-designated Registry Number. 
Lab Name: TESTAMERICA BURLINGTON

Contract: $8 \mathrm{E}-00302$

Lab code: STLV Case No.: MORRIL Mod. Ref No.: SDG No.: 200-1702

Matrix: (SOIL/SED/WATER) Water

Sample wt/vol: 25.0 $(\mathrm{g} / \mathrm{mL}) \mathrm{mL}$

Level: (TRACE/LOW/MED) TRACE

응 Moisture: not dec.

GC Column: DB-624 ID: $0.20 \quad(\mathrm{~mm})$

Soil Extract Volume: (uL)

Purge Volume: 25.0
Lab Sample ID: 200-1702-5

Lab File ID: JBOC10.D

Date Received: 09/24/2010

Date Analyzed: 09/29/2010

Dilution Factor: 1.0

Soil Aliquot Volume: (uL)

\begin{tabular}{|c|c|c|c|}
\hline CAS NO. & COMPOUND & $\begin{array}{l}\text { CONCENTRATION UNITS: } \\
(\mathrm{ug} / \mathrm{L} \text { or } \mathrm{ug} / \mathrm{kg}) \mathrm{ug} / \mathrm{L}\end{array}$ & $Q$ \\
\hline $75-71-8$ & Dichlorodifluoromethane & 0.50 & $\overline{\mathrm{U}}$ \\
\hline $74-87-3$ & Chloromethane & 0.50 & $\mathrm{U}$ \\
\hline $75-01-4$ & Vinyl chloride & 0.50 & $\mathrm{U}$ \\
\hline $74-83-9$ & Bromomethane & 0.50 & U \\
\hline $75-00-3$ & Chloroethane & 0.50 & $\mathrm{U}$ \\
\hline $75-69-4$ & Trichlorofluoromethane & 0.50 & $\mathrm{U}$ \\
\hline $75-35-4$ & 1,1-Dichloroethene & 0.50 & $\overline{\mathrm{U}}$ \\
\hline $76-13-1$ & 1,1,2-Trichloro-1,2,2-trifluoroethane & 0.50 & $\mathrm{U}$ \\
\hline $67-64-1$ & Acetone & 6.8 & B \\
\hline $75-15-0$ & Carbon disulfide & 0.17 & $\mathrm{JB}$ \\
\hline $79-20-9$ & Methyl acetate & 0.50 & $\mathrm{U}$ \\
\hline $75-09-2$ & Methylene Chloride & 0.50 & $\mathrm{U}$ \\
\hline $156-60-5$ & trans-1,2-Dichloroethene & 0.50 & $\mathrm{U}$ \\
\hline $1634-04-4$ & Methyl tert-butyl ether & 0.50 & $\mathrm{U}$ \\
\hline $75-34-3$ & 1,1-Dichloroethane & 0.50 & $\mathrm{U}$ \\
\hline $156-59-2$ & cis-1,2-Dichloroethene & 0.50 & $\bar{U}$ \\
\hline $78-93-3$ & 2-Butanone & 5.0 & $\bar{U}$ \\
\hline $74-97-5$ & Bromochloromethane & 0.50 & $\bar{U}$ \\
\hline $67-66-3$ & Chloroform & 0.16 & $\mathrm{~J}$ \\
\hline $71-55-6$ & 1,1,1-Trichloroethane & 0.50 & $\mathrm{U}$ \\
\hline $110-82-7$ & Cyclohexane & 0.50 & $\mathrm{U}$ \\
\hline $56-23-5$ & Carbon tetrachloride & 0.50 & $\mathrm{U}$ \\
\hline $71-43-2$ & Benzene & 0.035 & $\mathrm{~J}$ \\
\hline $107-06-2$ & 1,2-Dichloroethane & 0.50 & $\mathrm{U}$ \\
\hline
\end{tabular}

Report 1,4-Dioxane for Low-Medium VOA analysis only 
$1 B$ - FORM I VOA-2

VOLATILE ORGANICS ANALYSIS DATA SHEET
EPA SAMPLE NO. MRQCTB-W-30032
Lab Name: TESTAMERICA BURLINGTON

Lab Code: STLV Case No.: MORRIL Mod. Ref No.:
Contract: $8 \mathrm{E}-00302$ SDG No.: 200-1702
Matrix: (SOIL/SED/WATER) water

Sample wt/vol: 25.0 $(\mathrm{g} / \mathrm{mL}) \mathrm{mL}$

Level: (TRACE/LOW/MED) TRACE

\% Moisture: not dec.

GC Column: DB-624 ID: $0.20 \quad(\mathrm{~mm})$

Soil Extract Volume: (UL)

Purge Volume: 25.0
Lab Sample ID: 200-1702-5

Lab File ID: JBOC10.D

Date Received: 09/24/2010

Date Analyzed: 09/29/2010

Dilution Factor: 1.0

Soil Aliquot volume: (uL)

\begin{tabular}{|c|c|c|c|}
\hline CAS NO. & COMPOUND & $\begin{array}{l}\text { CONCENTRATION UNITS: } \\
(\mathrm{ug} / \mathrm{L} \text { or } \mathrm{ug} / \mathrm{kg}) \mathrm{ug} / \mathrm{L}\end{array}$ & $Q$ \\
\hline $79-01-6$ & Trichloroethene & 0.50 & $\overline{\mathrm{U}}$ \\
\hline $108-87-2$ & Methylcyclohexane & 0.50 & $\mathrm{U}$ \\
\hline $78-87-5$ & 1,2-Dichloropropane & 0.50 & $\mathrm{U}$ \\
\hline $75-27-4$ & Bromodichloromethane & 0.15 & $\mathrm{~J}$ \\
\hline $10061-01-5$ & cis-1,3-Dichloropropene & 0.50 & $\mathrm{U}$ \\
\hline $108-10-1$ & 4-Methyl-2-pentanone & 5.0 & $\mathrm{U}$ \\
\hline $108-88-3$ & Toluene. & 0.17 & $\mathrm{~J}$ \\
\hline $10061-02-6$ & trans-1,3-Dichloropropene & 0.50 & $\mathrm{U}$ \\
\hline $79-00-5$ & $1,1,2$-Trichloroethane & 0.50 & $\mathrm{U}$ \\
\hline $127-18-4$ & Tetrachloroethene & 0.50 & $\mathrm{U}$ \\
\hline $591-78-6$ & 2-Hexanone & 5.0 & $\mathrm{U}$ \\
\hline $124-48-1$ & Dibromochloromethane & 0.078 & $\mathrm{~J}$ \\
\hline $106-93-4$ & 1,2-Dibromoethane & 0.50 & $\mathrm{U}$ \\
\hline $108-90-7$ & Chlorobenzene & 0.50 & $\mathrm{U}$ \\
\hline $100-41-4$ & Ethylbenzene & 0.50 & U \\
\hline $95-47-6$ & o-XYlene & 0.055 & $\mathrm{~J}$ \\
\hline $179601-23-1$ & $\mathrm{~m}, \mathrm{p}$-xylene & 0.088 & $\mathrm{~J}$ \\
\hline $100-42-5$ & styrene & 0.50 & $\mathrm{U}$ \\
\hline $75-25-2$ & Bromoform & 0.50 & $\mathrm{U}$ \\
\hline $98-82-8$ & Isopropylbenzene & 0.50 & $\mathrm{U}$ \\
\hline $79-34-5$ & $1,1,2,2$-Tetrachloroethane & 0.50 & $\mathrm{U}$ \\
\hline $541-73-1$ & 1,3-Dichlorobenzene & 0.50 & $\mathrm{U}$ \\
\hline $106-46-7$ & 1,4-Dichlorobenzene & 0.50 & $\bar{U}$ \\
\hline $95-50-1$ & 1,2-Dichlorobenzene & 0.50 & $\mathrm{U}$ \\
\hline $96-12-8$ & 1,2-Dibromo-3-Chloropropane & 0.50 & $\mathrm{U}$ \\
\hline $120-82-1$ & $1,2,4$-Trichlorobenzene & 0.50 & $\mathrm{U}$ \\
\hline $87-61-6$ & 1,2,3-Trichlorobenzene & 0.50 & $\mathrm{U}$ \\
\hline
\end{tabular}


IJ - FORM I VOA-TIC

VOLATILE ORGANICS ANALYSIS DATA SHEET

TENTATIVELY IDENTIEIED COMPOUNDS
EPA SAMPLE NO.

MRQCTB-W-30032

Lab Name: TESTAMERICA BURLINGTON

Contract: $8 \mathrm{E}-00302$

Iab Code: STLV Case No.: MORRIL Mod. Ref No.: SDG No.: 200-1702

Matrix: (SOIL/SED/WATER) Water

Sample wt/vol: 25.0

Level: (TRACE or LOW/MED) TRACE

\% Moisture: not dec.

GC Column: DB-624 ID $: 0.20 \quad(\mathrm{~mm})$

Soil Extract Volume: (uI)

CONCENTRATION UNITS: (ug/L or ug/kg) ug/L

$\frac{(\mathrm{g} / \mathrm{mL}) \mathrm{mL}}{\mathrm{TRACE}}$

Lab Sample ID: 200-1702-5

Lab File ID: JBOC10.D

Date Received: 09/24/2010

Date Analyzed: 09/29/2010

Dilution Factor: 1.0

Soil Aliquot volume: (uL)

Purge volume: 25.0 (mL)

\begin{tabular}{l|l|l|r|r|r|}
\cline { 2 - 6 } & \multicolumn{1}{|c|}{ CAS NUMBER } & \multicolumn{1}{|c|}{ COMPOUND NAME } & RT & EST. CONC. & $Q$ \\
\cline { 2 - 6 } 01 & & Unknown & 6.93 & 2.6 & $\mathrm{~B} \mathrm{X} \mathrm{J}$ \\
\cline { 2 - 6 } 03 & $541-05-9$ & Cyclotrisiloxane, hexamethyl- & 7.88 & 0.59 & $\mathrm{~B} \mathrm{~J}$ N \\
\cline { 2 - 6 } & E9667961 & Total Alkanes & N/A & & \\
\hline
\end{tabular}

1EPA-designated Registry Number. 
Lab Name: TESTAMERICA BURLINGTON

Lab Code: STLV Case No.: MORRIL Mod. Ref No.:
Contract: 8E-00302

SDG No.: 200-1702
Matrix: (SOIL/SED/WATER) Water

Sample wt/vol: 25.0 $(\mathrm{g} / \mathrm{mL}) \mathrm{mL}$

Level: (TRACE/LOW/MED) TRACE

\% Moisture: not dec.

GC Column: DB-624

ID: 0.20

Soil Extract Volume:

Purge "Volume: 25.0
Lab Sample ID: 200-1702-7

Lab File ID: JBOC16.D

Date Received: 09/24/2010

Date Analyzed: 09/29/2010

Dilution Factor: 1.0

Soil Aliquot Volume: (uL)

\begin{tabular}{|c|c|c|c|}
\hline CAS NO. & COMPOUND & $\begin{array}{l}\text { CONCENTRATION UNITS: } \\
(\mathrm{ug} / \mathrm{L} \text { or } \mathrm{ug} / \mathrm{kg}) \mathrm{ug} / \mathrm{L}\end{array}$ & Q \\
\hline $75-71-8$ & Dichlorodifluoromethane & 0.50 & $\overline{\mathrm{U}}$ \\
\hline $74-87-3$ & Chloromethane & 0.50 & $\mathrm{U}$ \\
\hline $75-01-4$ & Vinyl chloride & 0.50 & $\overline{\mathrm{U}}$ \\
\hline $74-83-9$ & Bromomethane & 0.50 & $\mathrm{U}$ \\
\hline $75-00-3$ & Chloroethane & 0.50 & $\mathrm{U}$ \\
\hline $75-69-4$ & Trichlorofluoromethane & 0.50 & $\mathrm{U}$ \\
\hline $75-35-4$ & 1,1-Dichloroethene & 0.50 & $\mathrm{U}$ \\
\hline $76-13-1$ & 1;1,2-Trichloro-1,2,2-trifluoroethane & 0.50 & $\mathrm{U}$ \\
\hline $67-64-1$ & Acetone & 2.5 & $\mathrm{~J} B$ \\
\hline $75-15-0$ & Carbon disulfide & 0.089 & $\mathrm{~J} B$ \\
\hline $79-20-9$ & Methyl acetate & 0.50 & $\mathrm{U}$ \\
\hline $75-09-2$ & Methylene Chloride & 0.50 & $\mathrm{U}$ \\
\hline $156-60-5$ & trans-1,2-Dichloroethene & 0.50 & $\mathrm{U}$ \\
\hline $1634-04-4$ & Methyl tert-butyl ether & 0.50 & $\mathrm{U}$ \\
\hline $75-34-3$ & 1,1-Dichloroethane & 0.50 & $\mathrm{U}$ \\
\hline $156-59-2$ & cis-1,2-Dichloroethene & 0.50 & $\mathrm{U}$ \\
\hline $78-93-3$ & 2-Butanone & 5.0 & $\mathrm{U}$ \\
\hline $74-97-5$ & Bromochloromethane & 0.50 & $\mathrm{U}$ \\
\hline $67-66-3$ & Chloroform & 0.50 & $\mathrm{U}$ \\
\hline $71-55-6$ & 1,1,1-Trichloroethane & 0.50 & U \\
\hline $110-82-7$ & Cyclohexane & 0.50 & $\mathrm{U}$ \\
\hline $56-23-5$ & Carbon tetrachloride & 0.50 & $\mathrm{U}$ \\
\hline $71-43-2$ & Benzene & 0.50 & $\mathrm{U}$ \\
\hline $107-06-2$ & 1,2-Dichloroethane & 0.50 & $\mathrm{U}$ \\
\hline
\end{tabular}

Report 1,4-Dioxane for Low-Medium VOA analysis only 
EPA SAMPLE NO. VHBLKO1
Lab Name: TESTAMERICA BURLINGTON

Lab Code: STLV

Matrix: (SOIL/SED/WATER)

Sample wt/vol: 25.0

Level: (TRACE/LOW/MED) TRACE

: Moisture: not dec.

GC Column: DB-624 ID: 0.20 (mm)

Soil Extract Volume: (uL)

Purge volume: 25.0 $(\mathrm{mL})$
Contract: $8 \mathrm{E}-00302$

SDG No.: 200-1702

Lab Sample ID: 200-1702-7

Lab File ID: JBOC16.D

Date Received: 09/24/2010

Date Analyzed: 09/29/2010

Dilution Factor: 1.0

Soil Aliquot Volume: (uI)

\begin{tabular}{|c|c|c|c|}
\hline CAS NO. & COMPOUND & $\begin{array}{l}\text { CONCENTRATION UNITS: } \\
(\mathrm{ug} / \mathrm{L} \text { or } u g / \mathrm{kg}) \mathrm{ug} / \mathrm{L}\end{array}$ & $Q$ \\
\hline $79-01-6$ & Trichloroethene & 0.50 & $\mathrm{U}$ \\
\hline $108-87-2$ & Methylcyclohexane & 0.50 & $\mathrm{U}$ \\
\hline $78-87-5$ & 1,2-Dichloropropane & 0.50 & $\overline{\mathrm{U}}$ \\
\hline $75-27-4$ & Bromodichloromethane & 0.50 & $\mathrm{U}$ \\
\hline $10061-01-5$ & cis-1,3-Dichloropropene & 0.50 & $\mathrm{U}$ \\
\hline $108-10-1$ & 4-Methyl-2-pentanone & 5.0 & $\mathrm{U}$ \\
\hline $108-88-3$ & Toluene & 0.50 & $\mathrm{U}$ \\
\hline $10061-02-6$ & trans-1,3-Dichloropropene & 0.50 & $\mathrm{U}$ \\
\hline $79-00-5$ & $1,1,2$-Trichloroethane & 0.50 & $\mathrm{U}$ \\
\hline $127-18-4$ & Tetrachloroethene & 0.50 & $\mathrm{U}$ \\
\hline $591-78-6$ & 2-Hexanone & 5.0 & $\mathrm{U}$ \\
\hline $124-48-1$ & Dibromochloromethane & 0.50 & $\mathrm{U}$ \\
\hline $106-93-4$ & 1,2-Dibromoethane & 0.50 & $\mathrm{U}$ \\
\hline $108-90-7$ & Chlorobenzene & 0.50 & $\mathrm{U}$ \\
\hline $100-41-4$ & Ethylbenzene & 0.50 & $\mathrm{U}$ \\
\hline $95-47-6$ & o-Xylene & 0.50 & $\mathrm{U}$ \\
\hline $179601-23-1$ & m,p-xylene & 0.50 & $\mathrm{U}$ \\
\hline $100-42-5$ & Styrene & 0.50 & $\mathrm{U}$ \\
\hline $75-25-2$ & Bromoform & 0.50 & $\mathrm{U}$ \\
\hline $98-82-8$ & Isopropylbenzene & 0.50 & $\mathrm{U}$ \\
\hline $79-34-5$ & $1,1,2,2$-Tetrachloroethane & 0.50 & $\bar{U}$ \\
\hline $541-73-1$ & 1,3-Dichlorobenzene & 0.50 & $\mathrm{U}$ \\
\hline $106-46-7$ & 1,4-Dichlorobenzene & 0.50 & $\mathrm{U}$ \\
\hline $95-50-1$ & 1,2-Dichlorobenzene & 0.50 & $\mathrm{U}$ \\
\hline $96-12-8$ & 1,2-Dibromo-3-Chloropropane & 0.50 & $\mathrm{U}$ \\
\hline $120-82-1$ & $1,2,4$-Trichlorobenzene & 0.50 & $\bar{U}$ \\
\hline $87-61-6$ & $1,2,3$-Trichlorobenzene & 0.50 & $\bar{U}$ \\
\hline
\end{tabular}


IJ - FORM I VOA-TIC

VOLATILE ORGANICS ANALYSIS DATA SHEET

TENTATIVELY IDENTIEIED COMPOUNDS
EPA SAMPLE NO.

VHBLKO1

Lab Name: TESTAMERICA BURLINGTON

Contract: $8 \mathrm{E}-00302$

Lab Code: STLV

Case No.: MORRIL Mod. Ref No.:

SDG No.: 200-1702

Matrix: (SOIL/SED/WATER) Water

Sample wt/vol: 25.0

$(\mathrm{g} / \mathrm{mL}) \mathrm{mL}$

Level: (TRACE or LOW/MED) TRACE

\% Moisture: not dec.

GC Column: DB-624 ID: 0.20 (mm)

Soil Extract Volume: (UL)
Lab Sample ID: 200-1702-7

Lab File ID: JBOC16.D

Date Received: 09/24/2010

Date Analyzed: 09/29/2010

Dilution Factor: 1.0

Soil Aliquot Volume: (uL)

Purge Volume: 25.0 (mL)

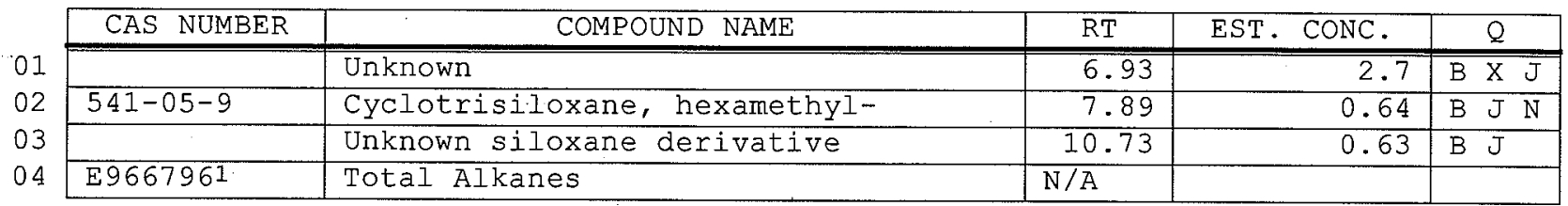


$6 A$ - FORM VI VOA-1

VOLATILE ORGANICS INITIAL CALIBRATION DATA

Lab Name: TESTAMERICA BURLINGTON

Contract: $8 \mathrm{E}-00302$

Lab Code: STLV Case No.: MORRIL Mod. Ref No.: SDG No.: 200-1702

Instrument ID: J.i Calibration Date(s): 09/27/2010 09/27/2010

Heated Purge: ( $\mathrm{Y} / \mathrm{N}) \mathrm{N}$ Calibration Time(s): 1544 1736

Purge Volume: 25.0 $(\mathrm{mL})$

GC Column: DB-624 ID $: 0.20$ (mm) Length: 25

(m)

\begin{tabular}{|c|c|c|c|c|c|c|c|}
\hline \multirow{2}{*}{$\begin{array}{l}\text { LAB FILE ID: } \\
\text { RRF } 5.0=\text { JBO0 4.D }\end{array}$} & \multirow{2}{*}{\multicolumn{3}{|c|}{$\begin{array}{l}\text { RRF0.5 }=\text { JBO02.D } \\
\text { RRF10 }=\text { JBO05.D }\end{array}$}} & \multirow{2}{*}{\multicolumn{4}{|c|}{$\begin{array}{l}\text { RRF1.0 }=\text { JBO03.D } \\
\text { RRF20 }=J \text { JO06.D }\end{array}$}} \\
\hline & & & & & & & \\
\hline COMPOUND & RRF0. 5 & RRF 1.0 & RRF5. 0 & RRF10 & RRF20 & $\overline{\mathrm{RRF}}$ & 吾 RSD \\
\hline Dichlorodifluoromethane & 0.457 & 0.441 & 0.445 & 0.453 & 0.436 & 0.446 & 1.9 \\
\hline Chloromethane & 0.371 & 0.315 & 0.323 & 0.333 & 0.315 & 0.331 & 7.1 \\
\hline Vinyl chloride & 0.420 & 0.379 & 0.360 & 0.361 & 0.349 & 0.374 & 7.4 \\
\hline Bromomethane & 0.180 & 0.109 & 0.119 & 0.139 & 0.154 & 0.140 & 20.2 \\
\hline Chloroethane & 0.242 & 0.183 & 0.225 & 0.235 & 0.229 & 0.223 & 10.4 \\
\hline Trichlorofluoromethane & 0.587 & 0.587 & 0.574 & 0.575 & 0.550 & 0.574 & 2.7 \\
\hline 1,1-Dichloroethene & 0.298 & 0.284 & 0.274 & 0.278 & 0.272 & 0.281 & 3.7 \\
\hline $\begin{array}{l}1,1,2 \text {-Trichloro- } \\
1,2,2 \text {-trifluoroethane }\end{array}$ & 0.329 & 0.324 & 0.315 & 0.319 & 0.311 & 0.319 & 2.2 \\
\hline Acetone & 0.017 & 0.014 & 0.012 & 0.011 & 0.011 & 0.013 & 19.6 \\
\hline Carbon disulfide & 0.985 & 0.779 & 0.755 & 0.777 & 0.748 & 0.809 & 12.3 \\
\hline Methyl acetate & 0.039 & 0.043 & 0.036 & 0.034 & 0.033 & 0.037 & 10.4 \\
\hline Methylene Chloride & 0.240 & 0.208 & 0.210 & 0.211 & 0.207 & 0.215 & 6.5 \\
\hline trans-1,2-Dichloroethene & 0.313 & 0.299 & 0.297 & 0.305 & 0.295 & 0.302 & 2.4 \\
\hline Methyl tert-butyl ether & 0.297 & 0.310 & 0.320 & 0.324 & 0.325 & 0.315 & 3.7 \\
\hline 1,1-Dichloroethane & 0.516 & 0.496 & 0.498 & 0.499 & 0.480 & 0.498 & 2.5 \\
\hline cis-1,2-Dichloroethene & 0.295 & 0.279 & 0.291 & 0.290 & 0.286 & 0.288 & 2.1 \\
\hline 2-Butanone & 0.019 & 0.019 & 0.020 & 0.020 & 0.020 & 0.020 & 3.1 \\
\hline Bromochloromethane & 0.091 & 0.083 & 0.086 & 0.085 & 0.084 & 0.086 & 3.9 \\
\hline Chloroform & 0.493 & 0.472 & 0.478 & 0.480 & 0.470 & 0.479 & 1.9 \\
\hline $1,1,1$-Trichloroethane & 0.695 & 0.649 & 0.635 & 0.650 & 0.630 & 0.652 & 3.9 \\
\hline Cyclohexane & 0.677 & 0.628 & 0.628 & 0.645 & 0.621 & 0.640 & 3.6 \\
\hline Carbon tetrachloride & 0.614 & 0.571 & 0.592 & $0 . \overline{602}$ & 0.590 & 0.594 & 2.7 \\
\hline Benzene & 1.624 & 1.539 & 1.495 & 1.516 & 1.505 & 1.536 & 3.4 \\
\hline 1,2-Dichloroethane. & 0.210 & 0.204 & 0.196 & 0.194 & 0.190 & 0.199 & 4.0 \\
\hline Trichloroethene & 0.435 & 0.400 & 0.397 & 0.400 & 0.393 & 0.405 & 4.2 \\
\hline Methylcyclohexane & 0.517 & 0.515 & 0.506 & 0.519 & 0.500 & 0.511 & 1.6 \\
\hline
\end{tabular}

Report 1,4-Dioxane for Low-Medium VOA analysis only 
$6 B$ - FORM VI VOA-2

VOLATILE ORGANICS INITIAL CALIBRATION DATA

Lab Name: TESTAMERICA BURLINGTON

Contract: $8 \mathrm{E}-00302$

Lab Code: STLV Case No.: MORRIL Mod. Ref No.: SDG No.: 200-1702

Instrument ID: J.i Calibration Date(s): 09/27/2010 09/27/2010

Heated Purge: ( $\mathrm{Y} / \mathrm{N}) \mathrm{N}$ Calibration Time(s): 1544 1736

Purge Volume: 25.0 (mL)

GC Column: DB-624

ID: 0.20

(mm) Length: 25

(m)

\begin{tabular}{|c|c|c|c|c|c|c|c|}
\hline LAB FILE ID: & \multirow{2}{*}{\multicolumn{3}{|c|}{$\begin{array}{l}\text { RRF0.5 }=\frac{\mathrm{JBO02.D}}{\mathrm{RRF10}}=\mathrm{JBO05. \textrm {D }}\end{array}$}} & \multirow{2}{*}{\multicolumn{3}{|c|}{$\begin{array}{l}\text { RRF1.0 }=\text { JB003.D } \\
\text { RRF20 JBO06.D }\end{array}$}} & \\
\hline $\mathrm{RRF} 5.0=\mathrm{JBO04.D}$ & & & & & & & \\
\hline COMPOUND & $\mathrm{RRF} 0.5$ & RRE1.0 & RRE5.0 & RRF10 & RRE20 & $\overline{\mathrm{RRF}}$ & $\because \mathrm{RSD}$ \\
\hline 1,2-Dichloropropane & 0.302 & 0.279 & 0.281 & 0.293 & 0.277 & 0.287 & 3.7 \\
\hline Bromodichloromethane & 0.348 & 0.350 & 0.348 & 0.341 & 0.342 & 0.346 & 1.2 \\
\hline cis-1,3-Dichloropropene & 0.419 & 0.406 & 0.413 & 0.414 & 0.416 & 0.414 & 1.2 \\
\hline 4-Methyl-2-pentanone & 0.056 & 0.061 & 0.062 & 0.062 & 0.062 & 0.061 & 4.2 \\
\hline Toluene & 1.688 & 1.564 & 1.601 & 1.637 & 1.614 & 1.621 & 2.8 \\
\hline trans-1,3-Dichloropropene & 0.268 & 0.281 & 0.287 & 0.296 & 0.302 & 0.287 & 4.6 \\
\hline $1,1,2$-Trichloroethane & 0.133 & 0.137 & 0.135 & 0.136 & 0.135 & 0.135 & 1.0 \\
\hline Tetrachloroethene & 0.393 & 0.364 & 0.347 & 0.364 & 0.356 & 0.365 & 4.8 \\
\hline 2-Hexanone & 0.038 & 0.038 & 0.042 & 0.040 & 0.041 & 0.040 & 4.7 \\
\hline Dibromochloromethane & 0.187 & 0.176 & 0.182 & 0.186 & 0.187 & 0.184 & 2.4 \\
\hline 1,2-Dibromoethane & 0.126 & 0.124 & 0.128 & 0.127 & 0.127 & 0.126 & 1.1 \\
\hline Chlorobenzene & 1.007 & 0.940 & 0.949 & 0.956 & 0.959 & 0.962 & 2.7 \\
\hline Ethylbenzene & 1.882 & 1.824 & 1.869 & 1.926 & 1.934 & 1.887 & 2.4 \\
\hline o-Xylene & 0.647 & 0.640 & 0.650 & 0.667 & 0.653 & 0.651 & 1.5 \\
\hline $\mathrm{m}, \mathrm{p}$-Xylene & 0.738 & 0.713 & 0.713 & 0.742 & 0.740 & 0.729 & 2.0 \\
\hline Styrene & 0.853 & 0.862 & $0 . \overline{934}$ & 0.964 & 0.983 & 0.919 & 6.4 \\
\hline Bromoform & 0.172 & 0.162 & 0.176 & 0.184 & 0.185 & 0.176 & 5.3 \\
\hline Isopropylbenzene & 1.815 & 1.771 & 1.893 & 1.953 & 1.965 & 1.879 & 4.5 \\
\hline $1,1,2,2$-Tetrachloroethane & 0.115 & 0.109 & 0.122 & 0.121 & 0.123 & 0.118 & 4.8 \\
\hline 1,3-Dichlorobenzene & 1.567 & 1.480 & 1.462 & 1.499 & 1.433 & 1.488 & 3.4 \\
\hline 1,4-Dichlorobenzene & 1.544 & 1.481 & 1.445 & 1.434 & 1.378 & 1.456 & 4.2 \\
\hline 1,2-Dichlorobenzene & 1.115 & 1.141 & 1.116 & 1.105 & 1.059 & 1.107 & 2.7 \\
\hline 1,2-Dibromo-3-Chloropropane & 0.027 & 0.031 & 0.035 & 0.037 & 0.034 & 0.033 & 12.1 \\
\hline $1,2,4$-Trichlorobenzene & 0.678 & 0.678 & 0.679 & 0.704 & 0.688 & $0 . \overline{686}$ & 1.7 \\
\hline $1,2,3-T r i c h l o r o b e n z e n e$ & 0.455 & 0.443 & 0.449 & 0.466 & 0.455 & 0.454 & 1.9 \\
\hline
\end{tabular}


$6 C-$ FORM VI VOA-3

VOLATILE ORGANICS INITIAL CAIIBRATION DATA

\begin{tabular}{|c|c|c|c|c|c|c|c|}
\hline Lab Name: TESTAMERICA BURL] & ON & & Contract & $8 E-0$ & 02 & & \\
\hline Lab Code: STLV & MORRIL MO & d. $\operatorname{Ref}$ & o.: & SD & No.: 2 & $00-170$ & \\
\hline Instrument ID: $\underline{\mathrm{J} . i}$ & & Calibr & tion Dat & $e(s): 09$ & $/ 27 / 2010$ & $09 / 27$ & 2010 \\
\hline Heated Purge: $(Y / N)$ N & & Calibra & tion Tim & $e(s): 15$ & & 1736 & \\
\hline Purge volume: 25.0 & & $(\mathrm{~mL})$ & & & & & \\
\hline GC Column: $\mathrm{DB}-624$ & ID: 0.20 & $(\mathrm{~mm})$ & Length: & 25 & (m) & & \\
\hline LAB FILE ID: & $\operatorname{RRF} 0.5=\mathrm{J}$ & $3002 . D$ & & RRFI. & $=\mathrm{JBOO}$ & $3 . \mathrm{D}$ & \\
\hline $\mathrm{RRF} 5.0=\mathrm{JBO04.D}$ & $\operatorname{RRF} 10=\underline{J}$ & $3005 . D$ & & $\mathrm{RRF} 20$ & $=\mathrm{JBOO}$ & $6 . \mathrm{D}$ & \\
\hline COMPOUND & RRF0.5 & RRE1.0 & $\mathrm{RRF} 5.0$ & RRE10 & RRF20 & $\overline{\mathrm{RRF}}$ & $\because \mathrm{RSD}$ \\
\hline Vinyl Chloride-d3 & 0.358 & 0.336 & 0.320 & 0.324 & 0.313 & $0 . \overline{330}$ & 5.3 \\
\hline Chloroethane-d5 & 0.288 & 0.272 & 0.267 & 0.261 & 0.257 & 0.269 & 4.5 \\
\hline 1,1-Dichloroethene-d2 & 0.537 & 0.502 & 0.505 & 0.512 & 0.495 & 0.510 & 3.2 \\
\hline 2-Butanone-d5 & 0.021 & 0.020 & 0.021 & 0.021 & 0.020 & 0.021 & 1.4 \\
\hline Chloroform-d & 0.495 & 0.490 & 0.483 & 0.488 & 0.476 & 0.486 & 1.5 \\
\hline 1,2-Dichloroethane-d4 & 0.162 & 0.152 & 0.155 & 0.154 & 0.150 & 0.155 & 3.0 \\
\hline Benzene-d6 & 1.436 & 1.359 & 1.370 & 1.386 & 1.371 & 1.384 & 2.2 \\
\hline 1,2-Dichloropropane-d6 & 0.423 & 0.391 & 0.373 & 0.331 & 0.366 & 0.377 & 9.0 \\
\hline Toluene-d8 & 1.354 & 1.300 & 1.325 & 1.353 & 1.339 & 1.334 & 1.7 \\
\hline trans-1,3-Dichloropropene-d 4 & 0.244 & 0.248 & 0.258 & 0.262 & 0.262 & 0.255 & 3.2 \\
\hline $2-$ Hexanone-d5 & 0.020 & 0.023 & 0.024 & 0.025 & 0.025 & 0.023 & 9.6 \\
\hline $1,1,2,2-$ Tetrachloroethane-d2 & 0.111 & 0.121 & 0.122 & 0.120 & 0.118 & 0.118 & 3.7 \\
\hline $1,2-$ Dichlorobenzene-d4 & 0.721 & 0.687 & 0.696 & 0.682 & 0.663 & 0.690 & 3.1 \\
\hline
\end{tabular}

Report 1,4-Dioxane-d8 for Low-Medium VOA analysis only 
$7 A$ - FORM VII VOA-1

VOLATILE CONTINUING CALIBRATION DATA

Lab Name: TESTAMERICA BURLINGTON

Contract: $8 \mathrm{E}-00302$

Lab Code: STLV Case No.: MORRIL Mod. Ref No.:

SDG No.: 200-1702

Instrument ID: J.i

Calibration Date: 09/29/2010 Time: 0844

Lab File Id: JBOC03.D Init. Calib. Date(s):09/27/2010 09/27/2010

EPA Sample No. (VSTD\#\#\#\#): VSTD005JR

Init. Calib. Time(s) : 1544 1736

Heated Purge: ( $\mathrm{Y} / \mathrm{N}) \mathrm{N}$

GC Column: DB-624

ID: $0.20(\mathrm{~mm})$ Length: 25 (m)

Purge Volume: 25.0

$(\mathrm{mL})$

\begin{tabular}{|c|c|c|c|c|c|}
\hline COMPOUND & $\overline{\mathrm{RRF}}$ & $\operatorname{RRE} 5.0$ & $\begin{array}{l}\text { MIN } \\
\text { RRF }\end{array}$ & $\because \mathrm{D}$ & $\operatorname{MAX} \div \mathrm{D}$ \\
\hline Dichlorodifluoromethane & 0.446 & 0.456 & 0.010 & 2.3 & 40.0 \\
\hline Chloromethane & 0.331 & 0.315 & 0.010 & -5.1 & 40.0 \\
\hline Vinyl chloride & 0.374 & 0.353 & 0.010 & -5.6 & 30.0 \\
\hline Bromomethane & 0.140 & 0.113 & 0.100 & -19.6 & 30.0 \\
\hline Chiloroethane & 0.223 & 0.207 & 0.010 & -7.0 & 40.0 \\
\hline Trichlorofluoromethane & 0.574 & 0.576 & 0.010 & 0.3 & 40.0 \\
\hline 1,1-Dichloroethene & 0.281 & 0.276 & 0.100 & -1.7 & 30.0 \\
\hline 1,1,2-Trichloro-1,2,2-trifluoroethane & 0.319 & 0.320 & 0.010 & 0.1 & 40.0 \\
\hline Acetone & 0.013 & 0.012 & 0.010 & -9.0 & 40.0 \\
\hline Carbon disulfide & 0.809 & 0.773 & 0.010 & -4.5 & 40.0 \\
\hline Methyl acetate & 0.037 & 0.036 & 0.010 & -2.3 & 40.0 \\
\hline Methylene Chloride & 0.215 & 0.216 & 0.010 & 0.4 & 40.0 \\
\hline $\operatorname{trans}-1,2-$ Dichloroethene & 0.302 & 0.301 & 0.010 & -0.1 & 40.0 \\
\hline Methyl tert-butyl ether & 0.315 & 0.328 & 0.010 & 3.9 & 40.0 \\
\hline 1,1-Dichloroethane & 0.498 & 0.501 & 0.200 & 0.6 & 30.0 \\
\hline cis-1,2-Dichloroethene & 0.288 & 0.299 & 0.010 & 3.9 & 40.0 \\
\hline 2-Butanone & 0.020 & 0.020 & 0.010 & 3.2 & 40.0 \\
\hline Bromochloromethane & 0.086 & 0.087 & 0.050 & 1.5 & 30.0 \\
\hline Chloroform & 0.479 & 0.484 & 0.200 & 1.1 & 30.0 \\
\hline $1,1,1$-Trichloroethane & 0.652 & 0.659 & 0.100 & 1.1 & 30.0 \\
\hline Cyclohexane & 0.640 & 0.649 & 0.010 & 1.4 & 40.0 \\
\hline Carbon tetrachloride & 0.594 & 0.596 & 0.100 & 0.5 & 30.0 \\
\hline Benzene & 1.536 & 1.530 & 0.400 & -0.4 & 30.0 \\
\hline 1,2-Dichloroethane & 0.199 & 0.198 & 0.100 & -0.5 & 30.0 \\
\hline Trichloroethene & 0.405 & 0.405 & 0.300 & -0.1 & 30.0 \\
\hline Methylcyclohexane & 0.511 & 0.522 & 0.010 & 2.1 & 40.0 \\
\hline
\end{tabular}

Report 1,4-Dioxane for Low/Medium VOA analysis only 
$7 B$ - FORM VII VOA-2

VOLATILE CONTINUING CALIBRATION DATA

Lab Name: TESTAMERICA BURLINGTON

Lab Code: STIV Case No.: MORRIL Mod. Ref No.:
Instrument ID: J.i

Lab File Id: JBOC03.D

EPA Sample No. (VSTD\#\#\#\#) : VSTD005JR

Heated Purge: ( $Y / N)$ N

Purge Volume: 25.0
Contract: $8 \mathrm{E}-00302$

SDG No.: 200-1702

Calibration Date: 09/29/2010 Time: 0844 Init. Calib. Date(s):09/27/2010 09/27/2010

Init. Calib. Time(s): $1544 \quad 1736$ ID: $0.20(\mathrm{~mm})$ Length: 25 (m) (mI)

\begin{tabular}{|c|c|c|c|c|c|}
\hline COMPOUND & $\overline{\mathrm{RRF}}$ & RRE5.0 & $\begin{array}{l}\text { MIN } \\
\text { RRF }\end{array}$ & $\% \mathrm{D}$ & $\operatorname{MAX} \div \mathrm{D}$ \\
\hline 1,2-Dichloropropane & 0.287 & 0.294 & 0.010 & 2.5 & 40.0 \\
\hline Bromodichloromethane & 0.346 & 0.351 & 0.200 & 1.7 & 30.0 \\
\hline cis-1,3-Dichloropropene & 0.414 & 0.411 & 0.200 & -0.7 & 30.0 \\
\hline 4-Methyl-2-pentanone & 0.061 & 0.062 & 0.010 & 2.6 & 40.0 \\
\hline Toluene & 1.621 & 1.625 & 0.400 & 0.3 & 30.0 \\
\hline trans-1,3-Dichloropropene & 0.287 & 0.294 & 0.100 & 2.4 & 30.0 \\
\hline 1,1,2-Trichloroethane & 0.135 & 0.141 & 0.100 & 4.1 & 30.0 \\
\hline Tetrachloroethene & 0.365 & 0.361 & 0.100 & -1.1 & 30.0 \\
\hline 2-Hexanone & 0.040 & 0.041 & 0.010 & 2.1 & 40.0 \\
\hline Dibromochloromethane & 0.184 & 0.181 & 0.100 & -1.5 & 30.0 \\
\hline 1,2-Dibromoethane & 0.126 & 0.125 & 0.010 & $\cdots \quad-0.9$ & 40.0 \\
\hline Chlorobenzene & 0.962 & 0.968 & 0.500 & 0.6 & 30.0 \\
\hline Ethylbenzene & 1.887 & 1.903 & 0.100 & 0.8 & 30.0 \\
\hline o-Xylene & 0.651 & 0.643 & 0.300 & $-1 \cdot 3$ & 30.0 \\
\hline m,p-xylene & 0.729 & 0.741 & 0.300 & 1.6 & 30.0 \\
\hline styrene & 0.919 & 0.934 & 0.300 & 1.6 & 30.0 \\
\hline Bromoform & 0.176 & 0.178 & 0.050 & 1.2 & 30.0 \\
\hline Isopropylbenzene & 1.879 & 1.904 & 0.010 & 1.3 & 40.0 \\
\hline $1,1,2,2$-Tetrachloroethane & 0.118 & 0.123 & 0.100 & 3.9 & 30.0 \\
\hline 1,3-Dichlorobenzene & 1.488 & 1.495 & 0.400. & 0.5 & 30.0 \\
\hline 1,4-Dichlorobenzene & 1.456 & 1.456 & 0.400 & -0.1 & 30.0 \\
\hline 1,2-Dichlorobenzene & 1.107 & 1.133 & 0.400 & 2.3 & 30.0 \\
\hline 1,2-Dibromo-3-Chloropropane & 0.033 & 0.035 & 0.010 & 8.3 & 40.0 \\
\hline $1,2,4$-Trichlorobenzene & 0.686 & 0.694 & 0.200 & 1.3 & 30.0 \\
\hline 1,2,3-Trichlorobenzene & 0.454 & 0.459 & 0.200 & 1.2 & 30.0 \\
\hline
\end{tabular}


7C - FORM VII VOA-3

VOLATILE CONTINUING CALIBRATION DATA

Lab Name: TESTAMERICA BURLINGTON

Contract: $8 \mathrm{E}-00302$

Lab Code: STLV Case No.: MORRIL Mod. Ref No.: SDG No.: 200-1702

Instrument ID: J.i

Calibration Date: 09/29/2010 Time: 0844

Lab File Id: JBOC03.D Init. Calib. Date(s):09/27/2010 09/27/2010

EPA Sample No. (VSTD\#\#\#): VSTD005JR

Init. Calib. Time(s): $1544 \quad 1736$

Heated Purge: ( $\mathrm{Y} / \mathrm{N}) \mathrm{N}$

GC Column: DB-624

ID $: \underline{0.20}(\mathrm{~mm})$ Length: 25 (m)

Purge Volume: 25.0

(mL)

\begin{tabular}{|c|c|c|c|c|c|}
\hline COMPOUND & $\overline{\mathrm{RRE}}$. & RRE5. 0 & $\begin{array}{l}\text { MIN } \\
\text { RRF }\end{array}$ & $\because \mathrm{D}$ & MAX $\div D$ \\
\hline Vinyl Chloride-d3 & 0.330 & 0.328 & 0.010 & -0.8 & 30.0 \\
\hline Chloroethane-d5 & 0.269 & 0.267 & 0.010 & -0.7 & 40.0 \\
\hline 1,1-Dichloroethene-d2 & 0.510 & 0.513 & 0.010 & 0.5 & 30.0 \\
\hline 2-Butanone-d5 & 0.021 & 0.021 & 0.010 & 0.8 & 40.0 \\
\hline Chloroform-d & 0.486 & 0.488 & 0.010 & 0.3 & 30.0 \\
\hline 1,2-Dichloroethane-d4 & 0.155 & 0.153 & 0.010 & -1.0 & 30.0 \\
\hline Benzene-d6 & 1.384 & 1.387 & 0.010 & 0.2 & 30.0 \\
\hline 1,2-Dichloropropane-d6 & 0.377 & 0.335 & 0.010 & -11.2 & 40.0 \\
\hline Toluene-d8 & 1.334 & 1.352 & 0.010 & 1.3 & 30.0 \\
\hline trans-1,3-Dichloropropene-d4 & 0.255 & 0.258 & 0.010 & 1.3 & 30.0 \\
\hline 2-Hexanone-d5 & 0.023 & 0.024 & 0.010 & 3.3 & 40.0 \\
\hline $1,1,2,2$-Tetrachloroethane-d2 & 0.118 & 0.122 & 0.010 & 3.1 & 30.0 \\
\hline 1,2-Dichlorobenzene-d4 & 0.690 & 0.694 & 0.010 & 0.6 & 30.0 \\
\hline
\end{tabular}

Report 1,4-Dioxane-d8 for Low/Medium.VOA analysis only 
$7 A$ - FORM VII VOA-1

VOLATILE CONTINUING CALIBRATION DATA

Lab Name: TESTAMERICA BURLINGTON

Contract: $8 \mathrm{E}-00302$

Lab Code: STLV Case No.: MORRIL Mod. Ref No.: SDG No.: 200-1702

Instrument ID: J.i

Calibration Date: 09/29/2010 Time: 1553

Lab File Id: JBOC17.D Init. Calib. Date(s):09/27/2010 09/27/2010

EPA Sample No. (VSTD\#\#\#\#) : VSTD005RJ

Init. Calib. Time(s): 1544 1736

Heated Purge: ( $\mathrm{Y} / \mathrm{N}) \mathrm{N}$ GC Column: DB-624 ID: $0.20(\mathrm{~mm})$ Length: 25 (m)

Purge Volume: 25.0 (mL)

\begin{tabular}{|c|c|c|c|c|c|}
\hline COMPOUND & $\overline{\mathrm{RRF}}$ & RRF5.0 & $\begin{array}{l}\text { MIN } \\
\text { RRF } \\
\end{array}$ & $\because \mathrm{D}$ & $\operatorname{MAX} \div \mathrm{D}$ \\
\hline Dichlorodifluoromethane & 0.446 & 0.445 & 0.010 & -0.4 & 50.0 \\
\hline Chloromethane & 0.331 & 0.325 & 0.010 & -1.8 & 50.0 \\
\hline Vinyl chloride & 0.374 & 0.358 & 0.010 & -4.1 & 50.0 \\
\hline Bromomethane & 0.140 & 0.105 & 0.010 & -24.8 & 50.0 \\
\hline Chloroethane & 0.223 & 0.205 & 0.010 & -8.0 & 50.0 \\
\hline Trichlorofluoromethane & 0.574 & 0.573 & 0.010 & -0.3 & 50.0 \\
\hline 1,1-Dichloroethene & 0.281 & 0.276 & 0.010 & -1.9 & 50.0 \\
\hline 1,1,2-Trichloro-1,2,2-trifluoroethane & 0.319 & 0.311 & 0.010 & -2.6 & 50.0 \\
\hline Acetone & 0.013 & 0.012 & 0.010 & -10.0 & 50.0 \\
\hline Carbon disulfide & 0.809 & 0.754 & 0.010 & -6.8 & 50.0 \\
\hline Methyl acetate & 0.037 & 0.036 & 0.010 & -2.2 & 50.0 \\
\hline Methylene Chloride & 0.215 & 0.214 & 0.010 & -0.7 & 50.0 \\
\hline trans-1,2-Dich1oroethene & 0.302 & 0.297 & 0.010 & -1.5 & 50.0 \\
\hline Methyl tert-butyl ether & 0.315 & 0.338 & 0.010 & 7.1 & 50.0 \\
\hline 1,1-Dichloroethane & 0.498 & 0.498 & 0.010 & 0.0 & 50.0 \\
\hline cis-1,2-Dichloroethene & 0.288 & 0.287 & 0.010 & -0.4 & 50.0 \\
\hline 2-Butanone & 0.020 & 0.019 & 0.010 & -2.0 & 50.0 \\
\hline Bromochloromethane & 0.086 & 0.087 & 0.010 & 1.4 & 50.0 \\
\hline Chloroform & 0.479 & 0.483 & 0.010 & 0.8 & 50.0 \\
\hline 1,1,1-Trichloroethane & 0.652 & 0.628 & 0.010 & -3.6 & 50.0 \\
\hline Cyclohexane & 0.640 & 0.621 & 0.010 & -3.0 & 50.0 \\
\hline Carbon tetrachloride & 0.594 & 0.590 & 0.010 & -0.6 & 50.0 \\
\hline Benzene & 1.536 & 1.517 & 0.010 & -1.2 & 50.0 \\
\hline 1,2-Dichloroethane & 0.199 & 0.194 & 0.010 & -2.2 & 50.0 \\
\hline Trichloroethene & 0.405 & 0.398 & 0.010 & -1.9 & 50.0 \\
\hline Methylcyclohexane & 0.511 & 0.513 & 0.010 & 0.4 & 50.0 \\
\hline
\end{tabular}

Report 1,4-Dioxane for Low/Medium VOA analysis only 
$7 B$ - FORM VII VOA-2

VOLATILE CONTINUING CALIBRATION DATA

Lab Name: TESTAMERICA BURIINGTON

Contract: $8 \mathrm{E}-00302$

Lab Code: STLV Case No.: MORRIL Mod. Ref No.: SDG No.: 200-1702

Instrument ID: J.i

Calibration Date: 09/29/2010 Time: 1553

Lab File Id: JBOC17.D Init. Calib. Date(s):09/27/2010 09/27/2010

EPA Sample No. (VSTD\#\#\#\#): VSTD005RJ

Init. Calib. Time(s): 1544 1736

Heated Purge: ( $\mathrm{Y} / \mathrm{N}) \mathrm{N}$ GC Column: DB-624 ID: $0.20(\mathrm{~mm})$ Length: 25 (m)

Purge Volume: 25.0 (mI)

\begin{tabular}{|c|c|c|c|c|c|}
\hline COMPOUND & $\overline{\mathrm{RRF}}$ & RRE5.0 & $\begin{array}{l}\text { MINN } \\
\text { RRE }\end{array}$ & $\because \mathrm{D}$ & $\operatorname{MAX} \div \mathrm{D}$ \\
\hline 1,2-Dichloropropane & 0.287 & 0.284 & 0.010 & -1.1 & 50.0 \\
\hline Bromodichloromethane & 0.346 & 0.347 & 0.010 & 0.5 & 50.0 \\
\hline cis-1,3-Dichloropropene & 0.414 & 0.416 & 0.010 & 0.6 & 50.0 \\
\hline 4-Methyl-2-pentanone & 0.061 & 0.063 & 0.010 & 4.0 & 50.0 \\
\hline Toluene & 1.621 & 1.604 & 0.010 & -1.0 & 50.0 \\
\hline trans-1,3-Dichloropropene & 0.287 & 0.298 & 0.010 & 4.0 & 50.0 \\
\hline 1,1,2-Trichloroethane & 0.135 & 0.140 & 0.010 & 3.8 & 50.0 \\
\hline Tetrachloroethene & 0.365 & 0.359 & 0.010 & -1.7 & 50.0 \\
\hline 2-Hexanone & 0.040 & 0.042 & 0.010 & 4.7 & 50.0 \\
\hline Dibromochloromethane & 0.184 & 0.186 & 0.010 & 1.2 & 50.0 \\
\hline 1,2-Dibromoethane & 0.126 & 0.130 & 0.010 & 2.8 & 50.0 \\
\hline Chlorobenzene & 0.962 & 0.947 & 0.010 & -1.5 & 50.0 \\
\hline Ethylbenzene & 1.887 & 1.870 & 0.010 & -0.9 & 50.0 \\
\hline o-Xylene & 0.651 & 0.659 & 0.010 & 1.2 & 50.0 \\
\hline$m, p-x y l e n e$ & 0.729 & 0.731 & 0.010 & 0.3 & 50.0 \\
\hline Styrene & 0.919 & 0.955 & 0.010 & 3.9 & 50.0 \\
\hline Bromoform & 0.176 & 0.184 & 0.010 & 4.7 & 50.0 \\
\hline Isopropylbenzene & 1.879 & 1.882 & 0.010 & 0.1 & 50.0 \\
\hline $1,1,2,2$-Tetrachloroethane & 0.118 & 0.123 & 0.010 & 4.3 & 50.0 \\
\hline 1,3-Dichlorobenzene & 1.488 & 1.451 & 0.010 & -2.5 & 50.0 \\
\hline 1,4-Dichlorobenzene & 1.456 & 1.395 & 0.010 & -4.2 & 50.0 \\
\hline 1,2-Dichlorobenzene & 1.107 & 1.104 & 0.010 & -0.3 & 50.0 \\
\hline 1,2-Dibromo-3-Chloropropane & 0.033 & 0.035 & 0.010 & 6.6 & 50.0 \\
\hline $1,2,4$-Trichlorobenzene & 0.686 & 0.666 & 0.010 & -2.8 & 50.0 \\
\hline 1,2,3-Trichlorobenzene & 0.454 & 0.436 & 0.010 & -3.9 & 50.0 \\
\hline
\end{tabular}


Lab Name: TESTAMERICA BURLINGTON

Contract: $8 \mathrm{E}-00302$

Lab Code: STLV Case No.: MORRIL Mod. Ref No.: SDG No.: 200-1702

Instrument ID: J.i

Calibration Date: 09/29/2010 Time: 1553

Lab File Id: JBOC17. D Init. Calib. Date(s):09/27/2010 09/27/2010

EPA Sample No. (VSTD\#\#\#\#): VSTD005RJ

Init. Calib. Time(s): 1544 1736

Heated Purge: ( $Y / N) \mathrm{N}$ GC Column: DB-624 ID: $0.20(\mathrm{~mm})$ Length: 25

(m)

Purge Volume: 25.0

(mL)

\begin{tabular}{|c|c|c|c|c|c|}
\hline COMPOUND & $\overline{\mathrm{RRF}}$ & RRF5. 0 & $\begin{array}{l}\text { MIN } \\
\text { RRF }\end{array}$ & $\because \mathrm{D}$ & MAX $\% \mathrm{D}$ \\
\hline Vinyl Chloride-d3 & 0.330 & 0.322 & 0.010 & -2.6 & 50.0 \\
\hline Chloroethane-d5 & 0.269 & 0.263 & 0.010 & -2.4 & 50.0 \\
\hline 1,1-Dichloroethene-d2 & 0.510 & 0.507 & 0.010 & -0.6 & 50.0 \\
\hline 2-Butanone-d5 & 0.021 & 0.021 & 0.010 & 2.9 & 50.0 \\
\hline Chloroform-d & 0.486 & 0.488 & 0.010 & 0.4 & 50.0 \\
\hline 1,2-Dichloroethane-d4 & 0.155 & 0.157 & 0.010 & 1.5 & 50.0 \\
\hline Benzene-d6 & 1.384 & 1.390 & 0.010 & 0.4 & 50.0 \\
\hline 1,2-Dichloropropane-d6 & 0.377 & 0.379 & 0.010 & 0.6 & 50.0 \\
\hline Toluene-d8 & 1.334 & 1.337 & 0.010 & 0.2 & 50.0 \\
\hline trans-1,3-Dichloropropene-d4 & 0.255 & 0.264 & 0.010 & 3.5 & 50.0 \\
\hline $2-$ Hexanone-d5 & 0.023 & 0.026 & 0.010 & 9.4 & 50.0 \\
\hline $1,1,2,2$-Tetrachloroethane-d2 & 0.118 & 0.124 & 0.010 & 4.8 & 50.0 \\
\hline 1,2-Dichlorobenzene-d4 & 0.690 & 0.698 & 0.010 & 1.1 & 50.0 \\
\hline
\end{tabular}

Report 1,4-Dioxane-d8 for Low/Medium VOA analysis only 
Lab Name: TESTAMERICA BURLINGTON

Contract: $8 \mathrm{E}-00302$

Lab Code: STLV Case No.: MORRIL Mod. Ref No.: SDG No.: 200-1702

Matrix: (SOIL/SED/WATER) water

Sample wt/vol: 25.0 $(\mathrm{g} / \mathrm{mL}) \mathrm{mL}$

Level: (TRACE/LOW/MED) TRACE

o Moisture: not dec.

GC Column: DB-624 ID: 0.20 (mm)

Soil Extract Volume: (uL)

Purge volume: 25.0 (mL)
Lab Sample ID: MB 200-7244/5

Lab File ID: JBOC05.D

Date Received:

Date Analyzed: 09/29/2010

Dilution Factor: 1.0

Soil Aliquot Volume: (uL)

\begin{tabular}{|l|l|c|c|}
\hline CAS NO. & COMpound & $\begin{array}{c}\text { CONCENTRATION UNITS: } \\
\text { (ug/L or ug/kg) ug/L }\end{array}$ & $\mathrm{Q}$ \\
\hline \hline $75-71-8$ & Dichlorodifluoromethane & 0.50 & $\mathrm{U}$ \\
\hline $74-87-3$ & Chloromethane & 0.50 & $\mathrm{U}$ \\
\hline $75-01-4$ & Vinyl chloride & 0.50 & $\mathrm{U}$ \\
\hline $74-83-9$ & Bromomethane & 0.50 & $\mathrm{U}$ \\
\hline $75-00-3$ & Chloroethane & 0.50 & $\mathrm{U}$ \\
\hline $75-69-4$ & Trichlorofluoromethane & 0.50 & $\mathrm{U}$ \\
\hline $75-35-4$ & $1,1-$ Dichloroethene & 0.50 & $\mathrm{U}$ \\
\hline $76-13-1$ & 1,1,2-Trichloro-1,2,2-trifluoroethane & 0.50 & $\mathrm{U}$ \\
\hline $67-64-1$ & Acetone & 2.9 & $\mathrm{~J}$ \\
\hline $75-15-0$ & Carbon disulfide & 0.074 & $\mathrm{~J}$ \\
\hline $79-20-9$ & Methyl acetate & 0.50 & $\mathrm{U}$ \\
\hline $75-09-2$ & Methylene Chloride & 0.50 & $\mathrm{U}$ \\
\hline $156-60-5$ & trans-1,2-Dichloroethene & 0.50 & $\mathrm{U}$ \\
\hline $1634-04-4$ & Methyl tert-butyl ether & 0.50 & $\mathrm{U}$ \\
\hline $75-34-3$ & $1,1-$ Dichloroethane & 0.50 & $\mathrm{U}$ \\
\hline $156-59-2$ & cis-1,2-Dichloroethene & 0.50 & $\mathrm{U}$ \\
\hline $78-93-3$ & 2-Butanone & 5.0 & $\mathrm{U}$ \\
\hline $74-97-5$ & Bromochloromethane & 0.50 & $\mathrm{U}$ \\
\hline $67-66-3$ & Chloroform & 0.50 & $\mathrm{U}$ \\
\hline $71-55-6$ & 1,1,1-Trichloroethane & 0.50 & $\mathrm{U}$ \\
\hline $110-82-7$ & Cyclohexane & 0.50 & $\mathrm{U}$ \\
\hline $56-23-5$ & Carbon tetrachloride & 0.50 & $\mathrm{U}$ \\
\hline $71-43-2$ & Benzene & 0.50 & $\mathrm{U}$ \\
\hline $107-06-2$ & 1,2-Dichloroethane & 0.50 & $\mathrm{U}$ \\
\hline
\end{tabular}

Report 1,4-Dioxane for Low-Medium VOA analysis only 
$1 B$ - FORM I VOA-2

VOLATILE ORGANICS ANALYSIS DATA SHEET
EPA SAMPLE NO.

VBLKJR
Lab Name: TESTAMERICA BURLINGTON

Lab Code: STLV

Case No.: MORRIL Mod. Ref No.:
Contract: $8 \mathrm{E}-00302$

SDG No.: 200-1702
Matrix: (SOIL/SED/WATER) Water

Sample wt/vol: 25.0 $(\mathrm{g} / \mathrm{mL}) \mathrm{mL}$

Level: (TRACE/LOW/MED) TRACE

응 Moisture: not dec.

GC Column: DB-624 ID: $0.20 \quad(\mathrm{~mm})$

Soil Extract Volume: (uL)

Purge Volume: 25.0 $(\mathrm{mL})$
Lab Sample ID: MB 200-7244/5

Lab File ID: JBOC05.D

Date Received:

Date Analyzed: 09/29/2010

Dilution Factor: 1.0

Soil Aliquot Volume:

(UL)

\begin{tabular}{|c|c|c|c|}
\hline CAS NO. & COMPOUND & $\begin{array}{l}\text { CONCENTRATION UNITS: } \\
(\mathrm{ug} / \mathrm{L} \text { or } \mathrm{ug} / \mathrm{kg}) \mathrm{ug} / \mathrm{L}\end{array}$ & $Q$ \\
\hline $79-01-6$ & Trichloroethene & 0.50 & $\overline{\mathrm{U}}$ \\
\hline $1.08-87-2$ & Methylcyclohexane & 0.50 & $\mathrm{U}$ \\
\hline $78-87-5$ & 1,2-Dichloropropane & 0.50 & $\mathrm{U}$ \\
\hline $75-27-4$ & Bromodichloromethane & 0.50 & $\mathrm{U}$ \\
\hline $10061-01-5$ & cis-1,3-Dichloropropene & 0.50 & $\mathrm{U}$ \\
\hline $108-10-1$ & 4-Methyl-2-pentanone & 5.0 & $\mathrm{U}$ \\
\hline $108-88-3$ & Toluene & 0.50 & $\mathrm{U}$ \\
\hline $10061-02-6$ & trans-1,3-Dichloropropene & 0.50 & $\mathrm{U}$ \\
\hline $79-00-5$ & $1,1,2$-Trichloroethane & 0.50 & $\mathrm{U}$ \\
\hline $127-18-4$ & Tetrachloroethene & 0.50 & $\mathrm{U}$ \\
\hline $591-78-6$ & 2-Hexanone & 5.0 & $\mathrm{U}$ \\
\hline $124-48-1$ & Dibromochloromethane & 0.50 & $\mathrm{U}$ \\
\hline $106-93-4$ & 1,2-Dibromoethane & 0.50 & $\mathrm{U}$ \\
\hline $108-90-7$ & Chlorobenzene & 0.50 & $\mathrm{U}$ \\
\hline $100-41-4$ & Ethylbenzene & 0.50 & $\mathrm{U}$ \\
\hline $95-47-6$ & o-xylene & 0.50 & $\bar{U}$ \\
\hline $179601-23-1$ & $\mathrm{~m}, \mathrm{p}$-xylene & 0.50 & $\mathrm{U}$ \\
\hline $100-42-5$ & styrene & 0.50 & $\mathrm{U}$ \\
\hline $75-25-2$ & Bromoform & 0.50 & $\mathrm{U}$ \\
\hline $98-82-8$ & Isopropylbenzene & 0.50 & $\mathrm{U}$ \\
\hline $79-34-5$ & $1,1,2,2$-Tetrachloroethane & 0.50 & $\mathrm{U}$ \\
\hline $541-73-1$ & 1,3-Dichlorobenzene & 0.50 & $\mathrm{U}$ \\
\hline $106-46-7$ & 1,4-Dichlorobenzene & 0.50 & $\mathrm{U}$ \\
\hline $95-50-1$ & 1,2-Dichlorobenzene & 0.50 & $\mathrm{U}$ \\
\hline $96-12-8$ & 1,2-Dibromo-3-Chloropropane & 0.50 & $\mathrm{U}$ \\
\hline $120-82-1$ & $1,2,4$-Trichlorobenzene & 0.50 & $\mathrm{U}$ \\
\hline $87-61-6$ & $1,2,3$-Trichlorobenzene & 0.041 & $\bar{J}$ \\
\hline
\end{tabular}


$1 \mathrm{~J}$ - FORM I VOA-TIC

VOLATILE ORGANICS ANALYSIS DATA SHEET TENTATIVELY IDENTIFIED COMPOUNDS
EPA SAMPLE NO.

VBLKJR

Lab Name: TESTAMERICA BURLINGTON

Contract: $8 \mathrm{E}-00302$

Lab Code: STLV

Case No.: MORRIL Mod. Ref No.: SDG No.: 200-1702

Matrix: (SOIL/SED/WATER) water

Sample wt/vol: 25.0 $(\mathrm{g} / \mathrm{mL}) \mathrm{mL}$

Level: (TRACE or LOW/MED) TRACE

\% Moisture: not dec.

GC Column: DB-624 ID: 0.20 (mm)

Soil Extract Volume: (uL)

CONCENTRATION UNITS: (ug/I or $u g / \mathrm{kg}) \mathrm{ug} / \mathrm{L}$
Lab Sample ID: MB 200-7244/5

Lab File ID: JBOC05.D

Date Received:

Date Analyzed: 09/29/2010

Dilution Factor: 1.0

Soil Aliquot Volume: (uL)

Purge Volume: 25.0

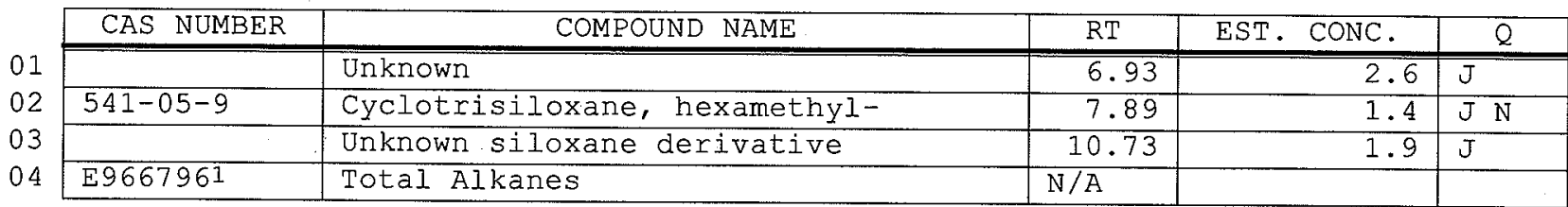

1 EPA-designated Registry Number. 
$1 A$ - FORM I VOA-1

VOLATILE ORGANICS ANALYSIS DATA SHEET
EPA SAMPLE NO.

VIBLKJZ
I ab Name: TESTAMERICA BURLINGTON

Lab Code: STLV

Case No.: MORRIL Mod. Ref No.:
Contract: 8E-00302

SDG No.: 200-1702
Matrix: (SOIL/SED/WATER) Water

Sample wt/vol: 25.0 $(\mathrm{g} / \mathrm{mL}) \mathrm{mL}$

Level: (TRACE/LOW/MED) TRACE

․ㅡㅇ Moisture: not dec.

GC Column: $\mathrm{DB}-624$ ID: $0.20 \quad(\mathrm{~mm})$

Soil Extract Volume:

Purge volume: 25.0
Lab Sample ID: VIBLK 200-7244/15

Lab File ID: JBOC15.D

Date Received:

Date Analyzed: 09/29/2010

Dilution Factor: 1.0

Soil Aliquot Volume: (uL)

\begin{tabular}{|c|c|c|c|}
\hline CAS NO. & COMPOUND & $\begin{array}{l}\text { CONCENTRATION UNITS: } \\
(\mathrm{ug} / \mathrm{L} \text { or } \mathrm{ug} / \mathrm{kg}) \mathrm{ug} / \mathrm{I}\end{array}$ & $Q$ \\
\hline $75-71-8$ & Dichlorodifluoromethane & 0.50 & $\overline{\mathrm{U}}$ \\
\hline $74-87-3$ & Chloromethane & 0.50 & $\mathrm{U}$ \\
\hline $75-01-4$ & Vinyl chloride & 0.50 & $\bar{U}$ \\
\hline $74-83-9$ & Bromomethane & 0.50 & $\bar{U}$ \\
\hline $75-00-3$ & Chloroethane & 0.50 & $\bar{U}$ \\
\hline $75-69-4$ & Trichlorofluoromethane & 0.50 & $\mathrm{U}$ \\
\hline $75-35-4$ & 1,1-Dichloroethene & 0.50 & $\mathrm{U}$ \\
\hline $76-13-1$ & $1,1,2$-Trichloro-1,2,2-trifluoroethane & 0.50 & $\mathrm{U}$ \\
\hline $67-64-1$ & Acetone & 1.7 & $\mathrm{~J}$ \\
\hline $75-15-0$ & Carbon disulfide & 0.10 & $\mathrm{~J}$ \\
\hline $79-20-9$ & Methyl acetate & 0.50 & $\mathrm{U}$ \\
\hline $75-09-2$ & Methylene Chloride & 0.50 & $\mathrm{U}$ \\
\hline $156-60-5$ & trans-1,2-Dichloroethene & 0.50 & $\mathrm{U}$ \\
\hline $1634-04-4$ & Methyl tert-butyl ether & 0.50 & $\mathrm{U}$ \\
\hline $75-34-3$ & 1,1-Dichloroethane & 0.50 & $\bar{U}$ \\
\hline $156-59-2$ & Cis-1,2-Dichloroethene & 0.50 & $\mathrm{U}$ \\
\hline $78-93-3$ & 2-Butanone & 5.0 & $\mathrm{U}$ \\
\hline $74-97-5$ & Bromochloromethane & 0.50 & $\mathrm{U}$ \\
\hline $67-66-3$ & Chloroform & 0.50 & $\mathrm{U}$ \\
\hline $71-55-6$ & 1,1,1-Trichloroethane & 0.50 & $\mathrm{U}$ \\
\hline $110-82-7$ & Cyclohexane & 0.50 & $\mathrm{U}$ \\
\hline $56-23-5$ & Carbon tetrachloride & 0.50 & $\mathrm{U}$ \\
\hline $71-43-2$ & Benzene & 0.50 & $\overline{\mathrm{U}}$ \\
\hline $107-06-2$ & 1,2-Dichloroethane & 0.50 & $\bar{U}$ \\
\hline
\end{tabular}

Report 1,4-Dioxane for Low-Medium VOA analysis only 
Lab Name: TESTAMERICA BURLINGTON

Lab Code: STLV

Case No.: MORRIL Mod. Ref No.:
Contract: $8 \mathrm{E}-00302$

SDG No.: 200-1702
Sample wt/vol: 25.0 $(\mathrm{g} / \mathrm{mL}) \mathrm{mL}$

Level: (TRACE/LOW/MED) TRACE

․ Moisture: not dec.

GC Column: DB-624

Soil Extract Volume: ID: $0.20 \quad(\mathrm{~mm})$

Purge Volume: 25.0 (UL) (mL)
Lab Sample ID: VIBLK 200-7244/15

Lab File ID: JBOC15.D

Date Received:

Date Analyzed: 09/29/2010

Dilution Factor: 1.0

Soil Aliquot Volume:

(uL)

\begin{tabular}{|c|c|c|c|}
\hline CAS NO. & COMPOUND & $\begin{array}{l}\text { CONCENTRATION UNITS: } \\
(\mathrm{ug} / \mathrm{L} \text { or } \mathrm{ug} / \mathrm{kg}) \mathrm{ug} / \mathrm{L} \\
\end{array}$ & $\mathrm{Q}$ \\
\hline $79-01-6$ & Trichloroethene & 0.50 & $\overline{\mathrm{U}}$ \\
\hline $108-87-2$ & Methylcyclohexane & 0.50 & $\mathrm{U}$ \\
\hline $78-87-5$ & 1,2-Dichloropropane & 0.50 & $\mathrm{U}$ \\
\hline $75-27-4$ & Bromodichloromethane & 0.50 & $\mathrm{U}$ \\
\hline $10061-01-5$ & cis-1,3-Díchloropropene & 0.50 & $\mathrm{U}$ \\
\hline $108-10-1$ & 4-Methyl-2-pentanone & 5.0 & $\mathrm{U}$ \\
\hline $108-88-3$ & Toluene & 0.50 & $\mathrm{U}$ \\
\hline $10061-02-6$ & trans-1,3-Dichloropropene & 0.50 & $\mathrm{U}$ \\
\hline $79-00-5$ & 1,1,2-Trichloroethane & 0.50 & $\mathrm{U}$ \\
\hline $127-18-4$ & Tetrachloroethene & 0.50 & $\mathrm{U}$ \\
\hline $591-78-6$ & 2-Hexanone & 5.0 & $\mathrm{U}$ \\
\hline $124-48-1$ & Dibromochloromethane & 0.50 & $\mathrm{U}$ \\
\hline $106-93-4$ & 1,2-Dibromoethane & 0.50 & $\mathrm{U}$ \\
\hline $108-90-7$ & Chlorobenzene & 0.50 & $\mathrm{U}$ \\
\hline $100-41-4$ & Ethylbenzene & 0.50 & $\mathrm{U}$ \\
\hline $95-47-6$ & o-xylene & 0.50 & U \\
\hline $179601-23-1$ & $m, p-x y l e n e$ & 0.50 & $\mathrm{U}$ \\
\hline $100-42-5$ & Styrene & 0.50 & $\mathrm{U}$ \\
\hline $75-25-2$ & Bromoform & 0.50 & $\mathrm{U}$ \\
\hline $98-82-8$ & Isopropylbenzene & 0.50 & $\mathrm{U}$ \\
\hline $79-34-5$ & $1,1,2,2$-Tetrachloroethane & 0.50 & $\mathrm{U}$ \\
\hline $541-73-1$ & 1,3-Dichlorobenzene & 0.50 & $\mathrm{U}$ \\
\hline $106-46-7$ & 1,4-Dichlorobenzene & 0.50 & $\mathrm{U}$ \\
\hline $95-50-1$ & 1,2-Dichlorobenzene & 0.50 & $\mathrm{U}$ \\
\hline $96-12-8$ & 1,2-Dibromo-3-Chloropropane & 0.50 & $\mathrm{U}$ \\
\hline $120-82-1$ & $1,2,4$-Trichlorobenzene & 0.50 & $\mathrm{U}$ \\
\hline $87-61-6$ & 1,2,3-Trichlorobenzene & 0.50 & $\mathrm{U}$ \\
\hline
\end{tabular}


$1 \mathrm{~J}$ - FORM I VOA-TIC

VOLATILE ORGANICS ANALYSIS DATA SHEET

TENTATIVELY IDENTIFIED COMPOUNDS
EPA SAMPLE NO.

VIBLKJZ
Lab Name: TESTAMERICA BURLINGTON

Contract: $8 E-00302$

Lab Code: STLV

Case No.: MORRIL Mod. Ref No.:

\section{Matrix: (SOIL/SED/WATER) Water}

Sample wt/vol: 25.0

Level: (TRACE or LOW/MED) TRACE

\% Moisture: not dec.

GC Column: $\mathrm{DB}-624$

Soil Extract Volume: ID $: 0.20$ $(\mathrm{mm})$

(UL)

CONCENTRATION UNITS: (ug/L or $\mathrm{ug} / \mathrm{kg}$ ) ug/L SDG .No.: 200-1702

Lab Sample ID: VIBLK 200-7244/15

Lab File ID: JBOC15.D

Date Received:

Date Analyzed: 09/29/2010

Dilution Factor: 1.0

Soil Aliquot volume: (uL)

Purge Volume: 25.0 (mL)

\begin{tabular}{l|l|l|l|r|r|}
\hline \multicolumn{1}{c|}{ CAS NUMBER } & \multicolumn{1}{|c|}{ COMPOUND NAME } & RT & ẼST. CONC. & $\mathrm{Q}$ \\
\cline { 2 - 6 } 01 & & Unknown & 6.93 & 2.6 & $\mathrm{~B} \mathrm{X} \mathrm{J}$ \\
\cline { 2 - 6 } 02 & E9667961 & Total Alkanes & N/A & & \\
\hline
\end{tabular}

IEPA-designated Registry Number. 
Supplement 4:

Sample Documentation from TestAmerica Laboratories, Inc., for Indoor Air Samples Samples 


\section{Radon Analytical Data}




\section{8}

Send results to: Clyde Dennis Argọne National Lab

MATRIX: Radon Test Kits RECEIVING LAB: Air CheK Inc PROJECT/SITE: Morrill KS SAMPLER(S) (Signature) DATE OF COLLECTION

CTION SAMPLE ID NUMBER(S)

Aag $12-16,2010$ Aus $12-16,2010$ Aas $11-16,2010$ Ans $12-16,2010$ Ang $12-16,2010$ Ang $\frac{12-16}{12-16}, \frac{2010}{2010}$ Ang $12-16,2010$ An $11-16,2010$ Acs $12-16,2010$ Ang $12-16,2010$ Aas $11-16,2010$ Aas $12-16,2010$ Aag $11-16,2010$ Aas 12-16,2010 Aag $12-16,2010$ Relinquished by (Signature) $\longrightarrow$

Relinquished by (Signature)

\begin{tabular}{|l|l|l}
\hline$Y$ & $N$ & \\
\hline & & \\
\hline & & \\
\hline & & \\
\hline & &
\end{tabular}

5805159 $5805 / 60$ 5805170 $5805 / 62$ 5805166 5805165 5805171 5805176 5805161

5805175

5805157

5805150 5805169 5805164 5805177 Date 1 Time $8 / 17 / 10 \quad 15,00$

FOR LAB USE ONLY

Sample containers were intact when received.

Sample labels, Tags and COC agree. $9700 \mathrm{~S}$ Cass A.ve

\begin{tabular}{|l|l|l|l}
\hline Date & Time & Received for Laboratory by & Date
\end{tabular} BLDG 203 RM B-110 Lemont IL 60439-4803 cdennis@anl.gov

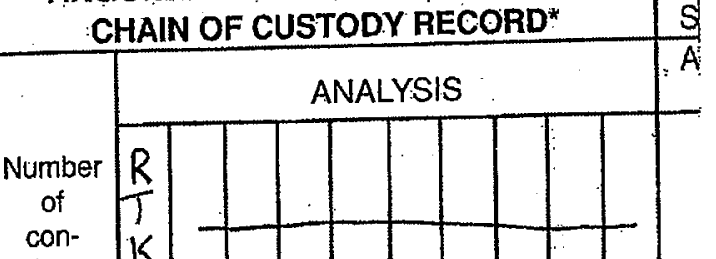

\section{REMARKS}

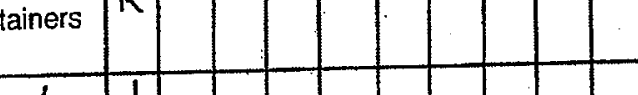

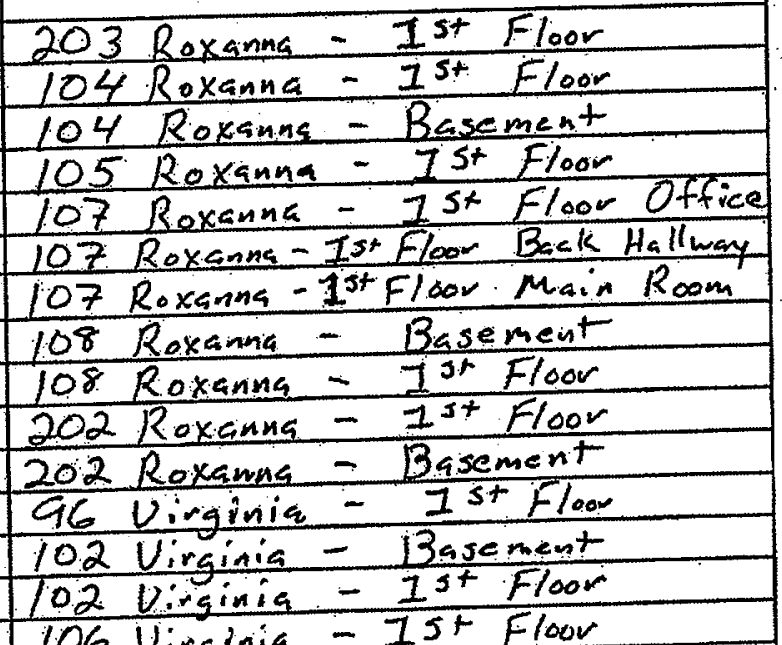

106 Virginia - $75 t$ Floor

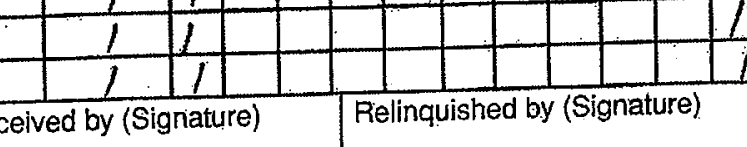

Date

Time

\section{Remarks} \\ $\longrightarrow$ ):}

*A sample is under custody if: 1. It is in your possession; or,

It is in your view, after having been in yoụr possession; or

3. It was in your possession and you locked it up; or,

4. It is in a designated secure area.

Argonne Nation labels, Tags and CoC agree. 
From: Air Chek Inc [mailto:apacheeplus.airchek.com] On Behalf of Air Chek, Inc.

Sent: Wednesday, August 18, $20101: 11$ PM

To: Dennis, Clyde B.

Subject: Radon Test Report \#5694017,5694020,5694027,

08/18/10 ACTIVATED CHARCOAL RADON TE
* Radon Test Result $=1.4 \mathrm{pCi} / \mathrm{L}$
* Test started $08 / 12 / 10$ at $1: 00 \mathrm{pm}$
* Test Ended $08 / 16 / 10$ at $2: 00 \mathrm{pm}$
* Location 1 1st Floor

203 ROXANNA ST MORRILL KS

,

* * INTERPRETING YOUR TEST RESULT

The USEPA states that there is little short-term risk with test results in this range $(0.6$ to $1.9 \mathrm{pCi} / \mathrm{L})$. However, because radon levels fluctuate daily as well as seasonally, you may want to retest during another season. Additionally, if you make any structural changes or start to use a lower level of the building more frequently, you should test again.

You may be able to obtain additional information about radon related subjects by calling your state radon officer at. or call the "Radon Fix-It Line" at 800-644-6999 Monday thru Friday between NOON and 8 pm EST 
Original Message----

From: Air Chek Inc [mailto: apache@plus.airchek.com] On Behalf of Air Chek, Inc.

Sent: Wednesday, August 18, 2010 1:11 PM

To: Dennis, Clyde B.

Subject: Radon Test Report \#5694017,5694020,5694027,

08/18/10 ACTIVATED CHARCOAL RADON TEST \#5805160

* Radon Test Result $=1.9 \mathrm{pCi} / \mathrm{L}$

* Test started $08 / 12 / 10$ at 3:00 pm

* Test Ended 08/16/10 at 3:00 pm

* Location Ist Floor

104 ROXANNA ST MORRILL KS

* * INTERPRETING YOUR TEST RESULT

The USEPA states that there is little short-term risk with test results in this range $(0.6$ to $1.9 \mathrm{pCi} / \mathrm{L})$. However, because radon levels fluctuate daily as well as seasonally, you may want to retest during another season. Additionally, if you make any structural changes or start to use a lower level of the building more frequently, you should test again.

You may be able to obtain additional information about radon related subjects by calling your state radon officer at. Or call the "Radon Fix-It Line" at 800-644-6999 Monday thru Friday between NOON and 8 pm EST 
----Original Message----

From: Air Chek Inc [mailto:apacheeplus.airchek.com] on Behalf of Air Chek, Inc.

Sent: Wednesday, August 18, $20101: 11$ PM

To: Dennis, Clyde B.

Subject: Radon Test Report \#5694017,5694020,5694027,

08/18/10 ACTIVATED CHARCOAL RADON TEST \#5805170

* Radon Test Result $=2.3 \mathrm{pCi} / \mathrm{I}$

* Test started 08/11/10 at 3:00 pm

* Test Ended 08/16/10 at 3:00 pm

* Location Basement

104 ROXANNA ST MORRILI KS

* * INTERPRETING YOUR TEST RESULT

With results in this range $(2.0$ to $3.9 \mathrm{pCi} / \mathrm{L})$ the USEPA recommends that you conduct further tests to determine the true annual average. If the result remains between 2 and 4 there is little short-term risk, but you should consider fixing your home. Additionally, if you make any structural changes or start to use a lower level of the building more frequentiy, you should test again.

You may be able to obtain additional information about radon related subjects by calling your state radon officer at. or call the "Radon Fix-It Line" at 800-644-6999 Monday thru Friday between NOON and 8 pm EST 


\section{Online Test Results}

Test Number:

OK.

Help

\section{Test Number: $\mathbf{5 8 0 5 1 6 2}$ Result: $\mathbf{5 . 0}$ pCi/I}

- This test was recelved for analysis on $08 / 18 / 2010$

- The total exposure time was 94 hours

- Starting on 08/12/2010 at 5:00 pm

- Ending on 08/16/2010 at 3:00 pm

\section{EPA Recommendations}

The USEPA states that for test results in this range (4 to $8 \mathrm{pCi} / \mathrm{L}$ ) you should conduct either a short-or long-term follow-up measurement. If, however, this is a follow-up (confiming) test, it is recommended that you take remedial action to reduce these radon levels.

Click here for EPA Radon Publications

\section{Printed Reports?}

Your formal written report is being mailed to the address entered into our computer when the test kit was first purchased...OR...to the address that may have been printed on the sample packet by the end user.

You may use your Browser's print function to print out this abbreviated report or you have the option of calling or faxing our office to request a faxed copy. Additionally, you may click this link to send your request directly to our server's mail box.

Click here to contact your state radon office

$$
\text { lOS ROXANNA ST. - IST FLOOR }
$$


From: Air Chek Inc [mailto: apachedplus,airchek.com] On Behalf of Air Chek, Inc.

Sent: Wednesday, August 18, 2010 1:11 PM

To: Dennis, Clyde B.

Subject: Radon Test Report \#5694017,5694020,5694027,

08/18/10 ACTIVATED CHARCOAI RADON TEST \#5805166

* Radon Test Result $=3.0 \mathrm{pCi} / \mathrm{L}$

* Test Started $08 / 12 / 10$ at 5:00 pm

* Test Ended 08/16/10 at 2:00 pm

* Location 1st Floor

107 ROXANNA ST OFFICE MORRILL KS

,

* * INTERPRETING YOUR TEST RESULT

With results in this range $(2.0$ to $3.9 \mathrm{pCi} / L)$ the USEPA recommends that you conduct further tests to determine the true annual average. If the result remains between 2 and 4 there is little short-term risk, but you should consider fixing your home. Additionally, if you make any structural changes or start to use a lower. level of the building more frequently, you should test again.

You may be able to obtain additional information about radon related subjects by calling your state radon officer at.

Or call the "Radon Fix-It Line" at 800-644-6999 Monday thru Friday between NOON and 8 pm EST 
-----Original Message-----

From: Air Chek Inc [mailto:apacheeplus.airchek.com] On Behalf of Air Chek, Inc.

Sent: Wednesday, August 18, $20101: 11$ PM

To: Dennis, Clyde B.

Subject: Radon Test Report \#5694017,5694020,5694027,

08/18/10 ACTIVATED CHARCOAI RADON TH
* Radon Test Result $=3.0 \mathrm{pCi} / \mathrm{I}$
* Test Started $08 / 12 / 10$ at $5: 00 \mathrm{pm}$
* Test Ended $08 / 16 / 10$ at $2: 00 \mathrm{pm}$
* Location $\quad$ 1st Floor

107 ROXANNA ST MORRILL KS

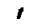

* INTERPRETING YOUR TEST RESULT

With results in this range $(2.0$ to $3.9 \mathrm{pCi} / L)$ the USEPA recommends that you conduct further tests to determine the true annual average. If the result remains between 2 and 4 there is little short-term risk, but you should consider fixing your home. Additionally, if you make any structural changes or start to use a lower level of the building more frequently, you should test again.

You may be able to obtain additional information about radon related subjects by calling your state radon officer at. Or call the "Radon Fix-It Line" at 800-644-6999 Monday thru Friday. between NOON and 8 pm EST 
-----Original Message-----

From: Air Chek Inc [mailto:apacheeplus.airchek.com] On Behalf of Air Chek, Inc.

Sent: Wednesday, August 18, 2010 1:11 PM

To: Dennis, Clyde B.

Subject: Radon Test Report \#5694017,5694020,5694027,

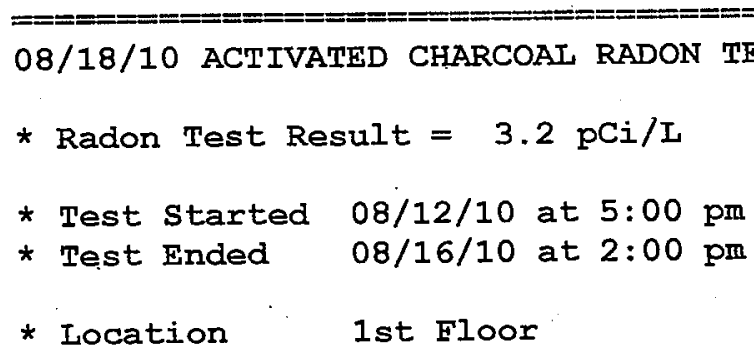

107 ROXANNA ST MAIN MORRILL KS

,

** INTERPRETING YOUR TEST RESULT

With results in this range $(2.0$ to $3.9 \mathrm{pCi} / L)$ the USEPA recommends that you conduct further tests to determine the true annual average. If the result remains between 2 and 4 there is little short-term risk, but you should consider fixing your home. Additionally, if you make any structural changes or start to use a lower level of the building more frequently, you should test again.

You may be able to obtain additional information about radon related subjects by calling your state radon officer at. Or call the "Radon Fix-It Line" at 800-644-6999 Monday thru Friday between NOON and 8 pm EST 
-----Original Message-----

From: Air Chek Inc [mailto:apacheeplus.airchek.com] On Behalf of Air Chek, Inc.

Sent: Wednesday, August 18, 2010 1:11 PM

To: Dennis, Clyde B.

Subject: Radon Test Report \#5694017,5694020,5694027,

08/18/10 ACTIVATED CHARCOAL RADON TEST \#5805176

* Radon Test Result $=8.5 \mathrm{pCi} / \mathrm{L}$

* Test Started 08/11/10 at 4:00 pm

* Test Ended 08/16/10 at 3:00 pm

* Location Basement

108 ROXANNA ST MORRILI KS

,

** INTERPRETING YOUR TEST RESULT

The USEPA states that for test results in this range (8 to $100 \mathrm{pCi} / \mathrm{L}$ ) you should conduct a short-term follow-up measurement within the next few weeks, under closed house conditions. A'long-term measurement is NOT recommended because additional exposure at these levels could pose an increased health risk. If, however this is a follow-up (confirming) test, it is recommended that you take remedial action to reduce these radon levels.

You may be able to obtain additional information about radon related subjects by calling your state radon officer at. Or call the "Radon Fix-It Line" at 800-644-6999 Monday thru Friday between NOON and 8 pm EST 
----Original Message-----

From: Air Chek Inc [mailto:apacheeplus.airchek.com] On Behalf of Air Chek, Inc.

Sent: Wednesday, August 18, $20101: 11$ PM

To: Dennis, Clyde B.

Subject: Radon Test Report \#5694017,5694020,5694027,

08/18/10 ACTIVATED CHARCOAL RADON TEST \#5805161

* Radon Test Result $=5.9 \mathrm{pCi} / \mathrm{L}$

* Test started $08 / 12 / 10$ at $4: 00 \mathrm{pm}$

* Test Ended 08/16/10 at 3:00 pm

* Location 1st Floor

108 ROXANNA ST MORRILI KS

,

* * INTERPRETING YOUR TEST RESULT

The USEPA states that for test results in this range (4 to $8 \mathrm{pCi} / \mathrm{I}$ ) you should conduct either a short- or long-term follow-up measurement. If, however, this is a follow-up (confirming) test, it is recommended that you take remedial action to reduce these radon levels.

You may be able to obtain additional information about radon related subjects by calling your state radon officer at.

Or call the "Radon Fix-It Line" at 800-644-6999 Monday thru Friday between NOON and 8 pm EST 
----Original Message----

From: Air Chek Inc [mailto:apache@plus.airchek.com] On Behalf Of Air Chek, Inc.

Sent: Wednesday, August 18, $20101: 11$ PM

To: Dennis, Clyde B.

Subject: Radon Test Report \#5694017,5694020,5694027,

08/18/10 ACTIVATED CHARCOAL RADON TEST \#5805175

* Radon Test Result $=1.3 \mathrm{pCi} / \mathrm{I}$

* Test started 08/12/10 at 1:00 pm

* Test Ended 08/16/10 at 2:00 pm

* Location 1st Floor

202 ROXANNA ST 1ST MORRILL KS

，

* * INTERPRETING YOUR TEST RESULT

The USEPA states that there is little short-term risk with test results in this range $(0.6$ to $1.9 \mathrm{pCi} / \mathrm{L})$. However, because radon levels fluctuate daily as well as seasonally, you may want to retest during another season. Additionally, if you make any structural changes or start to use a lower level of the building more frequently, you should test again.

You may be able to obtain additional information about radon related subjects by calling your state radon officer at. Or call the "Radon Fix-It Line" at 800-644-6999 Monday thru Friday between NOON and 8 pm EST 
-----Original Message-----

From: Air Chek Inc [mailto: apachelplus.airchek.com] On Behalf of Air Chek, Inc.

Sent: Wednesday, August 18, 2010 1:11 PM

To: Dennis, Clyde B.

Subject: Radon Test Report \#5694017,5694020,5694027,

\section{8/18/10 ACTIVATED CHARCOAI RADON TEST \#5805157}

* Rador Test Result $=1.4 \mathrm{pCi} / \mathrm{L}$

* Test Started 08/11/10 at 1:00 pm

* Test Ended 08/16/10 at 2:00 pm

* Location Basement

202 ROXANNA ST MORRILL KS

* * INTERPRETING YOUR TEST RESULT

The USEPA states that there is little short-term risk with test results in this range $(0.6$ to $1.9 \mathrm{pCi} / \mathrm{L})$. However, because radon levels fluctuate daily as well as seasonally, you may want to retest during another season. Additionally, if you make any structural changes or start to use a lower level of the building more frequently, you should test again.

You may be able to obtain additional information about radon related subjects by calling your state radon officer at. Or call the "Radon Fix-It Line" at 800-644-6999 Monday thru Friday between NOON and $8 \mathrm{pm}$ EST 
----Original Message-----

From: Air Chek Inc [mailto:apacheeplus.airchek.com] on Behalf of Air Chek, Inc.

Sent: Wednesday; August 18, $20101: 11$ PM

To: Dennis, Clyde B.

Subject: Radon Test Report \#5694017,5694020,5694027,

08/18/10 ACTIVATED CHARCOAI RADON TEST \#5805150

* Radon Test Result $=0.8 \mathrm{pCi} / \mathrm{L}$

* Test started 08/12/10 at 2:00 pm

* Test Ended 08/16/10 at 3:00 pm

* Location 1st Floor

96 VIRGINIA ST MORRIII KS

,

** INTERPRETING YOUR TEST RESULT

The USEPA states that there is little short-term risk with test results in this range $(0.6$ to $1.9 \mathrm{pCi} / I)$. However, because radon levels fluctuate daily as well as seasonally, you may want to retest during another season. Additionally, if you make any structural changes or start to use a lower level of the building more frequently, you should test again:

You may be able to obtain additional information about radon related subjects by calling your state radon officer at.

Or call the "Radon Fix-It Line" at 800-644-6999 Monday thru Friday

between NOON and 8 pm EST 
-----Original Message-----

From: Air Chek Inc [mailto:apacheeplus.airchek.com] on Behalf of Air Chek, Inc.

Sent: Wednesday, August 18, 2010 1:11 PM

To: Dennis, Clyde B.

Subject: Radon Test Report \#5694017,5694020,5694027,

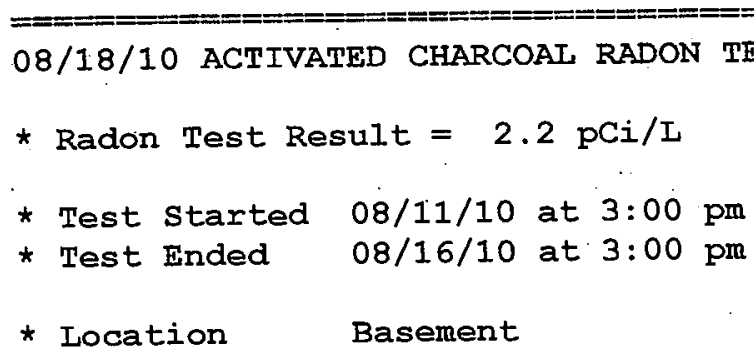

102 VIRGINIA ST MORRILI KS

* * INTERPRETING YOUR TEST RESULT

With results in this range $(2.0$ to $3.9 \mathrm{pCi} / \mathrm{I})$ the USEPA recommends that you conduct further tests to determine the true annual average. If the result remains between 2 and 4 there is little short-term risk, but you should consider fixing your home. Additionally, if you make any structural changes or start to use a lower level of the building more frequently, you should test again.

You may be able to obtain additional information about radon related subjects by calling your state radon officer at. Or call the "Radon Fix-It Line" at 800-644-6999 Monday thru Friday between NOON and 8 pm EST 
From: Air Chek Inc [mailto:apacheeplus.airchek.com] On Behalf of Air Chek, Inc.

Sent: Wednesday, August 18, 2010 1:11. PM

To: Dennis, Clyde B.

Subject: Radon Test Report \#5694017,5694020,5694027,

08/18/10 ACTIVATED CHARCOAL RADON TEST \#5805164

* Radon Test Result $=1.2 \mathrm{pCi} / \mathrm{L}$

* Test started $08 / 12 / 10$ at $3: 00 \mathrm{pm}$

* Test Ended 08/16/10 at 3:00 pm

* Location lst Floor

102 VIRGINIA ST MORRIII KS

,

** INTERPRETING YOUR TEST RESULT

The USEPA states that there is little short-term risk with test results in this range $(0.6$ to $1.9 \mathrm{pCi} / I)$. However, because radon levels fluctuate daily as well as seasonally, you may want to retest during another season. Additionally, if you make any structural changes or start to use a lower level of the building more frequently, you should test again.

You may be able to obtain additional information about radon related subjects by calling your state radon officer at. Or call the "Radon Fix-It Line" at 800-644-6999 Monday thru Friday between NOON and 8 pm EST 
-----Original Message-----

From: Air Chek Inc [mailto:apacheeplus.airchek.com] On Behalf of Air Chek, Inc.

Sent: Wednesday, August 18, 2010 1:11 PM

To: Dennis, Clyde B.

Subject: Radon Test Report \#5694017,5694020,5694027,

08/18/10 ACTIVATED CHARCOAT RADON TEST \#5805177

* Radon Test Result $=1.9 \mathrm{pCi} / \mathrm{L}$

* Test Started 08/12/10 at 3:00 pm

* Test Ended 08/16/10 at 3:00 pm

* Location 1st Floor

106 VIRGINIA ST 1ST MORRILI KS

,

** INTERPRETING YOUR TEST RESULT

The USEPA states that there is little short-term risk with test results in this range $(0.6$ to $1.9 \mathrm{pCi} / \mathrm{L})$. However, because radon levels fluctuate daily as well as seasonally, you may want to retest during another season. Additionally, if you make any structural changes or start to use a lower level of the building more frequently, you should test again.

You may be able to obtain additional information about radon related subjects by calling your state radon officer at.

or call the "Radon Fix-It Line" at 800-644-6999 Monday thru Friday

between NOON and 8 pm EST 


\section{TestAmerica \\ THE LEADER IN ENMTRONHENTAL TESTING}

ANALYTICAL REPORT

Job Number: 200-1136-1

SDG Number: MORRILL (200-1136)

Job Description: MORRILL (200-1136)

Contract Number: 8E-00302

For:

Argonne National Laboratory

9700 South Cass Avenue

Building 203

Office B-149

Argonne, IL 60439

Attention: Mr. Clyde Dennis

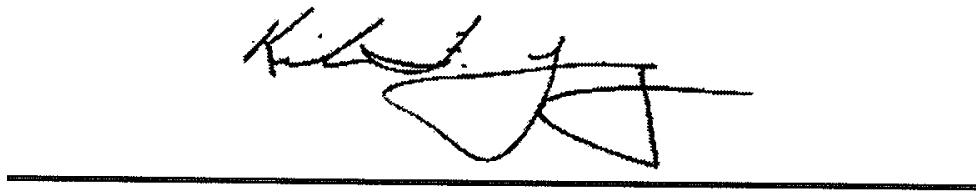

Kirk F Young

Project Manager I

kirk.young@testamericainc.com

$08 / 25 / 2010$

The test results in this report relate only to sample(s) as received by the laboratory. These test results were derived under a quality system that adheres to the requirements of NELAC. Pursuant to NELAC, this report may not be produced in full without written approval from the laboratory

TestAmerica Laboratories, Inc.

TestAmerica Burlington 30 Community Drive, Suite 11, South Burlington, VT 05403

Tel (802) 660-1990 Fax (802) 660-1919 www.testamericainc.com 


\section{Table of Contents}

Cover Title Page ............................. 1

Data Summaries ........................... 4

Report Narrative $\ldots \ldots \ldots \ldots \ldots \ldots \ldots \ldots \ldots \ldots, 4$

Shipping and Receiving Documents $\ldots \ldots \ldots \ldots \ldots \ldots \ldots \ldots$

Client Chain of Custody $\ldots \ldots \ldots \ldots \ldots \ldots \ldots \ldots \ldots \ldots \ldots \ldots \ldots \ldots$

Internal $\operatorname{Coc}$ (if required) $\ldots \ldots \ldots \ldots \ldots \ldots \ldots \ldots \ldots \ldots \ldots \ldots, 9$

Data Summaries ............................... 10

Sample Summary $\ldots \ldots \ldots \ldots \ldots \ldots \ldots \ldots \ldots \ldots \ldots \ldots, 10$

Method Summary $\ldots \ldots \ldots \ldots \ldots \ldots \ldots \ldots \ldots \ldots \ldots \ldots \ldots, 11$

Method / Analyst Summary $\ldots \ldots \ldots \ldots \ldots \ldots \ldots \ldots \ldots \ldots \ldots, 12$

Sample Datasheets $\ldots \ldots \ldots \ldots \ldots \ldots \ldots \ldots \ldots \ldots \ldots \ldots \ldots, 13$

QC Data Summary $\ldots \ldots \ldots \ldots \ldots \ldots \ldots \ldots \ldots \ldots \ldots \ldots \ldots \ldots \ldots, 29$

Data Qualifiers .................................... 30

Organic Sample Data ........................ 31

Air $-\mathrm{GC} / \mathrm{MS}$ VOA $\ldots \ldots \ldots \ldots \ldots \ldots \ldots \ldots \ldots \ldots \ldots \ldots \ldots, 31$

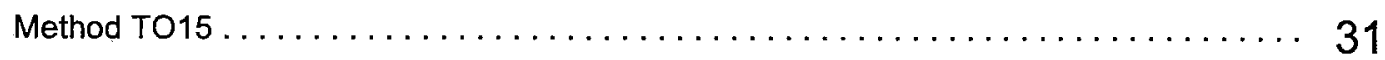

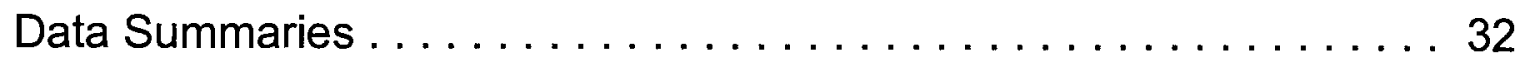

QC Association Summary $\ldots \ldots \ldots \ldots \ldots \ldots \ldots \ldots \ldots \ldots \ldots, 32$

Organic Sample Data ......................... 33

Air $-\mathrm{GC} / \mathrm{MS}$ VOA $\ldots \ldots \ldots \ldots \ldots \ldots \ldots \ldots \ldots \ldots \ldots \ldots \ldots \ldots \ldots, 33$

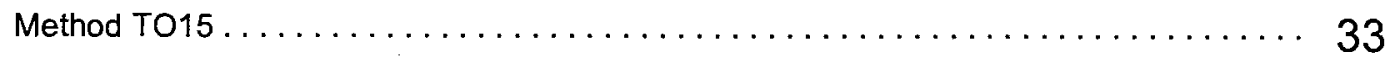

Method TO15 QC Summary ................................................ 33

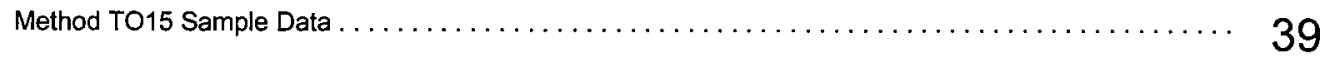

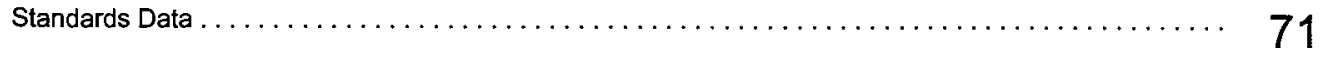

Method TO15 ICAL Data $\ldots \ldots \ldots \ldots \ldots \ldots \ldots \ldots \ldots \ldots \ldots \ldots \ldots \ldots \ldots \ldots \ldots \ldots \ldots \ldots \ldots$

Method TO15 CCAL Data $\ldots \ldots \ldots \ldots \ldots \ldots \ldots \ldots \ldots \ldots \ldots \ldots \ldots \ldots \ldots \ldots \ldots \ldots \ldots$ 


\section{Table of Contents}

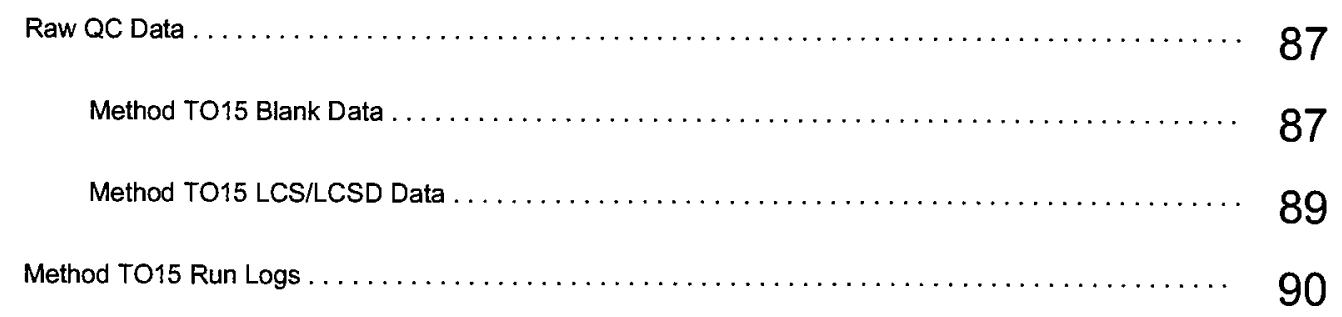

Shipping and Receiving Documents .................. 94

Sample Receipt Checklist . . . . . . . . . . . . . . . . . . 96

Organic Sample Data . . . . . . . . . . . . . . . . . . . . 97

Air - GC/MS VOA . . . . . . . . . . . . . . . . . . . 97

Method TO15 ........................................ 97

Method TO15 Prep Data .................................................... 97 


\section{Client: Argonne National Laboratory}

\section{Project: MORRILL (200-1136)}

\section{Report Number: 200-1136-1}

With the exceptions noted as flags or footnotes, standard analytical protocols were followed in the analysis of the sampla problems were encountered or anomalies observed. In addition all laboratory quality control samples were within establisho limits. Each sample in the sample set was analyzed without a dilution.

Calculations are performed before rounding to avoid round-off errors in calculated results.

All holing time werenet and proper preseration noted for thenethods pformed orthese samples, unles othense detailed in the individual sections below.

\section{RECEIPT}

The samples were received on 08/14D0@amentation of the condition of the samples at the time of receipt and exceptions at the laboratory's Sample Aceptance Policy si included rithe Shipping and Receiving section of this submittal.

The summa canisters and flow regulators that were used in the collection of the samples were prepared an TestAmerica Burlington.

The anister lable forareple 96 VIRGA SDFFICE id not atoh thénformation lisd onhet Cain-of-Custody (COC). Tbenister label listed a canister number of 5073. The COC listed a canister number of 3073 . The sample was logged in usb label that corresponded to the canister used for this sample.

The canister label for sample 202 ROXANNA ST BASEMENT did not match the information listed on the Chain-of-Custod canister label listed a canister number of 5087. The COC listed a canister number of 5082 . The sample was loggen from the label that corresponded to the canister used for this sample.

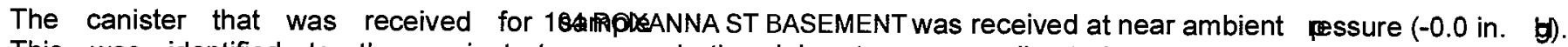
This was identified to the project team, and the laboratory was directed to proceed with thes RemaktisA of sa ST BASEMENT.

\section{VOLATILE ORGANIC COMPOUNDS IN AMBIENT AIR}

The samples in this sample set were analyzed for volatile organics by USEPA Method TO-15. The analytic analysis of the samples are reported in terms of parts per billion on a volume/volume basis (ppbv), with a results to a weight/volume basis $(\mathrm{g} / \mathrm{m} 3)$. Results are reported oly for those instrument responses ith derived values equa to or above the reporting limit. Estimated alues are not reorted for responses ith derived atues below the reporting limit.

The responses in the initial calibration for each target analyte met the 30 percent relative standard deviation response for each target analyte met the 30 percent difference criterion in the calibration check acquisition. analyses associated with the analytical work exhibited an acceptable internal standard performance. A laborato sample was analyzed in the analytical sequence, and there was an acceptable recovery of each target analy analysis. The analysis of the method blank associated with the analytical work was free of analyte contam 
TestAmerica Burlington

30 Community Drive

Sulle 11

South Burlington, VT 05403

phone 802-660-1890 fax 802-660-1919

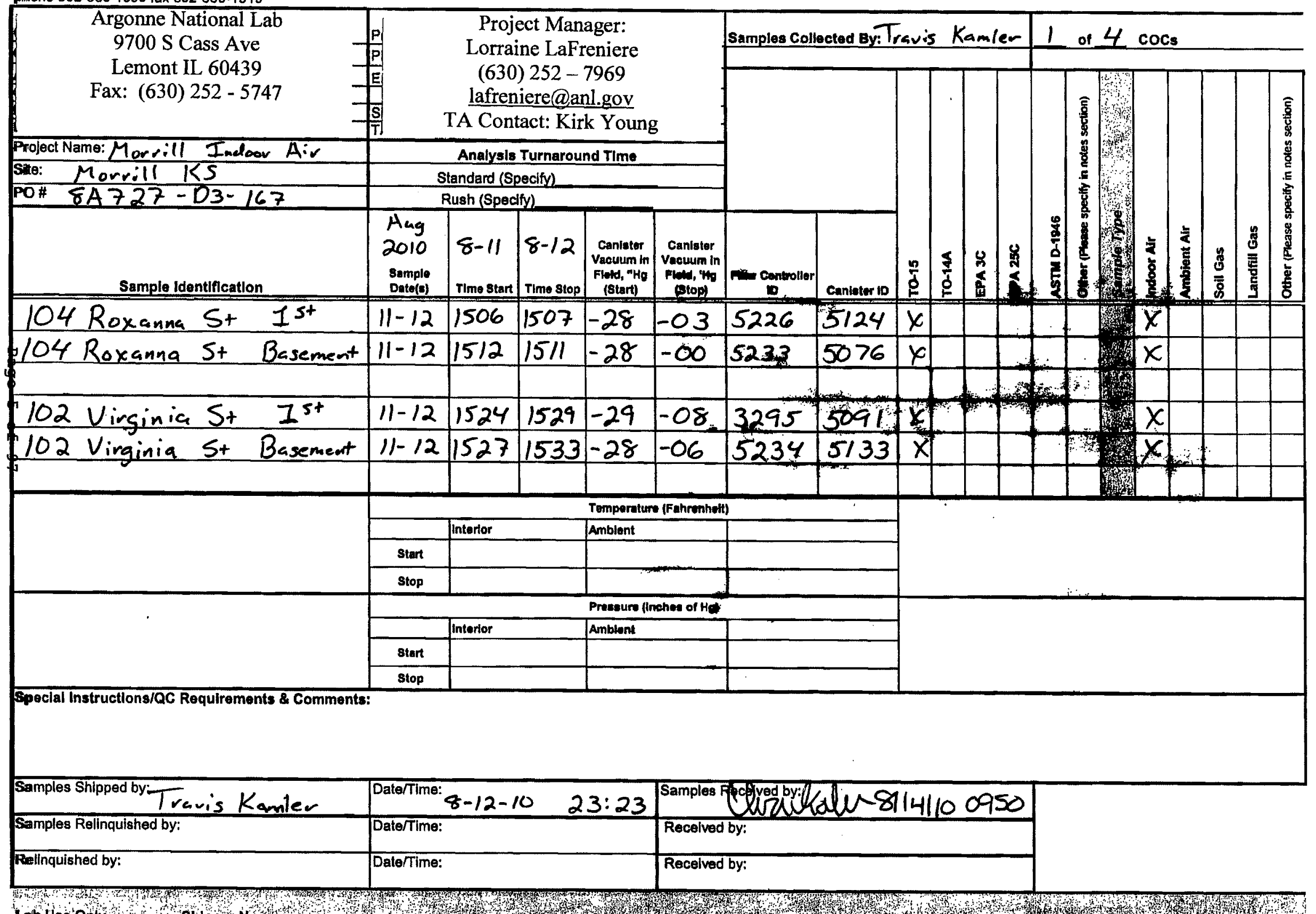

\section{Canister Samples Chain of Custody Record}

TestAmertca Analytical Testing Corp. essumes no llablitty with respect to the collection and shipment or these semples.

Analysls Turnaround Time

Standard (Specify)

Ang

(Start) Plow, th

.num.

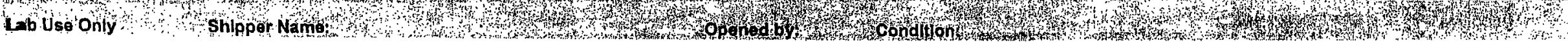


TocitAmorkea Burlington

30 Community Drive

Sulte 11

South Burtington, VT 05403

phone 802-660-1990 fax 802-660-1919

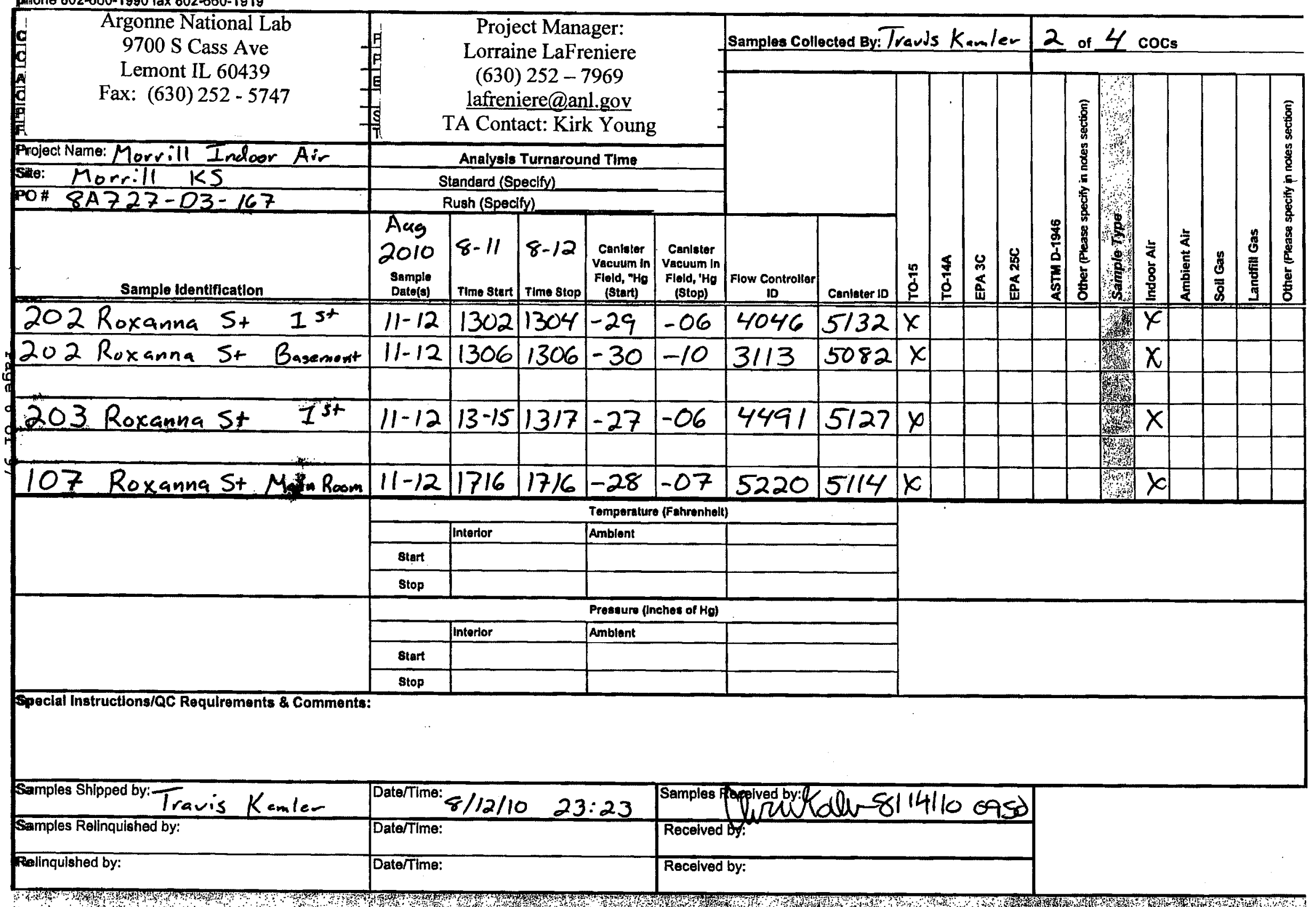

\section{Canister Samples Chain of Custody Record}

TestAmerice Analytical Testing Corp. essumes no llebilly with respect to the callectlon and shipment or these semplos. Lorraine LaFrenie (630) $252-7969$ lafreniere@anl.gov A Contact: Kirk Young Andard (Speclfy) 
TestAnterica Burlington

30 Community Drive

Sulte 11

South Burlington, VT 05403

phone 802-660-1990 fax 802-660-1919

Argonne National Lab 9700 S Cass Ave

Lemont IL 60439

Fax: (630) 252 - 5747

Project Name: Morrill Indoor Air

Sile: Morrill KS

PO\# 8A?27-D3-167

\section{Canister Samples Chain of Custody Record}

TestAmerica Analyllcal Testing Corp. assumes no llabillty with respect to the collection and shipment of these semples.

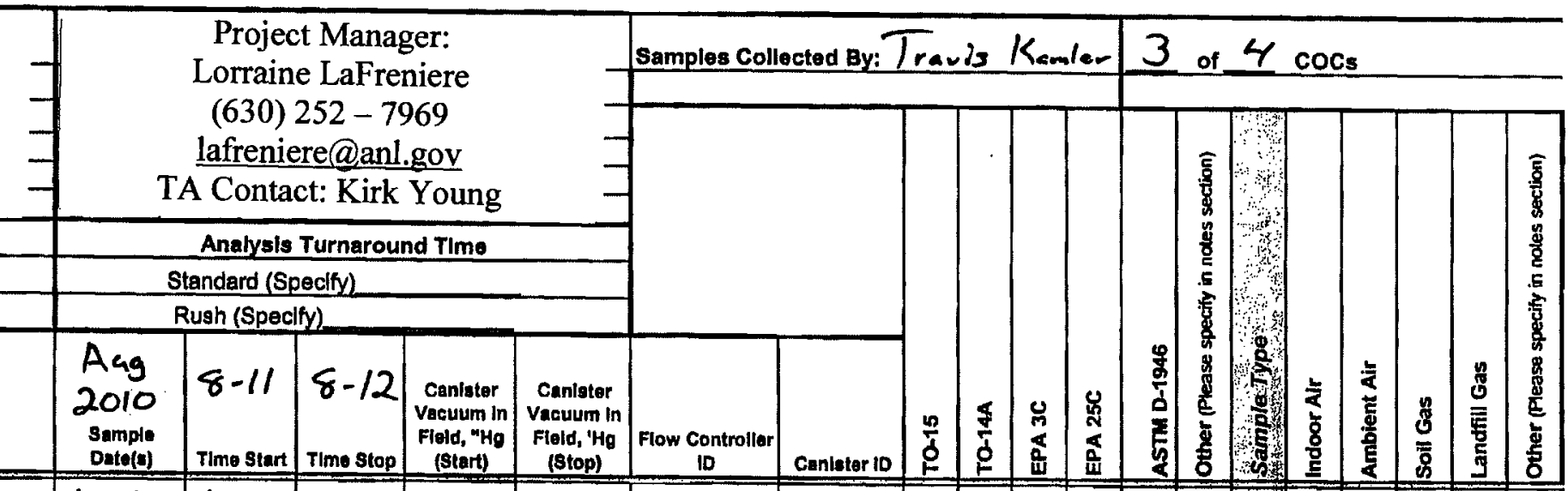

96 Virginia $5+$ Office

Ambient Air MWI

\begin{tabular}{l|l|l|l|}
$11-12$ & 1435 & 1434 \\
\hline
\end{tabular}

(Stop)

$-28$

$-043055$

$3073 x$

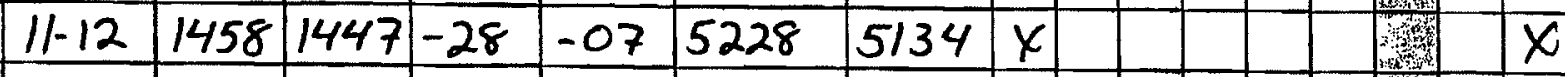

107 Roxanna $5+$ Office

\begin{tabular}{l|l|l|l|l|l|l|l}
$11-12$ & 1712 & 1711 & -28 & -06 & 5227 & 4550 & $x$
\end{tabular}

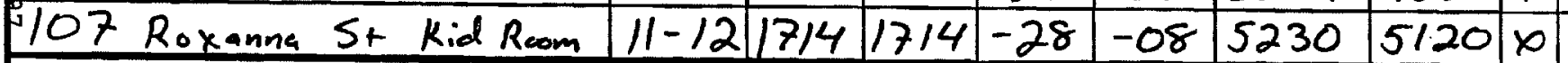

Tomperature (Fahrenheli)

\begin{tabular}{|c|c|c|c|}
\hline & & Tomperature (F ahrenheli) & \\
\hline & Intertor & Amblont & \\
\hline \multicolumn{4}{|l|}{ Start } \\
\hline \multicolumn{4}{|l|}{ Stop } \\
\hline \multicolumn{4}{|c|}{ Pressure (inches of $\mathrm{Hg}$ ) } \\
\hline & Intortor & Amblent & \\
\hline \multicolumn{4}{|l|}{ Btart } \\
\hline Stop & & 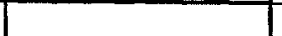 & \\
\hline
\end{tabular}

Special Instructions/QC Róquifiements a Comments:

\begin{tabular}{|c|c|c|}
\hline Samples Shlpped by: Travis $K_{\text {amler }}$ & Date/Time: $\delta / 12 / 10 \quad 23: 23$ & 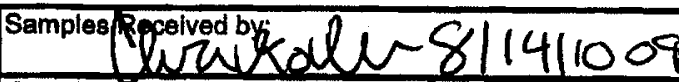 \\
\hline Samples Rellnquished by: & Date/Time: & Recelved by: \\
\hline Relinquished by: & Date/Time: & Recelved by: \\
\hline
\end{tabular}

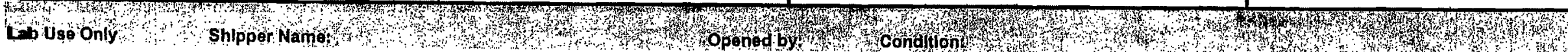


TestAmerica Burlington

30 Community Drive

Sulte 11

South Burllngton, VT 05403

phone 802-660-1990 fax 802-660-1919 Argonne National Lab 9700 S Cass Ave Lemont IL 60439 Fax: (630) 252 - 5747

Project Name: Morrill Indow Air Site: Marrill KS PO\# 84727 - D3- 167

\begin{tabular}{|c|c|c|c|c|c|c|c|c|c|c|c|c|c|c|c|c|c|c|}
\hline \multirow{3}{*}{$\begin{array}{l}\text { silte: Marrill KS } \\
\text { PO\# } 84727-D 3-167\end{array}$} & \multicolumn{5}{|c|}{ Analysis Turnaround Time } & \multirow[b]{4}{*}{$\begin{array}{c}\text { Fow Controliar } \\
\text { ID }\end{array}$} & \multirow[b]{4}{*}{ Canister iD } & \multirow[b]{4}{*}{$\frac{n}{\delta}$} & \multirow[b]{4}{*}{$\frac{\$}{d}$} & \multirow[b]{4}{*}{ 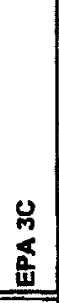 } & \multirow[b]{4}{*}{ 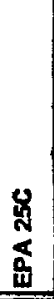 } & \multirow{4}{*}{. } & \multirow{4}{*}{ 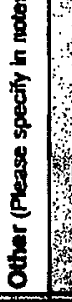 } & \multirow{4}{*}{8} & \multirow[b]{4}{*}{ 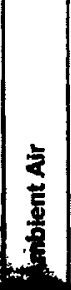 } & \multirow[b]{4}{*}{$\begin{array}{l}\frac{6}{6} \\
\overline{\bar{c}} \\
\end{array}$} & \multirow{4}{*}{ 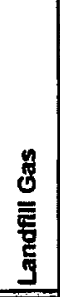 } & \multirow{4}{*}{ 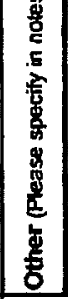 } \\
\hline & & tandard $(\mathrm{Sp}$ & ecify) & & & & & & & & & & & & & & & \\
\hline & & Lush (Sped) & & & & & & & & & & & & & & & & \\
\hline Sample Identification & $\begin{array}{l}\text { Ans } \\
2010 \\
\text { Sampla } \\
\text { Data(s) }\end{array}$ & $\begin{array}{l}8-11 \\
\text { Time stant }\end{array}$ & $\begin{array}{l}8-12 \\
\text { Time stop } \\
\end{array}$ & $\begin{array}{c}\text { Canistor } \\
\text { Vacuum in } \\
\text { Flold, wha } \\
\text { (Start) } \\
\end{array}$ & $\begin{array}{l}\text { Canister } \\
\text { Vacurum in } \\
\text { Field, ' } \mathrm{Hg} \\
\text { (blop) } \\
\end{array}$ & & & & & & & & & & & & & \\
\hline 108 Roxanna St $I^{\text {st }}$ & $11-12$ & 1540 & 1539 & -28 & -07 & 5238 & 5126 & $\mathrm{x}$ & & & & & m. & $x$ & & & & \\
\hline 108 Roxanna $5+$ Basement & $11-12$ & 1542 & 1543 & -28 & -07 & 5232 & 5090 & $x$ & & & & & thes & $\bar{x}$ & & & & \\
\hline 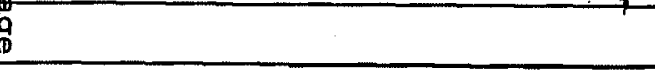 & & & & & & & & & & & & & ming & & & & & \\
\hline${ }_{0}^{\infty} / 06$ Virginia St $I^{5+}$ & $11-12$ & 1620 & 1451 & -28 & -08 & 5214 & 5088 & $x$ & & & & & W & $\chi$ & & & & \\
\hline (1) & & & & & & & & & & & & & & & & & & \\
\hline 105 Roxanna $5+$ & $11-12$ & 1724 & 1703 & -28 & -07 & 4062 & 5078 & $x$ & & & & & yty & $x$ & & & & \\
\hline & & & & Temperatur & ffahrenholl & & & & & & & & & & & & & \\
\hline & & Intertor & & Amblent & & & & & & & & & & & & & & \\
\hline & sturt & & & & & & & & & & & & & & & & & \\
\hline & Stop & & & & & & & & & & & & & & & & & \\
\hline & & & & Promeure fin & chos of $\mathrm{Ho}$ ) & & & & & & & & & & & & & \\
\hline & & Interlor & & Amblent & & & & & & & & & & & & & & \\
\hline & start & & & & & & & & & & & & & & & & & \\
\hline & Stop & & & & & & & & & & & & & & & & & \\
\hline
\end{tabular}

Special Instructions/QC Requiraments \& Comments:

\begin{tabular}{|l|l|l|}
\hline Samples Shipped by: Travis Kamlen & Date/Time: $8 / 12 / 10 \quad 23: 23$ \\
\hline Samples Rellnquished by: & Date/Time: & Date/Time: \\
\hline Ralinquished by: &
\end{tabular}

\section{Canister Smples Chain of Custody Record}

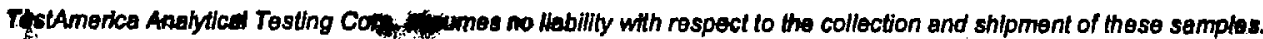

Project Manager:

Lorraine LaFreniere (630) $252-7969$

lafreniere@anl.gov TA Contact: Kirk Young
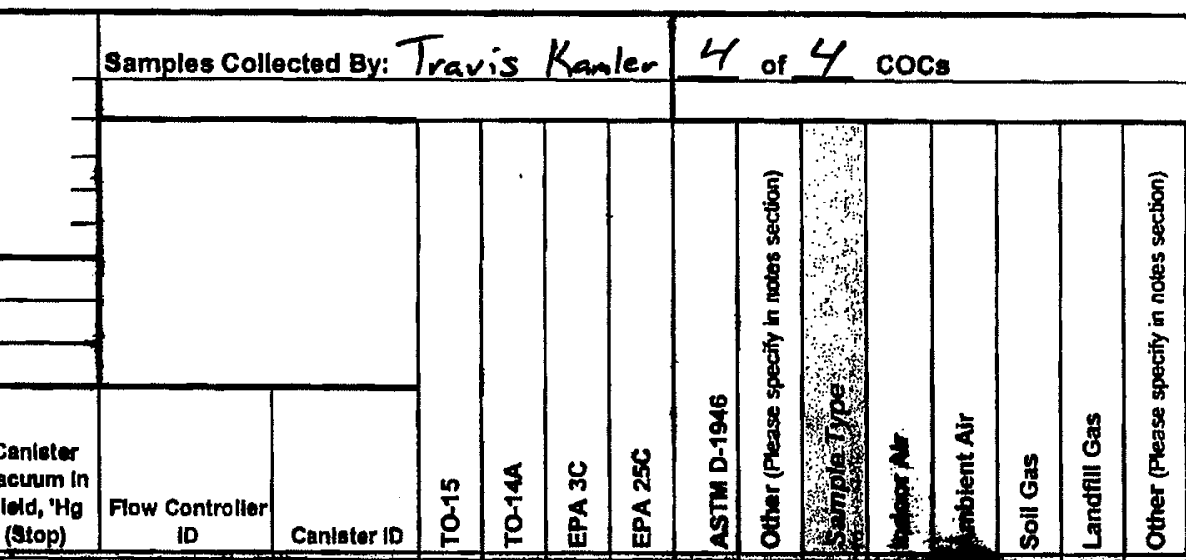
(n) 
TestAmerica Burlington

INTERNAL CHAIN OF CUSTODY LOG (ICOC)

\begin{tabular}{l} 
Project Information: \\
Log in \#: $2 \operatorname{CQ} Q-1136$ \\
\hline Client: $A R G L A B$ \\
\hline
\end{tabular}

\begin{tabular}{|l|l|}
\hline & LAB IDs: $2 C$ \\
\hline Samples associated with this log-in were placed into storage on
\end{tabular} $5+2$

Sample Custodian Signacie

Storage Location:

Air Starcge

Storage Condition: $\square$ Refrigeration Frozen

Specify storage location (refrigerator, freezer iD or lab location) for original sample containers Internal Transfer Information

\section{\begin{tabular}{|c|c|c|c|}
\hline Sample Type & Lab ID(s) & Transfer & Transfer \\
\hline
\end{tabular}}

\begin{tabular}{|c|c|c|}
\hline \multicolumn{2}{|c|}{ Sample Type } & Lab ID(s) \\
\hline Original $^{2}$ & Prepared & \\
\hline$\sim$ & & $1-16$ \\
\hline 6 & & $1-16$ \\
\hline
\end{tabular}

11

\begin{tabular}{|c|c|}
\hline $\begin{array}{c}\text { Transfer } \\
\text { Date }\end{array}$ & $\begin{array}{c}\text { Transfer } \\
\text { Time }^{2}\end{array}$ \\
\hline $8-17 \cdot 10$ & 1605 \\
\hline $8 \cdot 17 \cdot 10$ & 1632 \\
\hline 812.110 & 1000 \\
\hline
\end{tabular}

"1 $\$$ Ambient

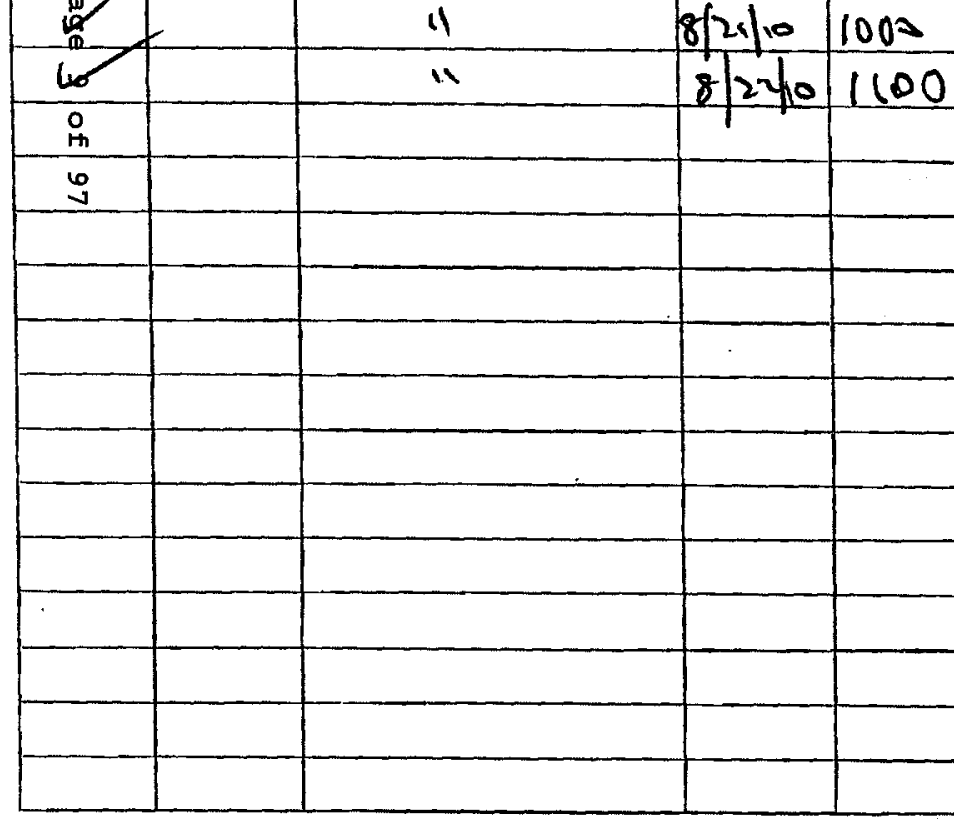

'Extract, cligestate, or any other prepared sample that is no longer in original sample container

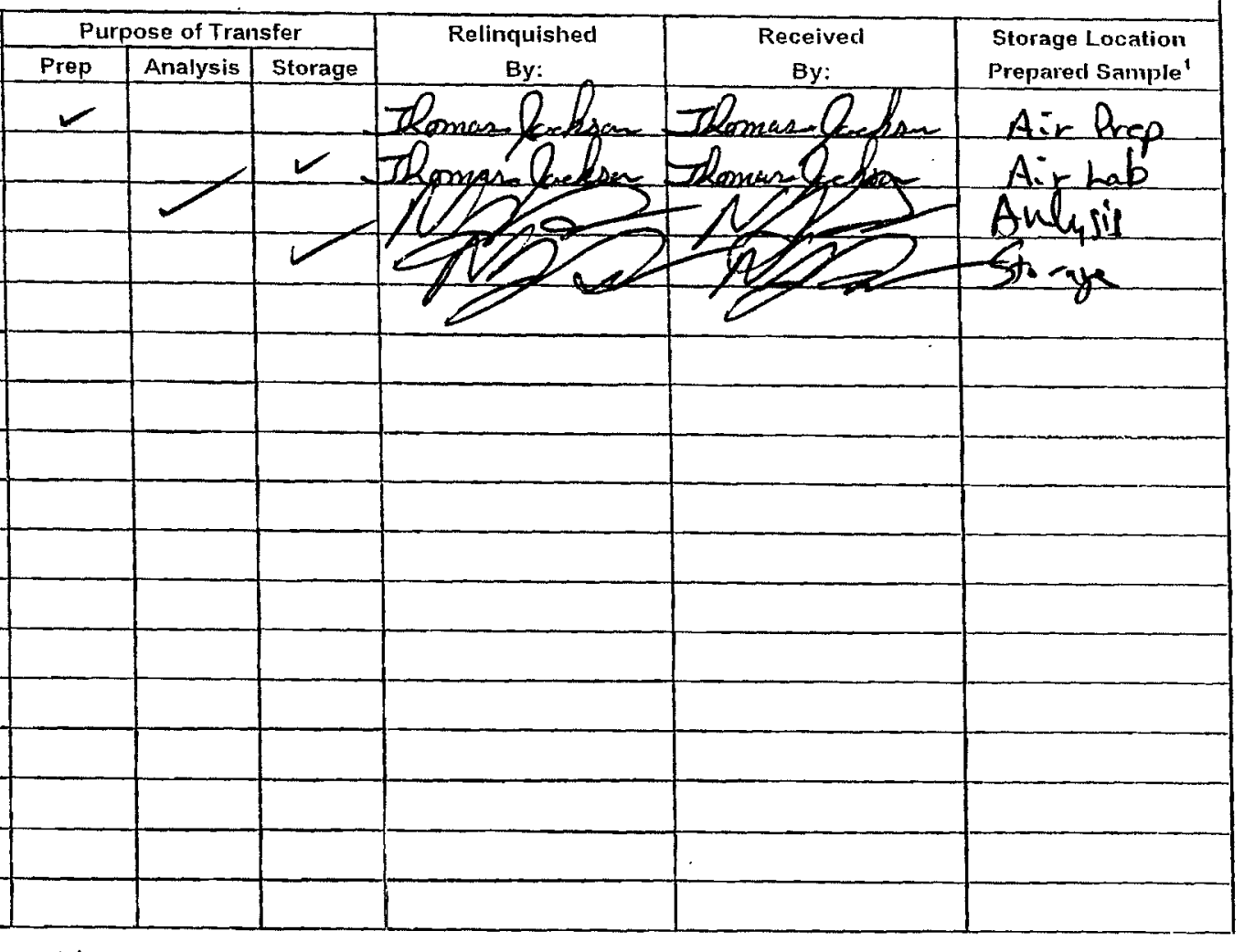

2 Mililary Time 


\section{SAMPLE SUMMARY}

Client: Argonne National Laboratory

Job Number: 200-1136-1

Sdg Number: MORRILL (200-1136)

\begin{tabular}{|c|c|c|c|c|c|c|}
\hline \multirow{2}{*}{$\begin{array}{l}\text { Lab Sample ID } \\
200-1136-1\end{array}$} & \multirow{2}{*}{$\frac{\text { Client Smple ID }}{104 \text { ROXANNA ST 1ST }}$} & \multirow{2}{*}{$\frac{\text { Client }}{\text { Air }}$} & \multicolumn{2}{|c|}{$\begin{array}{c}\text { Date/Time } \\
\text { Sampled }\end{array}$} & \multicolumn{2}{|c|}{$\begin{array}{l}\text { Date/Time } \\
\text { Received } \\
\end{array}$} \\
\hline & & & $08 / 12 / 2010$ & 1507 & $08 / 16 / 2010$ & 0950 \\
\hline $200-1136-2$ & $\begin{array}{l}104 \text { ROXANNA ST } \\
\text { BASEMENT }\end{array}$ & Air & $08 / 12 / 2010$ & 1511 & $08 / 16 / 2010$ & 0950 \\
\hline $200-1136-3$ & 102 VIRGINIA ST 1ST & Air & $08 / 12 / 2010$ & 1529 & $08 / 16 / 2010$ & 0950 \\
\hline $200-1136-4$ & $\begin{array}{l}102 \text { VIRGINIA ST } \\
\text { BASEMENT }\end{array}$ & Air & $08 / 12 / 2010$ & 1533 & $08 / 16 / 2010$ & 0950 \\
\hline $200-1136-5$ & 202 ROXANNA ST $1 \mathrm{ST}$ & Air & $08 / 12 / 2010$ & 1304 & $08 / 16 / 2010$ & 0950 \\
\hline $200-1136-6$ & $\begin{array}{l}202 \text { ROXANNA ST } \\
\text { BASEMENT }\end{array}$ & Air & $08 / 12 / 2010$ & 1306 & $08 / 16 / 2010$ & 0950 \\
\hline $200-1136-7$ & 203 ROXANNA ST 1ST & Air & $08 / 12 / 2010$ & 1317 & $08 / 16 / 2010$ & 0950 \\
\hline $200-1136-8$ & $\begin{array}{l}107 \text { ROXANNA ST MAIN } \\
\text { ROOM }\end{array}$ & Air & $08 / 12 / 2010$ & 1716 & $08 / 16 / 2010$ & 0950 \\
\hline $200-1136-9$ & 96 VIRGINIA ST OFFICE & Air & $08 / 12 / 2010$ & 1434 & $08 / 16 / 2010$ & 0950 \\
\hline $200-1136-10$ & AMBIENT AIR MW1 & Air & $08 / 12 / 2010$ & 1447 & $08 / 16 / 2010$ & 0950 \\
\hline $200-1136-11$ & $\begin{array}{l}107 \text { ROXANNA ST } \\
\text { OFFICE }\end{array}$ & Air & $08 / 12 / 2010$ & 1711 & $08 / 16 / 2010$ & 0950 \\
\hline $200-1136-12$ & $\begin{array}{l}107 \text { ROXANNA ST KID } \\
\text { ROOM }\end{array}$ & Air & $08 / 12 / 2010$ & 1714 & $08 / 16 / 2010$ & 0950 \\
\hline $200-1136-13$ & 108 ROXANNA ST 1ST & Air & $08 / 12 / 2010$ & 1539 & $08 / 16 / 2010$ & 0950 \\
\hline $200-1136-14$ & $\begin{array}{l}108 \text { ROXANNA ST } \\
\text { BASEMENT }\end{array}$ & Air & $08 / 12 / 2010$ & 1543 & $08 / 16 / 2010$ & 0950 \\
\hline $200-1136-15$ & 106 VIRGINIA ST 1ST & Air & $08 / 12 / 2010$ & 1451 & $08 / 16 / 2010$ & 0950 \\
\hline $200-1136-16$ & 105 ROXANNA ST 1ST & Air & $08 / 12 / 2010$ & 1703 & $08 / 16 / 2010$ & 0950 \\
\hline
\end{tabular}




\section{METHOD SUMMARY}

Client: Argonne National Laboratory

Job Number: 200-1136-1

Sdg Number: MORRILL (200-1136)

Description

Lab Location Method

Preparation Method

Matrix: Air

Volatile Organic Compounds in Ambient Air Collection via Summa Canister

TAL BUR

EPA TO-15

TAL BUR

Summa Canister

Lab References:

TAL BUR = TestAmerica Burlington

Method References:

$E P A=$ US Environmental Protection Agency 


\section{METHOD I ANALYST SUMMARY}

Client: Argonne National Laboratory

Job Number: $\quad 200-1136-1$

Sdg Number: MORRILL (200-1136)

Method

Analyst

Analyst ID

EPA TO-15

Valjevac, Sanel

SV

TestAmerica Burlington 


\section{Analytical Data}

Client: Argonne National Laboratory

Client Sample ID: 104 ROXANNA ST 1ST

Lab Sample ID:

Client Matrix:

200-1136-1

Air
Job Number:

200-1136-1

Sdg Number: MORRILL (200-1136)

Date Sampled: $\quad 08 / 12 / 20101507$

Date Received: 08/16/2010 0950
TO-15 Volatile Organic Compounds in Ambient Air

Method: TO-15

Preparation: Summa Canister

Dilution: 1.0

Date Analyzed: 08/21/2010 1810

Date Prepared: 08/21/2010 1810
Analysis Batch: 200-5449

\section{Instrument ID: Lab File ID:} Final Weight/Volume: $200 \mathrm{~mL}$ Injection Volume: 200

\begin{tabular}{|c|c|c|c|c|}
\hline Analyte & Result (ppb v/v) & Qualifier & MDL & $R L$ \\
\hline Chloroform & 0.22 & & 0.050 & 0.20 \\
\hline Carbon tetrachloride & 0.20 & U & 0.050 & 0.20 \\
\hline Analyte & Result (ug/m3) & Qualifier & MDL & $\mathrm{RL}$ \\
\hline $\begin{array}{l}\text { Chloroform } \\
\text { Carbon tetrachloride }\end{array}$ & $\begin{array}{l}1.1 \\
1.3\end{array}$ & U & $\begin{array}{l}0.24 \\
0.31\end{array}$ & 0.98 \\
\hline
\end{tabular}




\section{Analytical Data}

Client: Argonne National Laboratory

Job Number: 200-1136-1

Client Sample ID: 104 ROXANNA ST BASEMENT

Sdg Number: MORRILL (200-1136)

Lab Sample ID: $\quad$ 200-1136-2

Date Sampled: $\quad 08 / 12 / 20101511$

Client Matrix:

Air

Date Received: 08/16/2010 0950

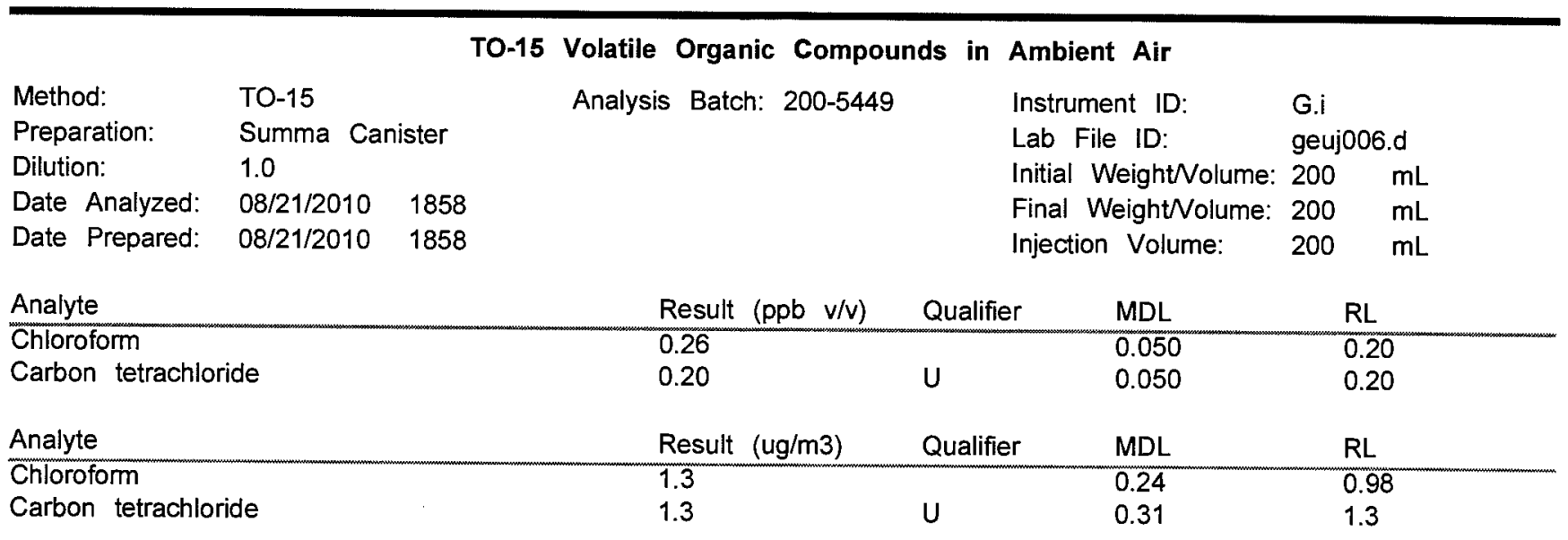




\section{Analytical Data}

Client: Argonne National Laboratory

Client Sample ID: 102 VIRGINIA ST 1ST

Lab Sample ID: 200-1136-3

Client Matrix: Air
Job Number: 200-1136-1

Sdg Number: MORRILL (200-1136)

Date Sampled: $\quad 08 / 12 / 20101529$

Date Received: 08/16/2010 0950

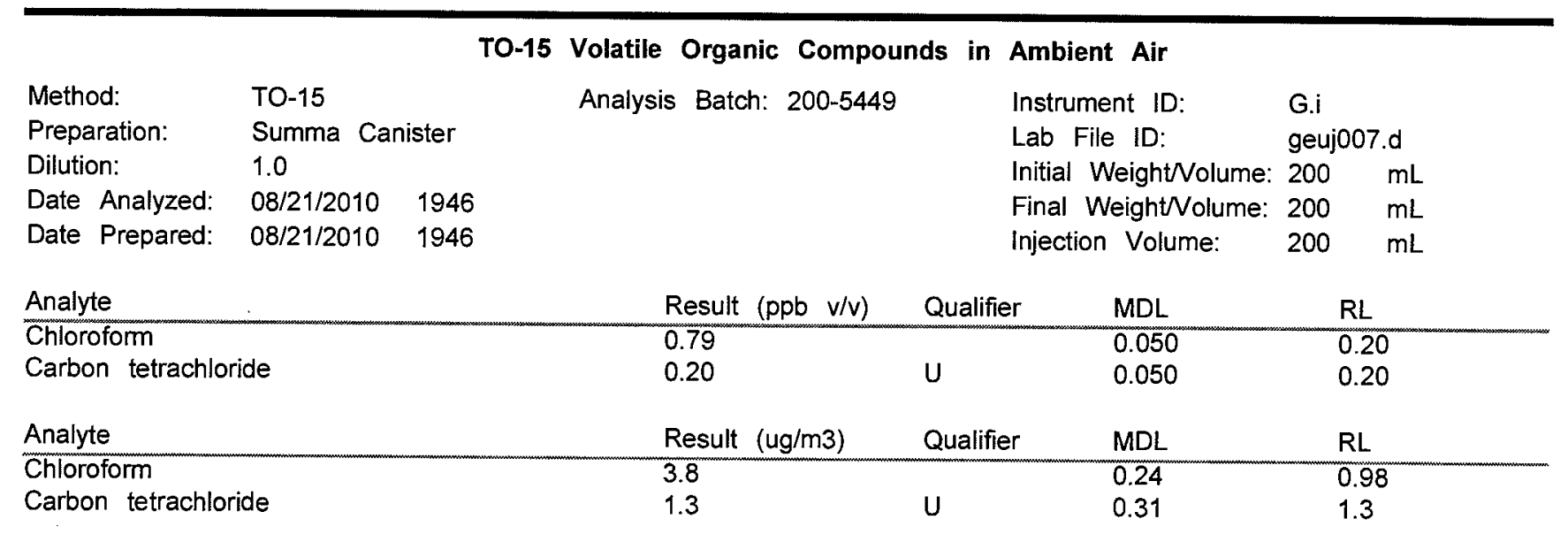




\section{Analytical Data}

Client: Argonne National Laboratory

Client Sample ID: 102 VIRGINIA ST BASEMENT

Lab Sample ID: 200-1136-4

Client Matrix: Air
Job Number: 200-1136-1

Sdg Number: MORRILL (200-1136)

Date Sampled: $\quad 08 / 12 / 20101533$

Date Received: 08/16/2010 0950

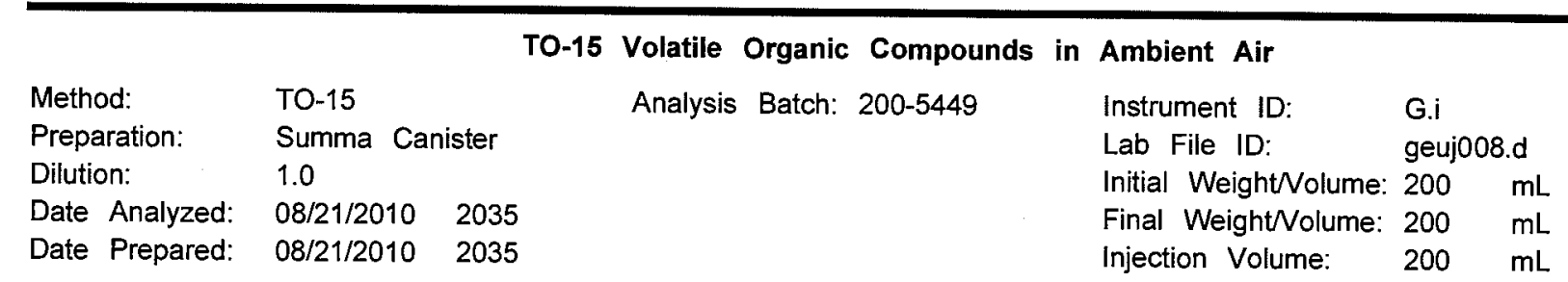

Analyte

Result (ppb v/v) Qualifier MDL

Chloroform

0.85

Carbon tetrachloride

0.20

U

0.050

$\mathrm{RL}$

0.20

0.20

Analyte

Result (ug/m3)

Qualifier

0.050

RL

Chloroform

4.1

Carbon tetrachloride

1.3

U

MDL
0.24
0.31

0.98

1.3 


\section{Analytical Data}

Client: Argonne National Laboratory

Job Number: 200-1136-1

Client Sample ID: 202 ROXANNA ST 1ST

Sdg Number: MORRILL (200-1136)

Lab Sample ID: 200-1136-5

Date Sampled: 08/12/2010 1304

Client Matrix: Air

Date Received: 08/16/2010 0950

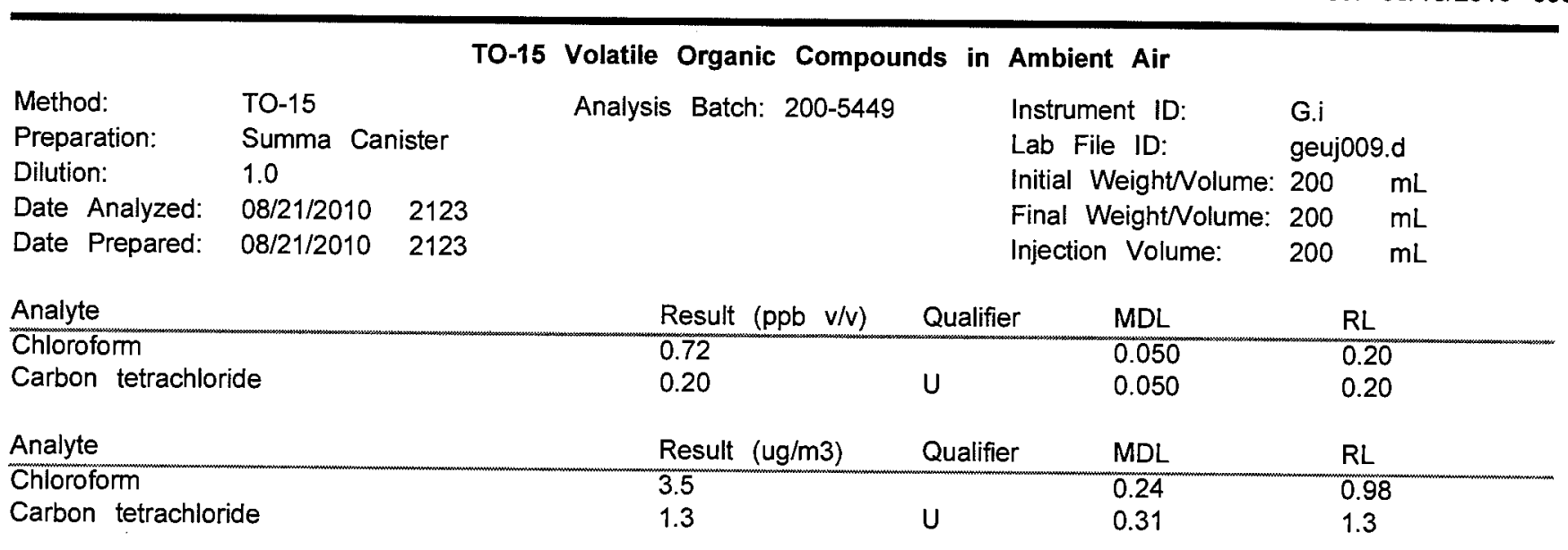




\section{Analytical Data}

Client: Argonne National Laboratory

Job Number: 200-1136-1

Client Sample ID: 202 ROXANNA ST BASEMENT

Sdg Number: MORRILL (200-1136)

Lab Sample ID: $\quad 200-1136-6$

Date Sampled: $\quad 08 / 12 / 20101306$

Client Matrix: Air

Date Received: $08 / 16 / 20100950$

TO-15 Volatile Organic Compounds in Ambient Air

$\begin{array}{llllll}\text { Method: } & \text { TO-15 } & \text { Analysis } & \text { Batch: } 200-5449 & \text { Instrument ID: } & \text { G.i } \\ \text { Preparation: } & \text { Summa Canister } & & \text { Lab File ID: } & \text { geuj010.d } \\ \text { Dilution: } & 1.0 & & \text { Initial Weight Nolume: } & 200 & \mathrm{~mL} \\ \text { Date Analyzed: } & 08 / 21 / 2010 \quad 2212 & & \text { Final Weight Volume: } & 200 & \mathrm{~mL} \\ \text { Date Prepared: } & 08 / 21 / 2010 & 2212 & \text { Injection Volume: } & 200 & \mathrm{~mL}\end{array}$

Analyte Result (ppb v/v) Qualifier. MDL

Chloroform

0.20

Carbon tetrachloride

0.20

U

0.050

$\mathrm{RL}$

Analyte Result (ug/m3)

Chloroform

0.98

Carbon tetrachloride

1.3

Qualifier

MDL

0.20

0.20

3

U

0.24

$\mathrm{RL}$

0.98




\section{Analytical Data}

Client: Argonne National Laboratory

Job Number: 200-1136-1

Client Sample ID: 203 ROXANNA ST 1ST

Sdg Number: MORRILL (200-1136)

Lab Sample ID: 200-1136-7

Date Sampled: 08/12/2010 1317

Client Matrix: Air

Date Received: 08/16/2010 0950

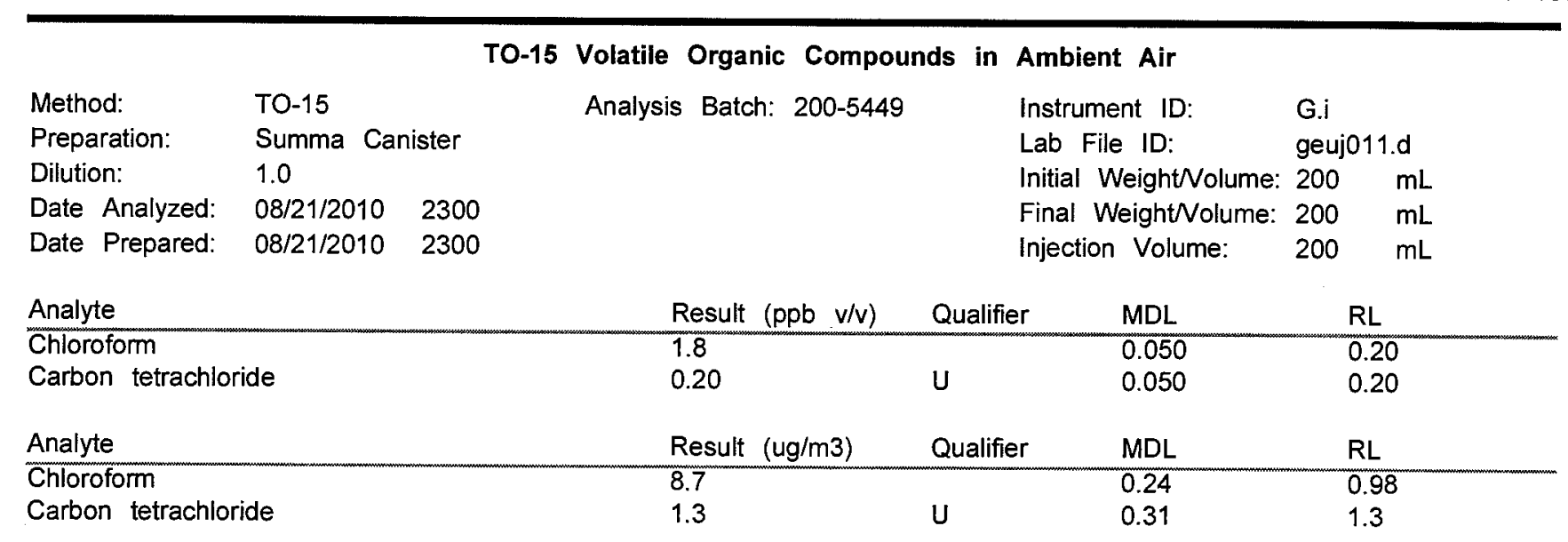




\section{Analytical Data}

Client: Argonne National Laboratory

Client Sample ID: 107 ROXANNA ST MAIN ROOM

$\begin{array}{ll}\text { Lab Sample ID: } & 200-1136-8 \\ \text { Client Matrix: } & \text { Air }\end{array}$
Job Number: 200-1136-1

Sdg Number: MORRILL (200-1136)

Date Sampled: $\quad 08 / 12 / 20101716$

Date Received: 08/16/2010 0950

TO-15 Volatile Organic Compounds in Ambient Air

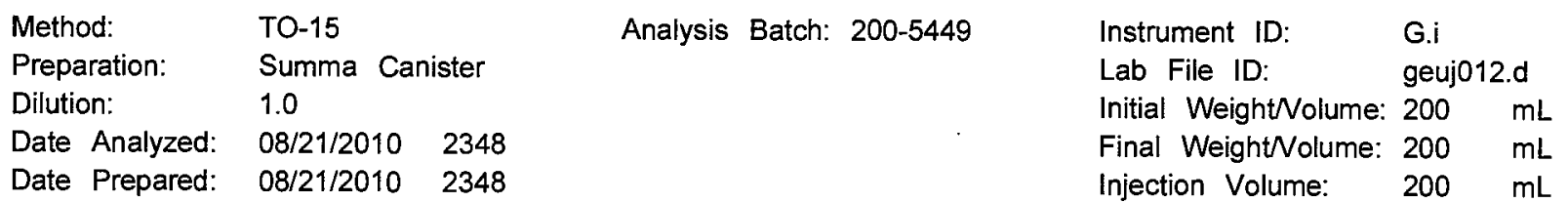

Analyte

\begin{tabular}{llll} 
Result (ppb V/V) & Qualifier & MDL & RL \\
\hline 0.20 & $U$ & 0.050 & 0.20 \\
0.20 & $U$ & 0.050 & 0.20
\end{tabular}

Carbon tetrachloride

Result (ug/m3)

0.98

Chloroform

0.98

Qualifier $\quad$ MDL

0.24

0.31

$\mathrm{RL}$

0.98

Carbon tetrachloride

1.3

U

1.3 


\section{Analytical Data}

Client: Argonne National Laboratory

Job Number:

200-1136-1

Client Sample ID: 96 VIRGINIA ST OFFICE

Sdg Number: MORRILL (200-1136)

Lab Sample ID: 200-1136-9

Date Sampled: $\quad 08 / 12 / 20101434$

Client Matrix:

Air

Date Received: 08/16/2010 0950

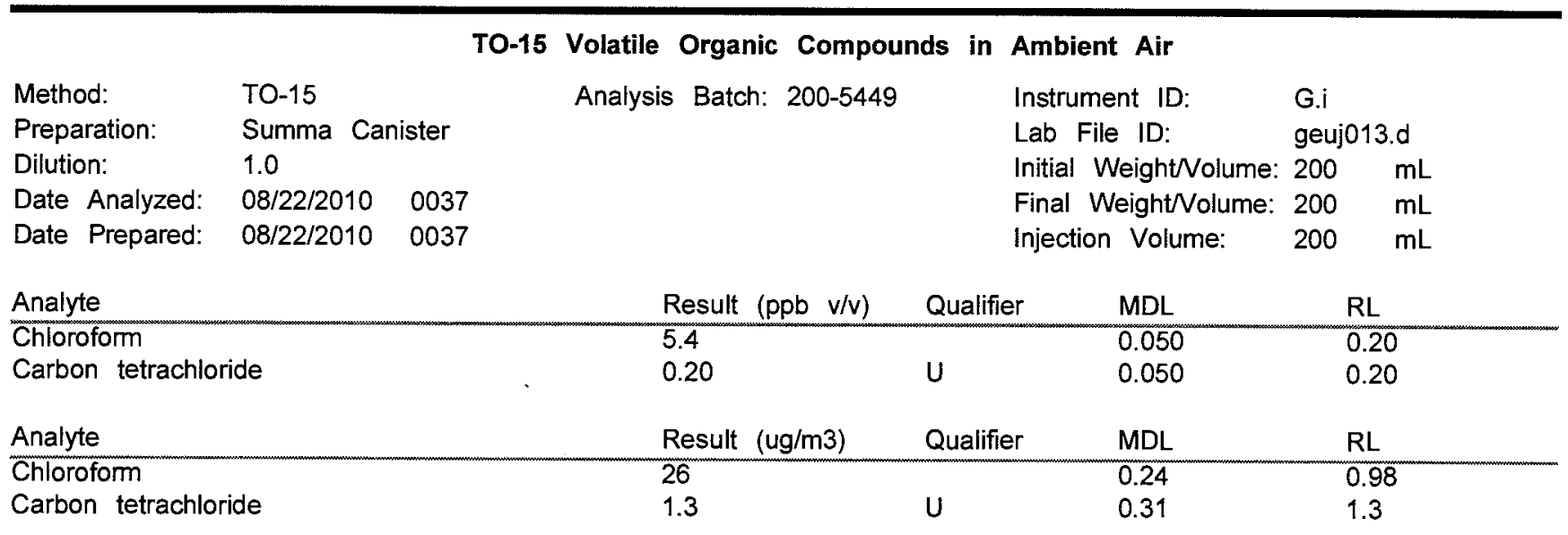




\section{Analytical Data}

Client: Argonne National Laboratory

Job Number: 200-1136-1

Client Sample ID: AMBIENT AIR MW1

Sdg Number: MORRILL (200-1136)

Lab Sample ID: 200-1136-10

Date Sampled: $\quad 08 / 12 / 20101447$

Client Matrix: Air

Date Received: 08/16/2010 0950

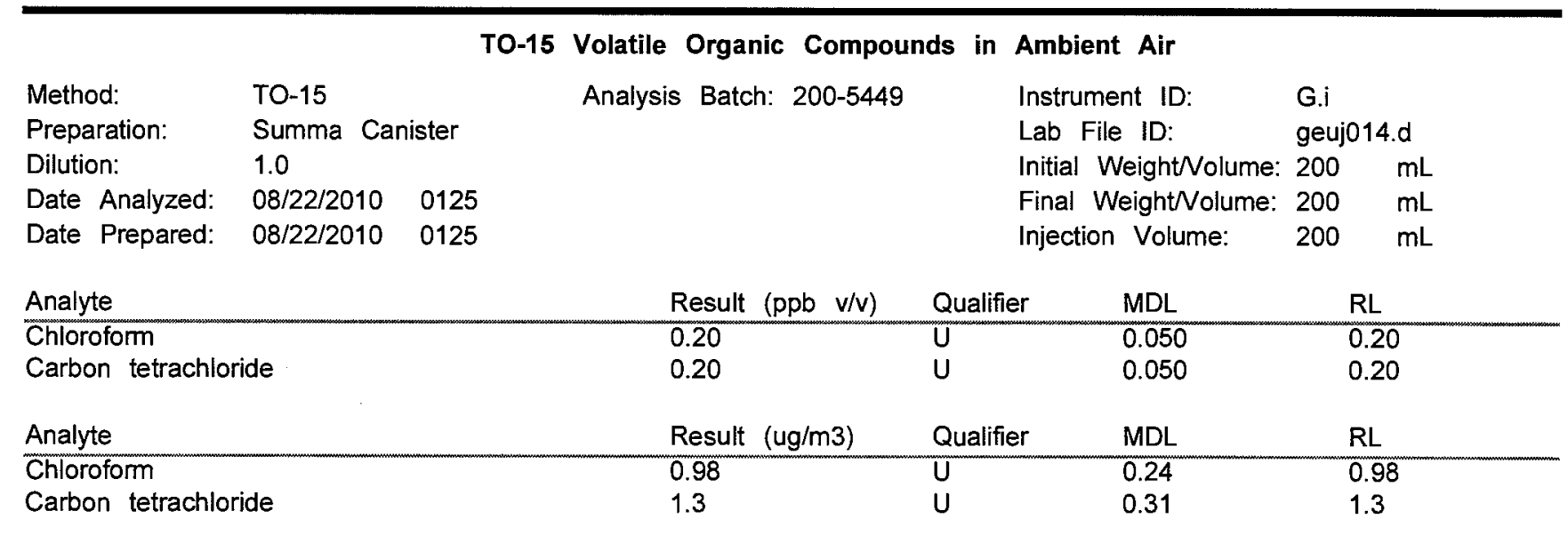




\section{Analytical Data}

Client: Argonne National Laboratory

Client Sample ID: 107 ROXANNA ST OFFICE

Lab Sample ID: 200-1136-11

Client Matrix: Air
Job Number: 200-1136-1

Sdg Number: MORRILL (200-1136)

Date Sampled: 08/12/2010 1711

Date Received: 08/16/2010 0950

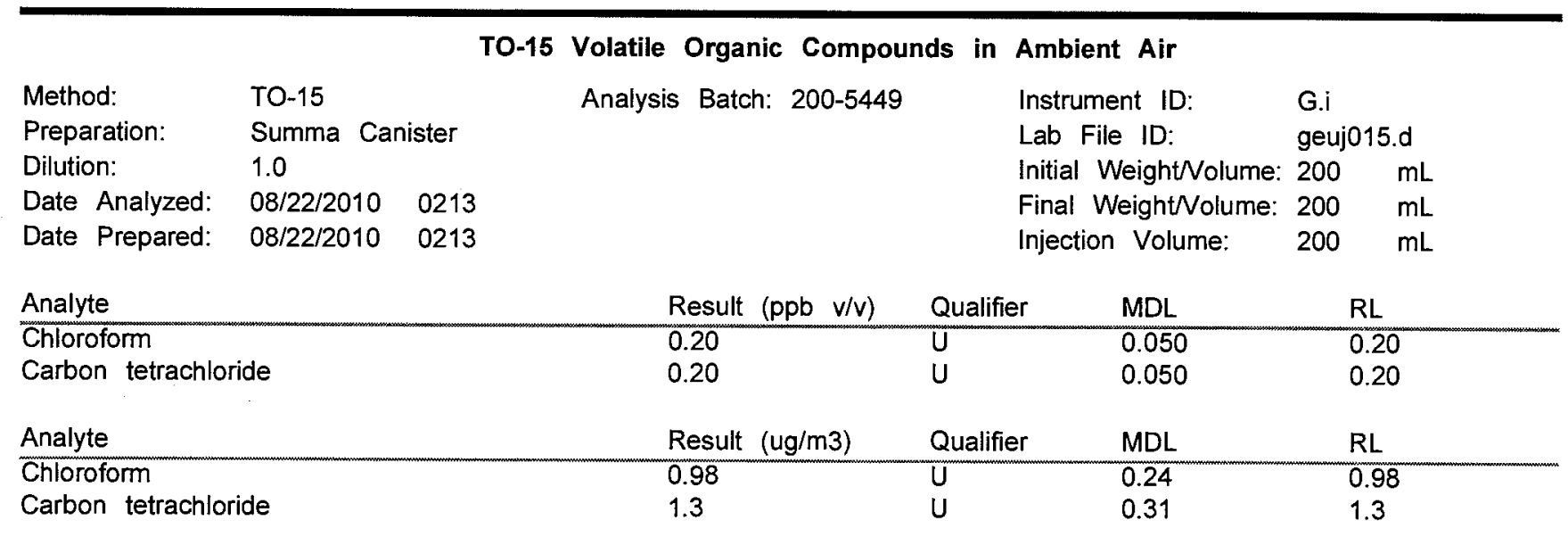




\section{Analytical Data}

Client: Argonne National Laboratory

Client Sample ID: 107 ROXANNA ST KID ROOM

Lab Sample ID: 200-1136-12

Client Matrix: Air
Job Number: 200-1136-1

Sdg Number: MORRILL (200-1136)

Date Sampled: 08/12/2010 1714

Date Received: 08/16/2010 0950

TO-15 Volatile Organic Compounds in Ambient Air

\begin{tabular}{|c|c|c|c|c|}
\hline Date Analyzed: & $08 / 22 / 2010$ & & Final Weight/Nolume: & 200 \\
\hline Date Prepared: & $08 / 22 / 2010$ & & Injection Volume: & 200 \\
\hline
\end{tabular}

Analyte

Result (ppb v/v) Qualifier MDL

Chloroform

0.20

Carbon tetrachloride

0.20

U

$\mathrm{RL}$

Analyte

Result (ug/m3)

Chloroform

0.98

Carbon tetrachloride

1.3

\begin{tabular}{ll} 
Qualifier & MDL \\
\hline$U$ & 0.24 \\
$U$ & 0.31
\end{tabular}

$\mathrm{RL}$

0.98

1.3 


\section{Analytical Data}

Client: Argonne National Laboratory

Client Sample ID: 108 ROXANNA ST 1ST

Lab Sample ID: 200-1136-13

Client Matrix: Air
Job Number: 200-1136-1

Sdg Number: MORRILL (200-1136)

Date Sampled: 08/12/2010 1539

Date Received: 08/16/2010 0950

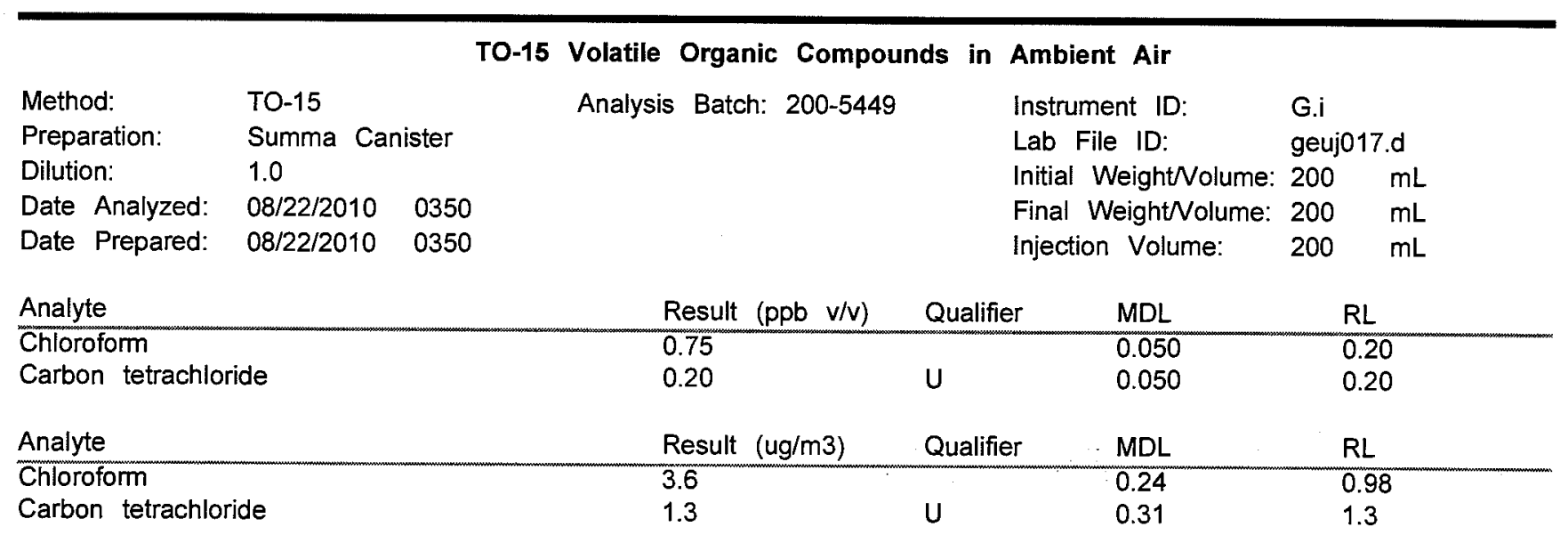




\section{Analytical Data}

Client: Argonne National Laboratory

Job Number: 200-1136-1

Sdg Number: MORRILL (200-1136)

Client Sample ID: 108 ROXANNA ST BASEMENT

Lab Sample ID: 200-1136-14

Date Sampled: 08/12/2010 1543

Client Matrix: Air

Date Received: 08/16/2010 0950

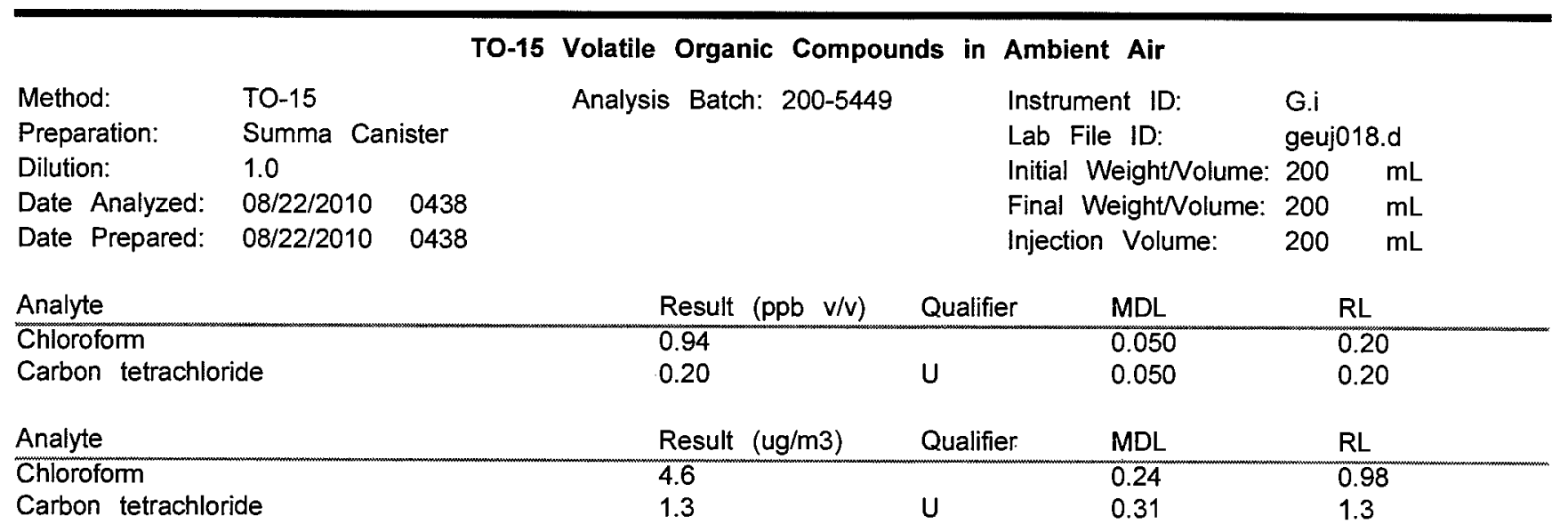




\section{Analytical Data}

Client: Argonne National Laboratory

Client Sample

Lab Sample ID:

Client Matrix:

ID: 106 VIRGINIA ST 1ST

200-1136-15

Air
Job Number: 200-1136-1

Sdg Number: MORRILL (200-1136)

Date Sampled: $\quad 08 / 12 / 20101451$

Date Received: 08/16/2010 0950

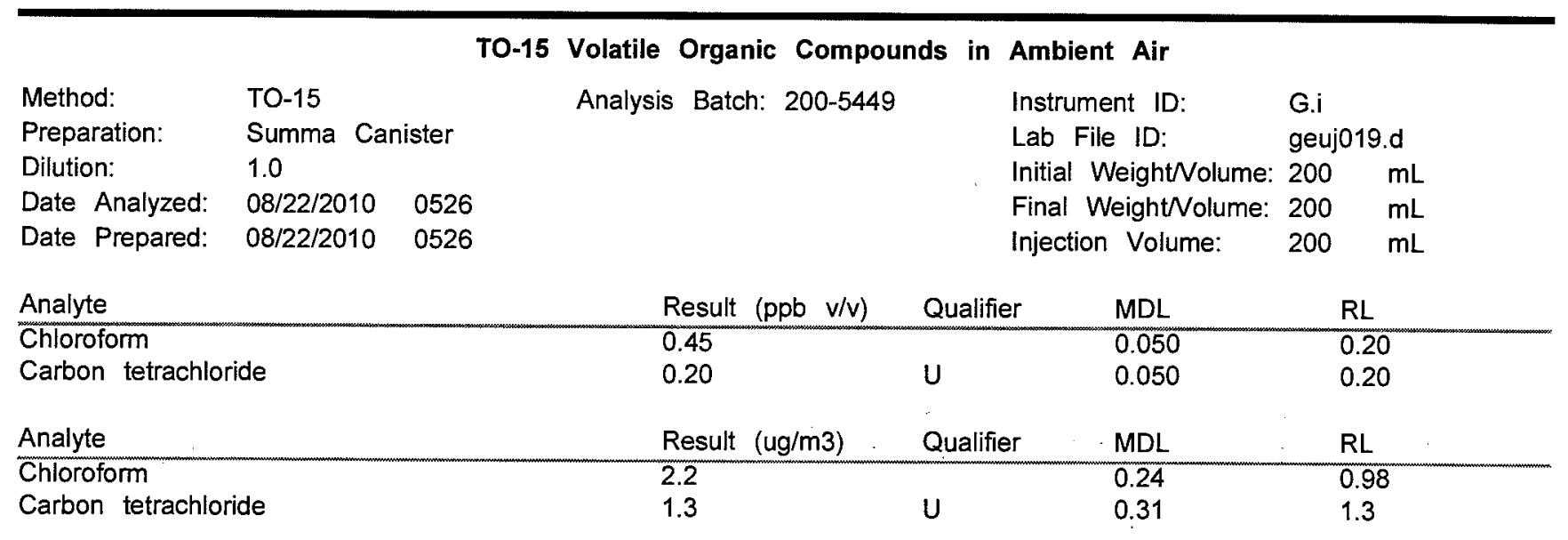




\section{Analytical Data}

Client: Argonne National Laboratory

Job Number: 200-1136-1

Client Sample ID: 105 ROXANNA ST 1ST

Sdg Number: MORRILL (200-1136)

Lab Sample ID: 200-1136-16

Date Sampled: 08/12/2010 1703

Client Matrix:

Air

Date Received: 08/16/2010 0950

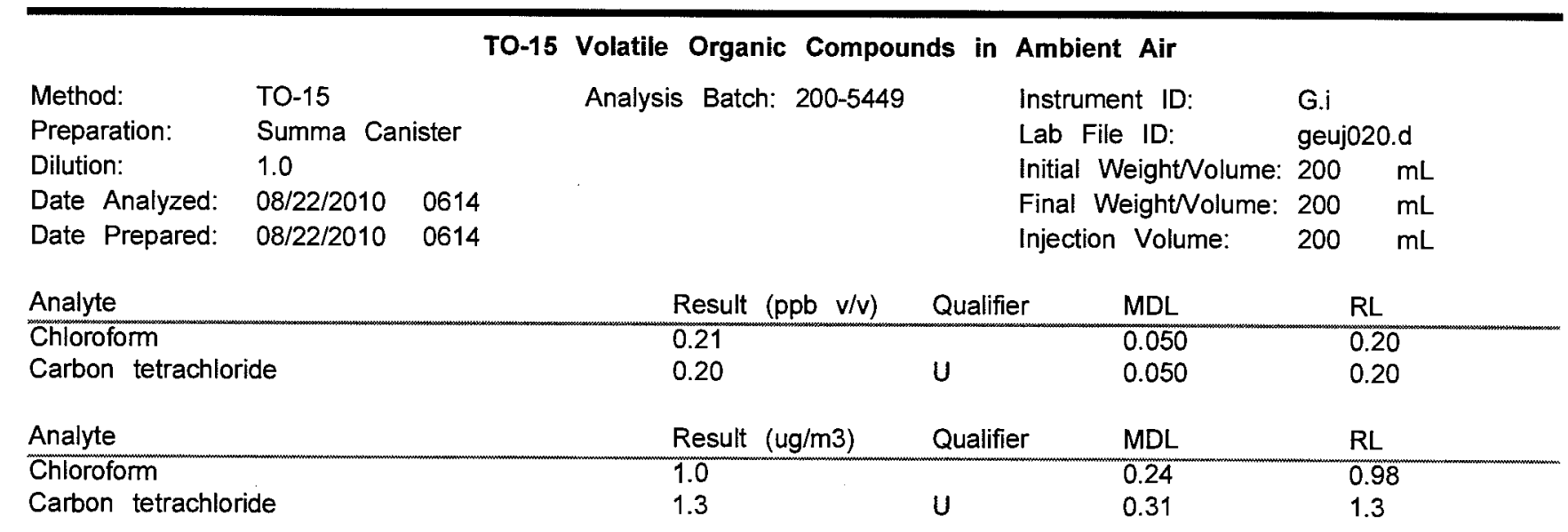




\section{Quality Control Results}

Client: Argonne National Laboratory

Method Blank - Batch: 200-5449

Lab Sample ID:MB 200-5449/4

Client Matrix: Air

Dilution: $\quad 1.0$

Date Analyzed: 08/21/2010 1721

Date Prepared: 08/21/2010 1721
Job Number: $\quad 200-1136-1$

Sdg Number: MORRILL (200-1136)

Method: D-15

Preparation: Summa Canister

Analysis Batch: 200-5449 Instrument ID: G.i

Prep Batch: N/A

Lab File ID: geuj004.d

Initial Weight Nolume: $200 \mathrm{~mL}$

Final WeightNolume: $200 \mathrm{~mL}$

Injection Volume: $200 \mathrm{~mL}$

\begin{tabular}{llccc} 
Analyte & Result & Qual & MDL & RL \\
\hline Chloroform & 0.20 & U & 0.050 & 0.20 \\
Carbon tetrachloride & 0.20 & U & 0.050 & 0.20
\end{tabular}

Method Blank - Batch: 200-5449

Method: D-15

Preparation: Summa Canister

Lab Sample ID:MB 200-5449/4

Client Matrix: Air

Dilution: $\quad 1.0$

Date Analyzed: 08/21/2010 1721

Date Prepared: 08/21/2010 1721
Analysis Batch: 200-5449 Instrument ID: G.i

Prep Batch: N/A

Units: ug/m3
Lab File ID: geuj004.d

Initial Weight/Volume: $200 \mathrm{~mL}$

Final WeightVolume: $200 \mathrm{~mL}$

Injection Volume: $200 \mathrm{~mL}$

\begin{tabular}{lllll} 
Analyte & Result & Qual & MDL & RL \\
\hline Chloroform & 0.98 & U & 0.24 & 0.98 \\
Carbon tetrachloride & 1.3 & U & 0.31 & 1.3
\end{tabular}

Lab Control Sample - Batch: 200-5449

Lab Sample ID:LCS 200-5449/3

Client Matrix: Air

Dilution: $\quad 1.0$

Date Analyzed: 08/21/2010 1633

Date Prepared: 08/21/2010 1633
Analysis Batch: 200-5449 Instrument ID: G.i

Prep Batch: N/A Lab File ID: geuj003.d

Units:ppb v/v
Method: D-15

Preparation: Summa Canister
Initial Weight/Volume: $200 \mathrm{~mL}$

Final Weight/Volume: $200 \mathrm{~mL}$ Injection Volume: $200 \mathrm{~mL}$

\begin{tabular}{|c|c|c|c|c|c|}
\hline Analyte & Spike Amount & Result & $\%$ Rec. & Limit & Qual \\
\hline Chloroform & 10.0 & 10.9 & 109 & $70-130$ & \\
\hline Carbon tetrachloride & 10.0 & 10.4 & 104 & $70-130$ & \\
\hline
\end{tabular}




\section{DATA REPORTING QUALIFIERS}

Client: Argonne National Laboratory

Job Number: 200-1136-1

Sdg Number: MORRILL (200-1136)

Lab Section

Air - GC/MS VOA
Qualifier

$\mathrm{U}$
Description

Indicates the analyte was analyzed for but not detected. 


\section{Method T015}

Volatile Organic Compounds (GC/MS) by Method T015 


\section{Quality Control Results}

Client: Argonne National Laboratory

Job Number: $\quad$ 200-1136-1

Sdg Number: MORRILL (200-1136)

\section{QC Association Summary}

\begin{tabular}{|c|c|c|c|c|c|}
\hline Lab Sample ID & Client & Sample ID & $\begin{array}{l}\text { Report } \\
\text { Basis }\end{array}$ & Client Matrix & Method \\
\hline \multicolumn{6}{|c|}{ Air - GC/MS VOA } \\
\hline \multicolumn{6}{|c|}{ Analysis Batch:200-5449 } \\
\hline LCS $200-5449 / 3$ & Lab & Control Sample & $\mathrm{T}$ & Air & TO-15 \\
\hline MB 200-5449/4 & Metr & hod Blank & $\mathrm{T}$ & Air & TO-15 \\
\hline $200-1136-1$ & 104 & ROXANNA ST $1 S T$ & $\mathrm{~T}$ & Air & TO-15 \\
\hline $200-1136-2$ & 104 & ROXANNA ST BASEMENT & $\mathrm{T}$ & Air & TO-15 \\
\hline $200-1136-3$ & 102 & VIRGINIA ST 1ST & $\mathrm{T}$ & Air & TO-15 \\
\hline $200-1136-4$ & 102 & VIRGINIA ST BASEMENT & $\mathrm{T}$ & Air & TO-15 \\
\hline $200-1136-5$ & 202 & ROXANNA ST 1ST & $\mathrm{T}$ & Air & TO-15 \\
\hline $200-1136-6$ & 202 & ROXANNA ST BASEMENT & $\mathrm{T}$ & Air & TO-15 \\
\hline $200-1136-7$ & 203 & ROXANNA ST 1ST & $\mathrm{T}$ & Air & TO-15 \\
\hline $200-1136-8$ & 107 & ROXANNA ST MAIN ROOM & $T$ & Air & TO-15 \\
\hline $200-1136-9$ & 96 & VIRGINIA ST OFFICE & $\mathbf{T}$ & Air & TO-15 \\
\hline $200-1136-10$ & $\mathrm{AMB}$ & BIENT AIR MW1 & $\mathrm{T}$ & Air & TO-15 \\
\hline $200-1136-11$ & 107 & ROXANNA ST OFFICE & $\mathrm{T}$ & Air & TO-15 \\
\hline $200-1136-12$ & 107 & ROXANNA ST KID ROOM & $\mathbf{T}$ & Air & TO-15 \\
\hline $200-1136-13$ & 108 & ROXANNA ST $1 \mathrm{ST}$ & $T$ & Air & TO-15 \\
\hline $200-1136-14$ & 108 & ROXANNA ST BASEMENT & $\mathrm{T}$ & Air & TO-15 \\
\hline $200-1136-15$ & 106 & VIRGINIA ST 1ST & $\mathrm{T}$ & Air & TO-15 \\
\hline $200-1136-16$ & 105 & ROXANNA ST $1 \mathrm{ST}$ & $\mathrm{T}$ & Air & TO-15 \\
\hline
\end{tabular}

\section{Report Basis}

$T=$ Total 
FORM III

AIR - GC/MS VOA LAB CONTROL SAMPLE RECOVERY

Lab Name: TestAmerica Burlington

SDG NO.: MORRILL (200-1136)

Matrix: Air

Lab ID: LCS 200-5449/3

\begin{tabular}{|c|c|c|c|c|c|}
\hline $\begin{array}{r}\text { COMPOUND } \\
\end{array}$ & $\begin{array}{c}\text { SPIKE } \\
\text { ADDED } \\
(\mathrm{ppb} \mathrm{v} / \mathrm{v})\end{array}$ & $\begin{array}{c}\text { LCS } \\
\text { CONCENTRATION } \\
(\mathrm{ppb} \mathrm{v} / \mathrm{v}) \\
\end{array}$ & $\begin{array}{c}\mathrm{LCS} \\
\% \\
\mathrm{REC} \\
\end{array}$ & $\begin{array}{l}\text { QC } \\
\text { LIMITS } \\
\text { REC } \\
\end{array}$ & $\#$ \\
\hline Chloroform & 10.0 & 10.9 & 109 & $70-130$ & \\
\hline Carbon tetrachloride & 10.0 & 10.4 & 104 & $70-130$ & \\
\hline
\end{tabular}

Job No.: 200-1136-1

Lab File ID: geuj003.d

Client ID:

\# Column to be used to flag recovery and RPD values

FORM III TO-15 
FORM IV

AIR - GC/MS VOA METHOD BLANK SUMMARY

Lab Name: TestAmerica Burlington

SDG NO.: MORRILL $(200-1136)$

Lab File ID: geuj004.d

Matrix: Air

Instrument ID: G.i

GC Column: RTX-624
Job No.: 200-1136-1

Lab Sample ID: MB 200-5449/4

Heated Purge: $(Y / N)$ N

Date Analyzed: 08/21/2010 17:21

THIS METHOD BLANK APPLIES TO THE FOLLOWING SAMPLES:

\begin{tabular}{|c|c|c|c|c|}
\hline CLIENT SAMPLE ID & LAB SAMPLE ID & $\begin{array}{l}\text { LAB } \\
\text { FILE ID }\end{array}$ & \multicolumn{2}{|c|}{ DATE ANALYZED } \\
\hline & LCS $200-5449 / 3$ & geuj003.d & $08 / 21 / 2010$ & $16: 33$ \\
\hline 104 ROXANNA ST 1 ST & $200-1136-1$ & geuj005.d & $08 / 21 / 2010$ & $18: 10$ \\
\hline 104 ROXANNA ST BASEMENT & $200-1136-2$ & geuj006.d & $08 / 21 / 2010$ & $18: 58$ \\
\hline 102 VIRGINIA ST 1ST & $200-1136-3$ & geuj007.d & $08 / 21 / 2010$ & $19: 46$ \\
\hline 102 VIRGINIA ST BASEMENT & $200-1136-4$ & geuj008.d & $08 / 21 / 2010$ & $20: 35$ \\
\hline 202 ROXANNA ST 1ST & $200-1136-5$ & geuj009.d & $08 / 21 / 2010$ & $21: 23$ \\
\hline 202 ROXANNA ST BASEMENT & $200-1136-6$ & geuj010.d & $08 / 21 / 2010$ & $22: 12$ \\
\hline 203 ROXANNA ST 1ST & $200-1136-7$ & geuj011.d & $08 / 21 / 2010$ & $23: 00$ \\
\hline 107 ROXANNA ST MAIN ROOM & $200-1136-8$ & geuj012.d & $08 / 21 / 2010$ & $23: 48$ \\
\hline 96 VIRGINIA ST OFFICE & $200-1136-9$ & geuj013.d & $08 / 22 / 2010$ & $00: 37$ \\
\hline AMBIENT AIR MWI & $200-1136-10$ & geuj014.d & $08 / 22 / 2010$ & $01: 25$ \\
\hline 107 ROXANNA ST OFFICE & $200-1136-11$ & geuj015.d & $08 / 22 / 2010$ & $02: 13$ \\
\hline 107 ROXANNA ST KID ROOM & $200-1136-12$ & geuj $016 . d$ & $08 / 22 / 2010$ & $03: 01$ \\
\hline 108 ROXANNA ST 1ST & $200-1136-13$ & geuj017.d & $08 / 22 / 2010$ & $03: 50$ \\
\hline 108 ROXANNA ST BASEMENT & $200-1136-14$ & geuj018.d & $08 / 22 / 2010$ & $04: 38$ \\
\hline 106 VIRGINIA ST 1 ST & $200-1136-15$ & geuj019.d & $08 / 22 / 2010$ & $05: 26$ \\
\hline 105 ROXANNA ST 1 ST & $200-1136-16$ & geuj020.d & $08 / 22 / 2010$ & $06: 14$ \\
\hline
\end{tabular}


FORM V

AIR - GC/MS VOA INSTRUMENT PERFORMANCE CHECK

Lab Name: TestAmerica Burlington

SDG NO.: MORRIII (200-1136)

Lab File ID: geu001.d

Instrument ID: G.i

Analysis Batch No.: 5008
Job No.: 200-1136-1

BFB Injection Date: 08/09/2010

BFB Injection Time: 14:23

\begin{tabular}{|c|c|c|}
\hline $\mathrm{M} / \mathrm{E}$ & ION ABUNDANCE CRITERIA & $\begin{array}{l}\text { \% RELATIVE } \\
\text { ABUNDANCE }\end{array}$ \\
\hline 50 & $8.0-40.0 \%$ of mass 95 & 11.9 \\
\hline 75 & $30.0-66.0 \%$ of mass 95 & 37.8 \\
\hline 95 & Base peak, $100 \%$ relative abundance & 100.0 \\
\hline 96 & $5.0-9.0 \%$ of mass 95 & 6.7 \\
\hline 173 & Less than $2.0 \%$ of mass 174 & $(0.4) 1$ \\
\hline 174 & $50.0-120.0 \%$ of mass 95 & 113.7 \\
\hline 175 & $4.0-9.0$ o of mass 174 & $(6.9) 1$ \\
\hline 176 & $93.0-101.0 \%$ of mass 174 & $(97.4) 1$ \\
\hline 177 & $5.0-9.0 \%$ of mass 176 & $(6.5) 2$ \\
\hline
\end{tabular}

1 -Value is $\frac{\circ}{0}$ mass 174

2-Value is $\frac{\circ}{\text { mass }} 176$

THIS CHECK APPLIES TO THE FOLLOWING SAMPLES, MS, MSD, BLANKS AND STANDARDS:

\begin{tabular}{|l|l|l|l|c|}
\hline \multirow{2}{*}{ CLIENT SAMPLE ID } & LAB SAMPLE ID & $\begin{array}{c}\text { LAB } \\
\text { FILE ID }\end{array}$ & $\begin{array}{c}\text { DATE } \\
\text { ANALYZED }\end{array}$ & $\begin{array}{c}\text { TIME } \\
\text { ANALYZED }\end{array}$ \\
\hline \hline & IC $200-5008 / 3$ & geu003.d & $08 / 09 / 2010$ & $15: 59$ \\
\hline & IC $200-5008 / 4$ & geu004.d & $08 / 09 / 2010$ & $16: 47$ \\
\hline & IC $200-5008 / 5$ & geu005.d & $08 / 09 / 2010$ & $17: 35$ \\
\hline & ICIS $200-5008 / 6$ & geu006.d & $08 / 09 / 2010$ & $18: 24$ \\
\hline & IC 200-5008/7 & geu007.d & $08 / 09 / 2010$ & $19: 12$ \\
\hline & IC $200-5008 / 8$ & geu008.d & $08 / 09 / 2010$ & $20: 01$ \\
\hline & IC $200-5008 / 9$ & geu009.d & $08 / 09 / 2010$ & $20: 50$ \\
\hline & ICV $200-5008 / 11$ & geu011.d & $08 / 09 / 2010$ & $22: 27$ \\
\hline
\end{tabular}


FORM V

AIR - GC/MS VOA INSTRUMENT PERFORMANCE CHECK

Lab Name: TestAmerica Burlington

SDG NO.: MORRILL (200-1136)

Lab File ID: geuj001.d

Instrument ID: G.i

Analysis Batch No.: 5449
Job No.: 200-1136-1

BEB Injection Date: 08/21/2010

BFB Injection Time: 14:59

\begin{tabular}{|c|c|c|}
\hline$M / E$ & ION ABUNDANCE CRITERIA & $\begin{array}{l}\% \text { RELATIVE } \\
\text { ABUNDANCE }\end{array}$ \\
\hline 50 & $8.0-40.0 \%$ of mass 95 & 15.3 \\
\hline 75 & $30.0-66.0 \%$ of mass 95 & 44.3 \\
\hline 95 & Base peak, $100 \%$ relative abundance & 100.0 \\
\hline 96 & $5.0-9.0 \%$ of mass 95 & 6.8 \\
\hline 173 & Less than $2.0 \%$ of mass 174 & $(0.5) 1$ \\
\hline 174 & $50.0-120.0 \%$ of mass 95 & 96.8 \\
\hline 175 & $4.0-9.0 \div$ of mass 174 & $(6.8) 1$ \\
\hline 176 & $93.0-101.0 \%$ of mass 174 & $(96.6) 1$ \\
\hline 177 & $5.0-9.0 \%$ of mass 176 & $(6.5) 2$ \\
\hline
\end{tabular}

THIS CHECK APPLIES TO THE FOLLOWING SAMPLES, MS, MSD, BLANKS AND STANDARDS:

\begin{tabular}{|l|l|l|c|c|}
\hline CLIENT SAMPLE ID & LAB SAMPLE ID & $\begin{array}{c}\text { LAB } \\
\text { FILE ID }\end{array}$ & $\begin{array}{c}\text { DATE } \\
\text { ANALYZED }\end{array}$ & $\begin{array}{c}\text { TIME } \\
\text { ANAYZED }\end{array}$ \\
\hline & CCVIS 200-5449/2 & geuj002.d & $08 / 21 / 2010$ & $15: 45$ \\
\hline & LCS 200-5449/3 & geuj003.d & $08 / 21 / 2010$ & $16: 33$ \\
\hline & MB 200-5449/4 & geuj004.d & $08 / 21 / 2010$ & $17: 21$ \\
\hline 104 ROXANNA ST 1ST & $200-1136-1$ & geuj005.d & $08 / 21 / 2010$ & $18: 10$ \\
\hline 104 ROXANNA ST BASEMENT & $200-1136-2$ & geuj006.d & $08 / 21 / 2010$ & $18: 58$ \\
\hline 102 VIRGINIA ST 1ST & $200-1136-3$ & geuj007.d & $08 / 21 / 2010$ & $19: 46$ \\
\hline $\begin{array}{l}102 \text { VIRGINIA ST } \\
\text { BASEMENT }\end{array}$ & $200-1136-4$ & geuj008.d & $08 / 21 / 2010$ & $20: 35$ \\
\hline 202 ROXANNA ST 1ST & $200-1136-5$ & geuj009.d & $08 / 21 / 2010$ & $21: 23$ \\
\hline 202 ROXANNA ST BASEMENT & $200-1136-6$ & geuj010.d & $08 / 21 / 2010$ & $22: 12$ \\
\hline 203 ROXANNA ST 1ST & $200-1136-7$ & geuj011.d & $08 / 21 / 2010$ & $23: 00$ \\
\hline $\begin{array}{l}107 \text { ROXANNA ST MAIN } \\
\text { ROOM }\end{array}$ & $200-1136-8$ & geuj012.d & $08 / 21 / 2010$ & $23: 48$ \\
\hline 96 VIRGINIA ST OFFICE & $200-1136-9$ & geuj013.d & $08 / 22 / 2010$ & $00: 37$ \\
\hline AMBIENT AIR MW1 & $200-1136-10$ & geuj014.d & $08 / 22 / 2010$ & $01: 25$ \\
\hline 107 ROXANNA ST OFFICE & $200-1136-11$ & geuj015.d & $08 / 22 / 2010$ & $02: 13$ \\
\hline 107 ROXANNA ST KID ROOM & $200-1136-12$ & geuj016.d & $08 / 22 / 2010$ & $03: 01$ \\
\hline 108 ROXANNA ST 1ST & $200-1136-13$ & geuj017.d & $08 / 22 / 2010$ & $03: 50$ \\
\hline 108 ROXANNA ST BASEMENT & $200-1136-14$ & geuj018.d & $08 / 22 / 2010$ & $04: 38$ \\
\hline 106 VIRGINIA ST 1ST & $200-1136-15$ & geuj019.d & $08 / 22 / 2010$ & $05: 26$ \\
\hline 105 ROXANNA ST 1ST & $200-1136-16$ & geuj020.d & $08 / 22 / 2010$ & $06: 14$ \\
\hline
\end{tabular}


FORM VIII

AIR - GC/MS VOA INTERNAL STANDARD AREA AND RETENTION TIME SUMMARY

Lab Name: TestAmerica Burlington

SDG No.: MORRILL $(200-1136)$

Sample No.: ICIS $200-5008 / 6$

Instrument ID: G.i

Lab File ID (Standard): geu006.d

Calibration ID: 1811
Job No.: 200-1136-1

Date Analyzed: 08/09/2010 18:24

GC Column: RTX-624

ID : $0.32(\mathrm{~mm})$

Heated Purge: ( $Y / N)$ N

\begin{tabular}{|c|c|c|c|c|c|c|c|}
\hline \multirow{2}{*}{\multicolumn{2}{|c|}{. }} & \multicolumn{2}{|l|}{$\overline{\mathrm{BCM}}$} & \multicolumn{2}{|l|}{ DF'B } & \multicolumn{2}{|l|}{ CBZ } \\
\hline & & AREA \# & RT \# & $\overline{\text { AREA \# }}$ & RT \# & AREA \# & RT \# \\
\hline INITIAI CALIBRATI & OINT & 673355 & 9.63 & 2867203 & 11.03 & 2840954 & 15.14 \\
\hline UPPER LIMIT & & 942697 & 9.96 & 4014084 & 11.36 & 3977336 & 15.47 \\
\hline LOWER LIMIT & & 404013 & 9.30 & 1720322 & 10.70 & 1704572 & 14.81 \\
\hline IAB SAMPLE ID & CLIENT SAMPLE ID & & & & & & \\
\hline ICV $200-5008 / 11$ & & 811655 & 9.63 & 3631468 & 11.03 & 3560459 & 15.14 \\
\hline
\end{tabular}

$\mathrm{BCM}=$ Bromochloromethane

$\mathrm{DFB}=1,4-\mathrm{Difluorobenzene}$

$\mathrm{CBZ}=$ Chlorobenzene-d5

Area Limit $=60 \%-140 \%$ of internal standard area

$\mathrm{RT}$ Limit $= \pm 0.33$ minutes of internal standard RT

\# Column used to flag values outside QC limits

FORM VIII TO- 15 
FORM VIII

AIR - GC/MS VOA INTERNAL STANDARD AREA AND RETENTION TIME SUMMARY

Lab Name: TestAmerica Burlington

SDG NO.: MORRILL (200-1136)

Sample No.: CCVIS 200-5449/2

Instrument ID: G.i

Lab Eile ID (Standard): geuj002.d

Calibration ID: 1811
Job No.: 200-1136-1

Date Analyzed: 08/21/2010 15:45

GC Column: RTX-624

ID : $0.32(\mathrm{~mm})$

Heated Purge: $(\mathrm{Y} / \mathrm{N}) \mathrm{N}$

\begin{tabular}{|c|c|c|c|c|c|c|c|}
\hline & \multicolumn{2}{|l|}{ BCM } & \multicolumn{2}{|l|}{ DFB } & \multicolumn{2}{|l|}{$\mathrm{CBZ}$} \\
\hline & & AREA \# & RT \# & AREA \# & RT \# & AREA \# & RT \# \\
\hline $12 / 24$ HOUR STD & & 571195 & 9.62 & 2493385 & 11.02 & 2371970 & 15.13 \\
\hline UPPER IIMIT & & 799673 & 9.95 & 3490739 & 11.35 & 3320758 & 15.46 \\
\hline LOWER LIMIT & & 342717 & 9.29 & 1496031 & 10.69 & 1423182 & 14.80 \\
\hline LAB SAMPLE ID & CLIENT SAMPLE ID & & & & & & \\
\hline ICS $200-5449 / 3$ & & 559401 & 9.61 & 2467576 & 11.02 & 2332418 & 15.13 \\
\hline MB 200-5449/4 & & 524031 & 9.61 & 2511942 & 11.02 & 2212003 & 15.13 \\
\hline $200-1136-1$ & 104 ROXANNA ST 1ST & 541203 & 9.61 & 2496092 & 11.02 & 2428782 & 15.13 \\
\hline $200-1136-2$ & $\begin{array}{l}104 \text { ROXANNA ST } \\
\text { BASEMENT }\end{array}$ & 532550 & 9.61 & 2416521 & 11.02 & 2356092 & 15.13 \\
\hline $200-1136-3$ & 102 VIRGINIA ST IST & 525679 & 9.62 & 2341245 & 11.02 & 2231322 & 15.13 \\
\hline $200-1136-4$ & $\begin{array}{l}102 \text { VIRGINIA ST } \\
\text { BASEMENT }\end{array}$ & 468049 & 9.61 & 2145283 & 11.02 & 2211224 & 15.13 \\
\hline $200-1136-5$ & 202 ROXANNA ST 1ST & 533572 & 9.61 & 2390295 & 11.02 & 2278712 & 15.13 \\
\hline $200-1136-6$ & $\begin{array}{l}202 \text { ROXANNA ST } \\
\text { BASEMENT }\end{array}$ & 550150 & 9.61 & 2487241 & 11.01 & 2399639 & 15.13 \\
\hline $200-1136-7$ & 203 ROXANNA ST 1ST & 558860 & 9.61 & 2546250 & 11.02 & 2481636 & 15.13 \\
\hline $200-1136-8$ & $\begin{array}{l}107 \text { ROXANNA ST MAIN } \\
\text { ROOM }\end{array}$ & 564360 & 9.62 & 2571229 & 11.02 & 2432231 & 15.13 \\
\hline $200-1136-9$ & 96 VIRGINIA ST OFFICE & 567628 & 9.62 & 2561540 & 11.02 & 2429193 & 15.13 \\
\hline $200-1136-10$ & AMBIENT AIR MWI & 575076 & 9.61 & 2571621 & 11.01 & 2425567 & 15.13 \\
\hline $200-1136-11$ & 107 ROXANNA ST OFFICE & 552936 & 9.61 & 2486175 & 11.02 & 2352362 & 15.13 \\
\hline $200-1136-12$ & $\begin{array}{l}107 \text { ROXANNA ST KID } \\
\text { ROOM }\end{array}$ & 567854 & 9.61 & 2582739 & 11.02 & 2484862 & 15.13 \\
\hline $200-1136-13$ & 108 ROXANNA ST IST & 561404 & 9.61 & 2549623 & 11.02 & 2427342 & 15.13 \\
\hline $200-1136-14$ & $\begin{array}{l}108 \text { ROXANNA ST } \\
\text { BASEMENT }\end{array}$ & 529128 & 9.61 & 2404173 & 11.02 & 2346753 & 15.13 \\
\hline $200-1136-15$ & 106 VIRGINIA ST 1ST & 525836 & 9.62 & 2342279 & 11.02 & 2118589 & 15.13 \\
\hline $200-1136-16$ & 105 ROXANNA ST IST & 539663 & 9.61 & 2455286 & 11.02 & 2390358 & 15.13 \\
\hline
\end{tabular}

$\mathrm{BCM}=$ Bromochloromethane

$\mathrm{DFB}=1,4-\mathrm{Difluorobenzene}$

$\mathrm{CBZ}=$ Chlorobenzene-d5

Area Limit $=60 \%-140 \%$ of internal standard area

$\mathrm{RT}$ Limit $= \pm 0.33$ minutes of internal standard RT

\# Column used to flag values outside QC limits

FORM VIII TO-15

Page 38 of 97 
FORM I

AIR - GC/MS VOA ORGANICS ANALYSIS DATA SHEET

Lab Name: TestAmerica Burlington

SDG NO.: MORRIIL (200-1136)

Client Sample ID: 104 ROXANNA ST 1ST

Matrix: Air

Analysis Method: TO-15

Sample wt/vol: $200(\mathrm{~mL})$

Soil Aliquot Vol:

Soil Extract vol.:

응 Moisture:

Analysis Batch No.: 5449
Job No.: 200-1136-1

Lab Sample ID: 200-1136-1

Lab File ID: geuj005.d

Date Collected: 08/12/2010 15:07

Date Analyzed: 08/21/2010 18:10

Dilution Factor: 1

GC Column: RTX-624

ID: $0.32(\mathrm{~mm})$

Level: (low/med) Low

Units: $\mathrm{ppb} \mathrm{v} / \mathrm{v}$

\begin{tabular}{|c|l|c|c|c|c|c|}
\hline CAS NO. & \multicolumn{1}{|c|}{ COMPOUND NAME } & $\begin{array}{c}\text { MOLECULAR } \\
\text { WEIGHT }\end{array}$ & RESULT & Q & RL & MDL \\
\hline \hline $67-66-3$ & Chloroform & 119.38 & 0.22 & & 0.20 & 0.050 \\
\hline $56-23-5$ & Carbon tetrachloride & 153.81 & 0.20 & U & 0.20 & 0.050 \\
\hline
\end{tabular}


FORM I

AIR - GC/MS VOA ORGANICS ANALYSIS DATA SHEET

Lab Name: TestAmerica Burlington

SDG NO.: MORRILI (200-1136)

Client sample ID: 104 ROXANNA ST $1 \mathrm{ST}$

Matrix: Air

Analysis Method: TO-15

Sample wt/vol: 200 (mL)

Soil Aliquot Vol:

Soil Extract vol.:

\% Moisture:

Analysis Batch No.: 5449

\begin{tabular}{|c|l|r|r|r|r|r|}
\hline CAS NO. & \multicolumn{1}{|c|}{ COMPOUND NAME } & $\begin{array}{c}\text { MOLECULAR } \\
\text { WEIGHT }\end{array}$ & RESULT & Q & RL ... & MDI \\
\hline \hline $67-66-3$ & Chloroform & 119.38 & 1.1 & & 0.98 & 0.24 \\
\hline $56-23-5$ & Carbon tetrachloride & 153.81 & 1.3 & $\mathrm{U}$ & 1.3 & 0.31 \\
\hline
\end{tabular}

Job No.: 200-1136-1

Lab Sample ID: 200-1136-1

Lab File ID: geuj005.d

Date Collected: 08/12/2010 15:07

Date Analyzed: 08/21/2010 18:10

Dilution Factor: 1

GC Column: RTX-624 ID $: 0.32(\mathrm{~mm})$

Level: (low/med) Low

Units: ug/m3 
FORM I

AIR - GC/MS VOA ORGANICS ANALYSIS DATA SHEET

Lab Name: TestAmerica Burlington

SDG NO.: MORRILL (200-1136)

Client Sample ID: 104 ROXANNA ST BASEMENT

Matrix: Air

Analysis Method: TO-15

Sample wt/vol: $200(\mathrm{~mL})$

Soil Aliquot Vol:

Soil Extract Vol.:

음 Moisture:

Analysis Batch No.: 5449

\begin{tabular}{|c|l|c|c|c|c|c|}
\hline \multicolumn{1}{|c|}{ CAS NO. } & \multicolumn{1}{|c|}{ COMPOUND NAME } & $\begin{array}{c}\text { MOLECULAR } \\
\text { WEIGHT }\end{array}$ & RESULT & Q & RL & MDI \\
\hline \hline $67-66-3$ & Chloroform & 119.38 & 0.26 & & 0.20 & 0.050 \\
\hline $56-23-5$ & Carbon tetrachloride & 153.81 & 0.20 & U & 0.20 & 0.050 \\
\hline
\end{tabular}

Job No.: 200-1136-1

Lab Sample ID: 200-1136-2

Lab File ID: geuj006.d

Date Collected: 08/12/2010 15:11

Date Analyzed: 08/21/2010 18:58

Dilution Factor: 1

GC Column: RTX-624 ID: $0.32(\mathrm{~mm})$

Level: (low/med) Low

Units: $\mathrm{ppb} \mathrm{v} / \mathrm{v}$ 
FORM I

AIR - GC/MS VOA ORGANICS ANALYSIS DATA SHEET

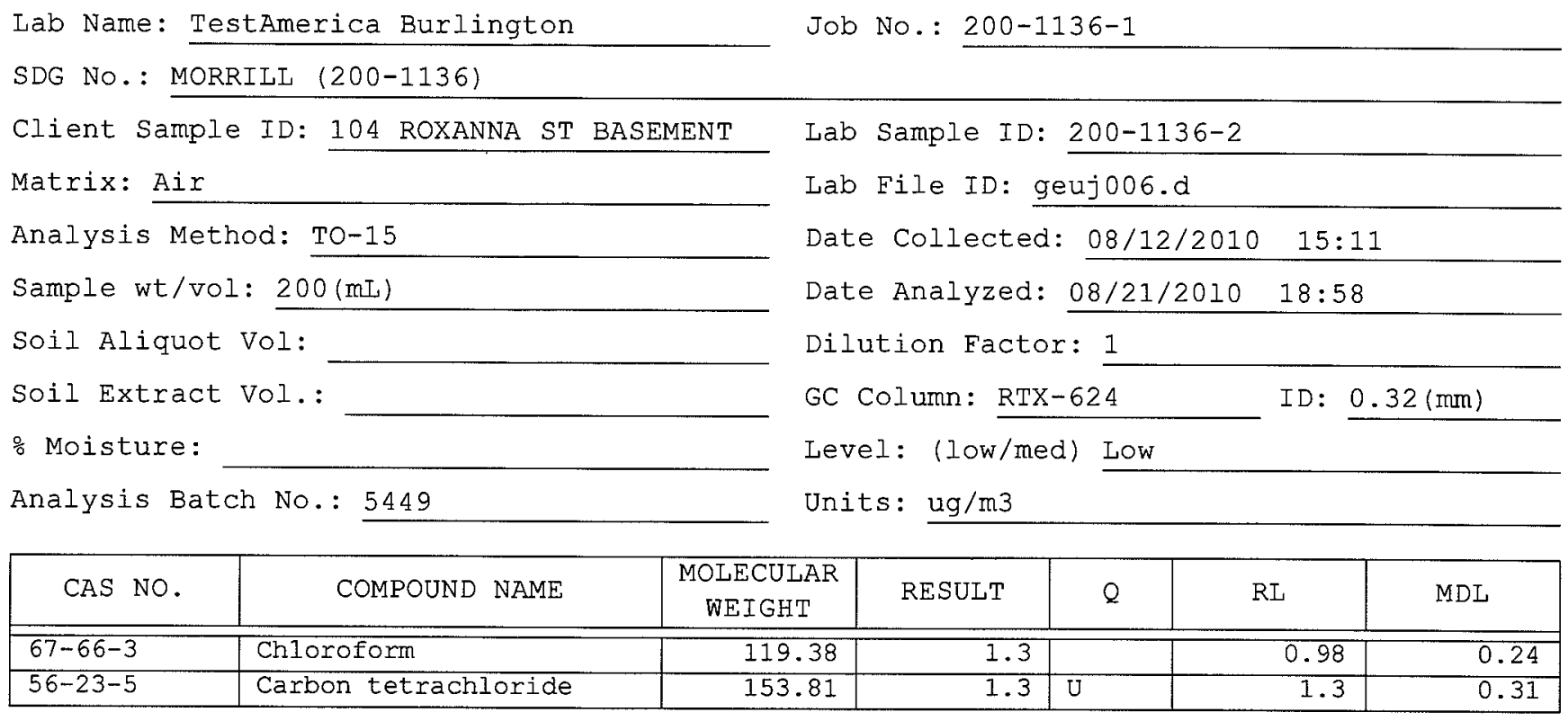


FORM I

AIR - GC/MS VOA ORGANICS ANALYSIS DATA SHEET

Iab Name: TestAmerica Burlington

SDG NO.: MORRILI (200-1136)

Client sample ID: 102 VIRGINIA ST 1ST

Matrix: Air

Analysis Method: TO-15

Sample wt/vol: $200(\mathrm{~mL})$

Soil Aliquot Vol:

Soil Extract Vol.:

\% Moisture:

Analysis Batch No.: 5449
Job No.: 200-1136-1

Iab Sample ID: 200-1136-3

Lab File ID: geuj007.d

Date Collected: 08/12/2010 15:29

Date Analyzed: 08/21/2010 19:46

Dilution Factor: 1

GC Column: RTX-624

ID: $0.32(\mathrm{~mm})$

Level: (low/med) Low

Units: ppb $v / v$

\begin{tabular}{|c|l|c|c|c|c|c|}
\hline CAS NO. & \multicolumn{1}{|c|}{ COMPOUND NAME } & $\begin{array}{c}\text { MOLECULAR } \\
\text { WEIGHT }\end{array}$ & RESULT & Q & RL & MDL \\
\hline \hline $67-66-3$ & Chloroform & 119.38 & 0.79 & & 0.20 & 0.050 \\
\hline $56-23-5$ & Carbon tetrachloride & 153.81 & 0.20 & U & 0.20 & 0.050 \\
\hline
\end{tabular}

FORM I TO-15 
FORM I

AIR - GC/MS VOA ORGANICS ANALYSIS DATA SHEET

Lab Name: TestAmerica Burlington

Job No.: $200-1136-1$

SDG NO.: MORRILL (200-1136)

Client Sample ID: 102 VIRGINIA ST 1ST

Lab Sample ID: 200-1136-3

Matrix: Air

Lab File ID: geuj007.d

Analysis Method: TO-15

Sample wt/vol: $200(\mathrm{~mL})$

Soil Aliquot Vol:

Date Collected: 08/12/2010 15:29

Date Analyzed: 08/21/2010 19:46

Soil Extract Vol.:

Dilution Factor: 1

GC Column: RTX-624

ID: $0.32(\mathrm{~mm})$

․ Moisture:

Analysis Batch No.: 5449

Level: (low/med) Low

Units: ug/m3

\begin{tabular}{|c|l|r|r|r|r|r|}
\hline CAS NO. & \multicolumn{1}{|c|}{ COMPOUND NAME } & $\begin{array}{c}\text { MOLECULAR } \\
\text { WEIGHT }\end{array}$ & RESULT & Q & RL & MDL \\
\hline $67-66-3$ & Chloroform & 119.38 & 3.8 & & 0.98 & 0.24 \\
\hline $56-23-5$ & Carbon tetrachloride & 153.81 & 1.3 & $\mathrm{U}$ & 1.3 & 0.31 \\
\hline
\end{tabular}

FORM I TO- 15 
FORM I

AIR - GC/MS VOA ORGANICS ANALYSIS DATA SHEET

Lab Name: TestAmerica Burlington

SDG NO.: MORRILL (200-1136)

Client Sample ID: 102 VIRGINIA ST BASEMENT

Matrix: Air

Analysis Method: TO-15

Sample wt/vol: $200(\mathrm{~mL})$

Soil Aliquot Vol:

Soil Extract vol.:

응 Moisture:

Analysis Batch No.: 5449
Job No.: 200-1136-1

Lab Sample ID: 200-1136-4

Lab File ID: geuj008.d

Date Collected: 08/12/2010 15:33

Date Analyzed: 08/21/2010 20:35

Dilution Factor: 1

GC Column: RTX-624

ID: $0.32(\mathrm{~mm})$

Level: (low/med) Low

Units: $\mathrm{ppb} \mathrm{v} / \mathrm{v}$

\begin{tabular}{|l|l|c|c|c|c|c|}
\hline \multicolumn{1}{|c|}{ CAS NO. } & \multicolumn{1}{|c|}{ COMPOUND NAME } & $\begin{array}{c}\text { MOLECULAR } \\
\text { WEIGHT }\end{array}$ & RESULT & Q & RL & MDI \\
\hline \hline $67-66-3$ & Chloroform & 119.38 & 0.85 & & 0.20 & 0.050 \\
\hline $56-23-5$ & Carbon tetrachloride & 153.81 & 0.20 & U & 0.20 & 0.050 \\
\hline
\end{tabular}


FORM I

AIR - GC/MS VOA ORGANICS ANALYSIS DATA SHEET

Lab Name: TestAmerica Burlington

SDG NO.: MORRILL (200-1136)

Client Sample ID: 102 VIRGINIA ST BASEMENT

Matrix: Air

Analysis Method: TO-15

Sample wt/vol: $200(\mathrm{~mL})$

Soil Aliquot Vol:

Soil Extract Vol.:

응 Moisture:

Analysis Batch No.: 5449
Job No.: 200-1136-1

Lab Sample ID: 200-1136-4

Lab File ID: geuj008.d

Date Collected: 08/12/2010 15:33

Date Analyzed: 08/21/2010 20:35

Dilution Factor: 1

GC Column: RTX-624

ID: $0.32(\mathrm{~mm})$

Level: (low/med) Low

Units: $\mathrm{ug} / \mathrm{m} 3$

\begin{tabular}{|c|l|r|r|r|r|r|}
\hline CAS NO. & \multicolumn{1}{|c|}{ COMPOUND NAME } & $\begin{array}{c}\text { MOLECULAR } \\
\text { WEIGHT }\end{array}$ & RESULT & Q & RL & MDL \\
\hline \hline $67-66-3$ & Chloroform & 119.38 & 4.1 & & 0.98 & 0.24 \\
\hline $56-23-5$ & Carbon tetrachloride & 153.81 & 1.3 & U & 1.3 & 0.31 \\
\hline
\end{tabular}


FORM I

AIR - GC/MS VOA ORGANICS ANALYSIS DATA SHEET

Lab Name: TestAmerica Burlington

SDG NO.: MORRIIL (200-1136)

Client Sample ID: 202 ROXANNA ST 1ST

Matrix: Air

Analysis Method: TO-15

Sample wt/vol: 200 (mL)

Soil Aliquot Vol:

Soil Extract Vol.:

응 Moisture:

Analysis Batch No.: 5449
Job No.: 200-1136-1

Lab Sample ID: 200-1136-5

Lab File ID: geuj009.d

Date Collected: 08/12/2010 13:04

Date Analyzed: 08/21/2010 21:23

Dilution Factor: 1

GC Column: RTX-624

ID: $0.32(\mathrm{~mm})$

Level: (low/med) Low

Units: $\mathrm{ppb} \mathrm{v} / \mathrm{v}$

\begin{tabular}{|c|l|r|r|r|r|r|}
\hline CAS NO. & \multicolumn{1}{|c|}{ COMPOUND NAME } & $\begin{array}{c}\text { MOLECULAR } \\
\text { WEIGHT }\end{array}$ & RESULT & Q & RI & MDL \\
\hline $67-66-3$ & Chloroform & 119.38 & 0.72 & & 0.20 & 0.050 \\
\hline $56-23-5$ & Carbon tetrachloride & 153.81 & 0.20 & U & 0.20 & 0.050 \\
\hline
\end{tabular}

FORM I TO-15 
FORM I

AIR - GC/MS VOA ORGANICS ANAIYSIS DATA SHEET

Lab Name: TestAmerica Burlington

SDG NO.: MORRILL (200-1136)

Client Sample ID: 202 ROXANNA ST 1 ST

Matrix: Air

Analysis Method: TO-15

Sample wt/vol: $200(\mathrm{~mL})$

Soil Aliquot Vol:

Soil Extract Vol.:

음 Moisture:

Analysis Batch No.: 5449
Job No.: 200-1136-1

Lab Sample ID: 200-1136-5

Lab File ID: geuj009.d

Date Collected: 08/12/2010 13:04

Date Analyzed: 08/21/2010 21:23

Dilution Factor: 1

GC Column: RTX-624

ID: $0.32(\mathrm{~mm})$

Level: (low/med) Low

Units: $u g / \mathrm{m} 3$

\begin{tabular}{|c|l|r|r|r|r|r|}
\hline CAS NO. & \multicolumn{1}{|c|}{ COMPOUND NAME } & $\begin{array}{c}\text { MOIECULAR } \\
\text { WEIGHT }\end{array}$ & RESULT & Q & RI & MDI \\
\hline $67-66-3$ & Chloroform & 119.38 & 3.5 & & 0.98 & 0.24 \\
\hline $56-23-5$ & Carbon tetrachloride & 153.81 & 1.3 & $\mathrm{U}$ & 1.3 & 0.31 \\
\hline
\end{tabular}


FORM I

AIR - GC/MS VOA ORGANICS ANALYSIS DATA SHEET

Lab Name: TestAmerica Burlington

SDG NO.: MORRILL (200-1136)

Client Sample ID: 202 ROXANNA ST BASEMENT

Matrix: Air

Analysis Method: TO-15

Sample wt/vol: $200(\mathrm{~mL})$

Soil Aliquot Vol:

Soil Extract Vol.:

\% Moisture:

Analysis Batch No.: 5449
Job No.: $200-1136-1$

Lab Sample ID: 200-1136-6

Lab File ID: geuj010.d

Date collected: 08/12/2010 13:06

Date Analyzed: 08/21/2010 22:12

Dilution Factor: 1

GC Column: RTX-624

Level: (low/med) Low

Units: $\mathrm{ppb} \mathrm{v} / \mathrm{v}$

\begin{tabular}{|c|l|c|r|r|r|r|}
\hline CAS NO. & \multicolumn{1}{|c|}{ COMPOUND NAME } & $\begin{array}{c}\text { MOLECULAR } \\
\text { WEIGHT }\end{array}$ & RESULT & Q & RL & MDL \\
\hline \hline $67-66-3$ & Chloroform & 119.38 & 0.20 & U & 0.20 & 0.050 \\
\hline $56-23-5$ & Carbon tetrachloride & 153.81 & 0.20 & U & 0.20 & 0.050 \\
\hline
\end{tabular}


FORM I

AIR - GC/MS VOA ORGANICS ANALYSIS DATA SHEET

Lab Name: TestAmerica Burlington

SDG NO.: MORRILL (200-1136)

Client Sample ID: 202 ROXANNA ST BASEMENT

Matrix: Air

Analysis Method: TO-15

Sample wt/vol: $200(\mathrm{~mL})$

Soil Aliquot Vol:

Soil Extract Vol.:

응 Moisture:

Analysis Batch No.: 5449
Job No.: 200-1136-1

Lab Sample ID: 200-1136-6

Lab File ID: geuj010.d

Date Collected: 08/12/2010 13:06

Date Analyzed: 08/21/2010 22:12

Dilution Factor: 1

GC Column: RTX-624

Level: (low/med) Low

Units: ug/m3

\begin{tabular}{|c|l|r|r|r|r|r|}
\hline CAS NO. & \multicolumn{1}{|c|}{ COMPOUND NAME } & $\begin{array}{c}\text { MOLECULAR } \\
\text { WEIGHT }\end{array}$ & RESULT & Q & RL & MDL \\
\hline $67-66-3$ & Chloroform & 119.38 & 0.98 & $\mathrm{U}$ & 0.98 & 0.24 \\
\hline $56-23-5$ & Carbon tetrachloride & 153.81 & 1.3 & $\mathrm{U}$ & 1.3 & 0.31 \\
\hline
\end{tabular}

FORM I TO- 15 
FORM I

AIR - GC/MS VOA ORGANICS ANALYSIS DATA SHEET

Lab Name: TestAmerica Burlington

SDG NO.: MORRILL (200-1136)

Client Sample ID: 203 ROXANNA ST 1ST

Matrix: Air

Analysis Method: TO-15

Sample wt/vol: $200(\mathrm{~mL})$

Soil Aliquot Vol:

Soil Extract Vol.:

\% Moisture:

Analysis Batch No.: 5449
Job No.: 200-1136-1

Lab Sample ID: 200-1136-7

Lab File ID: geuj011.d

Date Collected: 08/12/2010 13:17

Date Analyzed: 08/21/2010 23:00

Dilution Factor: 1

GC Column: RTX-624

ID: $0.32(\mathrm{~mm})$

Level: (low/med) Low

Units: $\mathrm{ppb} \mathrm{v} / \mathrm{v}$

\begin{tabular}{|c|l|r|r|r|r|r|}
\hline CAS NO. & COMPOUND NAME & $\begin{array}{c}\text { MOLECULAR } \\
\text { WEIGHT }\end{array}$ & RESULT & Q & RL & MDL \\
\hline \hline $67-66-3$ & Chloroform & 119.38 & 1.8 & & 0.20 & 0.050 \\
\hline $56-23-5$ & Carbon tetrachloride & 153.81 & 0.20 & U & 0.20 & 0.050 \\
\hline
\end{tabular}


FORM I

AIR - GC/MS VOA ORGANICS ANALYSIS DATA SHEET

Lab Name: TestAmerica Burlington

Job No.: 200-1136-1

SDG NO.: MORRILL (200-1136)

Client Sample ID: 203 ROXANNA ST 1ST

Lab Sample ID: 200-1136-7

Matrix: Air

Lab File ID: geuj011.d

Analysis Method: TO-15

Date collected: 08/12/2010 13:17

Sample wt/vol: $200(\mathrm{mI})$

Soil Aliquot Vol:

Date Analyzed: 08/21/2010 23:00

Soil Extract Vol.:

Dilution Factor: 1

GC Column: RTX-624

ID: $0.32(\mathrm{~mm})$

음 Moisture:

Analysis Batch No.: 5449

Level: (low/med) Low

Units: ug/m3

\begin{tabular}{|c|l|r|r|r|r|r|}
\hline CAS NO. & \multicolumn{1}{|c|}{ COMPOUND NAME } & $\begin{array}{c}\text { MOLECULAR } \\
\text { WEIGHT }\end{array}$ & RESULT & Q & RL & MDL \\
\hline \hline $67-66-3$ & Chloroform & 119.38 & 8.7 & & 0.98 & 0.24 \\
\hline $56-23-5$ & Carbon tetrachloride & 153.81 & 1.3 & U & 1.3 & 0.31 \\
\hline
\end{tabular}


FORM I

AIR - GC/MS VOA ORGANICS ANALYSIS DATA SHEET

Lab Name: TestAmerica Burlington

SDG NO.: MORRILL (200-1136)

Client sample ID: 107 ROXANNA ST MAIN ROOM

Matrix: Air

Analysis Method: To-15

Sample wt/vol: $200(\mathrm{~mL})$

Soil Aliquot Vol:

Soil Extract Vol.:

응 Moisture:

Analysis Batch No.: 5449
Job No.: 200-1136-1

Lab Sample ID: 200-1136-8

Lab File ID: geuj012.d

Date Collected: 08/12/2010 17:16

Date Analyzed: 08/21/2010 23:48

Dilution Factor: 1

GC Column: RTX-624

ID: $0.32(\mathrm{~mm})$

Level: (low/med) Low

Units: $\mathrm{ppb} \mathrm{v} / \mathrm{v}$

\begin{tabular}{|c|l|r|r|r|r|r|}
\hline CAS NO. & \multicolumn{1}{|c|}{ COMPOUND NAME } & $\begin{array}{c}\text { MOLECULAR } \\
\text { WEIGHT }\end{array}$ & RESULT & Q & RL & MDL \\
\hline $67-66-3$ & Chloroform & 119.38 & 0.20 & U & 0.20 & 0.050 \\
\hline $56-23-5$ & Carbon tetrachloride & 153.81 & 0.20 & U & 0.20 & 0.050 \\
\hline
\end{tabular}


FORM I

AIR - GC/MS VOA ORGANICS ANALYSIS DATA SHEET

Lab Name: TestAmerica Burlington

SDG NO.: MORRIII (200-1136)

Client Sample ID: 107 ROXANNA ST MAIN ROOM

Matrix: Air

Analysis Method: TO-15

Sample wt/vol: $200(\mathrm{~mL})$

Soil Aliquot Vol:

Soil Extract Vol.:

\% Moisture:

Analysis Batch No.: 5449
Job No.: 200-1136-1

Lab Sample ID: 200-1136-8

Lab File ID: geuj012.d

Date Collected: 08/12/2010 17:16

Date Analyzed: 08/21/2010 23:48

Dilution Factor: 1

GC Column: RTX-624

ID: $0.32(\mathrm{~mm})$

Level: (low/med) Low

Units: $\mathrm{ug} / \mathrm{m} 3$

\begin{tabular}{|c|l|r|r|r|r|r|}
\hline \multicolumn{1}{|c|}{ CAS NO. } & \multicolumn{1}{|c|}{ COMPOUND NAME } & $\begin{array}{c}\text { MOLECULAR } \\
\text { WEIGHT }\end{array}$ & RESULT & Q & RL & MDL \\
\hline \hline $67-66-3$ & Chloroform & 119.38 & 0.98 & U & 0.98 & 0.24 \\
\hline $56-23-5$ & Carbon tetrachloride & 153.81 & 1.3 & U & 1.3 & 0.31 \\
\hline
\end{tabular}


FORM I

AIR - GC/MS VOA ORGANICS ANALYSIS DATA SHEET

Lab Name: TestAmerica Burlington

Job No.: 200-1136-1

SDG NO.: MORRILI $(200-1136)$

Client Sample ID: 96 VIRGINIA ST OFFICE

Lab Sample ID: 200-1136-9

Matrix: Air

Lab File ID: geuj013.d

Analysis Method: TO-15

Date Collected: 08/12/2010 14:34

Sample wt/vol: $200(\mathrm{~mL})$

Soil Aliquot Vol:

Date Analyzed: 08/22/2010 00:37

Soil Extract Vol.:

\% Moisture:

Analysis Batch No.: 5449

Dilution Factor: 1

GC Column: RTX-624

ID $: 0.32(\mathrm{~mm})$

Level: (low/med) Low

Units: $\mathrm{ppb} \mathrm{v} / \mathrm{v}$

\begin{tabular}{|c|l|r|r|r|r|r|}
\hline CAS NO. & \multicolumn{1}{|c|}{ COMPOUND NAME } & $\begin{array}{c}\text { MOLECULAR } \\
\text { WEIGHT }\end{array}$ & RESULT & Q & RL & MDL \\
\hline $67-66-3$ & Chloroform & 119.38 & 5.4 & & 0.20 & 0.050 \\
\hline $56-23-5$ & Carbon tetrachloride & 153.81 & 0.20 & U & 0.20 & 0.050 \\
\hline
\end{tabular}

FORM I TO-15 
EORM I

AIR - GC/MS VOA ORGANICS ANALYSIS DATA SHEET

Lab Name: TestAmerica Burlington

SDG No.: MORRILL (200-1136)

Client sample ID: 96 VIRGINIA ST OFFICE

Matrix: Air

Analysis Method: TO-15

Sample wt/vol: $200(\mathrm{~mL})$

Soil Aliquot Vol:

Soil Extract Vol.:

\% Moisture:

Analysis Batch No.: 5449
Job No.: 200-1136-1

Lab Sample ID: 200-1136-9

Lab File ID: geuj013.d

Date Collected: 08/12/2010 14:34

Date Analyzed: 08/22/2010 00:37

Dilution Factor: 1

GC Column: RTX-624

ID: $0.32(\mathrm{~mm})$

Level: (low/med) Low

Units: ug/m3

\begin{tabular}{|c|l|r|r|r|r|r|}
\hline CAS NO. & \multicolumn{1}{|c|}{ COMPOUND NAME } & $\begin{array}{c}\text { MOLECULAR } \\
\text { WEIGHT }\end{array}$ & RESULT & Q & RI & MDL \\
\hline $67-66-3$ & Chloroform & 119.38 & 26 & & 0.98 & 0.24 \\
\hline $56-23-5$ & Carbon tetrachloride & 153.81 & 1.3 & $\mathrm{U}$ & 1.3 & 0.31 \\
\hline
\end{tabular}

FORM I TO-15 
FORM I

AIR - GC/MS VOA ORGANICS ANALYSIS DATA SHEET

Lab Name: TestAmerica Burlington

SDG NO.: MORRILL (200-1136)

Client Sample ID: AMBIENT AIR. MW1

Matrix: Air

Analysis Method: TO-15

Sample wt/vol: $200(\mathrm{~mL})$

Soil Aliquot Vol:

Soil Extract Vol.:

\% Moisture:

Analysis Batch No.: 5449
Job No.: 200-1136-1

Lab Sample ID: 200-1136-10

Lab File ID: geuj014.d

Date Collected: 08/12/2010 14:47

Date Analyzed: 08/22/2010 01:25

Dilution Factor: 1

GC Column: RTX-624

ID: $0.32(\mathrm{~mm})$

Level: (low/med) Low

Units: $\mathrm{ppb} \mathrm{v} / \mathrm{v}$

\begin{tabular}{|c|l|r|r|r|r|r|}
\hline CAS NO. & \multicolumn{1}{|c|}{ COMPOUND NAME } & $\begin{array}{c}\text { MOLECULAR } \\
\text { WEIGHT }\end{array}$ & RESULT & Q & RL & MDL \\
\hline \hline $67-66-3$ & Chloroform & 119.38 & 0.20 & U & 0.20 & 0.050 \\
\hline $56-23-5$ & Carbon tetrachloride & 153.81 & 0.20 & U & 0.20 & 0.050 \\
\hline
\end{tabular}


FORM I

AIR - GC/MS VOA ORGANICS ANALYSIS DATA SHEET

Lab Name: TestAmerica Burlington

SDG NO.: MORRILI (200-1136)

Client sample ID: AMBIENT AIR MW1

Matrix: Air

Analysis Method: TO-15

Sample wt/vol: $200(\mathrm{~mL})$

Soil Aliquot Vol:

Soil Extract Vol.:

\% Moisture:

Analysis Batch No.: 5449
Job No.: 200-1136-1

Lab Sample ID: 200-1136-10

Lab File ID: geuj014.d

Date Collected: 08/12/2010 14:47

Date Analyzed: 08/22/2010 01:25

Dilution Factor: 1

GC Column: RTX-624

ID: $0.32(\mathrm{~mm})$

Level: (low/med) Low

Units : ug/m3

\begin{tabular}{|c|l|r|r|r|r|r|}
\hline CAS NO. & \multicolumn{1}{|c|}{ COMPOUND NAME } & $\begin{array}{c}\text { MOLECULAR } \\
\text { WEIGHT }\end{array}$ & RESULT & Q & RI & MDI \\
\hline \hline $67-66-3$ & Chloroform & 119.38 & 0.98 & U & 0.98 & 0.24 \\
\hline $56-23-5$ & Carbon tetrachloride & 153.81 & 1.3 & U & 1.3 & 0.31 \\
\hline
\end{tabular}


FORM I

AIR - GC/MS VOA ORGANICS ANALYSIS DATA SHEET

Lab Name: TestAmerica Burlington

SDG NO.: MORRILL (200-1136)

Client Sample ID: 107 ROXANNA ST OFFICE

Matrix: Air

Analysis Method: TO-15

Sample wt/vol: $200(\mathrm{~mL})$

Soil Aliquot Vol:

Soil Extract Vol.:

․ Moisture:

Analysis Batch No.: 5449
Job No.: 200-1136-1

Lab Sample ID: 200-1136-11

Lab File ID: geuj015.d

Date Collected: 08/12/2010 17:11

Date Analyzed: 08/22/2010 02:13

Dilution Factor: 1

GC Column: RTX-624

ID $: 0.32(\mathrm{~mm})$

Level: (low/med) Low

Units: $\mathrm{ppb} v / \mathrm{v}$

\begin{tabular}{|c|l|c|c|c|c|c|}
\hline CAS NO. & \multicolumn{1}{|c|}{ COMPOUND NAME } & $\begin{array}{c}\text { MOLECULAR } \\
\text { WEIGHT }\end{array}$ & RESULT & Q & RI & MDL \\
\hline $67-66-3$ & Chloroform & 119.38 & 0.20 & U & 0.20 & 0.050 \\
\hline $56-23-5$ & Carbon tetrachloride & 153.81 & 0.20 & U & 0.20 & 0.050 \\
\hline
\end{tabular}

FORM I TO-15 
FORM I

AIR - GC/MS VOA ORGANICS ANALYSIS DATA SHEET

Lab Name: TestAmerica Burlington

Job No.: $200-1136-1$

SDG NO.: MORRILL $(200-1136)$

Client Sample ID: 107 ROXANNA ST OFFICE

Lab Sample ID: 200-1136-11

Matrix: Air

Analysis Method: TO-15

Lab File ID: geuj015.d

Sample wt/vol: $200(\mathrm{~mL})$

Date Collected: 08/12/2010 17:11

Soil Aliquot Vol:

Date Analyzed: 08/22/2010 02:13

Soil Extract Vol.:

Dilution Factor: 1

\% Moisture:

GC Column: RTX -624

ID : $0.32(\mathrm{~mm})$

Analysis Batch No.: 5449

Level: (low/med) Low

Units: $\mathrm{ug} / \mathrm{m} 3$

\begin{tabular}{|c|l|r|r|r|r|r|}
\hline \multicolumn{1}{|c|}{ CAS NO. } & \multicolumn{1}{|c|}{ COMPOUND NAME } & $\begin{array}{c}\text { MOLECULAR } \\
\text { WEIGHT }\end{array}$ & RESULT & Q & RI & MDL \\
\hline \hline $67-66-3$ & Chloroform & 119.38 & 0.98 & J & 0.98 & 0.24 \\
\hline $56-23-5$ & Carbon tetrachloride & 153.81 & 1.3 & U & 1.3 & 0.31 \\
\hline
\end{tabular}

FORM I TO-15 
FORM I

AIR - GC/MS VOA ORGANICS ANALYSIS DATA SHEET

Lab Name: TestAmerica Burlington

SDG NO.: MORRILL (200-1136)

Client Sample ID: 107 ROXANNA ST KID ROOM

Matrix: Air

Analysis Method: TO-15

Sample wt/vol: $200(\mathrm{~mL})$

Soil Aliquot Vol:

Soil Extract Vol.:

응 Moisture:

Analysis Batch No.: 5449
Job No.: 200-1136-1

Lab Sample ID: 200-1136-12

Lab File ID: geuj016.d

Date Collected: 08/12/2010 17:14

Date Analyzed: 08/22/2010 03:01

Dilution Factor: 1

GC Column: RTX-624 ID: $0.32(\mathrm{~mm})$

Level: (low/med) Low

Units: $\mathrm{ppb} \mathrm{v} / \mathrm{v}$

\begin{tabular}{|c|l|r|r|r|r|r|}
\hline CAS NO. & \multicolumn{1}{|c|}{ COMPOUND NAME } & $\begin{array}{c}\text { MOLECULAR } \\
\text { WEIGHT }\end{array}$ & RESULT & Q & RI & MDI \\
\hline \hline $67-66-3$ & Chloroform & 119.38 & 0.20 & U & 0.20 & 0.050 \\
\hline $56-23-5$ & Carbon tetrachloride & 153.81 & 0.20 & U & 0.20 & 0.050 \\
\hline
\end{tabular}


FORM I

AIR - GC/MS VOA ORGANICS ANALYSIS DATA SHEET

Lab Name: TestAmerica Burlington

Job No.: 200-1136-1

SDG NO.: MORRILL (200-1136)

Client Sample ID: 107 ROXANNA ST KID ROOM

Lab Sample ID: 200-1136-12

Matrix: Air

Analysis Method: TO-15

Sample wt/vol: $200(\mathrm{~mL})$

Soil Aliquot Vol:

Soil Extract Vol.:

\% Moisture:

Analysis Batch No.: 5449

Lab File ID: geuj016.d

Date Collected: 08/12/2010 17:14

Date Analyzed: 08/22/2010 03:01

Dilution Factor: 1

GC Column: RTX-624

ID: $0.32(\mathrm{~mm})$

Level: (low/med) Low

Units: ug/m3

\begin{tabular}{|c|l|r|r|r|r|r|}
\hline CAS NO. & \multicolumn{1}{|c|}{ COMPOUND NAME } & $\begin{array}{c}\text { MOLECULAR } \\
\text { WEIGHT }\end{array}$ & RESULT & Q & RL & MDL \\
\hline $67-66-3$ & Chloroform & 119.38 & 0.98 & $\mathrm{U}$ & 0.98 & 0.24 \\
\hline $56-23-5$ & Carbon tetrachloride & 153.81 & 1.3 & $\mathrm{U}$ & 0.31 \\
\hline
\end{tabular}


FORM I

AIR - GC/MS VOA ORGANICS ANALYSIS DATA SHEET

Lab Name: TestAmerica Burlington

SDG NO.: MORRILL (200-1136)

Client sample ID: 108 ROXANNA ST 1ST

Matrix: Air

Analysis Method: TO-15

Sample wt/vol: $200(\mathrm{~mL})$

Soil Aliquot Vol:

Soil Extract Vol.:

\% Moisture:

Analysis Batch No.: 5449

\begin{tabular}{|c|l|c|c|c|c|c|}
\hline \multicolumn{1}{|c|}{ CAS NO. } & \multicolumn{1}{|c|}{ COMPOUND NAME } & $\begin{array}{c}\text { MOLECULAR } \\
\text { WEIGHT }\end{array}$ & RESULT & Q & RL & MDL \\
\hline \hline $67-66-3$ & Chloroform & 119.38 & 0.75 & & 0.20 & 0.050 \\
\hline $56-23-5$ & Carbon tetrachloride & 153.81 & 0.20 & U & 0.20 & 0.050 \\
\hline
\end{tabular}

Job No.: 200-1136-1

Lab Sample ID: 200-1136-13

Lab File ID: geuj017.d

Date Collected: 08/12/2010 15:39

Date Analyzed: 08/22/2010 03:50

Dilution Factor: 1

GC Column: RTX-624

ID: $0.32(\mathrm{~mm})$

Level: (low/med) Low

Units: ppb v/v 
FORM I

AIR - GC/MS VOA ORGANICS ANALYSIS DATA SHEET

Lab Name: TestAmerica Burlington

SDG NO.: MORRILL (200-1136)

Client Sample ID: 108 ROXANNA ST 1ST

Matrix: Air

Analysis Method: TO-15

Sample wt/vol: $200(\mathrm{~mL})$

Soil Aliquot Vol:

Soil Extract Vol.:

음 Moisture:

Analysis Batch No.: 5449
Job No.: 200-1136-1

Lab Sample ID: 200-1136-13

Lab File ID: geuj017.d

Date Collected: 08/12/2010 15:39

Date Analyzed: 08/22/2010 03:50

Dilution Factor: 1

GC Column: RTX-624

ID: $0.32(\mathrm{~mm})$

Level: (low/med) Low

Units: ug/m3

\begin{tabular}{|c|l|r|r|r|r|r|}
\hline CAS NO. & \multicolumn{1}{|c|}{ COMPOUND NAME } & $\begin{array}{c}\text { MOLECULAR } \\
\text { WEIGHT }\end{array}$ & RESULT & Q & RL & MDL \\
\hline $67-66-3$ & Chloroform & 119.38 & 3.6 & & 0.98 & 0.24 \\
\hline $56-23-5$ & Carbon tetrachloride & 153.81 & 1.3 & U & 1.3 & 0.31 \\
\hline
\end{tabular}

FORM I TO-15 
FORM I

AIR - GC/MS VOA ORGANICS ANALYSIS DATA SHEET

Lab Name: TestAmerica Burlington

SDG NO.: MORRILL (200-1136)

Client Sample ID: 108 ROXANNA ST BASEMENT

Matrix: Air

Analysis Method: TO-15

Sample wt/vol: $200(\mathrm{~mL})$

Soil Aliquot Vol:

Soil Extract Vol.:

\% Moisture:

Analysis Batch No.: 5449

\begin{tabular}{|c|l|c|c|c|c|c|}
\hline CAS NO. & \multicolumn{1}{|c|}{ COMPOUND NAME } & $\begin{array}{c}\text { MOLECULAR } \\
\text { WEIGHT }\end{array}$ & RESULT & Q & RL & MDL \\
\hline \hline $67-66-3$ & Chloroform & 119.38 & 0.94 & & 0.20 & 0.050 \\
\hline $56-23-5$ & Carbon tetrachloride & 153.81 & 0.20 & U & 0.20 & 0.050 \\
\hline
\end{tabular}

Job No.: 200-1136-1

Lab Sample ID: 200-1136-14

Lab File ID: geuj018.d

Date Collected: 08/12/2010 15:43

Date Analyzed: 08/22/2010 04:38

Dilution Factor: 1

GC Column: RTX-624

ID $: 0.32(\mathrm{~mm})$

Level: (low/med) Low

Units: $\mathrm{ppb} \mathrm{v} / \mathrm{v}$ 
FORM I

AIR - GC/MS VOA ORGANICS ANALYSIS DATA SHEET

Lab Name: TestAmerica Burlington

SDG NO.: MORRILI (200-1136)

Client Sample ID: 108 ROXANNA ST BASEMENT

Matrix: Air

Analysis Method: TO-15

Sample wt/vol: $200(\mathrm{~mL})$

Soil Aliquot Vol:

Soil Extract Vol.:

$\%$ Moisture:

Analysis Batch No.: 5449

\begin{tabular}{|c|l|r|r|r|r|r|}
\hline CAS NO. & \multicolumn{1}{|c|}{ COMPOUND NAME } & $\begin{array}{c}\text { MOLECULAR } \\
\text { WEIGHT }\end{array}$ & RESULT & Q & RL & MDL \\
\hline \hline $67-66-3$ & Chloroform & 119.38 & 4.6 & & 0.98 & 0.24 \\
\hline $56-23-5$ & Carbon tetrachloride & 153.81 & 1.3 & $\mathrm{U}$ & 1.3 & 0.31 \\
\hline
\end{tabular}

Job No.: 200-1136-1

Lab Sample ID: 200-1136-14

Lab File ID: geuj018.d

Date Collected: 08/12/2010 15:43

Date Analyzed: 08/22/2010 04:38

Dilution Factor: 1

GC Column: RTX-624

ID: $0.32(\mathrm{~mm})$

Level: (low/med) Low

Units: $\mathrm{ug} / \mathrm{m} 3$ 
FORM I

AIR - GC/MS VOA ORGANICS ANALYSIS DATA SHEET

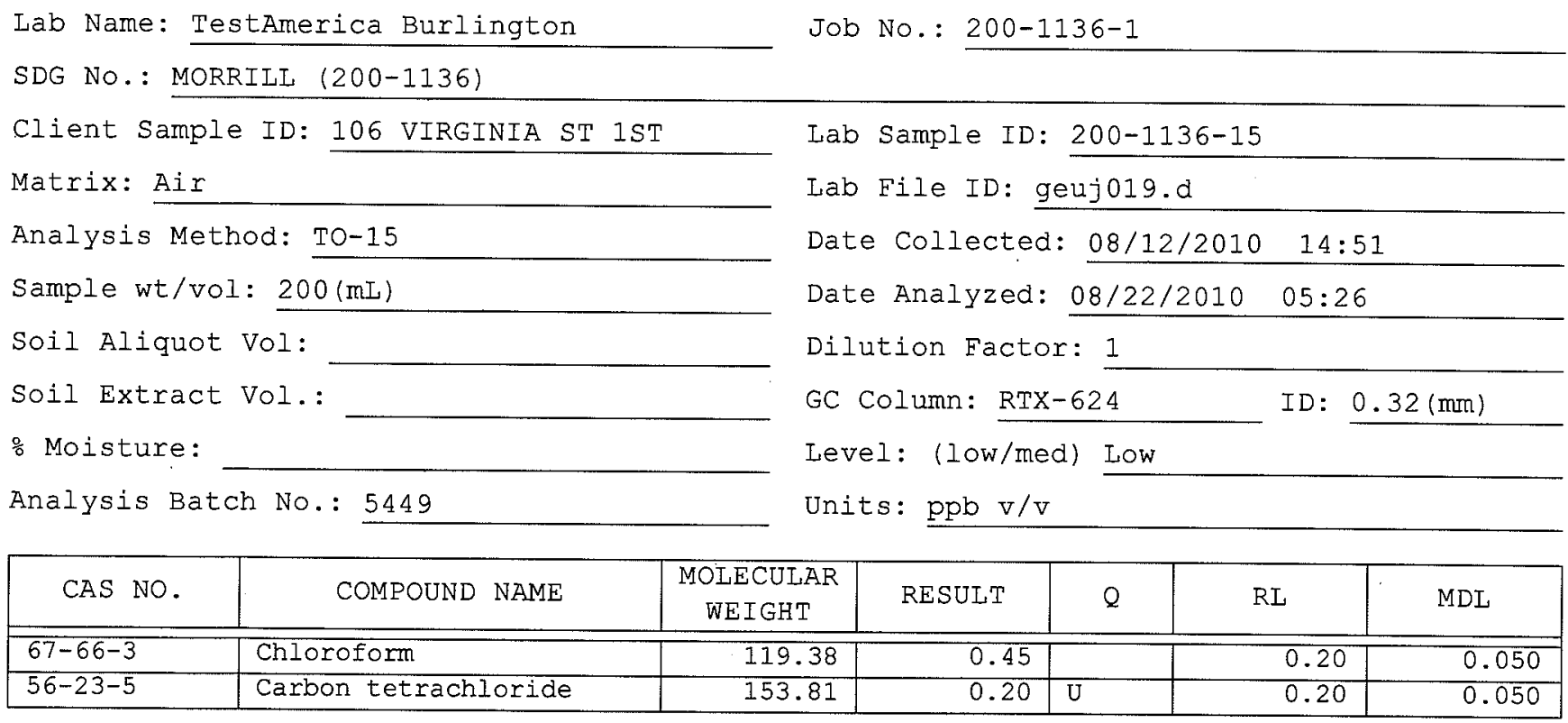


EORM I

AIR - GC/MS VOA ORGANICS ANALYSIS DATA SHEET

Iab Name: TestAmerica Burlington

SDG No.: MORRILL $(200-1136)$

Client Sample ID: 106 VIRGINIA ST 1ST

Matrix: Air

Analysis Method: TO-15

Sample wt/vol: $200(\mathrm{~mL})$

Soil Aliquot Vol:

Soil Extract Vol.:

응 Moisture:

Analysis Batch No.: 5449
Job No.: 200-1136-1

Lab Sample ID: 200-1136-15

Lab File ID: geuj019.d

Date Collected: 08/12/2010 14:51

Date Analyzed: 08/22/2010 05:26

Dilution Factor: 1

GC Column: RTX-624

ID : $0.32(\mathrm{~mm})$

Level: (low/med) Low

Units: ug/m3

\begin{tabular}{|c|l|r|r|r|r|r|}
\hline CAS NO. & \multicolumn{1}{|c|}{ COMPOUND NAME } & $\begin{array}{c}\text { MOLECULAR } \\
\text { WEIGHT }\end{array}$ & RESULT & Q & RL & MDL \\
\hline $67-66-3$ & Chloroform & 119.38 & 2.2 & & 0.98 & 0.24 \\
\hline $56-23-5$ & Carbon tetrachloride & 153.81 & 1.3 & $\mathrm{U}$ & 1.3 & 0.31 \\
\hline
\end{tabular}

FORM I TO-15 
FORM I

AIR - GC/MS VOA ORGANICS ANALYSIS DATA SHEET

Lab Name: TestAmerica Burlington

SDG NO.: MORRILI (200-1136)

Client Sample ID: 105 ROXANNA ST 1ST

Matrix: Air

Analysis Method: TO-15

Sample wt/vol: $200(\mathrm{~mL})$

Soil Aliquot Vol:

Soil Extract Vol.:

\% Moisture:

Analysis Batch No.: 5449
Job No.: 200-1136-1

Lab Sample ID: 200-1136-16

Lab File ID: geuj020.d

Date Collected: 08/12/2010 17:03

Date Analyzed: 08/22/2010 06:14

Dilution Factor: 1

GC Column: RTX-624

ID: $0.32(\mathrm{~mm})$

Level: (low/med) Low

Units: $\mathrm{ppb} \mathrm{v} / \mathrm{v}$

\begin{tabular}{|l|l|c|r|r|r|r|}
\hline \multicolumn{1}{|c|}{ CAS NO. } & \multicolumn{1}{|c|}{ COMPOUND NAME } & $\begin{array}{c}\text { MOLECULAR } \\
\text { WEIGHT }\end{array}$ & RESULT & Q & RI & MDI \\
\hline \hline $67-66-3$ & Chloroform & 119.38 & 0.21 & & 0.20 & 0.050 \\
\hline $56-23-5$ & Carbon tetrachloride & 153.81 & 0.20 & $\mathrm{U}$ & 0.20 & 0.050 \\
\hline
\end{tabular}


FORM I

AIR - GC/MS VOA ORGANICS ANALYSIS DATA SHEET

Lab Name: TestAmerica Burlington

SDG NO.: MORRILL (200-1136)

Client Sample ID: 105 ROXANNA ST 1ST

Matrix: Air

Analysis Method: TO-15

Sample wt/vol: $200(\mathrm{~mL})$

Soil Aliquot Vol:

Soil Extract Vol.:

\% Moisture:

Analysis Batch No.: 5449
Job No.: 200-1136-1

Lab Sample ID: 200-1136-16

Lab File ID: geuj020.d

Date Collected: 08/12/2010 17:03

Date Analyzed: 08/22/2010 06:14

Dilution Factor: 1

GC Column: RTX-624

ID: $0.32(\mathrm{~mm})$

Level: (low/med) Low

Units: ug/m3

\begin{tabular}{|c|l|c|r|r|r|r|}
\hline CAS NO. & \multicolumn{1}{|c|}{ COMPOUND NAME } & $\begin{array}{c}\text { MOLECULAR } \\
\text { WEIGHT }\end{array}$ & RESULT & Q & RL & MDL \\
\hline $67-66-3$ & Chloroform & 119.38 & 1.0 & & 0.98 & 0.24 \\
\hline $56-23-5$ & Carbon tetrachloride & 153.81 & 1.3 & $\mathrm{U}$ & 1.3 & 0.31 \\
\hline
\end{tabular}


FORM VI

AIR - GC/MS VOA INITIAL CALIBRATION DATA INTERNAL STANDARD CURVE EVALUTION

Lab Name: TestAmerica Burlington

Job No.: 200-1136-1

Analy Batch No.: 5008

SDG No.: MORRILL $(200-1136)$

Instrument ID: G.i GC Column: RTX-624 ID: 0.32 (mm)

Calibration Start Date: 08/09/2010 15:59 Calibration End Date: 08/09/2010 20:50 Calibration ID: 1811

Calibration Files:

$\begin{array}{llll}\text { LEVEL: } & \text { LAB SAMPLE ID: } & \text { LAB FILE } \\ \text { Level } & 1 & \text { IC } 200-5008 / 3 & \text { geu003.d } \\ \text { Level } & 2 & \text { IC } 200-5008 / 4 & \text { geu004.d } \\ \text { Level } & 3 & \text { IC } 200-5008 / 5 & \text { geu005.d } \\ \text { Level } & 4 & \text { ICIS } 200-5008 / 6 & \text { geu006.d } \\ \text { Level } & 5 & \text { IC } 200-5008 / 7 & \text { geu007.d } \\ \text { Level } & 6 & \text { IC } 200-5008 / 8 & \text { geu008.d } \\ \text { Leve1 } & 7 & \text { IC } 200-5008 / 9 & \text { geu009.d }\end{array}$

\begin{tabular}{|c|c|c|c|c|c|c|c|c|c|c|c|c|c|c|c|c|c|}
\hline \multirow[t]{2}{*}{ ANALYTE } & \multicolumn{5}{|c|}{$\mathrm{RRF}$} & \multirow{2}{*}{$\begin{array}{l}\text { CURVE } \\
\text { TYPE }\end{array}$} & \multicolumn{3}{|c|}{ COEFICIENT } & \multirow[t]{2}{*}{$\#$} & \multirow[t]{2}{*}{ MIN RRE } & \multirow[t]{2}{*}{8 RSD } & \multirow[t]{2}{*}{$\#$} & \multirow{2}{*}{$\begin{array}{l}\text { MAX } \\
\text { \&RSD }\end{array}$} & \multirow{2}{*}{$\begin{array}{c}\mathrm{R}^{\wedge} 2 \\
\mathrm{OR} C O D\end{array}$} & \multirow[t]{2}{*}{$\#$} & \multirow{2}{*}{$\begin{array}{l}\text { MIN } R^{\wedge} 2 \\
\text { OR COD }\end{array}$} \\
\hline & $\begin{array}{ll}\text { LVL } & 1 \\
\text { LVL } & 6 \\
\end{array}$ & $\begin{array}{l}\text { LVL } 2 \\
\text { LVL } 7\end{array}$ & LVL 3 & LVL 4 & LVL 5 & & B & M1 & M2 & & & & & & & & \\
\hline Propylene & 0.1896 & $\begin{array}{l}0.2084 \\
0.1699\end{array}$ & 0.2009 & 0.1808 & 0.2114 & Ave & & 0.1935 & & & & 8.4 & & 30.0 & & & \\
\hline Dichlorodifluoromethane & 1.4309 & $\begin{array}{l}1.6088 \\
1.2685\end{array}$ & 1.5317 & 1.4043 & 1.6362 & Ave & & 1.4801 & & & & 9.4 & & 30.0 & & & \\
\hline Freon 22 & 0.5532 & $\begin{array}{l}0.6524 \\
0.4945\end{array}$ & 0.5976 & 0.5460 & 0.6199 & Ave & & 0.5773 & & & & 9.9 & & 30.0 & & & \\
\hline 1,2-Dichlorotetrafluoroethane & $\begin{array}{l}1.5707 \\
1.3548 \\
\end{array}$ & $\begin{array}{l}1.5976 \\
1.1628 \\
\end{array}$ & 1.4963 & 1.3427 & 1.5204 & Ave & & 1.4350 & & & & 10.8 & & 30.0 & & & \\
\hline Chloromethane & 0.3120 & $\begin{array}{l}0.3622 \\
0.2793 \\
\end{array}$ & 0.3308 & 0.3025 & 0.3425 & Ave & & $0 . \overline{3216}$ & & & & 9.2 & & 30.0 & & & \\
\hline n-Butane & 0.4827 & $\begin{array}{l}0.5525 \\
0.4274 \\
\end{array}$ & 0.5164 & 0.4527 & 0.5310 & Ave & & 0.4938 & & & & 9.7 & & 30.0 & & & \\
\hline Vinyl chloride & $\begin{array}{l}0.5174 \\
0.4703 \\
\end{array}$ & $\begin{array}{l}0.5049 \\
0.4139 \\
\end{array}$ & 0.5032 & 0.4371 & 0.5127 & Ave & & 0.4799 & & & & 8.5 & & 30.0 & & & \\
\hline 1,3-Butadiene & $\begin{array}{l}0.3742 \\
0.3226 \\
\end{array}$ & $\begin{array}{l}0.3489 \\
0.2872 \\
\end{array}$ & 0.3469 & 0.3072 & 0.3535 & Ave & & 0.3343 & & & & 9.0 & & 30.0 & & & \\
\hline Bromomethane & $\begin{array}{l}1.0191 \\
0.8877 \\
\end{array}$ & $\begin{array}{l}0.9361 \\
0.8786 \\
\end{array}$ & 0.8862 & 0.8087 & 1.0248 & Ave & & 0.9202 & & & & 8.6 & & 30.0 & & & \\
\hline Chloroethane & 0.3630 & $\begin{array}{l}0.3724 \\
0.3276\end{array}$ & 0.2930 & 0.3185 & 0.3782 & Ave & & 0.3421 & & & & 10.0 & & 30.0 & & & \\
\hline Isopentane & $\begin{array}{l}0.6148 \\
0.6409 \\
\end{array}$ & $\begin{array}{l}0.6985 \\
0.5790 \\
\end{array}$ & 0.5659 & 0.5655 & 0.7117 & Äve & & 0.6252 & & & & 9.8 & & 30.0 & & & \\
\hline Bromoethene(Vinyl Bromide) & $\begin{array}{l}1.0110 \\
1.0533 \\
\end{array}$ & $\begin{array}{l}0.9752 \\
0.9502 \\
\end{array}$ & 0.9587 & 0.8774 & 1.1100 & Ave & & 0.9908 & & & & 7.6 & & .30 .0 & & & \\
\hline Trichlorofluoromethane & $\begin{array}{l}2.5952 \\
2.4647\end{array}$ & $\begin{array}{l}2.7470 \\
2.3632\end{array}$ & 2.3248 & 2.1885 & 2.6796 & Ave & & 2.4804 & & & & 8.2 & & 30.0 & & & \\
\hline n-Pentane & 1.0405 & $\begin{array}{l}1.2492 \\
0.9587 \\
\end{array}$ & 1.0480 & 1.0573 & 1.1451 & Ave & & 1.0831 & & & & 9.3 & & 30.0 & & & \\
\hline
\end{tabular}

Note: The ml coefficient is the same as Ave RRF for an Ave curve type.

FORM VI TO-15

Page 71 of 97 
Lab Name: TestAmerica Burlington

Job No.: 200-1136-1

Analy Batch No.: 5008

SDG No.: MORRILL (200-1136)

Instrument ID: G.i GC Column: RTX-624 ID: 0.32(mm)

Calibration Start Date: 08/09/2010 15:59 Calibration End Date: 08/09/2010 20:50 Calibration ID: 1811

\begin{tabular}{|c|c|c|c|c|c|c|c|c|c|c|c|c|c|c|c|c|c|}
\hline \multirow[t]{2}{*}{ ANALYTE } & \multicolumn{5}{|c|}{ RRE } & \multirow{2}{*}{$\begin{array}{l}\text { CURVE } \\
\text { TYPE }\end{array}$} & \multicolumn{3}{|c|}{ COEFEICIENT } & \multirow[t]{2}{*}{$\#$} & \multirow[t]{2}{*}{ MIN RRE } & \multirow[t]{2}{*}{$8 \mathrm{RSD}$} & \multirow[t]{2}{*}{$\#$} & \multirow{2}{*}{$\begin{array}{l}\text { MAX } \\
\text { \&RSD }\end{array}$} & \multirow{2}{*}{$\begin{array}{c}R^{\wedge} 2 \\
O R\end{array}$} & \multirow[t]{2}{*}{$\#$} & \multirow{2}{*}{$\begin{array}{c}\text { MIN } R^{\wedge} 2 \\
\text { OR } C O D\end{array}$} \\
\hline & $\begin{array}{ll}\text { LVL } & 1 \\
\text { LVL } & 6 \\
\end{array}$ & $\begin{array}{l}\text { LVL } 2 \\
\text { LVL } 7\end{array}$ & LVL 3 & LVL 4 & LVL 5 & & B & M1 & M2 & & & & & & & & \\
\hline Ethanol & 0.2345 & $\begin{array}{l}0.2621 \\
0.2199 \\
\end{array}$ & 0.2223 & 0.2457 & 0.2411 & Ave & & 0.2376 & & & & 6.6 & & 30.0 & & & \\
\hline Ethyl ether & $\begin{array}{l}0.4512 \\
0.4483\end{array}$ & $\begin{array}{l}0.4955 \\
0.4196 \\
\end{array}$ & 0.4102 & 0.4788 & 0.4496 & Ave & & 0.4504 & & & & 6.7 & & 30.0 & & & \\
\hline Acrolein & 0.2323 & 0.2090 & 0.2238 & 0.2530 & 0.2415 & Ave & & 0.2319 & & & & 7.2 & & 30.0 & & & \\
\hline Freon $\mathrm{TF}$ & $\begin{array}{l}1.8687 \\
1.7604\end{array}$ & $\begin{array}{l}2.0129 \\
1.7220\end{array}$ & 1.7375 & 1.8370 & 1.9303 & Ave & & 1.8384 & & & & 5.9 & & 30.0 & & & \\
\hline 1,1-Dichloroethene & $\begin{array}{l}1.0041 \\
0.8272 \\
\end{array}$ & $\begin{array}{l}0.9229 \\
0.8153 \\
\end{array}$ & 0.8000 & 0.8457 & 0.8922 & Ave & & 0.8725 & & & & 8.3 & & 30.0 & & & \\
\hline Acetone & 0.8084 & 0.7717 & 0.8062 & 0.9731 & 0.8211 & Ave & & 0.8361 & & & & 9.4 & & 30.0 & & & \\
\hline Isopropyl alcohol & 0.6465 & 0.6073 & 0.6339 & 0.6868 & 0.6762 & Ave & & 0.6501 & & & & 4.9 & & 30.0 & & & \\
\hline Carbon disulfide & 2.5480 & $\begin{array}{l}2.7102 \\
2.4220 \\
\end{array}$ & 2.4731 & 2.6201 & 2.7146 & Ave & & 2.5813 & & & & 4.7 & & 30.0 & & & \\
\hline 3-Chloropropene & $\begin{array}{l}0.7866 \\
0.7393 \\
\end{array}$ & $\begin{array}{l}0.8114 \\
0.6772 \\
\end{array}$ & 0.7392 & 0.7176 & 0.8045 & Ave & & 0.7537 & & & & 6.5 & & 30.0 & & & \\
\hline Acetonitrile & 0.4037 & 0.3852 & 0.3734 & 0.4035 & 0.4035 & Ave & & 0.3939 & & & & 3.5 & & 30.0 & & & \\
\hline Methylene Chloride & 0.7448 & $\begin{array}{l}1.0339 \\
0.6820 \\
\end{array}$ & 0.7610 & 0.7203 & 0.7902 & Äve & & 0.7887 & & & & 15.9 & & 30.0 & & & \\
\hline tert-Butyl alcohol & 1.0199 & 0.9648 & 0.9855 & 1.0274 & 1.0374 & Ave & & 1.0070 & & & & 3.0 & & 30.0 & & & \\
\hline Methyl tert-butyl ether & $\begin{array}{l}2.0786 \\
2.0084\end{array}$ & $\begin{array}{l}1.9529 \\
1.8276\end{array}$ & 1.7303 & 1.8981 & 1.8314 & Ave & & 1.9039 & & & & 6.3 & & 30.0 & & & \\
\hline trans-1,2-Dichloroethene & $\begin{array}{l}1.2960 \\
1.1237\end{array}$ & $\begin{array}{l}1.2057 \\
0.9981\end{array}$ & 1.1119 & 1.0439 & 1.1504 & Ave & & 1.1328 & & & & 8.7 & & 30.0 & & & \\
\hline n-Hexane & $\begin{array}{l}1.2054 \\
1.0285\end{array}$ & $\begin{array}{l}1.1205 \\
0.9538 \\
\end{array}$ & 1.0324 & 1.0195 & 1.0510 & Ave & & 1.0587 & & & & 7.7 & & 30.0 & & & \\
\hline 1,1-Dichloroethane & $\begin{array}{l}1.6562 \\
1.3786\end{array}$ & $\begin{array}{l}1.5139 \\
1.2673\end{array}$ & 1.4057 & 1.3118 & 1.3907 & Ave & & 1.4177 & & & & 9.2 & & 30.0 & & & \\
\hline Vinyl acetate & 1.4253 & 1.2705 & 1.3287 & 1.3678 & 1.3227 & Ave & & 1.3430 & & & & 4.3 & & 30.0 & & & \\
\hline cis-1,2-Dichloroethene & $\begin{array}{l}1.0727 \\
0.9539\end{array}$ & $\begin{array}{l}1.0245 \\
0.8842\end{array}$ & 0.9651 & 0.9159 & 0.9501 & Ave & & 0.9666 & & & & 6.6 & & 30.0 & & & \\
\hline Methyl Ethyl Ketone & 0.3237 & $\begin{array}{l}0.3634 \\
0.2905\end{array}$ & 0.2983 & 0.3143 & 0.2955 & Ave & & 0.3143 & & & & 8.6 & & 30.0 & & & \\
\hline Ethyl acetate & 0.0563 & 0.0505 & 0.0487 & 0.0516 & 0.0496 & Ave & & 0.0513 & & & & 5.8 & & 30.0 & & & \\
\hline
\end{tabular}

Note: The $m 1$ coefficient is the same as Ave RRE for an Ave curve type.

FORM VI TO-15 
FORM VI

AIR - GC/MS VOA INITIAL CALIBRATION DATA INTERNAL STANDARD CURVE EVALUTION

Lab Name: TestAmerica Burlington

SDG No.: MORRILL (200-1136)

Instrument ID: G.i

GC Column: RTX-624

ID: $0.32(\mathrm{~mm})$

Heated Purge: ( $Y / N)$ N

Calibration Start Date: 08/09/2010 15:59

Calibration End Date: 08/09/2010 20:50

Calibration ID: 1811

\begin{tabular}{|c|c|c|c|c|c|c|c|c|c|c|c|c|c|c|c|c|c|}
\hline \multirow[t]{2}{*}{ ANALYTE } & \multicolumn{5}{|c|}{ RRF } & \multirow{2}{*}{$\begin{array}{c}\text { CURVE } \\
\text { TYPE }\end{array}$} & \multicolumn{3}{|c|}{ COEFEICIENT } & \multirow[t]{2}{*}{$\#$} & \multirow[t]{2}{*}{ MIN RRF } & \multirow[t]{2}{*}{$8 \mathrm{RSD}$} & \multirow[t]{2}{*}{$\#$} & \multirow{2}{*}{$\begin{array}{l}\text { MAX } \\
\text { \& RSD }\end{array}$} & \multirow{2}{*}{\begin{tabular}{|c|}
$\mathrm{R}^{\wedge} 2$ \\
$\mathrm{OR}$ COD
\end{tabular}} & \multirow[t]{2}{*}{$\#$} & \multirow{2}{*}{$\begin{array}{l}\text { MIN } R^{\wedge} 2 \\
\text { OR COD }\end{array}$} \\
\hline & $\begin{array}{ll}\text { LVL } & 1 \\
\text { LVL } & 6 \\
\end{array}$ & $\begin{array}{l}\text { LVL } 2 \\
\text { LVL } 7\end{array}$ & LVL 3 & LVL 4 & LVL 5 & & B & M1 & M2 & & & & & & & & \\
\hline Tetrahydrofuran & 0.1328 & 0.1208 & 0.1312 & 0.1295 & 0.1152 & Ave & & 0.1259 & & & & 6.0 & & 30.0 & & & \\
\hline Chloroform & $\begin{array}{l}2.1180 \\
1.7978 \\
\end{array}$ & $\begin{array}{l}2.0245 \\
1.7704 \\
\end{array}$ & 1.8632 & 1.8484 & 1.8502 & Ave & & 1.8961 & & & & 6.7 & & 30.0 & & & \\
\hline Cyclohexane & $\begin{array}{l}0.2622 \\
0.2439 \\
\end{array}$ & $\begin{array}{l}0.2727 \\
0.2266 \\
\end{array}$ & 0.2753 & 0.2364 & 0.2393 & Ave & & 0.2509 & & & & 7.6 & & 30.0 & & & \\
\hline $1,1,1$-Trichloroethane & $\begin{array}{l}0.4808 \\
0.4652 \\
\end{array}$ & $\begin{array}{l}0.5118 \\
0.4457 \\
\end{array}$ & 0.5036 & 0.4556 & 0.4592 & Ave & & 0.4746 & & & & 5.3 & & 30.0 & & & \\
\hline Carbon tetrachloride & $\begin{array}{l}0.5304 \\
0.5274 \\
\end{array}$ & $\begin{array}{l}0.5991 \\
0.5186\end{array}$ & 0.5630 & 0.5669 & 0.5303 & Ave & & 0.5480 & & & & 5.3 & & 30.0 & & & \\
\hline $2,2,4$-Trimethylpentane & $\begin{array}{l}0.8331 \\
0.7124 \\
\end{array}$ & $\begin{array}{l}0.8535 \\
0.6547 \\
\end{array}$ & 0.8267 & 0.7678 & 0.6871 & Ave & & 0.7622 & & & & 10.3 & & 30.0 & & & \\
\hline Benzene & $\begin{array}{l}0.6241 \\
0.5493 \\
\end{array}$ & $\begin{array}{l}0.6393 \\
0.5273 \\
\end{array}$ & 0.6108 & 0.5538 & 0.5276 & Ave & & 0.5760 & & & & 8.2 & & 30.0 & & & \\
\hline 1,2-Dichloroethane & $\begin{array}{l}0.2486 \\
0.2339 \\
\end{array}$ & $\begin{array}{l}0.2648 \\
0.2235 \\
\end{array}$ & 0.2405 & 0.2385 & 0.2315 & Ave & & 0.2402 & & & & 5.6 & & 30.0 & & & \\
\hline n-Heptane & $\begin{array}{l}0.3402 \\
0.2263\end{array}$ & $\begin{array}{l}0.2870 \\
0.2047 \\
\end{array}$ & 0.2592 & 0.2353 & $0 . \overline{2292}$ & Ave & & 0.2546 & & & & 18.1 & & 30.0 & & & \\
\hline Trichloroethene & $\begin{array}{l}0.3378 \\
0.2863\end{array}$ & $\begin{array}{l}0.3389 \\
0.2711\end{array}$ & 0.3300 & 0.2864 & 0.2790 & Ave & & 0.3042 & & & & 9.8 & & 30.0 & & & \\
\hline 1,2-Dichloropropane & $\begin{array}{l}0.2152 \\
0.1811 \\
\end{array}$ & $\begin{array}{l}0.2330 \\
0.1690\end{array}$ & 0.1989 & 0.1837 & 0.1823 & Ave & & 0.1947 & & & & 11.5 & & 30.0 & & & \\
\hline Methy1 methacrylate & 0.1651 & $\begin{array}{l}0.1731 \\
0.1591 \\
\end{array}$ & 0.1589 & 0.1604 & 0.1596 & Ave & & 0.1627 & & & & 3.4 & & 30.0 & & & \\
\hline 1,4-Dioxane & 0.0712 & 0.0681 & 0.0773 & 0.0714 & 0.0760 & Āve & & 0.0728 & & & & 5.2 & & 30.0 & & & \\
\hline Dibromomethane & $\begin{array}{l}0.3817 \\
0.3256\end{array}$ & $\begin{array}{l}0.3800 \\
0.3253\end{array}$ & 0.3497 & 0.3096 & 0.3522 & Ave & & 0.3463 & & & & 8.0 & & 30.0 & & & \\
\hline Bromodichloromethane & $\begin{array}{l}0.4952 \\
0.4543 \\
\end{array}$ & $\begin{array}{l}0.5244 \\
0.4341 \\
\end{array}$ & 0.4893 & 0.4879 & 0.4665 & Ave & & 0.4788 & & & & 6.2 & & 30.0 & & & \\
\hline cis-1,3-Dichloropropene & $\begin{array}{l}0.3048 \\
0.3092\end{array}$ & $\begin{array}{l}0.3457 \\
0.2959\end{array}$ & 0.3052 & 0.3126 & 0.3103 & Ave & & 0.3120 & & & & 5.1 & & 30.0 & & & \\
\hline Methyl isobutyl ketone & 0.2452 & $\begin{array}{l}0.2545 \\
0.2323\end{array}$ & 0.2332 & 0.2474 & 0.2454 & Ave & & 0.2430 & & & & 3.5 & & 30.0 & & & \\
\hline n-octane & $\begin{array}{l}0.3628 \\
0.2817\end{array}$ & $\begin{array}{l}0.3797 \\
0.2371\end{array}$ & 0.3375 & 0.3102 & 0.2864 & Ave & & 0.3136 & & & & 15.9 & & 30.0 & & & \\
\hline Toluene & $\begin{array}{l}0.4331 \\
0.4143 \\
\end{array}$ & $\begin{array}{l}0.4922 \\
0.3950 \\
\end{array}$ & 0.4535 & 0.4087 & 0.3585 & Ave & & 0.4222 & & & & 10.2 & & 30.0 & & & \\
\hline trans-1,3-Dichloropropene & $\begin{array}{l}0.3252 \\
0.3190 \\
\end{array}$ & $\begin{array}{l}0.3311 \\
0.3088\end{array}$ & 0.3103 & 0.3325 & 0.3273 & Ave & & 0.3220 & & & & 3.0 & & 30.0 & & & \\
\hline
\end{tabular}

Note: The $m 1$ coefficient is the same as Ave RRF for an Ave curve type.

FORM VI TO-15

Page 73 of 97 
Lab Name: TestAmerica Bur1ington

SDG No.: MORRILL (200-1136)

Instrument ID: G.i

GC Column: $\mathrm{RTX}-624$ ID: $0.32(\mathrm{~mm})$

Calibration End Date: 08/09/2010 20:50
Heated Purge: ( $\mathrm{Y} / \mathrm{N}) \mathrm{N}$

Calibration ID: 1811

\begin{tabular}{|c|c|c|c|c|c|c|c|c|c|c|c|c|c|c|c|c|c|}
\hline \multirow[t]{2}{*}{ ANALYTE } & \multicolumn{5}{|c|}{ RRF } & \multirow{2}{*}{$\begin{array}{l}\text { CURVE } \\
\text { TYPE }\end{array}$} & \multicolumn{3}{|c|}{ COEFFICIENT } & \multirow[t]{2}{*}{$\#$} & \multirow[t]{2}{*}{ MIN RRE } & \multirow[t]{2}{*}{$\because \mathrm{RSD}$} & \multirow[t]{2}{*}{ \# } & \multirow{2}{*}{$\begin{array}{l}\text { MAX } \\
\text { \&RSD }\end{array}$} & \multirow{2}{*}{$\begin{array}{c}R^{\wedge} 2 \\
O R \quad C O D\end{array}$} & \multirow{2}{*}{$\#$} & \multirow{2}{*}{$\begin{array}{l}\text { MIN } R^{\wedge} 2 \\
\text { OR } \operatorname{COD}\end{array}$} \\
\hline & $\begin{array}{ll}\text { LVL } & 1 \\
\text { LVL } & 6 \\
\end{array}$ & $\begin{array}{l}\text { LVL } 2 \\
\text { LVL } 7 \\
\end{array}$ & LVL 3 & LVL 4 & LVL 5 & & B & M1 & M2 & & & & & & & & \\
\hline $1,1,2$-Trichloroethane & $\begin{array}{l}0.2367 \\
0.2308 \\
\end{array}$ & \begin{tabular}{l|}
0.2372 \\
0.2103 \\
\end{tabular} & 0.2431 & 0.2345 & 0.2132 & Ave & & 0.2294 & & & & 5.5 & & 30.0 & & & \\
\hline Tetrachloroethene & $\begin{array}{l}0.4933 \\
0.4842 \\
\end{array}$ & $\begin{array}{l}0.5060 \\
0.4704 \\
\end{array}$ & 0.5144 & 0.4521 & 0.4637 & Ave & & 0.4834 & & & & 4.7 & & 30.0 & & & \\
\hline Methyl Butyl Ketone (2-Hexanone) & 0.2401 & $\begin{array}{l}0.2564 \\
0.2286 \\
\end{array}$ & 0.2620 & 0.2552 & 0.2362 & Ave & & 0.2464 & & & & 5.4 & & 30.0 & & & \\
\hline Dibromochloromethane & $\begin{array}{l}0.5921 \\
0.6035 \\
\end{array}$ & $\begin{array}{l}0.5705 \\
0.5753 \\
\end{array}$ & 0.6146 & 0.5827 & 0.5549 & Aेve & & 0.5848 & & & & 3.5 & & 30.0 & & & \\
\hline 1,2-Dibromoethane & $\begin{array}{l}0.4985 \\
0.4782 \\
\end{array}$ & $\begin{array}{l}0.4788 \\
0.4525 \\
\end{array}$ & 0.4994 & 0.4625 & 0.4147 & Ave & & 0.4692 & & & & 6.3 & & 30.0 & & & \\
\hline Chlorobenzene & $\begin{array}{l}0.6333 \\
0.6218 \\
\end{array}$ & $\begin{array}{l}0.6638 \\
0.5922 \\
\end{array}$ & 0.6469 & 0.6363 & 0.5833 & Ave & & 0.6254 & & & & 4.6 & & 30.0 & & & \\
\hline Ethylbenzene & $\begin{array}{l}0.8389 \\
0.8605 \\
\end{array}$ & $\begin{array}{l}0.9235 \\
0.7362 \\
\end{array}$ & 0.9136 & 0.8615 & 0.7537 & Ave & & 0.8411 & & & & 8.6 & & 30.0 & & & \\
\hline n-Nonane & $\begin{array}{l}0.3672 \\
0.3060 \\
\end{array}$ & $\begin{array}{l}0.3685 \\
0.2393 \\
\end{array}$ & 0.3629 & 0.3291 & 0.2793 & Ave & & 0.3218 & & & & 15.5 & & 30.0 & & & \\
\hline $\mathrm{m}, \mathrm{p}-\mathrm{Xy}$ lene & $\begin{array}{l}0.3503 \\
0.3808 \\
\end{array}$ & $\begin{array}{l}0.4189 \\
0.3145 \\
\end{array}$ & 0.3838 & 0.3887 & 0.3206 & Ave & & 0.3654 & & & & 10.5 & & 30.0 & & & \\
\hline Xylene, o- & $\begin{array}{l}0.3617 \\
0.3943 \\
\end{array}$ & $\begin{array}{l}0.4411 \\
0.3402\end{array}$ & 0.3742 & 0.3620 & 0.3637 & Ave & & 0.3768 & & & & 8.7 & & 30.0 & & & \\
\hline Styrene & $\begin{array}{l}0.4856 \\
0.6156\end{array}$ & $\begin{array}{l}0.6029 \\
0.5343\end{array}$ & 0.5704 & 0.6176 & 0.5657 & Ave & & 0.5703 & & & & 8.4 & & 30.0 & & & \\
\hline Bromoform & $\begin{array}{l}0.5285 \\
0.7086 \\
\end{array}$ & $\begin{array}{l}0.5718 \\
0.6190 \\
\end{array}$ & 0.6209 & 0.6527 & 0.6213 & Ave & & 0.6175 & & & & 9.2 & & 30.0 & & & \\
\hline Cumene & $\begin{array}{l}0.9642 \\
1.0822\end{array}$ & $\begin{array}{l}1.0738 \\
0.9397 \\
\end{array}$ & 0.9993 & 1.0077 & 0.8958 & Ave & & 0.9947 & & & & 6.8 & & 30.0 & & & \\
\hline $1,1,2,2$-Tetrachloroethane & $\begin{array}{l}0.5561 \\
0.6050\end{array}$ & $\begin{array}{l}0.6098 \\
0.4946 \\
\end{array}$ & 0.5811 & 0.6221 & 0.5181 & Ave & & 0.5696 & & & & 8.5 & & 30.0 & & & \\
\hline n-Propylbenzene & \begin{tabular}{|l|}
1.0384 \\
1.2391 \\
\end{tabular} & $\begin{array}{l}1.2656 \\
0.9706 \\
\end{array}$ & 1.1588 & 1.1946 & 1.1144 & Ave & & 1.1402 & & & & 9.4 & & 30.0 & & & \\
\hline 1,2,3-Trichloropropane & 0.3986 & $\begin{array}{l}0.4419 \\
0.3145 \\
\end{array}$ & 0.3908 & 0.3892 & 0.3593 & Ave & & 0.3824 & & & & 11.1 & & 30.0 & & & \\
\hline n-Decane & 0.4154 & $\begin{array}{l}0.3955 \\
0.3174 \\
\end{array}$ & 0.4136 & 0.4124 & 0.3770 & Ave & & 0.3885 & & & & 9.7 & & 30.0 & & & \\
\hline 4-Ethyl toluene & $\begin{array}{l}0.9495 \\
1.1327\end{array}$ & $\begin{array}{l}0.9876 \\
0.9368 \\
\end{array}$ & 0.9817 & 1.0374 & 0.9842 & Ave & & 1.0014 & & & & 6.6 & & 30.0 & & & \\
\hline 2-Chlorotoluene & $\begin{array}{l}0.9721 \\
0.9951 \\
\end{array}$ & $\begin{array}{l}1.0274 \\
0.8215 \\
\end{array}$ & 0.9217 & 0.9318 & 0.8730 & Ave & & 0.9347 & & & & 7.6 & & 30.0 & & & \\
\hline $1,3,5$-Trimethylbenzene & $\begin{array}{l}0.7744 \\
0.9322 \\
\end{array}$ & $\begin{array}{l}0.8763 \\
0.7796 \\
\end{array}$ & 0.8107 & 0.8966 & 0.8026 & Ave & & 0.8389 & & & & 7.4 & & 30.0 & & & \\
\hline
\end{tabular}

Note: The $m 1$ coefficient is the same as Ave RRF for an Ave curve type.

FORM VI TO-15

Page 74 of 97 
AIR - GC/MS VOA INITIAL CALIBRATION DATA INTERNAL STANDARD CURVE EVALUTION

Lab Name: TestAmerica Burlington

SDG No.: MORRILL $(200-1136)$

Instrument ID: G.i

Calibration Start Date: 08/09/2010 15:59
Job No.: $200-1136-1$

Analy Batch No.: 5008

GC Column: RTX-624

ID: $0.32(\mathrm{~mm})$

Heated Purge: ( $\mathrm{Y} / \mathrm{N}) \mathrm{N}$

Calibration End Date: 08/09/2010 20:50

\begin{tabular}{|c|c|c|c|c|c|c|c|c|c|c|c|c|c|c|c|c|c|}
\hline \multirow[t]{2}{*}{ ANALYTE } & \multicolumn{5}{|c|}{ RRF } & \multirow{2}{*}{$\begin{array}{l}\text { CURVE } \\
\text { TYPE }\end{array}$} & \multicolumn{3}{|c|}{ COEFFICIENT } & \multirow[t]{2}{*}{$\#$} & \multirow[t]{2}{*}{ MIN RRE } & \multirow[t]{2}{*}{ \&RSD } & \multirow[t]{2}{*}{$\#$} & \multirow{2}{*}{$\begin{array}{c}\text { MAX } \\
\text { \& RSD }\end{array}$} & \multirow{2}{*}{$\begin{array}{c}R^{\wedge} 2 \\
O R \quad C O D\end{array}$} & \multirow[t]{2}{*}{$\#$} & \multirow{2}{*}{$\begin{array}{ll}\text { MIN } & R^{\wedge} 2 \\
\text { OR } & C O D\end{array}$} \\
\hline & $\begin{array}{ll}\text { LVL } 1 \\
\text { LVL } 6 \\
\end{array}$ & $\begin{array}{l}\text { LVL } 2 \\
\text { LVL } 7 \\
\end{array}$ & LVL 3 & LVL 4 & LVL 5 & & B & M1 & M2 & & & & & & & & \\
\hline tert-Butylbenzene & $\begin{array}{l}0.8179 \\
0.9432 \\
\end{array}$ & $\begin{array}{l}0.8392 \\
0.7772 \\
\end{array}$ & 0.7885 & 0.8347 & 0.7723 & Ave & & 0.8247 & & & & 7.1 & & 30.0 & & & \\
\hline $1,2,4$-Trimethylbenzene & $\begin{array}{l}0.7356 \\
0.9439 \\
\end{array}$ & $\begin{array}{l}0.9209 \\
0.7899 \\
\end{array}$ & 0.8199 & 0.8736 & 0.8202 & Ave & & 0.8434 & & & & 8.7 & & 30.0 & & & \\
\hline sec-Butylbenzene & $\begin{array}{l}.1585 \\
1.3174 \\
\end{array}$ & $\begin{array}{l}1.3021 \\
1.1014 \\
\end{array}$ & 1.2909 & 1.2283 & 1.1638 & Ave & & 1.2232 & & & & 6.9 & & 30.0 & & & \\
\hline 4-Isopropyltoluene & $\begin{array}{l}0.8407 \\
1.1534\end{array}$ & $\begin{array}{l}0.9538 \\
0.9699 \\
\end{array}$ & 1.0426 & 1.0392 & 1.0078 & Ave & & 1.0011 & & & & 9.6 & & 30.0 & & & \\
\hline 1,3-Dichlorobenzene & $\begin{array}{l}0.6680 \\
0.7898\end{array}$ & $\begin{array}{l}0.7240 \\
0.6945 \\
\end{array}$ & 0.7082 & 0.6909 & 0.7488 & Ave & & 0.7177 & & & & 5.7 & & 30.0 & & & \\
\hline 1,4-Dichlorobenzene & $\begin{array}{l}0.6406 \\
0.7961 \\
\end{array}$ & $\begin{array}{l}0.6933 \\
0.6935 \\
\end{array}$ & 0.6938 & 0.6817 & 0.7155 & Ave & & 0.7021 & & & & 6.7 & & 30.0 & & & \\
\hline Benzyl chloride & $\begin{array}{l}0.5310 \\
0.7781 \\
\end{array}$ & $\begin{array}{l}0.6036 \\
0.6961 \\
\end{array}$ & 0.6778 & 0.6097 & 0.6631 & Ave & & 0.6513 & & & & 12.1 & & 30.0 & & & \\
\hline n-Undecane & 0.4038 & 0.3169 & 0.3305 & 0.3436 & 0.3068 & Ave & & 0.3403 & & & & 11.2 & & 30.0 & & & \\
\hline n-Butylbenzene & $\begin{array}{l}0.6454 \\
0.9561 \\
\end{array}$ & $\begin{array}{l}0.7391 \\
0.7881 \\
\end{array}$ & 0.8627 & 0.9411 & 0.8302 & Ave & & 0.8232 & & & & 13.4 & & 30.0 & & & \\
\hline 1,2-Dichlorobenzene & $\begin{array}{l}0.5810 \\
0.7958 \\
\end{array}$ & $\begin{array}{l}0.6668 \\
0.6647 \\
\end{array}$ & 0.6519 & 0.7180 & 0.7086 & Ave & & 0.6838 & & & & 9.7 & & 30.0 & & & \\
\hline n-Dodecane & 0.2422 & 0.1192 & 0.2276 & 0.1918 & 0.2432 & Ave & & 0.2048 & & & & 25.5 & & 30.0 & & & \\
\hline $1,2,4$-Trichlorobenzene & 0.6131 & $\begin{array}{l}0.2983 \\
0.4196 \\
\end{array}$ & 0.3151 & 0.4474 & 0.4752 & Ave & & 0.4281 & & & & 26.9 & & 30.0 & & & \\
\hline Hexachlorobutadiene & $\begin{array}{l}0.3446 \\
0.6473 \\
\end{array}$ & $\begin{array}{l}0.4494 \\
0.3708 \\
\end{array}$ & 0.4512 & 0.5220 & 0.5218 & Ave & & 0.4724 & & & & 21.7 & & 30.0 & & & \\
\hline Naphthalene & 1.0232 & $\begin{array}{l}0.5077 \\
0.7285 \\
\end{array}$ & 0.5878 & 0.6459 & 0.8411 & Ave & & 0.7224 & & & & 25.9 & & 30.0 & & & \\
\hline $1,2,3$-Trichlorobenzene & $\begin{array}{l}0.3359 \\
0.5049 \\
\end{array}$ & $\begin{array}{l}0.2512 \\
0.2628 \\
\end{array}$ & 0.2621 & 0.2955 & 0.3993 & Ave & & 0.3303 & & & & 28.2 & & 30.0 & & & \\
\hline
\end{tabular}

Note: The $m 1$ coefficient is the same as Ave RRF for an Ave curve type.

FORM VI TO-15

Page 75 of 97 
FORM VI

AIR - GC/MS VOA INITIAL CALIBRATION DATA

INTERNAL STANDARD RESPONSE AND CONCENTRATION

Lab Name: TestAmerica Burlington

Job No.: $200-1136-1$

Analy Batch No.: 5008

SDG No.: MORRILL $(200-1136)$

Instrument ID: G.i

GC Column: RTX-624 ID: $0.32(\mathrm{~mm})$

Heated Purge: ( $\mathrm{Y} / \mathrm{N}) \mathrm{N}$

Calibration Start Date: 08/09/2010 15:59

Calibration End Date: 08/09/2010 20:50

Calibration ID: 1811

Calibration Files:

\begin{tabular}{lll|}
\hline LEVEL: & LAB SAMPLE ID: & LAB FILE ID: \\
Leve1 1 & IC 200-5008/3 & geu003.d \\
Level 2 & IC $200-5008 / 4$ & geu004.d \\
Leve1 3 & IC 200-5008/5 & geu005.d \\
Leve1 4 & ICIS $200-5008 / 6$ & geu006.d \\
Leve1 5 & IC $200-5008 / 7$ & geu007.d \\
Level 6 & IC $200-5008 / 8$ & geu0008.d \\
Level 7 & IC $200-5008 / 9$ & geu009.d \\
\hline
\end{tabular}

\begin{tabular}{|c|c|c|c|c|c|c|c|c|c|c|c|c|}
\hline \multirow[t]{2}{*}{ ANALYTE } & \multirow{2}{*}{$\begin{array}{l}\text { IS } \\
\text { REF }\end{array}$} & \multirow{2}{*}{$\begin{array}{l}\text { CURVE } \\
\text { TYPE }\end{array}$} & \multicolumn{5}{|c|}{ RESPONSE } & \multicolumn{5}{|c|}{ CONCENTRATION (PPB V/V) } \\
\hline & & & $\begin{array}{ll}\text { LVL } 1 \\
\text { LVL } & 6 \\
\end{array}$ & $\begin{array}{l}\text { LVL } 2 \\
\text { LVL } 7 \\
\end{array}$ & LVL 3 & LVL 4 & LVL 5 & LVL 6 & $\begin{array}{l}\text { LVL } 2 \\
\text { LVL } 7 \\
\end{array}$ & LVL 3 & LVL 4 & LVL 5 \\
\hline Propylene & $\mathrm{BCM}$ & Ave & 288236 & \begin{tabular}{r|}
6758 \\
515470 \\
\end{tabular} & 75946 & 121758 & 208877 & 20.0 & $\begin{array}{r}0.500 \\
40.0\end{array}$ & 5.00 & 10.0 & 15.0 \\
\hline Dichlorodifluoromethane & $\mathrm{BCM}$ & Ave & 2174775 & $\begin{array}{r}52179 \\
3848252\end{array}$ & 579101 & 945562 & 1616800 & 20.0 & $\begin{array}{r}0.500 \\
40.0\end{array}$ & 5.00 & 10.0 & 15.0 \\
\hline Freon 22 & $\mathrm{BCM}$ & Ave & 840854 & $\begin{array}{r}21159 \\
1500293\end{array}$ & 225949 & 367677 & 612545 & 20.0 & $\begin{array}{r}0.500 \\
40.0\end{array}$ & 5.00 & 10.0 & 15.0 \\
\hline 1,2-Dichlorotetrafluoroethane & BCM & Ave & $\begin{array}{r}20700 \\
2059073\end{array}$ & $\begin{array}{r}51816 \\
3527711\end{array}$ & 565699 & 904097 & 1502370 & $\begin{array}{r}0.200 \\
20.0\end{array}$ & $\begin{array}{r}0.500 \\
40.0\end{array}$ & 5.00 & 10.0 & 15.0 \\
\hline Chloromethane & $\mathrm{BCM}$ & Ave & 474232 & $\begin{array}{r}11749 \\
847404\end{array}$ & 125055 & 203672 & 338459 & 20.0 & $\begin{array}{r}40.0 \\
0.500 \\
40.0\end{array}$ & 5.00 & 10.0 & 15.0 \\
\hline n-Butane & BCM & Ave & 733646 & $\begin{array}{r}17921 \\
1296620\end{array}$ & 195228 & 304852 & 524673 & 20.0 & $\begin{array}{r}4.500 \\
40.0\end{array}$ & 5.00 & 10.0 & 15.0 \\
\hline Vinyl chloride & BCM & Ave & $\begin{array}{r}6818 \\
714769\end{array}$ & $\begin{array}{r}16376 \\
1255744\end{array}$ & 190261 & 294357 & 506636 & $\begin{array}{r}0.200 \\
20.0\end{array}$ & $\begin{array}{r}0.500 \\
40.0\end{array}$ & 5.00 & 10.0 & 15.0 \\
\hline 1,3-Butadiene & $\overline{B C M}$ & Ave & $\begin{array}{r}4932 \\
490294\end{array}$ & $\begin{array}{r}11315 \\
871242\end{array}$ & 131153 & 206861 & 349262 & $\begin{array}{r}0.200 \\
20.0\end{array}$ & $\begin{array}{r}4.500 \\
40.0\end{array}$ & 5.00 & 10.0 & 15.0 \\
\hline Bromomethane & $\mathrm{BCM}$ & Ave & $\begin{array}{r}13430 \\
1349245\end{array}$ & $\begin{array}{r}30362 \\
2665352\end{array}$ & 335056 & 544521 & 1012598 & $\begin{array}{r}0.200 \\
20.0\end{array}$ & $\begin{array}{r}0.500 \\
40.0\end{array}$ & 5.00 & 10.0 & 15.0 \\
\hline Chloroethane & BCM & Ave & 551748 & $\begin{array}{r}12080 \\
993710\end{array}$ & 110779 & 214449 & 373673 & 20.0 & $\begin{array}{r}0.500 \\
40.0\end{array}$ & 5.00 & 10.0 & 15.0 \\
\hline Isopentane & $\mathrm{BCM}$ & Ave & $\begin{array}{r}8102 \\
974091\end{array}$ & $\begin{array}{r}22655 \\
1756464\end{array}$ & 213965 & 380771 & 703229 & $\begin{array}{r}0.200 \\
20.0\end{array}$ & $\begin{array}{r}0.500 \\
40.0\end{array}$ & 5.00 & 10.0 & 15.0 \\
\hline Bromoethene (Vinyl Bromide) & BCM & Ave & $\begin{array}{r}13323 \\
1600934\end{array}$ & $\begin{array}{r}31630 \\
2882821\end{array}$ & 362439 & 590835 & 1096803 & $\begin{array}{r}0.200 \\
20.0\end{array}$ & $\begin{array}{r}0.500 \\
40.0\end{array}$ & 5.00 & 10.0 & 15.0 \\
\hline Trichlorofluoromethane & $\mathrm{BCM}$ & Ave & $\begin{array}{r}34201 \\
3746034 \\
\end{array}$ & $\begin{array}{r}89095 \\
7169411 \\
\end{array}$ & 878932 & 1473617 & 2647789 & $\begin{array}{r}0.200 \\
20.0 \\
\end{array}$ & $\begin{array}{r}0.500 \\
40.0 \\
\end{array}$ & 5.00 & 10.0 & 15.0 \\
\hline n-Pentane & $\mathrm{BCM}$ & Ave & 1581367 & $\begin{array}{r}40515 \\
2908349 \\
\end{array}$ & 396216 & 711959 & 1131503 & 20.0 & $\begin{array}{r}0.500 \\
40.0\end{array}$ & 5.00 & 10.0 & 15.0 \\
\hline Ethanol & $\mathrm{BCM}$ & Ave & 712936 & $\begin{array}{r}84999 \\
1668155\end{array}$ & 168125 & 248116 & 317635 & 40.0 & $\begin{array}{r}5.00 \\
100\end{array}$ & 10.0 & 15.0 & 20.0 \\
\hline
\end{tabular}


AIR - GC/MS VOA INITIAL CALIBRATION DATA

INTERNAL STANDARD RESPONSE AND CONCENTRATION

Lab Name: TestAmerica Burlington

Job No.: $200-1136-1$

SDG No.: MORRILL (200-1136)

Instrument ID: G.i

GC Column: RTX-624

ID $: 0.32(\mathrm{~mm})$

Calibration Start Date: 08/09/2010 15:59

Calibration End Date: 08/09/2010 20:50
Analy Batch No.: 5008

Heated Purge: ( $Y / N) \mathrm{N}$

Calibration ID: 1811

\begin{tabular}{|c|c|c|c|c|c|c|c|c|c|c|c|c|}
\hline \multirow[t]{2}{*}{ ANALYTE } & \multirow{2}{*}{$\begin{array}{c}\text { IS } \\
\text { REF }\end{array}$} & \multirow{2}{*}{$\begin{array}{l}\text { CURVE } \\
\text { TYPE }\end{array}$} & \multicolumn{5}{|c|}{ RESPONSE } & \multicolumn{5}{|c|}{ CONCENTRATION $($ RPB $\mathrm{V} / \mathrm{V})$} \\
\hline & & & $\begin{array}{ll}\text { LVL } 1 \\
\text { LVL } 6 \\
\end{array}$ & $\begin{array}{l}\text { LVL } 2 \\
\text { LVL } 7 \\
\end{array}$ & LVL 3 & LVL 4 & LVL 5 & $\begin{array}{l}\text { LVL } 1 \\
\text { LVL } 6 \\
\end{array}$ & $\begin{array}{l}\text { LVL } 2 \\
\text { LVL } 7 \\
\end{array}$ & LVL 3 & LVL 4 & LVL 5 \\
\hline Ethyl ether & $\overline{B C M}$ & Âve & $\begin{array}{r}5946 \\
681312 \\
\end{array}$ & \begin{tabular}{r|}
16072 \\
1272837
\end{tabular} & 155086 & 322384 & 444272 & $\begin{array}{r}0.200 \\
20.0\end{array}$ & $\begin{array}{r}0.500 \\
40.0\end{array}$ & 5.00 & 10.0 & 15.0 \\
\hline Acrolein & BCM & Ave & 353059 & 634190 & 84612 & 170358 & 238665 & 20.0 & 40.0 & 5.00 & 10.0 & 15.0 \\
\hline Freon $\mathrm{TF}$ & $\mathrm{BCM}$ & Ave & $\begin{array}{r}24627 \\
2675572 \\
\end{array}$ & $\begin{array}{r}65286 \\
5224132 \\
\end{array}$ & 656886 & 1236921 & 1907411 & $\begin{array}{r}0.200 \\
20.0\end{array}$ & $\begin{array}{r}0.500 \\
40.0\end{array}$ & 5.00 & 10.0 & 15.0 \\
\hline 1,1-Dichloroethene & $\mathrm{BCM}$ & Ave & $\begin{array}{r}13232 \\
1257220 \\
\end{array}$ & $\begin{array}{r}29934 \\
2473287 \\
\end{array}$ & 302440 & 569427 & 881594 & $\begin{array}{r}0.200 \\
20.0 \\
\end{array}$ & $\begin{array}{r}0.500 \\
40.0 \\
\end{array}$ & 5.00 & 10.0 & 15.0 \\
\hline Acetone & $\mathrm{BCM}$ & Ave & 1228704 & 2341058 & 304809 & 655209 & 811374 & 20.0 & 40.0 & 5.00 & 10.0 & 15.0 \\
\hline Isopropyl alcohol & $\mathrm{BCM}$ & Ave & 982556 & 1842509 & 239651 & 462461 & 668187 & 20.0 & 40.0 & 5.00 & 10.0 & 15.0 \\
\hline Carbon disulfide & $\mathrm{BCM}$ & Ave & 3872631 & $\begin{array}{r}87901 \\
7347699 \\
\end{array}$ & $9 \overline{34978}$ & 1764224 & 2682408 & 20.0 & $\begin{array}{r}.500 \\
40.0\end{array}$ & $5 . \overline{00}$ & 10.0 & 15.0 \\
\hline 3-Chloropropene & $\mathrm{BCM}$ & Ave & $\begin{array}{r}10366 \\
1123585 \\
\end{array}$ & $\begin{array}{r}26317 \\
2054346 \\
\end{array}$ & 279453 & 483217 & 794998 & $\begin{array}{r}0.200 \\
20.0\end{array}$ & $\begin{array}{r}0.500 \\
40.0 \\
\end{array}$ & 5.00 & 10.0 & 15.0 \\
\hline Acetonitrile & $\mathrm{BCM}$ & Ave & 613597 & 1168721 & 141165 & 271715 & 398754 & 20.0 & 40.0 & 5.00 & 10.0 & 15.0 \\
\hline Methylene Chloride & $\mathrm{BCM}$ & Ave & 1131976 & $\begin{array}{r}33535 \\
2069016 \\
\end{array}$ & 287700 & 485002 & 780849 & 20.0 & $\begin{array}{r}0.500 \\
40.0 \\
\end{array}$ & 5.00 & 10.0 & 15.0 \\
\hline tert-Butyl alcohol & $\mathrm{BCM}$ & Ave & 1550079 & 2927095 & 372598 & 691832 & 1025082 & 20.0 & 40.0 & 5.00 & 10.0 & 15.0 \\
\hline Methyl tert-butyl ether & $\mathrm{BCM}$ & Ave & $\begin{array}{r}27393 \\
3052532 \\
\end{array}$ & $\begin{array}{r}63341 \\
5544522 \\
\end{array}$ & 654181 & 1278088 & 1809679 & $\begin{array}{r}0.200 \\
20.0 \\
\end{array}$ & $\begin{array}{r}0.500 \\
40.0\end{array}$ & 5.00 & 10.0 & 15.0 \\
\hline trans-1,2-Dichloroethene & $\mathrm{BCM}$ & Ave & $\begin{array}{r}17079 \\
1707879 \\
\end{array}$ & $\begin{array}{r}39104 \\
3027905 \\
\end{array}$ & 420359 & 702894 & 1136767 & $\begin{array}{r}0.200 \\
20.0\end{array}$ & $\begin{array}{r}0.500 \\
40.0\end{array}$ & 5.00 & 10.0 & 15.0 \\
\hline n-Hexane & $\mathrm{BCM}$ & Ave & $\begin{array}{r}15885 \\
1563223 \\
\end{array}$ & $\begin{array}{r}36342 \\
2893553 \\
\end{array}$ & 390309 & 686519 & 1038583 & $\begin{array}{r}0.200 \\
20.0\end{array}$ & $\begin{array}{r}0.500 \\
40.0\end{array}$ & 5.00 & 10.0 & 15.0 \\
\hline 1,1-Dichloroethane & $\mathrm{BCM}$ & Äve & $\begin{array}{r}21826 \\
2095369 \\
\end{array}$ & $\begin{array}{r}49102 \\
3844560 \\
\end{array}$ & 531430 & 883295 & 1374223 & $\begin{array}{r}0.200 \\
20.0\end{array}$ & $\begin{array}{r}0.500 \\
40.0\end{array}$ & 5.00 & 10.0 & 15.0 \\
\hline Vinyl acetate & $\mathrm{BCM}$ & Ave & 2166345 & 3854464 & 502323 & 921003 & 1306973 & 20.0 & 40.0 & 5.00 & 10.0 & 15.0 \\
\hline cis-1,2-Dichloroethene & $\mathrm{BCM}$ & Ave & $\begin{array}{r}14137 \\
1449873\end{array}$ & $\begin{array}{r}33229 \\
2682587 \\
\end{array}$ & 364864 & 616747 & 938786 & $\begin{array}{r}0.200 \\
20.0\end{array}$ & $\begin{array}{r}0.500 \\
40.0\end{array}$ & 5.00 & 10.0 & 15.0 \\
\hline Methyl Ethyl Ketone & $\mathrm{BCM}$ & Ave & 491953 & $\begin{array}{r}11786 \\
881322 \\
\end{array}$ & 112766 & 211607 & 291995 & 20.0 & $\begin{array}{r}0.500 \\
40.0\end{array}$ & 5.00 & 10.0 & 15.0 \\
\hline Ethyl acetate & $\mathrm{BCM}$ & Ave & 85493 & 153120 & 18413 & 34732 & 49008 & 20.0 & 40.0 & 5.00 & 10.0 & 15.0 \\
\hline Tetrahydrofuran & DFB & Ave & 845473 & 1590340 & 197464 & 371403 & 509234 & 20.0 & 40.0 & 5.00 & 10.0 & 15.0 \\
\hline Chloroform & $\mathrm{BCM}$ & Ave & $\begin{array}{r}27912 \\
2732436 \\
\end{array}$ & $\begin{array}{r}65664 \\
5370961 \\
\end{array}$ & 704432 & 1244628 & 1828210 & $\begin{array}{r}0.200 \\
20.0 \\
\end{array}$ & $\begin{array}{r}0.500 \\
40.0\end{array}$ & 5.00 & 10.0 & 15.0 \\
\hline
\end{tabular}


FORM VI

AIR - GC/MS VOA INITIAL CALIBRATION DATA

INTERNAL STANDARD RESPONSE AND CONCENTRATION

Lab Name: TestAmerica Burlington

SDG NO.: MORRILL $(200-1136)$

Instrument ID: G.i

Calibration Start Date: 08/09/2010 15:59
Job No.: $200-1136-1$

GC Column: RTX-624 ID: $0.32(\mathrm{~mm})$

Calibration End Date: 08/09/2010 20:50
Analy Batch No.: 5008

Heated Purge: ( $\mathrm{Y} / \mathrm{N}) \mathrm{N}$

Calibration ID: 1811

\begin{tabular}{|c|c|c|c|c|c|c|c|c|c|c|c|c|}
\hline \multirow[t]{2}{*}{ ANALYTE } & \multirow{2}{*}{$\begin{array}{l}\text { IS } \\
\text { REF }\end{array}$} & \multirow{2}{*}{$\begin{array}{l}\text { CURVE } \\
\text { TYPE }\end{array}$} & \multicolumn{5}{|c|}{ RESPONSE } & \multicolumn{5}{|c|}{ CONCENTRATION (PPB V/V) } \\
\hline & & & $\begin{array}{ll}\text { LVL } & 1 \\
\text { LVL } & 6 \\
\end{array}$ & $\begin{array}{l}\text { LVL } 2 \\
\text { LVL } 7 \\
\end{array}$ & LVL 3 & LVL 4 & LVL 5 & $\begin{array}{ll}\text { LVL } & 1 \\
\text { LVL } & 6 \\
\end{array}$ & $\begin{array}{l}\text { LVL } 2 \\
\text { LVL } 7 \\
\end{array}$ & LVL 3 & LVL 4 & LVL 5 \\
\hline Cyclohexane & DFB & Ave & $\begin{array}{r}15493 \\
1553081 \\
\end{array}$ & $\begin{array}{r}36900 \\
2984081\end{array}$ & 414357 & 677931 & 1057979 & $\begin{array}{r}0.200 \\
20.0\end{array}$ & $\begin{array}{r}0.500 \\
40.0\end{array}$ & 5.00 & 10.0 & 15.0 \\
\hline $1,1,1$-Trichloroethane & DFB & Ave & $\begin{array}{r}28412 \\
2962798\end{array}$ & $\begin{array}{r}69249 \\
5869275\end{array}$ & 757870 & 1306385 & 2030421 & $\begin{array}{r}0.200 \\
20.0\end{array}$ & $\begin{array}{r}0.500 \\
40.0\end{array}$ & 5.00 & 10.0 & 15.0 \\
\hline Carbon tetrachloride & DFB & Ave & $\begin{array}{r}31344 \\
3358914\end{array}$ & $\begin{array}{r}81052 \\
6828674\end{array}$ & 847368 & 1625421 & 2344844 & $\begin{array}{r}0.200 \\
20.0\end{array}$ & $\begin{array}{r}0.500 \\
40.0\end{array}$ & 5.00 & 10.0 & 15.0 \\
\hline $2,2,4-T r i m e t h y l p e n t a n e$ & DFB & Ave & $\begin{array}{r}49230 \\
4537061 \\
\end{array}$ & $\begin{array}{r}115482 \\
8620007 \\
\end{array}$ & 1244257 & 2201579 & 3038011 & $\begin{array}{r}0.200 \\
20.0 \\
\end{array}$ & $\begin{array}{r}0.500 \\
40.0 \\
\end{array}$ & 5.00 & 10.0 & 15.0 \\
\hline Benzene & DFB & Ave & $\begin{array}{r}36881 \\
3498673 \\
\end{array}$ & $\begin{array}{r}86498 \\
6943202 \\
\end{array}$ & 919191 & 1587792 & 2332614 & $\begin{array}{r}0.200 \\
20.0 \\
\end{array}$ & $\begin{array}{r}0.500 \\
40.0 \\
\end{array}$ & 5.00 & 10.0 & 15.0 \\
\hline 1,2-Dichloroethane & DFB & Ave & $\begin{array}{r}14690 \\
1489785\end{array}$ & $\begin{array}{r}35831 \\
2942707 \\
\end{array}$ & 361941 & 683875 & 1023418 & $\begin{array}{r}0.200 \\
20.0\end{array}$ & $\begin{array}{r}0.500 \\
40.0\end{array}$ & 5.00 & 10.0 & 15.0 \\
\hline n-Heptane & DFB & Ave & $\begin{array}{r}20100 \\
1441312\end{array}$ & $\begin{array}{r}38837 \\
2695786 \\
\end{array}$ & 390034 & 574688 & 1013354 & $\begin{array}{r}0.200 \\
20.0\end{array}$ & $\begin{array}{r}0.500 \\
40.0\end{array}$ & 5.00 & 10.0 & $\overline{15.0}$ \\
\hline Trichloroethene & DFB & Ave & $\begin{array}{r}19962 \\
1823598\end{array}$ & $\begin{array}{r}45849 \\
3569291 \\
\end{array}$ & 496704 & 821238 & 1233457 & $\begin{array}{r}0.200 \\
20.0\end{array}$ & $\begin{array}{r}0.500 \\
40.0\end{array}$ & 5.00 & 10.0 & 15.0 \\
\hline 1,2-Dichloropropane & DFB & Ave & $\begin{array}{r}12719 \\
1153338 \\
\end{array}$ & $\begin{array}{r}31521 \\
2225305 \\
\end{array}$ & 299355 & 526722 & 805840 & $\begin{array}{r}0.200 \\
20.0 \\
\end{array}$ & $\begin{array}{r}0.500 \\
40.0 \\
\end{array}$ & 5.00 & 10.0 & 15.0 \\
\hline Methyl methacrylate & DFB & Ave & 1051364 & $\begin{array}{r}23423 \\
2094834 \\
\end{array}$ & 239201 & 459854 & 705572 & 20.0 & $\begin{array}{r}.500 \\
40.0 \\
\end{array}$ & 5.00 & 10.0 & 15.0 \\
\hline 1,4-Dioxane & DFB & Ave & 453188 & 896519 & 116322 & 204725 & 336180 & 20.0 & 40.0 & 5.00 & 10.0 & 15.0 \\
\hline Dibromomethane & DFB & Ave & $\begin{array}{r}22557 \\
2073633 \\
\end{array}$ & $\begin{array}{r}51408 \\
4283754 \\
\end{array}$ & 526320 & 887734 & 1557371 & $\begin{array}{r}0.200 \\
20.0 \\
\end{array}$ & $\begin{array}{r}0.500 \\
40.0 \\
\end{array}$ & 5.00 & 10.0 & 15.0 \\
\hline Bromodichloromethane & DFB & Ave & $\begin{array}{r}29260 \\
2893397 \\
\end{array}$ & $\begin{array}{r}70946 \\
5715878 \\
\end{array}$ & 736405 & 1399004 & 2062637 & $\begin{array}{r}0.200 \\
20.0\end{array}$ & $\begin{array}{r}0.500 \\
40.0 \\
\end{array}$ & 5.00 & 10.0 & 15.0 \\
\hline cis-1,3-Dichloropropene & DFB & Ave & $\begin{array}{r}18011 \\
1969386 \\
\end{array}$ & $\begin{array}{r}46769 \\
3896346 \\
\end{array}$ & 459350 & 896188 & 1372032 & $\begin{array}{r}0.200 \\
20.0 \\
\end{array}$ & $\begin{array}{r}0.500 \\
40.0 \\
\end{array}$ & 5.00 & 10.0 & 15.0 \\
\hline Methyl isobutyl ketone & DFB & Ave & 1561713 & $\begin{array}{r}34427 \\
3058407 \\
\end{array}$ & 350983 & 709252 & 1084991 & 20.0 & $\begin{array}{r}0.500 \\
40.0 \\
\end{array}$ & 5.00 & 10.0 & 15.0 \\
\hline n-octane & DFB & Ave & $\begin{array}{r}21440 \\
1794064 \\
\end{array}$ & $\begin{array}{r}51367 \\
3122000 \\
\end{array}$ & 508010 & 889483 & 1266134 & $\begin{array}{r}0.200 \\
20.0 \\
\end{array}$ & $\begin{array}{r}0.500 \\
40.0\end{array}$ & 5.00 & 10.0 & 15.0 \\
\hline Toluene & CBZ & Ave & $\begin{array}{r}24762 \\
2531555 \\
\end{array}$ & $\begin{array}{r}66253 \\
5017401 \\
\end{array}$ & 619174 & 1161021 & 1639275 & $\begin{array}{r}0.200 \\
20.0\end{array}$ & $\begin{array}{r}0.500 \\
40.0 \\
\end{array}$ & 5.00 & 10.0 & 15.0 \\
\hline trans $-1,3$-Dichloropropene & DFB & Ave & $\begin{array}{r}19218 \\
2031538 \\
\end{array}$ & $\begin{array}{r}44798 \\
4065626 \\
\end{array}$ & 466998 & 953387 & 1447109 & $\begin{array}{r}0.200 \\
20.0 \\
\end{array}$ & $\begin{array}{r}0.500 \\
40.0\end{array}$ & 5.00 & 10.0 & 15.0 \\
\hline $1,1,2$-Trichloroethane & CBZ & Ave & $\begin{array}{r}13532 \\
1410103 \\
\end{array}$ & $\begin{array}{r}31933 \\
2670884 \\
\end{array}$ & 331906 & 666215 & 975078 & $\begin{array}{r}0.200 \\
20.0 \\
\end{array}$ & $\begin{array}{r}0.500 \\
40.0 \\
\end{array}$ & 5.00 & 10.0 & 15.0 \\
\hline Tetrachloroethene & CBZ & Ave & $\begin{array}{r}28204 \\
2958869 \\
\end{array}$ & $\begin{array}{r}68114 \\
5975217 \\
\end{array}$ & 702242 & 1284411 & 2120362 & $\begin{array}{r}0.200 \\
20.0\end{array}$ & $\begin{array}{r}0.500 \\
40.0 \\
\end{array}$ & 5.00 & 10.0 & 15.0 \\
\hline Methyl Butyl Retone (2-Hexanone) & CBZ & Ave & 1467381 & $\begin{array}{r}34513 \\
2904042 \\
\end{array}$ & 357662 & 724992 & 1079914 & 20.0 & $\begin{array}{r}0.500 \\
40.0\end{array}$ & 5.00 & 10.0 & 15.0 \\
\hline
\end{tabular}


FORM VI

AIR - GC/MS VOA INITIAL CALIBRATION DATA

INTERNAL STANDARD RESPONSE AND CONCENTRATION

Lab Name: TestAmerica Burlington

Job No. : 200-1136-1

Analy Batch No.: 5008

SDG NO.: MORRILL $(200-1136)$

Instrument ID: G.i

GC Column: RTX-624 ID: $0.32(\mathrm{~mm})$

Calibration Start Date: 08/09/2010 15:59

Calibration End Date: 08/09/2010 20:50

Heated Purge: ( $Y / N)$ N

Calibration ID: 1811

\begin{tabular}{|c|c|c|c|c|c|c|c|c|c|c|c|c|}
\hline \multirow[t]{2}{*}{ ANALYTE } & \multirow{2}{*}{$\begin{array}{c}\text { IS } \\
\text { REF }\end{array}$} & \multirow{2}{*}{$\begin{array}{c}\text { CURVE } \\
\text { TYPE }\end{array}$} & \multicolumn{5}{|c|}{ RESPONSE } & \multicolumn{5}{|c|}{ CONCENTRATION (PPB V/V) } \\
\hline & & & $\begin{array}{ll}\text { LVL } & 1 \\
\text { LVL } & 6 \\
\end{array}$ & $\begin{array}{l}\text { LVI } 2 \\
\text { LVI } 7 \\
\end{array}$ & IVL 3 & LVL 4 & LVL 5 & $\begin{array}{ll}\text { LVL } 1 \\
\text { LVL } 6 \\
\end{array}$ & $\begin{array}{ll}\text { LVL } & 2 \\
\text { LVL } & 7 \\
\end{array}$ & LVL 3 & LVL 4 & IVL 5 \\
\hline Dibromochloromethane & $\mathrm{CBZ}$ & Ave & $\begin{array}{r}33849 \\
3687892 \\
\end{array}$ & $\begin{array}{r}76792 \\
7308048 \\
\end{array}$ & 839097 & 1655429 & 2537494 & $\begin{array}{r}0.200 \\
20.0\end{array}$ & $\begin{array}{r}0.500 \\
40.0 \\
\end{array}$ & 5.00 & 10.0 & 15.0 \\
\hline 1,2-Dibromoethane & $\mathrm{CBZ}$ & Ave & $\begin{array}{r}28499 \\
2922131 \\
\end{array}$ & $\begin{array}{r}64454 \\
5748101 \\
\end{array}$ & 681795 & 1313978 & 1896387 & $\begin{array}{r}0.200 \\
20.0\end{array}$ & $\begin{array}{r}0.500 \\
40.0\end{array}$ & 5.00 & 10.0 & 15.0 \\
\hline Chlorobenzene & CBZ & Àve & $\begin{array}{r}36208 \\
3799764 \\
\end{array}$ & $\begin{array}{r}89355 \\
7522646 \\
\end{array}$ & 883145 & 1807807 & 2667052 & $\begin{array}{r}0.200 \\
20.0 \\
\end{array}$ & $\begin{array}{r}0.500 \\
40.0 \\
\end{array}$ & 5.00 & 10.0 & 15.0 \\
\hline Ethylbenzene & $\mathrm{CBZ}$ & Ave & $\begin{array}{r}47964 \\
5258382 \\
\end{array}$ & $\begin{array}{r}124308 \\
9351695 \\
\end{array}$ & 1247264 & 2447468 & 3446635 & $\begin{array}{r}0.200 \\
20.0 \\
\end{array}$ & $\begin{array}{r}0.500 \\
40.0 \\
\end{array}$ & 5.00 & 10.0 & 15.0 \\
\hline n-Nonane & CBZ & Ave & $\begin{array}{r}20991 \\
1869696 \\
\end{array}$ & $\begin{array}{r}49608 \\
3039801 \\
\end{array}$ & 495488 & 935033 & 1276996 & $\begin{array}{r}0.200 \\
20.0\end{array}$ & $\begin{array}{r}0.500 \\
40.0\end{array}$ & 5.00 & 10.0 & 15.0 \\
\hline $\mathrm{m}, \mathrm{p}$-Xylene & $\mathrm{CBZ}$ & Ave & $\begin{array}{r}40050 \\
4653794 \\
\end{array}$ & $\begin{array}{r}112790 \\
7990425 \\
\end{array}$ & 1047813 & 2208657 & 2932192 & $\begin{array}{r}0.400 \\
40.0 \\
\end{array}$ & $\begin{array}{l}1.00 \\
80.0\end{array}$ & 10.0 & 20.0 & 30.0 \\
\hline Xylene, o- & $\mathrm{CBZ}$ & Ave & $\begin{array}{r}20681 \\
2409776 \\
\end{array}$ & $\begin{array}{r}59374 \\
4322092 \\
\end{array}$ & 510788 & 1028469 & 1663074 & $\begin{array}{r}0.200 \\
20.0\end{array}$ & $\begin{array}{r}0.500 \\
40.0\end{array}$ & 5.00 & 10.0 & 15.0 \\
\hline Styrene & CBZ & Ave & $\begin{array}{r}27764 \\
3761800 \\
\end{array}$ & $\begin{array}{r}81153 \\
6787125 \\
\end{array}$ & 778659 & 1754558 & 2586716 & $\begin{array}{r}0.200 \\
20.0\end{array}$ & $\begin{array}{r}0.500 \\
40.0\end{array}$ & 5.00 & 10.0 & 15.0 \\
\hline Bromoform & $\mathrm{CBZ}$ & Ave & $\begin{array}{r}30215 \\
4330022 \\
\end{array}$ & $\begin{array}{r}76966 \\
7862466\end{array}$ & 847700 & 1854263 & $2 \overline{840945}$ & $\begin{array}{r}0.200 \\
20.0\end{array}$ & $\begin{array}{r}0.500 \\
40.0\end{array}$ & 5.00 & 10.0 & 15.0 \\
\hline Cumene & $\mathrm{CBZ}$ & Ave & $\begin{array}{r}55123 \\
6613116 \\
\end{array}$ & $\begin{array}{r}144549 \\
11936616 \\
\end{array}$ & 1364184 & 2862915 & 4096271 & $\begin{array}{r}0.200 \\
20.0\end{array}$ & $\begin{array}{r}0.500 \\
40.0\end{array}$ & 5.00 & 10.0 & 15.0 \\
\hline $1,1,2,2$-Tetrachloroethane & $\mathrm{CBZ}$ & Ave & $\begin{array}{r}31795 \\
3697318 \\
\end{array}$ & $\begin{array}{r}82087 \\
6283079 \\
\end{array}$ & 793343 & 1767402 & 2369263 & $\begin{array}{r}0.200 \\
20.0\end{array}$ & $\begin{array}{r}0.500 \\
40.0\end{array}$ & 5.00 & 10.0 & 15.0 \\
\hline n-Propylbenzene & $\overline{\mathrm{CBZ}}$ & Ave & $\begin{array}{r}59368 \\
7572189 \\
\end{array}$ & $\begin{array}{r}170366 \\
12329069 \\
\end{array}$ & 1582032 & 3393880 & 5095918 & $\begin{array}{r}0.200 \\
20.0\end{array}$ & $\begin{array}{r}0.500 \\
40.0\end{array}$ & 5.00 & 10.0 & 15.0 \\
\hline 1,2,3-Trichloropropane & $\overline{\mathrm{CBZ}}$ & Ave & 2436051 & $\begin{array}{r}59491 \\
3994650 \\
\end{array}$ & 533538 & 1105749 & 1642816 & 20.0 & $\begin{array}{r}0.500 \\
40.0\end{array}$ & 5.00 & 10.0 & 15.0 \\
\hline n-Decane & $\mathrm{CBZ}$ & Ave & 2538531 & $\begin{array}{r}53241 \\
4032325\end{array}$ & 564622 & 1171518 & 1723726 & 20.0 & $\begin{array}{r}0.500 \\
40.0\end{array}$ & 5.00 & 10.0 & 15.0 \\
\hline 4-Ethyltoluene & $\mathrm{CBZ}$ & Ave & $\begin{array}{r}54282 \\
6921574\end{array}$ & $\begin{array}{r}132948 \\
11899547\end{array}$ & 1340266 & 2947275 & 4500371 & $\begin{array}{r}0.200 \\
20.0\end{array}$ & $\begin{array}{r}0.500 \\
40.0\end{array}$ & 5.00 & 10.0 & 15.0 \\
\hline 2-Chlorotoluene & CBZ & Ave & $\begin{array}{r}55578 \\
6081121\end{array}$ & $\begin{array}{r}138302 \\
10435157\end{array}$ & 1258300 & 2647243 & 3991987 & $\begin{array}{r}0.200 \\
20.0\end{array}$ & $\begin{array}{r}0.500 \\
40.0\end{array}$ & 5.00 & 10.0 & 15.0 \\
\hline $1,3,5$-Trimethylbenzene & $\mathrm{CBZ}$ & Ave & $\begin{array}{r}44272 \\
5696713 \\
\end{array}$ & $\begin{array}{r}117959 \\
9903377\end{array}$ & 1106738 & 2547264 & 3670194 & $\begin{array}{r}0.200 \\
20.0\end{array}$ & $\begin{array}{r}0.500 \\
40.0\end{array}$ & 5.00 & 10.0 & 15.0 \\
\hline tert-Butylbenzene & $\overline{\mathrm{CBZ}}$ & Ave & $\begin{array}{r}46762 \\
5763621\end{array}$ & $\begin{array}{r}112968 \\
9871967\end{array}$ & 1076466 & 2371323 & 3531374 & $\begin{array}{r}0.200 \\
20.0\end{array}$ & $\begin{array}{r}0.500 \\
40.0\end{array}$ & 5.00 & 10.0 & 15.0 \\
\hline $1,2,4$-Trimethylbenzene & $\overline{\mathrm{CBZ}}$ & Ave & $\begin{array}{r}42053 \\
5768081\end{array}$ & $\begin{array}{r}123966 \\
10033554\end{array}$ & 1119283 & 2481900 & 3750619 & $\begin{array}{r}0.200 \\
20.0\end{array}$ & $\begin{array}{r}0.500 \\
40.0\end{array}$ & 5.00 & 10.0 & 15.0 \\
\hline sec-Butylbenzene & $\mathrm{CBZ}$ & Ave & $\begin{array}{r}66231 \\
8050567 \\
\end{array}$ & $\begin{array}{r}175276 \\
13990355 \\
\end{array}$ & 1762309 & 3489425 & 5321661 & $\begin{array}{r}0.200 \\
20.0 \\
\end{array}$ & $\begin{array}{r}0.500 \\
40.0 \\
\end{array}$ & 5.00 & 10.0 & 15.0 \\
\hline 4-Isopropyltoluene & $\mathrm{CBZ}$ & Ave & $\begin{array}{r}48064 \\
7048124 \\
\end{array}$ & $\begin{array}{r}128391 \\
12320506 \\
\end{array}$ & 1423375 & 2952439 & 4608248 & $\begin{array}{r}0.200 \\
20.0 \\
\end{array}$ & $\begin{array}{r}0.500 \\
40.0 \\
\end{array}$ & 5.00 & 10.0 & 15.0 \\
\hline
\end{tabular}

FORM VI TO-15

Page 79 of 97 
FORM VI

AIR - GC/MS VOA INITIAL CALIBRATION DATA

INTERNAL STANDARD RESPONSE AND CONCENTRATION

Lab Name: TestAmerica Burlington

Job No.: 200-1136-1

Analy Batch No.: 5008

SDG No.: MORRILL (200-1136)

Instrument ID: G.i

GC Column: RTX- 524

ID: $0.32(\mathrm{~mm})$

Calibration Start Date: 08/09/2010 15:59

Calibration End Date: 08/09/2010 20:50

Heated Purge: ( $\mathrm{Y} / \mathrm{N}) \mathrm{N}$

\begin{tabular}{|c|c|c|c|c|c|c|c|c|c|c|c|c|}
\hline \multirow[t]{2}{*}{ ANALYTE } & \multirow{2}{*}{$\begin{array}{c}\text { IS } \\
\text { REF }\end{array}$} & \multirow{2}{*}{$\begin{array}{l}\text { CURVE } \\
\text { TYPE }\end{array}$} & \multicolumn{5}{|c|}{ RESPONSE } & \multicolumn{5}{|c|}{ CONCENTRATION (PPB V/V) } \\
\hline & & & $\begin{array}{ll}\text { LVL } 1 \\
\text { LVL } 6 \\
\end{array}$ & $\begin{array}{l}\text { LVL } 2 \\
\text { LVL } 7 \\
\end{array}$ & LVL 3 & LVL 4 & LVL 5 & $\begin{array}{l}\text { LVL } 1 \\
\text { LVL } 6 \\
\end{array}$ & $\begin{array}{l}\text { LVL } 2 \\
\text { LVL } 7\end{array}$ & LVL 3 & LVL 4 & LVL 5 \\
\hline 1,3-Dichlorobenzene & $\mathrm{CBZ}$ & Ave & $\begin{array}{r}38190 \\
4826587 \\
\end{array}$ & $\begin{array}{r}97457 \\
8821500 \\
\end{array}$ & 966869 & 1962812 & 3423926 & $\begin{array}{r}0.200 \\
20.0\end{array}$ & $\begin{array}{r}0.500 \\
40.0 \\
\end{array}$ & 5.00 & 10.0 & 15.0 \\
\hline 1,4-Dichlorobenzene & CBZ & Ave & $\begin{array}{r}36624 \\
4864802 \\
\end{array}$ & $\begin{array}{r}93320 \\
8808761 \\
\end{array}$ & 947181 & 1936682 & 3271592 & $\begin{array}{r}0.200 \\
20.0\end{array}$ & $\begin{array}{r}0.500 \\
40.0 \\
\end{array}$ & 5.00 & 10.0 & 15.0 \\
\hline Benzyl chloride & $\mathrm{CBZ}$ & Ave & $\begin{array}{r}30356 \\
4755150 \\
\end{array}$ & $\begin{array}{r}81252 \\
8842627 \\
\end{array}$ & 925377 & 1732019 & 3032104 & $\begin{array}{r}0.200 \\
20.0 \\
\end{array}$ & $\begin{array}{r}0.500 \\
40.0 \\
\end{array}$ & 5.00 & 10.0 & 15.0 \\
\hline n-Undecane & $\mathrm{CBZ}$ & Ave & 2467773 & 4024884 & 451232 & 976238 & 1402872 & 20.0 & 40.0 & 5.00 & 10.0 & 15.0 \\
\hline n-Butylbenzene & $\mathrm{CBZ}$ & Ave & $\begin{array}{r}36897 \\
5842555 \\
\end{array}$ & $\begin{array}{r}99486 \\
10011478 \\
\end{array}$ & 1177788 & 2673719 & 3796218 & $\begin{array}{r}0.200 \\
20.0\end{array}$ & $\begin{array}{r}0.500 \\
40.0\end{array}$ & 5.00 & 10.0 & $15 . \overline{0}$ \\
\hline 1,2-Dichlorobenzene & CBZ & Ave & $\begin{array}{r}33218 \\
4863070 \\
\end{array}$ & $\begin{array}{r}89755 \\
8443166 \\
\end{array}$ & 889915 & 2039877 & 3240270 & $\begin{array}{r}0.200 \\
20.0\end{array}$ & $\begin{array}{r}0.500 \\
40.0\end{array}$ & 5.00 & 10.0 & 15.0 \\
\hline n-Dodecane & CBZ & Ave & 1479930 & 1513689 & 310750 & 544872 & 1111986 & 20.0 & 40.0 & 5.00 & 10.0 & 15.0 \\
\hline 1,2,4-Trichlorobenzene & $\mathrm{CBZ}$ & Ave & 3746900 & $\begin{array}{r}40156 \\
5330236\end{array}$ & 430200 & 1271037 & 2173173 & 20.0 & $\begin{array}{r}0.500 \\
40.0\end{array}$ & 5.00 & 10.0 & 15.0 \\
\hline Hexachlorobutadiene & $\overline{C B Z}$ & Ave & $\begin{array}{r}19700 \\
3955785 \\
\end{array}$ & $\begin{array}{r}60498 \\
4710158 \\
\end{array}$ & 615968 & 1483034 & 2385965 & $\begin{array}{r}0.200 \\
20.0\end{array}$ & $\begin{array}{r}0.500 \\
40.0\end{array}$ & 5.00 & 10.0 & 15.0 \\
\hline Naphthalene & $\mathrm{CBZ}$ & Ave & 6252764 & $\begin{array}{r}68336 \\
9254416 \\
\end{array}$ & 802489 & 1834833 & 3846129 & 20.0 & $\begin{array}{r}0.500 \\
40.0\end{array}$ & 5.00 & 10.0 & 15.0 \\
\hline 1,2,3-Trichlorobenzene & $\overline{\mathrm{CBZ}}$ & Ave & $\begin{array}{r}19206 \\
3085488 \\
\end{array}$ & $\begin{array}{r}33817 \\
3338330 \\
\end{array}$ & 357812 & 839519 & 1826099 & $\begin{array}{r}0.200 \\
20.0 \\
\end{array}$ & $\begin{array}{r}0.500 \\
40.0 \\
\end{array}$ & 5.00 & 10.0 & 15.0 \\
\hline
\end{tabular}

Curve Type Legend:

Ave = Average ISTD 
FORM VII

AIR - GC/MS VOA CONTINUING CALIBRATION DATA

Lab Name: TestAmerica Burlington

SDG NO.: MORRILL $(200-1136)$

Lab Sample ID: ICV 200-5008/11

Instrument ID: G.i

GC Column: RTX-624

ID: $0.32(\mathrm{~mm})$

Lab File ID: geu011.d
Job No.: 200-1136-1

Calibration Date: 08/09/2010 22:27

Calib Start Date: 08/09/2010 15:59

Calib End Date: 08/09/2010 20:50

Conc. Units: $\mathrm{ppb} v / \mathrm{v}$ Heated Purge: (Y/N) N

\begin{tabular}{|c|c|c|c|c|c|c|c|c|}
\hline ANAIYTE & $\begin{array}{l}\text { CURVE } \\
\text { TYPE }\end{array}$ & AVE RRE & RRE & MIN RRF & $\begin{array}{c}\text { CALC } \\
\text { AMOUNT }\end{array}$ & $\begin{array}{c}\text { SPIKE } \\
\text { AMOUNT }\end{array}$ & $\stackrel{\circ}{\circ} \mathrm{D}$ & $\begin{array}{l}\text { MAX } \\
\because D\end{array}$ \\
\hline Propylene & Ave & 0.1935 & 0.1863 & & 9.63 & 10.0 & -3.7 & 30.0 \\
\hline Dichlorodifluoromethane & Ave & 1.480 & 1.427 & & 9.64 & 10.0 & -3.6 & 30.0 \\
\hline Freon 22 & Ave & 0.5773 & 0.5560 & & 9.63 & 10.0 & -3.7 & 30.0 \\
\hline $\begin{array}{l}\text { 1,2-Dichlorotetrafluoroethan } \\
\text { e }\end{array}$ & Ave & 1.435 & 1.369 & & 9.54 & 10.0 & -4.6 & 30.0 \\
\hline Chloromethane & Ave & 0.3216 & 0.3127 & & 9.72 & 10.0 & -2.8 & 30.0 \\
\hline n-Butane & Ave & 0.4938 & 0.4826 & & 9.77 & 10.0 & -2.3 & 30.0 \\
\hline Vinyl chloride & Ave & 0.4799 & 0.4756 & & 9.91 & 10.0 & -0.9 & 30.0 \\
\hline 1,3-Butadiene & Ave & 0.3343 & 0.3331 & & 9.96 & 10.0 & -0.4 & 30.0 \\
\hline Bromomethane & Ave & 0.9202 & 0.9007 & & 9.79 & 10.0 & -2.1 & 30.0 \\
\hline Chloroethane & Ave & 0.3421 & 0.2980 & & 8.71 & 10.0 & -12.9 & 30.0 \\
\hline Isopentane & Ave & 0.6252 & 0.5759 & & 9.21 & 10.0 & -7.9 & 30.0 \\
\hline Bromoethene (Vinyl Bromide) & Ave & 0.9908 & 0.9833 & & 9.92 & 10.0 & -0.8 & 30.0 \\
\hline Trichlorofluoromethane & Ave & 2.480 & 2.238 & & 9.02 & 10.0 & -9.8 & 30.0 \\
\hline n-Pentane & Ave & 1.083 & 0.9465 & & 8.74 & 10.0 & -12.6 & 30.0 \\
\hline Ethanol & Ave & 0.2376 & 0.1887 & & 11.9 & 15.0 & -20.6 & 30.0 \\
\hline Ethyl ether & Ave & 0.4504 & 0.3890 & & 8.63 & 10.0 & -13.6 & 30.0 \\
\hline Acrolein & Ave & 0.2319 & 0.1850 & & 7.98 & 10.0 & -20.2 & 30.0 \\
\hline Freon TF & Ave & 1.838 & 1.802 & & 9.80 & 10.0 & -2.0 & 30.0 \\
\hline 1,1-Dichloroethene & Ave & 0.8725 & 0.8445 & & 9.68 & 10.0 & -3.2 & 30.0 \\
\hline Acetone & Ave & 0.8361 & 0.8237 & & 9.85 & 10.0 & -1.5 & 30.0 \\
\hline Isopropyl alcohol & Ave & 0.6501 & 0.5517 & & 8.48 & 10.0 & -15.1 & 30.0 \\
\hline Carbon disulfide & Ave & 2.581 & 2.394 & & 9.27 & 10.0 & -7.3 & 30.0 \\
\hline 3-Chloropropene & Ave & 0.7537 & 0.6899 & & 9.15 & 10.0 & -8.5 & 30.0 \\
\hline Acetonitrile & Ave & 0.3939 & 0.3621 & & 9.19 & 10.0 & -8.1 & 30.0 \\
\hline Methylene Chloride & Ave & 0.7887 & 0.7575 & & 9.60 & 10.0 & -4.0 & 30.0 \\
\hline tert-Butyl alcohol & Ave & 1.007 & 0.8895 & & 8.83 & 10.0 & -11.7 & 30.0 \\
\hline Methyl tert-butyl ether & Ave & 1.904 & 1.808 & & 9.49 & 10.0 & -5.0 & 30.0 \\
\hline trans-1,2-Dichloroethene & Ave & 1.133 & 1.067 & & 9.42 & 10.0 & -5.8 & 30.0 \\
\hline n-Hexane & Ave & 1.059 & 0.996 & & 9.41 & 10.0 & -5.9 & 30.0 \\
\hline 1,1-Dichloroethane & Ave & 1.418 & 1.350 & & 9.52 & 10.0 & -4.8 & 30.0 \\
\hline Vinyl acetate & Ave & 1.343 & 1.312 & & 9.77 & 10.0 & -2.3 & 30.0 \\
\hline cis-1,2-Dichloroethene & Ave & 0.9666 & 0.9591 & & 9.92 & 10.0 & -0.8 & 30.0 \\
\hline Methyl Ethyl Ketone & Ave & 0.3143 & 0.3087 & & 9.82 & 10.0 & -1.8 & 30.0 \\
\hline Ethyl acetate & Ave & 0.0513 & 0.0506 & & 9.86 & 10.0 & -1.4 & 30.0 \\
\hline Tetrahydrofuran & Ave & 0.1259 & 0.1170 & & 9.29 & 10.0 & -7.1 & 30.0 \\
\hline Chloroform & Ave & 1.896 & 1.786 & & 9.42 & 10.0 & -5.8 & 30.0 \\
\hline Cyclohexane & Ave & 0.2509 & 0.2376 & & 9.47 & 10.0 & -5.3 & 30.0 \\
\hline 1,1,1-Trichloroethane & Ave & 0.4746 & 0.4302 & & 9.06 & 10.0 & -9.4 & 30.0 \\
\hline Carbon tetrachloride & Ave & 0.5480 & 0.4777 & & 8.72 & 10.0 & -12.8 & 30.0 \\
\hline $2,2,4$-Trimethylpentane & Ave & 0.7622 & 0.7089 & & 9.30 & 10.0 & -7.0 & 30.0 \\
\hline
\end{tabular}


FORM VII

AIR - GC/MS VOA CONTINUING CALIBRATION DATA

Lab Name: TestAmerica Burlington

SDG No.: MORRILL (200-1136)

Lab Sample ID: ICV 200-5008/11

Instrument ID: G.i

GC Column: RTX-624

ID: $0.32(\mathrm{~mm})$

Lab File ID: geu011.d
Job No.: 200-1136-1

Calibration Date: 08/09/2010 22:27

Calib Start Date: 08/09/2010 15:59

Calib End Date: 08/09/2010 20:50

Conc. Units: ppb v/v Heated Purge: ( $Y / N)$ N

\begin{tabular}{|c|c|c|c|c|c|c|c|c|}
\hline ANALYTE & $\begin{array}{l}\text { CURVE } \\
\text { TYPE }\end{array}$ & AVE RRF & RRF & MIN RRF & $\begin{array}{c}\text { CALC } \\
\text { AMOUNT }\end{array}$ & $\begin{array}{c}\text { SPIKE } \\
\text { AMOUNT }\end{array}$ & $\% \mathrm{D}$ & $\begin{array}{l}\text { MAX } \\
\% D\end{array}$ \\
\hline Benzene & Ave & 0.5760 & 0.4929 & & 8.55 & 10.0 & -14.4 & 30.0 \\
\hline 1,2-Dichloroethane & Ave & 0.2402 & 0.2043 & & 8.50 & 10.0 & -14.9 & 30.0 \\
\hline n-Heptane & Ave & 0.2546 & 0.2141 & & 8.41 & 10.0 & -15.9 & 30.0 \\
\hline Trichloroethene & Ave & 0.3042 & 0.2658 & & 8.74 & 10.0 & -12.6 & 30.0 \\
\hline 1,2-Dichloropropane & Ave & 0.1947 & 0.1717 & & 8.82 & 10.0 & -11.8 & 30.0 \\
\hline Methyl methacrylate & Ave & 0.1627 & 0.1454 & & 8.93 & 10.0 & -10.6 & 30.0 \\
\hline 1,4-Dioxane & Ave & 0.0728 & 0.0566 & & 7.77 & 10.0 & -22.3 & 30.0 \\
\hline Dibromomethane & Ave & 0.3463 & 0.3094 & & 8.93 & 10.0 & -10.7 & 30.0 \\
\hline Bromodichloromethane & Ave & 0.4788 & 0.4344 & & 9.07 & 10.0 & -9.3 & 30.0 \\
\hline cis-1,3-Dichloropropene & Ave & 0.3120 & 0.2771 & & 8.88 & 10.0 & -11.2 & 30.0 \\
\hline Methyl isobutyl ketone & Ave & 0.2430 & 0.2023 & & 8.32 & 10.0 & -16.8 & 30.0 \\
\hline n-Octane & Ave & 0.3136 & 0.2513 & & 8.01 & 10.0 & -19.9 & 30.0 \\
\hline Toluene & Ave & 0.4222 & 0.3659 & & 8.67 & 10.0 & -13.3 & 30.0 \\
\hline trans-1, 3-Dichloropropene & Ave & 0.3220 & 0.2658 & & 8.25 & 10.0 & -17.5 & 30.0 \\
\hline $1,1,2$-Trichloroethane & Ave & 0.2294 & 0.1904 & & 8.30 & 10.0 & -17.0 & 30.0 \\
\hline Tetrachloroethene & Ave & 0.4834 & 0.4506 & & 9.32 & 10.0 & -6.8 & 30.0 \\
\hline $\begin{array}{l}\text { Methyl Butyl Ketone } \\
\text { (2-Hexanone) }\end{array}$ & Ave & 0.2464 & 0.1942 & & 7.88 & 10.0 & -21.2 & 30.0 \\
\hline Dibromochloromethane & Ave & 0.5848 & 0.5498 & & 9.40 & 10.0 & -6.0 & 30.0 \\
\hline 1,2-Dibromoethane & Ave & 0.4692 & 0.4075 & & 8.68 & 10.0 & -13.2 & 30.0 \\
\hline Chlorobenzene & Ave & 0.6254 & 0.5581 & & 8.92 & 10.0 & -10.8 & 30.0 \\
\hline Ethylbenzene & Ave & 0.8411 & 0.7875 & & 9.36 & 10.0 & -6.4 & 30.0 \\
\hline n-Nonane & Ave & 0.3218 & 0.2978 & & 9.25 & 10.0 & -7.5 & 30.0 \\
\hline $\mathrm{m}, \mathrm{p}$-Xylene & Ave & 0.3654 & 0.3395 & & 18.6 & 20.0 & -7.1 & 30.0 \\
\hline Xylene, 0- & Ave & 0.3768 & 0.3284 & & 8.71 & 10.0 & -12.8 & 30.0 \\
\hline Styrene & Ave & 0.5703 & 0.5095 & & 8.93 & 10.0 & -10.7 & 30.0 \\
\hline Bromoform & Ave & 0.6175 & 0.6300 & & 10.2 & 10.0 & 2.0 & 30.0 \\
\hline Cumene & Ave & 0.9947 & 0.9157 & & 9.20 & 10.0 & -7.9 & 30.0 \\
\hline $1,1,2,2$-Tetrachloroethane & Ave & 0.5696 & 0.4644 & & 8.15 & 10.0 & -18.5 & 30.0 \\
\hline n-Propylbenzene & Ave & 1.140 & 1.056 & & 9.26 & 10.0 & -7.4 & 30.0 \\
\hline 1,2,3-Trichloropropane & Ave & 0.3824 & 0.3329 & & 8.70 & 10.0 & -12.9 & 30.0 \\
\hline n-Decane & Ave & 0.3885 & 0.3578 & & 9.21 & 10.0 & -7.9 & 30.0 \\
\hline 4-Ethyltoluene & Ave & 1.001 & 0.9458 & & 9.44 & 10.0 & -5.6 & 30.0 \\
\hline 2-Chlorotoluene & Ave & $0.9 \overline{347}$ & 0.8409 & & 8.99 & 10.0 & -10.0 & 30.0 \\
\hline $1,3,5$-Trimethylbenzene & Ave & 0.8389 & 0.7536 & & 8.98 & 10.0 & -10.2 & 30.0 \\
\hline tert-Butylbenzene & Ave & 0.8247 & 0.7616 & & 9.23 & 10.0 & -7.7 & 30.0 \\
\hline $1,2,4$-Trimethylbenzene & Ave & 0.8434 & 0.7302 & & 8.66 & 10.0 & -13.4 & 30.0 \\
\hline sec-Butylbenzene & Ave & 1.223 & 1.099 & & 8.99 & 10.0 & -10.1 & 30.0 \\
\hline 4-Isopropyltoluene & Ave & 1.001 & 0.9492 & & 9.48 & 10.0 & -5.2 & 30.0 \\
\hline 1,3-Dichlorobenzene & Ave & 0.7177 & 0.6348 & & 8.84 & 10.0 & -11.5 & 30.0 \\
\hline 1,4-Dichlorobenzene & Ave & 0.7021 & 0.6318 & & 9.00 & 10.0 & -10.0 & 30.0 \\
\hline
\end{tabular}


FORM VII

AIR - GC/MS VOA CONTINUING CALIBRATION DATA

Lab Name: TestAmerica Burlington

Job No.: 200-1136-1

SDG No.: MORRILL (200-1136)

Lab Sample ID: ICV 200-5008/11

Calibration Date: 08/09/2010 22:27

Instrument ID: G.i

GC Column: RTX-624

ID $: 0.32(\mathrm{~mm})$

Lab File ID: geu011.d

Calib Start Date: 08/09/2010 15:59

Calib End Date: 08/09/2010 20:50

Conc. Units: ppb v/v Heated Purge: (Y/N) N

\begin{tabular}{|c|c|c|c|c|c|c|c|c|}
\hline ANALYTE & $\begin{array}{l}\text { CURVE } \\
\text { TYPE }\end{array}$ & AVE RRE & RRF & MIN RRF & $\begin{array}{c}\text { CALC } \\
\text { AMOUNT }\end{array}$ & $\begin{array}{l}\text { SPIKE } \\
\text { AMOUNT }\end{array}$ & $\because \mathrm{D}$ & $\begin{array}{l}\text { MAX } \\
\div D\end{array}$ \\
\hline Benzyl chloride & Ave & 0.6513 & 0.5193 & & 7.97 & 10.0 & -20.3 & 30.0 \\
\hline n-Undecane & Ave & 0.3403 & 0.2328 & & 6.84 & 10.0 & $-31.6^{*}$ & 30.0 \\
\hline n-Butylbenzene & Ave & 0.8232 & 0.7768 & & 9.43 & 10.0 & -5.6 & 30.0 \\
\hline 1,2-Dichlorobenzene & Ave & 0.6838 & 0.5904 & & 8.63 & 10.0 & -13.7 & 30.0 \\
\hline n-Dodecane & Ave & 0.2048 & 0.1553 & & 7.58 & 10.0 & -24.2 & 30.0 \\
\hline $1,2,4$-Trichlorobenzene & Ave & 0.4281 & 0.3247 & & 7.58 & 10.0 & -24.2 & 30.0 \\
\hline Hexachlorobutadiene & Ave & 0.4724 & 0.3811 & & 8.06 & 10.0 & -19.3 & 30.0 \\
\hline Naphthalene & Ave & 0.7224 & 0.5901 & & 8.17 & 10.0 & -18.3 & 30.0 \\
\hline $1,2,3$-Trichlorobenzene & Ave & 0.3303 & 0.2695 & & 8.16 & 10.0 & -18.4 & 30.0 \\
\hline
\end{tabular}


FORM VII

AIR - GC/MS VOA CONTINUING CALIBRATION DATA

Lab Name: TestAmerica Burlington

SDG NO.: MORRILI (200-1136)

Lab Sample ID: CCVIS 200-5449/2

Instrument ID: G.i

GC Column: RTX-624

ID: $0.32(\mathrm{~mm})$

Lab File ID: geuj002.d

\begin{tabular}{|c|c|c|c|c|c|c|c|c|}
\hline ANALYTE & $\begin{array}{l}\text { CURVE } \\
\text { TYPE }\end{array}$ & AVE RRF & RRF & MIN RRF & $\begin{array}{c}\text { CALC } \\
\text { AMOUNT }\end{array}$ & $\begin{array}{l}\text { SPIKE } \\
\text { AMOUNT }\end{array}$ & $\frac{\circ}{0} \mathrm{D}$ & $\begin{array}{l}\text { MAX } \\
\% D\end{array}$ \\
\hline Propylene & Ave & 0.1935 & 0.2931 & & 15.1 & 10.0 & $51.5 *$ & 30.0 \\
\hline Dichlorodifluoromethane & Ave & 1.480 & 1.921 & & 13.0 & 10.0 & 29.8 & 30.0 \\
\hline Freon 22 & Ave & 0.5773 & 0.7879 & & 13.6 & 10.0 & $36.5 *$ & 30.0 \\
\hline$e^{1,2-D i c h l o r o t e t r a f l u o r o e t h a n}$ & $\overline{\text { Ave }}$ & 1.435 & 1.737 & & 12.1 & 10.0 & 21.1 & 30.0 \\
\hline Chloromethane & Ave & 0.3216 & 0.4052 & & 12.6 & 10.0 & 26.0 & 30.0 \\
\hline n-Butane & Ave & 0.4938 & 0.6306 & & 12.8 & 10.0 & 27.7 & 30.0 \\
\hline Vinyl chloride & Ave & 0.4799 & 0.5518 & & 11.5 & 10.0 & 15.0 & 30.0 \\
\hline 1,3-Butadiene & Ave & 0.3343 & 0.3740 & & 11.2 & 10.0 & 11.9 & 30.0 \\
\hline Bromomethane & Ave & 0.9202 & 0.8710 & & 9.46 & 10.0 & -5.3 & 30.0 \\
\hline Chloroethane & Ave & 0.3421 & 0.3455 & & 10.1 & 10.0 & 1.0 & 30.0 \\
\hline Isopentane & Ave & 0.6252 & 0.6927 & & 11.1 & 10.0 & 10.8 & 30.0 \\
\hline Bromoethene (Vinyl Bromide) & Ave & 0.9908 & 0.8908 & & 8.99 & 10.0 & -10.1 & 30.0 \\
\hline Trichlorofluoromethane & Ave & 2.480 & 2.568 & & 10.4 & 10.0 & 3.5 & 30.0 \\
\hline n-Pentane & Ave & 1.083 & 1.180 & & 10.9 & 10.0 & 8.9 & 30.0 \\
\hline Ethanol & Ave & 0.2376 & 0.2442 & & 15.4 & 15.0 & 2.8 & 30.0 \\
\hline Ethyl ether & Ave & 0.4504 & 0.4437 & & 9.85 & 10.0 & -1.5 & 30.0 \\
\hline Acrolein & $\overline{\text { Ave }}$ & 0.2319 & 0.2216 & & 9.55 & 10.0 & -4.4 & 30.0 \\
\hline Freon $\mathrm{TF}$ & Ave & 1.838 & 1.742 & & 9.48 & 10.0 & -5.2 & 30.0 \\
\hline 1,1-Dichloroethene & Ave & 0.8725 & 0.7757 & & 8.89 & 10.0 & -11.1 & 30.0 \\
\hline Acetone & Ave & 0.8361 & 1.071 & & 12.8 & 10.0 & 28.1 & 30.0 \\
\hline Isopropyl alcohol & Ave & 0.6501 & 0.7067 & & 10.9 & 10.0 & 8.7 & 30.0 \\
\hline Carbon disulfide & Ave & 2.581 & 2.460 & & 9.53 & 10.0 & -4.7 & 30.0 \\
\hline 3-Chloropropene & Ave & 0.7537 & 0.8117 & & 10.8 & 10.0 & 7.7 & 30.0 \\
\hline Acetonitrile & Ave & 0.3939 & 0.4462 & & 11.3 & 10.0 & 13.3 & 30.0 \\
\hline Methylene Chloride & Ave & 0.7887 & 0.8296 & & 10.5 & 10.0 & 5.2 & 30.0 \\
\hline tert-Butyl alcohol & Ave & 1.007 & 1.182 & & 11.7 & 10.0 & 17.4 & 30.0 \\
\hline Methyl tert-butyl ether & Ave & 1.904 & 1.924 & & 10.1 & 10.0 & 1.1 & 30.0 \\
\hline trans-1,2-Dichloroethene & Ave & 1.133 & 1.202 & & 10.6 & 10.0 & 6.2 & 30.0 \\
\hline n-Hexane & Ave & 1.059 & 1.097 & & 10.4 & 10.0 & 3.6 & 30.0 \\
\hline 1,1-Dichloroethane & Ave & 1.418 & 1.500 & & 10.6 & 10.0 & 5.8 & 30.0 \\
\hline Vinyl acetate & Ave & 1.343 & 1.491 & & 11.1 & 10.0 & 11.0 & 30.0 \\
\hline cis-1,2-Dichloroethene & Ave & 0.9666 & 0.9207 & & 9.52 & 10.0 & -4.8 & 30.0 \\
\hline Methyl Ethyl Ketone & Ave & 0.3143 & 0.2869 & & 9.13 & 10.0 & -8.7 & 30.0 \\
\hline Ethyl acetate & Ave & 0.0513 & 0.0453 & & 8.82 & 10.0 & -11.8 & 30.0 \\
\hline Tetrahydrofuran & Ave & 0.1259 & 0.1313 & & 10.4 & 10.0 & 4.3 & 30.0 \\
\hline Chloroform & Ave & 1.896 & 2.004 & & 10.6 & 10.0 & 5.7 & 30.0 \\
\hline 1,1,1-Trichloroethane & Ave & 0.4746 & 0.5028 & & 10.6 & 10.0 & 5.9 & 30.0 \\
\hline Cyclohexane & Ave & 0.2509 & 0.2425 & & 9.66 & 10.0 & -3.4 & 30.0 \\
\hline Carbon tetrachloride & Ave & 0.5480 & 0.5647 & & 10.3 & 10.0 & 3.1 & 30.0 \\
\hline $2,2,4$-Trimethylpentane & Ave & 0.7622 & 0.8018 & & 10.5 & 10.0 & 5.2 & 30.0 \\
\hline
\end{tabular}

FORM VII TO-15
Job No.: 200-1136-1

Calibration Date: 08/21/2010 15:45

Calib Start Date: 08/09/2010 15:59

Calib End Date: 08/09/2010 20:50

Conc. Units: ppb v/v Heated Purge: ( $Y / N)$ N 
FORM VII

AIR - GC/MS VOA CONTINUING CALIBRATION DATA

Lab Name: TestAmerica Burlington

SDG No.: MORRILL (200-1136)

Lab Sample ID: CCVIS 200-5449/2

Instrument ID: G.i

GC Column: RTX-624

ID: $0.32(\mathrm{~mm})$

Lab File ID: geuj002.d

\begin{tabular}{|c|c|c|c|c|c|c|c|c|}
\hline ANALYTE & $\begin{array}{c}\text { CURVE } \\
\text { TYPE }\end{array}$ & AVE RRF & RRF & MIN RRF & $\begin{array}{c}\text { CALC } \\
\text { AMOUNT }\end{array}$ & $\begin{array}{c}\text { SPIKE } \\
\text { AMOUNT }\end{array}$ & $\because \mathrm{D}$ & $\begin{array}{l}\text { MAX } \\
\frac{\circ}{\circ} \mathrm{D}\end{array}$ \\
\hline Benzene & Ave & 0.5760 & 0.5632 & & 9.77 & 10.0 & -2.2 & 30.0 \\
\hline 1,2-Dichloroethane & Ave & 0.2402 & 0.2836 & & 11.8 & 10.0 & 18.1 & 30.0 \\
\hline n-Heptane & Ave & 0.2546 & 0.2776 & & 10.9 & 10.0 & 9.0 & 30.0 \\
\hline Trichloroethene & Ave & 0.3042 & 0.3023 & & 9.93 & 10.0 & -0.6 & 30.0 \\
\hline 1,2-Dichloropropane & Ave & 0.1947 & 0.1913 & & 9.82 & 10.0 & -1.8 & 30.0 \\
\hline Methyl methacrylate & Ave & 0.1627 & 0.1398 & & 8.59 & 10.0 & -14.1 & 30.0 \\
\hline 1,4-Dioxane & Ave & 0.0728 & 0.0660 & & 9.07 & 10.0 & -9.3 & 30.0 \\
\hline Dibromomethane & Ave & 0.3463 & 0.3007 & & 8.68 & 10.0 & -13.2 & 30.0 \\
\hline Bromodichloromethane & Ave & 0.4788 & 0.4908 & & 10.2 & 10.0 & 2.5 & 30.0 \\
\hline cis-1,3-Dichloropropene & Ave & 0.3120 & 0.3172 & & 10.2 & 10.0 & 1.7 & 30.0 \\
\hline Methyl isobutyl ketone & Ave & 0.2430 & 0.2567 & & 10.6 & 10.0 & 5.6 & 30.0 \\
\hline n-Octane & Ave & 0.3136 & 0.3485 & & 11.1 & 10.0 & 11.1 & 30.0 \\
\hline Toluene & Ave & 0.4222 & 0.4032 & & 9.55 & 10.0 & -4.5 & 30.0 \\
\hline trans-1,3-Dichloropropene & Ave & 0.3220 & 0.3226 & & 10.0 & 10.0 & 0.2 & 30.0 \\
\hline 1,1,2-Trichloroethane & Ave & 0.2294 & 0.2199 & & 9.58 & 10.0 & -4.2 & 30.0 \\
\hline Tetrachloroethene & Ave & 0.4834 & 0.4449 & & 9.20 & 10.0 & -8.0 & 30.0 \\
\hline $\begin{array}{l}\text { Methyl Butyl Ketone } \\
\text { (2-Hexanone) }\end{array}$ & Ave & 0.2464 & 0.2595 & & 10.5 & 10.0 & 5.3 & 30.0 \\
\hline Dibromochloromethane & Ave & 0.5848 & 0.5696 & & 9.74 & 10.0 & -2.6 & 30.0 \\
\hline 1,2-Dibromoethane & Ave & 0.4692 & 0.4479 & & 9.54 & 10.0 & -4.5 & 30.0 \\
\hline Chlorobenzene & Ave & 0.6254 & 0.5854 & & 9.36 & 10.0 & -6.4 & 30.0 \\
\hline Ethylbenzene & Ave & 0.8411 & 0.8564 & & 10.2 & 10.0 & 1.8 & 30.0 \\
\hline n-Nonane & Ave & 0.3218 & 0.3453 & & 10.7 & 10.0 & 7.3 & 30.0 \\
\hline $\mathrm{m}, \mathrm{p}$-Xylene & Ave & 0.3654 & 0.3477 & & 19.0 & 20.0 & -4.8 & 30.0 \\
\hline Xylene, $0^{-}$ & Ave & 0.3768 & 0.3437 & & 9.12 & 10.0 & -8.8 & 30.0 \\
\hline styrene & Ave & 0.5703 & 0.5183 & & 9.09 & 10.0 & -9.1 & 30.0 \\
\hline Bromoform & Ave & 0.6175 & 0.5809 & & 9.41 & 10.0 & -5.9 & 30.0 \\
\hline Cumene & Ave & 0.9947 & 0.9789 & & 9.84 & 10.0 & -1.6 & 30.0 \\
\hline $1,1,2,2$-Tetrachloroethane & Ave & 0.5696 & 0.5422 & & 9.52 & 10.0 & -4.8 & 30.0 \\
\hline n-Propylbenzene & Ave & 1.140 & 1.148 & & 10.1 & 10.0 & 0.6 & 30.0 \\
\hline 1,2,3-Trichloropropane & Ave & 0.3824 & 0.3883 & & 10.2 & 10.0 & 1.5 & 30.0 \\
\hline n-Decane & Ave & 0.3885 & 0.4191 & & 10.8 & 10.0 & 7.9 & 30.0 \\
\hline 4-Ethyltoluene & Ave & 1.001 & 0.9842 & & 9.83 & 10.0 & -1.7 & 30.0 \\
\hline 2-Chlorotoluene & Ave & 0.9347 & 0.9220 & & 9.86 & 10.0 & -1.4 & 30.0 \\
\hline $1,3,5$-Trimethylbenzene & Ave & 0.8389 & 0.8176 & & 9.74 & 10.0 & -2.5 & 30.0 \\
\hline tert-Butylbenzene & Ave & 0.8247 & 0.7836 & & 9.50 & 10.0 & -5.0 & 30.0 \\
\hline $1,2,4$-Trimethylbenzene & Ave & 0.8434 & 0.8168 & & 9.68 & 10.0 & -3.2 & 30.0 \\
\hline sec-Butylbenzene & Ave & 1.223 & 1.171 & & 9.57 & 10.0 & -4.3 & 30.0 \\
\hline 4-Isopropyltoluene & Ave & 1.001 & 0.9534 & & 9.52 & 10.0 & -4.8 & 30.0 \\
\hline 1,3-Dichlorobenzene & Ave & 0.7177 & 0.6316 & & 8.80 & 10.0 & -12.0 & 30.0 \\
\hline 1,4-Dichlorobenzene & Ave & 0.7021 & 0.6238 & & 8.88 & 10.0 & -11.1 & 30.0 \\
\hline
\end{tabular}

Calibration Date: 08/21/2010 15:45

Calib Start Date: 08/09/2010 15:59

Calib End Date: 08/09/2010 20:50

Conc. Units: $\mathrm{ppb} v / \mathrm{v}$ Heated Purge: ( $\mathrm{Y} / \mathrm{N}) \mathrm{N}$

AX

.0

0.0

.0

0.0

.

.0

0.0

.0

.0

.0

0.0

.0

.0

0

.0

0.0

0.0

.0

.0

30.0

30.0

30.0

0.0

0.0

30.0

30.0

30.0 
FORM VII

AIR - GC/MS VOA CONTINUING CALIBRATION DATA

Lab Name: TestAmerica Burlington

SDG NO.: MORRILL (200-1136)

Lab Sample ID: CCVIS 200-5449/2

Instrument ID: G.i

GC Column: RTX-624

Lab File. ID: geuj002.d
Job No.: $200-1136-1$

Calibration Date: 08/21/2010 15:45

Calib start Date: 08/09/2010 15:59

ID $: 0.32(\mathrm{~mm})$

Calib End Date: 08/09/2010 20:50

Conc. Units: ppb v/v Heated Purge: ( $Y / N$ ) N

\begin{tabular}{|c|c|c|c|c|c|c|c|c|}
\hline ANALYTE & $\begin{array}{l}\text { CURVE } \\
\text { TYPE }\end{array}$ & AVE RRF & RRF & MIN RRE & $\begin{array}{c}\text { CAIC } \\
\text { AMOUNT }\end{array}$ & $\begin{array}{l}\text { SPIKE } \\
\text { AMOUNT }\end{array}$ & $\because \mathrm{D}$ & $\begin{array}{l}\text { MAX } \\
\frac{\circ}{D}\end{array}$ \\
\hline Benzyl chloride & Ave & 0.6513 & 0.5090 & & 7.81 & 10.0 & -21.8 & 30.0 \\
\hline n-Undecane & Ave & 0.3403 & 0.2965 & & 8.71 & 10.0 & -12.9 & 30.0 \\
\hline n-Butylbenzene & Ave & 0.8232 & 0.8127 & & 9.87 & 10.0 & -1.3 & 30.0 \\
\hline 1,2-Dichlorobenzene & Ave & 0.6838 & 0.6015 & & 8.79 & 10.0 & -12.0 & 30.0 \\
\hline n-Dodecane & Ave & 0.2048 & 0.2283 & & 11.1 & 10.0 & 11.5 & 30.0 \\
\hline 1,2,4-Trichlorobenzene & Ave & 0.4281 & 0.3191 & & 7.45 & 10.0 & -25.5 & 30.0 \\
\hline Hexachlorobutadiene & Ave & 0.4724 & 0.4309 & & 9.12 & 10.0 & -8.8 & 30.0 \\
\hline Naphthalene & Ave & 0.7224 & 0.5890 & & 8.15 & 10.0 & -18.5 & 30.0 \\
\hline 1,2,3-Trichlorobenzene & Ave & 0.3303 & 0.2614 & & 7.91 & 10.0 & -20.8 & 30.0 \\
\hline
\end{tabular}

FORM VII TO-15 
FORM I

AIR - GC/MS VOA ORGANICS ANALYSIS DATA SHEET

Lab Name: TestAmerica Burlington

SDG NO.: MORRILL (200-1136)

Client Sample ID:

Matrix: Air

Analysis Method: TO-15

Sample wt/vol: $200(\mathrm{~mL})$

Soil Aliquot Vol:

Soil Extract Vol.:

\% Moisture:

Analysis Batch No.: 5449

\begin{tabular}{|c|l|c|c|c|c|c|}
\hline \multicolumn{1}{|c|}{ CAS NO. } & \multicolumn{1}{|c|}{ COMPOUND NAME } & $\begin{array}{c}\text { MOLECULAR } \\
\text { WEIGHT }\end{array}$ & RESULT & Q & RL & MDL \\
\hline \hline $67-66-3$ & Chloroform & 119.38 & 0.20 & $\mathrm{U}$ & 0.20 & 0.050 \\
\hline $56-23-5$ & Carbon tetrachloride & 153.81 & 0.20 & $\mathrm{U}$ & 0.20 & 0.050 \\
\hline
\end{tabular}

Job No.: 200-1136-1

Lab Sample ID: MB 200-5449/4

Lab File ID: geuj004.d

Date Collected:

Date Analyzed: 08/21/2010 17:21

Dilution Factor: 1

GC Column: RTX-624

ID: $0.32(\mathrm{~mm})$

Level: (low/med) Low

Units: $\mathrm{ppb} \mathrm{v} / \mathrm{v}$ 
FORM I

AIR - GC/MS VOA ORGANICS ANALYSIS DATA SHEET

Lab Name: TestAmerica Burlington

SDG NO.: MORRIIL (200-1136)

Client sample ID:

Matrix: Air

Analysis Method: TO-15

Sample wt/vol: $200(\mathrm{~mL})$

Soil Aliquot Vol:

Soil Extract vol.:

\% Moisture:

Analysis Batch No.: 5449

\begin{tabular}{|c|l|r|r|r|r|r|}
\hline CAS NO. & \multicolumn{1}{|c|}{ COMPOUND NAME } & $\begin{array}{c}\text { MOLECULAR } \\
\text { WEIGHT }\end{array}$ & RESULT & Q & RI & MDI \\
\hline \hline $67-66-3$ & Chloroform & 119.38 & 0.98 & $\mathrm{U}$ & 0.98 & 0.24 \\
\hline $56-23-5$ & Carbon tetrachloride & 153.81 & 1.3 & $\mathrm{U}$ & 1.3 & 0.31 \\
\hline
\end{tabular}

Job No.: 200-1136-1

Lab Sample ID: MB 200-5449/4

Lab File ID: geuj004.d

Date Collected:

Date Analyzed: 08/21/2010 17:21

Dilution Factor: 1

GC Column: RTX-624

ID: $0.32(\mathrm{~mm})$

Level: (low/med) Low

Units: ug/m3 
FORM I

AIR - GC/MS VOA ORGANICS ANALYSIS DATA SHEET

Lab Name: TestAmerica Burlington

SDG No.: MORRILL (200-1136)

Client sample ID:

Matrix: Air

Analysis Method: TO-15

Sample wt/vol: $200(\mathrm{~mL})$

Soil Aliquot vol:

Soil Extract vol.:

․ㅡㅁ Moisture:

Analysis Batch No.: 5449
Job No.: 200-1136-1

Lab Sample ID: LCS 200-5449/3

Lab File ID: geuj003.d

Date Collected:

Date Analyzed: 08/21/2010 16:33

Dilution Factor: 1

GC Column: RTX-624

ID: $0.32(\mathrm{~mm})$

Level: (low/med) Low

Units: ppb $\mathrm{v} / \mathrm{v}$

\begin{tabular}{|c|l|c|c|c|c|c|}
\hline CAS NO. & \multicolumn{1}{|c|}{ COMPOUND NAME } & $\begin{array}{c}\text { MOIECULAR } \\
\text { WEIGHT }\end{array}$ & RESULT & Q & RI & MDL \\
\hline $67-66-3$ & Chloroform & 119.38 & 10.9 & & 0.20 & 0.050 \\
\hline $56-23-5$ & Carbon tetrachloride & 153.81 & 10.4 & & 0.20 & 0.050 \\
\hline
\end{tabular}


Lab Name: TestAmerica Burlington

SDG No.: MORRILL (200-1136)

Instrument ID: G.i

Analysis Batch Number: 5008
Job No.: 200-1136-1

Start Date: 08/09/2010 14:23

End Date: 08/10/2010 13:09

\begin{tabular}{|c|c|c|c|c|c|}
\hline LAB SAMPLE ID & CLIENT SAMPLE ID & DATE ANALYZED & $\begin{array}{c}\text { DILUTION } \\
\text { FACTOR }\end{array}$ & LAB FILE ID & COLUMN ID \\
\hline BFB $200-5008 / 1$ & & $08 / 09 / 2010 \quad 14: 23$ & 1 & geu001.d & $\begin{array}{ll}\text { RTX-624 } & 0.32(\mathrm{~mm})\end{array}$ \\
\hline VIBLK $200-5008 / 2$ & & $08 / 09 / 2010 \quad 15: 10$ & 1 & & $\mathrm{RTX}-624 \quad 0.32(\mathrm{~mm})$ \\
\hline IC $200-5008 / 3$ & & $08 / 09 / 2010 \quad 15: 59$ & 1 & geu003.d & $\begin{array}{ll}\mathrm{RTX}-624 & 0.32(\mathrm{~mm})\end{array}$ \\
\hline IC $200-5008 / 4$ & & $08 / 09 / 2010 \quad 16: 47$ & 1 & geu004.d & $\mathrm{RTX}-6240.32(\mathrm{~mm})$ \\
\hline IC $200-5008 / 5$ & & $08 / 09 / 2010$ & 1 & geu005.d & RTX-624 $0.32(\mathrm{~mm})$ \\
\hline ICIS $200-5008 / 6$ & & $08 / 09 / 2010 \quad 18: 24$ & 1 & geu006.d & RTX-624 $0.32(\mathrm{~mm})$ \\
\hline IC $200-5008 / 7$ & & $08 / 09 / 2010 \quad 19: 12$ & 1 & geu007.d & RTX-624 $0.32(\mathrm{~mm})$ \\
\hline IC $200-5008 / 8$ & & $08 / 09 / 2010 \quad 20: 01$ & 1 & geu008.d & $\mathrm{RTX}-6240.32(\mathrm{~mm})$ \\
\hline IC $200-5008 / 9$ & & $08 / 09 / 2010$ & 1 & geu009.d & RTX-624 $0.32(\mathrm{~mm})$ \\
\hline VIBLK $200-5008 / 10$ & & $08 / 09 / 2010 \quad 21: 38$ & 1 & & $\mathrm{RTX}-6240.32(\mathrm{~mm})$ \\
\hline ICV $200-5008 / 11$ & & $08 / 09 / 2010$ & 1 & geu011.d & RTX-624 $0.32(\mathrm{~mm})$ \\
\hline ZZZZZ & & $08 / 09 / 2010 \quad 23: 15$ & 1 & & $\begin{array}{lll}\text { RTX }-624 & 0.32(\mathrm{~mm})\end{array}$ \\
\hline $\mathrm{ZZZZZ}$ & & $08 / 10 / 2010$ & 1 & & $\begin{array}{lll}\text { RTX-624 } & 0.32(\mathrm{~mm})\end{array}$ \\
\hline $\mathrm{ZZZZZ}$ & & $08 / 10 / 2010 \quad 00: 52$ & 1 & & RTX-624 $0.32(\mathrm{~mm})$ \\
\hline $\mathrm{ZZZZZ}$ & & $08 / 10 / 2010$ & 1 & & $\begin{array}{ll}\mathrm{RTX}-624 & 0.32(\mathrm{~mm})\end{array}$ \\
\hline$\overline{Z Z Z Z Z}$ & & $08 / 10 / 2010 \quad 02: 30$ & 1 & & $\mathrm{RTX}-624 \quad 0.32(\mathrm{~mm})$ \\
\hline ZZZZZ & & $08 / 10 / 2010$ & 1 & & RTX-624 $0.32(\mathrm{~mm})$ \\
\hline$\overline{Z Z Z Z}$ & & $08 / 10 / 2010$ & 1 & & RTX-624 $0.32(\mathrm{~mm})$ \\
\hline $\mathrm{ZZZZZ}$ & & $08 / 10 / 2010$ & 1 & & $\mathrm{RTX}-624 \quad 0.32(\mathrm{~mm})$ \\
\hline $2 \mathrm{ZZZZ}$ & & $08 / 10 / 2010$ & 1 & & $\mathrm{RTX}-624 \quad 0.32(\mathrm{~mm})$ \\
\hline ZZZZZ & & $08 / 10 / 2010$ & 1 & & RTX-624 $0.32(\mathrm{~mm})$ \\
\hline$\overline{\mathrm{ZZZZZ}}$ & & $08 / 10 / 2010$ & 10 & & RTX-624 $0.32(\mathrm{~mm})$ \\
\hline $\mathrm{ZZZZZ}$ & & $08 / 10 / 2010$ & 10 & & RTX-624 $0.32(\mathrm{~mm})$ \\
\hline $\mathrm{ZZZZZ}$ & & $08 / 10 / 2010$ & 10 & & RTX-624 0.32 (mm) \\
\hline
\end{tabular}

TO-15 
Lab Name: TestAmerica Burlington

SDG NO.: MORRILL (200-1136)

Instrument ID: G.i

Analysis Batch Number: 5449
Job No.: 200-1136-1

Start Date: 08/21/2010 14:59

End Date: 08/22/2010 09:28

\begin{tabular}{|c|c|c|c|c|c|c|}
\hline LAB SAMPLE ID & CLIENT SAMPLE ID & DATE ANA. & YZED & $\begin{array}{c}\text { DILUTION } \\
\text { FACTOR }\end{array}$ & LAB FILE ID & COLUMN ID \\
\hline BFB 200-5449/1 & & $08 / 21 / 2010$ & $14: 59$ & 1 & geuj001.d & RTX-624 $0.32(\mathrm{~mm})$ \\
\hline CCVIS $200-5449 / 2$ & & $08 / 21 / 2010$ & $15: 45$ & 1 & geuj002.d & $\begin{array}{ll}\text { RTX-624 } & 0.32(\mathrm{~mm})\end{array}$ \\
\hline ICS $200-5449 / 3$ & & $08 / 21 / 2010$ & $16: 33$ & 1 & geuj003.d & $R T X-624 \quad 0.32(\mathrm{~mm})$ \\
\hline MB $200-5449 / 4$ & & $08 / 21 / 2010$ & $17: 21$ & 1 & geuj004.d & $\begin{array}{ll}R T X-624 & 0.32(\mathrm{~mm})\end{array}$ \\
\hline $200-1136-1$ & 104 ROXANNA ST 1ST & $08 / 21 / 2010$ & $18: 10$ & 1 & geuj005.d & $\begin{array}{lll}\text { RTX }-624 & 0.32(\mathrm{~mm})\end{array}$ \\
\hline $200-1136-2$ & $\begin{array}{l}104 \text { ROXANNA ST } \\
\text { BASEMENT }\end{array}$ & $08 / 21 / 2010$ & $18: 58$ & 1 & geuj006.d & $\begin{array}{ll}\text { RTX }-624 & 0.32(\mathrm{~mm})\end{array}$ \\
\hline $200-1136-3$ & 102 VIRGINIA ST 1 ST & $08 / 21 / 2010$ & $19: 46$ & 1 & geuj007.d & RTX-624 $0.32(\mathrm{~mm})$ \\
\hline $200-1136-4$ & $\begin{array}{l}102 \text { VIRGINIA ST } \\
\text { BASEMENT }\end{array}$ & $08 / 21 / 2010$ & $20: 35$ & 1 & geuj008.d & RTX-624 $0.32(\mathrm{~mm})$ \\
\hline $200-1136-5$ & 202 ROXANNA ST 1 ST & $08 / 21 / 2010$ & $21: 23$ & 1 & geuj009.d & $\begin{array}{ll}\text { RTX-624 } & 0.32(\mathrm{~mm})\end{array}$ \\
\hline $200-1136-6$ & $\begin{array}{l}202 \text { ROXANNA ST } \\
\text { BASEMENT }\end{array}$ & $08 / 21 / 2010$ & $22: 12$ & 1 & geuj010.d & RTX-624 $0.32(\mathrm{~mm})$ \\
\hline $200-1136-7$ & 203 ROXANNA ST IST & $08 / 21 / 2010$ & $23: 00$ & 1 & geuj011.d & RTX-624 $0.32(\mathrm{~mm})$ \\
\hline $200-1136-8$ & $\begin{array}{l}107 \text { ROXANNA ST MAIN } \\
\text { ROOM }\end{array}$ & $08 / 21 / 2010$ & $23: 48$ & 1 & geuj012.d & RTX-624 $0.32(\mathrm{~mm})$ \\
\hline $200-1136-9$ & 96 VIRGINIA ST OFFICE & $08 / 22 / 2010$ & $00: 37$ & 1 & geuj013.d & RTX-624 $0.32(\mathrm{~mm})$ \\
\hline $200-1136-10$ & AMBIENT AIR MW1 & $08 / 22 / 2010$ & $01: 25$ & 1 & geuj014.d & $\begin{array}{ll}\mathrm{RTX}-624 & 0.32(\mathrm{~mm})\end{array}$ \\
\hline $200-1136-11$ & 107 ROXANNA ST OFFICE & $08 / 22 / 2010$ & $02: 13$ & 1 & geuj015.d & $\mathrm{RTX}-624 \quad 0.32(\mathrm{~mm})$ \\
\hline $200-1136-12$ & $\begin{array}{l}107 \text { ROXANNA ST KID } \\
\text { ROOM }\end{array}$ & $08 / 22 / 2010$ & $03: 01$ & 1 & geuj016.d & RTX-624 $0.32(\mathrm{~mm})$ \\
\hline $200-1136-13$ & 108 ROXANNA ST 1ST & $08 / 22 / 2010$ & $03: 50$ & 1 & geuj017.d & $\mathrm{RTX}-624 \quad 0.32(\mathrm{~mm})$ \\
\hline $200-1136-14$ & $\begin{array}{l}108 \text { ROXANNA ST } \\
\text { BASEMENT }\end{array}$ & $08 / 22 / 2010$ & $04: 38$ & 1 & geuj018.d & RTX-624 $0.32(\mathrm{~mm})$ \\
\hline $200-1136-15$ & 106 VIRGINIA ST 1 ST & $08 / 22 / 2010$ & $05: 26$ & 1 & geuj019.d & $\operatorname{RTX}-624 \quad 0.32(\mathrm{~mm})$ \\
\hline $200-1136-16$ & 105 ROXANNA ST 1ST & $08 / 22 / 2010$ & $06: 14$ & 1 & geuj020.d & $\operatorname{RTX}-624 \quad 0.32(\mathrm{~mm})$ \\
\hline ZZZZZ & & $08 / 22 / 2010$ & $07: 02$ & 1 & & $\mathrm{RTX}-624 \quad 0.32(\mathrm{~mm})$ \\
\hline ZZZZZ & & $08 / 22 / 2010$ & $07: 51$ & 1 & & $\mathrm{RTX}-624 \quad 0.32(\mathrm{~mm})$ \\
\hline $\mathrm{zZZZZ}$ & & $08 / 22 / 2010$ & $08: 40$ & 1 & & $\begin{array}{ll}\mathrm{RTX}-624 & 0.32(\mathrm{~mm})\end{array}$ \\
\hline $\mathrm{ZZZZZ}$ & & $08 / 22 / 2010$ & $09: 28$ & 1 & & $\mathrm{RTX}-624 \quad 0.32(\mathrm{~mm})$ \\
\hline
\end{tabular}


GC/MS INSTRUMENT RUN LOG

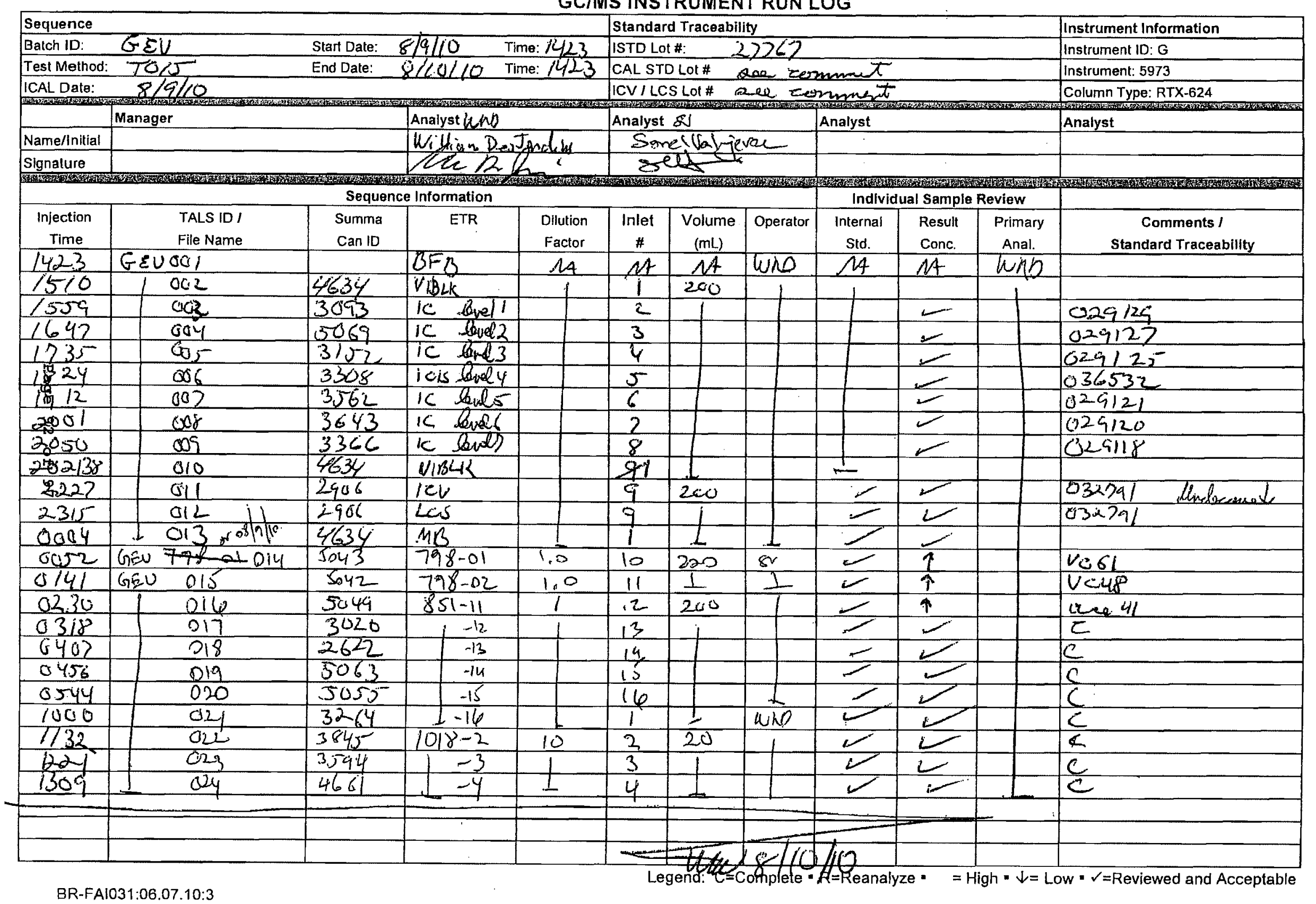


GC/MS INSTRUMENT RUN LOG

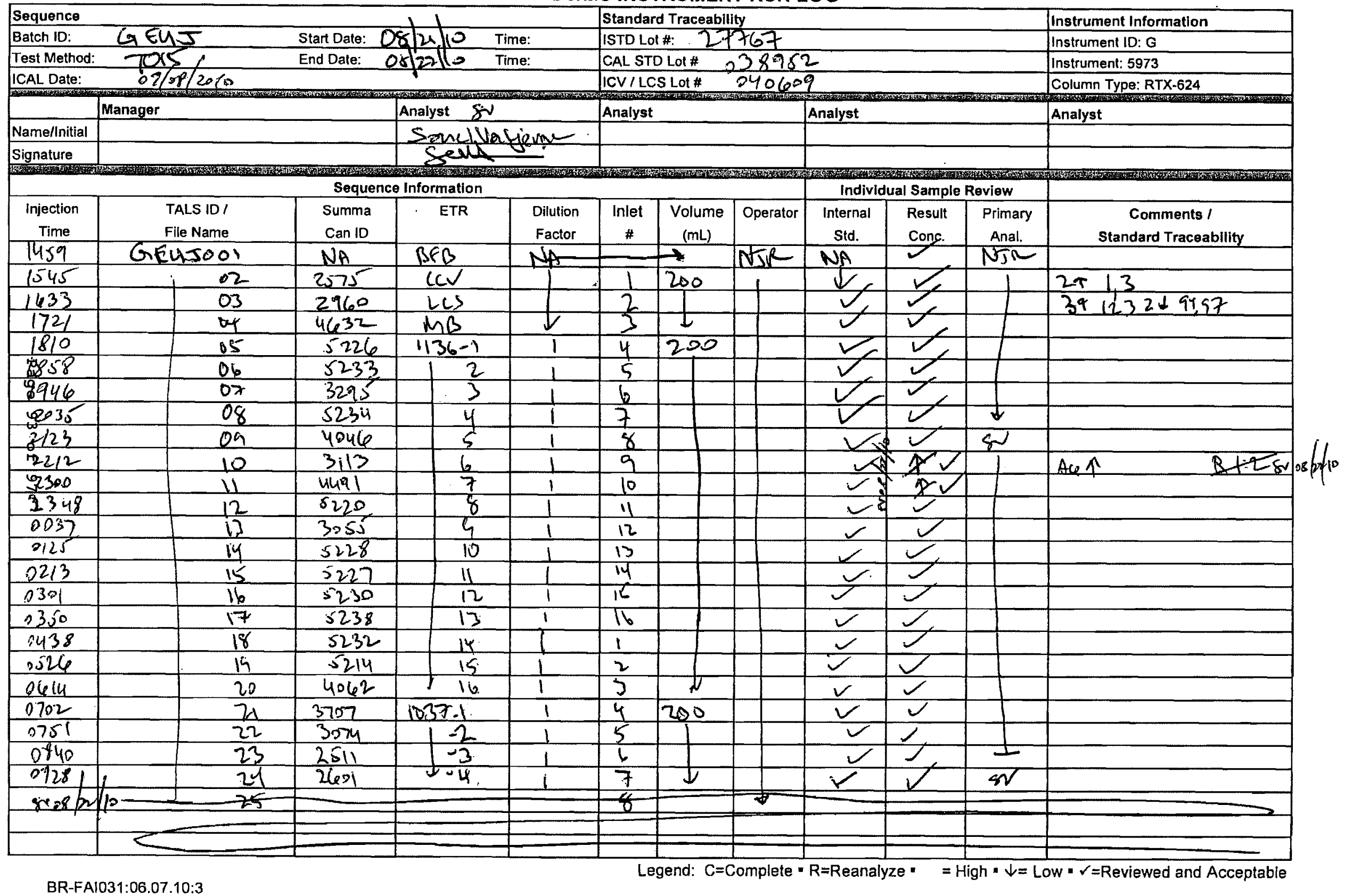




\section{Shipping and Receiving Documents}




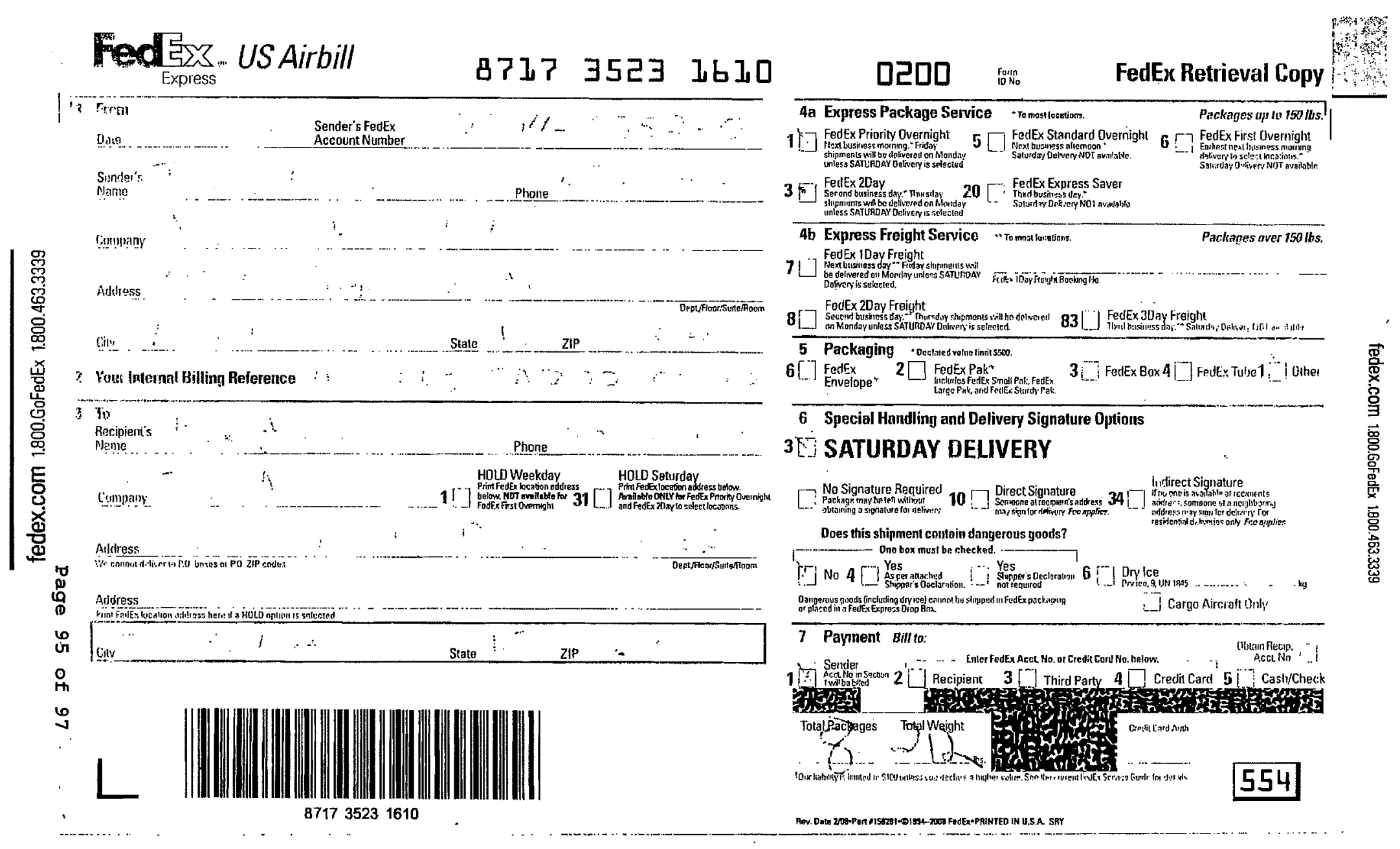




\section{Login Sample Receipt Check List}

Client: Argonne National Laboratory

Job Number: 200-1136-1

SDG Number: MORRILL (200-1136)

Login Number: 1136

List Source: TestAmerica Burlington

Creator: Kolb, Chris $M$

List Number: 1

Question

$T / F / N A$ Comment

Radioactivity either was not measured or, if measured, is at or belovil/A background

The cooler's custody seal, if present, is intact.

N/A

The cooler or samples do not appear to have been compromised orTrue tampered with.

Samples were received on ice. N/A

Cooler Temperature is acceptable. True

Cooler Temperature is recorded. True

$\mathrm{COC}$ is present. True

COC is filled out in ink and legible. True

$\mathrm{COC}$ is filled out with all pertinent information. True

Is the Field Sampler's name present on COC? True

There are no discrepancies between the sample IDs on the containefald Minor Discrepancies the COC.

Samples are received within Holding Time. True

Sample containers have legible labels. True

Containers are not broken or leaking. True

Sample collection date/times are provided. True

Appropriate sample containers are used. True

Sample bottles are completely filled. N/A

Sample Preservation Verified N/A

There is sufficient vol. for all requested analyses, incl. any requestedTrue MS/MSDs

VOA sample vials do not have headspace or bubble is $<6 \mathrm{~mm}\left(1 / 4^{\prime \prime}\right)$ Nith diameter.

If necessary, staff have been informed of any short hold time or quikA TAT needs

Multiphasic samples are not present. $\quad$ True

Samples do not require splitting or compositing. True 
Post-Sampling Air Canister Pressure Check Record

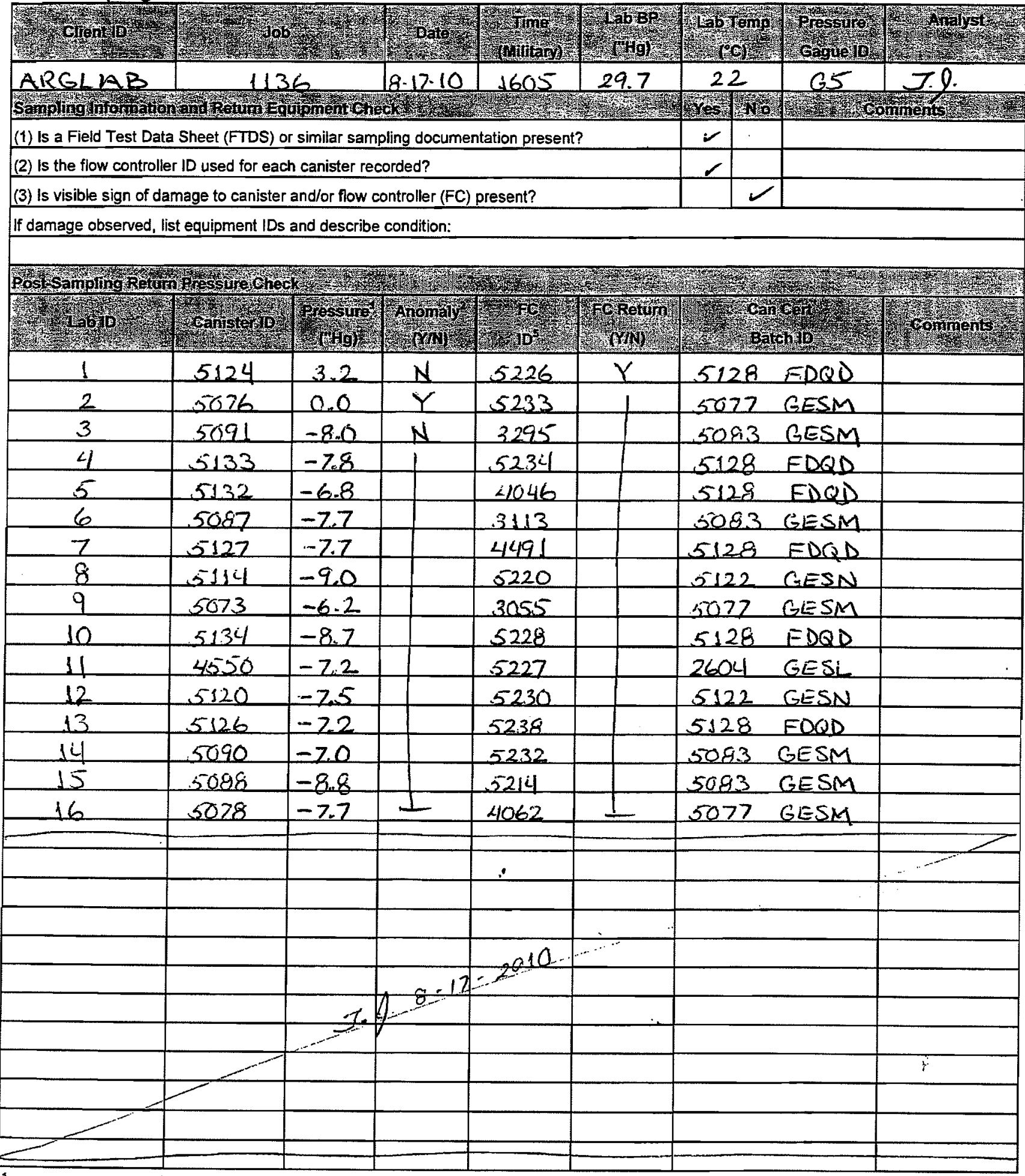

${ }^{1}$ Criteria: Return Pressure should be between -1 and -10 ("Hg)

${ }^{2}$ If return pressure is not within criteria, initiate anomaly report.

${ }^{3}$ Record the ID of the FC used for sampling if information is provided, otherwise leave blank. 
Argonne

\section{Environmental Science Division}

Argonne National Laboratory

9700 South Cass Avenue, Bldg. 203

Argonne, IL 60439-4843

www.anl.gov 